\title{
Survey of present data on photon structure functions and resolved photon processes
}

\author{
Maria Krawczyk and Andrzej Zembrzuski \\ Institute of Theoretical Physics, Warsaw University, Poland \\ and \\ Magdalena Staszel \\ Institute of Experimental Physics, Warsaw University, Poland
}

\begin{abstract}
Present data on the partonic content of the photon from LEP, TRISTAN and HERA accelerators are reviewed and the essential aspects of the underlying ideas and methods are pointed out. Results of the unpolarized photon structure

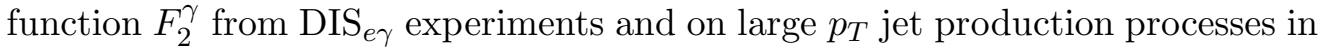
the resolved $\gamma \gamma$ collisions are presented for both real and virtual photons. The results of analysis of the hadronic final state accompanying the DIS er $_{\text {measure- }}$ ments, showing some discrepancies with the Monte Carlo models, are collected together and presented as a separate issue. Also results on the DIS er $_{\text {with }}$ leptonic final states are shown. The results from resolved real and virtual photon processes at HERA collider based on the single and double jet events, also charged particles and prompt photons, are presented. In the context of virtual photon processes the data for forward jet and forward particle production are included. In addition a short presentation of the recent data on the heavy quark content of the photon is given. Related topics - the polarized (spin dependent) structure functions for the real and virtual photon, the structure function of the electron and the photonic content of the proton are also shortly mentioned.
\end{abstract}

PACS numbers: 13.60. Fz, 12.38. Bx, 14.70. Bh

Keywords: structure functions of the photon, resolved photon processes, virtual photon, Quantum Chromodynamics 


\section{Contents}

\begin{tabular}{lll}
\hline & Introduction & 3
\end{tabular}

1.1 The concept of the "structure of the photon" . . . . . . . . . . . . . . 3

1.2 Recent measurements . . . . . . . . . . . . . . . . . . . . 4

1.3 The aim of the survey $\ldots \ldots \ldots \ldots \ldots$

1.4 The content of the survey . . . . . . . . . . . . . . . . . 6

\begin{tabular}{|lll|l|}
\hline 2 & Partonic content of the real photon & 8
\end{tabular}

2.1 Source of the real photons . . . . . . . . . . . . . . . . . . . . . . . . 9

2.2 DIS $S_{e \gamma}$ experiments . . . . . . . . . . . . . . . . . . . . . . . . . 9

2.2 .1 Theoretical description . . . . . . . . . . . . . . . . . . 10

2.2 .2 Measurements of $F_{2} \ldots \ldots \ldots \ldots \ldots$

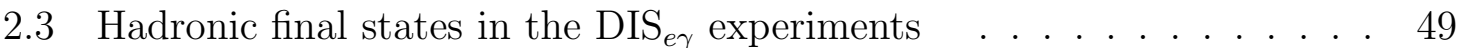

$2.3 .1 \quad$ Modelling of the hadronic final state in $\gamma^{*} \gamma$ collisions . . . . . . 49

2.3 .2 Measurements of the hadronic final state . . . . . . . . . . . 51

2.4 Large $p_{T}$ processes in $\gamma \gamma$ and $\gamma p$ collisions. Resolved photons . . . . . 77

2.4 .1 Theoretical description . . . . . . . . . . . . . 78

2.4 .2 Measurements of the resolved photon processes . . . . . . . . . 79

2.4 .3 Jet production in $\gamma \gamma$ collisions . . . . . . . . . . . . . . . . . . . 82

2.4 .4 Jet production in $\gamma p$ collisions . . . . . . . . . . . . . . . . . . . 98

2.4 .5 Prompt photon production in the resolved $\gamma p$ collision . . . . . 134

3 Partonic content of the virtual photon 140

3.1 The virtual photon-induced processes . . . . . . . . . . . . . . . . . . . 140

3.1 .1 The flux of virtual photons . . . . . . . . . . . . . . . . . . . 141

3.1 .2 The virtual photon as a (non-elementary) probe . . . . . . . . . 141

3.1 .3 The virtual photon as a target . . . . . . . . . . . . . . . . . . . 142

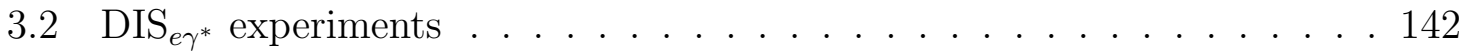

3.2.1 Theoretical description . . . . . . . . . . . . . . . . . . 143

3.2.2 Measurements of $F_{2}^{t}$. . . . . . . . . . . . . . . . . . . . . 144

3.3 Measurements of resolved virtual photon(s) processes . . . . . . . . . . 147

3.3.1 Jet production in $\gamma^{*} \gamma$ and $\gamma^{*} \gamma^{*}$ collisions . . . . . . . . . . . . . . 148

3.3 .2 Jet production in $\gamma^{*} p$ collisions . . . . . . . . . . . . . . . . . . . 148

3.4 Forward jet (particle) production in $\gamma^{*} p$ collisions and the resolved virtual photon contribution . . . . . . . . . . . . 168

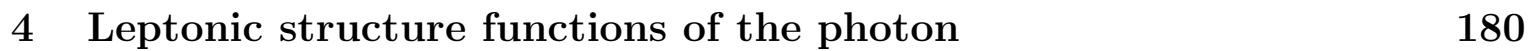

4.1 Theoretical description $\ldots \ldots \ldots \ldots$. . . . . . . . . . 180

4.2 Measurements of single and double-tag events . . . . . . . . . . . . . . 182

5 Selected results on the heavy quark content of the photon 195

$\begin{array}{lll}6 \text { Related topics } & 198\end{array}$

$6.1 \quad$ On the polarized photon structure functions . . . . . . . . . . . . . . . 198

6.2 On the structure function of the electron . . . . . . . . . . . . . . . . . 198

6.3 On the photon content of the proton . . . . . . . . . . . . . . . . . . . 198 
$\begin{array}{lll}7 \text { Summary and outlook } & 199\end{array}$

8 Appendix 201

8.1 Parton parametrizations for the real photon . . . . . . . . . . . . . 201

8.2 Parton parametrizations for the virtual photon . . . . . . . . . . . . 203

$\begin{array}{lll}9 & \text { References } & 205\end{array}$ 


\section{Introduction}

\subsection{The concept of the "structure of the photon"}

The photon is one of the basic elementary particles. This "horrendous-looking hypothesis of light-quanta" introduced in 1905 by Einstein has been based on the Planck's concept of quanta of energy dating from 1900. The photon properties, as follows from Quantum Electrodynamics (QED), are well known: the photon is a massless, chargeless object with a pointlike coupling to the charged fundamental particles. As such, it was used in the past and is still used at present as an ideal tool for probing structure of more complicated objects, for example hadrons, acting as a microscope with the resolution given by its wavelength.

The concept of "the structure of the light-quanta" which has appeared later and which is the topic of this work, makes people confused even today. However it is very natural in the quantum field theory, since a photon, as any elementary particle, can fluctuate into various states consisting of leptons, quarks, $W^{ \pm}$bosons, hadrons.... A transition to a particular observed state can be realized as a physical process due to the interaction with another object(s), for example, ... "through an interaction with a Coulomb field the photon could materialize as a pair of electrons, $\gamma \rightarrow e^{+} e^{-}$. Although not usually thought of in these terms, this phenomenon was the earliest manifestation of photon structure" [1].

Similarly the process $\gamma \rightarrow q \bar{q}$ leads to the "hadronic (partonic) structure of the photon" in the perturbative Quantum Chromodynamics (pQCD). This notion offers an effective way to describe hard and semihard processes involving photons. The hadronic properties of the photon become apparent also in the soft photon-hadron interactions, where the similarity between photon and vector mesons $\rho, \omega, \phi$ interaction is observed. Probing the "structure" of the photon can be performed in an analogous way as testing the structure of other objects - once a hard probe is at our disposal, the partonic structure can be revealed. Often the probe happens to be just another photon (..."an ideal tool"). Sometimes this leads to additional complications and even confusion when the partonic content of the photon is discussed.

Contrary to the nucleon case, the Quark-Parton Model (QPM or PM) gives the definite predictions for the hadronic structure functions $F_{1,2}^{\gamma}$ for the real photon, and for the related parton densities in the photon. They exhibit a logarithmic dependence on the $Q^{2}$ scale. The leading QCD corrections to these quantities can be summed up using the evolution equations, which, due to existence of the inhomogeneous (PM) term, can be solved in principle without any input, leading to the so called asymptotic solution. Despite this fact evolution equations are solved usually in an analogous way as for the nucleon, i.e. by introducing some experimental or model input, to cure the singular behaviour present in the asymptotic solution. The gross features of the parton densities in the photon present already in the PM, as the scaling violation and large quark densities at large $x_{B j}$, are unique for the photon (in general for gauge bosons), therefore they are treated as one of the basic tests of QCD. This holds to even larger extent for the structure functions of the virtual photon. Here the existence of the second scale, the "mass" of the virtual photon, makes it possible, at least in principle, to obtain the QCD predictions in the input-independent way. The QCD description of all basic processes induced by the real and virtual photons is now available with the 
NLO accuracy.

Basic ideas related to the considered topic are discussed in review articles, e.g. [1], [2].

\section{$1.2 \quad$ Recent measurements}

There are two basic types of inclusive processes where the structure of photon is tested in existing experiments:

- the deep inelastic scattering, DIS ${ }_{e \gamma}$ and $\mathrm{DIS}_{e \gamma^{*}}$, where the structure functions for a real and virtual photon are measured,

- the large $p_{T}$ jet (and particles) production in $\gamma p$ and $\gamma \gamma$ collisions, where individual quark and gluon densities in the photon may be probed (the so called resolved photon processes).

The early experiments of the first type were performed at PETRA and PEP $e^{+} e^{-}$ colliders almost twenty years ago, starting with the first measurement by the PLUTO Collaboration [4] in 1981. Recent results on the structure function $F_{2}^{\gamma}$, for a real $\gamma$, based on few years runs at LEP1, i.e. at the CM energy $\sim M_{Z}$, are now available in published or preliminary form. Also new data taken at LEP 1.5 (CM energy 130$136 \mathrm{GeV})$ and at LEP2 (161-172, 183, 189 and $202 \mathrm{GeV}$ ) have been or are being analysed. Final analyses appeared from the TRISTAN collider (CM energy $\sim 60$ $\mathrm{GeV}$ ). 円 Altogether the measurements cover a wide range of the (average) $Q^{2}$ from 0.2 to $\sim 390-400 \mathrm{GeV}^{2}$ a allowing to test the scaling violation pattern. The range of the $x_{B j}$ variable extends from $\sim 0.001$, where the possible rise of the structure function of the real photon can be observed, to 0.98. In earlier analyses the charm (sometimes also bottom) quark contribution to $F_{2}^{\gamma}$ was subtracted, later on it was included, although with a different treatment than for the light quarks (only the QPM term). Recently the first dedicated measurement of $F_{2, c}^{\gamma}$ has been performed at LEP2 for $0.0014<x_{B j}<0.87$ and $5<Q^{2}<100 \mathrm{GeV}^{2}$.

The measurement of the resolved real photon processes, i.e. production of large $p_{T}$ jets (also particles), started a decade later. First evidence for the resolved photon process was reported in 1992 by the AMY collaboration at TRISTAN [67]. Data have been and are still being taken in $\gamma \gamma$ collisions at the abovementioned $e^{+} e^{-}$machines and in the photoproduction processes at the $e^{ \pm} p$ collider HERA (with the CM energy $\sim 300-320 \mathrm{GeV}$ ). The latter one gave first results on the effective parton density (the light quark distributions combined with the gluon distributions) and on the gluonic content of the photon, showing the rise at small $x$, a part of the momentum of the photon taken by a parton. The probed range of $x$ is from $0.05-0.1$ to 1 , where $x$ equal to 1 corresponds to the direct interaction of the photon. The hard scale in such processes, $\tilde{Q}$, is usually given by $p_{T}$, and the range of the measured $p_{T}^{2}\left(=\tilde{Q}^{2}\right)$ extends from $\sim 20$ up to $1000 \mathrm{GeV}^{2}$. There appeared also first data on the prompt photon production, and on the $c$ and $b$ quark content of the photon, both from $e^{+} e^{-}$and $e^{ \pm} p$ collisions.

\footnotetext{
${ }^{1}$ In principle the measurement could be performed also at the SLC collider (at energy $\sim 91 \mathrm{GeV}$ ), but the luminosity is too low for a precise analysis of two-photon processes.

${ }^{2}$ The first high $Q^{2}$ results were obtained by the AMY Collaboration [5]
} 
An impressive progress has been observed during the last year in the measurements of the "structure of the virtual photon". Fifteen years after the first measurement of the $F_{2}^{\gamma^{*}}$ by PLUTO Collaboration [6], a new measurement has just been performed at LEP. A large amount of data concerning the resolved virtual photon comes from the ep collider HERA, with the virtualities of the photon spanning the range from 0.1 to $85 \mathrm{GeV}^{2}$. Here an effective parton distribution for a virtual photon has been extracted from the dijet production. Here also a new type of analysis related to the forward jet production in events with large $Q^{2}$ (DIS D -type events) is being performed, with the aim to test the underlying dynamics of the QCD radiation processes at the small $x_{B j}$ in the proton. The resolved virtual photon subprocesses contribute here as well and they should be included when describing the forward jet production.

The discrepancies between the data and Monte Carlo (MC) models in the hadronic final states in the photon-induced processes are observed by many collaborations. They are especially visible in the $\mathrm{DIS}_{e \gamma}$ experiments, where there is a need of unfolding, i.e. reconstruction of basic kinematical variable $x_{B j}$ from the visible energy of hadronic system $W$. The improvement in the unfolding has been obtained recently, still the dependence on the chosen MC used in the analysis cannot be avoided [7]. Similar situation, i.e. the lack of proper description of hadronic final state, occurs also at the ep collider HERA. It was found there that implementation of the modified transverse momenta distribution of the partons in the $\mathrm{MC}$ programs helps to describe the data. The same modified MC models have been used recently in analysis of the $e^{+} e^{-}$ data from the LEP and TRISTAN colliders. The observed discrepancies may also be a signal of a new production mechanism not included in the standard perturbative QCD analysis, as for example the multiple interaction. This mechanism, when added, usually improves description of the data, both at the $e^{+} e^{-}$and $e p$ colliders. The situation is still not clear and it enforces the advanced study of various aspects of hadron production in the DIS $_{e \gamma}$ setup (see e.g. 8, 9, 11, []) and in the photon-induced jet production [12].

\subsection{The aim of the survey}

The aim of this survey is to present our knowledge on the structure of the photon, as it stands AD 2000. We chose to use the data as a guide through the field. We collected main results from precision measurements for the unpolarized real and virtual photons? performed during last few years at LEP, TRISTAN and HERA colliderst. In order to show the status and also the progress achieved in this rapidly expanding field of research in high energy physics, we present the very recent (mainly published) results, and also some of the previous measurements playing the role of the milestones in the field.

The survey is a collection of short presentations of the various experimental data without much detail on the experimental setup. Only basic information on the type of measurements for each entry, used Monte Carlo generators and, wherever possible, comparison with the theoretical predictions are given. This allows to compare different

\footnotetext{
${ }^{3}$ So far there is no data for the polarized photon structure functions nor for the individual polarized parton densities.

4 We refer to data taken up to 1998, and papers that appeared till June 2000.

${ }^{5} \mathrm{~A}$ comprehensive collection of the earlier data can be found in [13]; for the new results see [2] h, j.
} 
results in an easy way, and also to show various attempts to improve the Monte Carlo description of the data. Although the collection of the experimental results is the main body of the survey, we found it important to point out the essential aspects of the analyses and to mention also some new theoretical ideas and future measurements.

This survey is an extension of our first attempt [2] i, which covered the data which appeared up to early 1998 when the first measurements of some quantities related to the structure of photon have appeared, and also some problems in describing data have been recognized. The present overview contains much more data, and it reports new types of analyses undertaken to solve the observed problems ( already with some positive results).

\subsection{The content of the survey}

We start with the results of measurements of the hadronic structure function $F_{2}^{\gamma}$ of the unpolarized real photons in $\mathrm{DIS}_{\text {er }}$ experiments. The results of analysis of the hadronic final state accompanying these measurements are collected together and presented as a separate issue. We discuss also results on double-tag hadronic events $\left(\right.$ DIS $\left._{e \gamma^{*}}\right)$, and single and double-tag leptonic events. The results from resolved real and virtual photon processes at HERA collider based on the single and double jet events, and in addition on charged particles and prompt photons, are presented. In the context of virtual photon processes the HERA data for forward jet and forward particle production are included. In addition a short presentation of the very recent data on the heavy quark content of the photon is given to signal the new type of measurements. Related topics - the polarized photon structure functions, the structure function of the electron and the photonic content of the proton - are also shortly mentioned. In the Appendix we give a short description of the existing parton parametrizations for the real and the virtual photon. Bibliography contains, besides references to the data we describe, references to MC generators and the comprehensive list of theoretical papers divided into groups according to the topics.

We will not discuss the total cross sections, either for real or virtual photons, nor diffraction phenomena. The data from the dedicated studies of the structure of jets and remnant jets are omitted, a compromise between the completeness of the picture and the size of the presentation.

Each of the topics presented in the survey is preceded by a short review of the basic theoretical ideas, the description of the specific - for the discussed topic - type of analysis, used MC programs, the quality of the description in terms of the $\mathrm{MC}$ and theoretical predictions, and finally the notation. (In different types of measurements and in analyses made by different groups, different notation is used. We try to keep a consistent notation throughout the survey. The double role played by the virtual photon - serving as a probe of the structure of the target, and in some cases being a target itself, which may be resolved by final large $p_{T}$ hadrons/jets, causes additional problems, e.g. $Q^{2}$ versus $P^{2}$ notation for $\mathrm{DIS}_{e p}$ events at HERA.) In each entry we present individual measurements by the short description of results together with some representative figures, as well as comments/conclusions mainly quoted from original publications. (Standard names of the Monte Carlo generators and the acronyms of the parton parametrizations are used in the descriptions, see references and Appendix, respectively.) We also give tables with the measured values of the hadronic structure 
function $F_{2}^{\gamma}$ and for parton densities for the real and virtual photon.

We denote each entry by the name of the collaboration in alphabetic order, the publication year (trying to keep the chronological order) and the reference number listed in the bibliography, and in addition by the name of the collider. The conference presentations or submitted papers are labelled with conf in the header of the entry. By adding a small letter we distinguish among the entries published or presented by the same collaboration in the same year, in the alphabetic order corresponding to their appearance in the text, e.g. DELPHI 96a. 


\section{Partonic content of the real photon}

The deep inelastic scattering on the $\gamma$, DIS $_{e \gamma}$, with the hadronic final state produced in the single-tag events, corresponding to the $\gamma^{*} \gamma$ collision at $e^{+} e^{-}$colliders, is the main source of information on the partonic content of the real photon. For the unpolarized $e^{+} e^{-}$case, the hadronic structure functions $F_{1,2}^{\gamma}$ can be introduced to describe this process, in analogous way as for the proton in the DIS exp $_{\text {experiments. }}$

Contrary to the proton case, the (Quark) Parton Model gives definite predictions for $F_{1,2}^{\gamma}$, based on the fully calculable QED process $\gamma^{*} \gamma \rightarrow q \bar{q}$. These functions, and related quark densities in the photon, depend logarithmically on $Q^{2}$. The Parton Model contribution together with QCD corrections constitute the inhomogeneous evolution equations which can be solved without any extra input, leading to the definite QCD predictions both for $x_{B j}$ and $Q^{2}$ dependence. This was why the photon structure functions were treated at the beginning as a unique test of QCD, allowing also, in principle, for an extraction of $\Lambda_{Q C D}$. The pointlike (called also asymptotic) solution obtained in this way suffers, however, from the singularities at small $x_{B j}$, calling for an additional contribution of nonperturbative (hadronic) origin. Even after proper implementation of the hadronic contribution the specific properties of $F_{2}^{\gamma}$, namely the large quark density at large $x_{B j}$ and logarithmic rise with $Q^{2}$ remain, providing a very well defined (a "must") test of QCD.

First measurements of $F_{2}^{\gamma}$ were performed at PETRA and PEP in early eighties 13. Recently new data from LEP (LEP1 with the CM energy $\sqrt{s} \sim 90 \mathrm{GeV}, \mathrm{LEP} 1.5$ with 130-136 GeV and LEP2 with 161-202 GeV) and TRISTAN (with the CM energy $58 \mathrm{GeV}$ ) $e^{+} e^{-}$colliders have appeared. They reach very small $x_{B j} \sim 10^{-3}$ (at small $Q^{2}$ ), and also very high $<Q^{2}>\sim 400 \mathrm{GeV}^{2}$ regions. In sec. 2.2 .2 we collect these results for the hadronic structure function $F_{2}^{\gamma}$ of the real photon.

In extracting $F_{2}^{\gamma}$ the unfolding of the true kinematical variables is a real challenge. Specific problems which occur here, special data analyses and related experimental results are discussed in the separate section (sec. 2.3). The contributions due to the resolved, both target and probe, photons happen to be also of relevance for proper unfolding of $F_{2}^{\gamma}$, see below. On top of it the target is always slightly virtual, which influences the extraction of $F_{2}^{\gamma}$. The problem how to correct for the offshellness of the target photon is easier to handle for a leptonic structure function $F_{2}^{\gamma(Q E D)}$, based on the process $\gamma^{*} \gamma \rightarrow l^{+} l^{-}$, see corresponding results in sec. $¥$.

The description of the heavy quark content of the photon deserves a comment. At very high scale $\left(\sim Q^{2}\right)$ the c and b-quark, or even t-quark play a role of another (massless) parton in the photon. At the present relatively low scales it is often enough to use just the (Q)PM description of the massive $c$ (and b) - quark contribution to $F_{2}^{\gamma}$. In early measurements heavy quark contributions were even subtracted from data, as they are not given by the pointlike solution of the QCD evolution equation. It is only recently that the charm part of $F_{2}^{\gamma}, F_{2, c}^{\gamma}$, was measured at LEP in the dedicated experiment. These and other very recent results on the heavy quark content of the photon are shortly commented in sec. 5 .

The other source, of growing importance, of information on the partonic content of the real photon is the hard particle(s) or jet production in $\gamma \gamma$ collision at the $e^{+} e^{-}$colliders or $\gamma p$ collision at ep HERA collider $(\sqrt{s} \sim 300-320 \mathrm{GeV})$. These hard processes were observed first at TRISTAN and HERA. Here, as in a typical hard 
hadron-hadron process, the individual partons of parent particles (e.g. two photons in $e^{+} e^{-}$or photon and proton in the photoproduction at ep collision) interact and the corresponding parton densities in the photon may be extracted from data. The collection of the results for these resolved real photon processes is presented in sec. 2.4. For corresponding results for a virtual photon see sec. 3 .

Neither the structure functions of the polarized real photon nor the corresponding resolved polarized photon processes have been measured so far; sec. 6.1 contains a short note on this topic.

\subsection{Source of the real photons}

In the present experiments there are no beams of high energy real photons. Therefore testing of the "structure" of the real photon is performed at the $e^{+} e^{-}$collisions, both in the $\mathrm{DIS}_{e \gamma}$ and the resolved $\gamma \gamma$ (with both photons real) processes, or in photoproduction at $e^{ \pm} p$ colliders. In all these cases the flux of 'initial real' photons arises from the electron or positron beams. The flux of these (almost) real photons in present experimental setups for unpolarized electron (positron) beams can be approximated by the Weizsäcker - Williams (WW, sometimes called also the Equivalent Photon Approximation, EPA) formula [14, 2]:

$$
f_{\gamma / e}(z)=\frac{\alpha}{2 \pi} \frac{1+(1-z)^{2}}{z} \ln \frac{P_{\max }^{2}}{P_{\min }^{2}}-\frac{2(1-z)}{z}\left(1-\frac{P_{\min }^{2}}{P_{\max }^{2}}\right),
$$

where $z$ denotes the fraction of the electron energy taken by the photon. $P_{\text {min }}^{2}=$ $m_{e}^{2} z^{2} /(1-z)$ with $m_{e}$ denoting the electron mass, and $P_{\max }^{2}$ stands for the maximal value of the photon virtuality appropriate for the experimental setup?. Eq. 1 gives the typical soft bremsstrahlung spectrum. Note that in $e \gamma$ or $\gamma \gamma$ options planned for the future Linear Colliders, where the real photons would be produced in the Compton backscattering process, the corresponding flux is expected to be much harder [16]. Here also a flux of photons with definite polarization can be obtained in an easy way.

\subsection{DIS $_{e \gamma}$ experiments}

In this section we consider the standard DIS $_{e \gamma}$ measurements in unpolarized $e \gamma$ collisions based on the process (fig. 四):

$$
e(k) \gamma(p) \rightarrow e\left(k^{\prime}\right) \text { hadrons }
$$

at $e^{+} e^{-}$colliders (single tagged events) [2]. Here the target is a real (in practice almost real, with $p^{2} \sim 0$ ) photon, radiated by the initial electron】.

In the $\mathrm{DIS}_{e \gamma}$ process (2) the real photon with four-momentum $p$, and the virtuality $P^{2}=-p^{2}=0$, is probed by the highly virtual photon with four-momentum $q=k-k^{\prime}$, where $k$ and $k^{\prime}$ are the four-momenta of the initial and final electron, and a large virtuality $Q^{2}=-q^{2}>0$.

\footnotetext{
${ }^{6}$ The critical discussion of this approach for the $\gamma \gamma$ processes in $e^{+} e^{-}$collisions at LEP 2 can be found in 15].

${ }^{7}$ For simplicity we will use the symbol $e$ for both electron and positron.

${ }^{8}$ The positive square of the four-momentum of the exchanged particle will be called its virtuality.
} 


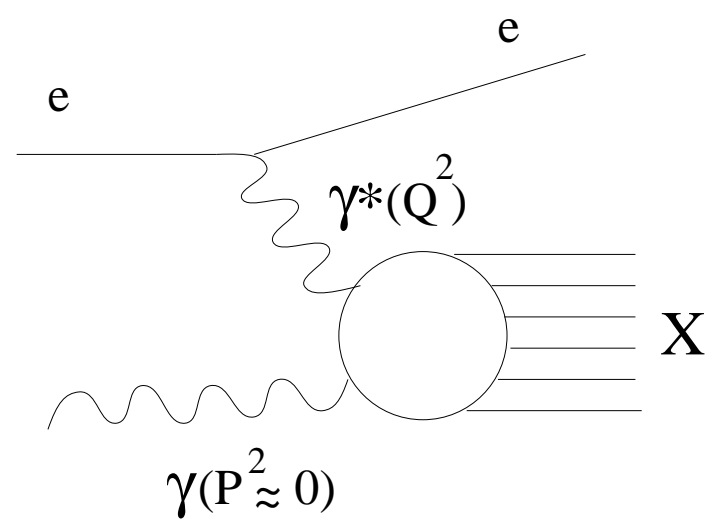

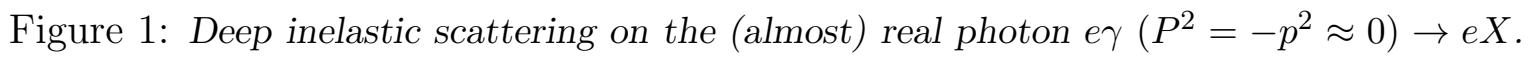
The photon probe has a large virtuality $Q^{2}=-q^{2}>0$.

The differential cross section for the process (2) is given by:

$$
\begin{array}{r}
\frac{d \sigma^{e \gamma \rightarrow e X}}{d x_{B j} d y}=\frac{4 \pi \alpha^{2}(2 p \cdot k)}{Q^{4}}\left[(1-y) F_{2}^{\gamma}+x_{B_{j}} y^{2} F_{1}^{\gamma}\right] \\
=\frac{2 \pi \alpha^{2}(2 p \cdot k)}{Q^{4}}\left[\left(1+(1-y)^{2}\right) F_{2}^{\gamma}-y^{2} F_{L}^{\gamma}\right]
\end{array}
$$

with the relation among the transverse $F_{T}^{\gamma}\left(=F_{1}^{\gamma}\right)$ and longitudinal $F_{L}^{\gamma}$ structure functions: $F_{2}^{\gamma}=F_{L}^{\gamma}+2 x_{B j} F_{1}^{\gamma}[1]$. The standard DIS variables are defined by

$$
x_{B j}=\frac{Q^{2}}{2 p \cdot q}, \quad y=\frac{p \cdot q}{p \cdot k},
$$

where $x_{B j}$ is a standard Bjorken variable, while $y$, in case of a massive target (e.g. proton) denotes the scaled energy of the exchanged photon in the target rest frame. In practice, in $D I S_{\text {er }}$ measurements the variable $y$ is small and the cross section (3, 1) is effectively saturated by $F_{2}^{\gamma}$. Note that in $e^{+} e^{-}$collisions there is always a small offshellness of the photon target, i.e. $p^{2}=-P^{2} \neq 0$, and there may appear in addition the third structure function, $F_{3}^{\gamma}$ [17]. After integration over the azimuthal angle between two scattering planes of the scattered $e^{+}$and $e^{-}$it disappears however from the cross section in the single-tag measurements, since it enters the cross section as the term $\left.F_{3}^{\gamma} \cos (2 \bar{\phi})\right)$. See sec. 3 for a discussion on virtual photon measurements where $P^{2}$ is kept far from zero. Discussion on the additional structure functions measured for a leptonic final state can be found in sec. 4 .

\subsubsection{Theoretical description}

In the (Quark) Parton Model (denoted as QPMflor PM) one assumes that the hadronic final state in eq. (2) is due to the production of quark pairs: $q_{i}$ and $\bar{q}_{i}\left(i=1,2 \ldots N_{f}\right.$, with $N_{f}$ being the number of quark flavours) with the fractional charge $Q_{i}$. The $F_{2}^{\gamma}$ is obtained by the integration over the four-momentum of the outgoing quarks with respect to the target photon direction. The full (QPM or the lowest order) expression

\footnotetext{
${ }^{9}$ Usually in QPM quarks are treated as massive.
} 
for $F_{2}^{\gamma}$, keeping the terms with quark mass $m_{q_{i}}$, is given by the Bethe-Heitler formula [2]:

$$
\begin{aligned}
& F_{2}^{\gamma}=\frac{\alpha}{\pi} N_{c} \sum_{i=1}^{N_{f}} Q_{i}^{4} x_{B j}\left[\left(-1+8 x_{B j}\left(1-x_{B j}\right)-x_{B j}\left(1-x_{B j}\right) \frac{4 m_{q_{i}}^{2}}{Q^{2}}\right) \beta\right. \\
& \left.+\left[x_{B j}^{2}+\left(1-x_{B j}\right)^{2}+x_{B j}\left(1-3 x_{B j}\right) \frac{4 m_{q_{i}}^{2}}{Q^{2}}-x_{B j}^{2} \frac{8 m_{q_{i}}^{2}}{Q^{2}}\right] \ln \frac{1+\beta}{1-\beta}\right],
\end{aligned}
$$

where the quark velocity $\beta$, in the $\gamma^{*} \gamma \mathrm{CM}$ system, is given by

$$
\beta=\sqrt{1-\frac{4 m_{q_{i}}^{2}}{s}}=\sqrt{1-\frac{4 m_{q_{i}}^{2} x_{B j}}{Q^{2}\left(1-x_{B j}\right)}} .
$$

The squared energy of the $\gamma^{*} \gamma$ collision, $s$, equal to the square of the invariant mass of the hadronic system, $W^{2}$, is given by

$$
s=W^{2}=\frac{Q^{2}}{x_{B j}}\left(1-x_{B j}\right) .
$$

In the limit of $s$ well above threshold, i.e. for $\beta \approx 1$, which for fixed $x_{B j}$ (not too small and not too close to 1) corresponds to the Bjorken limit, one gets

$$
\ln \frac{1+\beta}{1-\beta} \approx \ln \frac{Q^{2}\left(1-x_{B j}\right)}{m_{q_{i}}^{2} x_{B j}} .
$$

The structure function $F_{2}^{\gamma}$ can be approximated in such case by

$$
F_{2}^{\gamma}=\frac{\alpha}{\pi} N_{c} \sum_{i=1}^{N_{f}} Q_{i}^{4} x_{B j}\left[\left[x_{B j}^{2}+\left(1-x_{B j}\right)^{2}\right] \ln \frac{W^{2}}{m_{q_{i}}^{2}}+8 x_{B j}\left(1-x_{B j}\right)-1\right]
$$

and it can be used to define the quark densities in the photon:

$$
F_{2}^{\gamma}=x_{B j} \sum_{i=1}^{2 N_{f}} Q_{i}^{2} q_{i}^{\gamma}\left(x_{B j}, Q^{2}\right)
$$

In the above formulae $N_{c}=3$ denotes the number of colors and $Q_{i}$ - the fractional quark charge. In the last equation a (natural) assumption, that quark and antiquark distributions in photon are the same, has been introduced. Note that

- $F_{2}^{\gamma}$ is calculable in the (Q)PM, in contrast to the structure function of hadrons, e.g. the nucleon structure function $F_{2}^{N}$.

- $F_{2}^{\gamma}$ is proportional to $\alpha$, the fine structure coupling constant $(\approx 1 / 137)$. There is an overall logarithmic dependence on the energy scale squared $W^{2}$ (or $Q^{2}$, see eq. (8)). A large value of $F_{2}^{\gamma}$, or in other words a large quark density, is predicted for large $x_{B j}$.

- $F_{2}^{\gamma}$ is a sum of the quark contributions, each containing the factor $Q_{i}^{4}$, so the individual quark density is proportional to $Q_{i}^{2}$ (see eqs. (10, 11)). 
The longitudinal structure function $F_{L}^{\gamma}$ is not zero in PM, in contrast to the corresponding function for hadrons, and it is scale invariant:

$$
F_{L}^{\gamma}=\frac{\alpha}{\pi} N_{c} \sum_{i=1}^{N_{f}} Q_{i}^{4} x_{B j}\left[4 x_{B j}\left(1-x_{B j}\right)\right] .
$$

Note that also the third structure function is scale invariant:

$$
F_{3}^{\gamma}=\frac{\alpha}{\pi} N_{c} \sum_{i=1}^{N_{f}} Q_{i}^{4} x_{B j}\left[-x_{B j}^{2}\right]
$$

In the leading logarithmic approximation (LLA) the Callan-Gross relation $F_{2}^{\gamma}=$ $2 x_{B j} F_{1}^{\gamma}$ holds as in the case of hadrons.

In this approximation the PM formula for the quark density is given by

$$
\left.q_{i}^{\gamma}\left(x_{B j}, Q^{2}\right)\right|_{P M} ^{L L}=\frac{\alpha}{2 \pi} N_{c} Q_{i}^{2}\left[x_{B j}^{2}+\left(1-x_{B j}\right)^{2}\right] \ln \frac{Q^{2}}{\Lambda_{Q C D}^{2}} .
$$

Since the LL contribution corresponds to the on-shell quarks one can treat $x_{B j}$ in the above formula as equal to the part of four-momentum of the initial photon carried by the quark; this latter variable is usually denoted by $x_{\gamma}$ (or simply $x$ ). Note also that in the above formula instead of a quark mass there appears the QCD scale $\Lambda_{Q C D}$. Therefore this expression allows to describe all the light quark (light as compared to the scale $W^{2}$ ) contributions to $F_{2}^{\gamma}$ in a universal way. Heavy quark contributions should be treated separately, according to the QPM (eqs. 6 and 7), as long as $Q^{2}$ is not considerably larger than $m_{q}^{2}$ [2].

An additional $Q^{2}$ dependence will appear in $F_{2}^{\gamma}$ and further in $q_{i}^{\gamma}$ due to the QCD corrections. Here also the gluonic content of the photon, $G^{\gamma}$, appears. Large logarithmic corrections $\sim \ln Q^{2}$ can be described by the Dokshitzer-Gribov-Lipatov-AltarelliParisi (DGLAP) type of equations [25, 19] or by other techniques [2, 18, 20, 21, 22] in the leading logarithm approximation LLA, in the next-to-leading logarithm approximation NLLA, or with a higher accuracy (NNLLA,...).

The inhomogeneous DGLAP equations for the (massless) parton densities in the real photon can be represented in the following way (below $q_{i}^{\gamma}$ is used for both quarks and antiquarks):

$$
\begin{gathered}
\left.\frac{\partial q_{i}^{\gamma}\left(x, Q^{2}\right)}{\partial \ln Q^{2}}=\frac{\alpha}{2 \pi} Q_{i}^{2} P_{q \gamma}+\frac{\alpha_{s}}{2 \pi} \int_{x}^{1} \frac{d y}{y}\left[P_{q q}\left(\frac{x}{y}\right) q_{i}^{\gamma}\left(y, Q^{2}\right)\right)+P_{q g}\left(\frac{x}{y}\right) G^{\gamma}\left(y, Q^{2}\right)\right] \\
\frac{\partial G^{\gamma}\left(x, Q^{2}\right)}{\partial \ln Q^{2}}=0+\frac{\alpha_{s}}{2 \pi} \int_{x}^{1} \frac{d y}{y}\left[P_{g q}\left(\frac{x}{y}\right) \sum_{i=1}^{2 N_{f}} q_{i}^{\gamma}\left(y, Q^{2}\right)+P_{g g}\left(\frac{x}{y}\right) G^{\gamma}\left(y, Q^{2}\right)\right]
\end{gathered}
$$

with the standard splitting functions $P_{q q}, P_{q g}, P_{g q}, P_{g g}$ and in addition with the function

$$
P_{q \gamma}=N_{c}\left[x^{2}+(1-x)^{2}\right]
$$

describing the splitting of the photon into quarks (from eq. 14). 
The dominant LL $\left(Q^{2}\right)$ contribution in a general solution of the eqs. (15) (corresponding to the one-loop expressions for $\alpha_{s}$ and for the splitting functions) is coming from the strong ordering of the transverse momenta of radiated gluons along the chain between the target and the probe particles.

Note that it is possible to solve the above equations, based on the pointlike couplings only, without the initial conditions, assuming the $\operatorname{LL}\left(Q^{2}\right)$ or $\operatorname{NLL}\left(Q^{2}\right)$ behaviour of the solution [18]. Obtained in this way the, so called, asymptotic solutions, which give definite predictions for both the $x_{B j}$ and $Q^{2}$ dependence of the $q_{i}^{\gamma}, G^{\gamma}$ and $F_{2}^{\gamma}$. The logarithmic dependence of this solutions on the QCD parameter $\Lambda_{Q C D}$ would, in principle, allow to extract this basic parameter from $F_{2}^{\gamma}$ data. However these pointlike solutions have a singular behaviour at small $x$ [22], and in practice while solving the equations (14) the initial conditions have to be assumed from a model (e.g. VMD) or taken from measurements, at some (low) $Q_{0}^{2}$ scale [23. This way nonperturbative contributions enter, which regularize somehow the unphysical behaviour of the purely pointlike solution.

At this stage the parton parametrizations for the photon, can be constructed. For light quarks and a gluon the procedure is based on eqs. (15), while treatment of the heavy quarks depends on the scale. At present energies (or $Q^{2}$ scales) for $c, b$ quark contributions the QPM formula (eq. 6), with possible QCD corrections, is applied close to the threshold, see also sec. 5. In the Appendix some details of existing parton parametrizations for a real photon are given.

In pre-QCD times, the hadronic structure of the photon has been solely attributed to the vector meson component $(\rho, \omega, \phi)$ in the real photon. Therefore for the matrix element of the photon between e.g. the nucleon states, the following representation by the corresponding matrix elements for the $\rho$ meson current was assumed [24]:

$$
<N\left|J_{\mu}\right| N>=-\frac{m_{\rho}^{2}}{g_{\rho}} \frac{1}{q^{2}-m_{\rho}^{2}}<N\left|\rho_{\mu}\right| N>+\ldots
$$

That was the basic assumption of the Vector Meson Dominance (VMD) model or, if higher than $\phi$ vector meson states were included, of the Generalized VMD (GVMD) [24, [1]. Once a photon "becomes" a hadron, the cross section for $\gamma \gamma \rightarrow$ hadrons is dominated by soft exchange processes, as for any other hadron-hadron collisions (soft VMD contribution). This mechanism is also present in $\mathrm{DIS}_{e \gamma}$ case, leading to nonperturbative (or hadronic) contribution to the measured structure function $F_{2}^{\gamma}$, as well as to $q^{\gamma}$ or $G^{\gamma}$ densities.

It is clear that the VMD not only models the soft photon-induced processes, but, being built into the parton densities, it is also important in the description of the hard large $p_{T}$ processes involving resolved photons. For the general discussion of various components of the photon, with less conventional description of the photon-induced processes and the photon structure functions, see ref. [3] and the Appendix.

Parton distributions of the photon do not fulfill the, standard for hadrons, "momentum" sum rule formulated for quarks and gluons. The reason for this is that $\gamma$ can interact directly, being a parton itself. Various attempts to introduce a usefull momentum sum rule for the photon were undertaken, see [26], [162] and [169.

\footnotetext{
${ }^{10}$ denoted also VDM and GVDM
} 
Finally we would like to mention specific problems when counting the order of the perturbation (leading logarithms) in the higher order calculations for the photoninduced processes. This is already seen in eq. 6, where the QPM formula contains both LL and NLL terms, both being, however, of the QED origin. The presence of the inhomogeneous term in the evolution equations for the quark densities in the photon (15) leads to further complications in the interpretation of what is meant by the LO and the NLO QCD analysis. For a recent discussion, see [27, 135], and also comments in secs. 2.4.1 and 2.4.5.

For future discussion (sec. 3.4) let us mention other than the DGLAP approaches to the deep inelastic scattering processes (for any target). They may become important if the region of small $x_{B j}$ is tested in DIS experiments. In such case large logarithms $\sim \ln \left(1 / x_{B j}\right)$ appear, and summing them to all orders becomes necessary. The corresponding evolution equation, Balitsky-Fadin-Kuraev-Lipatov equation (BFKL), leading to LL $\left(1 / x_{B j}\right)$ terms does not rely on the strong ordering in the transverse momenta along the chain, allowing for the parton emission with large transverse momenta 28 2 . The combined approach was also proposed (Catani-Ciafaloni-FioraniMarchesini equation) 30]. Note that BFKL and CCFM approaches should apply for any target as the DGLAP does, since they correspond to the QCD chain to be attached to the target particle.

\subsubsection{Measurements of $F_{2}^{\gamma}$}

Measurements of the $F_{2}^{\gamma}\left(x_{B j}, Q^{2}\right)$ for the real photon base on the single tagged events at $e^{+} e^{-}$colliders (i.e. DIS $_{e \gamma}$ events, see eq. (2) and fig. (1). The bulk of the hadrons is produced at small angles to the incoming $e^{+} e^{-}$beams, and remains undetected. Due to the particle losses in the experiment not $x_{B j}$ but the quantity $x_{v i s}$ is measured,

$$
x_{v i s}=\frac{Q^{2}}{Q^{2}+W_{v i s}^{2}}
$$

where $W_{v i s}$ is the invariant mass of the visible hadronic system. Since the target photon has always the nonzero virtuality $P^{2}$, the measured quantity is in fact

$$
x_{v i s}=\frac{Q^{2}}{Q^{2}+W_{v i s}^{2}+P^{2}} .
$$

In order to reconstruct $x_{B j}$ from a measured value for $x_{v i s}$, an unfolding procedure is needed. To this end, in some analyses the related variables, $W_{\text {rec }}, x_{r e c}$, are also used, see e.g. L3 98a, OPAL 2000. They are reconstructed from the measured four-momenta of electrons with the constraints from the transverse momentum conservation. Even using the above technique, the value of $W_{\text {rec }}$ is still generally smaller than the true value due to e.g. energy loss in the forward regions. Therefore new variables $W_{\text {cor }}$ and $x_{c o r}$ are formed to correct for this fact, see OPAL 2000. The very important improvement in the description of the data has been achieved due to the recently proposed two-dimensional unfolding, with the energy in the forward cone used as the second variable [9]. It was used in ALEPH 99b,conf, and OPAL 2000 leading to much smaller errors [7, 11]. The proper inclusion of the nonzero virtuality of the target photon [2] should lead to the further improvement [0] (see sec. 4 where this effect for the

\footnotetext{
${ }^{11}$ The NLO BFKL [29] calculations demonstrate large corrections to the LO BFKL results.

${ }^{12}$ The procedure "is not well defined if the photon is not pointlike" 10].
} 
leptonic structure functions is discussed). The unfolding can be performed using the traditional method based on the linear scale or using the approach with the logarithmic scale, especially useful for extracting $F_{2}^{\gamma}$ in the small $x_{B j}$ region 31 .

In order to perform reliable unfolding of the structure function $F_{2}^{\gamma}$ from the cross section for $\gamma^{*} \gamma \rightarrow$ hadrons, the corresponding events are grouped into classes of similar topologies (see discussion in [5], [8] and e.g. TOPAZ 94, OPAL 97a, DELPHI 96b,conf), which roughly coincide with the contributions from QPM, VMD and RPC (RPC - Resolved Photon Contribution). First analyses applied the FKP approach, with the pointlike QCD contribution assumed for light quarks, QPM description of the heavy quarks, and VMD contribution for the soft hadronic production. The $p_{T}^{0}$ parameter separates the pointlike and the hadronic ( $\rho$-type) configuration of the quarks in the target photon (at the first vertex level). The additional parameter $p_{T}^{\text {min }}$, in the FKP approach of the same order as $p_{T}^{0}$, is introduced as a cutoff for the perturbative $2 \rightarrow 2$ subprocesses QCD calculations. More details on the modelling of the hadronic final state can be found in sec. 2.3.

Nowadays the Monte Carlo generators HERWIG and PYTHIA, adapted for DIS er in 1995 (see e.g. OPAL 97a), can describe these contributions (using the cutoff $\left.p_{T}^{m i n}\right)$ with any of the existing parton parametrizations for the photon. It is no longer necessary for the resolved $\gamma$ term to fit an empirical $p_{T}^{0}$ parameter to the data before unfolding, as in the parton density in the photon both nonperturbative (e.g. à la VMD) and the perturbative contributions are included. The PYTHIA and modified HERWIG ("HERWIG + power law- $p_{T}$ ") programs with broader $p_{t}$ distribution $d p_{t}^{2} / p_{t}^{2}+\tilde{p}_{t 0}^{2}$ 巴马 originally introduced to improve description of $\gamma p$ processes (ZEUS 95c), seem to describe $\gamma \gamma$ data better than the default versions (e.g. [59]). On the other hand, the TWOGAM generator, based on QPM + VMD + RPC contributions is successfully used to describe the newest $F_{2}^{\gamma}$ data although with a large $p_{T}^{\min }$ parameter, $\sim 2.3$ - 3.5 GeV, instead of the default value $1.8 \mathrm{GeV}(\mathbf{L 3} \mathbf{9 9 a})$. The general purpose generator PHOJET based on the Dual Parton Model is also used successfully in the $F_{2}^{\gamma}$ measurements (with $p_{T}^{\min }=2.5 \mathrm{GeV}$ ). The large dependence on the chosen Monte Carlo model leads to large errors in the $F_{2}^{\gamma}$ data points, or even to two sets of data points from the same event sample (L3 98a). The multi-dimensional unfolding method reduces the dependence on MC models, as it was found in very recent analyses (OPAL 2000 and ALEPH 99b,conf), see sec. 2.3.

The $F_{2}^{\gamma}$ measurements at small $x_{B j}$ are presented in DELPHI 96a, OPAL 97b, OPAL 2000 and L3 98a, with the lowest value $x_{B j}$ (center of bin value) 0.001 (0.0022). The low $Q^{2}$ measurements at $\mathrm{LEP}\left(<Q^{2}>\sim 2 \mathrm{GeV}^{2}\right)$ were reported in OPAL 97b, L3 98a, OPAL 2000 while the largest $<Q^{2}>=400 \mathrm{GeV}^{2}$ data are discussed in DELPHI 98,conf. Note also that in the earlier measurements the $c$ (and $b$ ) quark contribution was usually subtracted from the structure function $F_{2}^{\gamma}$ (e.g. OPAL 94). For the dedicated measurement of $F_{2, c}^{\gamma}$ reported in 1999 by OPAL collaboration, see sec. 5 .

The general features of $F_{2}^{\gamma}$, as far as $x_{B j}$ and $\log Q^{2}$ dependences are concerned, agree with the theoretical expectations, although the precision of the data does not allow in many cases to distinguish between existing parton parametrizations and clarify

\footnotetext{
${ }^{13}$ The parameter $\tilde{p}_{t 0} \sim 0.66 \mathrm{GeV}$ should not be confused with $p_{T}^{0}$ separating the soft and hard hadronic processes within the FKP approach.
} 
the small $x_{B j}$ behaviour of $F_{2}^{\gamma}$. The parton densities which give, at present, the best description of the $F_{2}^{\gamma}$ data are GRV and SaS1D [7].

As we have already pointed out, the study of the hadronic final state together with the possibly resolved (real and virtual) photon contribution became part of the measurements of the structure function for the photon at $e^{+} e^{-}$colliders (details of the studies of the hadronic final state will be presented in the next section). Here we would only like to stress that some discrepancies have been found for certain distributions, e.g. the hadronic energy flow. This fact is included in the estimation of the uncertainty of the measured function $F_{2}^{\gamma}$.

We start the presentation of the $F_{2}^{\gamma}$ data from the LEP collider, then the TRISTAN data are discussed. Figures and tables with corresponding numbers for the $F_{2}^{\gamma}$ as a function of $x_{B j}$ at fixed $\left\langle Q^{2}>\right.$, and also for $F_{2}^{\gamma}$ averaged over relevant $x_{B j}$ ranges are given. Collective figures of $F_{2}^{\gamma}$ versus $x_{B j}$ and $F_{2}^{\gamma}$ versus $Q^{2}$ containing all existing results are presented at the end of this section. 


\section{DATA}

\section{-ALEPH 99a 33 (LEP 1)}

Data on $F_{2}^{\gamma}$ for $x_{B j}$ from 0.005 to 0.97 and $Q^{2}$ between 6 and $3000 \mathrm{GeV}^{2}$, in three $Q^{2}$ bins, were collected in the period 1991-95. An analysis of the hadronic final state was performed using the QPM+VMD model and the "HERWIG $5.9+$ power law $p_{t}$ " involving GRV LO parton parametrizations (see next section for details).

The unfolded results for $F_{2}^{\gamma}$ as a function of $x_{B j}$ are presented in table 1 and in figs. 2, 3, 4, where the comparison with predictions of the parton parametrizations: AFG HO, LAC 1 and GRV LO is made. The values of $F_{2}^{\gamma}$ averaged over $x_{B j}$ (for 0.1 $\left.<x_{B j}<0.6\right)$ for three values of $<Q^{2}>$ are given in table 2 .

Table 1:

\begin{tabular}{|c|c|c|}
\hline $\begin{array}{c}<Q^{2}> \\
{\left[G e V^{2}\right]}\end{array}$ & $x_{B j}$ & $\begin{array}{c}F_{2}^{\gamma} / \alpha \\
\text { (stat. }+ \text { syst. })\end{array}$ \\
\hline 9.9 & $0.005-0.08$ & $0.30 \pm 0.02 \pm 0.02$ \\
& $0.08-0.2$ & $0.40 \pm 0.03 \pm 0.07$ \\
& $0.2-0.4$ & $0.41 \pm 0.05 \pm 0.09$ \\
& $0.4-0.8$ & $0.27 \pm 0.13 \pm 0.09$ \\
\hline 20.7 & $0.009-0.120$ & $0.36 \pm 0.02 \pm 0.05$ \\
& $0.12-0.27$ & $0.34 \pm 0.03 \pm 0.11$ \\
& $0.27-0.50$ & $0.56 \pm 0.05 \pm 0.10$ \\
& $0.50-0.89$ & $0.45 \pm 0.11 \pm 0.05$ \\
\hline 284 & $0.03-0.35$ & $0.65 \pm 0.1 \pm 0.09$ \\
& $0.35-0.65$ & $0.70 \pm 0.16 \pm 0.19$ \\
& $0.65-0.97$ & $1.28 \pm 0.26 \pm 0.26$ \\
\hline
\end{tabular}

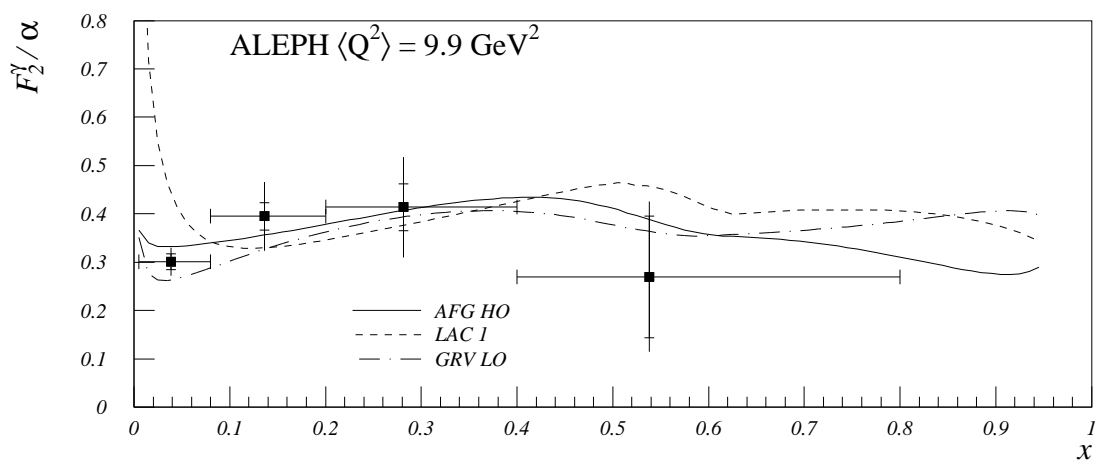

Figure 2: The unfolded structure function $F_{2}^{\gamma} / \alpha$ as a function of $x_{B j}$ for $<Q^{2}>=9.9$ $\mathrm{GeV}^{2}$. Comparison with predictions of different parton parametrizations: $A F G \mathrm{HO}$ (solid line), LAC 1(dashed line), GRV LO (dot-dashed line) (from 33]). 


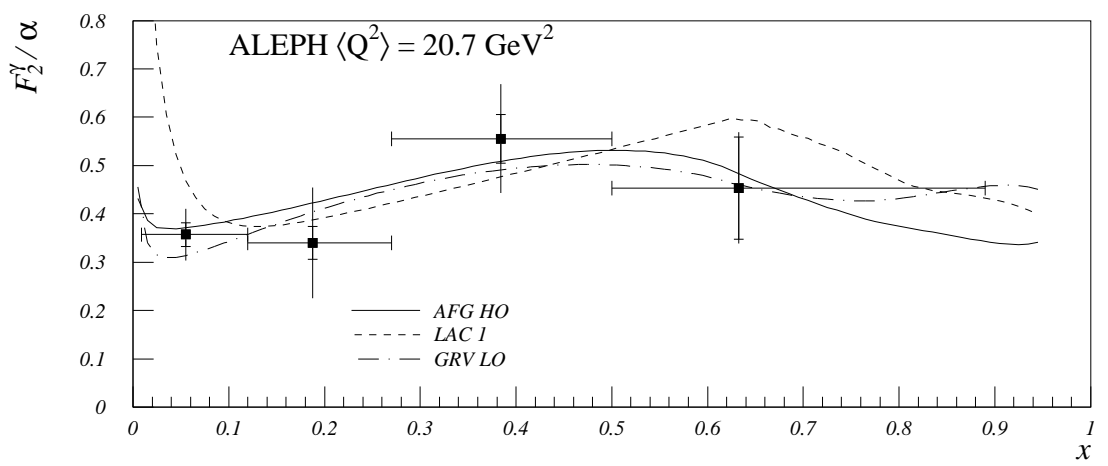

Figure 3: The same as in fig. [2 for $<Q^{2}>=20.7 \mathrm{GeV}^{2}$ (from 33]).

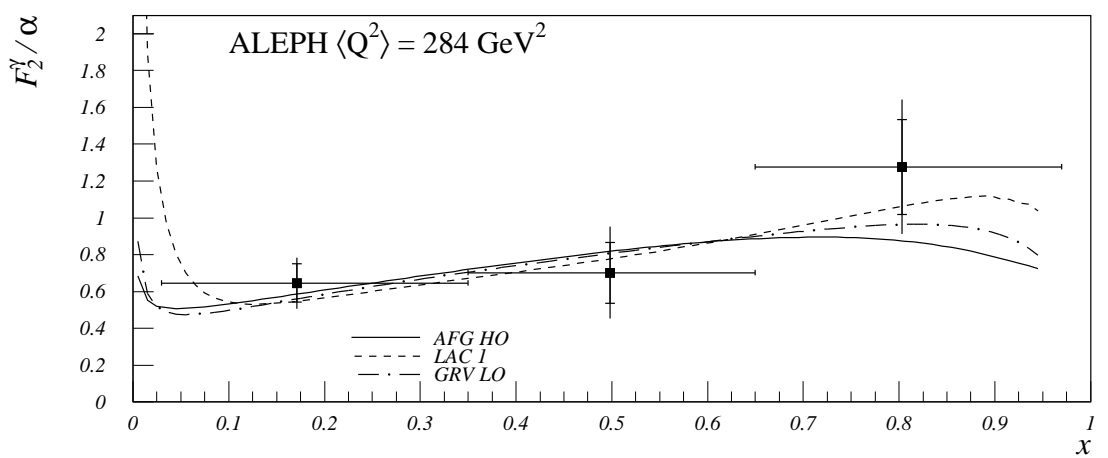

Figure 4: The same as in fig. [ 2 for $\left\langle Q^{2}>=284 \mathrm{GeV}^{2}\right.$ (from [33]).

Table 2:

\begin{tabular}{|c|c|}
\hline $\begin{array}{c}<Q^{2}> \\
{\left[\mathrm{GeV}^{2}\right]}\end{array}$ & $\begin{array}{c}<F_{2}^{\gamma} / \alpha> \\
(\text { tot })\end{array}$ \\
\hline 9.9 & $0.38 \pm 0.05$ \\
\hline 20.7 & $0.50 \pm 0.05$ \\
\hline 284 & $0.68 \pm 0.12$ \\
\hline
\end{tabular}

The $Q^{2}$ dependence of $F_{2}^{\gamma}$ (from the $0.1<x_{B j}<0.6$ range) has also been measured (not shown) and a good agreement with the OPAL 97c measurements was found.

The $F_{2}^{\gamma}$ data were compared to different parametrizations and the $\chi^{2}$ test was performed. LAC1 and 2 and WHIT 4,5 and 6, all containing a large gluon density at low $x$, show large $\chi^{2}$.

Comment: "Comparisons to parametrized parton density functions show that those containing a large gluon content are inconsistent with the data."

\section{-ALEPH 99b,conf [34 (LEP 2)}

The measurement of $F_{2}^{\gamma}$ at $\sqrt{s}=183 \mathrm{GeV}$ based on the 1997 data was performed using a two-dimensional unfolding method (the principle of maximum entropy) [9]. The two $Q^{2}$ ranges: $7-24 \mathrm{GeV}^{2}$ and $17-200 \mathrm{GeV}^{2}$ were studied. In the analysis "HERWIG $5.9+$ 
power law $p_{t}$ " with the GRV LO and SaS1D parton parametrizations and in addition the PHOJET model were used.

The hadronic final state analysis was performed (see next section for details) and it was found that the unfolding procedure reduces the dependence on Monte Carlo models, and that "PHOJET is in poor agreement with the data for the higher $Q^{2}$ range".

The unfolded results for $F_{2}^{\gamma}$ as a function of $x_{B j}$ are presented in fig. 5 for $<Q^{2}>=$ 13.7 $\mathrm{GeV}^{2}$ and $56.5 \mathrm{GeV}^{2}$ and in table 3 (only for lower $Q^{2}$ ).
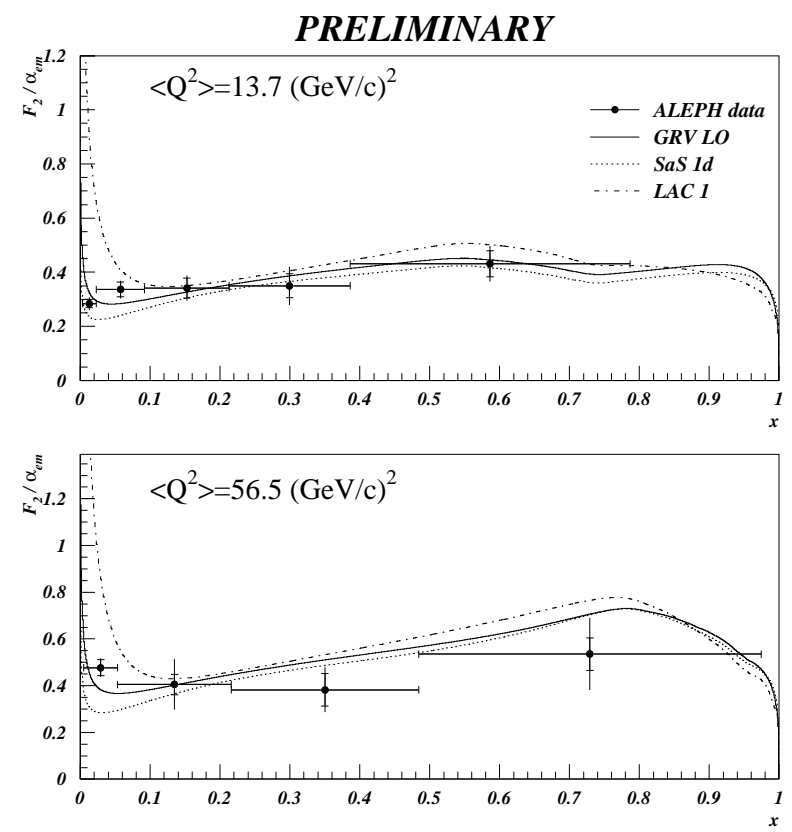

Figure 5: The unfolded structure function $F_{2}^{\gamma} / \alpha$ as a function of $x_{B j}$ for $<Q^{2}>=13.7 \mathrm{GeV}^{2}$ and $56.5 \mathrm{GeV}^{2}$. Comparison with predictions of different parton parametrizations: GRV LO (solid line), SaS1D (dotted line), LAC1 (dot-dashed line) (from 34]).

Table 3:

\begin{tabular}{|c|c|c|}
\hline $\begin{array}{c}<Q^{2}> \\
{\left[\mathrm{GeV}^{2}\right]}\end{array}$ & $x_{B j}$ & $\begin{array}{c}F_{2}^{\gamma} / \alpha \\
\text { (stat. }+ \text { syst. }) \text { in } \%\end{array}$ \\
\hline 13.7 & $0.003-0.023$ & $0.28 \pm 5.3 \pm 6.3$ \\
& $0.023-0.092$ & $0.34 \pm 7.5 \pm 5.3$ \\
& $0.092-0.213$ & $0.34 \pm 10.8 \pm 8.0$ \\
& $0.213-0.386$ & $0.35 \pm 13.6 \pm 15.7$ \\
& $0.386-0.786$ & $0.43 \pm 11.1 \pm 10.5$ \\
\hline
\end{tabular}

Comment: The new unfolding method was used in the analysis. It "leads to smaller statistical errors and a reduced model dependence compared to one-dimensional procedure". The GRV LO parametrization describes the $F_{2}^{\gamma}$ data well. At low x, "the SaS1D parametrization is found to be lower than data, whereas the LAC 1 parametrization is considerably higher...".

•DELPHI 96a [35] (LEP 1) 
Data on $F_{2}^{\gamma}$ were taken in the period 1991-93 for $Q^{2}$ between 4 and $30 \mathrm{GeV}^{2}\left(<Q^{2}>\right.$ $\left.=12 \mathrm{GeV}^{2}\right)$ and for $x_{B j}$ down to 0.003. The so called $F_{2}^{\gamma(Q E D)}$ was also measured for nonzero virtuality $P^{2}$ of the target photon (see sec. 4.2 for details). Estimated target photon virtuality $P^{2}$ was used in the unfolding of $F_{2}^{\gamma}$. The TWOGAM event generator was used to simulate QPM events, another event generator was used to obtain the QCD correction (LL) to the pointlike contribution for light quarks in the FKP approach. The GVMD and the pointlike (FKP) contributions were studied with $p_{T}^{0}$ $=0.1$ and $0.5 \mathrm{GeV}$. Results for $F_{2}^{\gamma}$ for the light quarks are presented in fig. 6 and in table 田.

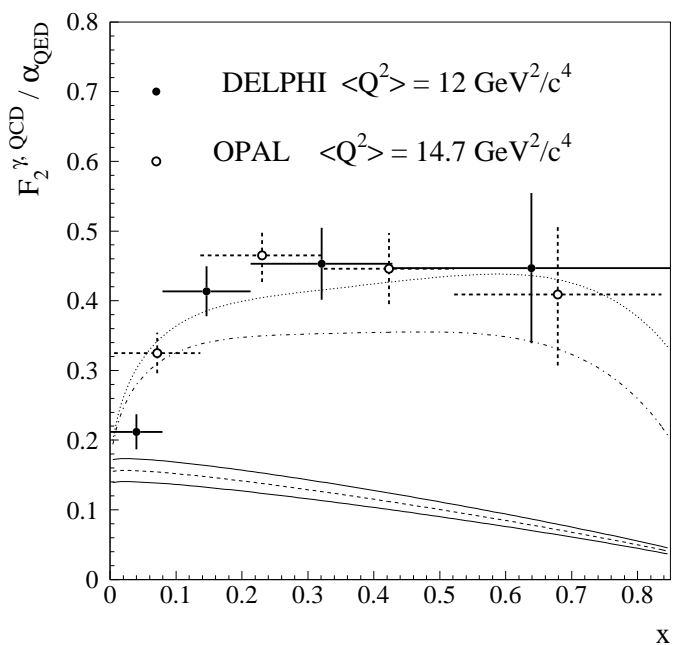

Figure 6: The unfolded $F_{2}^{\gamma} / \alpha$ for the light quarks together with OPAL 94 data. The curves show the sum of the GVMD model prediction multiplied by the threshold factor 1- $x_{B j}$ and the prediction of the FKP parametrization, with the parameter $p_{T}^{0}$ equal $0.1 \mathrm{GeV}$ (upper line) and $0.5 \mathrm{GeV}$ (lower line). The bottom curves show separately the GVMD contribution with different target masses (from [35]).

Table 4:

\begin{tabular}{|c|c|c|}
\hline $\begin{array}{c}<Q^{2}> \\
{\left[\mathrm{GeV}^{2}\right]}\end{array}$ & $x_{B j}$ & $\begin{array}{c}F_{2}^{\gamma} / \alpha \\
(\text { stat. }+ \text { syst. })\end{array}$ \\
\hline 12 & $0.003-0.080$ & $0.21 \pm 0.03 \pm 0.06$ \\
& $0.080-0.213$ & $0.41 \pm 0.04 \pm 0.05$ \\
& $0.213-0.428$ & $0.45 \pm 0.05 \pm 0.05$ \\
& $0.428-0.847$ & $0.45 \pm 0.11 \pm 0.10$ \\
\hline
\end{tabular}

The averaged value of $F_{2}^{\gamma} / \alpha$ over the $x_{B j}$ range between 0.3 and 0.8 was extracted:

\begin{tabular}{|c|c|}
\hline $\begin{array}{c}<Q^{2}> \\
{\left[\mathrm{GeV}^{2}\right]}\end{array}$ & $<F_{2}^{\gamma} / \alpha>$ \\
\hline 12 & $0.45 \pm 0.08$ \\
\hline
\end{tabular}

For comparison with other measurements of the $Q^{2}$ dependence of the $F_{2}^{\gamma}$, see fig.7.7.

\footnotetext{
${ }^{14}$ It was found that although $<P^{2}>=0.13 \mathrm{GeV}^{2}$, a fixed value of $0.04 \mathrm{GeV}^{2}$ fits the data better.
} 


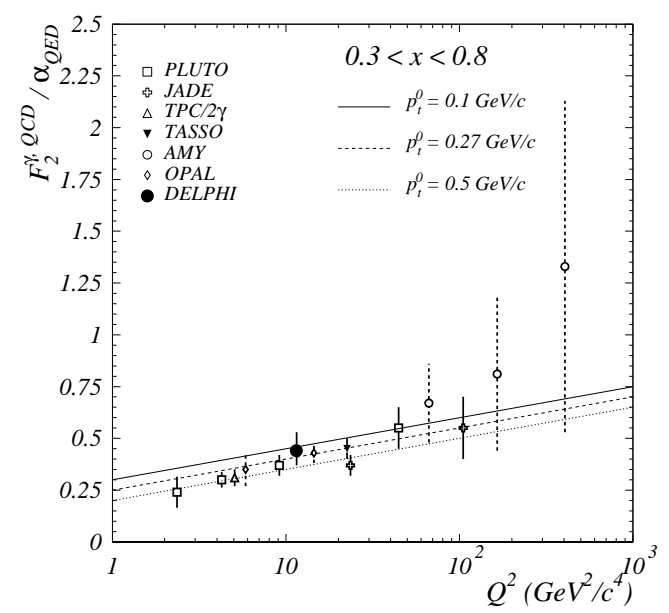

Figure 7: $F_{2}^{\gamma} / \alpha$ averaged over $x_{B j}$ between 0.3 and 0.8 as a function of $Q^{2}$. The curves show the FKP parametrization predictions for different values of the parameter $p_{T}^{0}$ (from [35]).

The study of the $F_{2}^{\gamma}$ behaviour at $\left\langle Q^{2}\right\rangle=12 \mathrm{GeV}^{2}$ in the low $x_{B j}$ domain leads to the following results (fig. 8 and table 5):

Table 5:

\begin{tabular}{|c|c|c|}
\hline $\begin{array}{c}<Q^{2}> \\
{\left[\mathrm{GeV}^{2}\right]}\end{array}$ & $x_{B j}$ & $\begin{array}{c}F_{2}^{\gamma} / \alpha \\
\text { (stat. }+ \text { syst. })\end{array}$ \\
\hline 12 & $0.003-0.046$ & $0.24 \pm 0.03 \pm 0.07$ \\
& $0.046-0.117$ & $0.41 \pm 0.05 \pm 0.08$ \\
& $0.117-0.350$ & $0.46 \pm 0.17 \pm 0.09$ \\
\hline
\end{tabular}

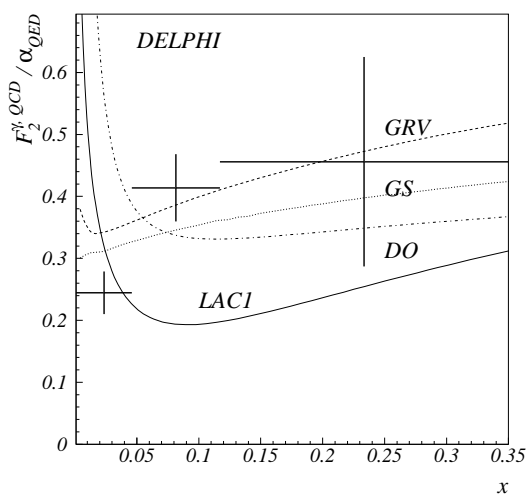

Figure 8: The unfolded $F_{2}^{\gamma} / \alpha$ for $\left\langle Q^{2}>=12 \mathrm{GeV}^{2}\right.$ as a function of $x_{B j}$ with the LO parametrizations LAC1, GS, DO, and GRV (from [35]).

Comment: The effect of the nonzero $P^{2}$ was included in the unfolding of $F_{2}^{\gamma}$. No rise of $F_{2}^{\gamma}$ at small $x_{B j}$ has been found. The GRV and GS leading order parametrizations of the quark density in the photon are in agreement with the data.

\section{-DELPHI 96b,conf [36] (LEP 1)}

The measurement of the photon structure function $F_{2}^{\gamma}$ at $<Q^{2}>=13$ and $106 \mathrm{GeV}^{2}$ (data collected in the years 1991-95) for $x_{B j}$ down to 0.003 together with a study of 
the hadronic final state (see next section) is reported. Three types of subprocesses have been considered: QPM (direct term with $m_{q} \neq 0$ for all quarks), soft hadronic GVMD or VMD (the TPC/2 $\gamma$-type parametrization) and the resolved target photon contribution (RPC) with GS2 parton parametrization. In addition the effects of the resolved probe photon were studied. The unfolding was done in the linear, and, for better sensitivity to low- $x_{B j}$, logarithmic scale in $x_{B j}$.

Fig. 9 shows the $x_{B j}$ dependence of $F_{2}^{\gamma}$ for two $Q^{2}$ samples, compared with the predictions of various parton parametrizations.
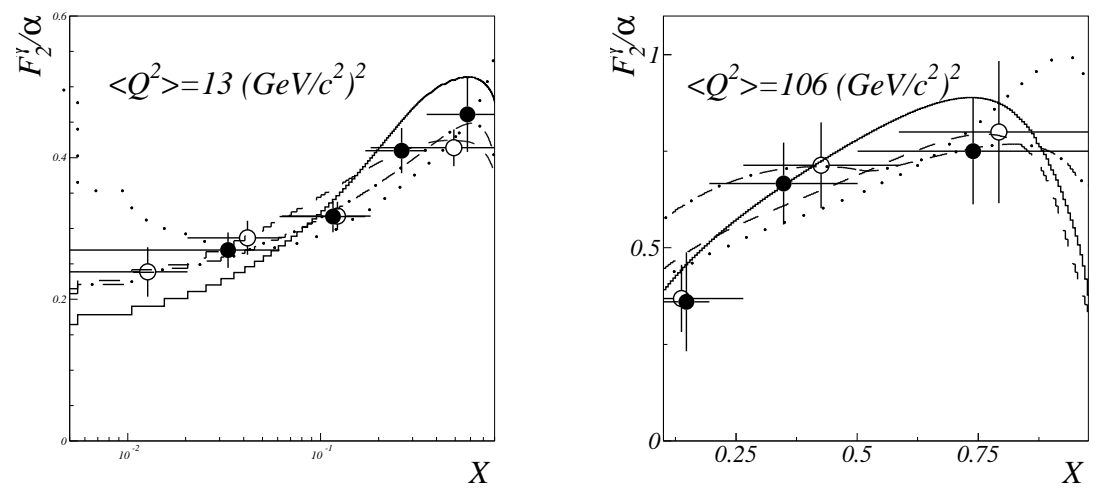

Figure 9: Unfolded $F_{2}^{\gamma} / \alpha$ as a function of $x_{B j}$ compared with $Q P M+G V M D+R P C$ (with parton parametrization GS2) (solid line) and predictions of the SaS4 (dashed line), GS2 (dash-dotted) and GRV3 (dotted) parametrizations (from [3]]).

The value of $F_{2}^{\gamma} / \alpha$ averaged in the range $0.3<x_{B j}<0.8$ was extracted for $Q^{2}=$ 13 and $106 \mathrm{GeV}^{2}$ (see table 6).

Table 6:

\begin{tabular}{|c|c|}
\hline $\begin{array}{c}<Q^{2}> \\
{\left[\mathrm{GeV}^{2}\right]}\end{array}$ & $\begin{array}{c}<F_{2}^{\gamma} / \alpha> \\
\text { (stat. }+ \text { syst. })\end{array}$ \\
\hline 13 & $0.38 \pm 0.031 \pm 0.016$ \\
106 & $0.576 \pm 0.081 \pm 0.076$ \\
\hline
\end{tabular}

Comment: The importance of the final hadronic state topology was noticed and the resolved photon contribution was included. The study of a linear and logarithmic unfolding was performed. 


\section{-DELPHI 97a,conf [37] (LEP 1, LEP 2)}

A study of $F_{2}^{\gamma}$ in the $Q^{2}$ range between 3 and $150 \mathrm{GeV}^{2}$, based on data from the 199495 runs for energies around the $Z^{0}$ mass and from 1996 for energies between 161 and $172 \mathrm{GeV}$ is presented. An analysis of the hadronic final state is performed using the TWOGAM Monte Carlo program, where QPM (with $N_{f}=4$ ), soft hadronic VMD and RPC (with the GS2 parton parametrization, both single and double resolved photon terms) parts are included (see also next section).

The unfolded results for $F_{2}^{\gamma}$ as a function of $x_{B j}$ and $Q^{2}$ are presented in figs. 10a and 19b, respectively.
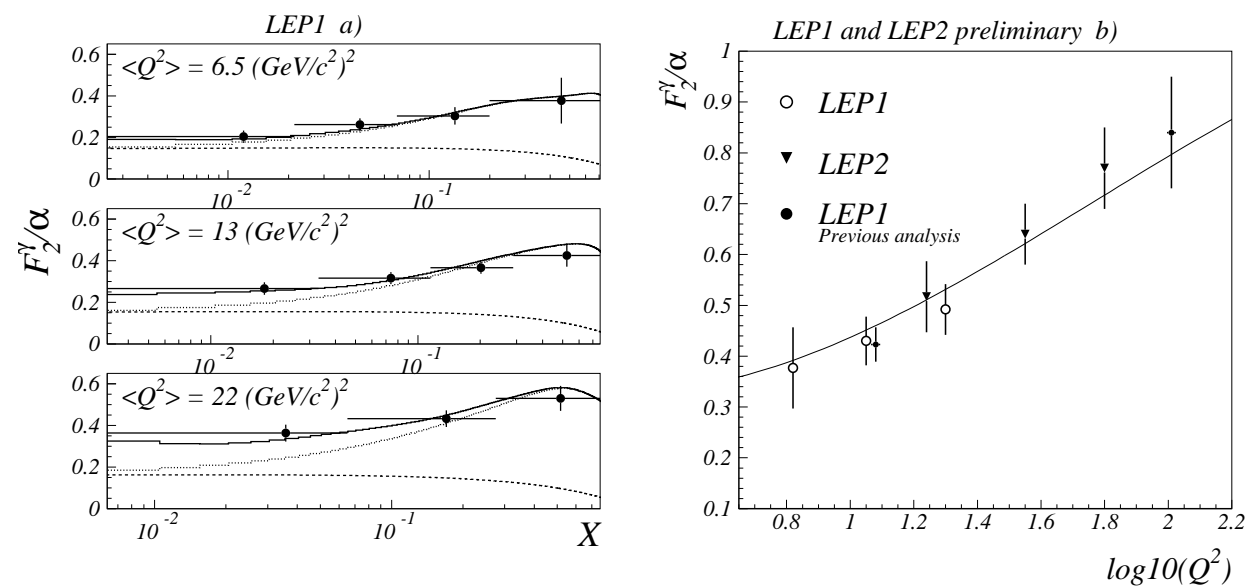

Figure 10: a) $F_{2}^{\gamma} / \alpha$ versus $x_{B j}$ from the DELPHI experiment based on the LEP 1 data for three values of $\left\langle Q^{2}\right\rangle: 6.5,13$, and $22 \mathrm{GeV}^{2}$. The solid line corresponds to $Q P M+G V M D+R P C$ (with with parton parametrization GS2), dotted - QPM+GVMD and dashed-GVMD. b) $F_{2}^{\gamma} / \alpha$ averaged over $x_{B j}$ from 0.3 to 0.8 as a function of $Q^{2}$. Results obtained from the LEP1 and LEP2 data are shown together with results of a previous analysis of the LEP1 data (from [37]).

Comment: "There is some indication of deviations of TWOGAM predictions for the data in the high $Q^{2}$ region."

\section{-DELPHI 98,conf [38, 39] (LEP 2)}

Data on $F_{2}^{\gamma}$ were taken in the period 1996-1998 for $Q^{2}$ between 10 and $1000 \mathrm{GeV}^{2}$ (for the energy 163-188 GeV). The three components were applied: the QPM for all quarks simulated by the TWOGAM generator, the soft hadronic VMD contribution and RPC for single and double resolved photon processes, and a good description was obtained (see also next section). The data are shown in table 7 and in fig. 11. 
Table 7:

\begin{tabular}{|c|c|c|}
\hline $\begin{array}{c}<Q^{2}> \\
{\left[\mathrm{GeV}^{2}\right]}\end{array}$ & $x_{B j}$ & $\begin{array}{c}F_{2}^{\gamma} / \alpha \\
\text { (stat. }+ \text { syst. })\end{array}$ \\
\hline 21 & $0.01-0.1$ & $0.33 \pm 0.01 \pm 0.03$ \\
& $0.1-0.3$ & $0.41 \pm 0.03 \pm 0.02$ \\
& $0.3-0.8$ & $0.51 \pm 0.05 \pm 0.04$ \\
\hline 42 & $0.01-0.1$ & $0.41 \pm 0.01 \pm 0.03$ \\
& $0.1-0.3$ & $0.48 \pm 0.02 \pm 0.02$ \\
& $0.3-0.8$ & $0.59 \pm 0.03 \pm 0.04$ \\
\hline 99 & $0.01-0.1$ & $0.45 \pm 0.06 \pm 0.02$ \\
& $0.1-0.3$ & $0.52 \pm 0.05 \pm 0.03$ \\
& $0.3-0.8$ & $0.73 \pm 0.05 \pm 0.03$ \\
\hline 400 & $0.01-0.1$ & $0.5 \pm 0.3 \pm 0.1$ \\
& $0.1-0.3$ & $0.70 \pm 0.2 \pm 0.2$ \\
& $0.3-0.8$ & $1.0 \pm 0.1 \pm 0.3$ \\
\hline
\end{tabular}
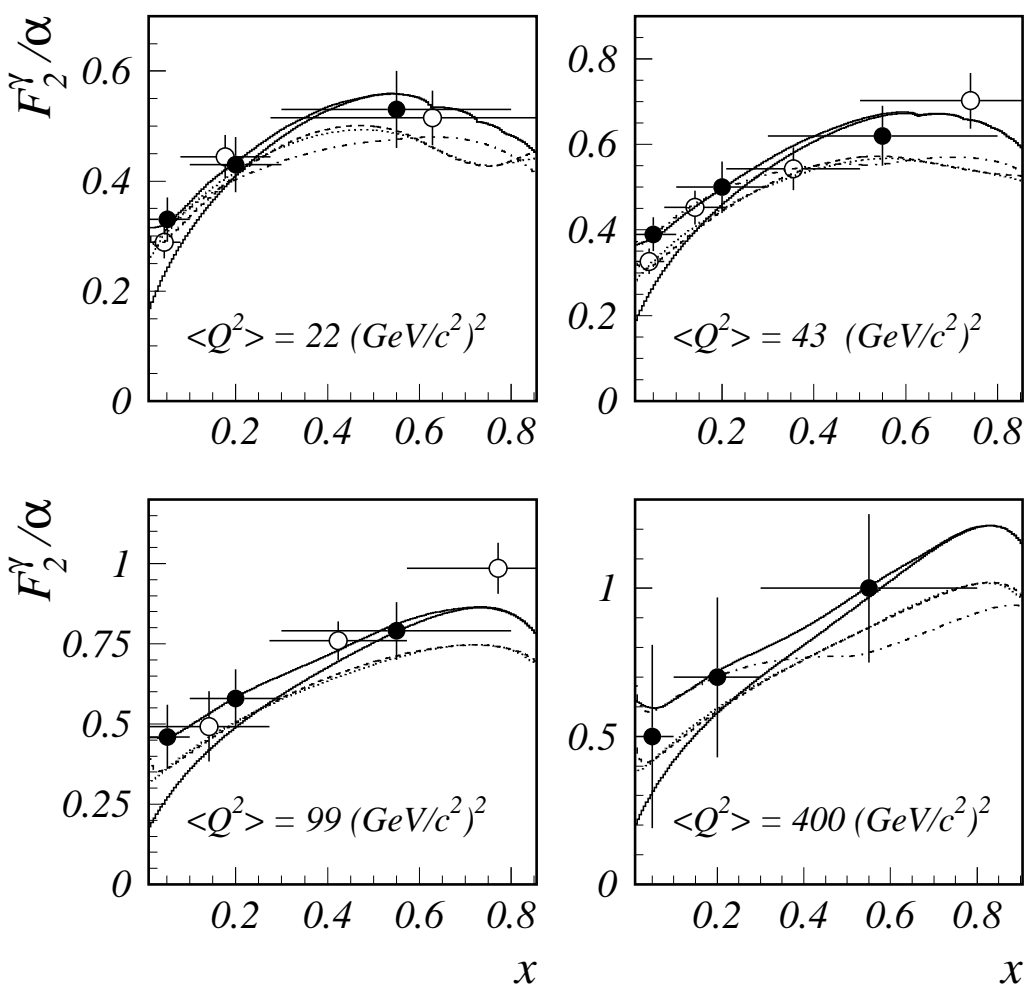

Figure 11: The DELPHI structure function $F_{2}^{\gamma} / \alpha$ as a function of $x_{B j}$ at $\left\langle Q^{2}\right\rangle=22,43$, 99 and $400 \mathrm{GeV}^{2}$. Comparison with the parton parametrizations: GS (dash - dotted line), GRV (dashed line) and SaS (dotted line); TWOGAM prediction is the solid line (from [39]). 
The $Q^{2}$ dependence was studied in two $x_{B j}$ ranges:

$$
\text { for } 0.01<x_{B j}<0.1 \quad F_{2}^{\gamma} / \alpha=(0.12 \pm 0.02)+(0.073 \pm 0.009) \ln Q^{2} \text {, }
$$

while for $0.3<x_{B j}<0.8 \quad F_{2}^{\gamma} / \alpha=(0.09 \pm 0.05)+(0.14 \pm 0.02) \ln Q^{2}$.

The data for $F_{2}^{\gamma} / \alpha$ as a function of $Q^{2}$ are presented in figs. 12, 13 in comparison with the Monte Carlo predictions and LO calculations. A suppression factor was introduced to describe the high $Q^{2}$ data.

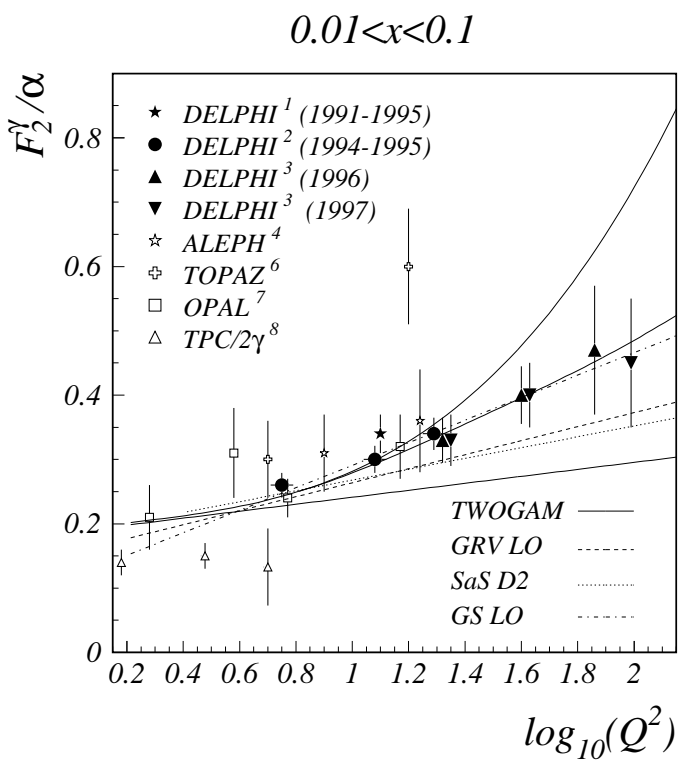

Figure 12: The DELPHI results for $F_{2}^{\gamma} / \alpha$ as a function of $Q^{2}$ for $0.01<x_{B j}<0.1$ compared with other data (from [39]).

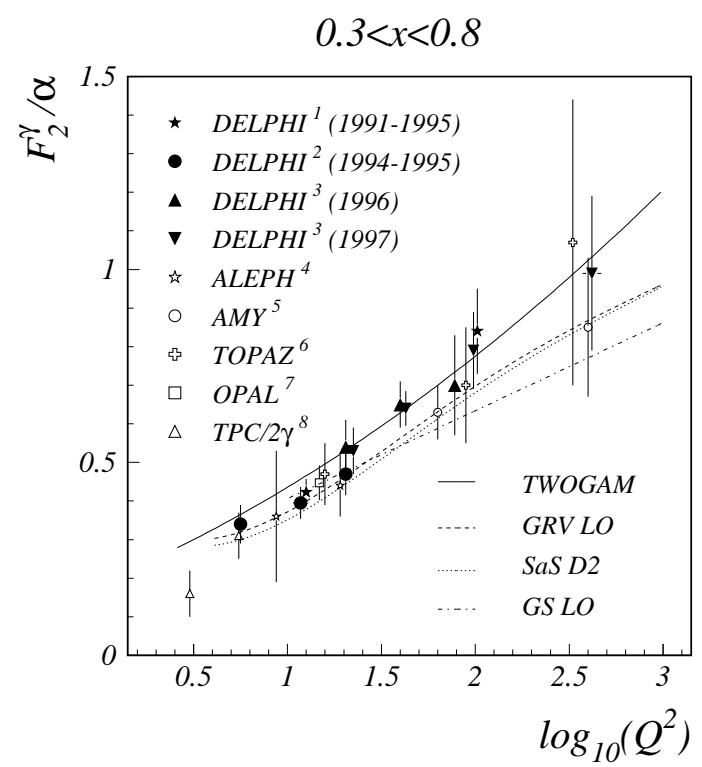

Figure 13: The DELPHI results for $F_{2}^{\gamma} / \alpha$ as a function of $Q^{2}$ for $0.3<x_{B j}<0.8$ compared with other data (from [39]). 
Comment: "Prediction for the RPC component tends to have too high cross section for the high $Q^{2}$ " and the low $x_{B j}$. "For the first time TWOGAM has been tuned to get a good agreement in the region of high $Q^{2}$.

\section{-L3 98a 40 (LEP 1)}

The $F_{2}^{\gamma}$ data for $1.2 \leq Q^{2} \leq 9.0 \mathrm{GeV}^{2}$ collected in years 1991-95 are reported. Two bins: $<Q^{2}>=1.9 \mathrm{GeV}^{2}$ in the $x_{B j}$ range $0.002<x_{B j}<0.1$ and $<Q^{2}>=5 \mathrm{GeV}^{2}$ in the $x_{B j}$ range $0.005<x_{B j}<0.2$ were analysed. To improve the measurement of $W_{v i s}$ the kinematics of the tagged electron was included (the transverse momentum conservation). In the analysis three Monte Carlo generators: PHOJET 1.05c $\left(p_{T}^{\min }=\right.$ $2.5 \mathrm{GeV})$, HERWIG 5.9 and TWOGAM $\left(p_{T}^{\min }=2.3 \mathrm{GeV}\right)$ have been used. They led to different results for $x_{B j}, p_{T}$ distributions and the energy flow (for more details see next section).

Two (!) data sets for $F_{2}^{\gamma}$ based on two Monte Carlo analyses: the PHOJET and TWOGAM, are given. They differ up to $14 \%$ at high $x_{B j}$ and up to $28 \%$ at low $x_{B j}$. The results are presented in fig. 14 and in table 8 (the values of $F_{2}^{\gamma} / \alpha$ are given at the centre of the $x_{B j}$ bin).

Table 8:

\begin{tabular}{|c|c|c|c|}
\hline $\begin{array}{c}<Q^{2}> \\
{\left[\mathrm{GeV}^{2}\right]}\end{array}$ & $x_{B j}$ & $\begin{array}{c}F_{2}^{\gamma} / \alpha(\text { set } 1) \\
(\text { stat. }+ \text { syst. })\end{array}$ & $\begin{array}{c}F_{2}^{\gamma} / \alpha(\text { set } 2) \\
(\text { stat. }+ \text { syst. })\end{array}$ \\
\hline 1.9 & $0.002-0.005$ & $0.184 \pm 0.009 \pm 0.013$ & $0.231 \pm 0.011 \pm 0.016$ \\
& $0.005-0.010$ & $0.179 \pm 0.007 \pm 0.009$ & $0.199 \pm 0.008 \pm 0.010$ \\
& $0.010-0.020$ & $0.176 \pm 0.006 \pm 0.006$ & $0.191 \pm 0.007 \pm 0.006$ \\
& $0.020-0.030$ & $0.191 \pm 0.008 \pm 0.004$ & $0.193 \pm 0.008 \pm 0.004$ \\
& $0.030-0.050$ & $0.193 \pm 0.008 \pm 0.007$ & $0.199 \pm 0.008 \pm 0.007$ \\
& $0.050-0.100$ & $0.185 \pm 0.007 \pm 0.015$ & $0.206 \pm 0.008 \pm 0.017$ \\
\hline 5 & $0.005-0.010$ & $0.307 \pm 0.021 \pm 0.035$ & $0.394 \pm 0.027 \pm 0.045$ \\
& $0.010-0.020$ & $0.282 \pm 0.014 \pm 0.027$ & $0.318 \pm 0.016 \pm 0.031$ \\
& $0.020-0.040$ & $0.263 \pm 0.011 \pm 0.015$ & $0.277 \pm 0.012 \pm 0.016$ \\
& $0.040-0.060$ & $0.278 \pm 0.013 \pm 0.007$ & $0.279 \pm 0.013 \pm 0.007$ \\
& $0.060-0.100$ & $0.270 \pm 0.012 \pm 0.008$ & $0.275 \pm 0.012 \pm 0.008$ \\
& $0.100-0.200$ & $0.252 \pm 0.011 \pm 0.029$ & $0.287 \pm 0.013 \pm 0.032$ \\
\hline
\end{tabular}




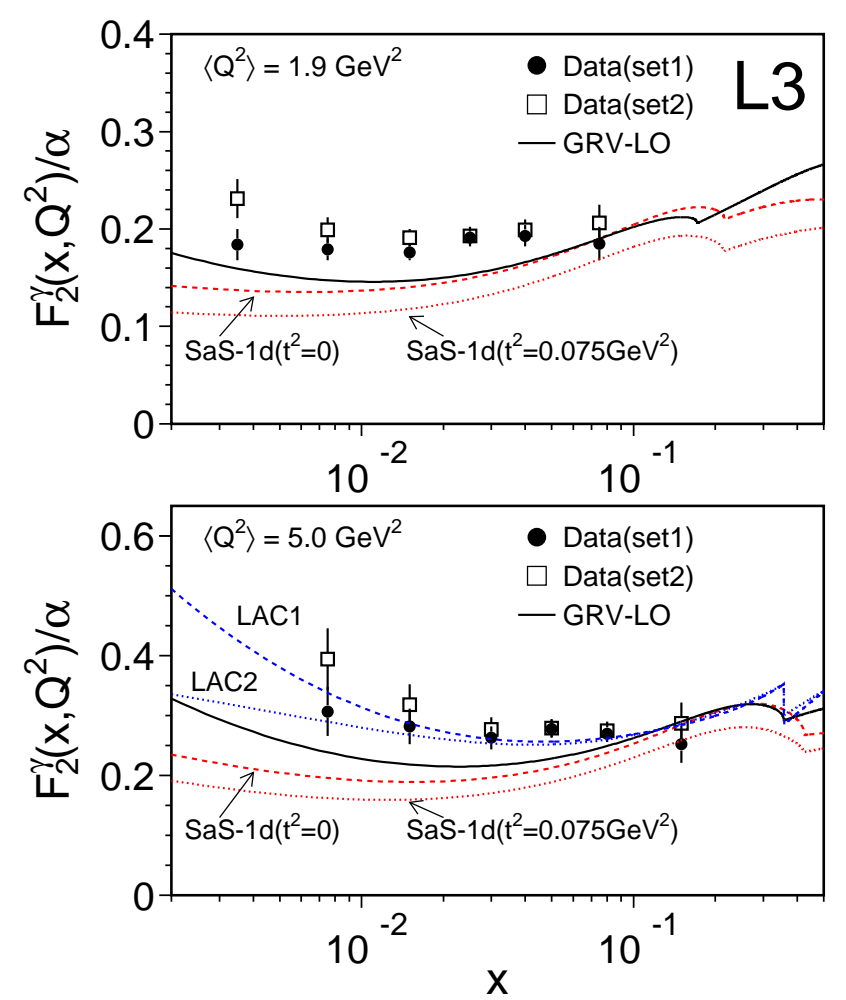

Figure 14: Two sets of data for $F_{2}^{\gamma} / \alpha$ versus $x_{B j}$ for two values of $\left\langle Q^{2}>\right.$ : 1.9 and 5 $\mathrm{GeV}^{2}$. Set 1 (2) corresponds to the unfolding based on PHOJET (TWOGAM). The solid line corresponds to GRV LO, dashed - SaS1D (for $P^{2}=0, P^{2}$ is denoted here as $t^{2}$ ) and dotted - $\operatorname{SaS1D}\left(P^{2}=t^{2}=0.075 \mathrm{GeV}^{2}\right)$ parton distributions. For the $Q^{2}=5 \mathrm{GeV}^{2}$ in addition the LAC1 and LAC2 predictions are shown (from 44]).

The $Q^{2}$ - dependence was also studied; results for $0.01<x_{B j}<0.1$ are presented in table 9 (see also fig. 15), for the two sets of data.

Table 9:

\begin{tabular}{|c|c|c|}
\hline $\begin{array}{c}<Q^{2}> \\
{\left[\mathrm{GeV}^{2}\right]}\end{array}$ & $\begin{array}{c}F_{2}^{\gamma} / \alpha(\text { set } 1) \\
\text { (stat. }+ \text { syst. })\end{array}$ & $\begin{array}{c}F_{2}^{\gamma} / \alpha(\text { set } 2) \\
\text { (stat. }+ \text { syst. })\end{array}$ \\
\hline 1.5 & $0.173 \pm 0.004 \pm 0.009$ & $0.196 \pm 0.005 \pm 0.010$ \\
2.4 & $0.195 \pm 0.005 \pm 0.004$ & $0.208 \pm 0.005 \pm 0.004$ \\
3.8 & $0.245 \pm 0.006 \pm 0.007$ & $0.252 \pm 0.006 \pm 0.007$ \\
6.6 & $0.278 \pm 0.009 \pm 0.013$ & $0.292 \pm 0.009 \pm 0.014$ \\
\hline
\end{tabular}

The following $Q^{2}$ - dependence was found for set1:

$$
F_{2}^{\gamma}\left(Q^{2}\right) / \alpha=(0.131 \pm 0.012 \pm 0.021)+(0.079 \pm 0.011 \pm 0.009) \ln \left(Q^{2} /(\mathrm{GeV})^{2}\right.
$$




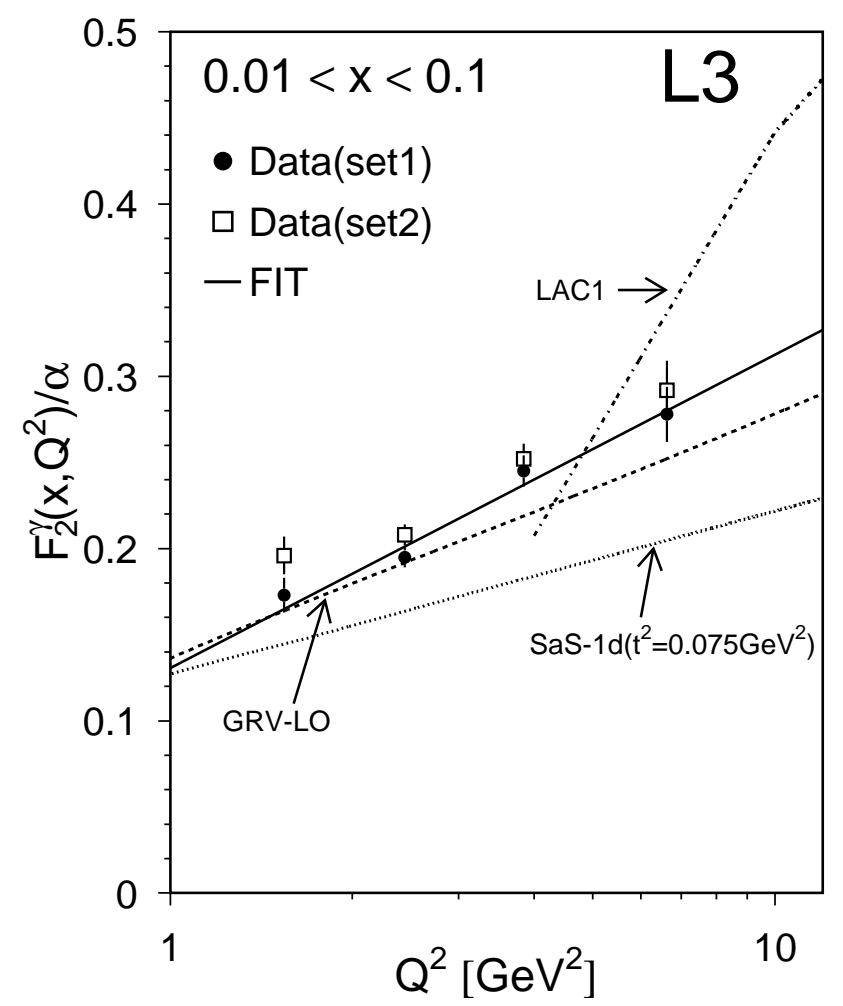

Figure 15: Two sets of data for $F_{2}^{\gamma} / \alpha$ as a function of $Q^{2}$. Set 1 (2) corresponds to the unfolding based on PHOJET (TWOGAM). The solid line corresponds to the fit to set 1, dashed line denotes the GRV LO parton parametrization prediction, dotted - SaS1D $\left(P^{2}=0.075 \mathrm{GeV}^{2}, P^{2}\right.$ is denoted here as $\left.t^{2}\right)$ and dot-dashed corresponds to LAC1 (LAC2 is similar to LAC1) (from 40]).

The $F_{2}^{\gamma}$ data are not corrected for the virtuality $P^{2}$ of the target photon since this correction is model dependent: $7.5 \%$ for GVMD and 10-20\% for SaS1D. As estimated by PHOJET and TWOGAM (QPM in TWOGAM), $<P^{2}>=0.075(0.087) \mathrm{GeV}^{2}$ [10.

Comment: "Because of the large discrepancies with the data, we do not use HERWIG for the results." "At low values of $x_{B j}$ the data are above the prediction of the GRV LO and SaS1D models (...). The LAC model can reproduce the $x_{B j}$ behaviour of $F_{2}^{\gamma}$ at $<Q^{2}>=5 \mathrm{GeV}^{2}$ but it predicts too fast a rise of $F_{2}^{\gamma}$ as a function of $\ln Q^{2}$ ".

Because of the significant differences in results for $F_{2}^{\gamma}$ obtained using different Monte Carlo for the first time two different data sets were 'derived' from the same sample of events.

\section{-L3 99a 41 (LEP 2)}

Results of the measurements of $F_{2}^{\gamma}$ at the CM energy $183 \mathrm{GeV}$ in the interval $9<$ $Q^{2}<30 \mathrm{GeV}^{2}, x_{B j}$ from 0.01 to 0.5 are presented (data collected in 1997). Three Monte Carlo generators were used: TWOGAM $\left(p_{T}^{\min }=3.5 \mathrm{GeV}\right)$ and PHOJET $1.05 \mathrm{c}$ $\left(p_{T}^{\min }=2.5 \mathrm{GeV}\right)$ with JAMVG (the latter used for the charm quark contribution, as in PHOJET the charm quark is treated as massless). The $p_{T}^{0}$ cutoff in TWOGAM needed to describe data is $3.5 \mathrm{GeV}$, since the $p_{T}^{\min }=2.3 \mathrm{GeV}$ used in previous analysis (L3 98a) "produces a too large cross section for hard processes in the high $Q^{2}$ region 433". The hadronic final state is reasonably described by the used Monte Carlo generators. 
Comparison was made with the SaS1D, GRV LO, GRV HO and LAC1 predictions. The values of $F_{2}^{\gamma} / \alpha$ are not corrected for $P^{2} \neq 0$.

The results for $F_{2}^{\gamma} / \alpha$ obtained using PHOJET are given in table 10 and in fig. 16.

Table 10:

\begin{tabular}{|c|r|c|}
\hline $\begin{array}{c}<Q^{2}> \\
{\left[\mathrm{GeV}^{2}\right]}\end{array}$ & \multicolumn{1}{|c|}{$x_{B j}$} & $\begin{array}{c}F_{2}^{\gamma} / \alpha \\
\text { stat. }+ \text { syst. })\end{array}$ \\
\hline 10.8 & $0.01-0.1$ & $0.30 \pm 0.02 \pm 0.03$ \\
& $0.1-0.2$ & $0.35 \pm 0.03 \pm 0.02$ \\
& $0.2-0.3$ & $0.30 \pm 0.04 \pm 0.10$ \\
\hline 15.3 & $0.01-0.1$ & $0.37 \pm 0.02 \pm 0.03$ \\
& $0.1-0.2$ & $0.42 \pm 0.04 \pm 0.01$ \\
& $0.2-0.3$ & $0.42 \pm 0.05 \pm 0.05$ \\
& $0.3-0.5$ & $0.35 \pm 0.05 \pm 0.08$ \\
\hline 23.1 & $0.01-0.1$ & $0.40 \pm 0.03 \pm 0.03$ \\
& $0.1-0.2$ & $0.44 \pm 0.04 \pm 0.04$ \\
& $0.2-0.3$ & $0.47 \pm 0.05 \pm 0.02$ \\
& $0.3-0.5$ & $0.44 \pm 0.05 \pm 0.11$ \\
\hline
\end{tabular}

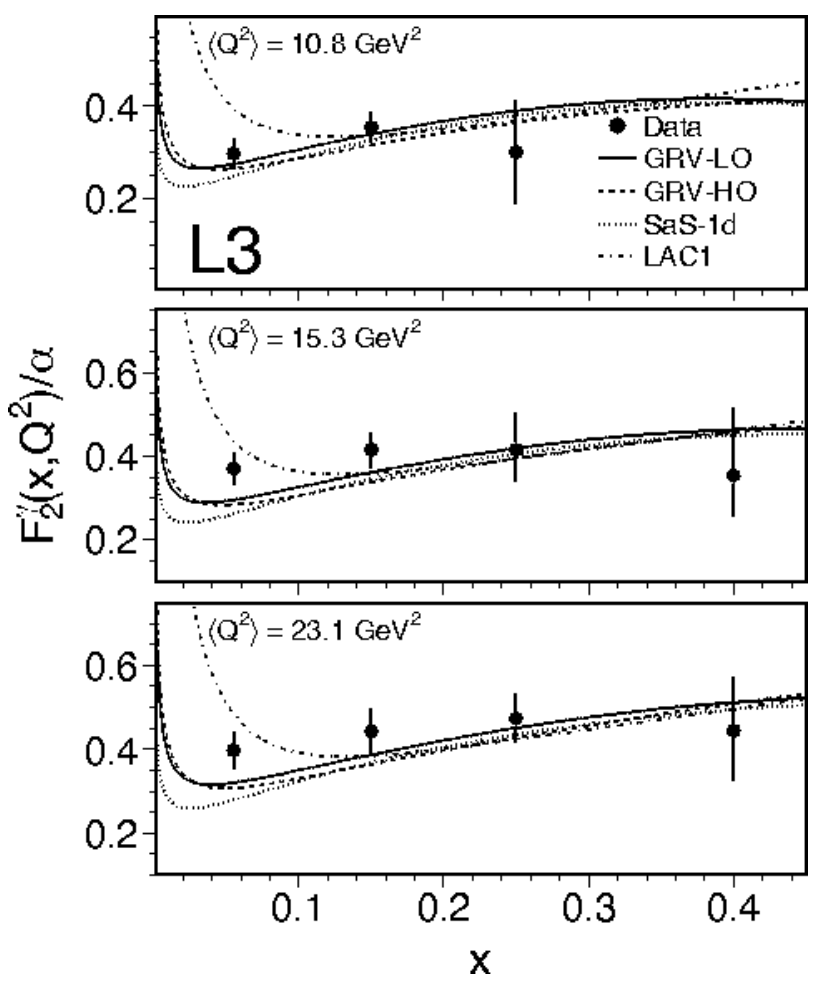

Figure 16: $F_{2}^{\gamma} / \alpha$ as a function of $x_{B j}$ for three $Q^{2}$ bins unfolded with PHOJET (plus $J A M V G)$. The data are compared with predictions of the GRV LO, GRV HO, SaS1D and LAC1 parton parametrizations (from [41]).

The $Q^{2}$ dependence of $F_{2}^{\gamma}$ was studied as well, results are shown in fig. 17 . 


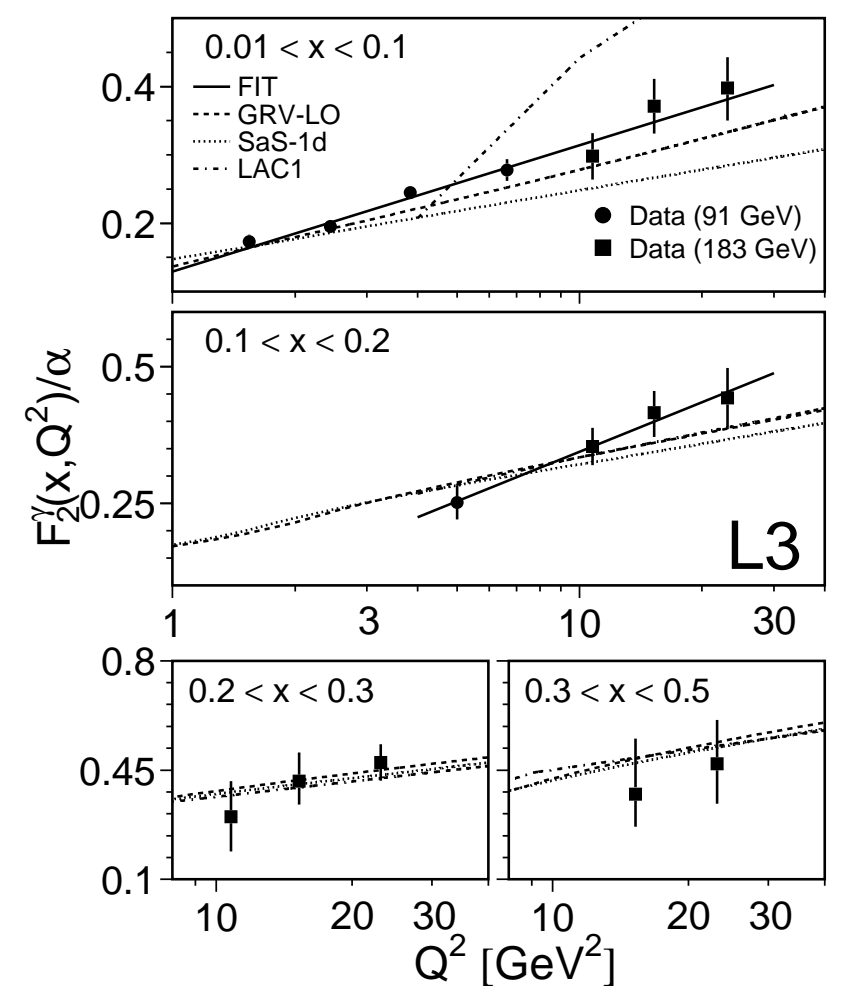

Figure 17: $F_{2}^{\gamma} / \alpha$ as a function of $Q^{2}$ for different $x_{B j}$ regions from LEP1 and LEP2 data (the unfolding based on PHOJET with JAMVG). The solid line corresponds to the fit, the GRV LO parton parametrization prediction is denoted as a dashed line, SaS1D - dotted, and dot-dashed line corresponds to the LAC1 prediction (from [4]]).

The $Q^{2}$ - dependence was found to be, for $0.01<x_{B j}<0.1$ :

$$
F_{2}^{\gamma}\left(Q^{2}\right) / \alpha=(0.13 \pm 0.01 \pm 0.02)+(0.08 \pm 0.009 \pm 0.009) \ln \left(Q^{2} / \mathrm{GeV}\right)^{2}
$$

and for $0.1<x_{B j}<0.2$ :

$$
F_{2}^{\gamma}\left(Q^{2}\right) / \alpha=(0.04 \pm 0.008 \pm 0.008)+(0.13 \pm 0.03 \pm 0.03) \ln \left(Q^{2} / \mathrm{GeV}\right)^{2}
$$

Comment: High $p_{T}^{\text {min }}$ cutoff (3.5 GeV) in TWOGAM is needed.

"The values of $F_{2}^{\gamma} / \alpha$ are not corrected for the fact that $P^{2}$ is not strictly equal to zero". "The agreement between data and the different Monte Carlo predictions is good except at pseudorapidity values, $\eta>3$ and $\eta<-1$ ", note however that these regions contribute negligibly, when $F_{2}^{\gamma}$ is extracted.

"At low $x_{B j}$, the rise of the $F_{2}^{\gamma}$ (with $Q^{2}$ ) is larger than predicted by the GRV and SaS1D models, thus requiring a modification of the gluon density in the photon structure function $F_{2}^{\gamma}$."

\section{-L3 2000 42] (LEP 1)}

The measurement of the structure function for the real photon was performed based on 1991-1995 data for large $Q^{2}$, between 40 to $500 \mathrm{GeV}^{2}$ with $<Q^{2}>=120 \mathrm{GeV}^{2}$. The virtual photon structure function was studied as well, see sec. 3.2 . 
The $F_{2}^{\gamma}$ data from single-tag events were obtained using the JAMVG generator modelling the QPM with $N_{f}=4$, the PHOJET $1.05 \mathrm{c}$ with a cutoff $p_{T}^{m i n}=2.5 \mathrm{GeV}$ and TWOGAM with $p_{T}^{\text {min }}=3.5 \mathrm{GeV}$ generating three processes: QPM, VMD and QCD resolved photon contributions. The hadronic final state was investigated (see next section for details), and it is properly described by JAMVG (QPM) model for $x_{B j}>0.5$ and by PHOJET for smaller $x_{B j}$.

The $F_{2}^{\gamma}$ data are presented in table 11 and fig. 18, where the results are compared with the predictions of QPM and of QCD calculations, the latter being needed to describe the $x_{B j}$ region below 0.5 .

Table 11:

\begin{tabular}{|c|c|c|}
\hline $\begin{array}{c}<Q^{2}> \\
{\left[G e V^{2}\right]}\end{array}$ & $x_{B j}$ & $\begin{array}{c}F_{2}^{\gamma} / \alpha \\
\text { stat. }+ \text { syst. })\end{array}$ \\
\hline 120 & $0.05-0.20$ & $0.66 \pm 0.08 \pm 0.06$ \\
& $0.20-0.40$ & $0.81 \pm 0.08 \pm 0.08$ \\
& $0.40-0.60$ & $0.76 \pm 0.12 \pm 0.07$ \\
& $0.60-0.80$ & $0.85 \pm 0.14 \pm 0.08$ \\
& $0.80-0.98$ & $0.91 \pm 0.19 \pm 0.09$ \\
\hline
\end{tabular}

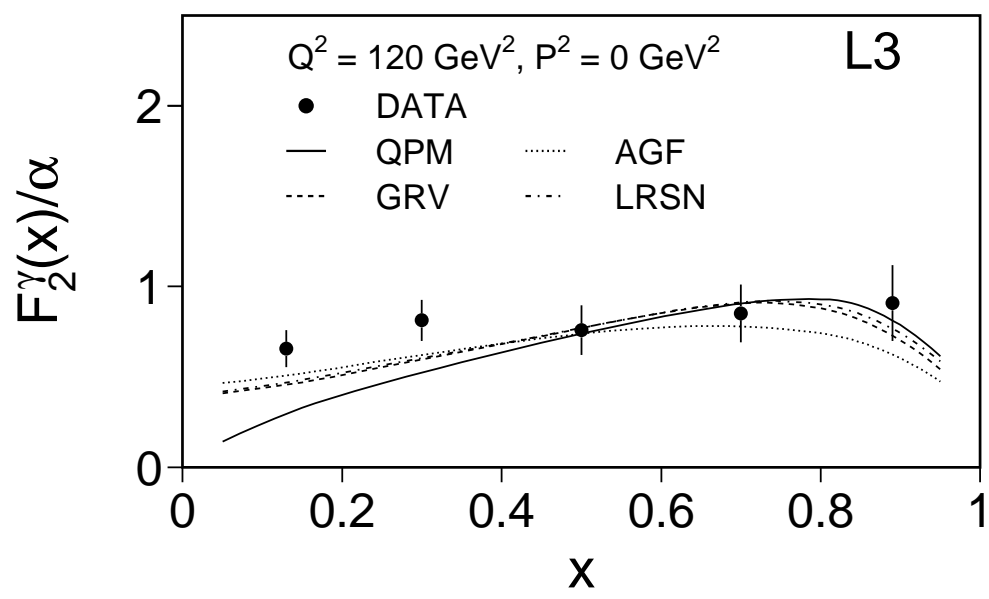

Figure 18: $F_{2}^{\gamma} / \alpha$ as a function of $x_{B j}$ for $\left\langle Q^{2}>=120 \mathrm{GeV}^{2}\right.$ from single-tag events. The solid line corresponds to the QPM prediction, the GRV LO parton parametrization prediction is denoted as a dashed line, AGF - dotted line, and dot-dashed line corresponds to the LRSN prediction (from [42]).

The averaged values of $\left\langle F_{2}^{\gamma} / \alpha>\right.$ as a function of $\left\langle Q^{2}>\right.$ are presented in table 12 for two $x_{B j}$ ranges and in fig. 19 . 
Table 12:

\begin{tabular}{|c|c|c|}
\hline $\begin{array}{c}<Q^{2}> \\
{\left[G e V^{2}\right]}\end{array}$ & $\begin{array}{c}<F_{2}^{\gamma} / \alpha> \\
(\text { stat. }+ \text { syst. })\end{array}$ & $\begin{array}{c}<F_{2}^{\gamma} / \alpha> \\
(\text { stat. }+ \text { syst. })\end{array}$ \\
\hline 60 & $0.73 \pm 0.11 \pm 0.07$ & $0.66 \pm 0.09 \pm 0.06$ \\
90 & $0.89 \pm 0.13 \pm 0.09$ & $0.79 \pm 0.14 \pm 0.08$ \\
125 & $0.85 \pm 0.11 \pm 0.09$ & $0.88 \pm 0.12 \pm 0.08$ \\
225 & $1.01 \pm 0.25 \pm 0.10$ & $1.18 \pm 0.22 \pm 0.11$ \\
\hline
\end{tabular}

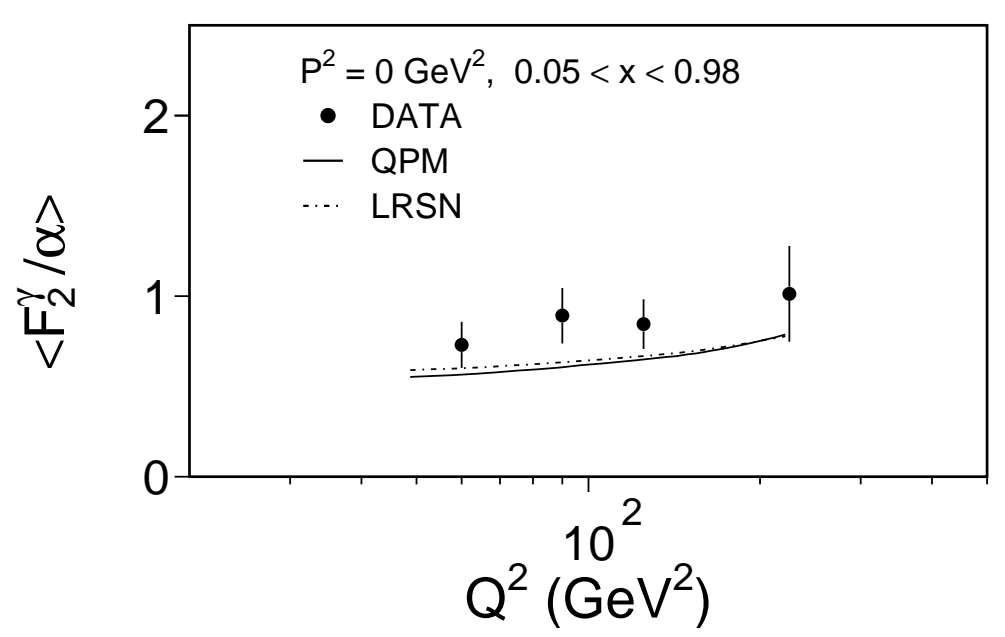

Figure 19: The $Q^{2}$ dependence of $F_{2}^{\gamma} / \alpha$ averaged over $x_{B j}=0.05-0.98$ for single-tag data. Comparison with the QPM prediction (solid line) and the LRSN NLO calculation (dashed line) (from [42]).

The virtuality of the photon is estimated to be $P^{2}=0.014 \mathrm{GeV}^{2}$.

Comment: "The structure function $F_{2}^{\gamma}(x)$ of real photons shows an excess at low $x_{B j}$ over QPM and over several QCD calculations."

\section{-OPAL 94 [44] (LEP 1)}

The measurement of $F_{2}^{\gamma}$ at $\left\langle Q^{2}>=5.9\right.$ and $14.7 \mathrm{GeV}^{2}$ was performed using data from the period 1990-92. The new Monte Carlo TWOGEN program was used to generate events according to chosen formula for $F_{2}^{\gamma}\left(x, Q^{2}, P^{2}\right)$ or $F_{2}^{\gamma}\left(x, Q^{2}\right)$. The VMD with two different distributions of $q \bar{q}$ pair (peripheral and QPM - type) and pointlike QCD contributions for light quarks (in the FKP approach) were studied. The cutoff parameter $p_{T}^{0}$ separating these contributions was determined to be $0.27 \pm 0.10 \mathrm{GeV}$ for both $Q^{2}$ values. The Monte Carlo model with this parameter value was then used to unfold $F_{2}^{\gamma}$. See also sec. 2.3.

Results for unfolded $F_{2}^{\gamma}$, with the charm contribution calculated using QPM, compared with the PLUTO and TPC $/ 2 \gamma$ data are presented in fig. 20. 

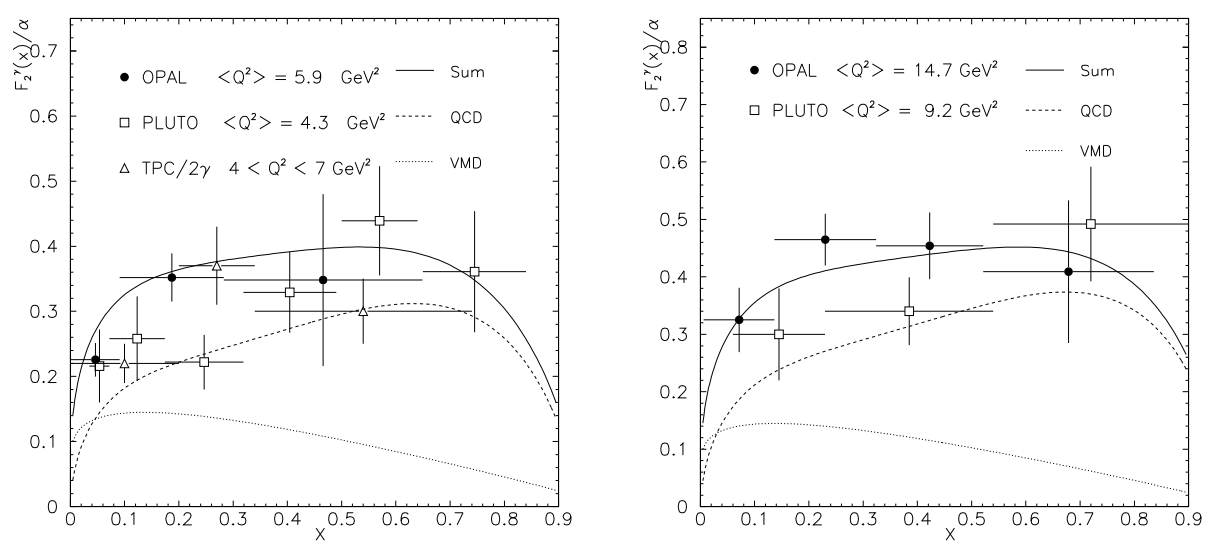

Figure 20: The results for unfolded charm subtracted $F_{2}^{\gamma} / \alpha$ shown and compared with other experiments at similar $\left\langle Q^{2}\right\rangle$ values. The curves show contributions of (left) VMD (dots), $Q C D$-based model (dashes) and their sum (line) for $<Q^{2}>=5.9 \mathrm{GeV}^{2}$, (right) $<Q^{2}>=14.7$ $\mathrm{GeV}^{2}$ (from [4]).

Comment: In the analysis a simple formula $F_{2}^{\gamma} / \alpha=0.2(1-x)$ was applied to describe the VMD contribution. The TWOGEN with a $Q P M$ formula for $F_{2}^{\gamma}\left(x, Q^{2}, P^{2}\right)$ was used to obtain the correction for the $P^{2} \neq 0$.

"No increase of $F_{2}^{\gamma}(x)$ is observed" at small $x_{B j}$.

-OPAL 97a 45] (LEP 1)

The measurement of $F_{2}^{\gamma}$ was done for two samples, $6<Q^{2}<30 \mathrm{GeV}^{2}$ and $60<Q^{2}<$ $400 \mathrm{GeV}^{2}$, using all data at the $Z$ peak (years 1990-95) with new type of unfolding as compared to OPAL 94. The detailed analysis of the hadronic final states was performed and sizeable discrepancies with the expectations were found especially at low $x_{B j}$ (see also next section). The influence of the choice of different Monte Carlo generators (HERWIG 5.8d, PYTHIA 5.718, also for comparisons and systematic checks the F2GEN) on the unfolded $F_{2}^{\gamma}$ was studied. The dependence of $F_{2}^{\gamma}$ on the $P^{2}$ values was estimated based on the SaS1D parametrization.

The results for unfolded $F_{2}^{\gamma}$ are presented in fig. 21a,b,c and in table 13 (the value of $F_{2}^{\gamma} / \alpha$ is given at the centre of the $x_{B j}$ bin). 
Table 13:

\begin{tabular}{|c|c|c|}
\hline $\begin{array}{c}<Q^{2}> \\
{\left[\mathrm{GeV}^{2}\right]}\end{array}$ & $x_{B j}$ & $\begin{array}{c}F_{2}^{\gamma} / \alpha \\
\text { (stat. }+ \text { syst. })\end{array}$ \\
\hline 7.5 & $0.001-0.091$ & $0.28 \pm 0.02_{-0.10}^{+0.03}$ \\
& $0.091-0.283$ & $0.32 \pm 0.02_{-0.13}^{+0.08}$ \\
& $0.283-0.649$ & $0.38 \pm 0.04_{-0.21}^{+0.06}$ \\
\hline 14.7 & $0.006-0.137$ & $0.38 \pm 0.01_{-0.13}^{+0.06}$ \\
& $0.137-0.324$ & $0.41 \pm 0.02_{-0.03}^{+0.06}$ \\
& $0.324-0.522$ & $0.41 \pm 0.03_{-0.11}^{+0.08}$ \\
& $0.522-0.836$ & $0.54 \pm 0.05_{-0.13}^{+0.31}$ \\
\hline 135.0 & $0.100-0.300$ & $0.65 \pm 0.09_{-0.06}^{+0.33}$ \\
& $0.300-0.600$ & $0.73 \pm 0.08_{-0.08}^{+0.04}$ \\
& $0.600-0.800$ & $0.72 \pm 0.10_{-0.07}^{+0.81}$ \\
\hline
\end{tabular}
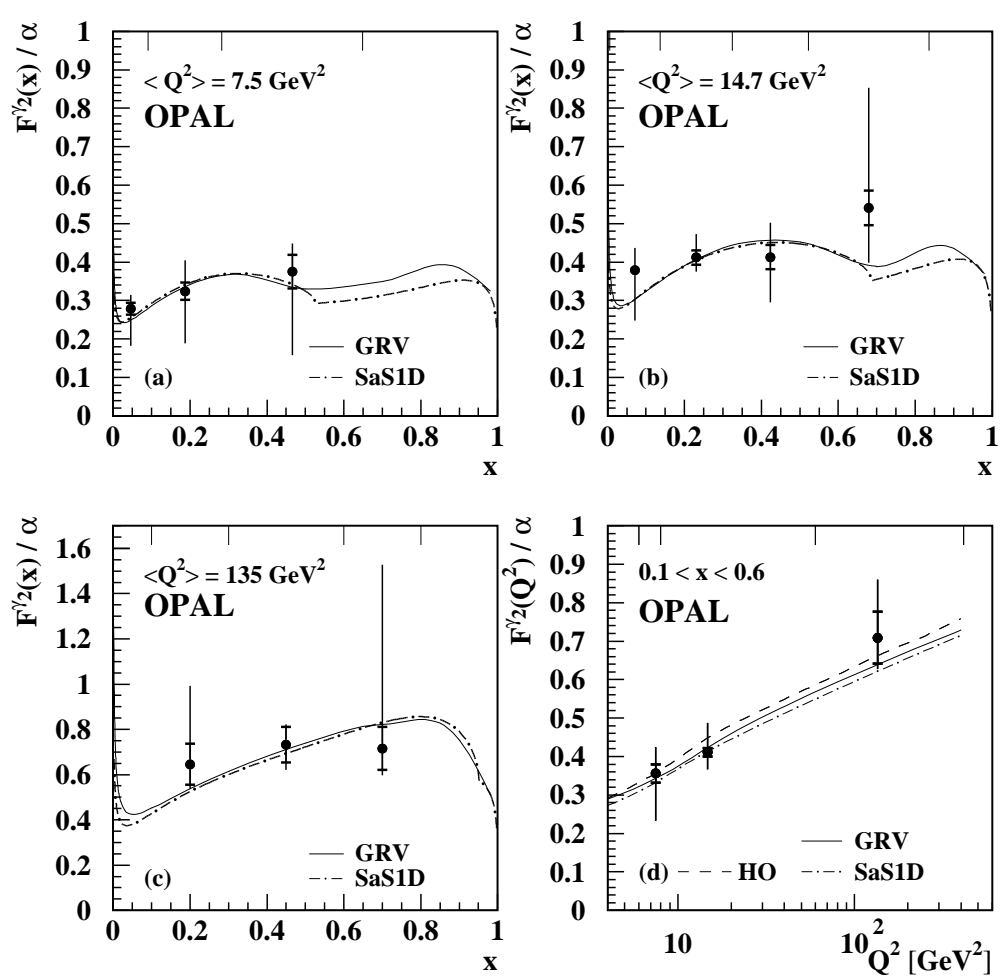

Figure 21: The $F_{2}^{\gamma} / \alpha$ data from the OPAL experiment for the number of flavours $N_{f}=4$. Curves in (a)-(d) show predictions of the GRV and SaS1D parametrizations (from [45]).

The measurement of $F_{2}^{\gamma} / \alpha$ as a function of $Q^{2}$ averaged over the $x_{B j}$ range, $0.1<$ $x_{B j}<0.6$, gives results shown in fig. 21d and in table 14.

The slope $d\left(F_{2}^{\gamma} / \alpha\right) / d \ln Q^{2}$ is measured to be $0.13_{-0.04}^{+0.06}$.

Comment: New measurements of $F_{2}^{\gamma}\left(x, Q^{2}\right)$ are presented, "allowing for the first time for uncertainties in the description of the final state by different Monte Carlo models". The $F_{2}^{\gamma}$ is not correced for $P^{2}$. Large discrepancies between the hadronic energy flow data and Monte Carlo simulations are observed at low $Q^{2}$ for $x_{v i s}<0.1$, when the results are presented versus pseudorapidity or azimuthal angle (see next section). 
Table 14:

\begin{tabular}{|c|c|}
\hline $\begin{array}{c}<Q^{2}> \\
{\left[\mathrm{GeV}^{2}\right]}\end{array}$ & $\begin{array}{c}<F_{2}^{\gamma} / \alpha> \\
\text { (stat. }+ \text { syst. })\end{array}$ \\
\hline 7.5 & $0.36 \pm 0.02_{-0.12}^{+0.06}$ \\
14.7 & $0.41 \pm 0.01_{-0.04}^{+0.08}$ \\
135.0 & $0.71 \pm 0.07_{-0.05}^{+0.14}$ \\
\hline
\end{tabular}

\section{-OPAL 97b 46 (LEP 1)}

The measurement of $F_{2}^{\gamma}$ (years 1993-94) was done for $1.1<Q^{2}<2.5 \mathrm{GeV}^{2}$ and $2.5<Q^{2}<6.6 \mathrm{GeV}^{2}$ (average $Q^{2}=1.86$ and $3.76 \mathrm{GeV}^{2}$ ) as a function of $x_{B j}$, reaching the lowest, at that time, measured (center of bin on a logarithmic scale) value: $x_{B j}=0.004$. For a better sensitivity on the low $x_{B j}$ region the unfolding procedure was performed on a logarithmic scale. Final state topology was analysed as well (using the HERWIG 5.18d, PYTHIA 5.722, F2GEN - both with the pointlike and the peripheral $q \bar{q}$ distributions generators). The GRV LO and SaS1D parton parametrizations were used in the analysis. Charm contribution is treated differently in different MC programs. Discrepancy between the data (hadron energy flow) and results from the Monte Carlo generators, as well as between different models was found for low $x_{v i s}<0.05$ (see sec. 2.3).

The obtained values of $F_{2}^{\gamma} / \alpha$ are given in table 15 and shown in fig. 22 together with early measurements from PLUTO and TPC/ $2 \gamma$.

Table 15:

\begin{tabular}{|c|r|c|}
\hline $\begin{array}{c}<Q^{2}> \\
{\left[\mathrm{GeV}^{2}\right]}\end{array}$ & \multicolumn{1}{|c|}{$x_{B j}$} & $\begin{array}{c}F_{2}^{\gamma} / \alpha \\
\text { stat. }+ \text { syst. })\end{array}$ \\
\hline 1.86 & $0.0025-0.0063$ & $0.27 \pm 0.03_{-0.07}^{+0.05}$ \\
& $0.0063-0.020$ & $0.22 \pm 0.02_{-0.05}^{+0.02}$ \\
& $0.020-0.040$ & $0.20 \pm 0.02_{-0.02}^{+0.09}$ \\
& $0.040-0.100$ & $0.23 \pm 0.02_{-0.05}^{+0.03}$ \\
\hline 3.76 & $0.0063-0.020$ & $0.35 \pm 0.03_{-0.08}^{+0.08}$ \\
& $0.020-0.040$ & $0.29 \pm 0.03_{-0.06}^{+0.06}$ \\
& $0.040-0.100$ & $0.32 \pm 0.02_{-0.05}^{+0.07}$ \\
& $0.100-0.200$ & $0.32 \pm 0.03_{-0.04}^{+0.08}$ \\
\hline
\end{tabular}



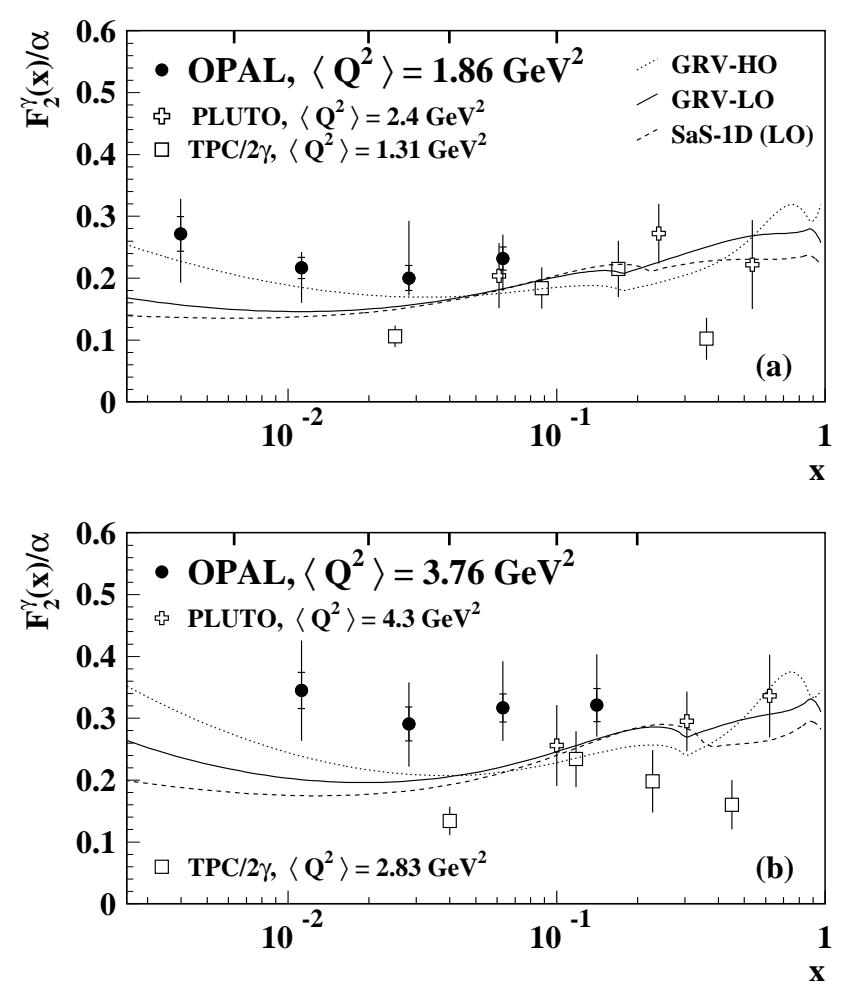

Figure 22: The OPAL $F_{2}^{\gamma} / \alpha$ data (circles) as a function of $x_{B j}$ for $<Q^{2}>=1.86 \mathrm{GeV}^{2}$ (a) and $\left\langle Q^{2}\right\rangle=3.76 \mathrm{GeV}^{2}$ (b). Also data from PLUTO (crosses) for $\left\langle Q^{2}>=2.4,4.3 \mathrm{GeV}^{2}\right.$ and TPC $/ 2 \gamma$ (squares) for $\left\langle Q^{2}>=1.31,2.83 \mathrm{GeV}^{2}\right.$ are shown. The curves show predictions of the GRV HO (dots), GRV LO (line), and SaS1D (dashed) parametrizations. The range of the $x_{B j}$-bins of the OPAL results are marked at the tops of figures (from [46]).

Comment: No correction for $P^{2} \neq 0$ was made. GRV HO is consistent with the low-x $O P A L$ results in the lower $Q^{2}$ bin, but at higher $Q^{2}$ it underestimates the low $x$ OPAL data. GRV LO and SaS1D describe the unfolded results worse than GRV HO. Shapes of measured $F_{2}^{\gamma}$ are flat within the errors, but a small rise in the low $x$ region is not excluded.

-OPAL 97c [47 (LEP 2)

New data on $F_{2}^{\gamma}$ from LEP 2 at the CM energies 161-172 $\mathrm{GeV}$ were collected in 1996 in two samples $Q^{2}=6-20 \mathrm{GeV}^{2}\left(0.004<x_{B j}<0.76\right)$ and $Q^{2}=20-100 \mathrm{GeV}^{2}$ $\left(0.012<x_{B j}<0.94\right)$. Also the distribution of the final hadronic energy flow was studied using HERWIG 5.9, PYTHIA 5.718 and F2GEN, see next section. The LEP1 OPAL 97a data were used for comparison. For unfolding the Monte Carlo based on HERWIG program with the GRV parton parametrization was used. Two types of binning were performed, with $\left\langle Q^{2}>=9,14.5,30\right.$ and $59 \mathrm{GeV}^{2}$, and with $<Q^{2}>$ $=11$ (combined samples 9 and $14.5 \mathrm{GeV}^{2}$ ) and $41 \mathrm{GeV}^{2}\left(30\right.$ and $59 \mathrm{GeV}^{2}$ ).

The unfolded $F_{2}^{\gamma} / \alpha$ as a function of $x_{B j}$ and $Q^{2}$ are presented in figs. 23 and 24 and in tables 16, 17. 
Table 16:

\begin{tabular}{|c|r|c|}
\hline $\begin{array}{c}<Q^{2}> \\
{\left[G e V^{2}\right]}\end{array}$ & \multicolumn{1}{|c|}{$x_{B j}$} & $\begin{array}{c}F_{2}^{\gamma} / \alpha \\
\text { stat. }+ \text { syst. })\end{array}$ \\
\hline 9 & $0.02-0.1$ & $0.33 \pm 0.03_{-0.06}^{+0.06}$ \\
& $0.100-0.250$ & $0.29 \pm 0.04_{-0.05}^{+0.04}$ \\
& $0.250-0.600$ & $0.39 \pm 0.08_{-0.10}^{+0.30}$ \\
\hline 14.5 & $0.02-0.1$ & $0.37 \pm 0.03_{-0.01}^{+0.16}$ \\
& $0.100-0.250$ & $0.42 \pm 0.05_{-0.14}^{+0.04}$ \\
& $0.250-0.600$ & $0.39 \pm 0.06_{-0.11}^{+0.10}$ \\
\hline 30 & $0.050-0.100$ & $0.32 \pm 0.04_{-0.02}^{+0.11}$ \\
& $0.100-0.350$ & $0.52 \pm 0.05_{-0.13}^{+0.06}$ \\
& $0.350-0.600$ & $0.41 \pm 0.09_{-0.05}^{+0.20}$ \\
& $0.600-0.800$ & $0.46 \pm 0.15_{-0.14}^{+0.39}$ \\
\hline 59 & $0.050-0.100$ & $0.37 \pm 0.06_{-0.07}^{+0.28}$ \\
& $0.100-0.350$ & $0.44 \pm 0.07_{-0.07}^{+0.08}$ \\
& $0.350-0.600$ & $0.48 \pm 0.09_{-0.10}^{+0.16}$ \\
& $0.600-0.800$ & $0.51 \pm 0.14_{-0.02}^{+0.48}$ \\
\hline
\end{tabular}

Table 17:

\begin{tabular}{|c|c|}
\hline $\begin{array}{c}<Q^{2}> \\
{\left[\mathrm{GeV}^{2}\right]}\end{array}$ & $\begin{array}{c}<F_{2}^{\gamma} / \alpha> \\
\text { (stat. }+ \text { syst. })\end{array}$ \\
\hline 9 & $0.36 \pm 0.05_{-0.06}^{+0.08}$ \\
14.5 & $0.41 \pm 0.04_{-0.01}^{+0.04}$ \\
30 & $0.48 \pm 0.05_{-0.07}^{+0.06}$ \\
59 & $0.46 \pm 0.06_{-0.04}^{+0.07}$ \\
\hline
\end{tabular}



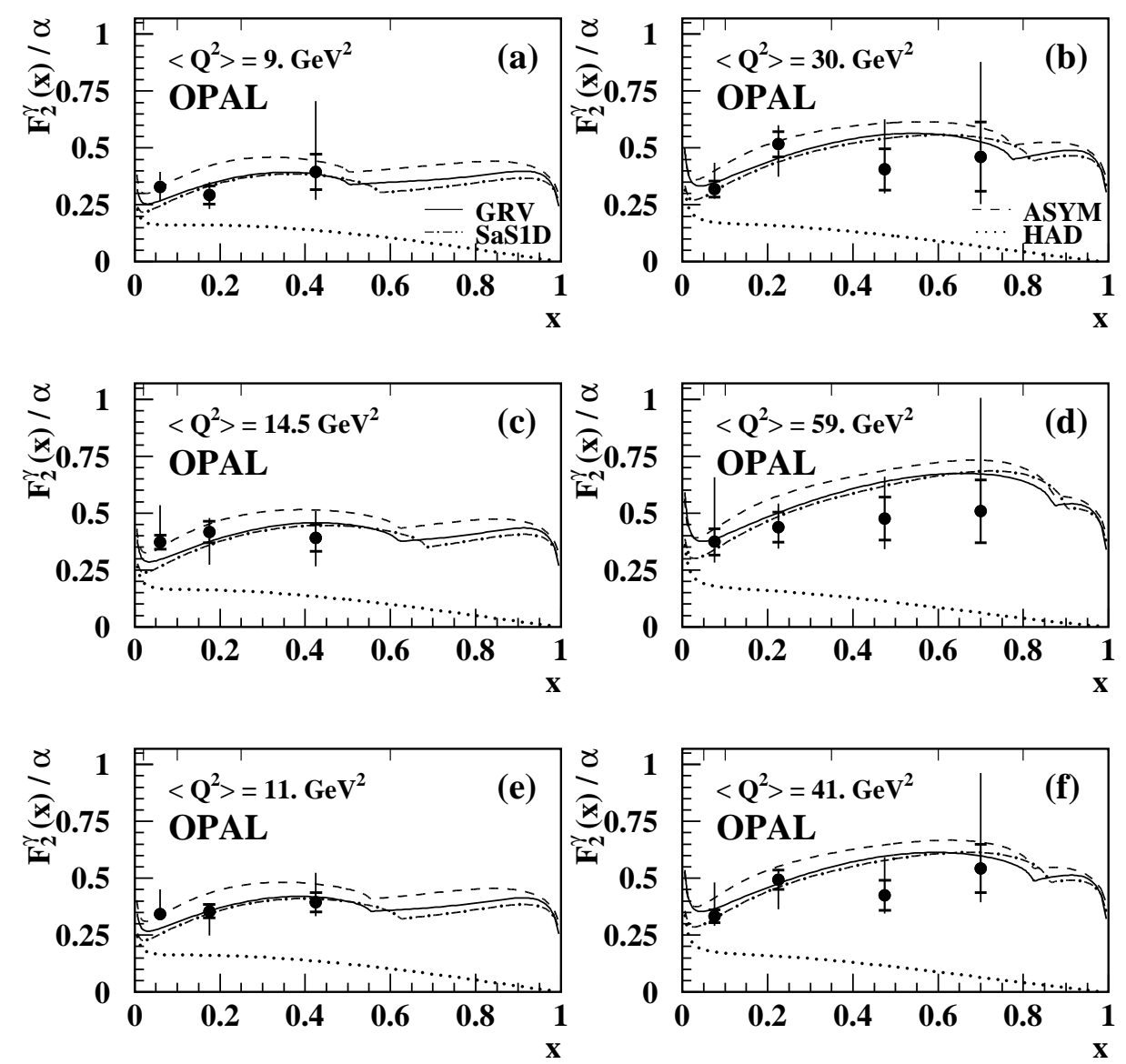

Figure 23: The $x_{B j}$-dependence of $F_{2}^{\gamma} / \alpha$ for different values of $\left\langle Q^{2}>\right.$ : 9, 30, 14.5, 59, 11, and $41 \mathrm{GeV}^{2}$. For comparison the GRV LO (solid line) and SaS1D (dot-dashed line) parametrizations are shown; dotted line represents the hadronic component and the dashed one the (augmented) asymptotic solution (ASYM) (from [4]).

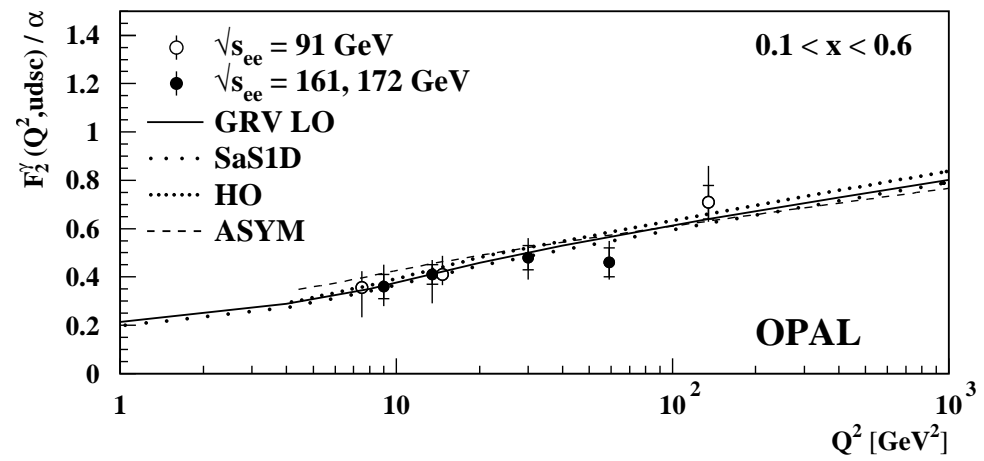

Figure 24: The $Q^{2}$ - dependence of $F_{2}^{\gamma} / \alpha$ averaged over $0.1<x_{B j}<0.6$, for the energy 91 $\mathrm{GeV}$ (open circles) and for the energies 161, $172 \mathrm{GeV}$ (full circles). Predictions of the QCD calculation are shown by the lines: solid (GRV LO), dotted (SaS1D), and double-dotted (HO, based on the GRV HO parametrization for light quarks); dashed line corresponds to the (augmented) asymptotic solution (ASYM) (from [47]).

A fit to the new $F_{2}^{\gamma}$ data at the energies $161-172 \mathrm{GeV}$ and the previous OPAL 97a set at $91 \mathrm{GeV}$ for $Q^{2}$ from 7.5 to $135 \mathrm{GeV}^{2}$, averaged over the $x_{B j}$ range of $0.1-0.6$ 
(see fig. 24), has the form:

$$
F_{2}^{\gamma}\left(Q^{2}\right) / \alpha=\left(0.16 \pm 0.05_{-0.16}^{+0.17}\right)+\left(0.10 \pm 0.02_{-0.02}^{+0.05}\right) \ln \left(Q^{2} / \mathrm{GeV}^{2}\right) .
$$

A special study of the $Q^{2}$ dependence of $F_{2}^{\gamma}$ is performed for different $x_{B j}$ ranges, see fig. 25.

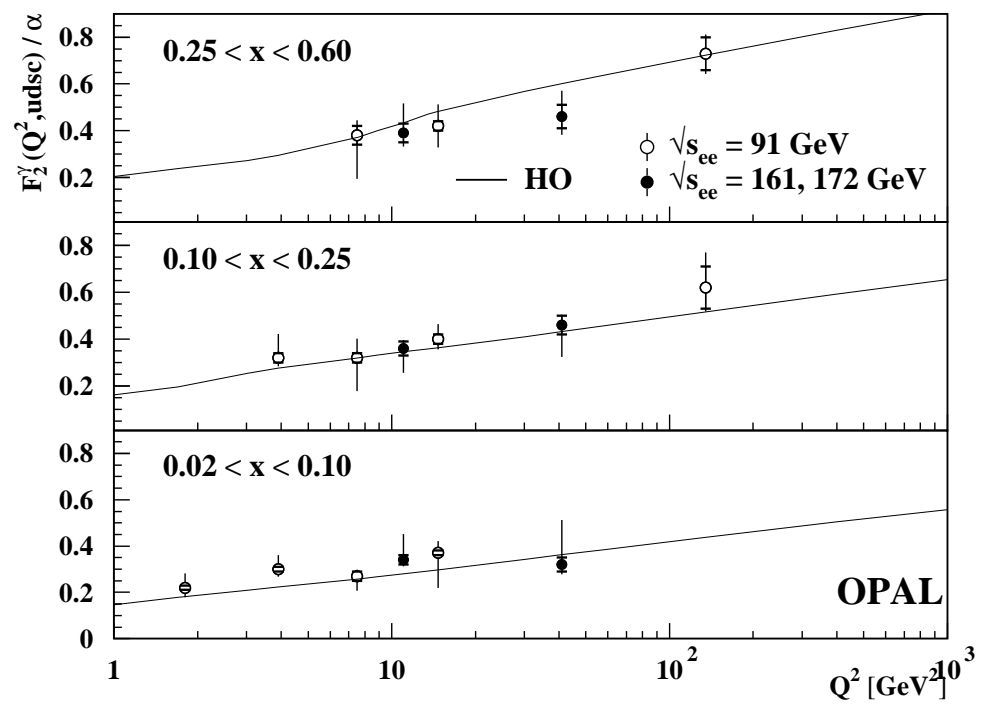

Figure 25: The $Q^{2}$ dependence of $F_{2}^{\gamma} / \alpha$ in three $x_{B j}$ bins. The data are compared to HO predictions based on the GRV HO parametrization (from [47]).

Comment: No correction for $P^{2} \neq 0$ was made. Discrepancies are observed in the hadronic energy flow between the data and the HERWIG and PYTHIA simulations, especially at $x_{v i s}<0.1$. Accuracy of the data does not allow to see the expected different slope of $F_{2}^{\gamma}$ versus $\ln Q^{2}$ for different $x_{B j}$ ranges.

\section{-OPAL 2000 [48] (LEP 1, LEP 2)}

The low $x_{B j}$ data of $F_{2}^{\gamma}$ obtained at the CM energies $91 \mathrm{GeV}, 183$ and $189 \mathrm{GeV}$ (in 1993-5 and 1997-8) were collected in the partly overlapping $Q^{2}$ ranges: $1.5-30 \mathrm{GeV}^{2}$ at LEP1 and 7-30 $\mathrm{GeV}^{2}$ at LEP2. Three samples: LEP1 SW $\left(<Q^{2}>=1.9\right.$ and 3.7 $\left.\mathrm{GeV}^{2}\right)$, LEP1 FD $\left(<Q^{2}>=8.9\right.$ and $\left.17.5 \mathrm{GeV}^{2}\right)$ and LEP2 SW $\left(<Q^{2}>=10.7\right.$ and $17.8 \mathrm{GeV}^{2}$ ) were studied. In the analysis new Monte Carlo programs and improved unfolding methods were introduced. The final state was studied using PHOJET 1.05, HERWIG 5.9, "HERWIG $5.9+$ power law $p_{t}$ (dyn)" and F2GEN, with the GRV LO parametrization as an input function, see next section for details.

"HERWIG $5.9+$ power law $p_{t}$ (dyn)" and PHOJET 1.05, giving better description of OPAL data, were applied to obtain the $F_{2}^{\gamma}$ structure function. To reduce dependence of results on the implemented MC model, two-dimensional unfolding was performed with $x_{c o r}$ used as a first variable and $E_{T}^{\text {out }} / E_{\text {tot }}$ as a second one. To investigate the low- $x_{B j}$ range, the unfolding of the $x_{B j}$ variable was done on a logarithmic scale.

The values of $F_{2}^{\gamma} / \alpha$ were obtained as an average of results of the unfolding with "HERWIG $5.9+$ power law $p_{t}(\mathrm{dyn})$ " and PHOJET $1.05 \mathrm{c}$, see table $18\left(x_{B j}^{*}\right.$ corresponds to the log centre of the bin for $x_{B j}$ below the charm threshold. The $F_{2}^{\gamma} / \alpha$ was unfolded in the quoted bins and corrected to the $x_{B j}^{*}$ values). 
The unfolded $F_{2}^{\gamma} / \alpha$ as a function of $x_{B j}$ for $Q^{2}=1.9$ and $3.7 \mathrm{GeV}^{2}$ is slightly lower than the previous OPAL results obtained using HERWIG 5.8d (OPAL 97b), see fig. 26. In fig. 27 the comparison with other measurements and parton parametrizations predictions for $Q^{2}$ range $1.9-5 \mathrm{GeV}^{2}$ is shown.

The results for the unfolded $F_{2}^{\gamma}$ at $\left\langle Q^{2}>=8.9,10.7,17.5\right.$ and $17.8 \mathrm{GeV}^{2}$ (similar to the previous ones, OPAL 97b; comparison not shown) are compared in fig. 28 with results of other experiments and predictions of various parametrizations, in similar $Q^{2}$ range.

Table 18:

\begin{tabular}{|c|c|c|c|}
\hline $\begin{array}{c}<Q^{2}> \\
{\left[G e V^{2}\right]}\end{array}$ & $x_{B j}$ & $\begin{array}{c}x_{B j}^{*} \\
\text { log centre } \\
\text { of bin })\end{array}$ & $\begin{array}{c}F_{2}^{\gamma} / \alpha \\
\text { stat. }+ \text { syst. })\end{array}$ \\
\hline 1.9 & $0.0006-0.0028$ & 0.0012 & $0.269 \pm 0.027_{-0.034}^{+0.018}$ \\
& $0.0028-0.0143$ & 0.0063 & $0.177 \pm 0.009_{-0.017}^{+0.017}$ \\
& $0.0143-0.0724$ & 0.0322 & $0.179 \pm 0.007_{-0.006}^{+0.007}$ \\
& $0.0724-0.3679$ & 0.1124 & $0.227 \pm 0.010_{-0.012}^{+0.012}$ \\
\hline 3.7 & $0.0015-0.0067$ & 0.0032 & $0.269 \pm 0.033_{-0.033}^{+0.047}$ \\
& $0.0067-0.0302$ & 0.0143 & $0.232 \pm 0.013_{-0.021}^{+0.023}$ \\
& $0.0302-0.1353$ & 0.0639 & $0.259 \pm 0.010_{-0.013}^{+0.006}$ \\
& $0.1353-0.6065$ & 0.1986 & $0.296 \pm 0.014_{-0.022}^{+0.029}$ \\
\hline 8.9 & $0.0111-0.0498$ & 0.0235 & $0.221 \pm 0.017_{-0.026}^{+0.030}$ \\
& $0.0498-0.2231$ & 0.1054 & $0.308 \pm 0.014_{-0.012}^{+0.011}$ \\
& $0.2231-0.8187$ & 0.3331 & $0.379 \pm 0.022_{-0.015}^{+0.017}$ \\
\hline 10.7 & $0.0009-0.0050$ & 0.0021 & $0.362 \pm 0.045_{-0.039}^{+0.058}$ \\
& $0.0050-0.0273$ & 0.0117 & $0.263 \pm 0.015_{-0.030}^{+0.032}$ \\
& $0.0273-0.1496$ & 0.0639 & $0.275 \pm 0.011_{-0.030}^{+0.029}$ \\
& $0.1496-0.8187$ & 0.3143 & $0.351 \pm 0.012_{-0.016}^{+0.025}$ \\
\hline 17.5 & $0.0235-0.0821$ & 0.0439 & $0.273 \pm 0.028_{-0.039}^{+0.032}$ \\
& $0.0821-0.2865$ & 0.1534 & $0.375 \pm 0.023_{-0.020}^{+0.020}$ \\
& $0.2865-0.9048$ & 0.3945 & $0.501 \pm 0.027_{-0.019}^{+0.027}$ \\
\hline 17.8 & $0.0015-0.0074$ & 0.0033 & $0.428 \pm 0.061_{-0.071}^{+0.055}$ \\
& $0.0074-0.0369$ & 0.0166 & $0.295 \pm 0.019_{-0.020}^{+0.033}$ \\
& $0.0369-0.1827$ & 0.0821 & $0.336 \pm 0.013_{-0.042}^{+0.041}$ \\
& $0.1827-0.9048$ & 0.3483 & $0.430 \pm 0.013_{-0.025}^{+0.032}$ \\
\hline
\end{tabular}




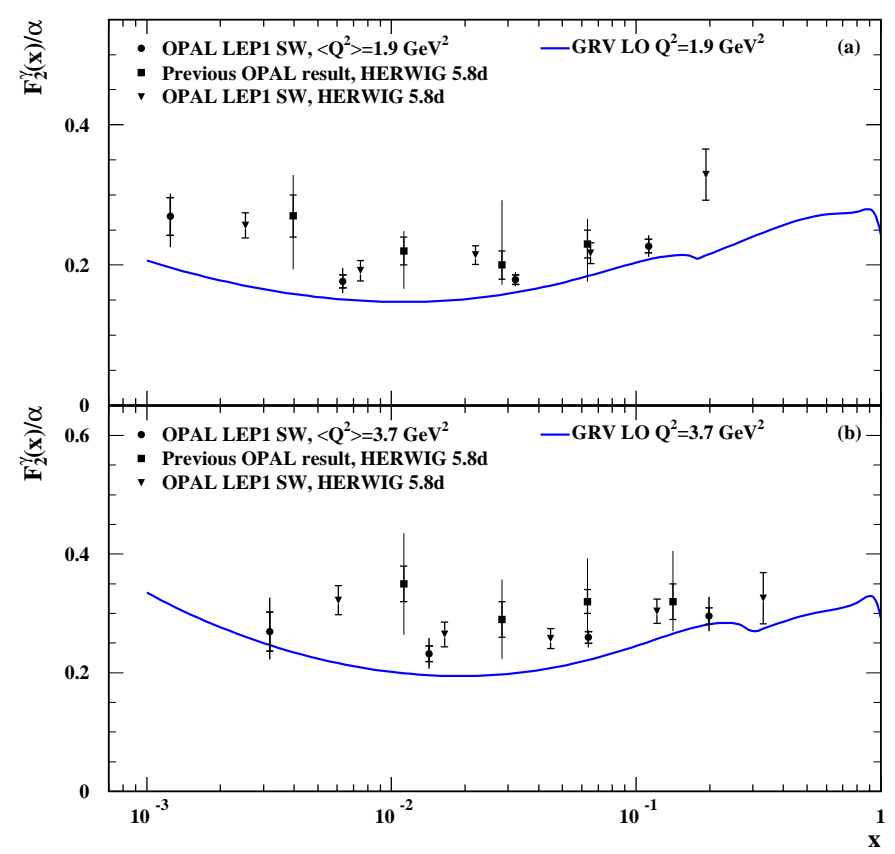

Figure 26: The $x_{B j}$-dependence of $F_{2}^{\gamma} / \alpha$ measured by OPAL for the LEP1 SW sample with $<Q^{2}>$ equal to a) $1.9 \mathrm{GeV}^{2}$ and b) $3.7 \mathrm{GeV}^{2}$. For comparison the previous $O P A L$ data (OPAL 97b) and the present sample, both obtained using HERWIG 5.8d, are presented. The GRV LO predictions are also shown (from [48]).

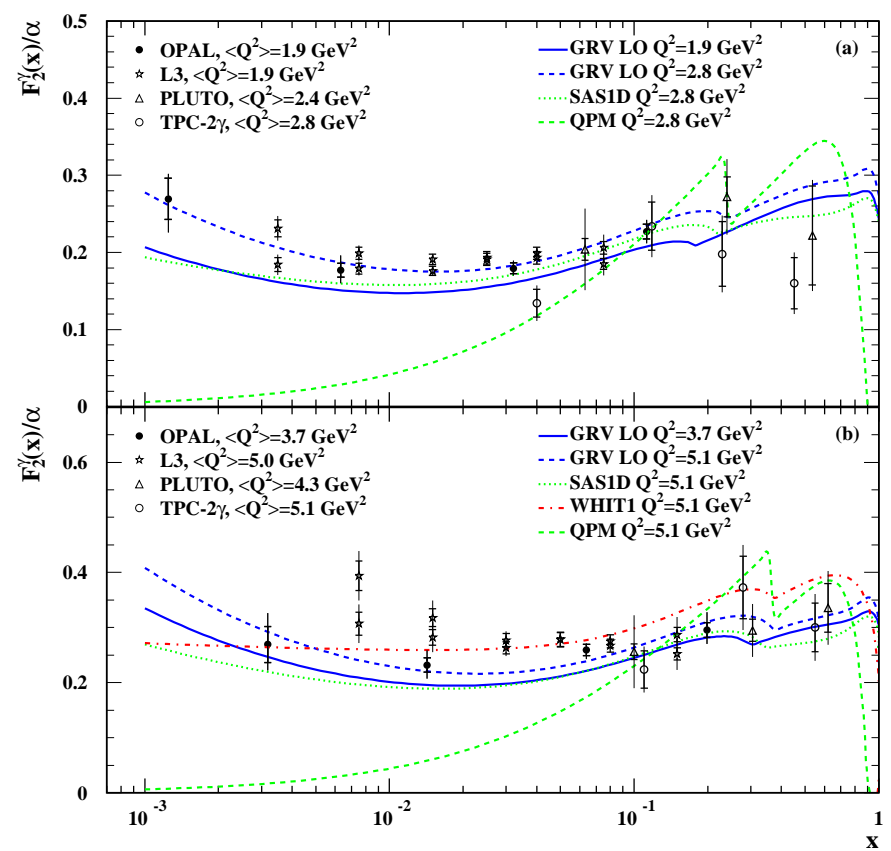

Figure 27: The $x_{B j}$-dependence of $F_{2}^{\gamma} / \alpha$ measured by OPAL for the LEP1 SW sample with $<Q^{2}>$ equal to a) 1.9 and b) $3.7 \mathrm{GeV}^{2}$. For comparison the (L3 98a), PLUTO [4] and $T P C / 2 \gamma$ [4] data are shown together with GRV LO, SaS1D and QPM parton parametrizations predictions (from [48]). 


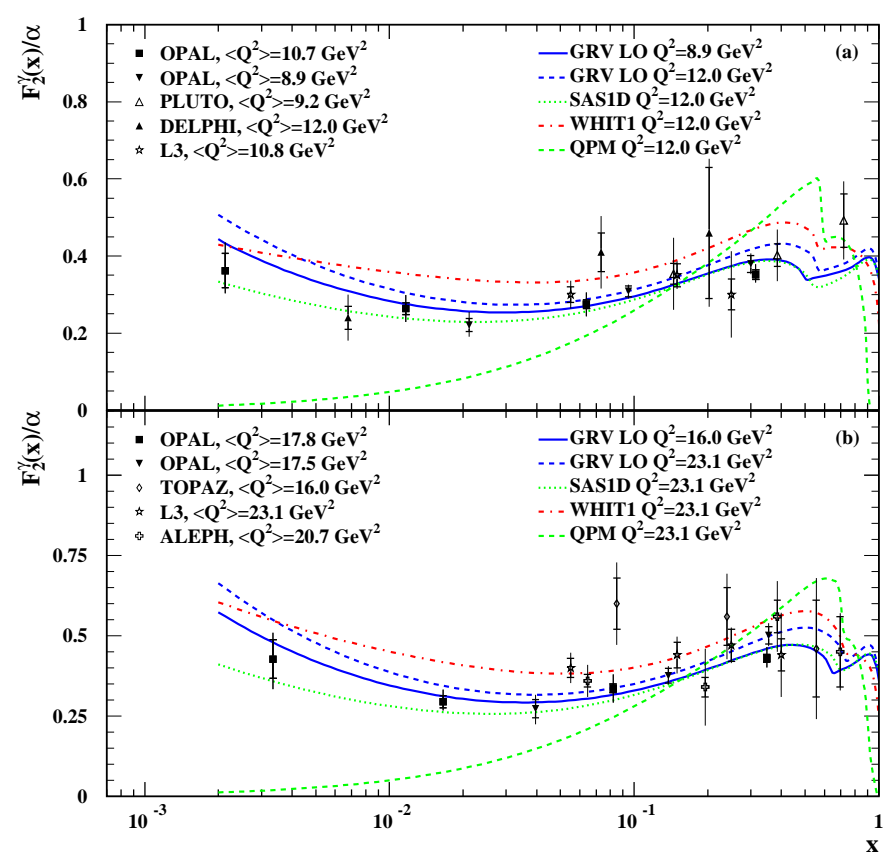

Figure 28: The $x_{B j}$-dependence of $F_{2}^{\gamma} / \alpha$ measured by $O P A L$ for $<Q^{2}>$ equal to a) 8.9 (LEP1 FD) and $10.7 \mathrm{GeV}^{2}$ (LEP2 SW), and b) 17.5 (LEP1 FD) and 17.8 (LEP2 SW) GeV ${ }^{2}$. For comparison the ALEPH 99a, DELPHI 96a, L3 99a, PLUTO [ब] and TOPAZ 94 data are shown with predictions of GRV LO, SaS1D, WHIT1 and QPM (from [48]).

Comment: No correction for $P^{2} \neq 0$ was made. The $F_{2}^{\gamma}$ measurement at "the lowest attainable $x_{B j}$ values", uses new MC models and two-dimensional unfolding, on a logarithmic scale.

"The GRV LO and SaS1D parametrizations are generally consistent with the OPAL data in all the accessible $x_{B j}$ and $Q^{2}$ regions, with the exception of the measurement at the lowest scale, $\left\langle Q^{2}\right\rangle=1.9$ GeV $V^{2}$, where GRV is too low."

The precision of the measurement is insufficient to determine whether or not there is a rise in $F_{2}^{\gamma}$ at low $x_{B j}$.

\section{-AMY 1.595 [49] (TRISTAN)}

The upgraded AMY 1.5 detector was used to perform a high $Q^{2}$ measurement of the photon structure function $F_{2}^{\gamma}$ (with averaged $Q^{2}=73$ and $390 \mathrm{GeV}^{2}$ ). For previous measurement done with AMY 1.0 see [5]. In the Monte Carlo analysis the FKP approach was used to describe light quarks (with $p_{T}^{0} \approx 0.5 \mathrm{GeV}$ ), the QPM for $\mathrm{c}$ and b-quarks, and VMD contribution for soft hadronic processes were introduced. Results for unfolded $F_{2}^{\gamma}$ are presented in table 19 and in fig. 29, where predictions of various parton parametrizations as well as individual components are shown. 
Table 19:

\begin{tabular}{|c|c|c|}
\hline $\begin{array}{c}<Q^{2}> \\
{\left[\mathrm{GeV}^{2}\right]}\end{array}$ & $<x_{B j}>$ & $\begin{array}{c}F_{2}^{\gamma} / \alpha \\
\text { (stat. }+ \text { syst. })\end{array}$ \\
\hline 73 & 0.25 & $0.65 \pm 0.08 \pm 0.06$ \\
& 0.50 & $0.60 \pm 0.16 \pm 0.03$ \\
& 0.75 & $0.65 \pm 0.11 \pm 0.08$ \\
\hline 390 & 0.31 & $0.94 \pm 0.23 \pm 0.10$ \\
& 0.69 & $0.82 \pm 0.16 \pm 0.11$ \\
\hline
\end{tabular}

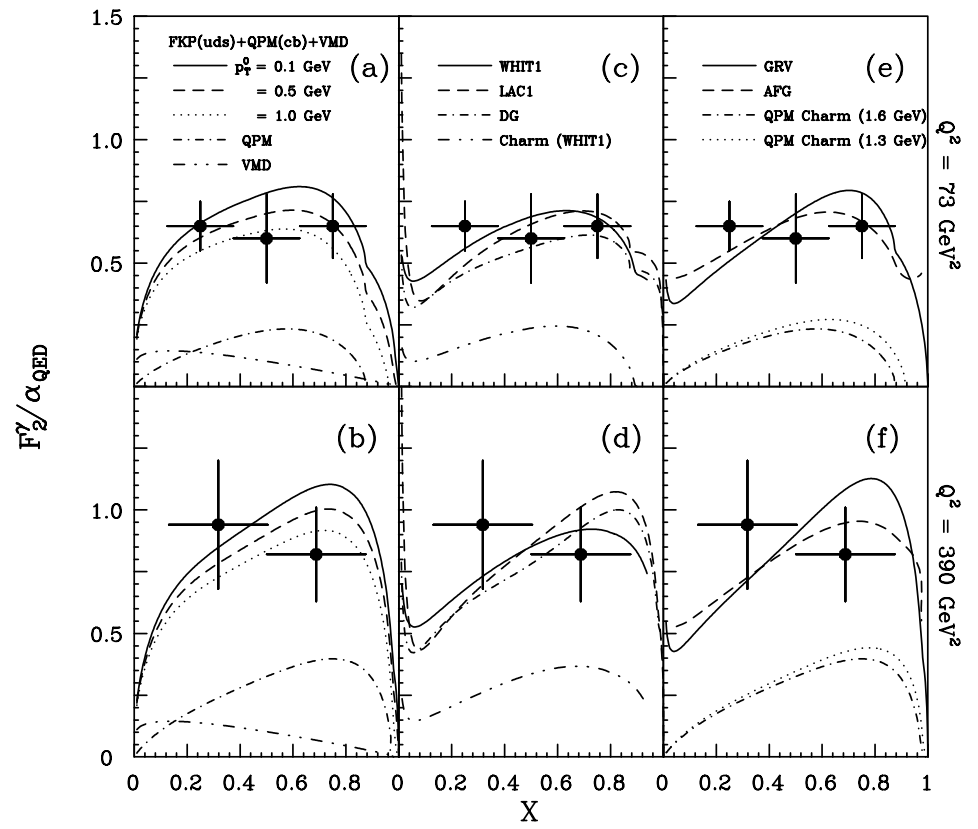

Figure 29: The $F_{2}^{\gamma} / \alpha$ data from the AMY collaboration. Comparison with the following contributions to the photon structure: a) and b) FKP (u,d,s) with various $p_{T}^{0}, Q P M(c$, b) with various charm masses, VMD. Comparison with parton parametrizations: c) and d) WHIT1, LAC1, DG; e) and f) GRV, AFG. Upper a),c),e) and lower b),d),f) figures correspond to averaged $Q^{2}=73 \mathrm{GeV}^{2}$ and $Q^{2}=390 \mathrm{GeV}^{2}$, respectively (from [49]).

The values of $F_{2}^{\gamma}$ averaged over $0.3<x_{B j}<0.8$ are shown in table 20 .

Table 20:

\begin{tabular}{|c|c|}
\hline $\begin{array}{c}<Q^{2}> \\
{\left[G e V^{2}\right]}\end{array}$ & $<F_{2}^{\gamma} / \alpha>$ \\
\hline 73 & $0.63 \pm 0.07$ \\
390 & $0.85 \pm 0.18$ \\
\hline
\end{tabular}

Comment: The observed $x_{B j}$-behaviour of $F_{2}^{\gamma}$ is consistent with the GRV parametrization and with the FKP one. The fitted parameter $p_{T}^{0}$ is equal to $0.51 \pm 0.39$ GeV if only 
the AMY data are included, and $0.45 \pm 0.07 \mathrm{GeV}$ if all available, at that time, $F_{2}^{\gamma}$ data are taken.

\section{•AMY 1.597 [50 (TRISTAN)}

The measurement of $F_{2}^{\gamma}$ at $\left\langle Q^{2}>=6.8 \mathrm{GeV}^{2}\right.$ was performed. In the Monte Carlo analysis the FKP approach was used to describe light quarks (with $p_{T}^{0} \approx 0.5 \mathrm{GeV}$ ), the QPM for c-quark, and VMD contribution for soft hadronic processes were introduced. The results for $F_{2}^{\gamma}$ versus $x_{B j}$, together with earlier data, are presented in fig. 30. A comparison of the data with various parametrizations is given in fig. 31 .

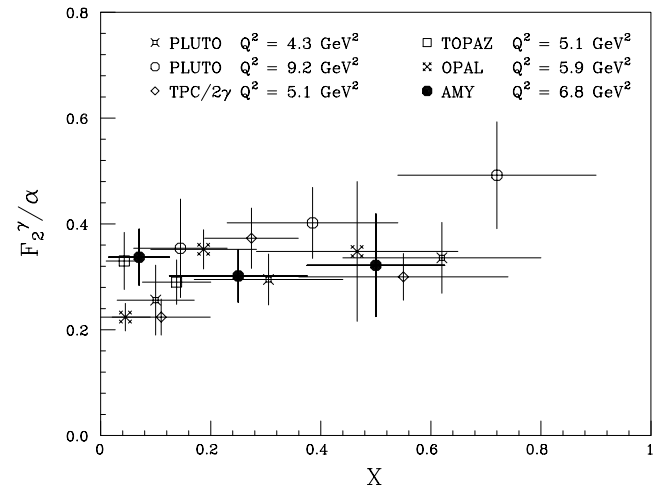

Figure 30: The $F_{2}^{\gamma} / \alpha$ data from the $A M Y$ collaboration in comparison with the results of other experiments at $Q^{2} \sim 4-9 \mathrm{GeV}^{2}$ (from [50]).
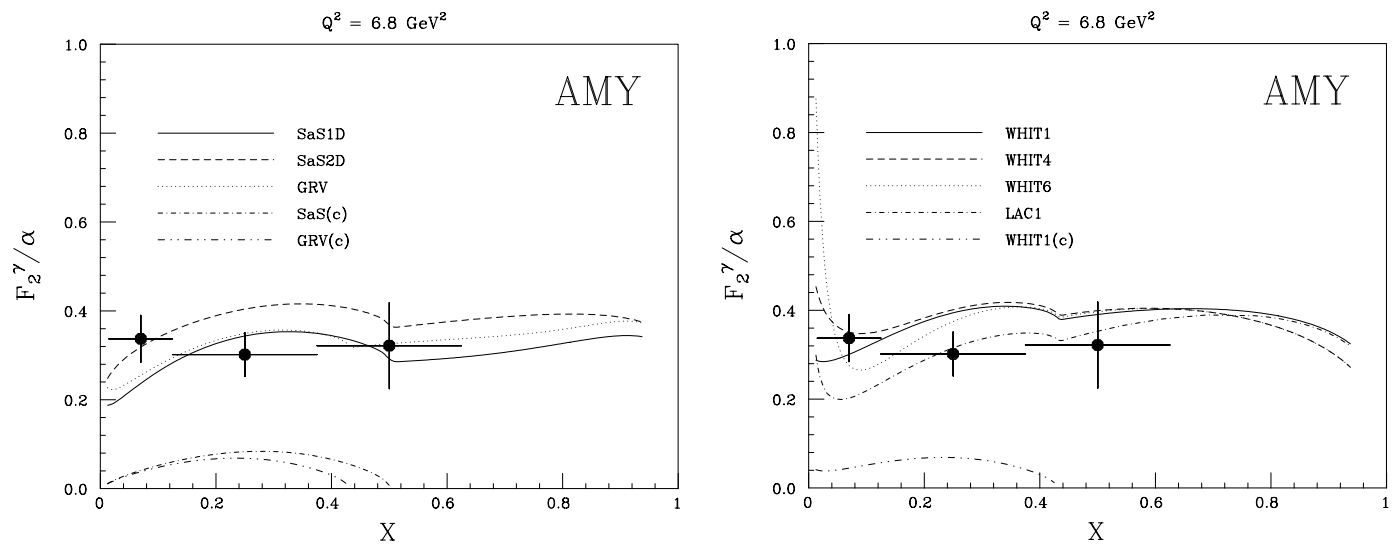

Figure 31: The $F_{2}^{\gamma} / \alpha$ as a function of $x_{B j}$. Left: Comparison with the SaS1D, SaS2D and GRV LO parton parametrization predictions. Also shown are the c-quark contributions from the SaS and GRV LO parametrizations. Right: Comparison with the WHIT1, WHIT4, WHIT6 and LAC1 predictions. The c-quark contribution is shown for the WHIT1 parametrization (from [50]).

Comment: "The $x_{B j}$ behaviour of the measured $F_{2}^{\gamma}$ is consistent with the QCD-based predictions such as SaS, GRV LO and WHIT models, but inconsistent with the LAC1 prediction for $x_{B j}$ values around 0.07."

-TOPAZ 94 [51] (TRISTAN)

The photon structure function $F_{2}^{\gamma}$ has been measured for averaged $Q^{2}$ values from 5.1 to 80 , and also $338 \mathrm{GeV}^{2}$. The Monte Carlo with FKP contribution included for light 
quarks (with $p_{T}^{0}=0.1-1 \mathrm{GeV}$ ), the QPM one for charm quark and VMD contribution for a soft particle production. The one and two-jet events ("hadronic" events with a stuck quark and the pointlike ones based on $\gamma^{*} \gamma \rightarrow q \bar{q}$, respectively) in this sample

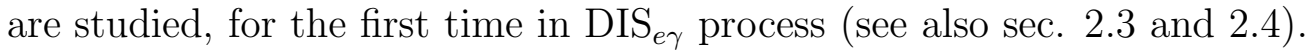

Table 21:

\begin{tabular}{|c|c|l|l|c|}
\hline $\begin{array}{c}<Q^{2}> \\
{\left[\mathrm{GeV}^{2}\right]}\end{array}$ & $\begin{array}{c}Q^{2} \\
{\left[\mathrm{GeV}^{2}\right]}\end{array}$ & $<x_{B j}>$ & $x_{B j}$ & $\begin{array}{c}F_{2}^{\gamma} / \alpha \\
\text { stat. }+ \text { syst. })\end{array}$ \\
\hline 5.1 & $3-10$ & 0.043 & $0.010-0.076$ & $0.33 \pm 0.02 \pm 0.05$ \\
& & 0.138 & $0.076-0.20$ & $0.29 \pm 0.03 \pm 0.03$ \\
\hline 16 & $10-30$ & 0.085 & $0.02-0.15$ & $0.60 \pm 0.08 \pm 0.06$ \\
& & 0.24 & $0.15-0.33$ & $0.56 \pm 0.09 \pm 0.04$ \\
& & 0.555 & $0.33-0.78$ & $0.46 \pm 0.15 \pm 0.06$ \\
\hline 80 & $45-130$ & 0.19 & $0.06-0.32$ & $0.68 \pm 0.26 \pm 0.05$ \\
& & 0.455 & $0.32-0.59$ & $0.83 \pm 0.22 \pm 0.05$ \\
& & 0.785 & $0.59-0.98$ & $0.53 \pm 0.21 \pm 0.05$ \\
\hline
\end{tabular}

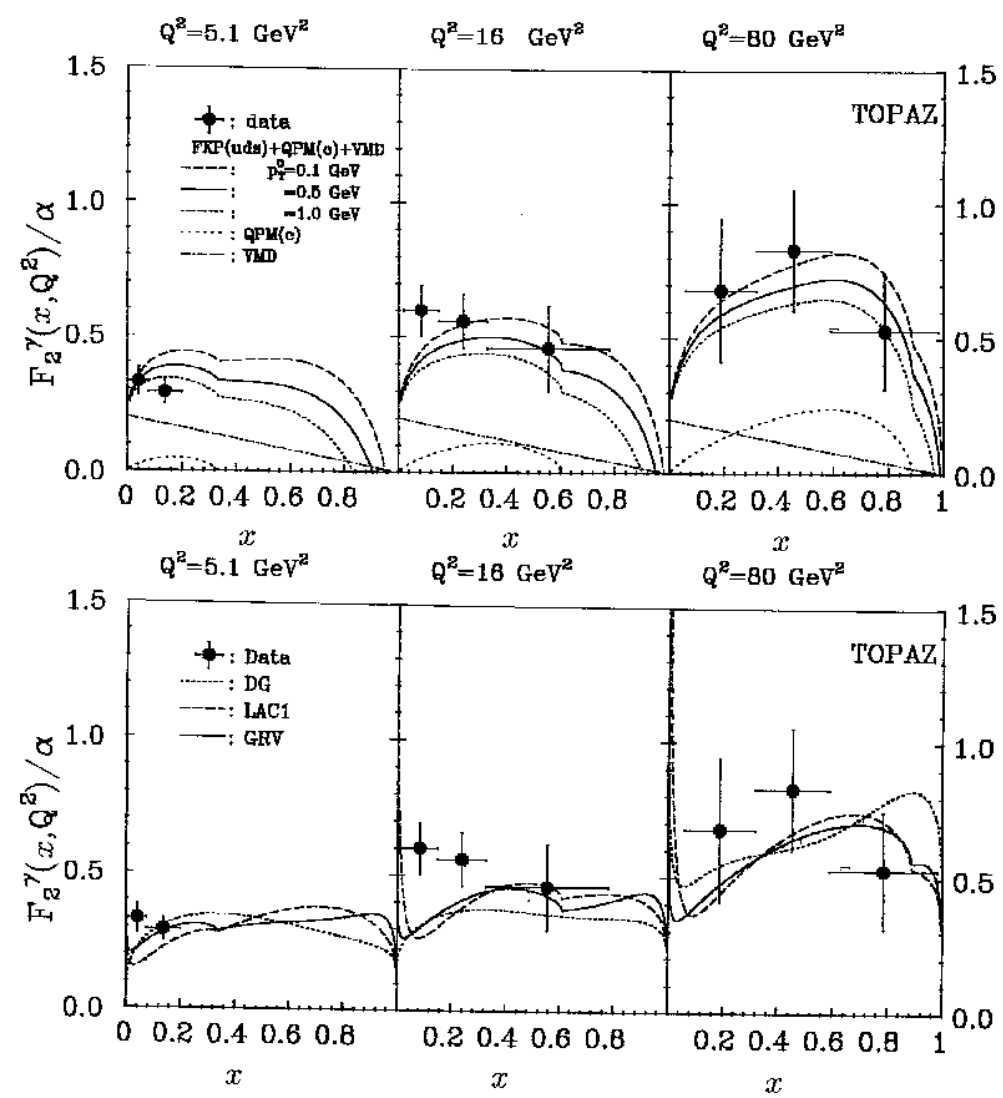

Figure 32: The $F_{2}^{\gamma} / \alpha$ versus $x_{B j}$ for $\left\langle Q^{2}>=5.1,16,80 \mathrm{GeV}^{2}\right.$ and a comparison with the prediction of $F K P(u, d, s), Q P M(c), V M D$ contributions with various $p_{T}^{0}$ parameters (upper set) and with parton parametrizations: DG, LAC1, GRV (lower set) (from [51]).

The results for $\left\langle Q^{2}>=5.1,16\right.$ and $80 \mathrm{GeV}^{2}$ are presented in table 21 and in fig. 32 where the individual components as well as predictions of various parton 
parametrizations are shown. To study the $Q^{2}$ dependence of $F_{2}^{\gamma}$, the averaged values of $F_{2}^{\gamma} / \alpha$ in the $x_{B j}$-range from 0.3 to 0.8 were extracted at $<Q^{2}>=16,80$ and 338 $\mathrm{GeV}^{2}$ (see table 22; the numbers in parentheses are results for the light quarks alone).

Table 22:

\begin{tabular}{|c|c|}
\hline $\begin{array}{c}<Q^{2}> \\
{\left[\mathrm{GeV}^{2}\right]}\end{array}$ & $F_{2}^{\gamma} / \alpha$ \\
\hline 16 & $0.47 \pm 0.08(0.38 \pm 0.08)$ \\
80 & $0.70 \pm 0.15(0.49 \pm 0.15)$ \\
338 & $1.07 \pm 0.37(0.72 \pm 0.37)$ \\
\hline
\end{tabular}

Comment: The final hadronic state described by $Q P M(c)+V M D+F K P(u, d, s)$ with $p_{T}^{0}=0.1,0.5$ and $1 \mathrm{GeV}$, and also by the GRV,DG and LAC1 parton parametrizations, was studied.

$* * * * *$

For an overall comparison the collective figures of $F_{2}^{\gamma} / \alpha$ versus $x_{B j}$ (fig. 33) and $F_{2}^{\gamma} / \alpha$ versus $Q^{2}$ (fig. 34), containing also earlier data not discussed here, are presented based on [52]. In these figures the comparison with theoretical predictions for $x_{B j}$ dependence of $F_{2}^{\gamma}$ based on the SaS1D (LO) and GRV NLO parton parametrizations (in fig. 34 in addition the prediction of the asymptotic solution) are shown. For comparison with theoretical predictions of the $Q^{2}$ evolution for different $x_{B j}$ ranges see also figs. 7, 10 b, 12, 13, 15, 17, 19, 21 d, 24, 25.

The effective parton density, as measured at HERA collider by the H1 group [53] in the jet production from resolved photons, is compared with the $F_{2}^{\gamma}$ data in fig. 35 as well. 


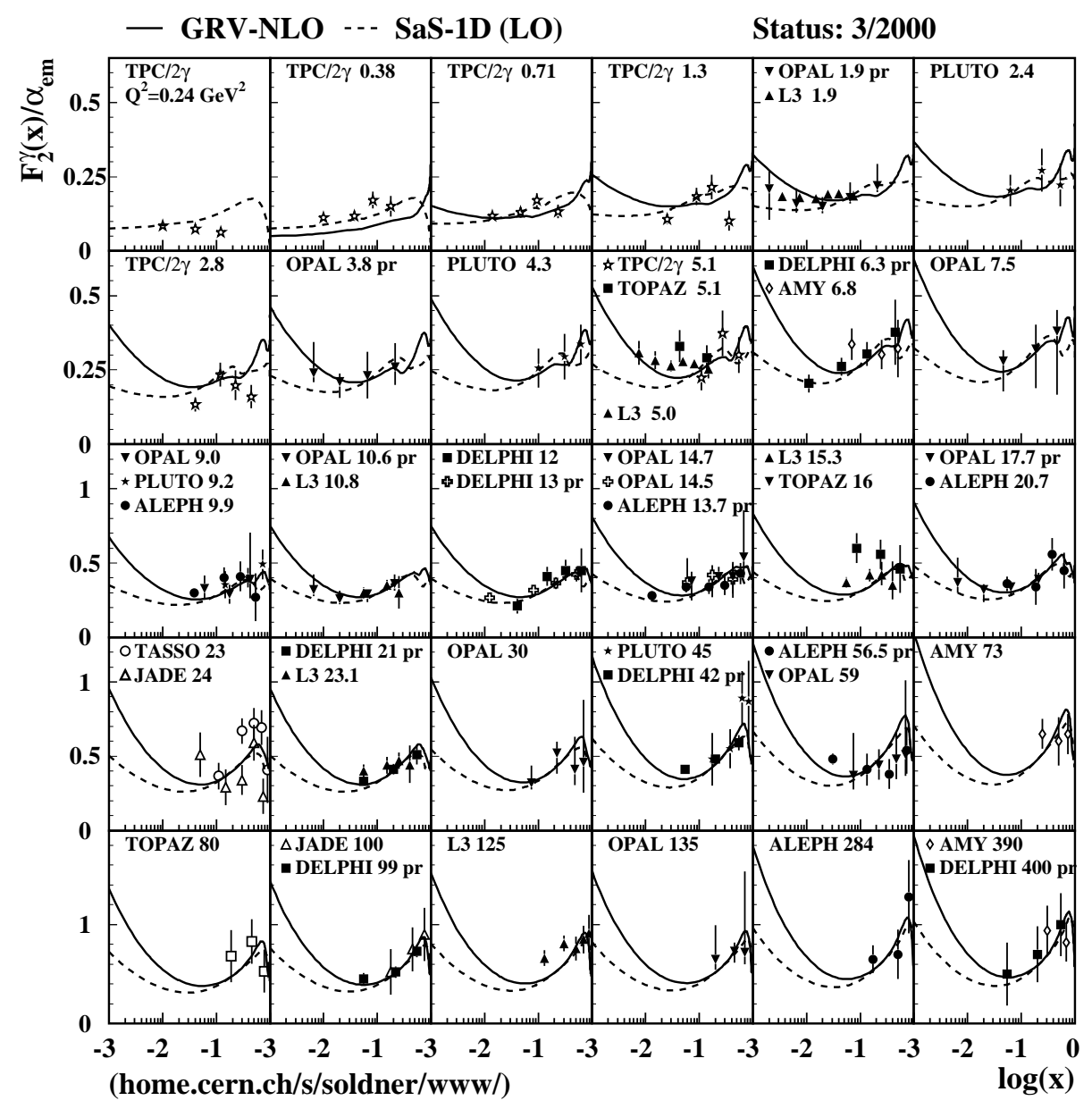

Figure 33: The photon structure function $F_{2}^{\gamma} / \alpha$ as a function of $x_{B j}$ in bins of $Q^{2}$ compared to the GRV NLO (solid line) and SaS1D (LO) (dashed line) parametrizations of parton distributions in the photon (from [52]). 


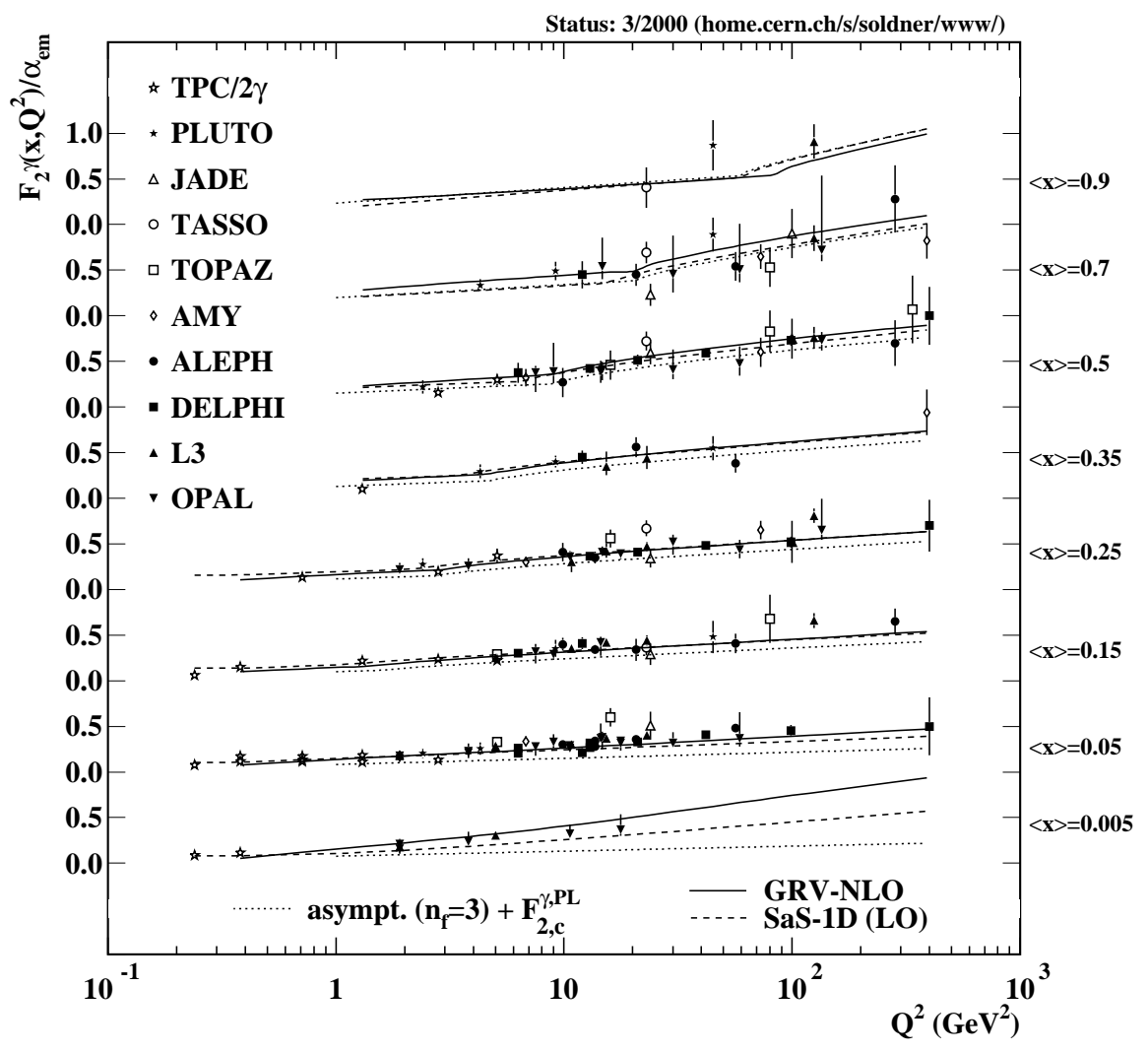

Figure 34: The photon structure function $F_{2}^{\gamma} / \alpha$ (data from various experiments) as a function of $Q^{2}$ (from [52]).

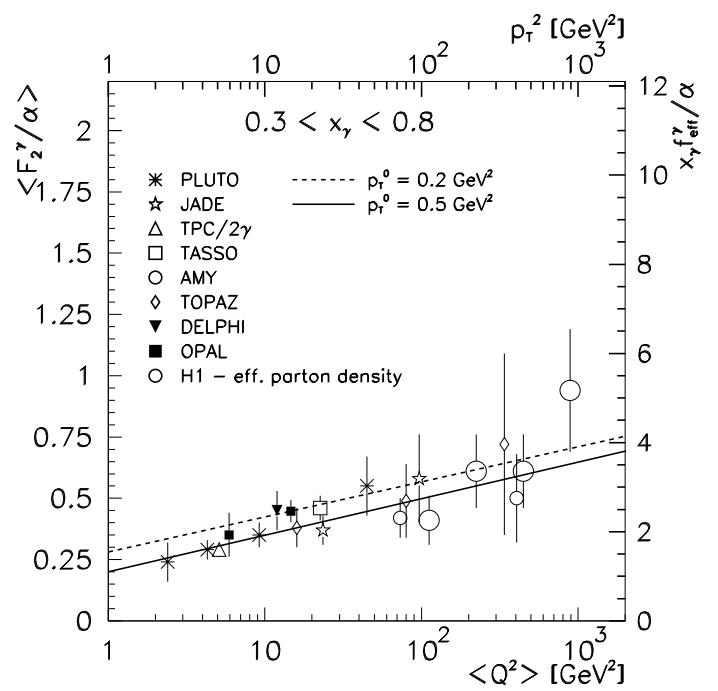

Figure 35: The scaling violation of the effective parton densities measured at HERA compared with the $Q^{2}$ dependence of $F_{2}^{\gamma}$ (averaged over the range $0.3<x_{B j}<0.8$ ) (from [53]). 


\subsection{Hadronic final states in the DIS $_{e \gamma}$ experiments}

Although a detailed analysis of hadronic final states, including those involving large $p_{T}$ particles/jets, has not been the main aim of DIS $_{e \gamma}$ experiments, it has proved crucial in extracting the unfolded $F_{2}^{\gamma}$ as mentioned in the previous section (sec. 2.2.2). The obtained experimental results, and problems that appear while describing the hadronic energy flow, transverse energy out of event plane, pseudorapidity and other distributions within the existing Monte Carlo models, deserve close attention and a separate treatment - this section is devoted to this subject.

The $F_{2}^{\gamma}$ corresponds to the cross section for hadron production in $\gamma^{*} \gamma$ collisions, eqs. (3, (4), and obviously the bulk of the contribution is due to the soft processes, i.e. production of particles with a relatively small transverse momentum $p_{T}$. One should be aware that in the hadronic final state in DIS $_{e \gamma}$ experiments two types of large scale may appear: $Q^{2}$ and $p_{T}^{2}$ [55]. The bulk of the data, as it was already mentioned, corresponds to events with not very large $p_{T}$; if for these events the relation $Q^{2} \gg p_{T}^{2} \gg P^{2}$ holds, the interpretation in terms of the photon interaction between one direct $\left(\gamma^{*}\right)$ and one resolved real $(\gamma)$ photon may be introduced. Then it is not clear what scale should be used in the parton density for a real photon, $f^{\gamma}\left(x, \tilde{Q}^{2}\right)$ : $\tilde{Q}^{2}=p_{T}^{2}, Q^{2}$, or ? Moreover the processes corresponding to $Q^{2} \ll p_{T}^{2}$ should be treated as being resolved from the point of view of both the real and the virtual photon.

Some of the problems that will appear here are common with those presented in sec. 3.3 for the jet production in $\gamma^{*} \gamma$ collision and in sec. 2.4 where dedicated measurements of the large $p_{T}$ jet production in real $\gamma \gamma$ and $\gamma p$ processes are presented.

\subsubsection{Modelling of the hadronic final state in $\gamma^{*} \gamma$ collisions}

In the $\mathrm{DIS}_{e \gamma}$ (single-tag) events the whole hadronic final state is not seen in the detector. A correct unfolding method is necessary to reconstruct the true kinematical variables (for definition see beginning of sec. 2.2.2). At this stage models to generate separate samples for the different components of the photon are needed, as we have already mentioned in sec. 2.2.2. The modelling is performed using different MC programs and it is still a large source of systematic errors in $F_{2}^{\gamma}$.

We start with a short description of the FKP approach used in the early analyses which bases on the QPM, (soft) VDM and RPC contributions. The QPM contribution is generated using the $e^{+} e^{-} \rightarrow e^{+} e^{-} q \bar{q}$ matrix element for massive quarks, for an arbitrary value of $p_{T}$ of produced quarks. The final state transverse momentum distribution is here $d \sigma / d p_{T}^{2} \sim p_{T}^{-4}$. The QPM was applied mainly to the $c$ and $b$ quark production. The (soft) VMD (called sometimes hadronic) contribution, in which the initial real photon is treated as the vector $\operatorname{meson}(\mathrm{s})$, say $\rho$, with the typical soft interaction with a photon probe, leads to the production of the final quarks (then hadrons) with the $d \sigma / d p_{T}^{2} \sim e^{-b p_{T}^{2}}$ distribution (where $b \sim 3-5 \mathrm{GeV}^{-2}$ ). This contribution should be negligible for the production of particles with large $p_{T}$ where, on the other hand, other contributions should be included. In the FKP approach 32 the cutoff parameter $p_{T}^{0}$ is built in to separate the mentioned above VMD component of the initial photon target and the resolved photon (QCD) contribution, which develops from the pointlike coupling of the $\gamma$ to the $q \bar{q}$ pair. This parameter, $p_{T}^{0}$, corresponds therefore to the intrinsic transverse momentum of the partons in the photon. The additional 
cutoff $p_{T}^{\min }$ is introduced in the analysis, to define hard $(2 \rightarrow 2)$ subprocesses where the pQCD can be used. In the description of the particle production with $p_{T}>p_{T}^{m i n}$ the resolved photon contribution is introduced (see also sec. 2.4). In the FKP approach, where the (soft) VMD contribution was introduced without the $Q^{2}$ dependence, both cutoff parameters are of the same order.

In the DELPHI analysis, the TWOGAM generator ("fixed" in 1993) was used, with the QPM, VMD for soft production and RPC contributions included within the FKP approach [36, 37]. This generator treats exactly the kinematics of scattered electron and positron, uses exact expression for the two photon luminosity function, and also in the (single and double) resolved process the kinematics of the partonic system is exact for any photon virtuality. The TWOGAM generator was used also in the last L3 measurements.

In many OPAL analysis the generator F2GEN (developed from TWOGEN used in older analyses) was used, which can generate events according to "any selected

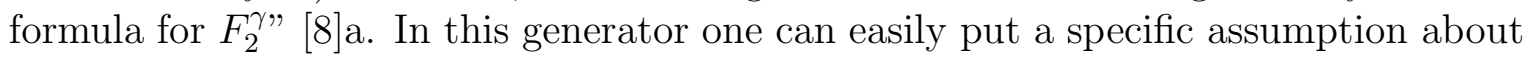
the final state. For example different angular distributions may be assumed in the $\gamma^{*} \gamma$ CM system for the produced pair of quarks: "pointlike", i.e. as for lepton pair from two real photons, "peripheral" with an exponential distribution of the transverse momentum, or the "perimiss" combination [45].

In recent analyses general purpose Monte Carlo generators are being used in analysis of hadronic final state. HERWIG and PYTHIA (the implementation of the SaS approach) apply chosen parton parametrizations. In the PHOJET generator, where hard interaction is described using QCD, the description of soft interaction bases on the Regge theory. The very recent ALEPH, L3, OPAL and TRISTAN $F_{2}$ data have been obtained using the modified HERWIG (PYTHIA) program, where the additional power distribution of the transverse momentum of partons in the photon, of the form $d p_{t}^{2} /\left(p_{t}^{2}+\tilde{p}_{t 0}^{2}\right)$, is included, see [59] and ALEPH 99a, ALEPH 99b,conf or OPAL 2000.

As far as the global hadronic variables like $W_{v i s}, Q^{2}, M_{j j}$ (invariant mass of two jets) and other distributions are concerned, there is a fairly good description of the data by existing Monte Carlo generators. The problems arose for the transverse energy out of the tag plane (the plane defined by the initial and tagged electrons) or for the energy flow per event as a function of pseudorapidity, $\eta=-\ln (\tan (\theta / 2))$ t. The discrepancies were very pronounced especially for small $x_{B j}$.

The first observation of the disagreement between the data and Monte Carlo models was made by the OPAL collaboration [45. "The serious discrepancies between the data and any of the available Monte Carlo models are seen both within the central region of the detector $(|\eta|<2.3)$, where the energy flow is well measured, and in the forward region, where the energy can only be sampled." It is clear that the unfolding of $F_{2}^{\gamma}$ "will have large errors as long as the energy flow from different models remains in clear disagreement with the energy flow in the data, in particular in the region of $x_{v i s}<0.1$ and $Q^{2}<30 \mathrm{GeV}^{2}$ (from [45]). A similar effect has been seen by now by other groups, see below.

The improved description of the mentioned data is obtained in the "HERWIG

\footnotetext{
${ }^{15}$ The polar angle is measured from the $z$-axis, and the tagged electron(positron) is by definition at the negative rapidity.
} 
$($ PYTHIA $)+$ power law $p_{t}$ " generators. The PHOJET 1.05 and TWOGAM generators can also satisfactorily describe the data (DELPHI, L3). Note however that the values of $p_{T}^{\text {min }}$ fitted in these analyses are high $(2.5-3.5 \mathrm{GeV})$. Another approach which usually improves the agreement with the data is the inclusion of multiple interaction. The effect is similar in all these approaches and consists in increasing $p_{T}$ in processes involving photons.

The recent progress in describing the hadronic final state in single-tag events at LEP by using the two-dimensional unfolding led to a reduction of the Monte Carlo modelling error (OPAL 2000). As follows from first combined results of the newly formed LEP-wide Two Photon Physics group, which used the "HERWIG $5.9+$ power law $p_{t}$ " and PHOJET 1.05, a good agreement is found between experiments in the central regions. "Unresolved problems remain in the small angle regions where the results of the different experiments showed greater discrepancies than could be accounted for by statistical fluctuations..." [11]. It was also found that to describe low $Q^{2}$ data an improved, modified model "HERWIG $5.9+$ power law $p_{t}$ (dyn)", with a dynamical limit $\tilde{p}_{\text {tmax }}^{2}=Q^{2}$ or $Q^{2}+p_{t}^{2}$ has to be introduced.

For the general discussion and data - Monte Carlo comparison in the context of modelling the hadronic final state see [8, 9], see also [7, 11] and [12], where the jet production in the photon-induced processes is studied.

\subsubsection{Measurements of the hadronic final state}

The analyses of the hadronic final state accompanying the measurements of $F_{2}^{\gamma}$ discussed in sec. 2.2.2, as well as the results from independent analyses of large $p_{T}$ hadron production in single tagged events are presented below. Variables used in the unfolding procedure are defined and discussed in sec. 2.2.2. Note that in some analyses the jet topology of the final state was studied (see e.g. TOPAZ 94, OPAL 97d,conf).

For the two photons involved, we introduce the following notation for their squared (positive) virtualities: $P_{1}^{2}\left(=Q^{2}\right)$ and $P_{2}^{2}\left(=P^{2}\right)$, with the relation to variables used in the discussed $\mathrm{DIS}_{e \gamma}$ scattering in parentheses, i.e. with the inequality $P_{1}^{2} \geq P_{2}^{2}$ understood. 


\section{DATA}

\section{-ALEPH 97a,conf [56] (LEP 1)}

Single tagged events were collected in years 1992-1994 at the average $Q^{2}=14.2 \mathrm{GeV}^{2}$. A dedicated study of the hadronic final state assuming the QPM (for $u, d, s$ and $c$ quarks) + VMD (for the target photon) and using the HERWIG 5.9 generator, with the GRV parametrizations, was performed. Also the modified HERWIG generator, "HERWIG $5.9+$ power law $p_{t}$ ", with $\tilde{p}_{t 0}=0.66 \mathrm{GeV}$ was used. A cone jet-finding algorithm was used in the analysis.

Results for energy flow are presented in figs. 36, 37. The discrepancy is observed in the energy flow in the positive pseudorapidity region. The approach "HERWIG 5.9 + power law $p_{t}$ " leads everywhere to a better description of the data.

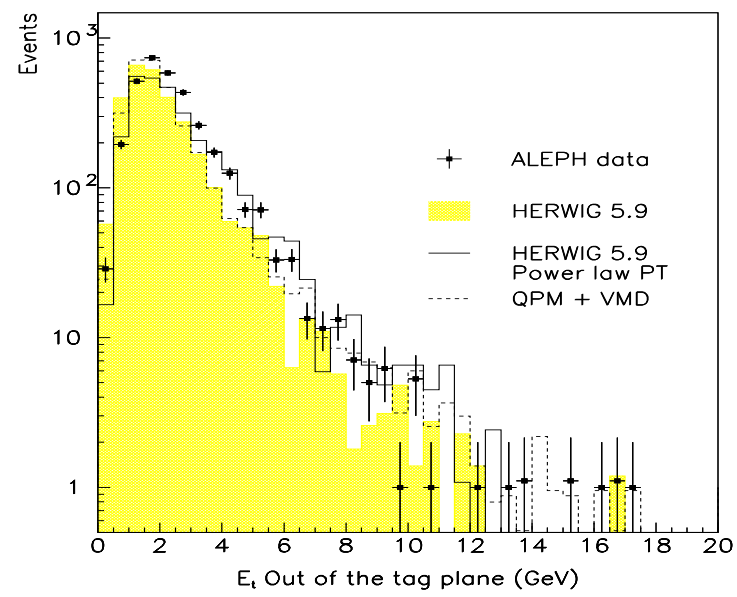

Figure 36: The energy (out of the plane of the tag and the beam) distribution. Comparison with different Monte Carlo models: HERWIG 5.9 (shaded), "HERWIG $5.9+$ power law $p_{t}$ " (solid line) and QPM + VMD (dashed line) (from [56]).

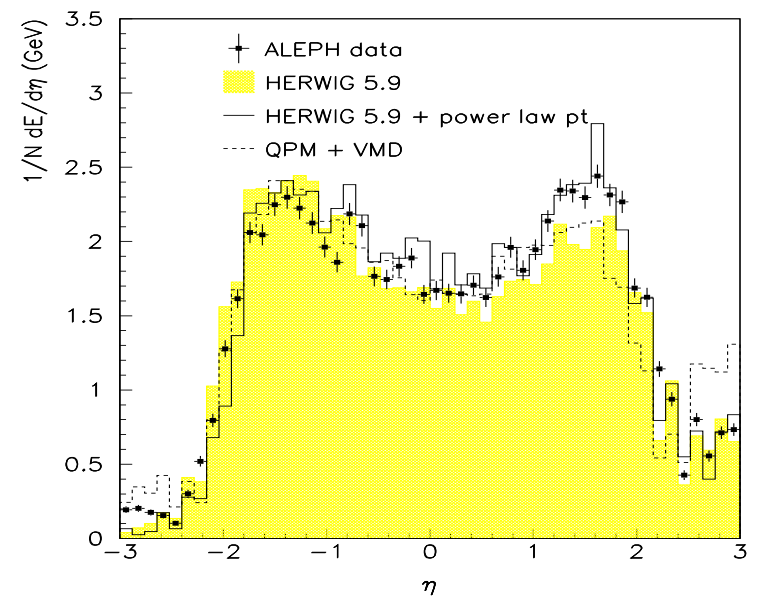

Figure 37: The energy flow versus pseudorapidity of the final hadrons. Comparison with different Monte Carlo models as in fig. 36 (from [56]).

In order to pin down the source of the observed discrepancy the analysis of the number of cone jets was performed in the hadronic final state (see fig. 38 for results). The 
standard HERWIG model, having additional production mechanisms with a resolved photon, should in principle give larger number of two-jet events than the QPM+VMD model. On the contrary, it leads to a lower number.

To check the presence of the single and double resolved photon events, the $x_{\gamma}$ and $x_{\text {tag }}$ distributions were measured (not shown). Note, that double resolved photon events contain the resolved virtual photon contribution (see also sec. 3). Also the energy not assigned to jets in two-jet events, $E_{2}^{\text {non-jet }}$, was studied (not shown). The "HERWIG + power law $p_{t}$ " approach was used successfully to describe these data.

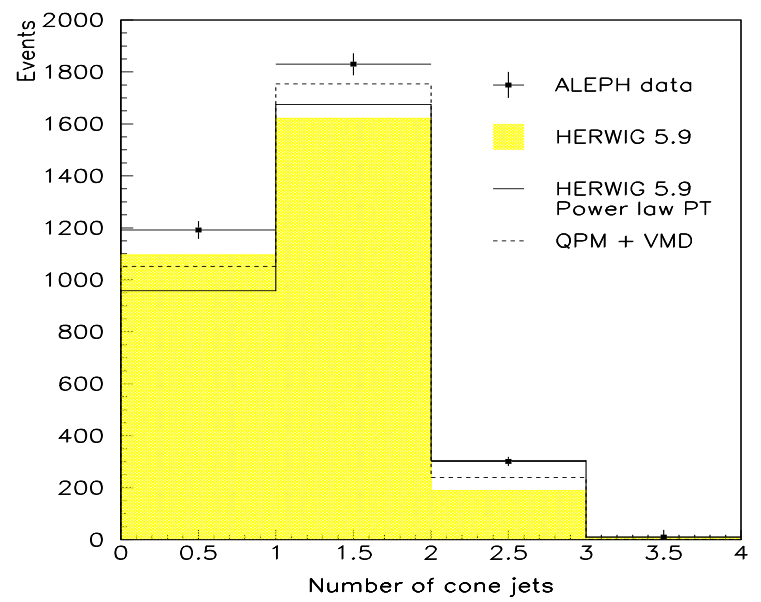

Figure 38: The number of events classified in four bins referring to various numbers of jets in an event. Comparison with different Monte Carlo models as in fig. 36 (from [56]).

Comment: "The ALEPH data confirms recent problems reported by OPAL in modelling the hadronic final state."

" 'HERWIG + power law $p_{t}$ ' is better at modelling the region of large $E_{t, \text { out }}$ " and the peak in the energy flow at positive rapidity.

The "double resolved processes are not present to an large extent in the data at this $Q^{2} "$.

\section{-ALEPH 99a 33 (LEP 1)}

Analysis of the hadronic final state during the $F_{2}^{\gamma}$ measurement is reported. Monte Carlo models: QPM (for all quarks) + VMD (for a target photon) and "HERWIG 5.9 + power law $p_{t}$ ", with GRV LO parametrization, were studied. Results are presented in figs. 39 and 40. 

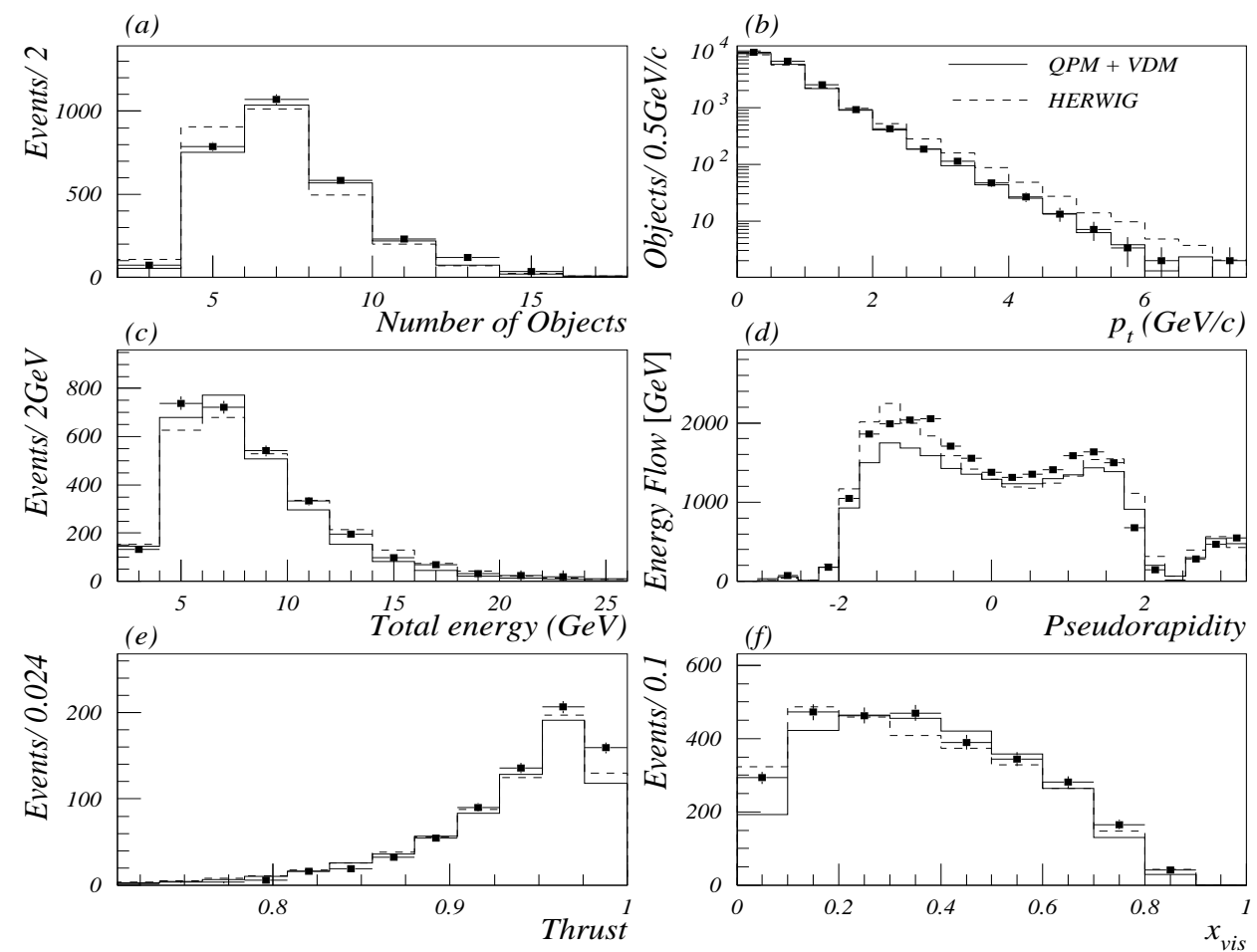

Figure 39: The data in which the scattered electron is detected (tagged) in luminosity calorimeter LCAL, compared with the QPM + VMD model (solid histogram) and the "HERWIG $5.9+$ power law $p_{t}$ " model (dashed histogram) (from [33]).
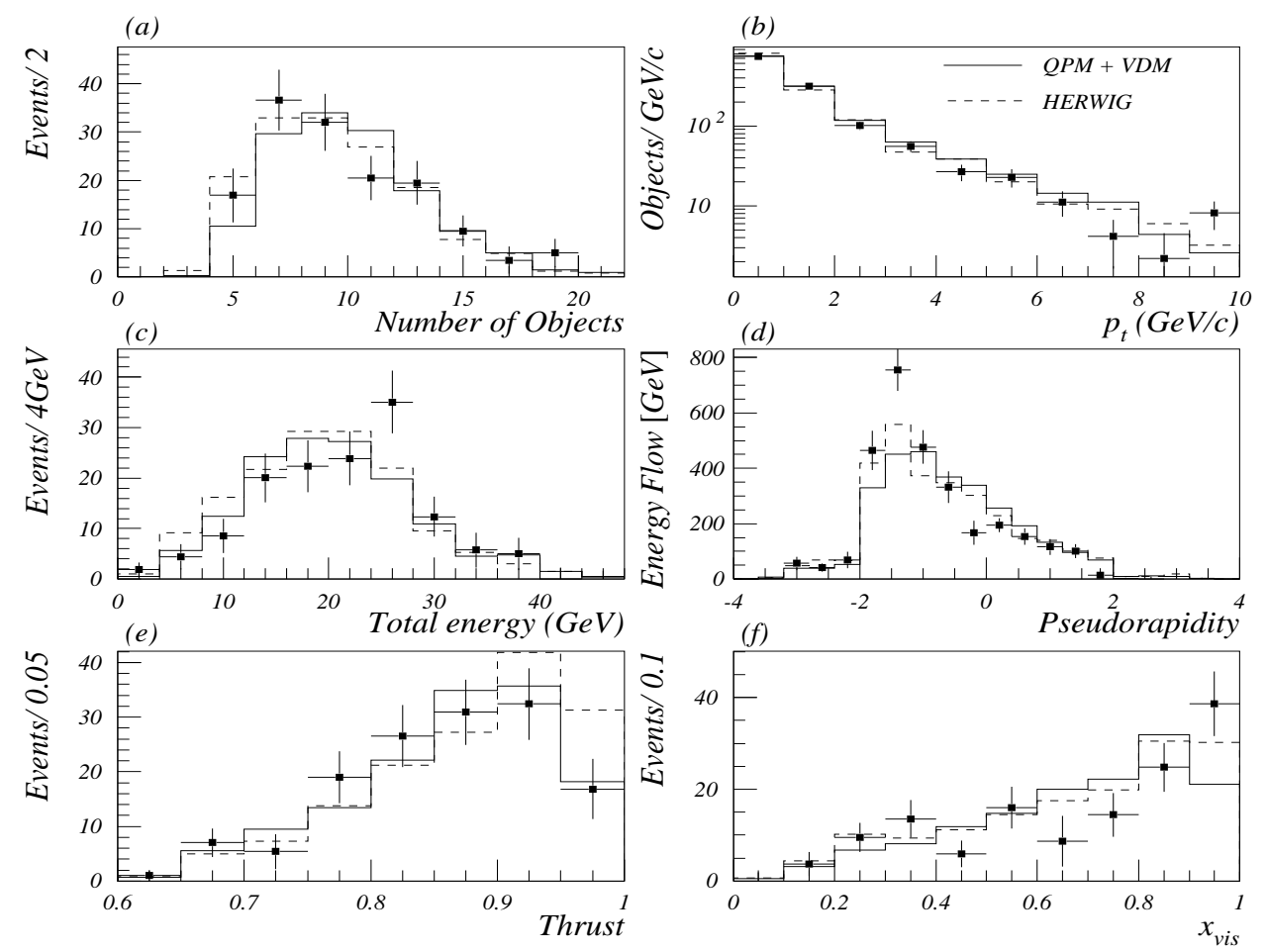

Figure 40: The data in which the scattered electron is detected (tagged) in electromagnetic calorimeter ECAL, compared with the QPM + VMD model (solid histogram) and the "HERWIG 5.9 + power law p " model (dashed histogram) (from [33]). 
Comment: The HERWIG program with modified $p_{t}$ distribution and the $Q P M+V M D$ model give "an acceptable description of the data".

\section{-ALEPH 99b,conf [34] (LEP 2)}

A new unfolding method was used in the measurement of $F_{2}^{\gamma}$ at $\sqrt{s}=183 \mathrm{GeV}$ based on 1997 data (see also previous section). Two samples of single-tag events with the tag defined as a cluster in one of the small angle luminosity calorimeters SICAL or LCAL, for $Q^{2}$ ranges: $7-24 \mathrm{GeV}^{2}$ and $17-200 \mathrm{GeV}^{2}$, were studied.

The new unfolding procedure, based on the principle of maximum entropy, was used to correct the distributions for finite resolution and acceptance. The unfolding was done for $x_{B j}$ and in additional for a second kinematical variable, $E_{17}$, defined as the total energy of particles produced at an angle with respect to the beam direction smaller than $17^{\circ}$. In fig. 41 results of unfolding of the $x$ variable for different values of $E_{17}$ obtained using "HERWIG $5.9+$ power law $p_{t}$ " (SaS1D) are presented (LCAL data). One can see that the small $E_{17}$ gives better result.
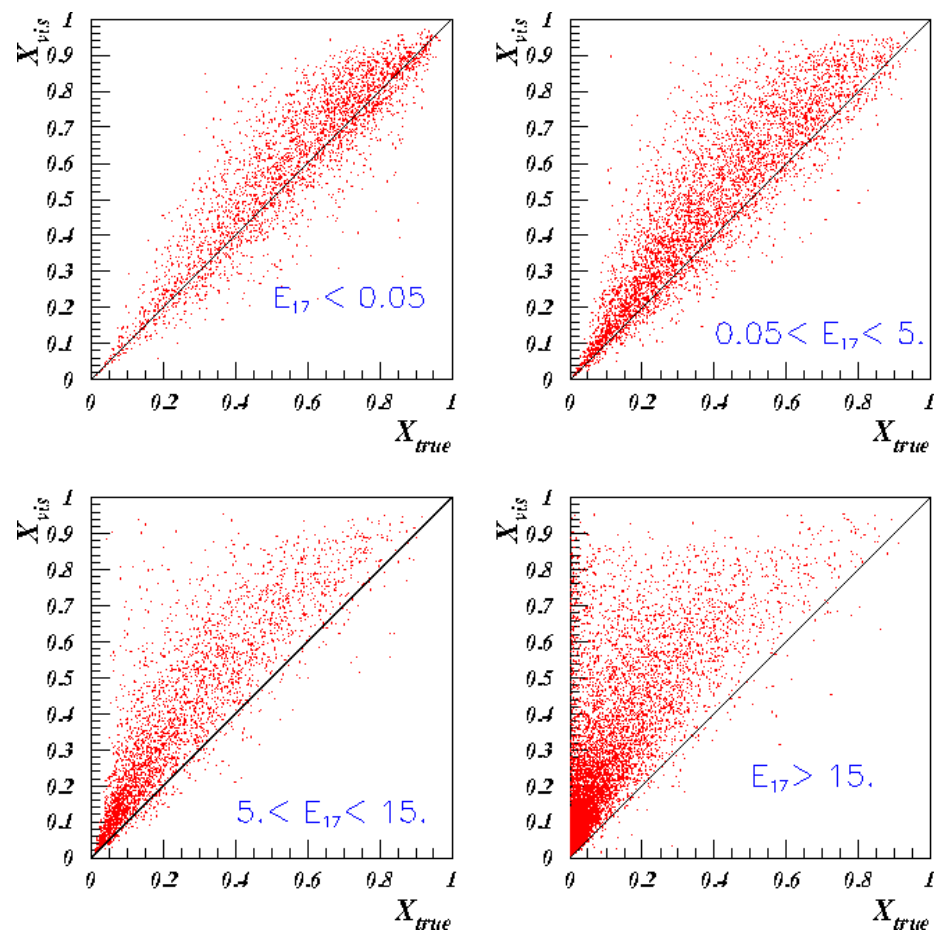

Figure 41: The relationship between reconstructed and true values for $x_{B j}$ for four bins of $E_{17}$ (from [3]]).

Although the two-variable unfolding reduces the dependence on the Monte Carlo, some model dependence still remains. This point was studied by comparing several kinematic distributions obtained using "HERWIG 5.9 + power law $p_{t}$ " with the GRV $\mathrm{LO}$ and SaS1D parton parametrizations and in addition using the PHOJET model. The SICAL-tag data (not shown) are described reasonably by the used models, while for LCAL data (fig. 42) "PHOJET is in poor agreement with the data". 


\section{PRELIMINARY}
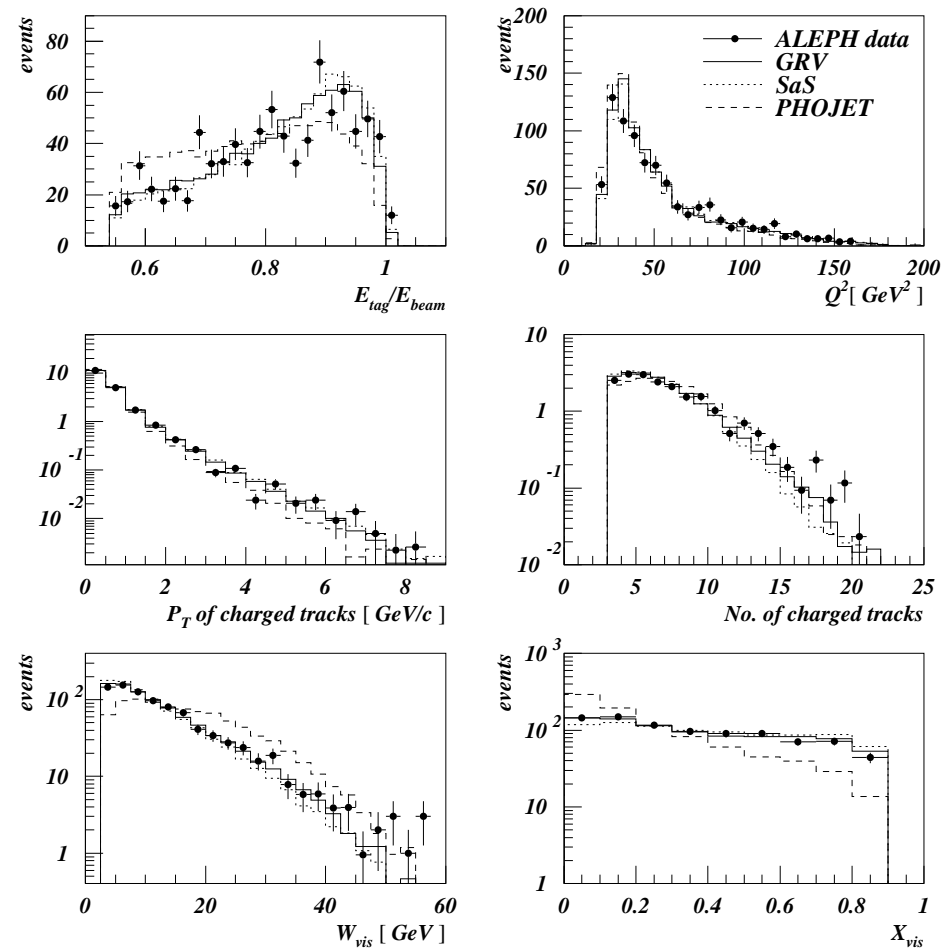

Figure 42: Comparison of data and simulations for the LCAL tagged data. Predictions of the "HERWIG $5.9+$ power law $p_{t}$ " model with two parton parametrizations: GRV (solid line), SaS1D (dotted line), and of PHOJET (dashed line) are shown (from [34]).

Comment: This unfolding method "leads to smaller statistical errors and a reduced model dependence compared to one-dimensional procedure".

\section{-DELPHI 95 [57] (LEP 1)}

First evidence of the hard scattering process in the single tagged events in the data from the 1991-1992 run is reported. The values of $E_{T}$ of observed jets were larger than $1.5 \mathrm{GeV}$, while the magnitude of the mass of virtual photon was equal to $\left\langle Q^{2}>\approx\right.$ $0.06 \mathrm{GeV}^{2}$. This corresponds to the standard resolved (almost real) photon process, from the point of view of both photons.

Comment: This is not a typical DIS experiment since the photon probe is almost real. The analysis is not typical, either, for the standard large $p_{T}$ jet study. "The data are consistent with the predictions for quark and gluon density functions in the GS parametrization. The sum of the contributions: VMD + QPM (Quark Parton Model) $+[Q C D-R P C]$ is needed in order to describe the data; the DO and LAC3 parametrizations do not adequately describe the data."

\section{-DELPHI 96b,conf [36] (LEP 1)}

The results for the averaged $Q^{2}=13$ and $106 \mathrm{GeV}^{2}$ were considered and compared with the TWOGAM generator, for $F_{2}^{\gamma}$ results see sec. 2.3. The QPM, GVMD contributions and RPC with the GS2 parton parametrization were included. 
Comment: It was shown that the resolved photon contributions are needed to obtain the description of the data.

\section{-DELPHI 97a,conf [37] (LEP1, LEP2)}

Analysis of the hadronic final state in the $F_{2}^{\gamma}$ measurement (see sec. 2.2.2) was performed for energies around the $Z^{0}$ mass, and for $161-172 \mathrm{GeV}$ (1996 run). The $<Q^{2}>=6.5,13,22$ and $17,34,63 \mathrm{GeV}^{2}$ were studied, respectively. The hadronic final state topology with events containing jets was studied using the TWOGAM generator. For various distributions obtained for LEP 2 data, see fig. 43. In fig. 44, the event energy flow as a function of the pseudorapidity is shown for LEP1 and LEP2 data.

Comment: "All variables were found to be in good agreement with TWOGAM prediction."

Note that the $p_{T}$ range of jets may be of the order of $<Q^{2}>$ for small $Q^{2}$ samples.
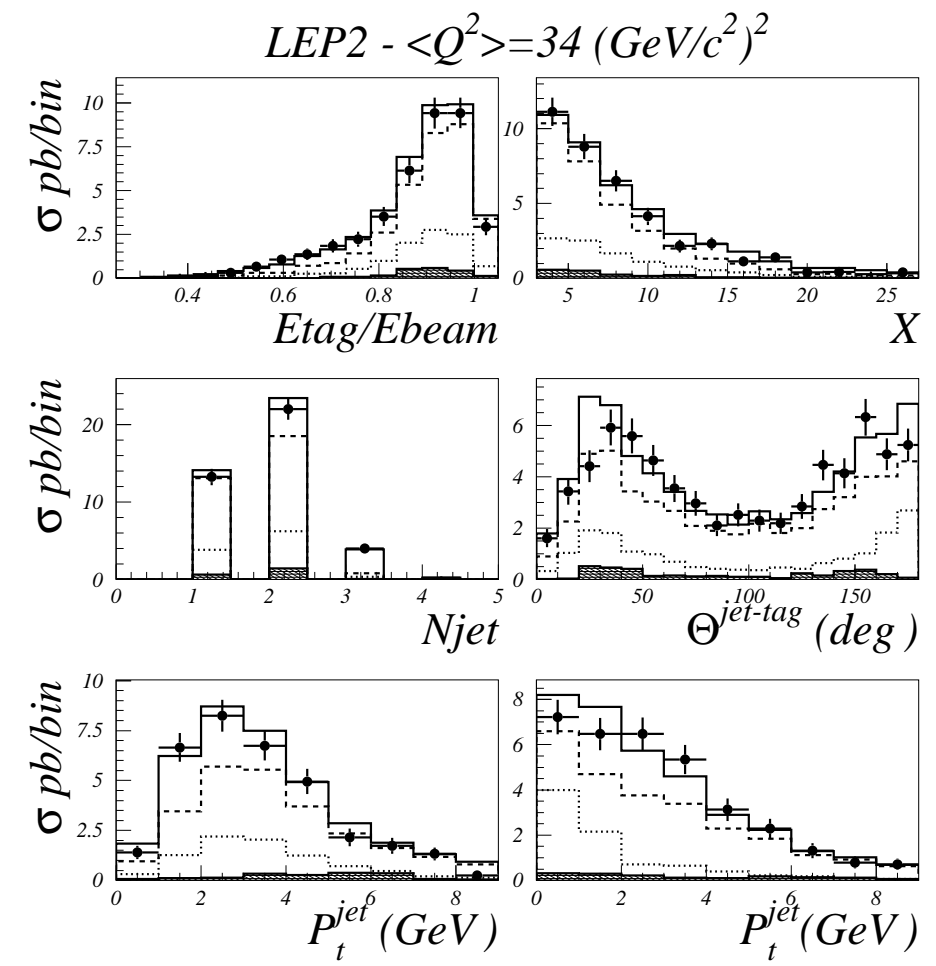

Figure 43: Comparison of data and the Monte Carlo prediction for $\left\langle Q^{2}>=34 \mathrm{GeV}^{2}\right.$ (LEP2) for the variables: (shown first left, then right in each row) Energy of tagged particle; The invariant mass; The number of jets; The jet angle with respect to the tagged particle; $p_{T}$ for jets in the same hemisphere as the tagged particle; $p_{T}$ for jets in the opposite hemisphere. Curves show the Monte Carlo predictions: GVMD+QPM+RPC (GS2) (solid line), GVMD+QPM (dots), GVMD (dashes) (from 37]). 


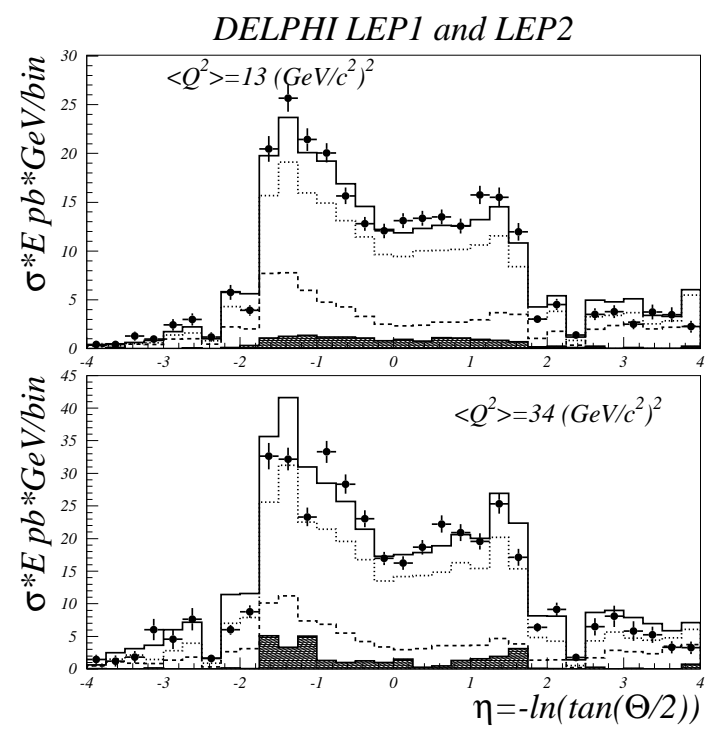

Figure 44: The event energy flow as a function of the pseudorapidity for $\left\langle Q^{2}\right\rangle=13 \mathrm{GeV}^{2}$ (LEP1) and $<Q^{2}>=34 \mathrm{GeV}^{2}$ (LEP2). The notations are as in fig. 43 (from [37).

\section{-DELPHI 98, conf [38, 39] (LEP 2)}

The hadronic final state analysis accompanied the $F_{2}^{\gamma}$ data taking in the period 1996-98 for $Q^{2}$ between 10 and $1000 \mathrm{GeV}^{2}$ (for the $e^{+} e^{-}$energy of $163-188 \mathrm{GeV}$ ), see sec. 2.2.2. The three-component description of DIS events was applied: the TWOGAM generator was used for the QPM (all quarks), the soft hadronic GVMD and RPC (with the GS2 parton densities in single and double resolved photon processes) contributions. The results are presented in figs. 45 and 46. Good description of the data was obtained. 


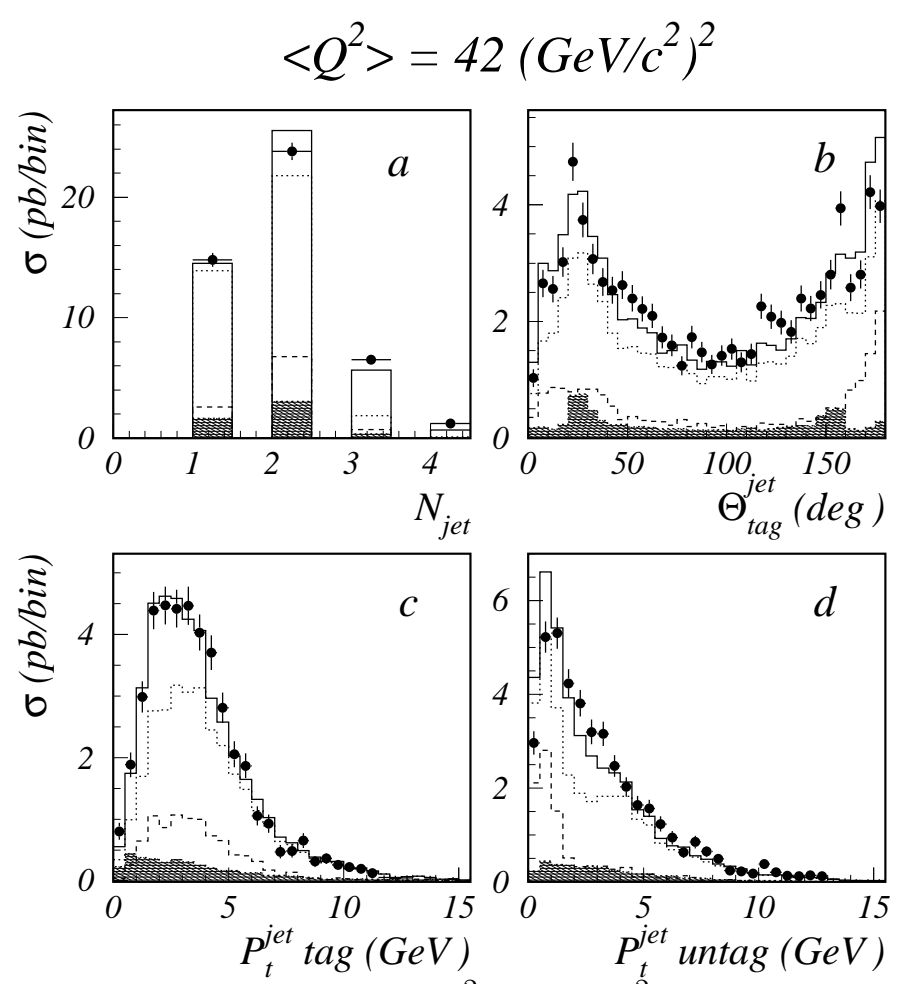

Figure 45: The cross section for averaged $Q^{2}=42 G e V^{2}$ as a function of a) The number of jets, b) the jet angle with respect to the tagged particle and of the transverse momentum of the jet for c) tagged and d) untagged events. Curves show the Monte Carlo predictions with $Q P M+G V M D+R P C$ (solid line), $Q P M+G V M D$ (dotted line) and GVMD (dashed line) (from [39]).

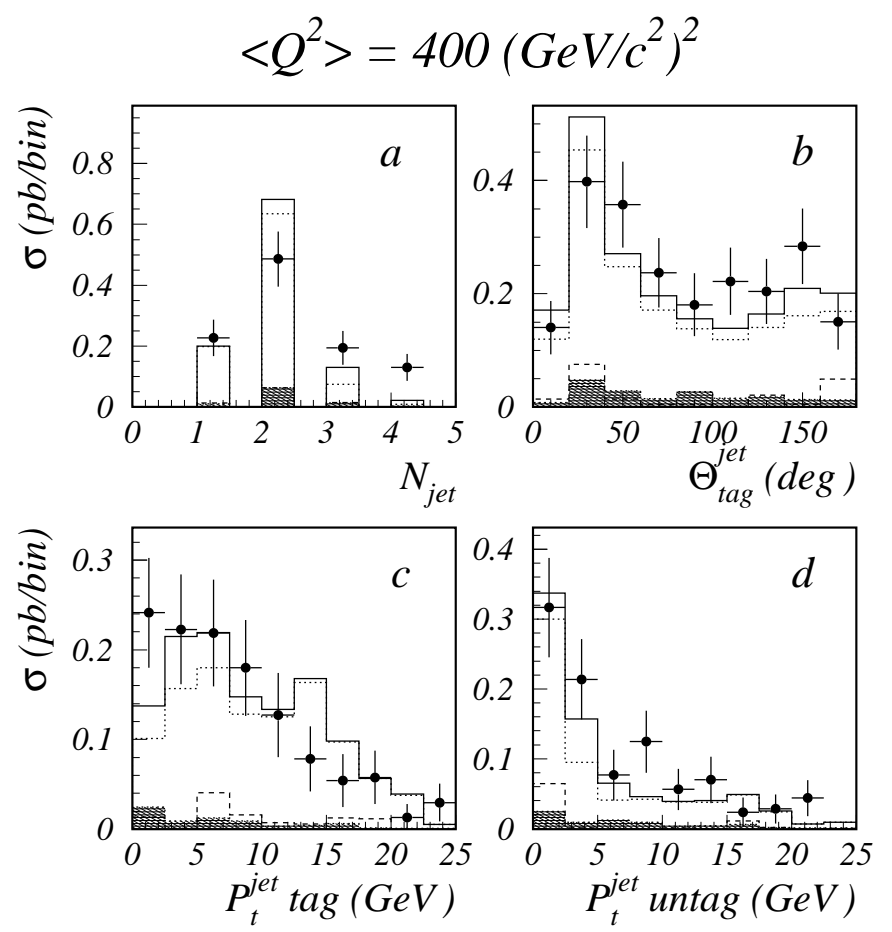

Figure 46: The cross section for averaged $Q^{2}=400 \mathrm{GeV}^{2}$ as a function of a) number of jets, b) the jet angle with respect to the tagged particle, and of the transverse momentum of the jet for c) tagged and d) untagged events. The notations are as in fig. 45 (from [39]). 


\section{-L3 98a 40] (LEP 1)}

The study of the hadronic final state was performed while the $F_{2}^{\gamma}$ data were collected in years 1991-95 for $1.2 \leq Q^{2} \leq 9.0 \mathrm{GeV}^{2}$ (see sec. 2.2.2). Two bins: $<Q^{2}>=1.9 \mathrm{GeV}^{2}$ in the $x_{B j}$ range $0.002<x_{B j}<0.1$ and $<Q^{2}>=5 \mathrm{GeV}^{2}$ in the $x_{B j}$ range $0.005<x_{B j}<$ 0.2 were analysed. A new method for reconstructing kinematical variables, based on imposing a transverse momentum conservation, was used in the analysis.

The invariant mass of the hadronic system obtained using PHOJET 1.05 - $\mathrm{W}_{\gamma \gamma}$, as well as $\mathrm{W}_{\text {vis }}$ and $\mathrm{W}_{\text {rec }}$ (from the new method) are compared in fig.47. In further analysis of $x_{B j}, p_{T}$ distributions and of the energy flow three Monte Carlo generators : PHOJET 1.05, HERWIG 5.9 and TWOGAM have been used. The results are shown in figs. 48 and 49 .

Two data sets for $F_{2}^{\gamma}$ based on the PHOJET and TWOGAM analyses are presented (they differ up to $28 \%$ ), see previous section.

Comment: "A significant improvement is seen in the $W_{\text {rec }}$ variable which uses the constraint of transverse momentum conservation.

$H E R W I G$ disagrees with data in the small $x_{B j}$ region." The distribution of the transverse momentum of charged particles and energy flow were reproduced by PHOJET and TWOGAM, the spectrum of HERWIG was found to be too soft.

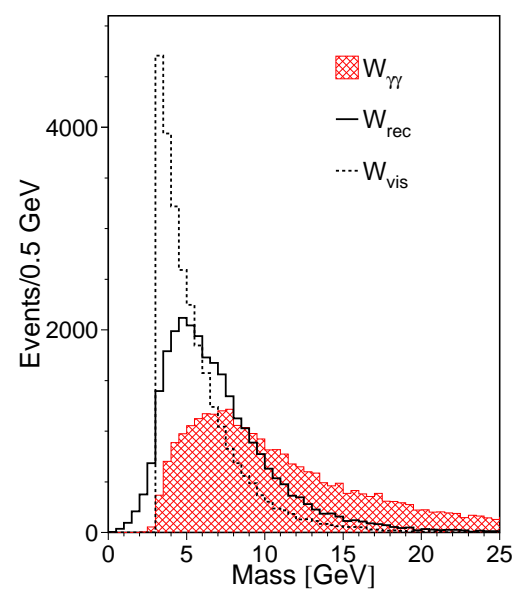

Figure 47: Monte Carlo (PHOJET) comparison of the generated two-photon mass $W_{\gamma \gamma}$ distribution with the distributions $W_{\text {vis }}$ and $W_{\text {rec }}$ (from [40]). 


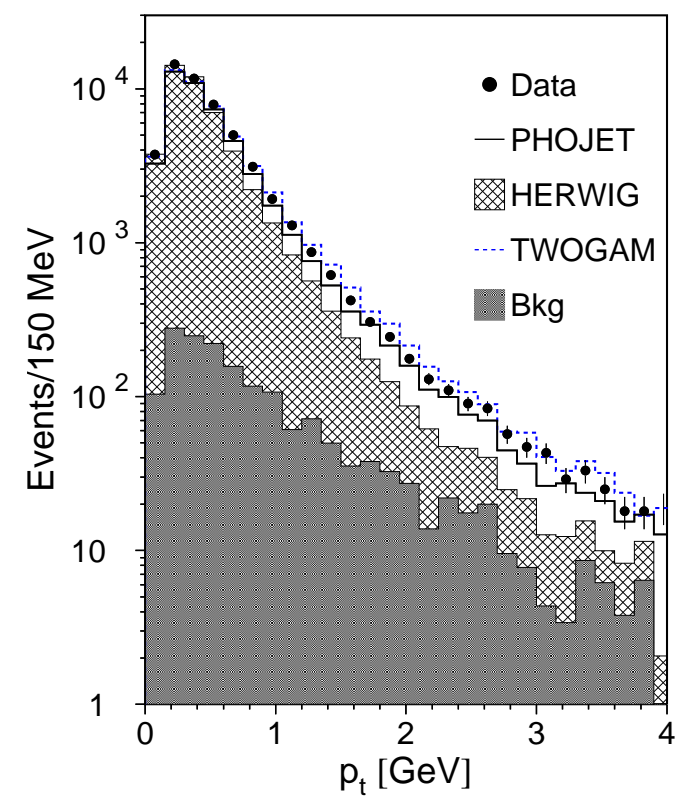

Figure 48: The $p_{T}$ distribution for charged hadrons: data and Monte Carlo predictions (from 40]).

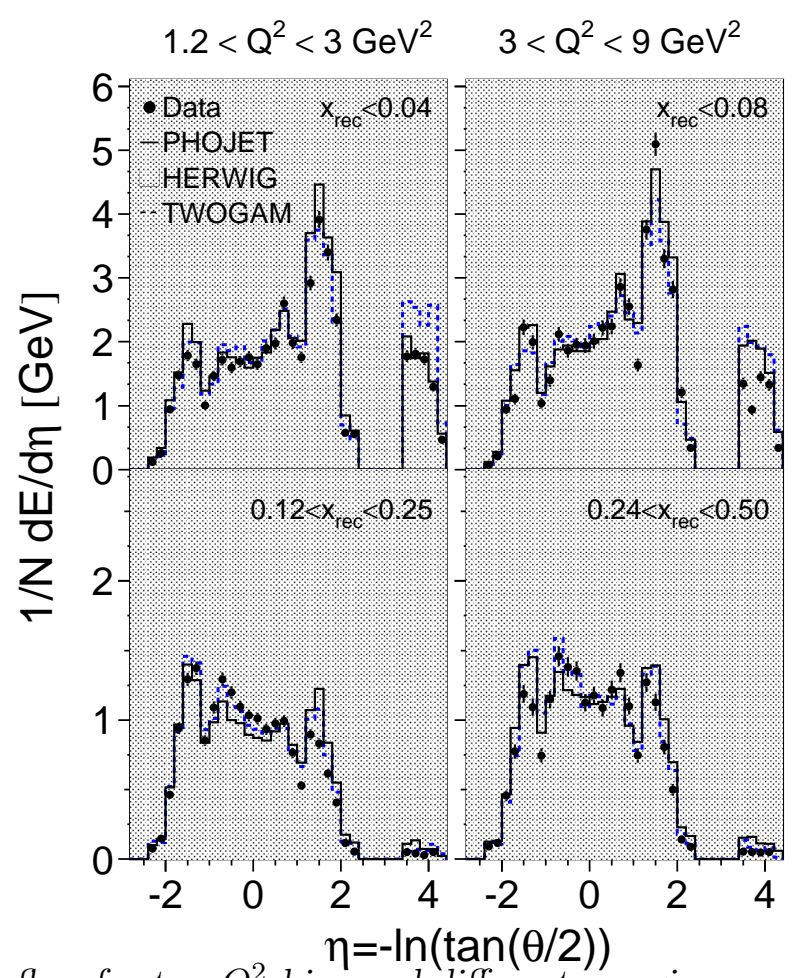

Figure 49: Energy flow for two $Q^{2}$ bins and different $x$ regions as a function of the pseudorapidity, with the polar angle defined with respect to the tagged electron. Comparison with three Monte Carlo generators is shown (from [40]).

\section{-L3 2000 42 (LEP 1)}

The analysis of the hadronic final state was performed in the measurement of the structure function for the real photon and virtual photon at $\sqrt{s}=89-92 \mathrm{GeV}$ (see also secs. 2.2 .2 and 3.2). The large $Q^{2}$ events, $Q^{2}$ between 40 to $500 \mathrm{GeV}^{2}$ with $<$ 
$Q^{2}>=120 \mathrm{GeV}^{2}$, were studied. The mean virtuality of the target photon is estimated to be $<P^{2}>=0.014 \mathrm{GeV}^{2}$ for single-tag events and $<P^{2}>=3.7 \mathrm{GeV}^{2}$ for double-tag events. "A new feature in the present work is a fit to two-photon kinematics imposed on each event."

Single-tag events were analysed using the JAMVG generator modelling the QPM contribution with $N_{f}=4$, the PHOJET $1.05 \mathrm{c}$ with a cutoff $p_{T}^{\min }=2.5 \mathrm{GeV}$ (and charm generated using JAMVG), and TWOGAM with $p_{T}^{\min }=3.5 \mathrm{GeV}$ generating three processes: QPM (all quarks), the soft hadronic VMD and QCD resolved photon contribution $(\mathrm{RPC})$. Kinematical variables were reconstructed in a fitting procedure applying constrains from four-momentum conservation. The correlation between generated and fitted values for the invariant mass and $x_{B j}$ is shown in fig. 50 .

The Monte Carlo predictions and the data are shown in figs. 51 and 52 for singletag events. The JAMVG (QPM) is in agreement with the data for $x_{B j}$ above 0.5. PHOJET agrees with the data at low $x_{B j}$. Similar distributions were studied for double tagged events, see fig. 53, where some disagreement is seen.
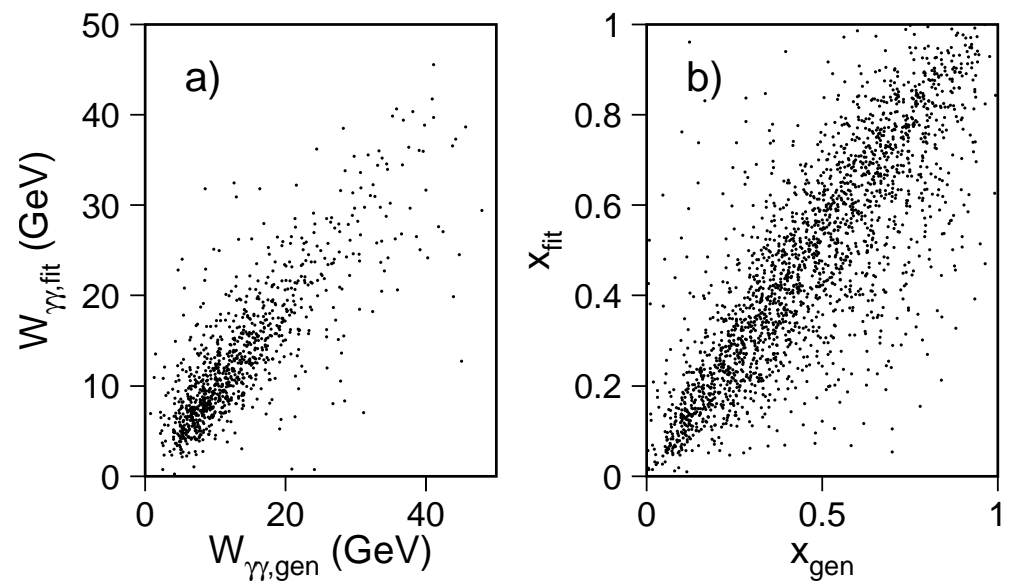

Figure 50: a) Correlation between values of $W$ generated and obtained in the unfolding after the fit, b) the same for $x_{B j}$. Based on the JAMVG Monte Carlo (from [42]). 

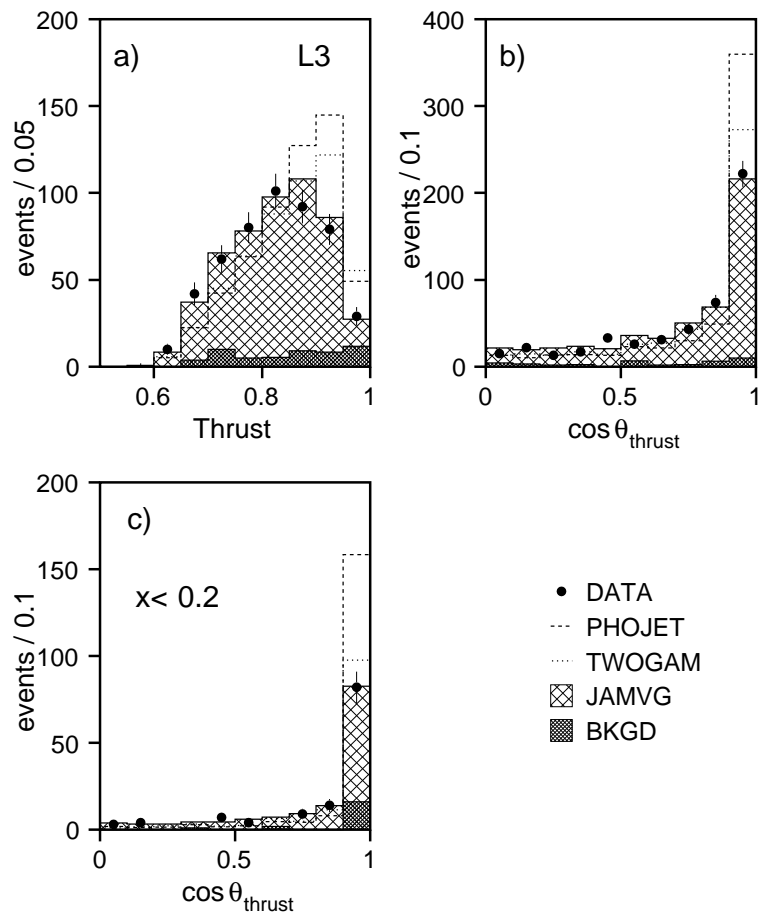

- DATA

PHOJET

TWOGAM

JAMVG

BKGD

Figure 51: Distributions for the single-tag data. Comparison with PHOJET, TWOGAM, JAMVG predictions and with the background estimation is shown. The Monte Carlo distributions are normalized to the same number of events as the data (from [42]).
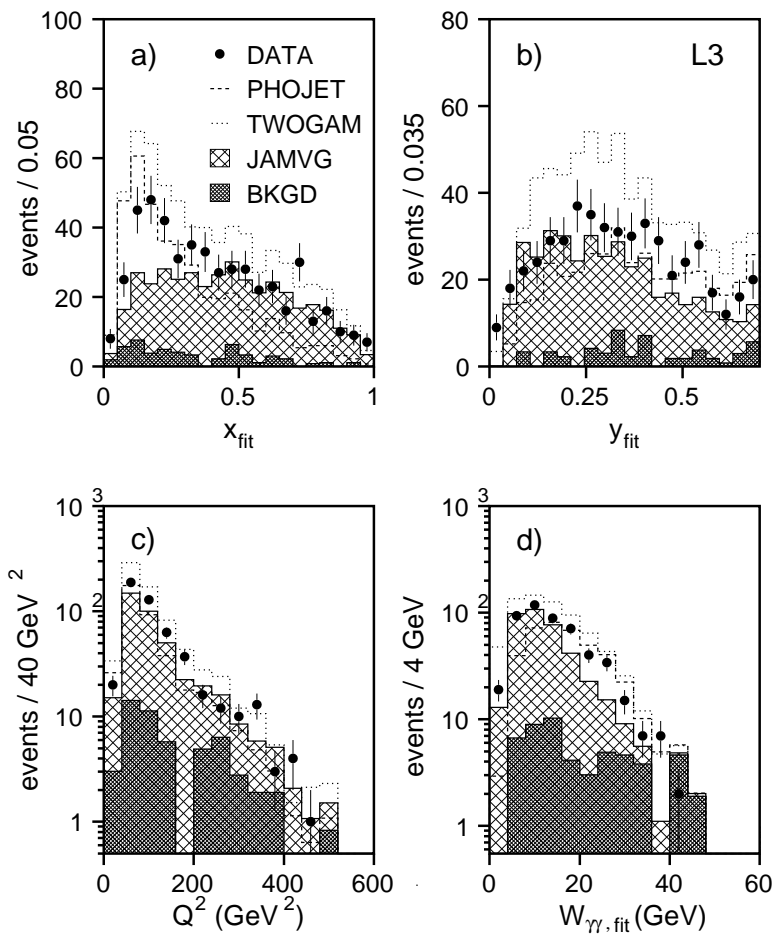

Figure 52: Results from the single-tag events. a) $x_{f i t}$ b) $y_{f i t}$ c) $Q^{2}$ and d) $W_{\text {fit }}$ distributions. Comparison with predictions of the PHOJET, TWOGAM and JAMVG models and with an estimation of the background is shown (from [42]). 

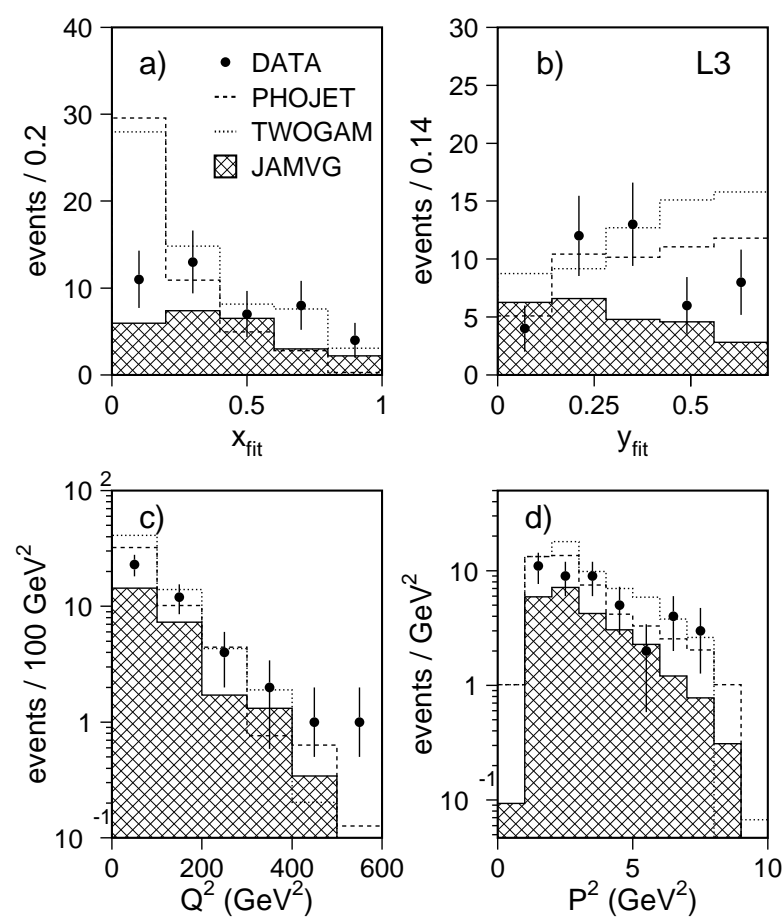

Figure 53: Results from double-tag events. a), b) and c) as in fig. 52, and d) the $P^{2}$ distribution (from [42]).

Comment: "...it is not clear if (this) successful analysis (i.e. unfolding) can be applied also to $Q^{2}$ and $x_{B j}$ regions not dominated by the direct process" (from [X]). "PHOJET and TWOGAM expectations exceed the data at low $x_{B j}$ values" for double-tag events.

-OPAL 94 [44] (LEP 1)

Data for $F_{2}^{\gamma}$ were collected in 1990-92 for averaged $Q^{2}=5.9$ and $14.7 \mathrm{GeV}^{2}$. Early analysis of the final hadronic state (the TWOGEN generator with the contribution based on QPM, the soft hadronic VMD process and on the FKP parametrization for the QCD contribution) was performed. The parameter $p_{T}^{0}=0.27 \pm 0.10 \mathrm{GeV}$ was determined.

\section{-OPAL 97a 45] (LEP 1)}

The hadronic final state was analysed in details in the measurement of $F_{2}^{\gamma}$ with one photon highly virtual $\left(Q^{2}\right.$ between 6-30 $\mathrm{GeV}^{2}$ - low $Q^{2}$ sample, and between 60-400 $\mathrm{GeV}^{2}$ - high $Q^{2}$ sample), the other being almost real. For generating the hadronic final state the Monte Carlo programs HERWIG 5.8d, PYTHIA, and for comparison F2GEN were used with the GRV and SaS1D parton parametrizations.

The energy $\left(E_{t, \text { out }}\right)$ transverse to the plane defined by the beam axis and the tag direction, and other quantities for the low $Q^{2}$ sample are presented in fig. 54. The discrepancy found for the $E_{v i s}$ and $E_{t, \text { out }}$ distributions in this sample (figs. $54 \mathrm{~b}$ and $\mathrm{d}$, respectively) is absent in the high $Q^{2}$ events (not presented).

The hadronic energy $E_{t, \text { out }}$ distributions in the three $x_{B j}$ bins are shown in fig. 55. The failure of the models in the low $Q^{2}$ region is most visible at low $x_{B j}$.

To establish a source of the discrepancy the energy flow per event in the low $Q^{2}$ sample was also studied as a function of the pseudorapidity (see fig. 56). The distribu- 
tion of pseudorapidity $\eta$ for the low $Q^{2}$ sample, corrected for the experimental effects, is shown in fig. 57.
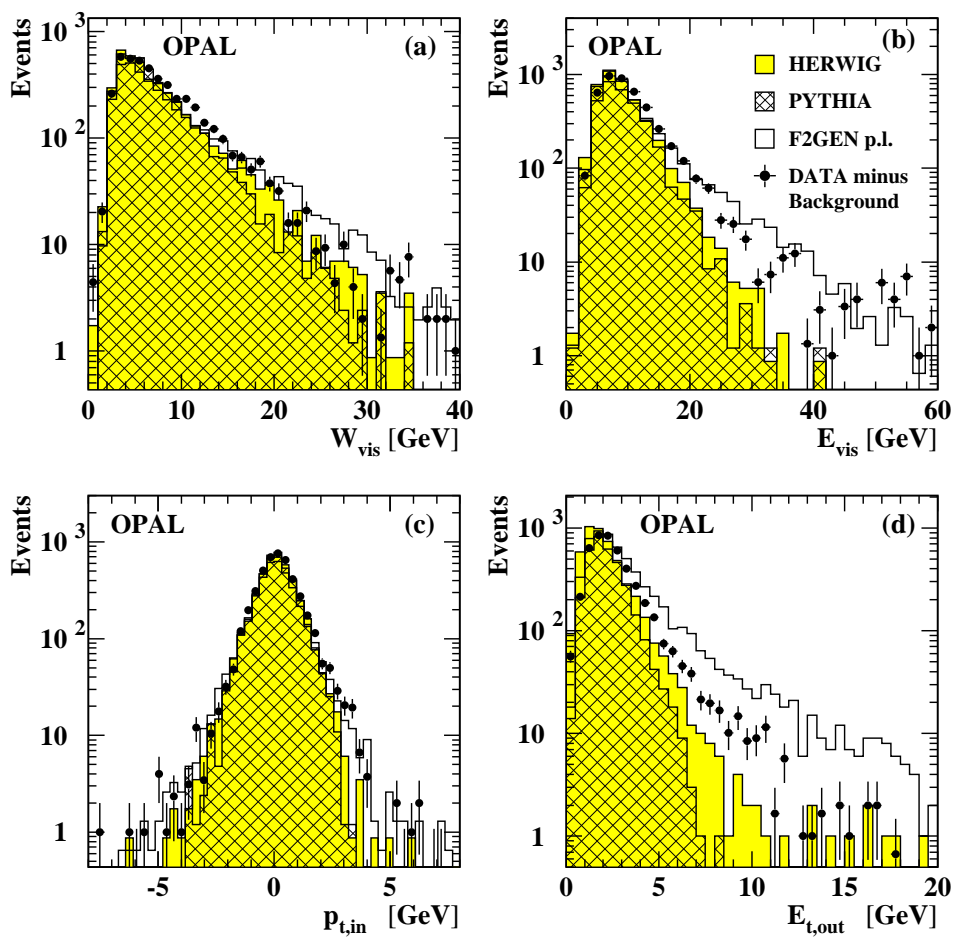

Figure 54: Comparison of the data event quantities in the low- $Q^{2}$ sample with HERWIG, PYTHIA and F2GEN (PL) Monte Carlo simulations. a) the distribution of the visible invariant mass; b) the total visible energy of the event; c) the transverse momentum of the event in the tag plane; d) the energy out of the tag plane (from 45]).

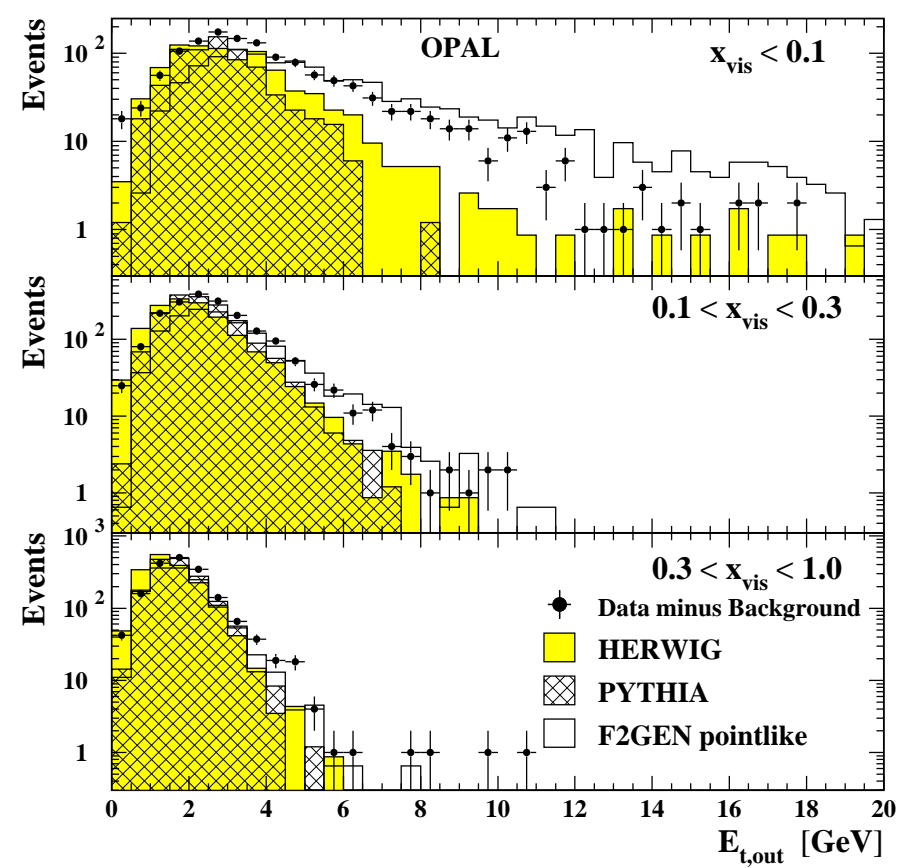

Figure 55: The energy transverse to the tag plane for three $x_{v i s}$ bins for the low $Q^{2}$ sample. Monte Carlo generators as in fig. 54 (from [45]). 


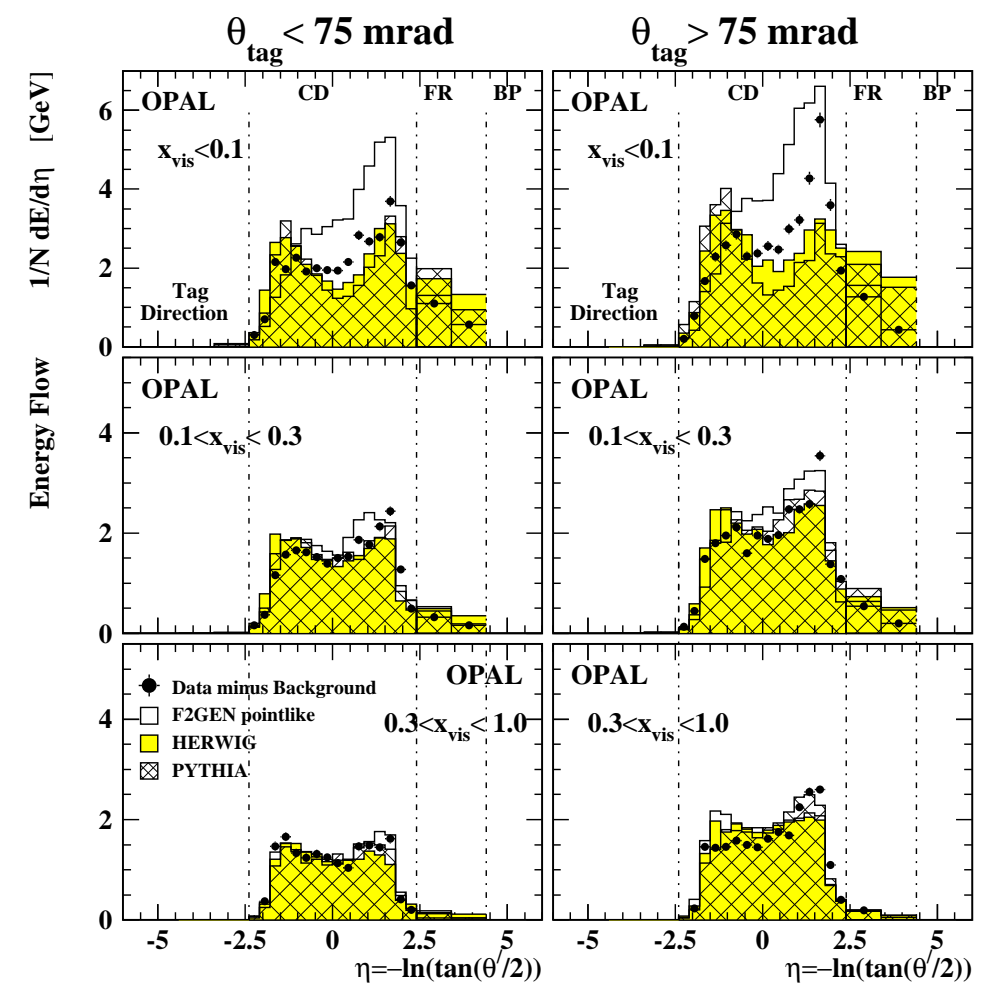

Figure 56: The hadronic energy flow per event as a function of the pseudorapidity $\eta$ for the data and various Monte Carlo simulations, in various ranges of $x_{v i s}$ and $\theta_{\text {tag }}$ for the low $Q^{2}$ sample (from [45].

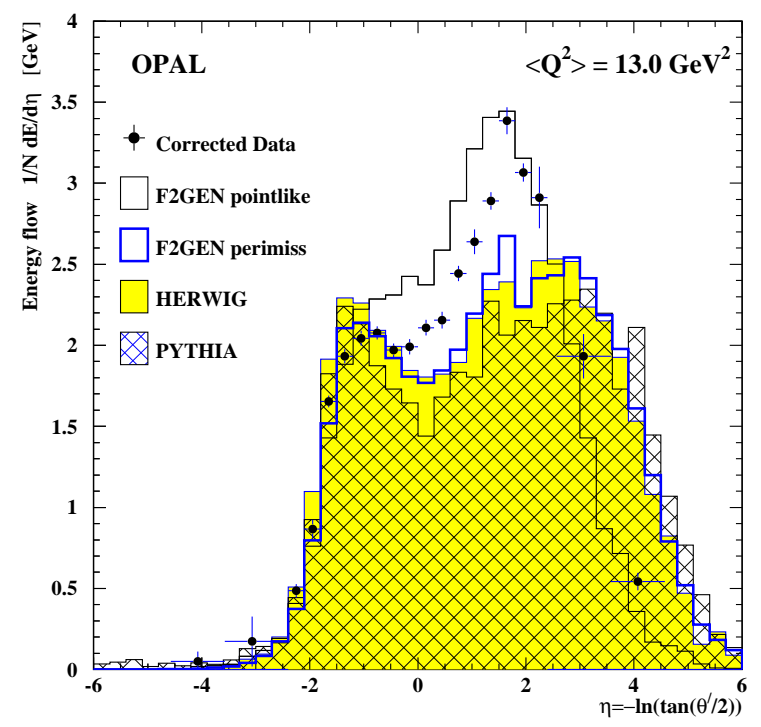

Figure 57: The measured energy flow in the low- $Q^{2}$ sample corrected for the detector inefficiencies, as a function of the pseudorapidity $\eta$, compared to the values generated by various Monte Carlo models (from [45]).

Comment: None of the generators represents the final state accurately; $E_{v i s}, E_{t, \text { out }}$ distributions as well as the hadronic flow per event show a clear discrepancy. 
"The failure of the models in the low $Q^{2}$ region is most marked at low $x_{B j}$."

The differences between the Monte Carlo models and the data in the low $Q^{2}$ region are more pronounced for the energy flow per event as a function of the pseudorapidity and the azimuthal angle.

"Particular attention will need to be given to the angular distribution of partons in $\gamma^{*} \gamma$ system".

The relation between $p_{T}^{2}$ and $Q^{2}$ in the low $Q^{2}$ sample may indicate a need to take into account the structure of the virtual photon.

"The differences between the data and the models contribute significantly to the systematic errors on $F_{2}^{\gamma}$ ".

\section{-OPAL 97b 46] (LEP 1)}

The measurement of $F_{2}^{\gamma}$ and the modelling of the $\gamma^{*} \gamma$ fragmentation into hadrons at low $Q^{2}$ region (1.1 to $6.6 \mathrm{GeV}^{2}$ ) and very small $x_{B j}$ - bins from 0.0025 to 0.2 is reported. The hadronic energy flow as a function of $\eta$ for three $x_{B j}$ regions is plotted in fig. 58.

Differences between the data and Monte Carlo models (HERWIG, PYTHIA, F2GEN) in the energy flow distributions versus the pseudorapidity (fig. 58) and in the summed energy transverse to the tag plane (not shown) are found for $x_{v i s}<0.05$.

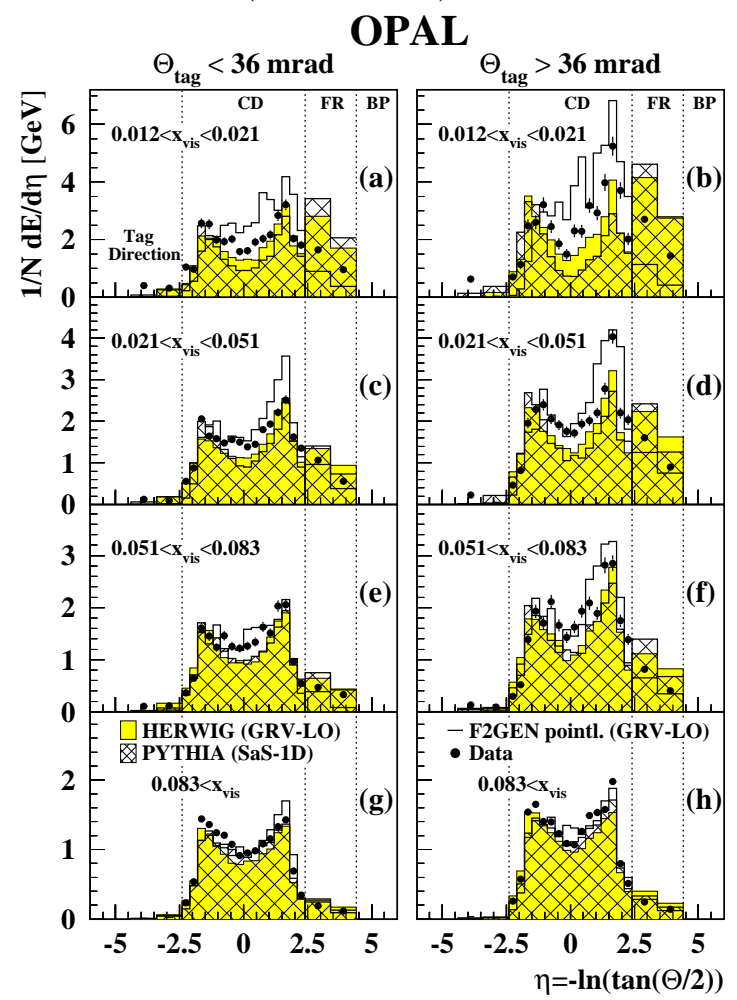

Figure 58: The measured hadronic energy flow as a function of the pseudorapidity. Comparison of data with various Monte Carlo predictions using the GRV LO and SaS1D parton parametrizations for three bins in $x_{v i s}$ and two bins in $\theta_{\text {tag }}$ (from [4]).

Comment: Discrepancy between data and Monte Carlo models in small-x region is found. 
-OPAL 97c [47] (LEP 2)

The data on $F_{2}^{\gamma}$ at the $\mathrm{CM}$ energies $161-172 \mathrm{GeV}$ were collected in 1996 in two samples: $Q^{2}=6-20 \mathrm{GeV}^{2}\left(0.02<x_{B j}<0.6\right)$ and $Q^{2}=20-100 \mathrm{GeV}^{2}\left(0.05<x_{B j}<0.8\right)$. The analysis is similar to that of OPAL 97a. The final hadronic energy flow was studied using the HERWIG, PYTHIA and F2GEN models. The LEP 1 data OPAL 97a, 97b were used for comparison. For unfolding the HERWIG program with the GRV parton parametrization was used. Two types of binning were performed, with $\left\langle Q^{2}\right\rangle$ $=9,14.5,30$ and $59 \mathrm{GeV}^{2}$, and with $<Q^{2}>=11$ and $41 \mathrm{GeV}^{2}$.

Comment: "A slightly better agreement between the predictions for the hadronic energy flow of the various models and the data in the region $0.1<x_{\text {vis }}<0.6$ is found for the data taken at $\sqrt{s_{e e}}=161-172 \mathrm{GeV}$ than for the data collected at $\sqrt{s_{e e}}=91 \mathrm{GeV}$. At $x_{v i s}<0.1$ significant differences persist, as the data prefer a more pointlike hadronic energy flow than assumed in either HERWIG or PYTHIA."

\section{-OPAL 97d,conf [58] (LEP 1)}

An analysis of the hadronic final state was done, in which the discrepancies between the data and predictions, reported in OPAL 97a 45, were examined from the point of view of the number of produced jets. The data for $Q^{2} \approx 6-30 \mathrm{GeV}^{2}$ taken in years 1994-95 were compared to the results of the HERWIG, PYTHIA and F2GEN generators.

The numbers of events for different numbers of jets, divided by the sum of all events, are presented in fig. 59.

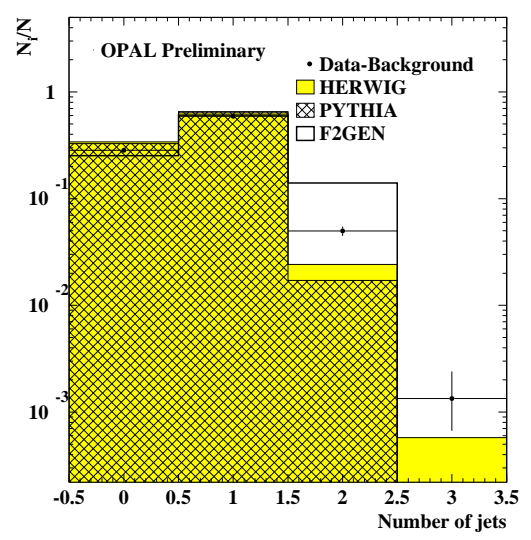

Figure 59: The fraction of events with 0-3 jets. The points show the data with the background subtracted, with statistical errors; histograms were obtained with the HERWIG, PYTHIA and F2GEN generators (from [58]).

The results of further studies of the energy flow versus the pseudorapidity $\eta$ for events with different number of jets are shown in fig. 60. 

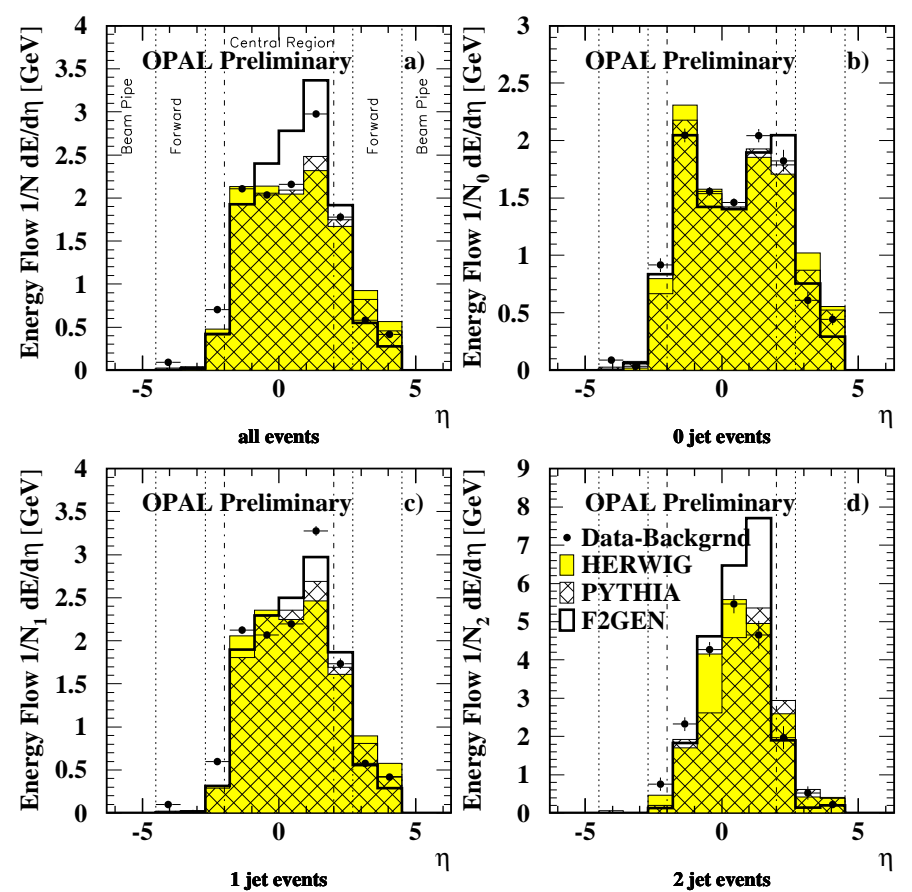

Figure 60: The hadronic energy flow per event in bins of the pseudorapidity $\eta$. Plots a), b), c) and $d$ ) show the average energy flow per event for summed events, and for events with 0 , 1 and 2 jets, respectively. The samples are represented as in fig. 59 (from [58]).

The number of events versus the energy transverse to the tag plane, obtained by the different generators and observed in the experiment, are compared in the fig. 61.
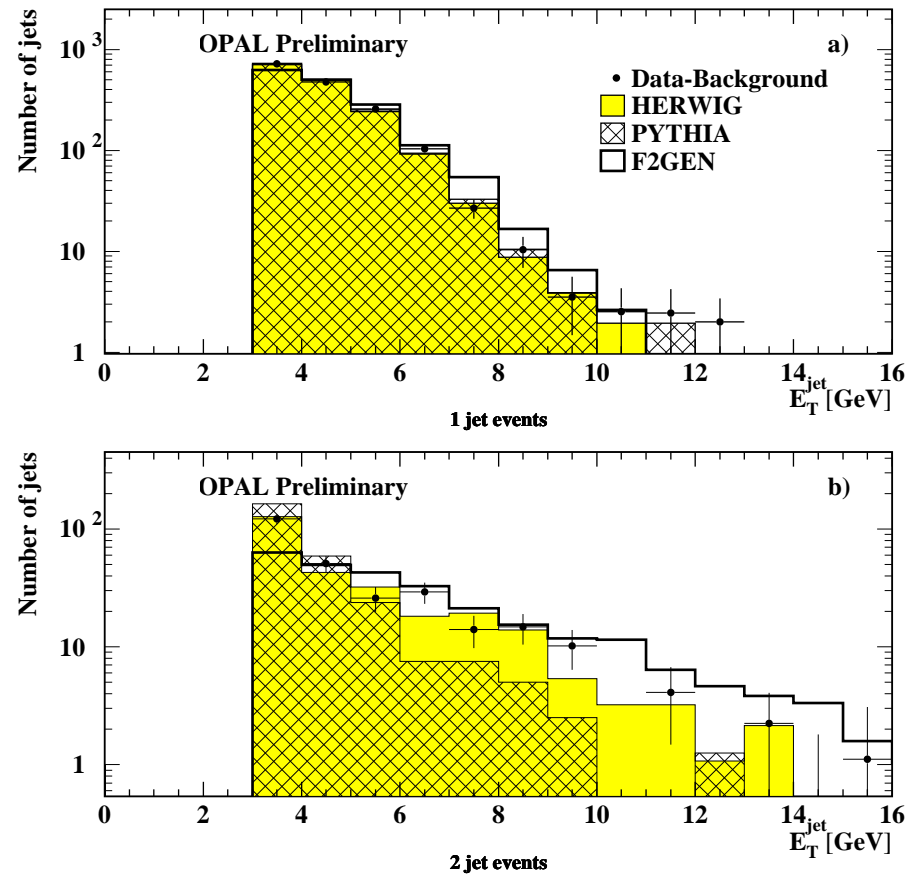

Figure 61: The number of a) one-jet and b) two-jet events as a function of the jet transverse energy, $E_{T}$. The HERWIG, PYTHIA and F2GEN events are normalized to the a) one-jet and b) two-jet data events (from [58]). 
Comment: "There is a marked difference between the data and the Monte Carlo samples in the number of events with 2 jets". "While the F2GEN sample, generated using the pointlike approximation, overestimates the 2 jet rate by a factor of 2.4, HERWIG and PYTHIA are too low by factors of 2.4 and 3.6 respectively."

Disagreement between the data and each of the generators for the hadronic energy flow versus the rapidity is seen for all types of events: 0, 1 and 2-jet.

"All of the Monte Carlo samples model well the $E_{t, o u t}$ distributions for events with 1 jet, but PYTHIA underestimates the $E_{t, \text { out }}$ of the events with 2 jets."

\section{-OPAL 2000 48] (LEP 1, LEP 2)}

Hadronic final state analysis accompanying measurement of $F_{2}^{\gamma}$ was performed at the CM energies 91, 183 and $189 \mathrm{GeV}$ (data from 1993-5 and 1997-8). Two detectors were used: the small-angle detector SW allowing to access the lowest $x_{B j}$ region, and the forward detector (FD) providing the LEP1 data in the same $Q^{2}$ range as the LEP2 data. Three samples were studied: LEP1 SW with $<Q^{2}>=1.9,3.7 \mathrm{GeV}^{2}$ and $0.0006<x_{B j}<0.6065$, LEP1 FD with $<Q^{2}>=8.9,17.5 \mathrm{GeV}^{2}$ and $0.0111<x_{B j}<$ 0.9048 and LEP2 SW with $<Q^{2}>=10.7,17.8 \mathrm{GeV}^{2}$ and $0.0009<x_{B j}<0.9048$. In the analyses new Monte Carlo programs, and improved unfolding method - the two-variable unfolding, were used. The unfolding of $x_{B j}$ was done on a logarithmic scale in order to study the low- $x_{B j}$ region in more detail.
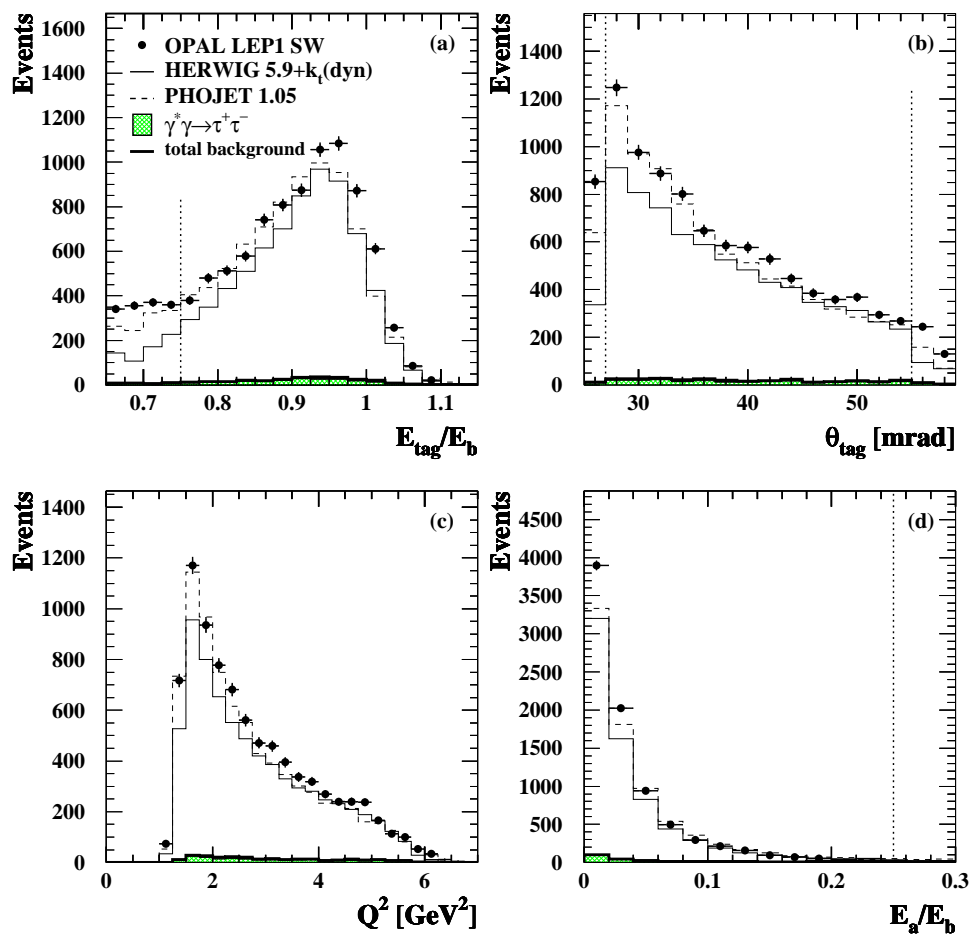

Figure 62: The comparison of the LEP1 SW data with MC predictions ("HERWIG $5.9+$ power law $p_{t}$ (dyn)" and PHOJET 1.05) for a) $E_{t a g} / E_{b}$, where $E_{\text {tag }}$ is the energy of tagged electron and $E_{b}$ is the energy of the beam, b) electron scattering angle $\left.\theta_{\text {tag }}, c\right) Q^{2}$ and $d$ ) $E_{a} / E_{b}$, where $E_{a}$ is the highest energy cluster opposite to the tag (from [48]).

The final hadronic state was studied using HERWIG 5.9, "HERWIG $5.9+$ power law $p_{t}$ (dyn)", F2GEN (all with GRV LO parametrization as an input function) and 
PHOJET 1.05 programs.

In fig. 62 the LEP1 SW data are compared to the "HERWIG $5.9+$ power law $p_{t}$ (dyn)" and PHOJET 1.05 distributions for variables related to the scattered electron. Both the generators underestimate the data (similar comparisons for LEP1 FD and LEP2 SW samples give better agreement - not shown).

For the variables related to the hadronic final state there are "large discrepancies" in comparison of data and Monte Carlo, what can be seen in fig. 63 where various measured distributions for the LEP1 SW sample are shown together with "HERWIG $5.9+$ power law $p_{t}(\mathrm{dyn}) "$ and PHOJET 1.05 simulations.
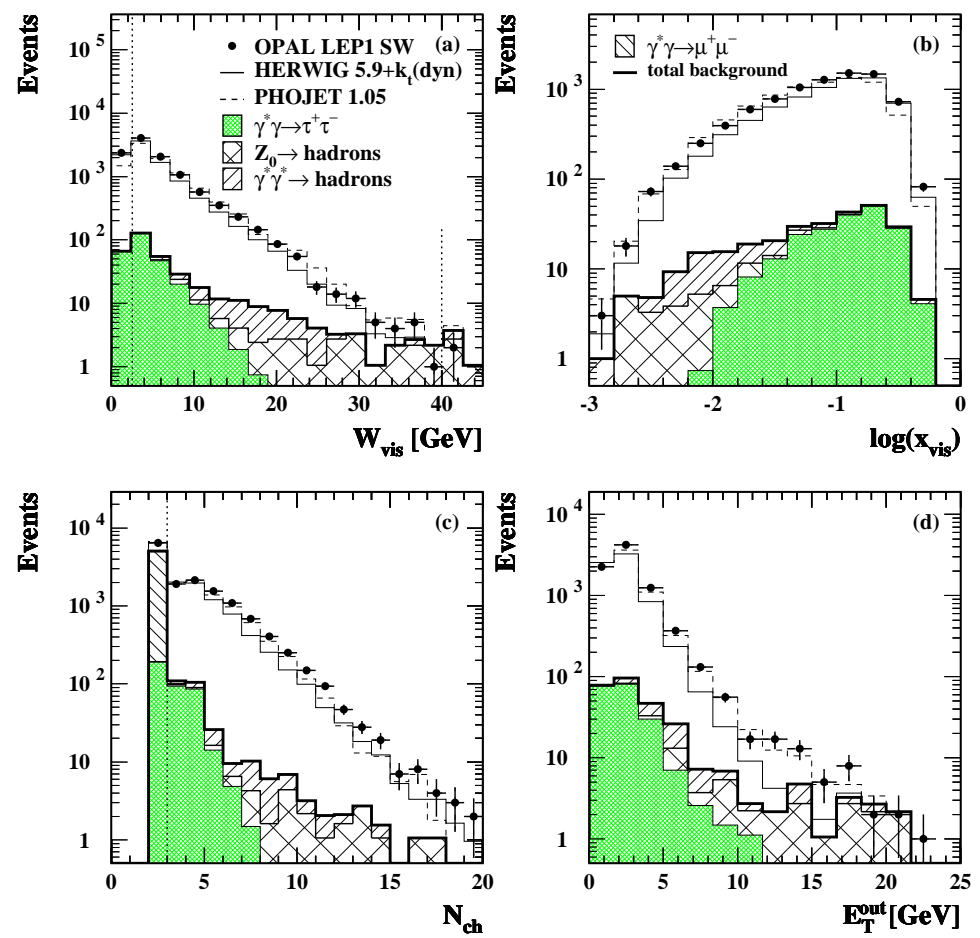

Figure 63: The comparison of the data (LEP1 SW) with the MC predictions ("HERWIG $5.9+$ power law $p_{t}$ (dyn)" and PHOJET 1.05) for a) invariant mass $\left.\left.W_{v i s}, b\right) \ln x_{v i s}, c\right)$ number of tracks in the event, and d) $E_{T}^{\text {out }}$ - the energy out of the tag plane. The dominant background contributions are also shown (from [48]).

The hadronic energy flow as a function of pseudorapidity in various MC models for LEP1 and LEP2 data is presented in fig. 64. The HERWIG 5.9 generally underestimates data, while F2GEN overestimates data in the rapidity range from -1 to 2.5. The PHOJET 1.05 gives the best description of the data for LEP1 SW and LEP1 FD samples. For LEP2 SW sample "HERWIG $5.9+$ power law $p_{t}$ (dyn)" seems to be in better agreement with data than PHOJET 1.05.

The correlation between the Monte Carlo generated invariant mass and its visible, reconstructed and corrected values was studied (not shown). 


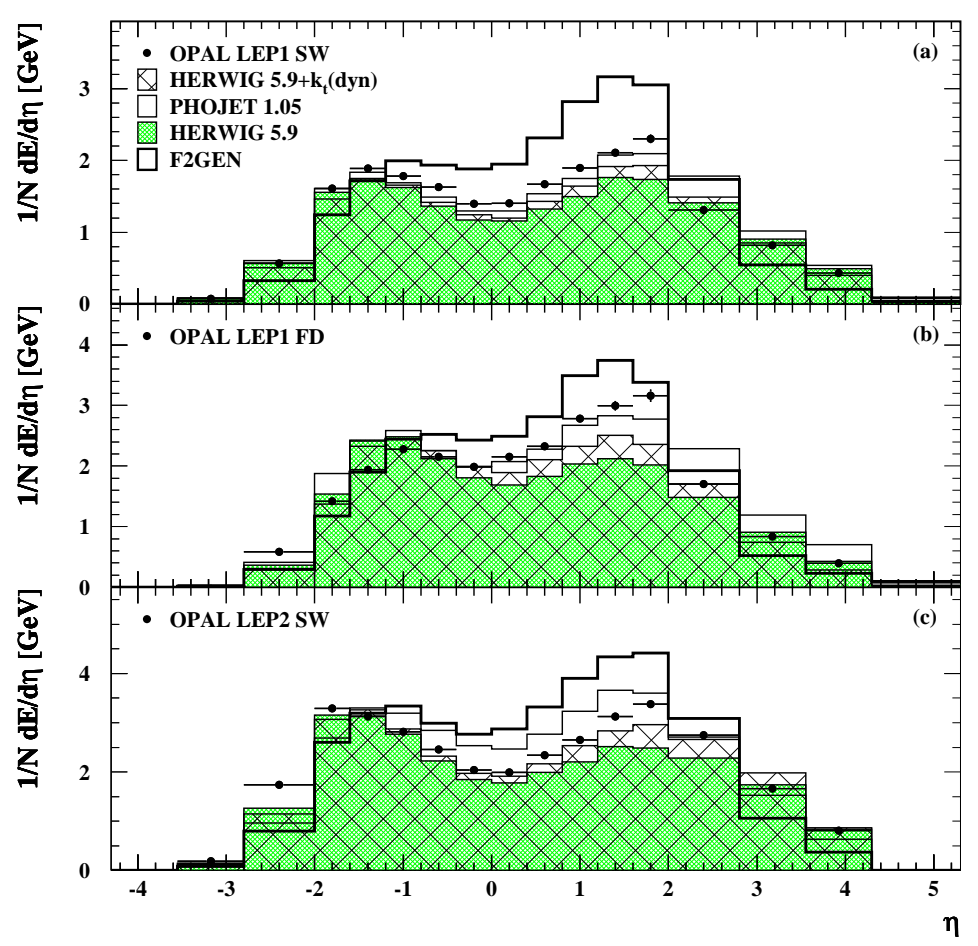

Figure 64: The energy flow per event as a function of the pseudorapidity is shown for the a) LEP1 SW, b) LEP1 FD and c) LEP2 SW samples in comparison with predictions of F2GEN, HERWIG 5.9, "HERWIG 5.9 + power law $p_{t}$ (dyn)" and PHOJET 1.05 (from [48]).

In fig. 65 the usefulness of two choices of the second, beside $x_{B j}$, unfolding variable is studied for the LEP1 SW sample. Both studied variables $E_{\text {for }} / E_{\text {tot }}$ and $E_{T}^{\text {out }} / E_{\text {tot }}$ are sensitive to the angular distribution of the final hadrons. Similar results for LEP1 FD and LEP2 SW were obtained as well (not shown).

Test of the unfolding methods was performed, see figs. 66 and 67, where $F_{2}^{\gamma}$ obtained using different MC models and unfolding variables is shown for the LEP1 SW and LEP2 SW samples, respectively. Similar test was performed for LEP1 FD data (not shown). These results allowed to choose the most reliable unfolding variables, which are $x_{c o r}$ and $E_{\text {for }} / E_{t o t}$. 

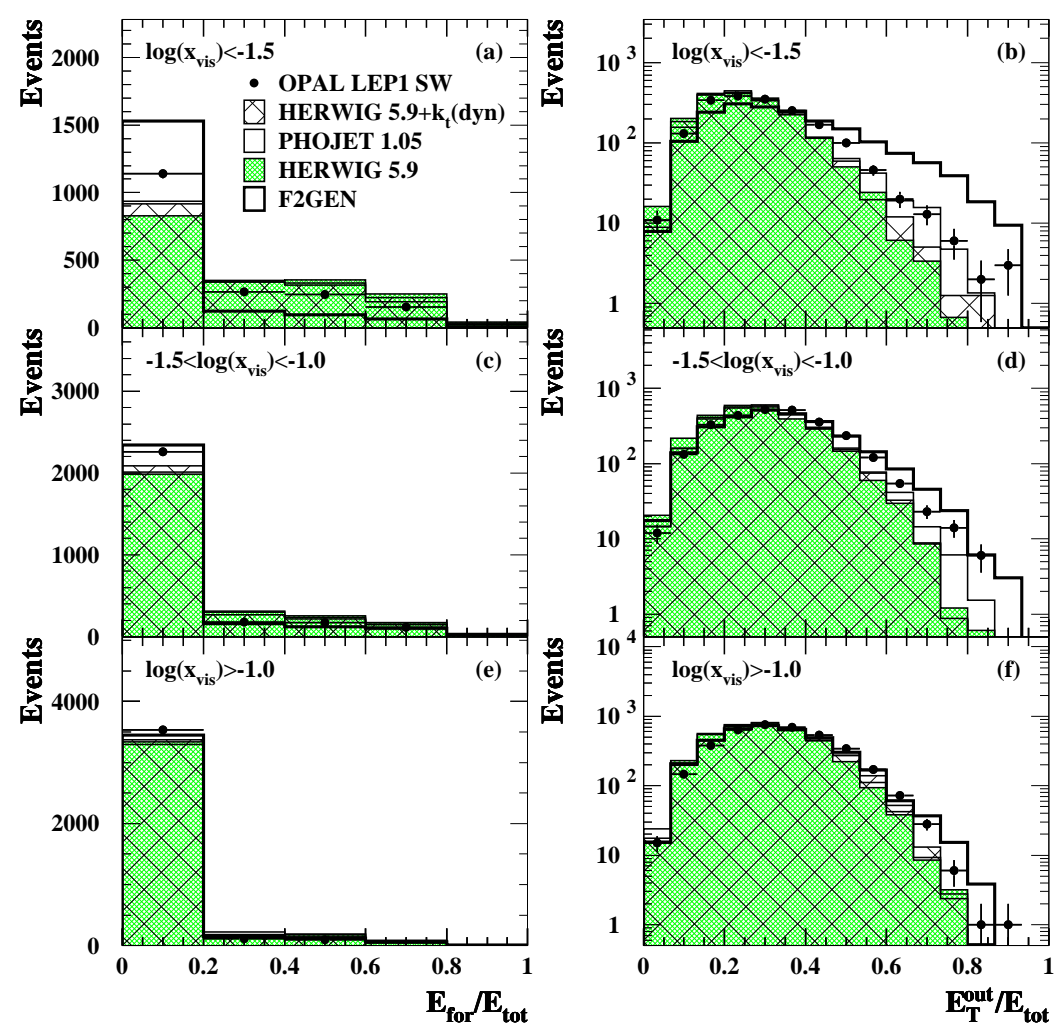

Figure 65: The distribution of the observed energy in the forward direction divided by the total observed energy, $E_{\text {for }} / E_{\text {tot }}$ (a,c,e), and the transverse energy out of the tag plane divided by the total observed energy, $E_{T}^{\text {out }} / E_{\text {tot }}(b, d, f)$ for three bins of $x_{v i s}$. The comparison of the data with the predictions of F2GEN, HERWIG 5.9, "HERWIG $5.9+$ power law $p_{t}$ (dyn)" and PHOJET 1.05 is shown (from 48]). 


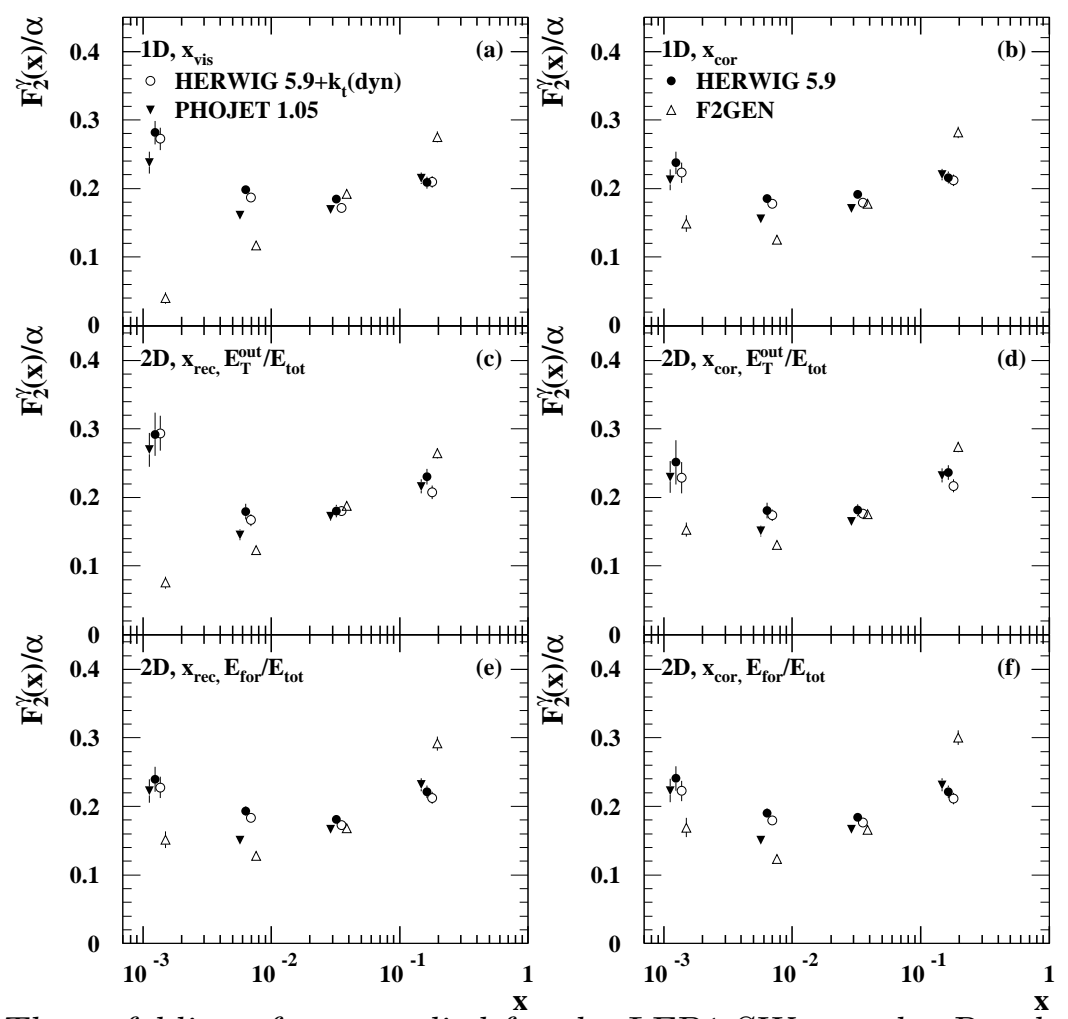

Figure 66: The unfolding of $x_{B j}$ studied for the LEP1 SW sample. Results based on onevariable unfolding with a) $x_{v i s}$, b) $x_{c o r}$ and on two-dimensional unfolding with c) $x_{r e c}$ and $\left.E_{T}^{o u t} / E_{t o t}, d\right) x_{c o r}$ and $E_{T}^{o u t} / E_{t o t}$, e) $x_{r e c}$ and $\left.E_{f o r} / E_{t o t}, f\right) x_{c o r}$ and $E_{f o r} / E_{t o t}$. The unfolding was done using F2GEN, HERWIG 5.9, "HERWIG $5.9+$ power law p " (dyn) and PHOJET 1.05 (from 48]). 

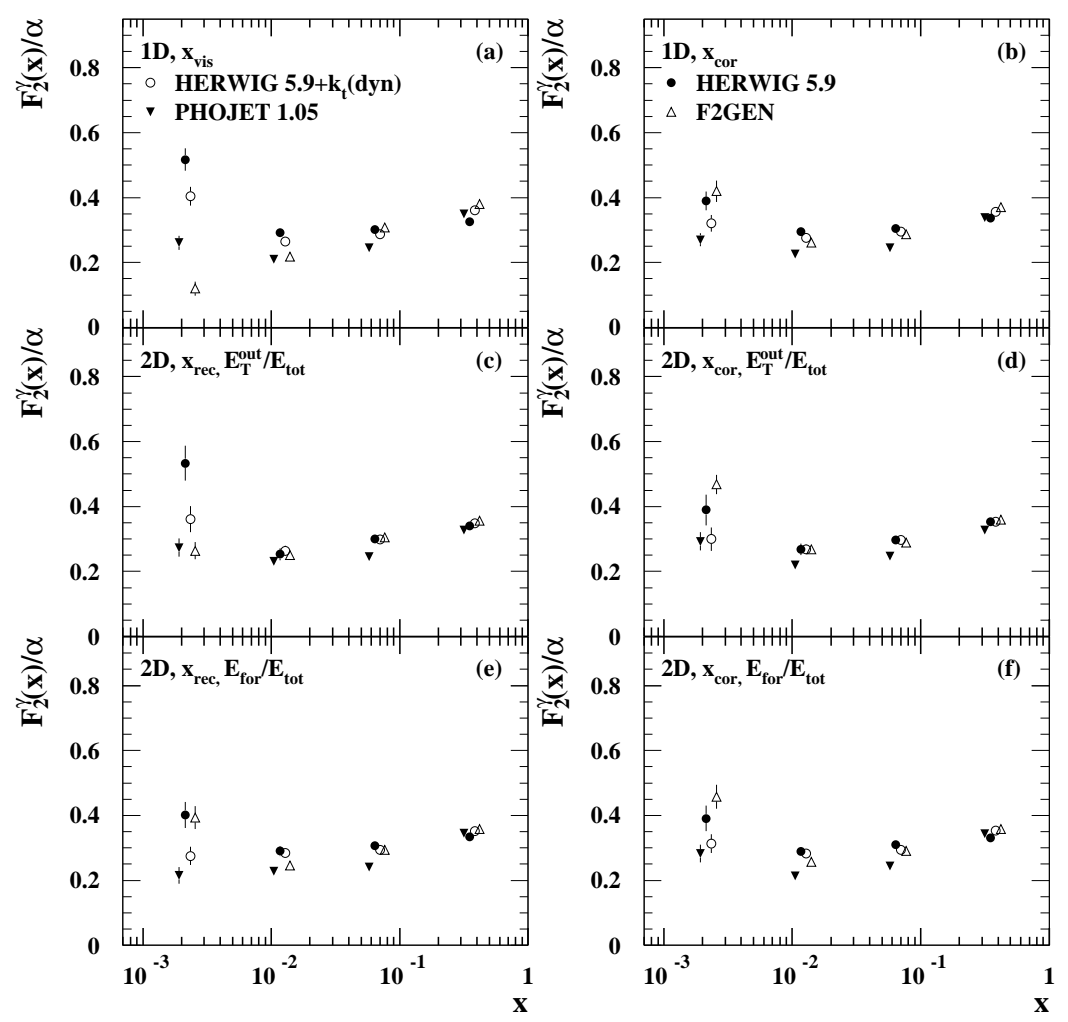

Figure 67: As in fig. 66 for the LEP2 SW sample (from 487).

Comment: "HERWIG 5.9 + power law $p_{t}$ (dyn)" and PHOJET 1.05 describe the OPAL data better than the F2GEN and HERWIG models.

To reduce the MC "modelling error, two-dimensional unfolding has been introduced, using $E_{T}^{o u t} / E_{\text {tot }}$ as a second unfolding variable."

"Monte Carlo modelling of the final state is still a significant source of systematic error, but it no longer dominates all other sources."

\section{-TOPAZ 94 [51] (TRISTAN)}

The jet production (one and two jets) has been studied for the first time in deep inelastic $e \gamma$ scattering $\left(3.0 \mathrm{GeV}^{2}<Q^{2}<30 \mathrm{GeV}^{2}\right)$, see also sec. 2.2.2. Events with the transverse momentum of jets between 2 and $8 \mathrm{GeV}$ and for the $|\eta|<0.7$ were studied using the jet cone algorithm with $\mathrm{R}=1$ (see next section for the definition of the jets). The Monte Carlo simulation was based on the VMD formula for the hadronic part. For the perturbative (pointlike) part a QCD model was used with FKP (uds) and QPM (c) contributions, with $p_{T}^{0}=0.5 \mathrm{GeV}$.

The pointlike and hadron-like configurations resulting in the different final-state jet topologies (two jets and single jet, respectively) were studied. The pointlike part was simulated with the same angular distribution as for $\gamma \gamma \rightarrow q \bar{q}$. The hadron-like part involves the struck quark along the $\gamma^{*}$ direction and the remnant one along the $\gamma$ direction.

Comment: The production of events with two high-p T $_{\text {jets }}$ is consistent with the point- 
like perturbative contribution.

"...an excess over the pointlike component is observed in the one-jet sample, which is direct experimental evidence for the existence of the hadron-like component in deep inelastic er scattering". 


\subsection{Large $p_{T}$ processes in $\gamma \gamma$ and $\gamma p$ collisions. Resolved pho- tons}

The structure function $F_{2}^{\gamma}$ for the real photon considered in sec. 2.2 is sensitive mainly to the combination of quark densities, and, moreover, due to its strong dependence on the charge, to the up-type quark distributions. Therefore it is of great importance

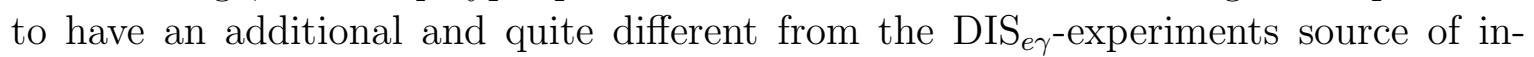
formation. Hard photon(s)-induced processes provide such a source, with the cross section given by a combination of different parton densities in the photon(s) convoluted with the cross sections for basic partonic subprocesses (see below). The role of these resolved photon processes, e.g. in determining the gluon density in the photon, is unique indeed. They are also crucial in probing the heavy quark content of the photon, in particular the charm quark density; this topic we discuss separately in sec. 5 .

As we have already mentioned, the large $p_{T}$ jet production in $\gamma \gamma$ collision leads to the complementary information on the photon structure to that coming from the $F_{2}^{\gamma}$. Moreover, the study of the hadronic final state, with the production of, predominantly, small $p_{T}$ particles (jets), is a standard part of the structure function analyses performed in $\mathrm{DIS}_{e \gamma}$ measurements at LEP and TRISTAN (see discussion in secs. 2.2 and 2.3).

The production cross section of the large $p_{T}$ jets, which are the hadronic representations of the hard partons produced in the basic subprocess, does depend on the applied jet definition. Although this introduces an additional uncertainty in the description of the data, the relatively high rate makes large $p_{T}$ jet production, apart from $F_{2}^{\gamma}$, the basic source of information on the partonic structure of $\gamma$. The main goal in such measurements is to extract the individual parton density in the real photon $\square$.

In this section we focus on the dedicated large $p_{T}$ single and double jet $\square$ production in the resolved $\gamma \gamma$ and $\gamma p$ collisions, where the real photon(s) may interact through its partons 8 . The bulk of data is coming from the HERA collider, where the photoproduction of jets with higher transverse momentum than at LEP and TRISTAN colliders was analysed. The data on the three or more jets, the photon remnant or the jet shape are also available.

Beside the jet production also the inclusive one-particle production is sensitive to the partonic content of the photon, but it depends in addition on the fragmentation functions, or, as in the prompt photon production, on an additional assumption (the isolation criteria). Nevertheless they play a crucial role in establishing the structure of the photon, as the newest charged particle production measurements at HERA, where the gluon content has been derived. In addition we collect also the results for the photoproduction of the prompt photon, which may consist in future an additional source of information on the gluon density in the photon. The prompt photon production was studied also in $\gamma \gamma$ collision at TRISTAN (see TOPAZ 98,conf).

In the previous section we have discussed the single tagged events with virtuality of

\footnotetext{
${ }^{16}$ At present most of the data deal with the almost real photon; for the jet production in processes with virtual photon, see sec. 3 .

${ }^{17}$ We count only jets from hard process, not remnant jets.

${ }^{18}$ The first evidence for the resolved photon contributions and a need for the gluonic content of photon in $\gamma \gamma$ collision can be found in AMY 92. See H1 92 and ZEUS 92 for a first evidence for hard processes in the $\gamma p$ collision.
} 
photons radiated by the tagged electrons larger or much larger than $1 \mathrm{GeV}^{2}$ (with the exception of one experiment, DELPHI 95). In this section the initial photons may be considered real, as the median virtuality of the photon(s) is smaller than $\Lambda_{Q C D}^{2}$, while the transverse momenta of the observed jets are chosen to be much larger than $\Lambda_{Q C D}$.

\subsubsection{Theoretical description}

Below we discuss shortly how the single and double jet production in the photoninduced processes is described in QCD. The specific case of the the charged hadron photoproduction at HERA will be described shortly in $\mathbf{H 1} \mathbf{9 9 a}$, and the prompt photon photoproduction will be discussed in the separate subsection 2.4.5.

The production of large $p_{T}$ jets (or individual particles) may proceed directly (the direct or the (Q)PM contribution), or as a result of partonic interaction between components of the photon(s) leading to resolved photon contribution. In some analyses the soft VMD-type contributions are included separately.

The resolved photon processes with large $p_{T}$ jets can be characterized by the scale of hardness $\tilde{Q}^{2}$, at which the photon is resolved. It is usually provided by the $p_{T}$ of the final jets, i.e. $\tilde{Q}^{2} \sim p_{T}^{2}$. (We introduce here the notation $\tilde{Q}^{2}$ in order to distinguish it from the DIS scale $Q^{2}$, which is equal to the virtuality of the photon probe.) To apply the perturbative QCD to such resolved almost real photons, i.e. with $P^{2} \sim 0$, the scale $\tilde{Q}^{2}$ should be much larger than $\Lambda_{Q C D}^{2}$. Events where virtualities fulfill the relation $\Lambda_{Q C D}^{2} \ll P^{2} \ll \tilde{Q}^{2}$ are discussed in the next section, where the concept and data on the structure of virtual photon will be introduced.
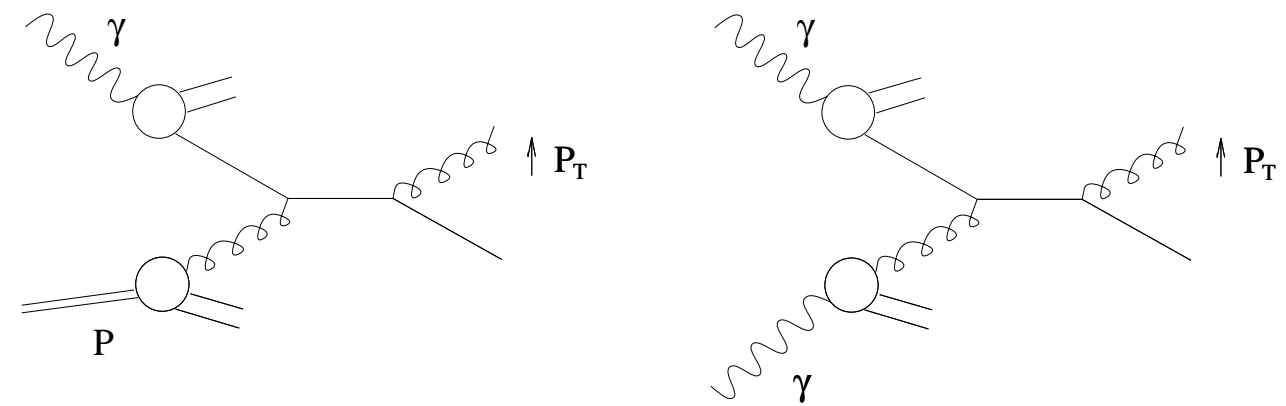

Figure 68: The inclusive large $p_{T}$ jet production in the resolved $\gamma p$ collision (left) and in the (double) resolved $\gamma \gamma$ collision (right).

Assuming the factorization between the hard subprocess cross section $\hat{\sigma}[9$ and the parton densities, the generic LO cross section for the two-jet production in $\gamma p$ collision (fig. 68, left) is given by

$$
d \sigma^{\gamma p \rightarrow \text { jet }_{1} j e t_{2} X}=\sum_{i, j} \iint d x_{\gamma} d x_{p} f_{i / \gamma}\left(x_{\gamma}, \tilde{Q}^{2}\right) f_{j / p}\left(x_{p}, \tilde{Q}^{2}\right) \widehat{\sigma}^{i j \rightarrow j^{j e t_{1}} \text { jet }_{2}}
$$

where $f_{i / \gamma}\left(x_{\gamma}, \tilde{Q}^{2}\right)$ describes the LL probability of finding a parton of a type " $i$ " in the photon. The corresponding function $f_{j / p}\left(x_{p}, \tilde{Q}^{2}\right)$ is related to the proton. The $x_{\gamma}\left(x_{p}\right)$

\footnotetext{
${ }^{19}$ The collection of useful formulae for the cross section $\hat{\sigma}$ for $2 \rightarrow 2$ body partonic processes can be found in 60 .
} 
variable is by definition the part of the four-momentum of the photon (proton) carried by its parton. The direct interaction of the initial photon corresponds to $x_{\gamma}=1$ and $f_{\gamma / \gamma}=\delta\left(1-x_{\gamma}\right)$. The scale of hardness $\tilde{Q}^{2}$ plays here a role of the factorization scale between the hard and soft processes in the $\gamma p$ collision. Note that the precise definition of the factorization scale can only be given in the QCD calculation going beyond the LO approach.

For the two-jet production in $\gamma \gamma$ collision, $\gamma \gamma \rightarrow$ jet $_{1}$ jet $_{2} X$ (fig. 68, right) a similar LO formula holds:

$$
d \sigma^{\gamma \gamma \rightarrow \text { jet }_{1}{ }^{j e t_{2} X}}=\sum_{i, j} \iint d x_{\gamma}^{1} d x_{\gamma}^{2} f_{i / \gamma}\left(x_{\gamma}^{1}, \tilde{Q}^{2}\right) f_{j / \gamma}\left(x_{\gamma}^{2}, \tilde{Q}^{2}\right) \hat{\sigma}^{i j \rightarrow j^{j e t_{1}} j^{j e t_{2}}} .
$$

If in the final state a specific hadron is measured, the fragmentation function will appear in addition in formulae $(20)$ and (21), together with the corresponding $z$ variable, describing the part of four-momenta of final parton taken by the final hadron.

In the NLO approach, needed to describe the present data, the NLL parton densities should be used and the $\hat{\sigma}$ should contain $\alpha_{S}$ corrections. Note that the separation between the direct and resolved photon contribution holds only in the LO approach. The theoretical predictions based on the LO [171] - [173] or NLO (HO) [174] - [188] QCD calculations are available for the inclusive one and two-jet photoproduction in $e^{+} e^{-}$and $e p$ experiments.

Here we would like to point out that the way of counting the order of the QCD calculation in the photon-induced processes is still a subject of discussion (see e.g. [27], [192] c and earlier papers [189] ef). The origin of the problem is the presence of the definite PM prediction, in form of $\alpha \log \left(Q^{2}\right)$ contribution, leading to the inhomogeneous term in the $Q^{2}$ evolution equations for the quark densities in the photon. One approach bases on the treatment of the quark density in the photon as being of order $q^{\gamma} \sim \alpha / \alpha_{s}$, while in the second one $q^{\gamma} \sim \alpha$. This difference leads to different sets of diagrams which formally should be included in the NLO calculations, see e.g. the prompt photon production at HERA, where two types of NLO predictions are compared to data (ZEUS 2000a). We will not discuss here this issue any further, and in the presentation of the results we will use the terminology describing the accuracy of the analysis, LO or NLO, as given in the original papers.

The multiple interaction between partons can also be taken into account in the analysis of the jet production in $\gamma \gamma$ and $\gamma p$ processes, in analogy to $p \bar{p}$ processes. Since the direct events should be free from such multiple interactions, the $\gamma \gamma$ and $\gamma p$ collisions may offer a unique laboratory to study this problem. The multiple interaction seems to be needed for a good description of the data, see below.

\subsubsection{Measurements of the resolved photon processes}

At present, the $\gamma \gamma$ collisions arising in $e^{+} e^{-}$colliders in no-tag 20 or antitagged conditions correspond to the $\gamma \gamma$ events where both real photons can be described by the Weizsäcker - Williams energy spectra. In the OPAL experiment the maximal squared target photon masses were equal $P_{1,2}^{2}=0.8 \mathrm{GeV}^{2}$, while in the ALEPH experiment around $7 \mathrm{GeV}^{2}$, and in TOPAZ $-2.6 \mathrm{GeV}^{2}$. Still, the median virtuality in $\gamma \gamma$ collision

\footnotetext{
${ }^{20}$ The name "untagged" is also used.
} 
is small enough (e.g. $10^{-4} \mathrm{GeV}^{2}$ in TOPAZ). In the $\gamma p$ scattering at ep collider HERA a similar Weizsäcker - Williams spectrum describes the flux of photons coming from the electron (in year 1996/7 the positron, in 1998 the electron again? $\mathrm{GeV}^{2}$ (but with the median $0.001 \mathrm{GeV}^{2}$ ), recently also $P^{2}<1 \mathrm{GeV}^{2}$ or below $0.01-0.02$ $\mathrm{GeV}^{2}$ if the dedicated detector is used.

Note that, contrary to the $\mathrm{DIS}_{e \gamma}$ experiments, in the resolved photon processes there is no one basic observable, analogous to $F_{2}^{\gamma}$. Usually the $p_{T}$ or $E_{T}$ and pseudorapidity $\eta$ distributions for final particle or jet are compared with the QCD predictions using a specific parton parametrization. The agreement with the data justifies a posteriori the correctness of the applied parton parametrization and of the QCD calculation. Only in few cases the parton distribution is extracted from data (and only for $\gamma p$ events, see below).

For comparison with QCD not only the above distributions are important but also the study of the structure of the jets (transverse energy flow around the jet axis, i.e. jet profile ) and the structure of the underlying event (jet pedestal) 2 , corresponding to the hadronic activity outside the jets where effects due to the beam remnants, from spectator partons, may be seen. Also an additional interaction between these remnants, leads to the higher energy level of the underlying event.

For a more detailed study of properties of the resolved photon processes the separation between the direct photon events, where the photon participates directly in the hard subprocess, $\left(x_{\gamma} \sim 1\right)$, and the resolved photon events, is needed.

In the two-jet events, $x_{p}$ and $x_{\gamma}$ (for HERA measurements) and $x_{\gamma}^{1,2}$ (for LEP and TRISTAN data) distributions, sensitive to parton densities, were studied, see e.g. ZEUS 95b, 98a and OPAL 97e. The angular distribution (e.g. in the dijet CM system) $d \sigma / d \cos \theta^{*}$, on the other hand, is expected not to be sensitive to these ingredients. So this measurement may help to verify the expectation of different angular distributions due to different subprocesses which correspond to the direct and resolved photon contributions, see ZEUS 96 for the first results, also OPAL 99a. The first extraction of the gluon density (H1 95a) and of the effective parton distribution $(\mathbf{H 1}$ 98) was reported by the H1 Collaboration. The data on prompt photon production have appeared recently both from the TRISTAN and HERA colliders.

The main Monte Carlo generators used by the experimental groups for these kinds of processes are the general purpose generators PYTHIA, HERWIG and PHOJET (with or without the multiple parton interactions). The DELPHI collaboration uses the TWOGAM generator to simulate the QPM, VMD and resolved photon contributions. All of these MC programs use the $p_{T}^{\text {min }}$ cutoffs, separating the soft and hard processes in the simulation, being fixed by the requirement to reproduce the total cross section. (In case of the multiple interaction $p_{T}^{m i}$ parameter is used instead of $p_{T}^{m i n}$ ). For both $e^{+} e^{-}$and $e p$ colliders the additional power law $p_{t}$ spectrum has been included recently in the HERWIG and PYTHIA programs. They were used in e.g. ALEPH 97a,conf, 99a, 99b,conf; OPAL 2000; TOPAZ 98,conf; ZEUS 95c (where the spectrum was first introduced).

The single and double jet samples were studied, reaching the highest $p_{T}$ of jets in

\footnotetext{
${ }^{21}$ Note that starting from 1998 new energies are accessible: $27.5 \mathrm{GeV}$ for $e^{-}$and $920 \mathrm{GeV}$ for $p$, see 2.3.4.

${ }^{22} i . e$. the transverse energy determined outside of the jet cone
} 
$\gamma \gamma$ processes up to $17 \mathrm{GeV}$. Jets at the HERA collider are observed up to $75 \mathrm{GeV}$ (ZEUS 98c,conf and 99b,conf).

The jet $E_{T}$ distributions are in general in agreement with the expectations for both single and double jet production, both at $e^{+} e^{-}$and $e^{ \pm} p$ colliders. In the pseudorapidity dependence the discrepancy is observed for the jet rates. The transverse energy flow around the jet axis, especially for small $E_{T}$ and small $x_{\gamma}$ is not properly described by existing Monte Carlo generators. Also for the two-jet events it has been found that distribution at the small $x_{\gamma}$ lacks proper description. The agreement with the data is improved when the additional power law $p_{t}$ spectrum is taken into account in the analysis, as in $F_{2}^{\gamma}$ and hadronic final state analysis. A need for extra $p_{t}$ in the simulation of the whole event can also be taken as a hint that the multiple scattering may be important in the photon-induced processes. The multiple interaction included in the experimental analysis is modelled usually as in the $p \bar{p}$ experiments (see e.g. H1 96a). Global study of the photon-induced jet production with the aim of tuning the general purpose Monte Carlo models was performed recently in [12]. "The main ingredients investigated in the tuning are the description of the "underlying event" and the choice of photon structure". The conclusion of this analysis is that "the agreement without the multiple interaction is very poor".

The strong dependence on the proper choice of the jet definition and the parameters like the cone size $R$ (see below), has also been observed in the jet production at HERA. There is a possible relation of this effect to the problem of describing the underlying events observed both in $e^{+} e^{-}$and in $e p$ collisions, that was mentioned above.

The following two jet definitions are used in the analyses reported below. The first is the jet cone algorithm with the fixed value of the cone variable $R$, defined as $R=\sqrt{(\delta \phi)^{2}+(\delta \eta)^{2}}$, with $\delta \phi$ and $\delta \eta$ describing the differences between the cone (jet) axis and the particle direction in the azimuthal angle and pseudorapidity, respectively. It is used in PUCELL and EUCELL algorithms. The second one corresponds to the $k_{T}$ - cluster algorithm on which the KTCLUS approach is built up. Beside $R$, also

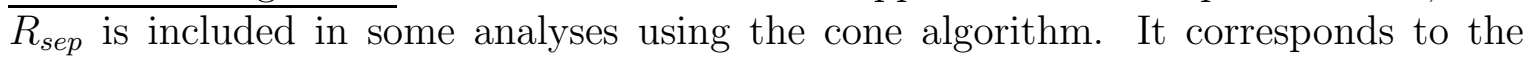
additional separation between partons, and the $R_{\text {sep }}=2 R$ means no restriction. (The discussion of jet definitions in the NLO calculation can be found e.g. in [187].)

The difference of the two jets' transverse energy $\Delta E_{T}=E_{T 1}-E_{T 2}$ and analogous difference for the pseudorapidities, $\Delta \eta=\left|\eta_{1}-\eta_{2}\right|$, are introduced in two-jet events. (For the analysis of the energy of the underlying event the variable $\delta \eta=\eta_{\text {cell }}-\eta$ is used.) In the analysis of two-jet events there appear also the average pseudorapidity $\bar{\eta}=\left(\eta_{1}+\eta_{2}\right) / 2\left(\right.$ denoted also by $\left.\eta_{\text {jet }(s)}\right)$ and the average transverse energy of jets, $\bar{E}_{T}$ $\left(\bar{E}_{j e t(s)}\right)$.

It is worth noticing the difference between the variables describing the produced parton and the corresponding quantities for the jet, representing the considered parton on the hadronic level. The unfolding procedure is needed in order to obtain the "true" partonic variables, e.g. the transverse momentum of the parton $\left(p_{t}\right)$ from the transverse momentum or energy of the jet $\left(p_{T} \text { or } E_{T}\right)^{23}$.

\footnotetext{
${ }^{23}$ We keep in subscripts small letters for quantities related to the partonic level
} 


\subsubsection{Jet production in $\gamma \gamma$ collisions}

Here we present the results for the jet production in $\gamma \gamma$ collisions, where one or two resolved photons may interact.

As in the previous section we will denote the virtualities of two involved photons by $P_{1}^{2}$ and $P_{2}^{2}$. The antitagged events at LEP correspond to $P^{2}<4 \mathrm{GeV}^{2}$, with median approximately $10^{-4} \mathrm{GeV}^{2}$. For the antitagging conditions in OPAL 97e the maximum value $P^{2}=0.8 \mathrm{GeV}^{2}$ was used. At TRISTAN the $P^{2}$ value in antitagging case lies between $10^{-8}$ and $2.6 \mathrm{GeV}^{2}$ with the mean value $\sim 10^{-4} \mathrm{GeV}^{2}$.

In $e^{+} e^{-}$machines the parton momentum fractions, variables $x_{\gamma}^{1,2}=x_{\gamma}^{ \pm}$, are determined from the two final jets with the highest $E_{T}$, according to the formulae:

$$
x_{\gamma}^{ \pm}=\frac{\sum_{\text {jets }}\left(E \pm p_{z}\right)}{\sum_{\text {hadrons }}\left(E \pm p_{z}\right)} .
$$

Variables referring to the dijet centre of mass system are (in OPAL 99b) denoted by star, e.g. $\eta^{*}$.

Below we present the LEP and TRISTAN results.

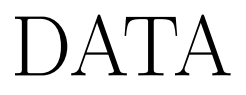

\section{-ALEPH 93 [62] (LEP 1)}

The presence of hard scattering processes is demonstrated in the sample from 19901991 data. The antitagging condition corresponds here to $<P_{1,2}^{2}>=0.23 \mathrm{GeV}^{2}$. The VMD, QPM and QCD contributions, the latter with $p_{T}^{\text {min }}$ cutoff, were studied using Vermasseren program for QPM, and TOPAZ MC generator for QCD part. The $p_{T}^{\min }$ was fitted to be $2.5 \pm 0.1 \pm 0.3$.

Comment: "The data are well described by a combination of three models, namely $V M D, Q P M$ and $Q C D "$.

The fitted $p_{T}^{\text {min }}$ parameter is "higher than the values previously obtained at TRISTAN but consistent with recent HERA photoproduction results".

\section{-DELPHI 94 63] (LEP 1)}

Data were collected in the period 1990-92 with $<P_{1}^{2}>\sim 0.12 \mathrm{GeV}^{2}$. Events are groupped into a minimum bias sample (sample I) and a high $p_{T}$ jet events sample (sample II). Jets, defined according to the Lund cluster algorithm, with $p_{T}$ greater than $1.75 \mathrm{GeV}$, and a polar angle between $40^{\circ}$ and $140^{\circ}$, were observed. The three component model (VMD for a fully nonperturbative contribution, QPM, and QCD with the $p_{T}^{\min }$ ) was used in the analysis.

Results for the jet $p_{T}$ distributions are shown in fig. 69. To model the QCD (resolved photon) contribution the FKP approach (see secs. 2.2.2, 2.3) was used with DG and DO parton parametrizations. For a comparison also the LAC1 and GS 
densities were used.

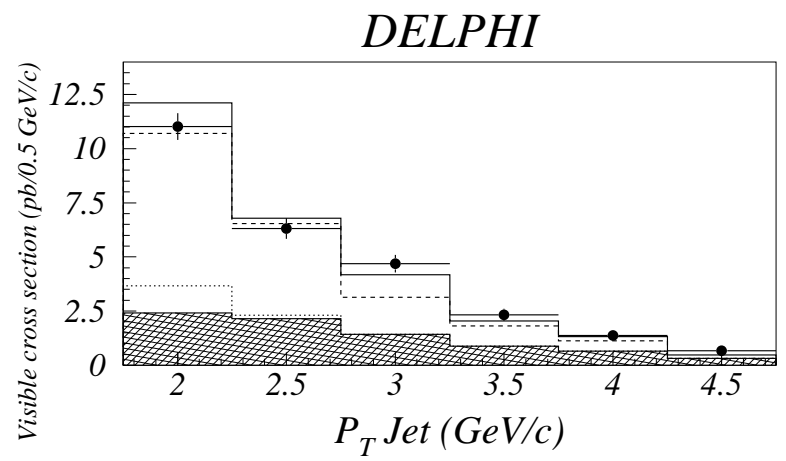

Figure 69: Distribution of jet transverse momentum compared with the Monte Carlo predictions (TWOGAM generator). Dark area - QPM only; dots - QPM + VMD; full line $Q P M+V M D+D G\left(p_{T}^{\text {min }}=1.45 \mathrm{GeV} / \mathrm{c}\right)$; dashed line $-Q P M+V M D+D O\left(p_{T}^{\text {min }}=1.22\right.$ $\mathrm{GeV} / \mathrm{c}$ ) (from 63]).

Comment: "A three-component model which includes QCD hard scattering subprocesses is needed to describe the data."

Remnant jets, generated along the direction of the initial quasi-real photon, are studied within the FKP approach.

"Each parametrization is associated to a special value of $p_{T}^{\min }$, constrained by the description of the visible total cross section".

\section{-DELPHI 95 [57] (LEP 1)}

The measurement of hard processes in $e \gamma$ collision was performed based on 1991-1992 data, as described in the previous section. The average virtuality of the photon from the tagged electron, registered by VSAT, was equal to $<Q^{2}\left(=P_{1}^{2}\right)>\approx 0.06 \mathrm{GeV}^{2}$ (single-tag events). The values of $E_{T}$ of observed jets were larger than $1.5 \mathrm{GeV}$.

Comment: "This study confirms recent results from no-tag experiments, indicating that the photon has a significant partonic content."

\section{-DELPHI 97b,conf [64] (LEP 1, LEP 2)}

Based on the LEP1 and LEP2 data (1995 and 1996 runs), with CM energy $172 \mathrm{GeV}$, various distributions for the hadronic final state in anti-tag events were measured. The TWOGAM generator was used in the analysis with soft VMD, QPM and QCD-RPC (with $p_{T}^{m i n}$ cutoff) contributions. Different parton parametrizations for the photon were used assuming the "leading order QCD factorization", which lead to different values of $p_{T}^{\min }: p_{T}^{\min }(G S 2)=1.88 \pm 0.02 \mathrm{GeV}$ and $p_{T}^{\min }(G R V)=1.58 \pm 0.018 \mathrm{GeV}$. 
Results are presented in fig. 70 .
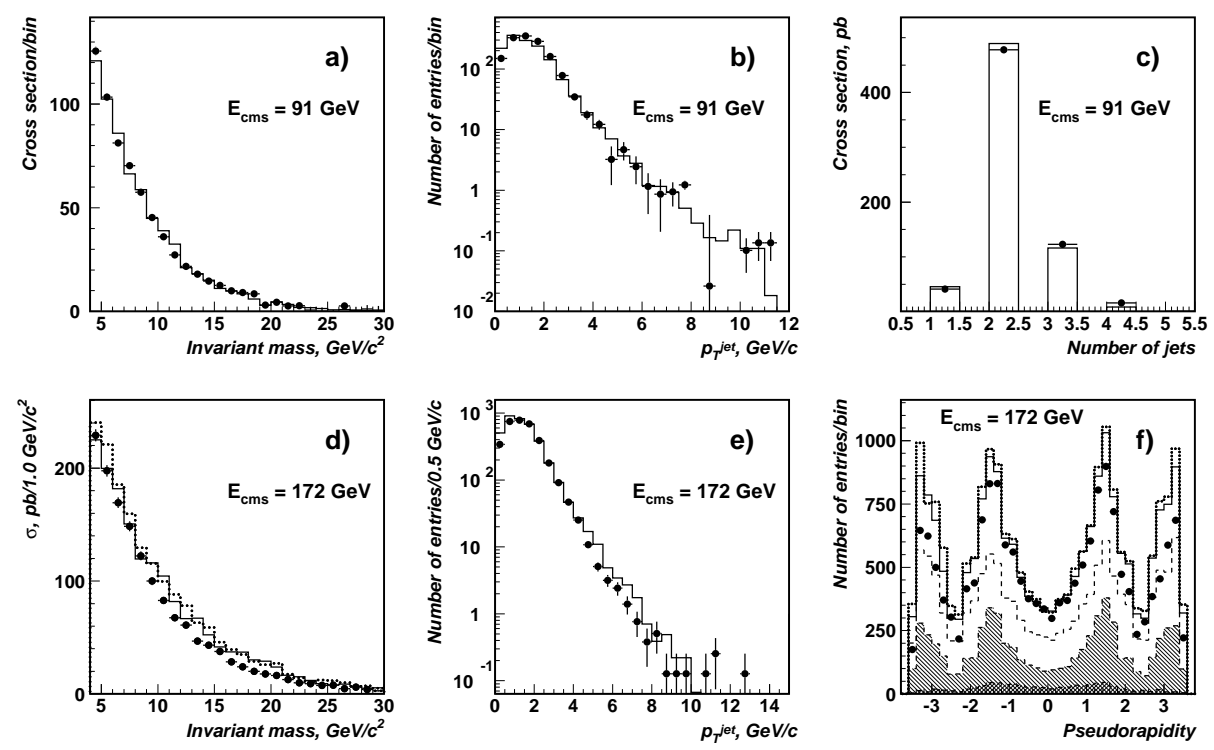

Figure 70: Distributions of: a), d) invariant mass, b), e) $p_{T}$, c) number of reconstructed jets, f) pseudorapidity. Solid and dotted lines - predictions based on VMD $+Q P M+R P C$ with the GS2 and GRV parton densities, respectively. The dashed line - RPC contribution with the GS2 parton densities; the hatched histogram - the VMD part, the double hatched - the QPM part. Upper (lower) figures correspond to $\sqrt{s}=91$ (172) GeV (from [64]).

Comment: "Monte Carlo prediction (based on TWOGAM generator) gives perfect agreement at CM energy $=91 \mathrm{GeV}$ and slightly exceeds data at $172 \mathrm{GeV} "$.

-OPAL 97e [65] (LEP 1.5)

The inclusive one and two-jet cross sections have been measured at $\sqrt{s_{e e}}=130$ and $136 \mathrm{GeV}$ (based on the 1995 run). The antitagging condition was applied to the initial photons corresponding to the maximum photon virtuality $P_{\max }^{2} \approx 0.8 \mathrm{GeV}^{2}$. The transverse energy of jets $E_{T}$ is taken to be larger than $3 \mathrm{GeV}$ and the pseudorapidity lies within $|\eta|<1$. Generators PYTHIA 5.721 (with the SaS1D parton parametrization) and PHOJET 1.05 (with the GRV LO parton parametrization) were used. The cone jet-finding algorithm with $\mathrm{R}=1$ was used (for the first time in photon - photon collisions at LEP).

For the two-photon events, the $x_{\gamma}^{ \pm}$distribution and the transverse energy flow around the jet, studied separately for the direct $\left(\min \left\{x_{\gamma}^{+}, x_{\gamma}^{-}\right\}>0.8\right)$ and the resolved $\left(\min \left\{x_{\gamma}^{+}, x_{\gamma}^{-}\right\}<0.8\right)$ photon contributions, were obtained (see fig. 71 and fig. 72, 
respectively).

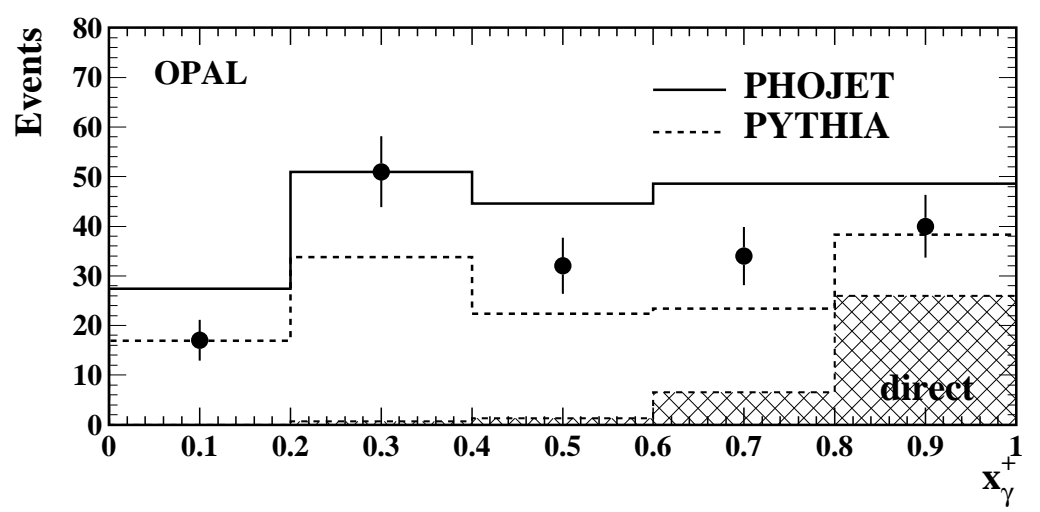

Figure 71: The number of two-jet events as a function of $x_{\gamma}^{+}$compared to the PHOJET (solid line) and PYTHIA (dashed line) simulations. The hatched histogram is the direct contribution to PYTHIA events (from [65]).
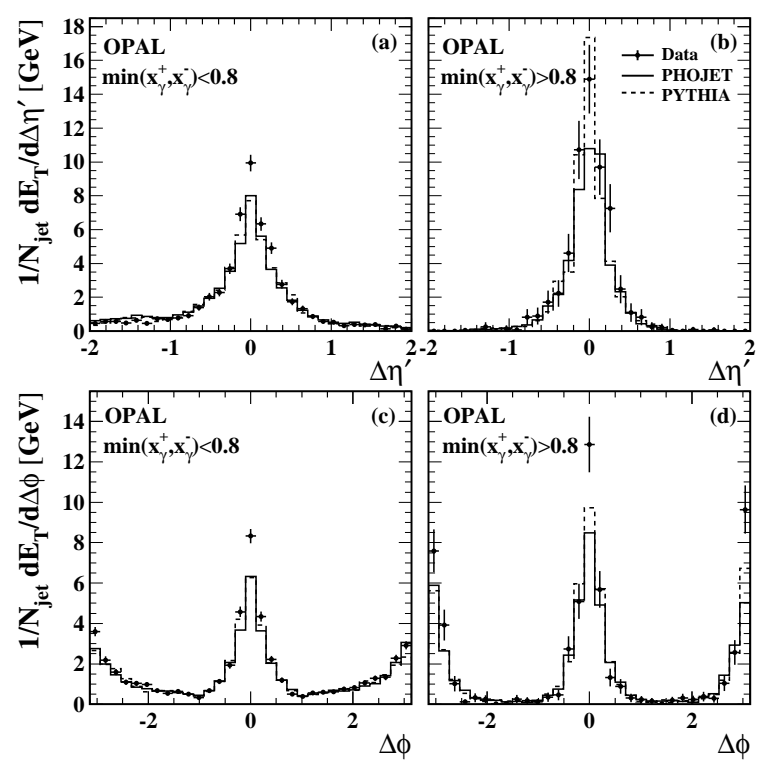

Figure 72: The transverse energy flow around the jets in two-jet events. b), d) Direct events $\left(\min \left\{x_{\gamma}^{+}, x_{\gamma}^{-}\right\}>0.8\right)$. a), c) Resolved events $\left(\min \left\{x_{\gamma}^{+}, x_{\gamma}^{-}\right\}<0.8\right)$. The data are compared with the PHOJET (solid line) and PYTHIA (dashed line) simulations. The rapidity $\delta \eta$ distribution is shown in a) and b), while the $\delta \phi$ angle distribution in c) and d) (from [65]).

The one-jet and two-jet cross sections $\frac{d \sigma}{d E_{T}}$ were measured up to $E_{T}=16 \mathrm{GeV}$. Results are presented in fig. 73 . 

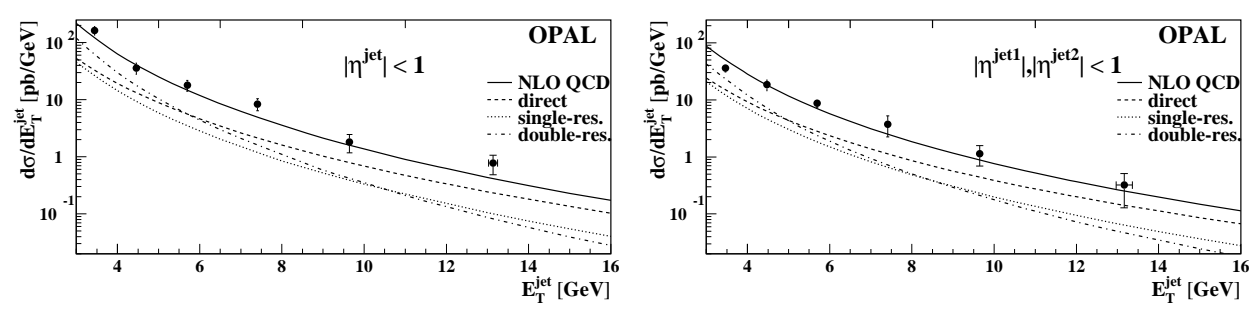

Figure 73: The inclusive one-jet (left) and two-jet (right) cross section as a function of $E_{T}$ for the jets with $|\eta|<1$ compared to the NLO calculations [182]. The solid line is the sum of direct, single resolved and double resolved contributions that are also shown separately (from [65]).

The $\eta$ distributions for one and two-jet events were studied as well (see fig. 74).
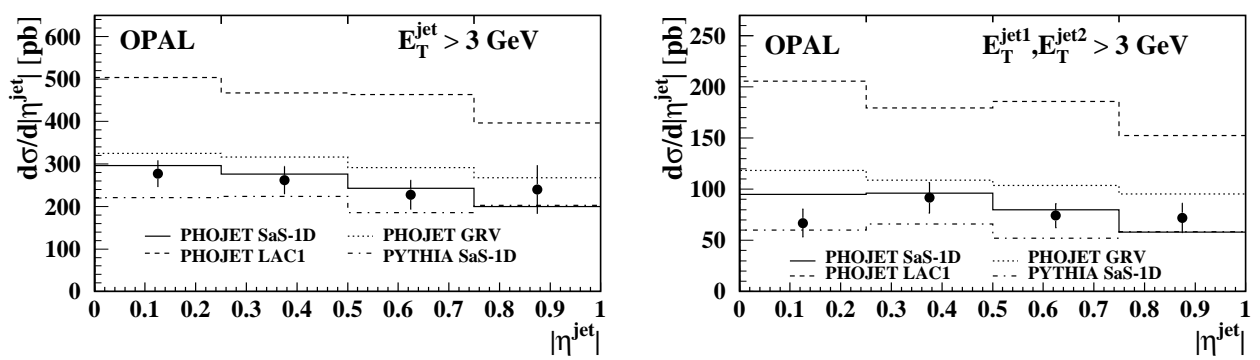

Figure 74: The inclusive one-jet (left) and two-jet (right) cross section as a function of $|\eta|$ for the jets with $E_{T}>3 \mathrm{GeV}$ compared to the LO QCD calculations of the PYTHIA (with the SaS1D, LAC1 and GRV parton distributions) and PHOJET (with the SaS1D parton distributions) generators (from [65]).

Comment: "The data on $\eta$ distributions agree well with NLO QCD calculations based on GRV parametrization. The GRV LO and SaS1D parametrizations describe the data equally well, the LAC 1, however, gives twice the observed value".

The large difference between Monte Carlo generators with a fixed parton parametrization, and for a particular Monte Carlo generator with different parton parametrizations is observed.

\section{-OPAL 99a 66] (LEP 2)}

The dijet production in two-photon collisions at $e^{+} e^{-}$energies 161 and $172 \mathrm{GeV}$ in the "antitagging condition" was measured. The transverse energy of the jets is taken to be $E_{T}>3 \mathrm{GeV}$ and the pseudorapidity lies within $|\eta|<2$. The Monte Carlo generators PYTHIA 5.722 and PHOJET 1.05c were used with the SaS1D and GRV LO parton parametrizations, respectively (with $p_{t}^{0}=1.4 \mathrm{GeV}$, as a default value). Both generators use the multiple interaction to model the underlying events (a default $\left.p_{T}^{m i}=1.4 \mathrm{GeV}\right)$. The cone jet-finding algorithm with $\mathrm{R}=1$ was used.

The contributions of direct and resolved photon subprocesses $\left(x_{\gamma}^{ \pm}<0.8\right)$ were studied for various distributions.

The $x_{\gamma}^{ \pm}$distributions were measured for different $\bar{E}_{T}$ bins, from 3 to $20 \mathrm{GeV}$, showing the large contribution of the resolved photon processes (fig. 75). 


\section{OPAL}
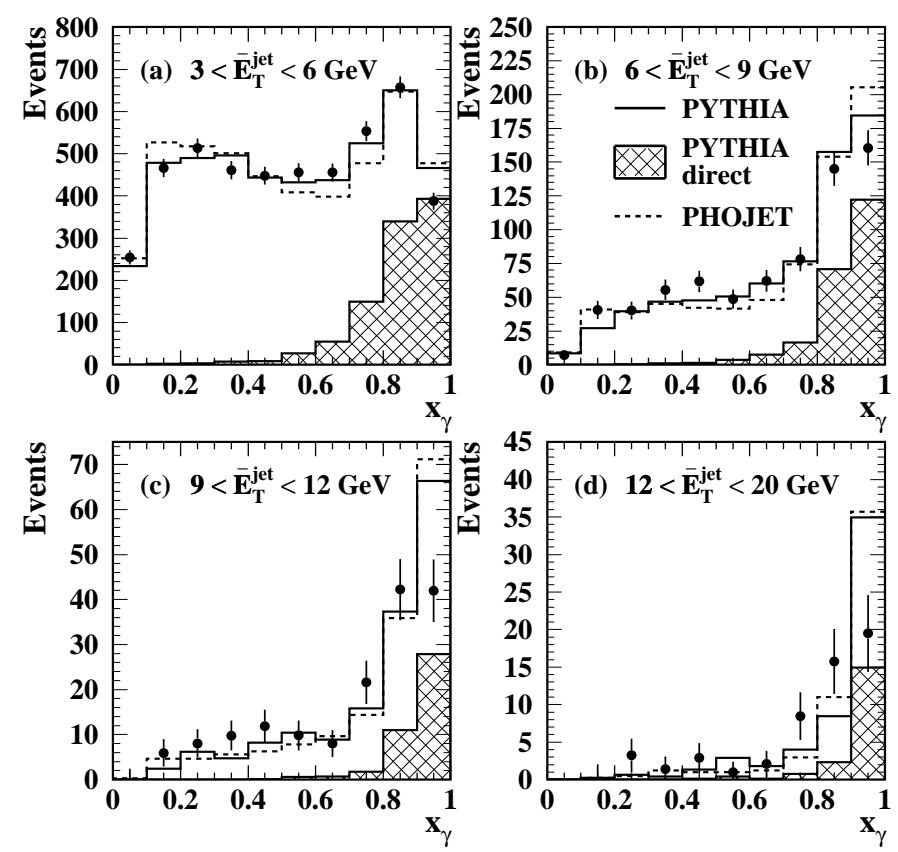

Figure 75: The uncorrected $x_{\gamma}$ distributions in bins of $\bar{E}_{T}$, compared to PYTHIA and PHOJET predictions (from [60]).

The transverse energy flow around the jet axis is presented in fig. 76 for the double resolved and direct photon processes. Larger hadronic activity for the resolved photon sample is observed as expected.

\section{OPAL}

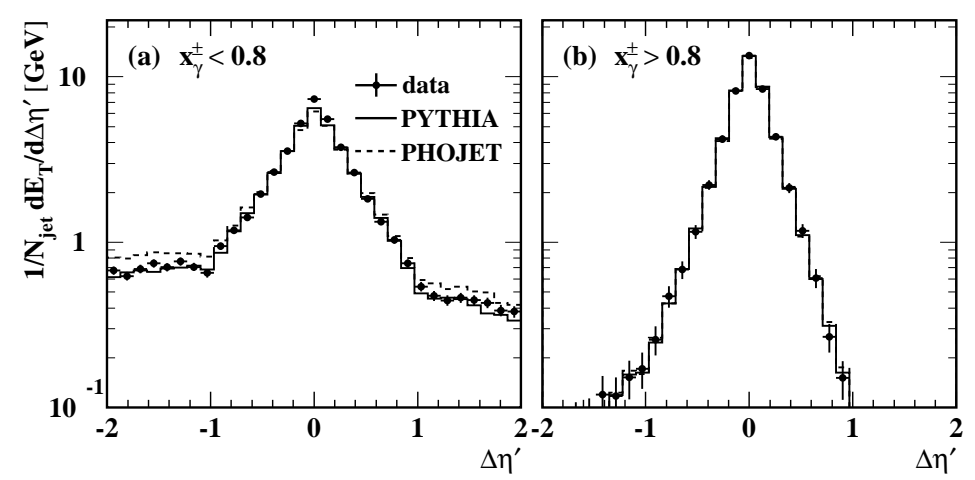

Figure 76: The transverse energy flow around the jet axis as a function of $\delta \eta$ in two-jet events; a) double resolved events, (b) direct events. Comparison with PYTHIA (solid line) and PHOJET (dashed line) (from [60]).

The angular dependence plotted separately for the three basic resolved photon subprocesses and for the direct events is presented in comparison with matrix element predictions 60] in fig. 77. A comparison with the NLO calculation with the GRV HO parton parametrization was also made (not shown). 


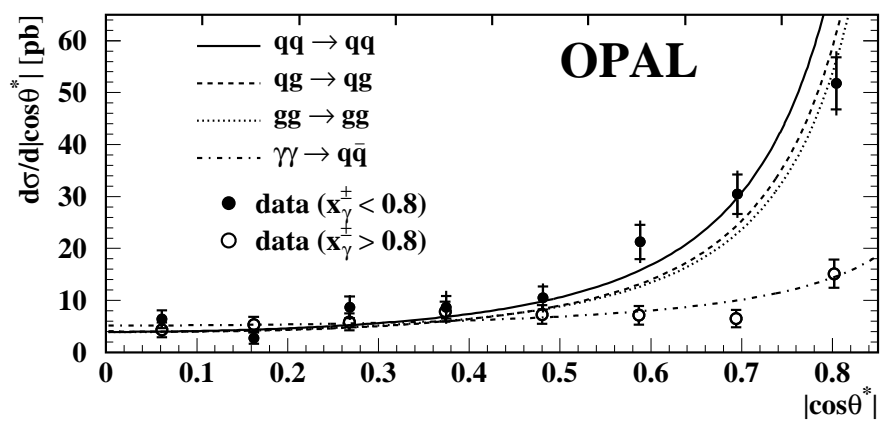

Figure 77: The angular distribution of events with $E_{T 1}=E_{T 2}$ and collinear in $\phi$, separated into the direct and the double resolved contributions, together with the LO QCD expectations based on matrix elements [60] (from 66]).

The cross sections for the direct, single resolved and double resolved two-jet events versus $E_{T}$, compared to the NLO calculation with the GRV HO parton parametrization, are shown in fig. 78 .

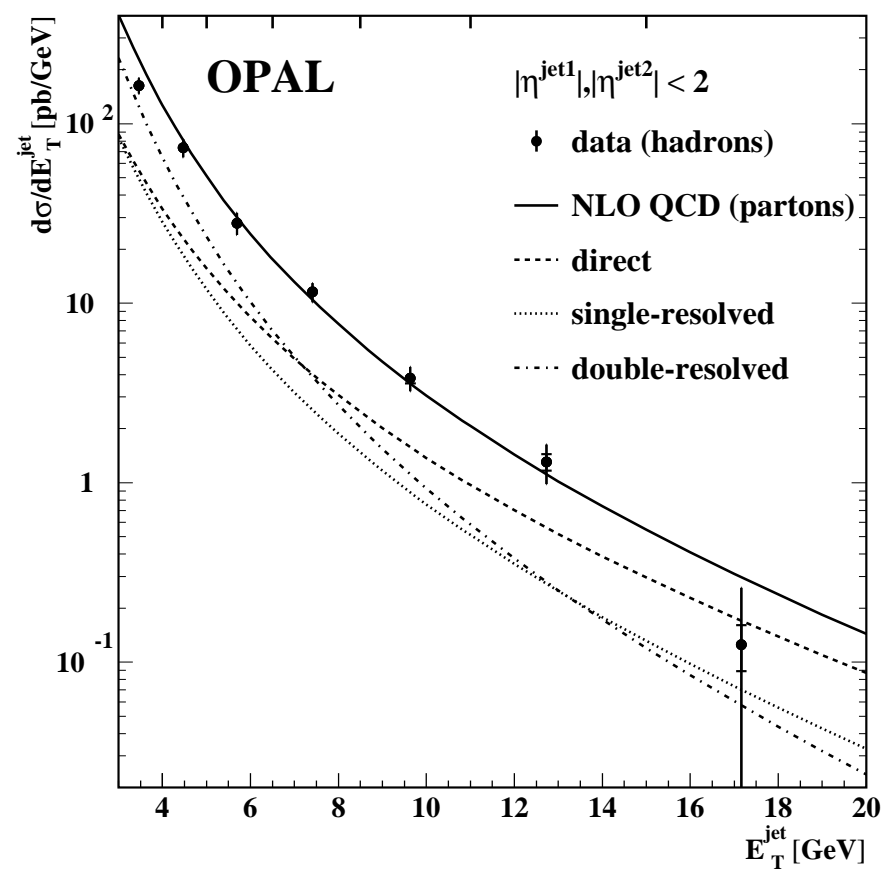

Figure 78: The inclusive two-jet cross section as a function of $E_{T}$ for jets with $|\eta|<2$, compared to the NLO calculations [182]. Solid line is the sum of the direct, single resolved and double resolved cross sections that are also shown separately (from [6]]).

The influence of the "underlying event" has been studied. The transverse energy flow outside the jets was measured as a function of $x_{\gamma}$, in order to study the possible contribution from multiple interactions. The Monte Carlo simulations (PYTHIA, PHOJET) were performed with various parametrizations and values of the $p_{t}^{m i}$ - cutoff parameter for multiple interactions (fig. 79)).

The two-jet pseudorapidity distribution is presented in fig. 80 together with the predictions from the PYTHIA and PHOJET generators, with various parton parametrizations. Here the asymmetric cuts were applied, to avoid infrared instability in the NLO 
calculations. The results are also shown for two samples, corresponding to the direct and double resolved photon processes.

\section{OPAL}

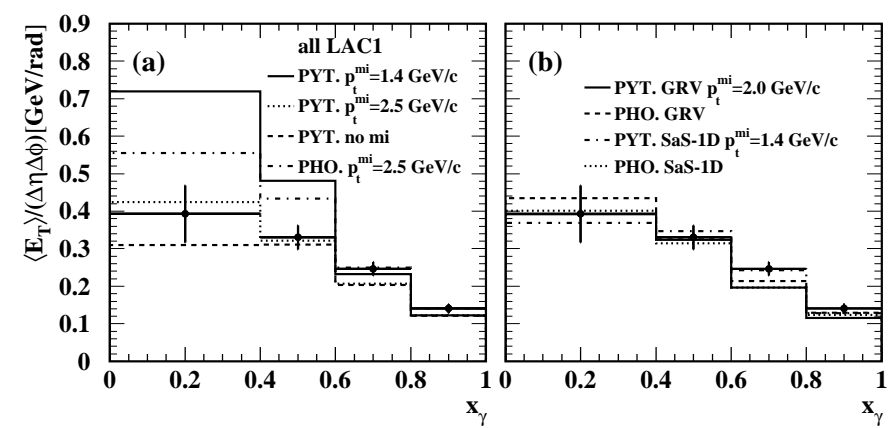

Figure 79: The transverse energy flow outside the jets for $\left|\eta^{*}\right|<1$ as a function of $x_{\gamma}$ for various parton parametrizations, $M C$ models and values of $p_{t}^{m i}$ (from [66]).

\section{OPAL}

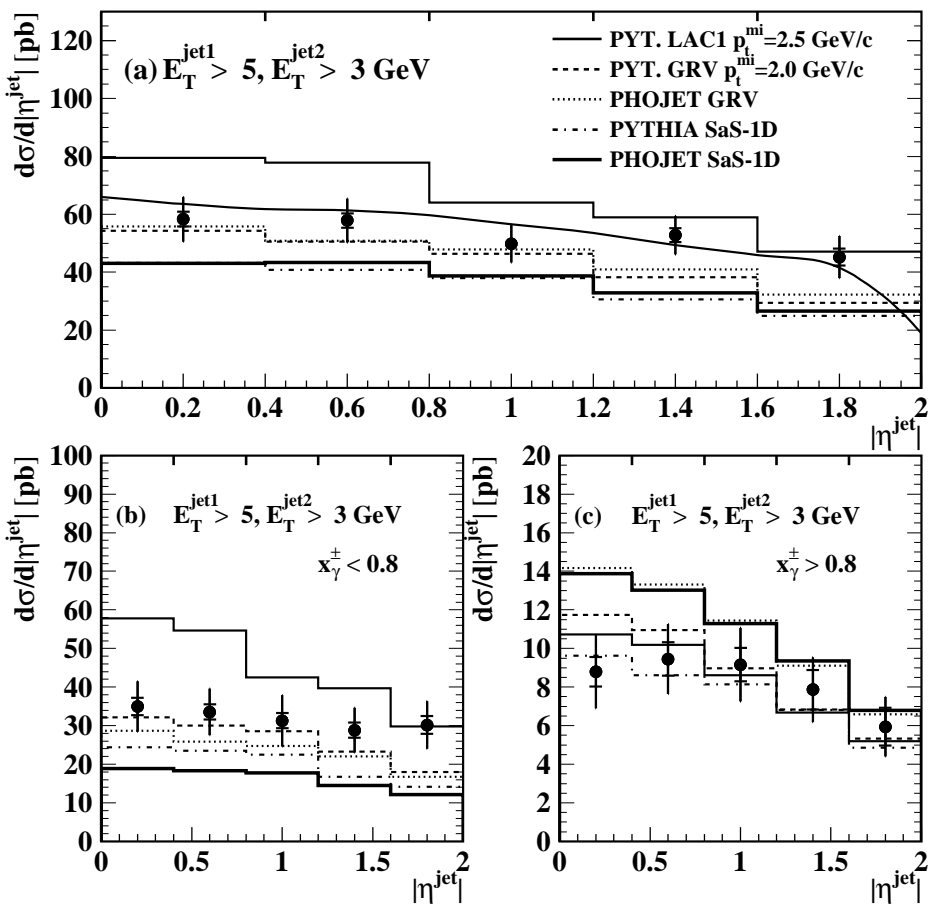

Figure 80: The inclusive two-jet cross sections as a function of $|\eta|$ for jets with $E_{T}^{j e t 1}>5$ $\mathrm{GeV}$ and $E_{T}^{\text {jet } 2}>3 \mathrm{GeV}$ : a) for all events, b) for direct events, c) for double resolved events (from [66]).

To reduce the influence from multiple interactions and hadronization effects the two-jet cross section was measured also for $E_{T}^{\text {jet1 }}>5 \mathrm{GeV}$ (not shown).

Comment: "The $E_{T}$ dependent two-jet cross section is in good agreement with NLO QCD calculation."

"Using PYTHIA and PHOJET the GRV LO parametrization is able to describe the two-jet cross section; prediction based on the SaS1D is too low, and that for LAC1 significantly too high." 
"Within the errors of the measurement we are unable to differentiate between models with and without multiple interactions."

\section{-AMY 1.092 [67] (TRISTAN)}

The measurement of the high $p_{T}$ hadron production in the quasi-real $\gamma \gamma$ collision, for the $e^{+} e^{-}$collision energy between 55 and $61.4 \mathrm{GeV}$, was performed. In the observed three and four-jet events there are one or two spectator jets coming from the resolved photon(s). The QPM+VMD contribution, and the MJET one, which corresponds to the RPC contributions, were included in the analysis. The DG parton parametrization was used with the $p_{T}^{m i n}$ equal to $1.6 \mathrm{GeV}$. Various distributions were studied, the results for the $p_{T}$ distribution are shown in fig. 81.

The sensitivity of the results to the gluonic content of the photon was studied.

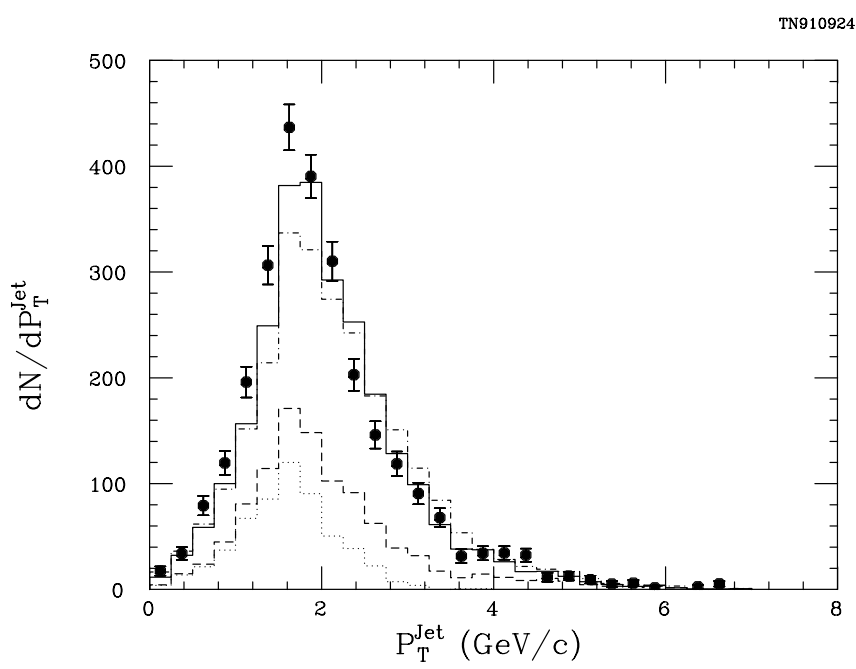

Figure 81: The $p_{T}$ distribution compared with the predictions of QPM (dotted histogram), $Q P M+V M D$ (dashed histogram) and $Q P M+V M D+M J E T$ (solid histogram) with $p_{T}^{\text {min }}$ $=1.6 \mathrm{GeV}$ (from [67]).

Comment: The "first clear" evidence is found for the hard scattering of hadronic constituents of photons in the photon - photon collisions at TRISTAN.

"...without the gluonic component it is impossible to reproduce the data".

\section{-AMY 1.594 68 (TRISTAN)}

AMY 1.5 (the upgraded AMY detector at the energy $60 \mathrm{GeV}$ ) measured the inclusive single and double jet cross section $\frac{d \sigma}{d p_{T}}$ in quasi-real $\gamma \gamma$ collisions. Jets with $p_{T}$ above $2.5 \mathrm{GeV}$ up to $8 \mathrm{GeV}$ and $|\eta|<1$ were studied. The QPM + GVMD (for the diffractive production of the hadrons) and the improved MJET generator for the resolved photon processes (using DG, LAC 1,2,3 and GRV parton parametrizations, and various $p_{T}^{\text {min }}$ parameters) were applied in the analysis. The cone algorithm with $\mathrm{R}=1$ was used.

In fig. 82 the $p_{T}$ distributions for one and two jets, for $|\eta|<1.0$, are presented. 

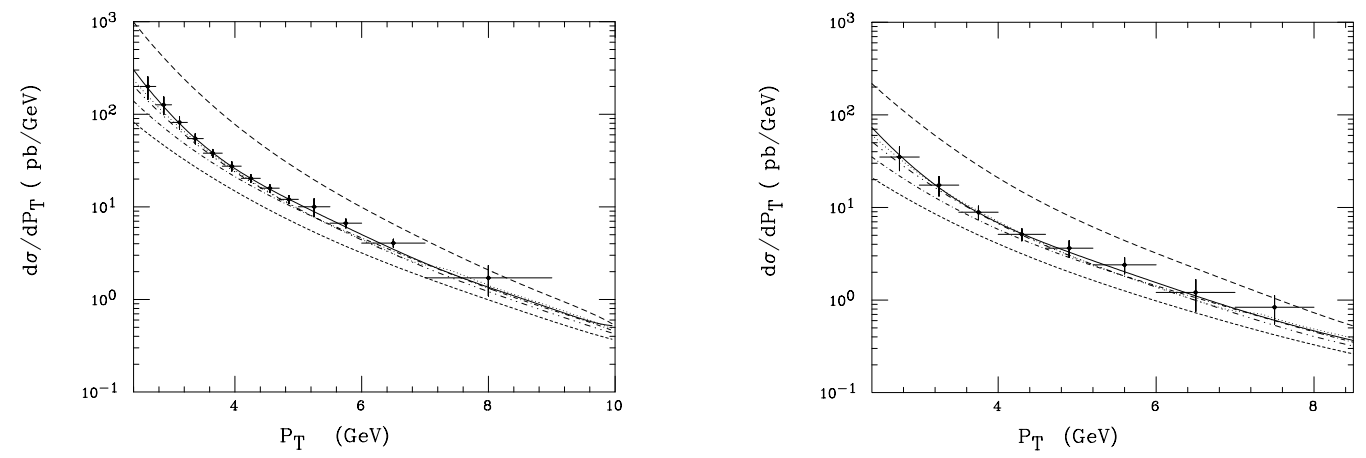

Figure 82: The inclusive jet cross section as a function of $p_{T}$ integrated over $|\eta|<1.0$ for one-jet (left) and two-jet events (right). The curves represent the sum of the QPM (direct) and MJET (resolved) cross sections using the parton parametrizations: LAC1 (full line), GRV LO (double dot-dashed), DG (dotted line), LAC3 (dashed line) and LAC1 without the gluon component (dot - dashed line). The short - dashed curve corresponds to the QPM cross section (from 68]).

The $\eta$ dependence for the jet with $p_{T}$ larger than 2.5 is presented in fig. 83.

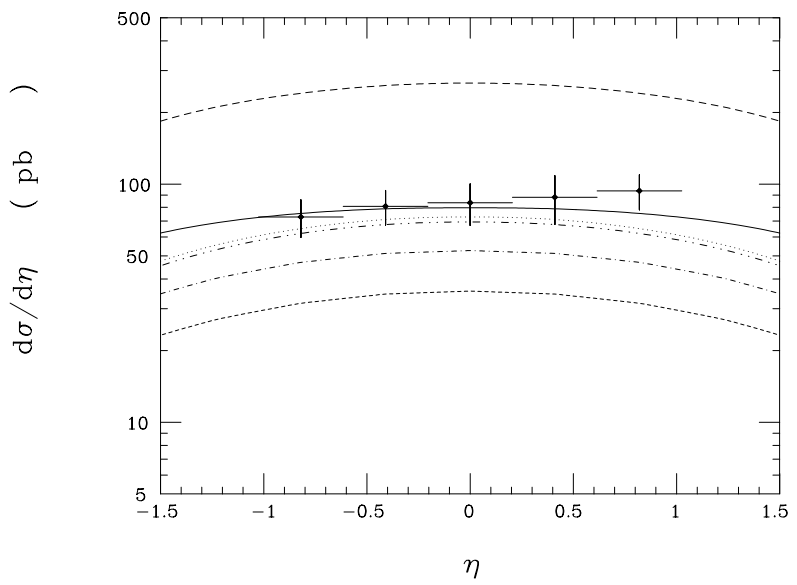

Figure 83: The inclusive jet cross section as a function of $\eta$ integrated over $\left|p_{T}\right| \geq 2.5 \mathrm{GeV}$. The lines convention is the same as in fig. 82 (from [68]).

Comment: "The data are in good agreement with LO QCD calculations based on either $L A C$ 1, DG or GRV parametrizations of the parton densities in the photon. The calculation based on LAC 3 disagrees with the data and we do not see the deviation that is observed in the $\eta$ distribution by the H1 experiment" (here denoted as $\mathbf{H 1} \mathbf{9 3}$ ).

\section{-TOPAZ 93 [69] (TRISTAN)}

The inclusive jet cross section $\frac{d \sigma}{d p_{T}}$ was measured at the collision energy $\sqrt{s_{e e}}=58 \mathrm{GeV}$ and virtuality of photon below $2.6 \mathrm{GeV}^{2}$. Jets with $p_{T}$ between $2.5 \mathrm{GeV}$ and 7.5 $\mathrm{GeV}$ and $|\eta|<0.7$ were analysed. The direct and resolved photon contributions were included, as well as the VMD one. The resolved contributions were calculated with various parton parametrizations (DO, DG and LAC1,2,3), using the $p_{T}^{\text {min }}$ parameter. The BASES/SPRING generator was used. To describe jets, the cone algorithm with $R=1$ was applied. 
The energy flow within the jet (per jet) as a function of $\delta \eta$ and $\delta \phi$ was also studied and found in agreement with the Monte Carlo simulation (not shown).

In figs. 84 and 85 the $p_{T}$ distribution of the jet and the energy flow per event as a function of the polar angle of the jet $(\theta)$ are presented, respectively. Fig. 86 shows the inclusive one-jet and two-jet cross sections.

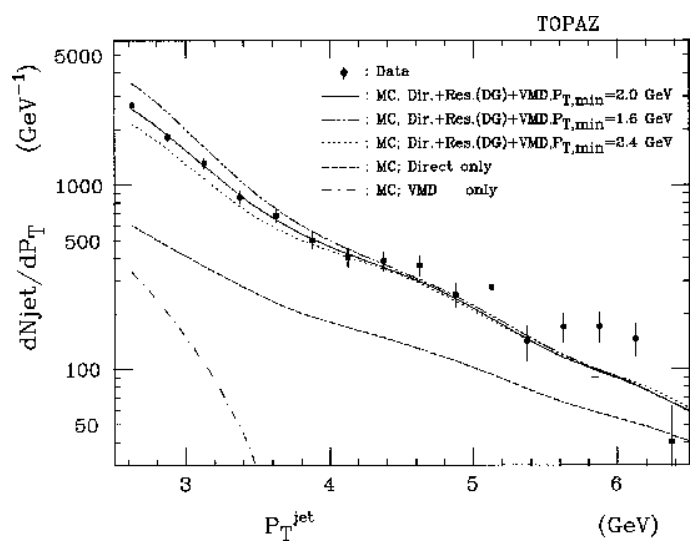

Figure 84: The jet $p_{T}$ distribution for a no-tag sample. Predictions are shown for the sum of direct + resolved photon (with the DG parton parametrization) + VMD processes for different $p_{T}^{m i n}$ values. Contributions of the direct (dashed line) and the VMD (dot - dashed line) processes are shown. Parton parametrization according to DG (from [69]).

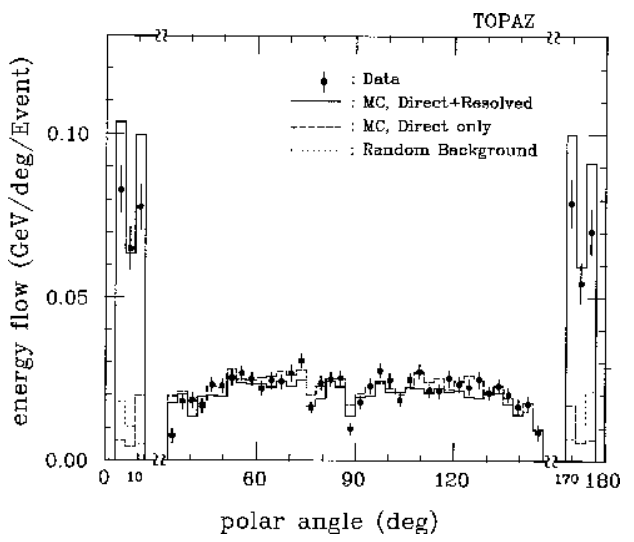

Figure 85: The energy flow versus the polar angle $\theta$ for the jet sample. The data are compared with the Monte Carlo predictions for the sum of the direct and the resolved processes (solid histogram) and the direct process only (dashed histogram) (from [69]). 

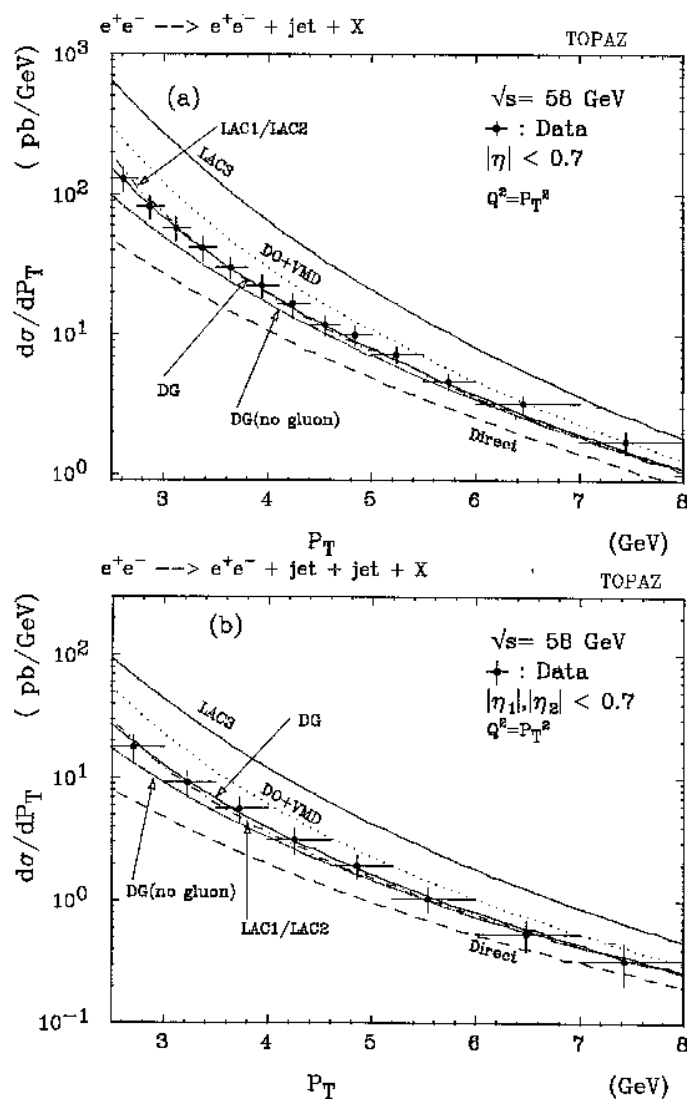

Figure 86: The inclusive a) jet and b) two-jet cross sections as a function of $p_{T}$ for the pseudorapidity $|\eta| \leq 0.7$. TOPAZ Collaboration results are compared with the predictions of different parton parametrizations: LAC3, DO +VMD, DG, DG (without gluon), LAC1 and LAC2. Also a direct contribution is shown (from [69]).

Comment: The polar angle distribution gives .."direct evidence of the presence of the resolved processes. The data exclude the parametrizations of $L A C 3$ and $D O+V M D$, which predict a very large gluon content even at large $x_{\gamma} . "$

\section{- TOPAZ 95,conf [70] (TRISTAN)}

The updated results of the jet study in (quasi) real $\gamma \gamma$ collisions are given, for a larger data sample than in [69]. Anti-tag condition corresponds to the maximum photon virtuality of $P_{\max }^{2}=2.6 \mathrm{GeV}^{2}$. Production of jets with $p_{T}$ between 1 (2) and $8 \mathrm{GeV}$, and $|\eta|<0.7$ was measured. The direct $(\mathrm{QPM})+\mathrm{VMD}+$ resolved (RPC) contributions were included in the analysis of the data. The parton density parametrization used is that of DG, and LAC1, with optimalized cutoff parameters $p_{T}^{\min }$.

The $p_{T}$ distribution for the inclusive jet production has been studied down to 1 $\mathrm{GeV}$, and the data show clearly the evidence for the resolved processes (see fig. 87). 


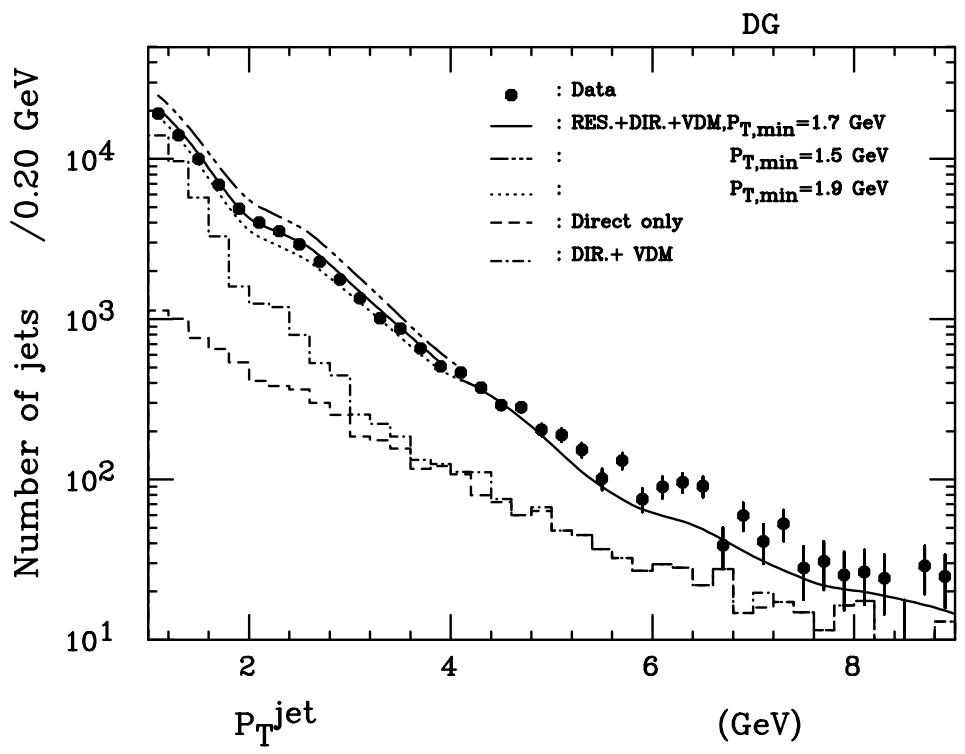

Figure 87: The inclusive jet distribution as a function of $p_{T}$ for $|\eta| \leq 0.7$. The solid line is a prediction of the direct + resolved (with DG parton parametrization) + VMD processes $\left(p_{T}^{\min }=1.7 \mathrm{GeV}\right)$. The contributions of the direct and of direct $+V M D$ processes are shown as dashed and dot-dashed histograms, respectively (from [7]]).

The evidence for the remnant-jet activity in small-angle region was observed. Fig. 88 shows the particle energy flow for the sample with two high - $p_{T}$ jets in the central region, where the energy deposit from direct (or VMD) processes is rare.

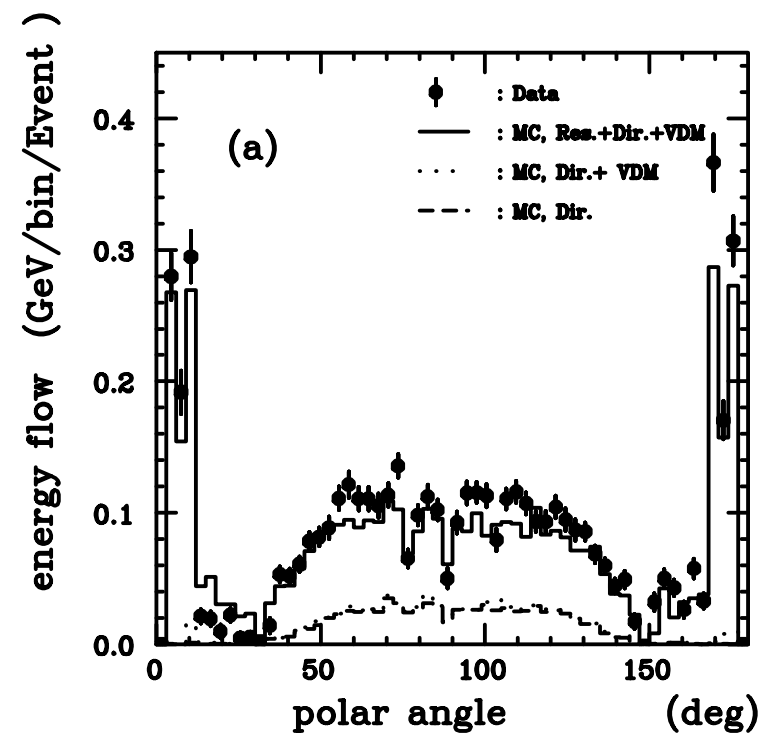

Figure 88: The energy flow distribution for the central two-jet sample with $|\eta| \leq 0.7$ and $p_{T} \geq 2 \mathrm{GeV}$. Comparison with prediction of Monte Carlo models as in fig. 87 (from [70]).

Comment: The existence of the resolved processes in $\gamma \gamma$ collisions is confirmed by the jet rate and the direct detection of the energy clusters coming from the remnant jet. The rate of the remnant jet is also consistent with the Monte Carlo expectations. 


\section{- TOPAZ 98,conf [71] (TRISTAN)}

A study of dijet production in (quasi) real $\gamma \gamma$ collisions (anti-tag conditions: $P^{2}$ from $10^{-8}$ to $2.6 \mathrm{GeV}^{2}$ with the mean value $\sim 10^{-4} \mathrm{GeV}^{2}$ ) at $\sqrt{s_{e e}}=58 \mathrm{GeV}$ based on the full data taken in the years 1990-95 was performed. (Also a prompt photon production cross section was measured.) Limits: $E_{T} \geq 3 \mathrm{GeV}$ and $E_{T} \geq 2 \mathrm{GeV}$ for two jets with the highest transverse energy were applied. The Monte Carlo programs PYTHIA (also with additional power law $p_{t}$ distribution) and the PHOJET with GRV LO and SaS1D parton distributions were used. The jets were reconstructed using a cone jet-finding algorithm with fixed value of cone radius $R=1.0$.

The study of the hadronic final state (the transverse energy distribution, the jet energy profile and the azimuthal angle dependence of the number of dijet events) was carried out by separating the direct and resolved sample by a $x_{\gamma}$ reconstruction method developed at HERA $\gamma p$ experiments (see e.g. sec. 2.2 and 2.4.4). The number of dijets as a function of $x_{\gamma}$ is shown in fig. 89. The predictions of the PYTHIA generator with the GRV LO structure function agree with the data, but that with SaS1D are too low by a factor 2 for $x_{\gamma}^{\min }$ (defined as $\left.\min \left(x_{\gamma}^{+}, x_{\gamma}^{-}\right)\right) \sim 0.5$. This observation appears for both PYTHIA and PHOJET programs, and also for different values of parameter $p_{T}^{\text {min }}$, varying from 1.6 to $2 \mathrm{GeV}$.
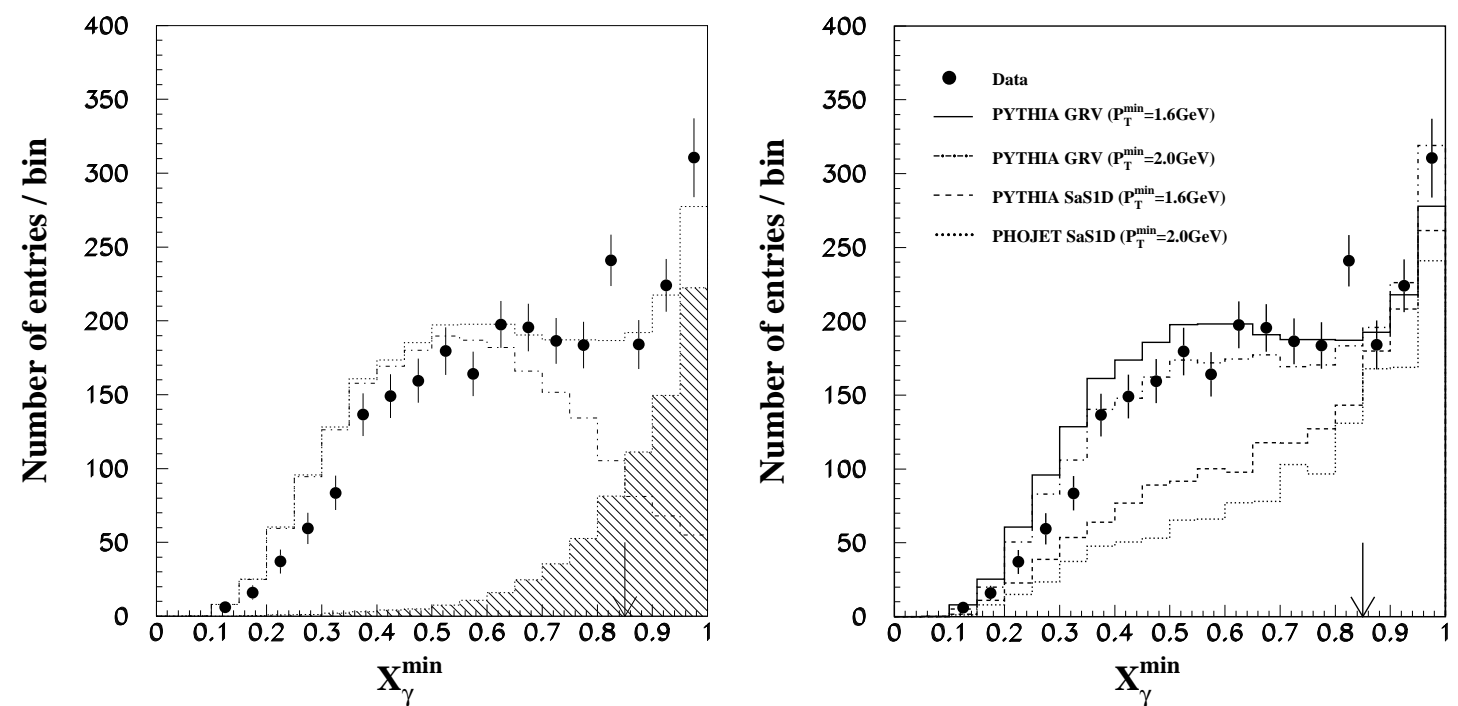

Figure 89: The $x_{\gamma}^{\text {min }}$ distribution of the dijet sample. Left: Comparison with the PYTHIA predictions with the GRV LO photon structure function; the shaded histogram - contribution from the direct process, the dashed one - from the resolved process, the dotted one - sum of both. Right: The same distribution compared with the various Monte Carlo predictions (with $p_{T}^{0}=1.6 \mathrm{GeV}$ or $2 \mathrm{GeV}$ ) (from [7]).

The measured $E_{T}$ distributions for the resolved - enriched sample $\left(x_{\gamma}^{\min }<0.8\right)$ and for the direct - enriched sample $\left(x_{\gamma}^{\min }>0.8\right)$ are shown in fig. 90 . 

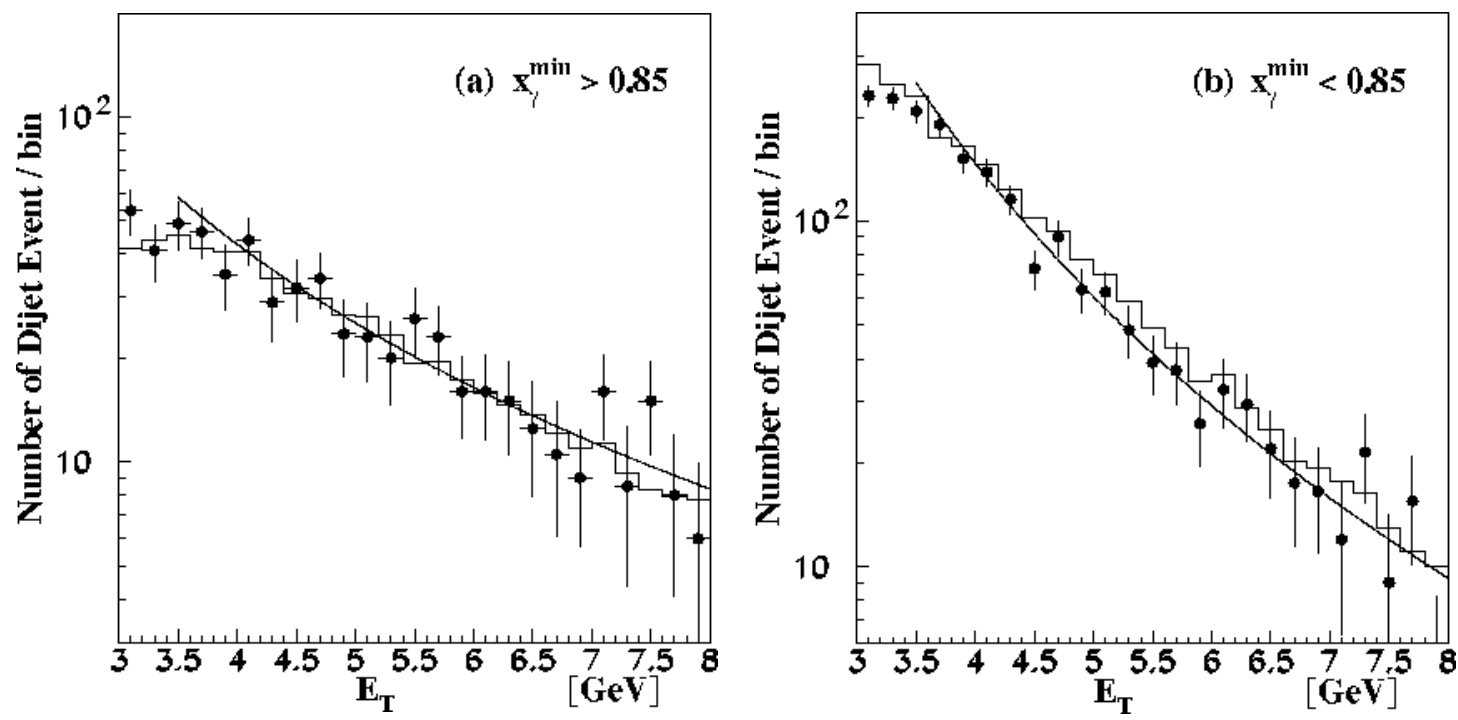

Figure 90: The $E_{T}$ distributions for (a) the direct - enriched sample and (b) the resolved enriched sample. The histograms are the PYTHIA predictions with the GRV LO structure function (from [71]).

The transverse energy flow as a function of the pseudorapidity $\delta \eta$ was measured with respect to the jet direction for different dijet samples, see fig. 91. The direct enriched sample is nearly symmetric and there is almost no activity outside the jet, while the resolved - enriched sample shows considerable activity outside the jet cone for $|\delta \eta|>1$. The energy flow outside the jets is well modelled by the PYTHIA generator.
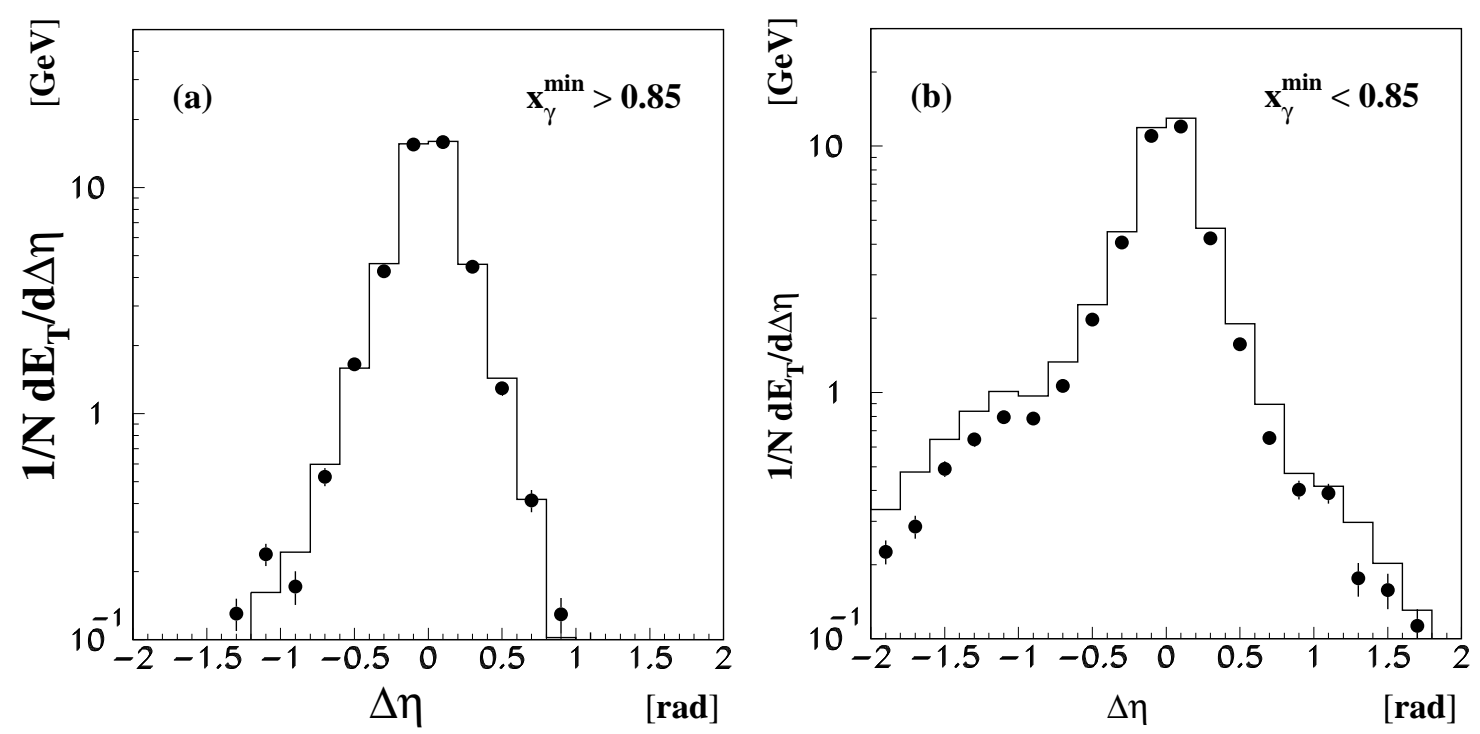

Figure 91: Transverse energy flow relative to the direction of each jet in dijet events as a function of $\delta \eta$ for (a) direct - enriched and (b) resolved - enriched sample. The histogram is the result of the PYTHIA simulation (from [71).

The transverse energy flow versus the azimuthal angle around the jet direction is shown in fig. 92 for the two above samples together with the Monte Carlo predictions 
with and without the multiple interactions (MI). The activity observed for the resolved - enriched sample is much larger than for the direct - enriched; at $|\delta \phi| \sim 1.5$ the difference is by a factor 8 .
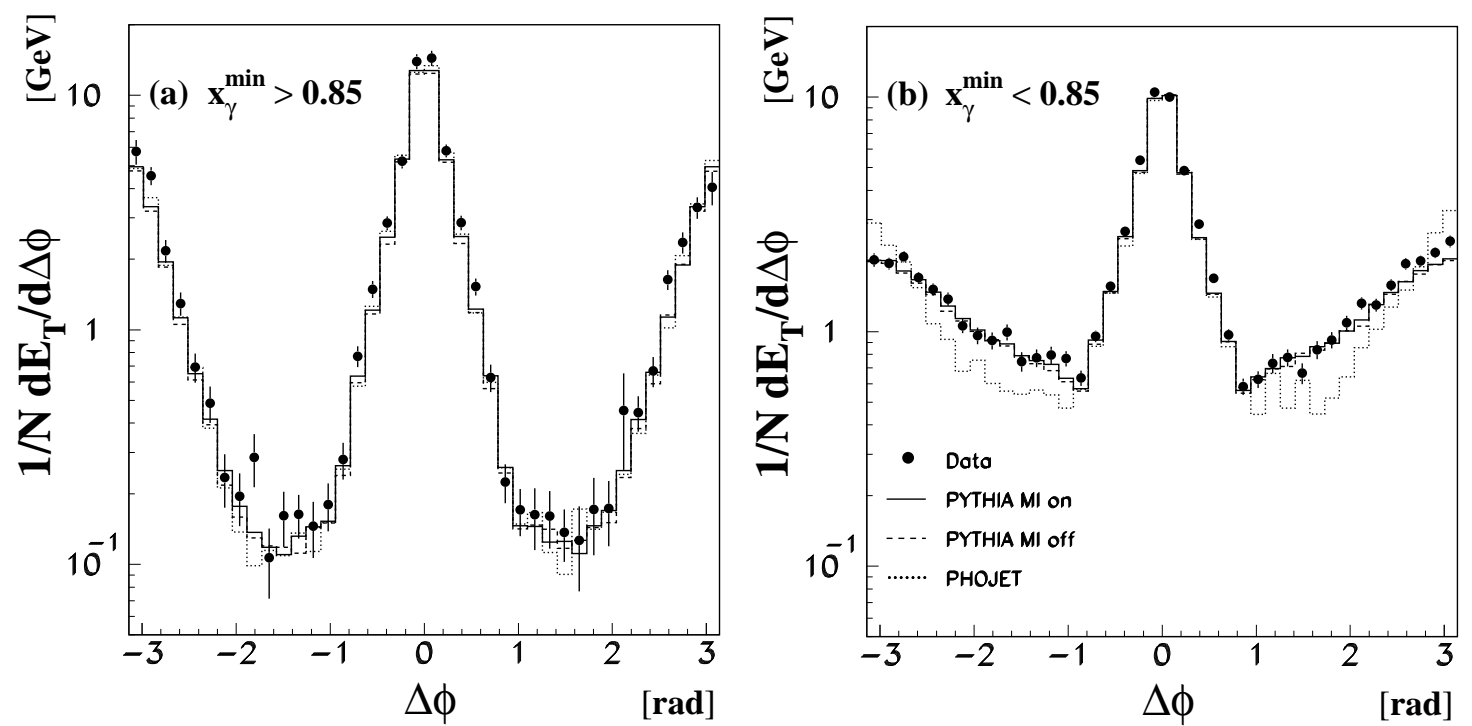

Figure 92: The transverse energy flow relative to each jet direction as a function of the azimuthal angle $\delta \phi$ for (a) direct - enriched and (b) resolved - enriched sample. The PYTHIA (with and without multiple interaction, solid and dashed line, respectively) and PHOJET (dotted line) predictions are shown (from [71]).

The dependence of the azimuthal angle difference between two jets with the highest $E_{T}$ was studied. Here the effect of the primordial $p_{t}$ distribution of partons - gaussian and the power-law type - was analysed using the PYTHIA program (with the GRV parton parametrization). These data (not shown) are reproduced very well when the initial state radiation (IR) is included and the power-law $p_{t}$ distribution is assumed.

Comment: Too low prediction for $x_{\gamma}$ distribution from the PYTHIA and PHOJET programs if based on the SaS1D parton parametrizations.

"The PYTHIA predictions on the direct and resolved processes reproduce the transverse energy flow reasonably well. While the PHOJET prediction for the resolved sample shows too small activity in the middle region between two jets, the direct sample is reproduced well."

The transverse energy flow around the jet shows that... "In $\gamma \gamma$ collisions at TRISTAN, the PYTHIA predictions with and without multiple interactions are almost identical, indicating that there are no effects of the multiple interaction at the TRISTAN energy region", although they have been found at HERA in $\gamma p$ reaction.

The data on the azimuthal angle between jets are very well described when the power law $p_{t}$ distribution in PYTHIA is assumed. 


\subsubsection{Jet production in $\gamma p$ collisions}

Here we present the data for the jet production in $\gamma p$ scattering taken at HERA collider at $\sqrt{s_{e p}} \sim 300 \mathrm{GeV}$, where only one photon may be resolved ${ }^{20}$. The photoproduction events correspond here to the limit of virtuality of the initial photon: $P^{2} \sim 4 \mathrm{GeV}^{2}$ (with median $0.001 \mathrm{GeV}^{2}$ ), at present also $\sim 1 \mathrm{GeV}^{2}$ or (with a special condition) $\sim$ 0.01-0.02 $\mathrm{GeV}^{2}$ (see below). We will concentrate below on the single and double jet production and in addition we will include in the presentation some results for the charged particle production in the context of extraction of the gluonic content of the photon.

The partonic variables, related both to the initial and final states in the hard partonic subprocesses, are reconstructed from the corresponding quantities for the final state hadronic jets. The relation between variables corresponding to these two levels depends on the order of the perturbative QCD calculation, as was mentioned before. The parton momentum fraction $x_{\gamma}^{j e t}$ (called also $x_{\gamma}^{v i s}$ or $x_{\gamma}^{o b s}$ ) is in the LO case equal to $x_{\gamma}$. In practice it is reconstructed using two jets with the highest transverse energy $E_{T}$ (or $E_{T}^{j e t}$ ) in the event, using the following relation:

$$
x_{\gamma}^{j e t}=\frac{E_{T 1} e^{-\eta_{1}}+E_{T 2} e^{-\eta_{2}}}{2 E_{\gamma}},
$$

where also jet pseudorapidities, $\eta=-\ln (\tan (\theta / 2))$, and the energy of the photon $E_{\gamma}$ $\left(=y E_{e}\right)$ enter. (The positive pseudorapidity corresponds to the proton direction.) The scaled energy $y$ of the initial photon (eq. 5) is measured from the transverse energy $E_{T}^{h}$ and pseudorapidity of hadrons $\eta^{h}$ according to the formula

$$
y=\frac{1}{2 E_{e}} \sum_{h} E_{T}^{h} e^{-\eta^{h}}
$$

where the sum is over the produced hadrons.

The rapidity in the $\gamma p$ CM system is usually denoted by $\eta^{*}$ (typically $\eta-\eta^{*} \sim 2$, where $\eta$ is the value in the laboratory frame). In the angular distribution analysis (ZEUS 96, 98d,conf) the polar angle in the dijet CM system, $\theta^{*}$, is introduced.

\section{DATA}

\section{$\bullet$ H1 92 72 (HERA)}

The evidence for the hard photoproduction of a jet and single particle with $E_{T}>10$ $\mathrm{GeV}$ is reported. The "soft" contribution and the hard processes were simulated, the latter ones using the PYTHIA program with the MT B1 parton parametrization for the proton and DG for the photon, and $p_{T}^{\text {min }}=2.5 \mathrm{GeV}$. The jet cone algorithm with $R<1$ was used in the analysis.

\footnotetext{
${ }^{24}$ The energy of the electron at the beginning of running of the HERA collider was equal to 26.7 $\mathrm{GeV}$; starting from 1994 the positron replaced the electron and its energy increased to $27.5 \mathrm{GeV}$, with the energy of the proton $820 \mathrm{GeV}$. Starting from 1998 the electron beam with energy $27.5 \mathrm{GeV}$ is used again while the proton energy is higher than before, $920 \mathrm{GeV}$.

${ }^{25}$ The $\gamma \gamma$ events leading to the large $p_{T}$ jets are rare at HERA.
} 
•H193 [73 (HERA)

First measurement of the inclusive jet cross section at the $e p$ collider HERA (based on the 1992 data) is reported for the photon virtuality $P^{2}$ smaller than $0.01 \mathrm{GeV}^{2}$. Events with the scaled energy $y$ of the initial photon between 0.25 and 0.7 were collected. The photoproduction of jet was studied for $E_{T}$ from 7 to $20 \mathrm{GeV}$ and the pseudorapidity interval $-1<\eta<1.5$. The PYTHIA 5.6 program (with the GRV LO parton parametrization for the proton and GRV LO for the photon) with the jet cone algorithm assuming $\mathrm{R}=1$ was used in the analysis.

The transverse energy flow around the jet axis was studied and the discrepancy was found in a form of too large transverse energy flow on the forward side of the jet (see fig. 93). Among others, multiple parton interactions were mentioned as a possible explanation of this effect.
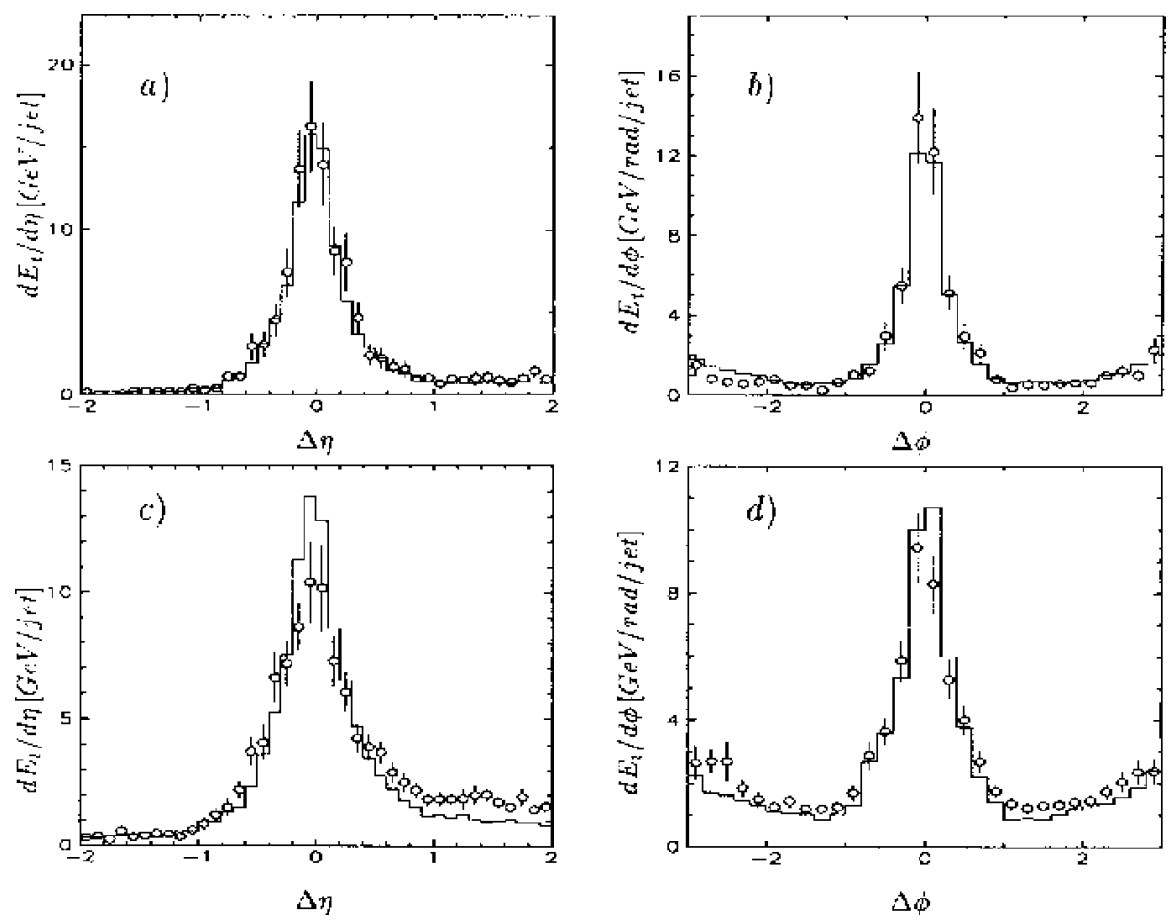

Figure 93: The transverse energy flow as a function of $\delta \eta$ (integrated over $|\delta \phi|<1.0$ ) (a, $c)$ and as a function of $\delta \phi$ (integrated over $|\delta \eta|<1.0)(b, d)$. Figs. a) and b) correspond to $-1.0<\eta<0.5, c$ ) and $d$ ) to $0.5<\eta<1.5$ (from [73]).

The inclusive jet cross sections $d \sigma / d E_{T}$ versus $E_{T}$ and $d \sigma / d \eta$ as a function of $\eta$ integrated over the corresponding range of the $\eta$ and $E_{T}$, respectively, were measured and compared with the $\mathrm{LO}$ calculation using the following parton parametrizations: LAC2, LAC3 and GRV LO for the photon, and GRV LO for the proton (see fig. 94). 

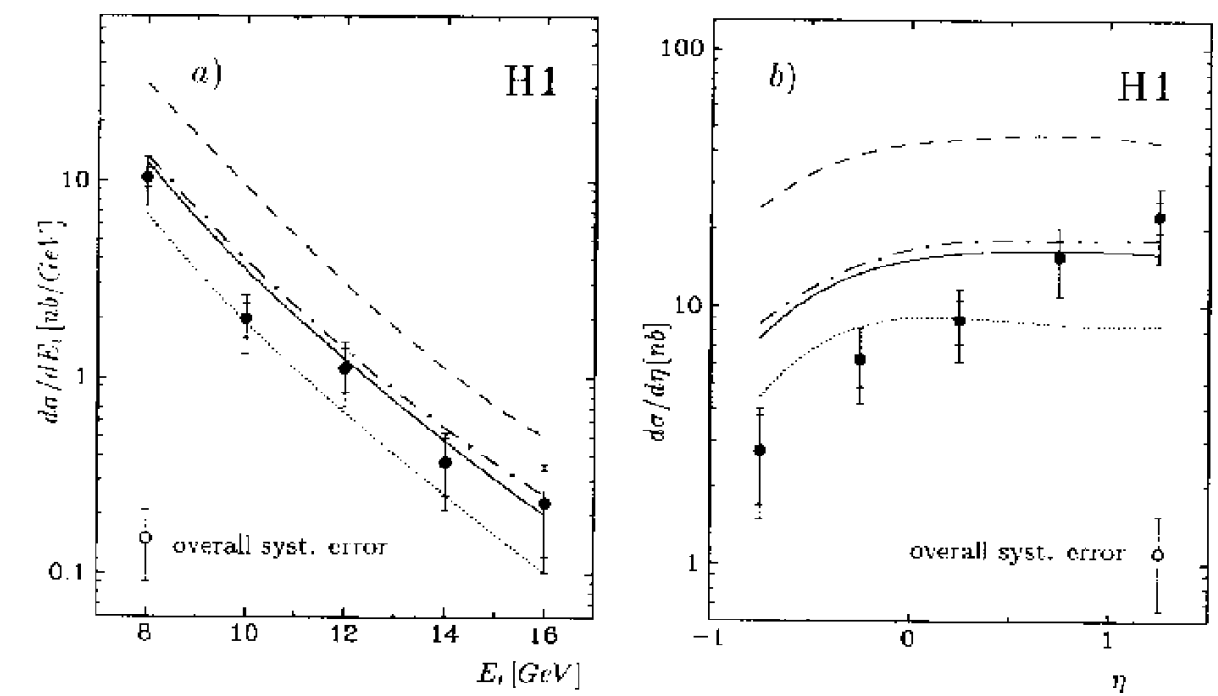

Figure 94: The inclusive jet $E_{T}$ spectrum integrated over the $\eta$ interval $-1.0<\eta<1.5$ (a) and inclusive $\eta$ spectrum (b) for jets with $E_{T}>7 \mathrm{GeV}$. The $L O Q C D$ predictions from the PYTHIA generator using parametrizations LAC3 (dashed line), LAC2 (dash-dotted line), GRV LO (full line) and GRV LO (without gluons, dotted line) (from [73]).

The shape of the $d \sigma / d E_{T}$ is well described in the range of $\eta$ between -1 and 1.5. It is a problem to describe the $\eta$ distribution of jets.

Comment: "In the (pseudorapidity) range 0.5 to 1.5 the data show larger average values of the transverse energy flow outside the jet cone on the forward side of the jet than predicted by the Monte Carlo."

$L A C 3$ gives cross section higher by factor 3 than data for the $E_{T}$ distribution of the jets.

"None of the models describe well the measured $\eta$ dependence (for jets)."

This discrepancy is absent in $\mathbf{H 1} \mathbf{9 6 \mathbf { a }}$ analysis, where multiple interactions are added in the Monte Carlo programs.

\section{-H1 95a [74] (HERA)}

The photoproduction of two-jet events (the 1993 data) was studied for the photon virtuality $P^{2}$ smaller than $0.01 \mathrm{GeV}^{2}$. The scaled energy $y$ of the initial photon was between 0.25 and 0.7 . The jet $E_{T}$ range from 7 to $20 \mathrm{GeV}$ and the pseudorapidity interval $0 \leq \eta \leq 2.5$ with $|\Delta \eta| \leq 1.2$ (between the most energetic jets) (see also $\mathbf{H 1}$ 96a) were investigated.

The cone jet-finding algorithm with $\mathrm{R}=1$ (and 0.7 for cross checks) was used. The PYTHIA 5.6 generator with the GRV LO parton parametrizations for the proton and the photon, with $p_{T}^{\text {min }}=2 \mathrm{GeV}$ was used in the analysis.

For the first time the inclusive (LO) cross sections were derived for the parton level, and the gluon density in the photon was measured.

The transverse energy flow around the jet direction per event versus the rapidity distance from the jet direction was studied for $7 \leq E_{T} \leq 8 \mathrm{GeV}$ and $0 \leq \eta \leq 1$ and found to be asymmetric and different for samples with $x_{\gamma}>0.4$ and $x_{\gamma}<0.4$. The data were compared with the PYTHIA (with MI) predictions (not shown).

The transverse energy flow versus the azimuthal angle around the jet direction and 
the transverse energy of the underlying events outside the jets (here named $E_{t}^{\text {pedestal }}$ ) was studied for $7 \leq E_{T} \leq 8 \mathrm{GeV}$ in a cone size $R=1$. Results (see fig. 95) compared to the prediction of the PYTHIA program (with and without MI) lead to the conclusion: "the multiple interaction gives an improved description".
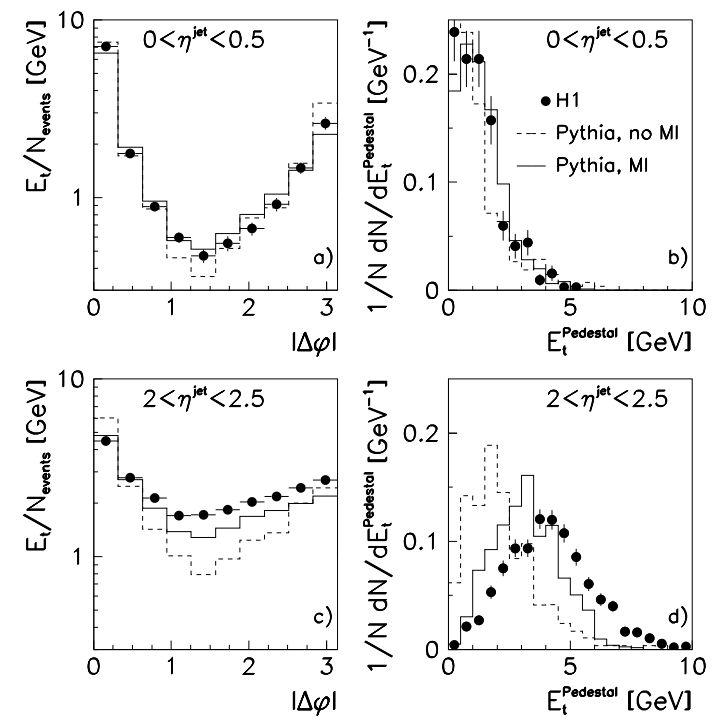

Figure 95: (a, c) The transverse energy flow versus the azimuthal angle with respect to the jet direction in two rapidity bins. ( $b, d)$ Distributions of the transverse energy measured outside of jets. Histograms show the PYTHIA simulations with (full line) and without (dashed line) the multiple interactions (from [74]).

To achieve the goal which was here the extraction of the gluon distribution, the single parton cross section $d \sigma / d p_{t}$ integrated over the parton rapidity range, as well as the single parton cross section $d \sigma / d \eta$ were studied and compared with the prediction based on the LO parametrizations for the photon: GRV, LAC 1 and LAC 3 (see fig. 96). 

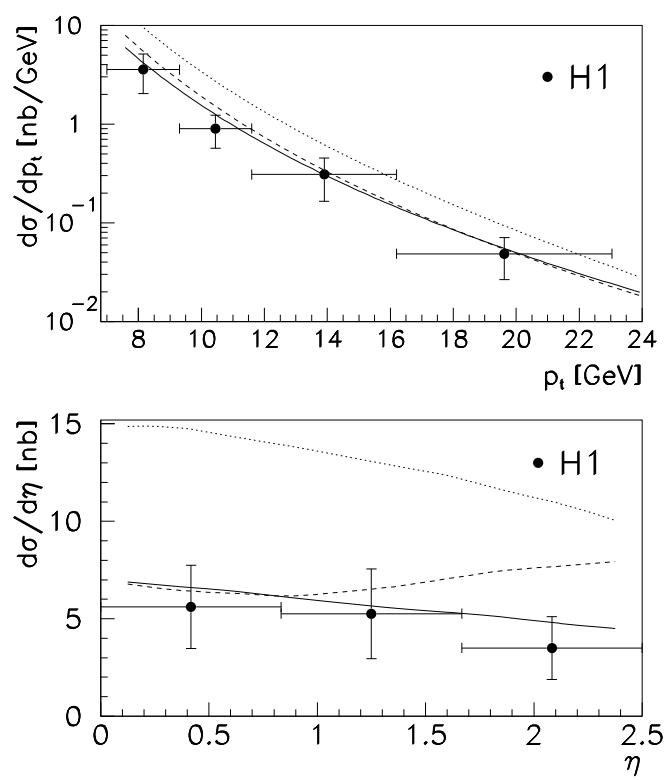

Figure 96: The single parton cross sections: (top) $d \sigma / d p_{t}$ integrated over the pseudorapidity range $0<\eta<2.5$, (bottom) $d \sigma / d \eta$ for $p_{t}>7 \mathrm{GeV}$. The solid line - the LO QCD calculation with the GRV LO parametrization for partons in the proton and the photon. The dashed (dotted) line - the same for the LAC1 (LAC3) parametrization for the photon (GRV LO for the proton) (from [7]).

To extract information on the subprocesses, the full two-jet kinematics was used and the distributions of $\Delta \eta, \Delta E_{T}$, and of $x_{\gamma}$ and $x_{p}$ were studied. In fig. 97 we present the distribution of $x_{\gamma}$.

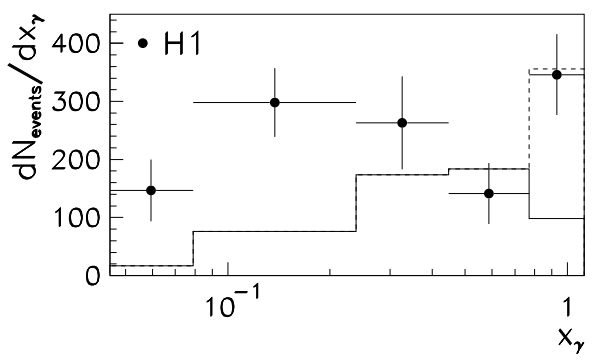

Figure 97: The distribution of $x_{\gamma}$ in the photon. The solid line - the contribution from the quark-resolved photon processes; the dashed line - the direct photon contribution, from the PYTHIA Monte Carlo (from 74]).

The (LO) gluon distribution in the photon was derived, at the average (factorization and renormalization) scale $<\tilde{Q}^{2}>=<p_{t}^{2}>=75 \mathrm{GeV}^{2}$ for $0.04 \leq x_{\gamma} \leq 1$, see table 23 and fig. 98 for results. 
Table 23:

\begin{tabular}{|c|l|r|}
\hline $\begin{array}{c}<\tilde{Q}^{2}> \\
{\left[G e V^{2}\right]}\end{array}$ & $<x_{\gamma}>$ & \multicolumn{1}{c|}{$\begin{array}{c}x_{\gamma} G\left(x_{\gamma}\right) / \alpha \\
\text { stat. }+ \text { syst. })\end{array}$} \\
\hline 75 & 0.059 & $1.92 \pm 0.87 \pm 1.68$ \\
& 0.14 & $1.19 \pm 0.34 \pm 0.59$ \\
& 0.33 & $0.26 \pm 0.24 \pm 0.33$ \\
& 0.59 & $-0.12 \pm 0.15 \pm 0.33$ \\
& 0.93 & $-0.08 \pm 0.61 \pm 0.30$ \\
\hline
\end{tabular}

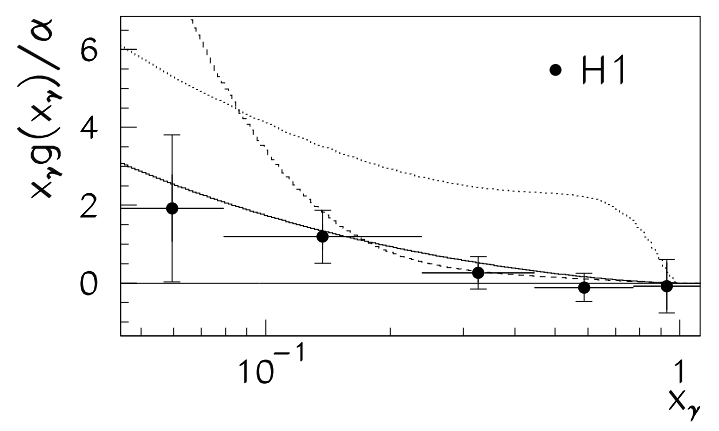

Figure 98: The gluon distribution extracted from the resolved photon processes at $<\tilde{Q}^{2}>=<p_{t}^{2}>=75 \mathrm{GeV}^{2}$. For comparison the GRV LO (full line), LAC1 (dashed) and LAC3 (dotted) gluon parametrizations are shown (from [74]).

Comment: "The multiple interaction option gives an improved description" of the jet profiles and pedestal distributions (still some "deviations from data at large jet rapidities $2<\eta<2.5$ " remain).

In the extracting the gluon density $q^{\gamma}$ was taken as determined by the two-photon experiments at LEP and TRISTAN, in the form given by the GRV LO parametrization. "A high gluon density at large parton momenta as suggested by the LAC 3 parametrization is clearly excluded. The strong rise of the LAC1 parametrization below $x_{\gamma} \leq 0.08$ is not supported."

\section{-H1 96a [75] (HERA)}

The jet production with $E_{T} \geq 7 \mathrm{GeV}$ (and $-1<\eta<2.5$ ) was measured in ep collisions (data from 1994) with the scaled photon energy $0.25<y<0.7$ and $P^{2}$ below 0.01 $\mathrm{GeV}^{2}$. The properties of the hadronic final state and the distribution of the transverse energy are studied in detail. The PYTHIA 5.7 (with $p_{T}^{\min }=2 \mathrm{GeV}$ ), HERWIG 5.8 $\left(p_{T}^{\min }=2 \mathrm{GeV}\right)$ and PHOJET $1.0\left(p_{T}^{\min }=3 \mathrm{GeV}\right)$ generators with the GRV LO parton parametrizations for the proton and the photon) were used in the analysis. The PYTHIA program were used also with MI, then with $p_{T}^{m i}=1.2 \mathrm{GeV}$. The cone algorithm with $\mathrm{R}=1$ was used.

The integration over the $\gamma p$ CM system pseudorapidity $-2.5<\eta^{*}<1$ leads to the total transverse event energy distribution shown in fig. 99 a (here $0.3<y<0.7$ ). The average transverse energy flow versus $\eta^{*}$ for the total $E_{T}$ range between 25 and 30 $\mathrm{GeV}$ was also measured (see fig. 99b). The shape of both distributions may indicate 
the need of the multiple interactions.
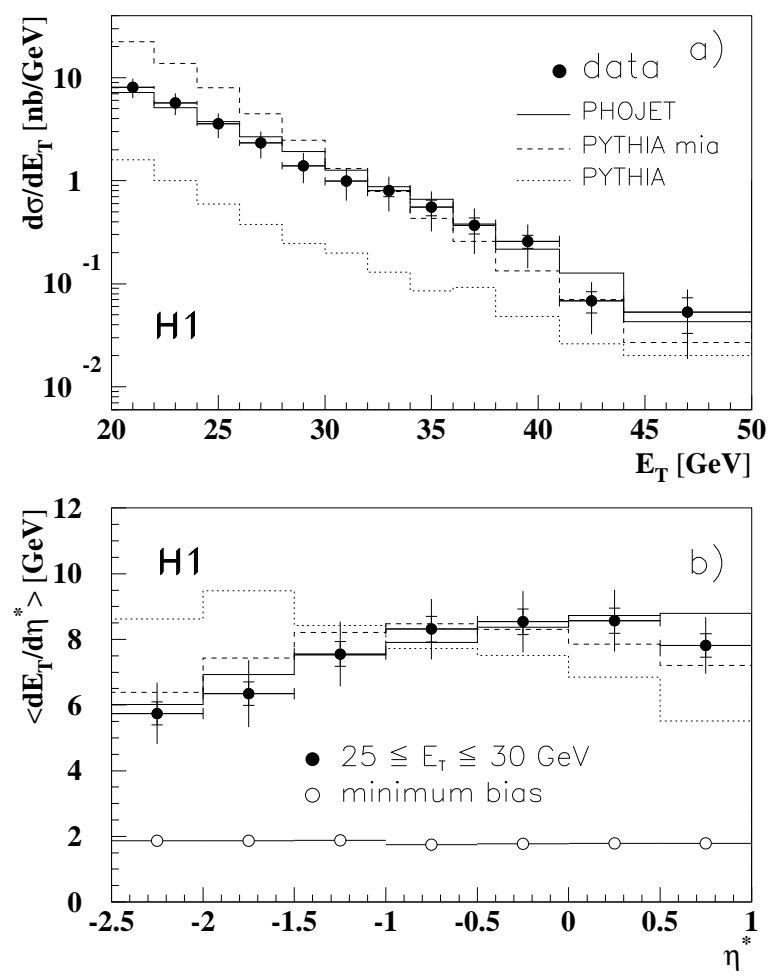

Figure 99: a) The differential transverse energy cross section integrated over the pseudorapidity $\left(-2.5 \leq \eta^{*} \leq 1\right)$. The histograms are the results of the simulations with interactions of the beam remnants (full line - PHOJET, dashed - PYTHIA) and without them (dotted line - PYTHIA). b) The corrected transverse energy flow versus $\eta^{*}\left(\eta^{*}>0\right.$ corresponds to the proton direction). The pseudorapidity range and histograms as in a) (from 75]).

To get an insight into the details of the considered events the transverse energy flow outside of the two jets with the highest $E_{T}$ was studied as a function of $x_{\gamma}$ for the $\left|\eta^{*}\right|<1$ and $\Delta \eta<1.2$. Results for the transverse energy density can be found in fig. 100.

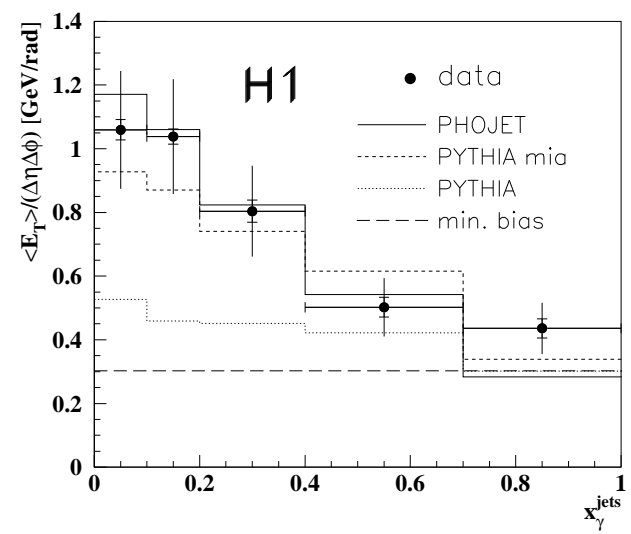

Figure 100: The corrected transverse energy density in the region $\left|\eta^{*}\right|<1$ outside the jets, as a function of $x_{\gamma}$. The histograms are as in fig. 99 (from 757).

The distribution of the transverse energy around the jet axis was also measured as a function of $\delta \phi$ (not shown). The jet width obtained in this analysis is similar to the 
corresponding quantity in the $p \bar{p}$ collision.

Results obtained for the jet cross section (here $0.25<y<0.7$ ) are presented in fig. 101a, where $d \sigma / d E_{T}$ for the jet production in two $\eta$ regions is shown, and in fig. 101 b, where the distributions $d \sigma / d \eta$ for the events with the transverse jet energy $E_{T}>7,11,15 \mathrm{GeV}$ are presented. The comparison with the PHOJET and PYTHIA simulations, with and without multiple interactions, was done for both kinds of distributions. Note that the rapidity distribution is sensitive rather to the photon structure functions, while the $E_{T}$ cross section to the matrix elements for the hard processes. Note also that "for $E_{T}$ bigger than $7 \mathrm{GeV}$ previous measurements (H1 93) suffered from a defect and are superceded by this new measurement".
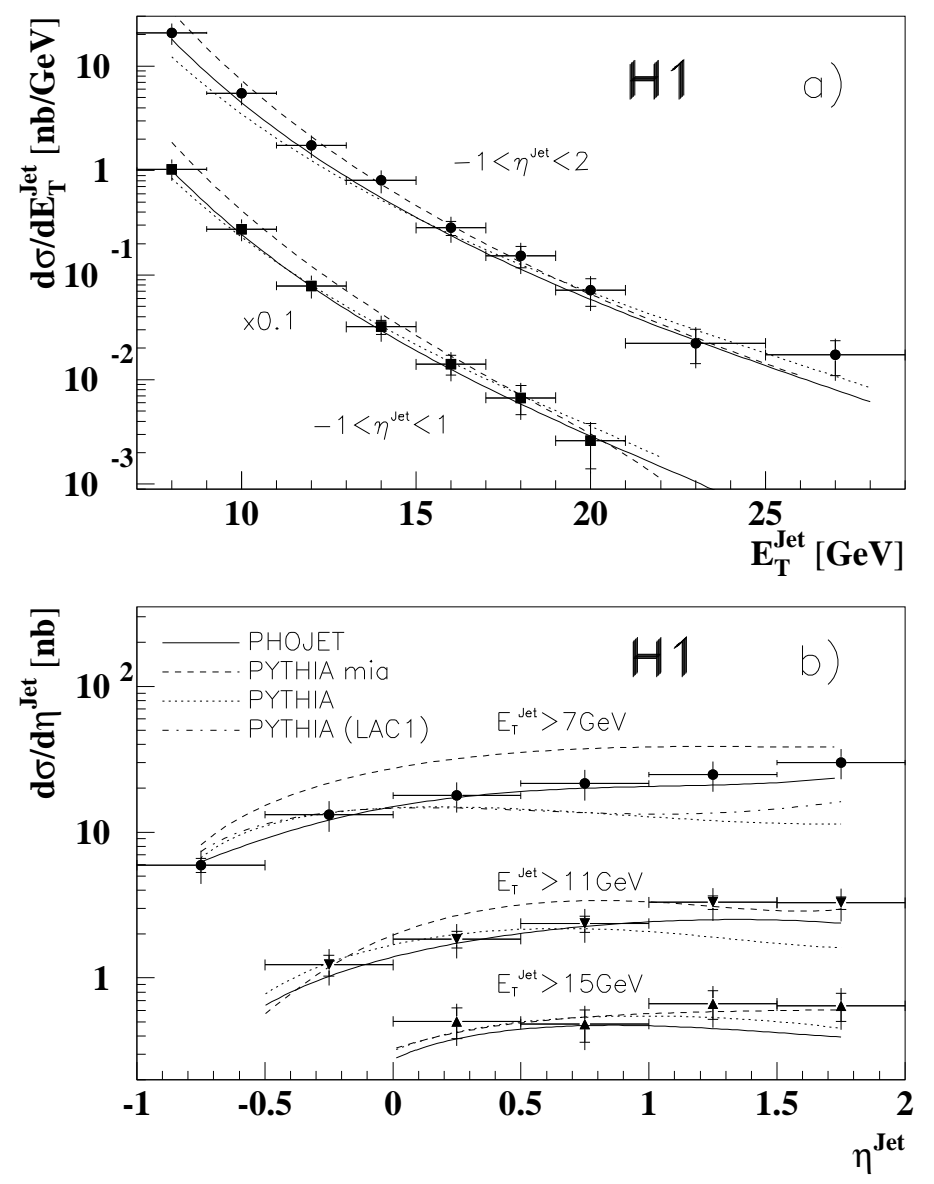

Figure 101: a) The cross section $d \sigma / d E_{T}$ for the jet production for $E_{T}>7 \mathrm{GeV}$, in two $\eta$ ranges: $-1<\eta<2$ and $-1<\eta<1$. The curves show the Monte Carlo simulations with interactions of the beam remnants (full line - PHOJET, dashed - PYTHIA) and without them (dotted - PYTHIA). b) Cross section $d \sigma / d \eta$ versus $\eta$ for different thresholds in $E_{T}$ : 7 , 11 and $15 \mathrm{GeV}$. The curves are as in a); additional dash-dotted curve - PYTHIA with the LAC1 parametrization (from 75]).

Comment: In addition to the primary hard scattering process, the interaction between the two beam remnants is included in the analysis (e.g. using PYTHIA with $p_{T}^{m i} \geq 1.2$ (for GRV LO) or $2.0 \mathrm{GeV}$ (LAC1)). It gives "adequate description of data" for the transverse energy versus pseudorapidity and the average energy flow obtained in this analysis. 
"For the first time the underlying event energy has been measured in jet events using direct and resolved photon probes."

"The strong influence of the underlying event energy on the measured cross section is demonstrated."

The multiple interaction seems to improve also $d \sigma / d E_{T}$ and $d \sigma / d \eta$ distributions for jets; within this approach the low $E_{T}$ and positive $\eta$ range is still not properly described by the two considered LO parton parametrizations: LAC1 and GRV within the PYTHIA program. PHOJET describes these data.

\section{•H1 98 |76" (HERA)}

The two-jet events (the 1994 data) corresponding to to $P^{2}$ lower than $4 \mathrm{GeV}^{2}$ and the scaled energy $y$ between 0.2 and 0.83 were measured. All jets have $E_{T}>7.5 \mathrm{GeV}$, with $\bar{E}_{T}$ ranges above $10 \mathrm{GeV}$ and $\Delta E_{T}<0.25 \bar{E}_{T}$, and pseudorapidity $0<\bar{\eta}<2$, with $\Delta \eta<1$. The cone algorithm with $\mathrm{R}=0.7$ was used. The PYTHIA 5.7 generator with the multiple interaction using the GRV LO parton parametrizations for the proton and the photon was used in the analysis $\left(p_{T}^{m i}=1.2 \mathrm{GeV}\right)$. The PHOJET 1.06 was also used with the same parton parametrizations (with the $p_{T}$ cutoff $2.5 \mathrm{GeV}$ ).

The double differential dijet cross sections, $d \sigma / d x_{\gamma} / d \log \left(E_{T}^{2} / E_{0}^{2}\right)$, as a function of $\bar{E}_{T}^{2}$ for few ranges of $x_{\gamma}$ are compared with the NLO QCD calculation [183] and PYTHIA (GRV) simulation, see fig. 102.

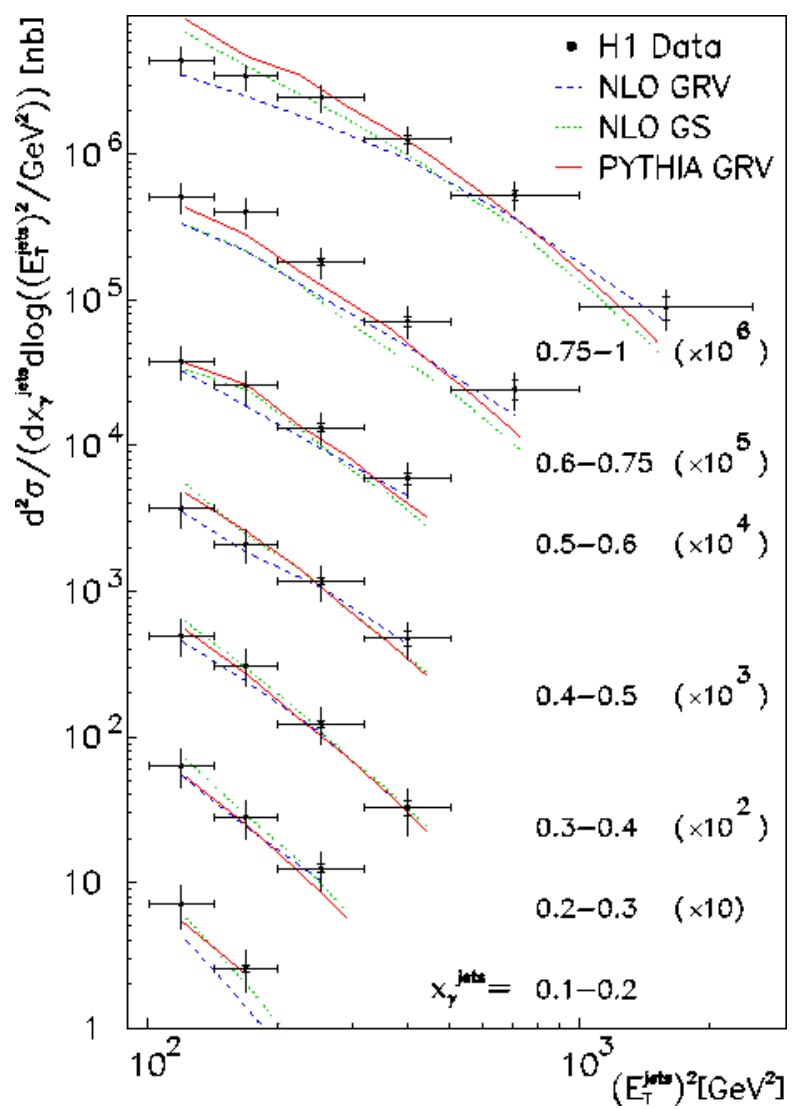

Figure 102: The double differential cross section as a function of the square of the averaged jet transverse energy for the different $x_{\gamma}$ bins, compared to the PYTHIA (GRV) and the NLO calculation (GRV and GS) (from 53]). 

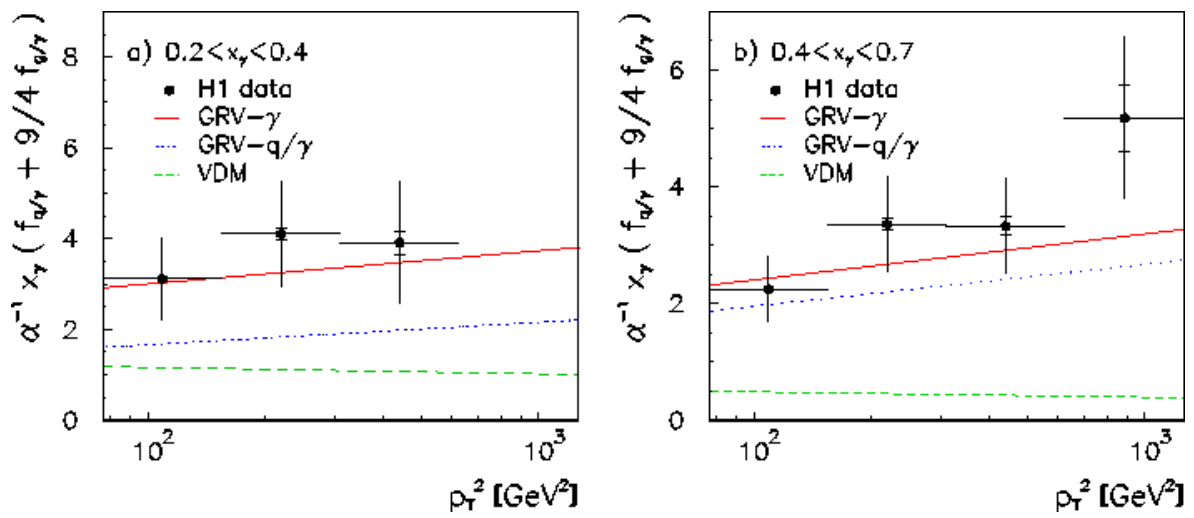

Figure 103: The effective parton density in the photon (from 537).

The method based on the Single Effective Subprocess Approximation [54] was used to extract, for the first time, the effective (LO) parton density of the photon:

$$
\alpha^{-1} x_{\gamma}\left(\tilde{q}^{\gamma}+\frac{9}{4} G^{\gamma}\right)
$$

with $\tilde{q}^{\gamma}=\sum\left(q^{\gamma}+\bar{q}^{\gamma}\right)$.

This was done for $63 \mathrm{GeV}^{2}<p_{t}^{2}<1000 \mathrm{GeV}^{2}$, for $0.2<x_{\gamma}<0.4$ and $0.4<x_{\gamma}<$ 0.7. The dependence of the effective parton density on the $\tilde{Q}^{2}\left(=p_{t}^{2}\right)$ scale is shown in fig. 103 and in table 24 (where the statistical and systematical errors are added in quadrature).

Table 24:

\begin{tabular}{|c|c|c|}
\hline$\left\langle x_{\gamma}\right\rangle$ & $\tilde{Q}^{2}\left[\mathrm{GeV}^{2}\right]$ & $x_{\gamma} \tilde{f}\left(x_{\gamma}\right) / \alpha$ \\
\hline 0.3 & 112 & $3.11 \pm 0.88$ \\
& 224 & $4.10 \pm 1.17$ \\
& 447 & $3.91 \pm 1.34$ \\
\hline 0.55 & 112 & $2.25 \pm 0.55$ \\
& 224 & $3.36 \pm 0.82$ \\
& 447 & $3.33 \pm 0.82$ \\
& 891 & $5.18 \pm 1.38$ \\
\hline
\end{tabular}

Comment: "Satisfactory overall description (of the double differential cross section for jet) except for $x_{\gamma}>0.6 "$.

"The effective parton distribution grows with the scale $p_{t}^{2}$, although the increase appears slightly steeper than expected from the GRV LO parametrization."

-H1 99a 777 (HERA)

The new method of extracting the gluon density in the (real) photon from the processes with charged particles is introduced. Events collected in 1994 with $0.3<y<0.7$, $P^{2}<0.01 \mathrm{GeV}^{2},|\eta|<1$ and $p_{T}>2,3 \mathrm{GeV}$ were used. The reconstruction of the $x_{\gamma}$ variables from the highest $p_{T}$ charged tracks follows closely the analysis H1 95a. 
The PYTHIA 5.7 (with and without MI) using the GRV LO parton parametrization for the proton, and for the photon : GRV LO, SaS1D, LAC1, with the corresponding $p_{T}^{\text {min }}$ cutoff $1.2 \mathrm{GeV}, 1.0 \mathrm{GeV}$ and $2 \mathrm{GeV}$. The $p_{T}$ and $\eta$ distributions were measured. The result on the $\mathrm{LO}$ gluon density at $\tilde{Q}^{2}=<p_{t}^{2}>=38 \mathrm{GeV}^{2}$ is presented in fig. 104 and compared with the jet data based on the 1993 runs at $<p_{t}^{2}>=75 \mathrm{GeV}^{2}$ (see also fig. 98).

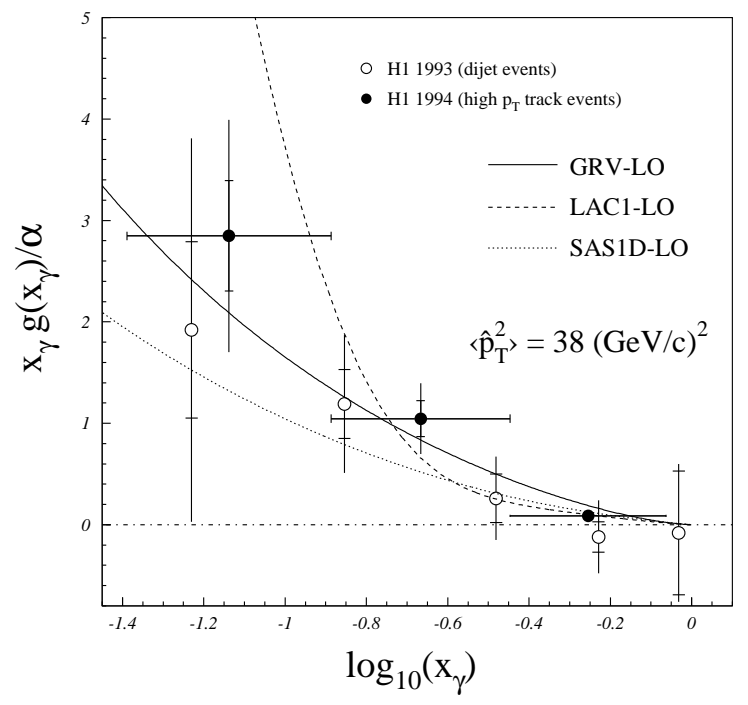

Figure 104: The LO gluon density data extracted from charged particles events at $<p_{T}^{2}>=38 \mathrm{GeV}^{2}(\bullet)$ together with predictions of the GRV LO, LAC1 LO and SaS1D $L O$ parametrizations. Also the $\mathbf{H 1}$ 95a [74] data extracted from dijet events at $\left\langle p_{t}^{2}\right\rangle=75$ $\mathrm{GeV}^{2}$ are shown (o) (from 77]).

\section{$\bullet$ H1 2000a 78 (HERA)}

The more precise dijet data taken in 1996 are analysed in regions $0.5<y<0.7$, $P^{2}<0.01 \mathrm{GeV}^{2}$ and $E_{T}>4 \mathrm{GeV}$ or $E_{T}>6 \mathrm{GeV}$. The rapidities lie in the range $-0.5<\bar{\eta}<2.5$ and $\Delta \eta<1$. In the cross section for $E_{T}>4 \mathrm{GeV}$ the cut on the invariant mass of the jets was introduced: $M_{j j}>12 \mathrm{GeV}$. The two Monte Carlo models were used : PHOJET and "PYTHIA $5.7+$ power law $p_{T}$ " (with $\tilde{p}_{t_{0}}=1.55$ $\mathrm{GeV}$. Multiple interaction was included in both generators. For PYTHIA $p_{T}^{\min }=$ $p_{T}^{m i}=1.2 \mathrm{GeV}$, while for PHOJET $p_{T}$ cutoff equals $2.5 \mathrm{GeV}$. The GRV LO parton parametrizations in the photon and the proton were used. The CDFCONE algorithm with $\mathrm{R}=0.7$ was assumed for jet reconstruction.

The measured cross section as a function of $x_{\gamma}$ for $E_{T}>4 \mathrm{GeV}$ is presented in fig. 105. Similar distribution for $E_{T}>6 \mathrm{GeV}$ (obtained after correcting for pedestal energy due to the underlying event) is shown in fig. 106, where the contribution from direct, and resolved - quark and gluon initiated - processes are indicated separately. 


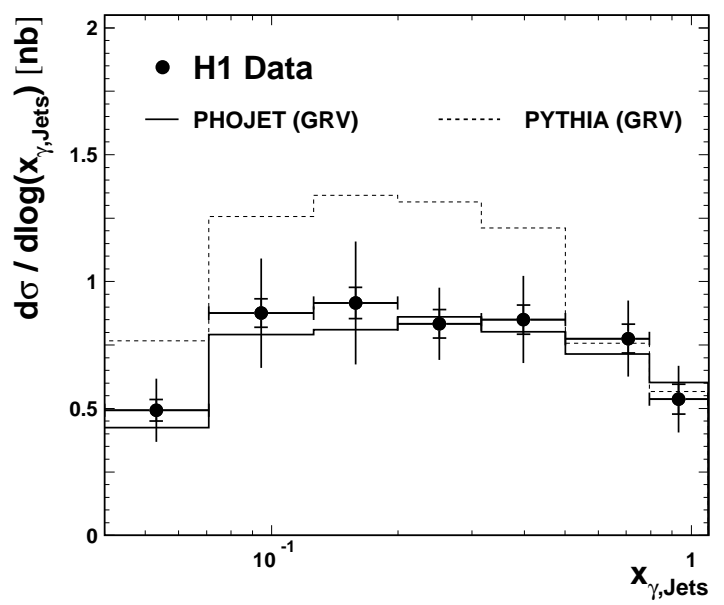

Figure 105: The $x_{\gamma}$ distribution of dijet events with $E_{T}>4 \mathrm{GeV}$ (from [78]).

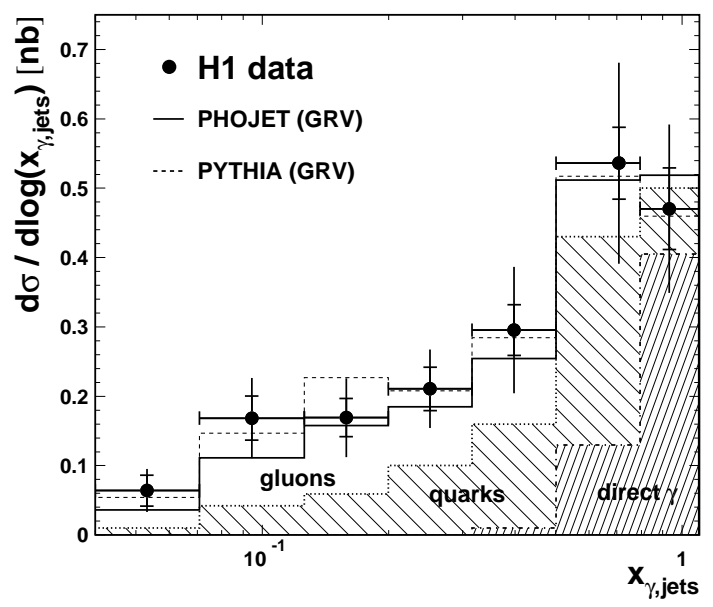

Figure 106: The $x_{\gamma}$ distribution of dijet events with $E_{T}>6 \mathrm{GeV}$ (from [78]).

Using the Single Effective Subprocess Approximation [54 the LO effective parton densities of the photon were determined as a function of $x_{\gamma}$ for $<\tilde{Q}^{2}>\left(=<p_{t}^{2}>\right)=74$ $\mathrm{GeV}^{2}$. The result is presented in fig. 107 (left) and in table 25. The gluon density is determined from the effective parton density by subtracting the quark densities (as given by GRV LO in agreement with $F_{2}^{\gamma}$ data). Results of this analysis are presented in fig. 107 (right) and in table 25. The obtained gluon density is in agreement with that from the single particle data presented in $\mathbf{H 1}$ 99a. 

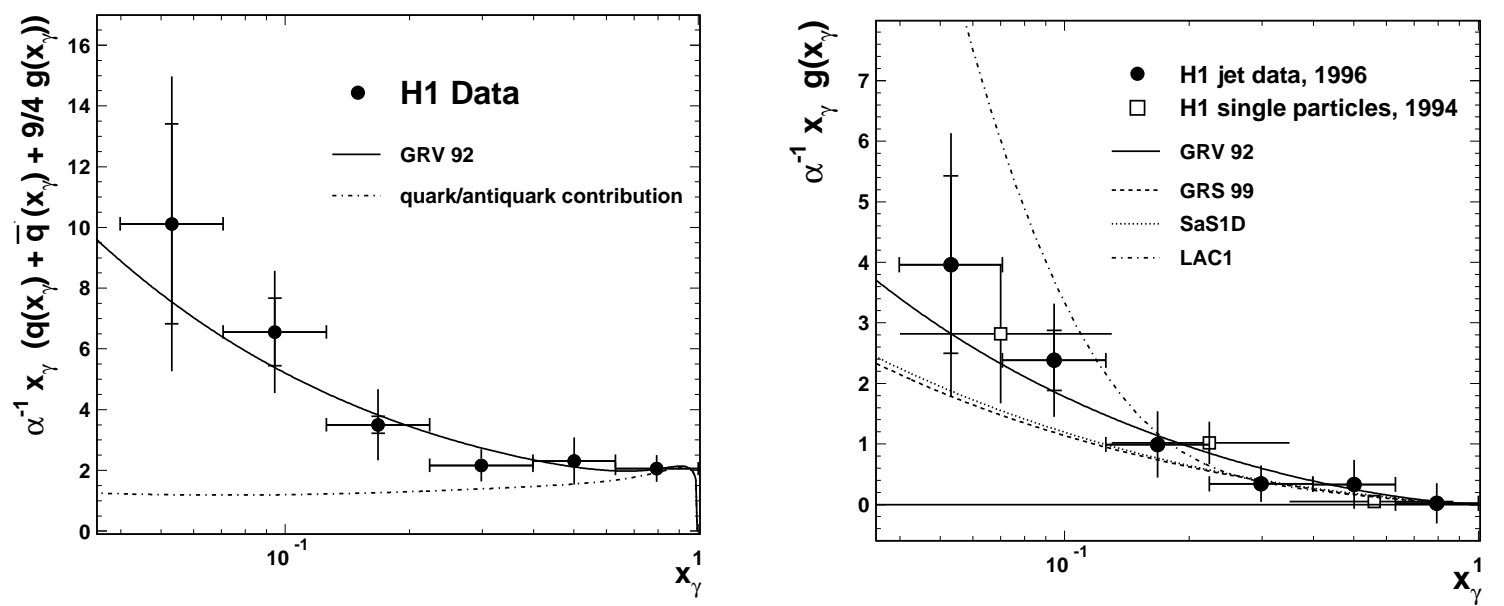

Figure 107: The $x_{\gamma}$ distributions at $<\tilde{Q}^{2}>=<p_{t}^{2}>=74 \mathrm{GeV}^{2}$ of the effective parton density (left) and the gluon density (right). The comparison with GRV LO (left) and with GRV LO, GRS LO, SaS1D, LAC1 predictions and the $\mathbf{H 1} \mathbf{9 9 a}$ data (right) is shown (from [78]).

Table 25:

\begin{tabular}{|c|c|c|c|}
\hline $\begin{array}{c}<\tilde{Q}^{2}> \\
{\left[\mathrm{GeV}^{2}\right]}\end{array}$ & $<x_{\gamma}>$ & $\begin{array}{c}x_{\gamma} f_{\gamma, \text { eff }}\left(x_{\gamma}\right) / \alpha \\
\text { total }(\text { stat. })\end{array}$ & $\begin{array}{c}x_{\gamma} G\left(x_{\gamma}\right) / \alpha \\
\text { total }(\text { stat. })\end{array}$ \\
\hline 74 & 0.053 & $10.1 \pm 4.9(3.3)$ & $4.0 \pm 2.1(1.4)$ \\
& 0.094 & $6.6 \pm 2.0(1.1)$ & $2.4 \pm 0.9(0.5)$ \\
& 0.17 & $3.5 \pm 1.2(0.3)$ & $0.99 \pm 0.55(0.12)$ \\
& 0.30 & $2.2 \pm 0.5(0.1)$ & $0.34 \pm 0.30(0.03)$ \\
& 0.50 & $2.3 \pm 0.8(0.2)$ & $0.33 \pm 0.40(0.08)$ \\
& 0.79 & $2.1 \pm 0.4(0.1)$ & $0.02 \pm 0.33(0.03)$ \\
\hline
\end{tabular}

Comment: The data reach parton fractional energies down to $x_{\gamma}=0.05$. Leading order QCD gives a good description of the $E_{T}>6 \mathrm{GeV}$ data (after subtraction of the underlying event energy) which makes possible a determination of the effective parton density in the photon. "This quantity is dominated by the gluon density for $x_{\gamma}<0.2$ which is found to rise strongly towards small $x_{\gamma} . "$ 
-ZEUS 92 [79] (HERA)

The evidence for the hard scattering (jet production and resolved photon processes) in the photoproduction has been found. The cross section for jets with $E_{T} \geq 10 \mathrm{GeV}$ at HERA was obtained. The PYTHIA and HERWIG generators, with GS and MT B1 parton parametrization for the photon and the proton, respectively, were used (with $p_{T}^{\min }=1.5 \mathrm{GeV}$ ). In the analysis the cone algorithm with $R=1$ was applied.

Comment: "Evidence for the photon remnant jets was found."

\section{-ZEUS 94 [80] (HERA)}

The measurement was based on the 1992 data for the single and double jet photoproduction, with $y$ between 0.2 and 0.7. For tagged events $P^{2}$ was below $0.02 \mathrm{GeV}^{2}$, otherwise below $4 \mathrm{GeV}^{2}$. The analysis of the direct and resolved photon processes was made using the HERWIG 5.7 and PYTHIA 5.6 generators. In generation of events the parton parametrization GRV for the photon and MRSD0 for the proton (in addition also DG, LAC and MRSD_) were used, with $p_{T}^{\min }=2.5 \mathrm{GeV}$. The jet-finding cone algorithm with $R=1$ was applied.
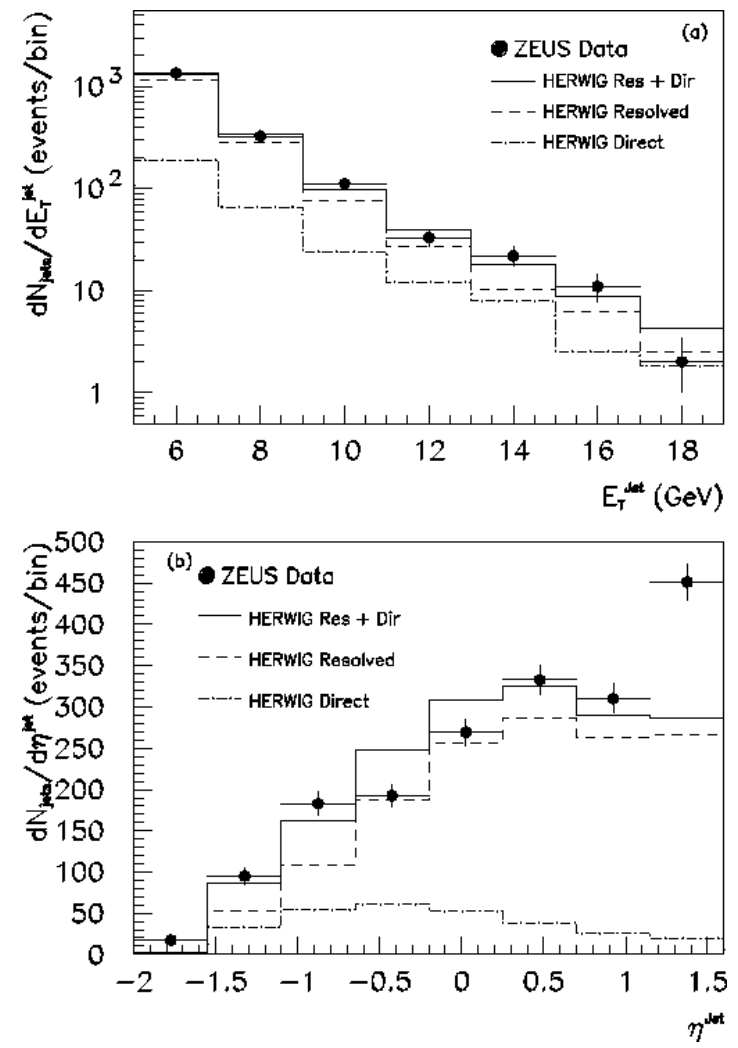

Figure 108: Inclusive jet distributions for (a) transverse energy of jets, (b) pseudorapidity of jets. Comparison with the HERWIG prediction for the direct and resolved contributions is shown (from [80]).

The results for the single jets' concerning the $E_{T}$ distribution up to $E_{T}=18 \mathrm{GeV}$, integrated over rapidity $\eta$ below 1.6 , are presented in fig. 108 a. Fig. 108b shows the 
$d \sigma / d \eta$ data where the disagreement with the Monte Carlo prediction occurs for the positive $\eta$.

Dijet production has been studied by selecting events with two or more jets with $E_{T}>5 \mathrm{GeV}$, for $\eta$ smaller than 1.6. (fig. 109).

The $x_{\gamma}$ and $x_{\text {proton }}$ distributions were studied as well for events with $|\Delta \eta|<1.5$, $|\Delta \phi|>120^{\circ}$ and the invariant mass of two jets $M_{i j}$ larger than $16 \mathrm{GeV}$ (not shown).
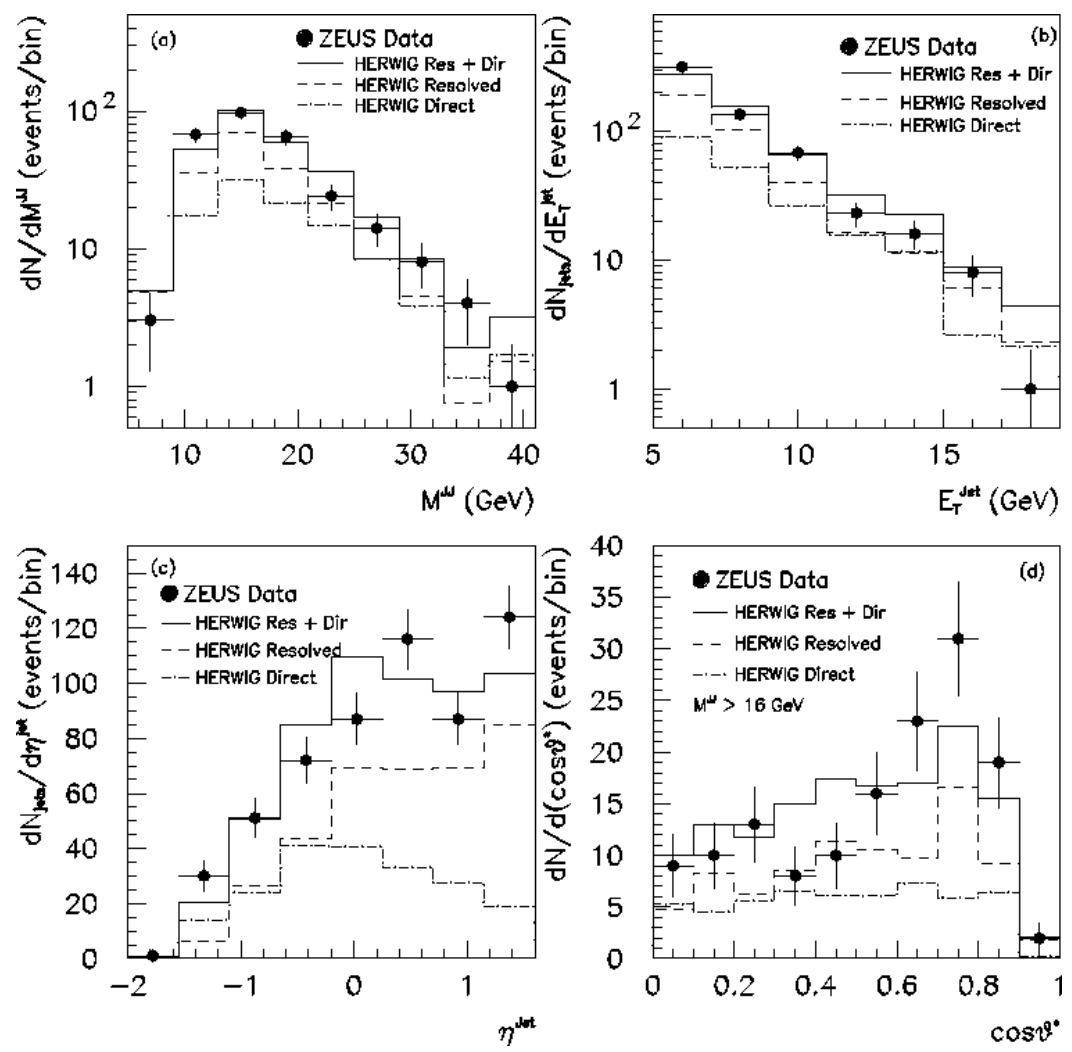

Figure 109: Kinematic distributions for events with two or more jets: (a) the jet pair invariant mass, (b) the transverse energy of jets, (c) the pseudorapidity, (d) $\cos \theta^{*}$ of jet angles in jet-jet $C M$ with respect to the proton momentum for events with $M_{i j}>16 \mathrm{GeV}$. The comparison with the Monte Carlo simulations is shown (from 80]).

\section{-ZEUS 95a 81] (HERA)}

The 1993 data for the production of at least one jet with $E_{T}>6 \mathrm{GeV}$ (up to $41 \mathrm{GeV}$ ) are presented. Events correspond to: $P^{2}$ below $4 \mathrm{GeV}^{2}, y$ between 0.2 and 0.85 and the jet pseudorapidity between -1 and 2. The PYTHIA 5.6 and HERWIG 5.7 generators with the GRV parametrization for the photon and MRSD_ for the proton (and in addition LAC1 and $\mathrm{MRSD}_{0}$ ) were used. The diffraction contribution was modelled by the POMPYT program $\left(p_{T}^{\text {min }}=3 \mathrm{GeV}\right)$.

The cone algorithm with $\mathrm{R}=1$ was assumed for jets.

The transverse energy flow around jet axis was studied. Results in three $\eta$ ranges are presented in fig. 110, where "there is some discrepancy for the forward-going jets in the $\delta \eta>1 "$.

The $E_{T}$ distributions integrated over two different pseudorapidity ranges and $d \sigma / d \eta$ distribution integrated above three $E_{T}$ thresholds: 8, 11 and $17 \mathrm{GeV}$ were measured. The results are presented in figs. 111 and 112, respectively, and compared to the 
PYTHIA prediction (for LAC1, GRV HO, ACFGP HO, GS parton parametrizations), with $p_{T}^{\min }=5 \mathrm{GeV}$. The discrepancies between the measurement and the LO QCD prediction of PYTHIA are restricted to low $E_{T}$, very forward jet.

ZEUS 1993
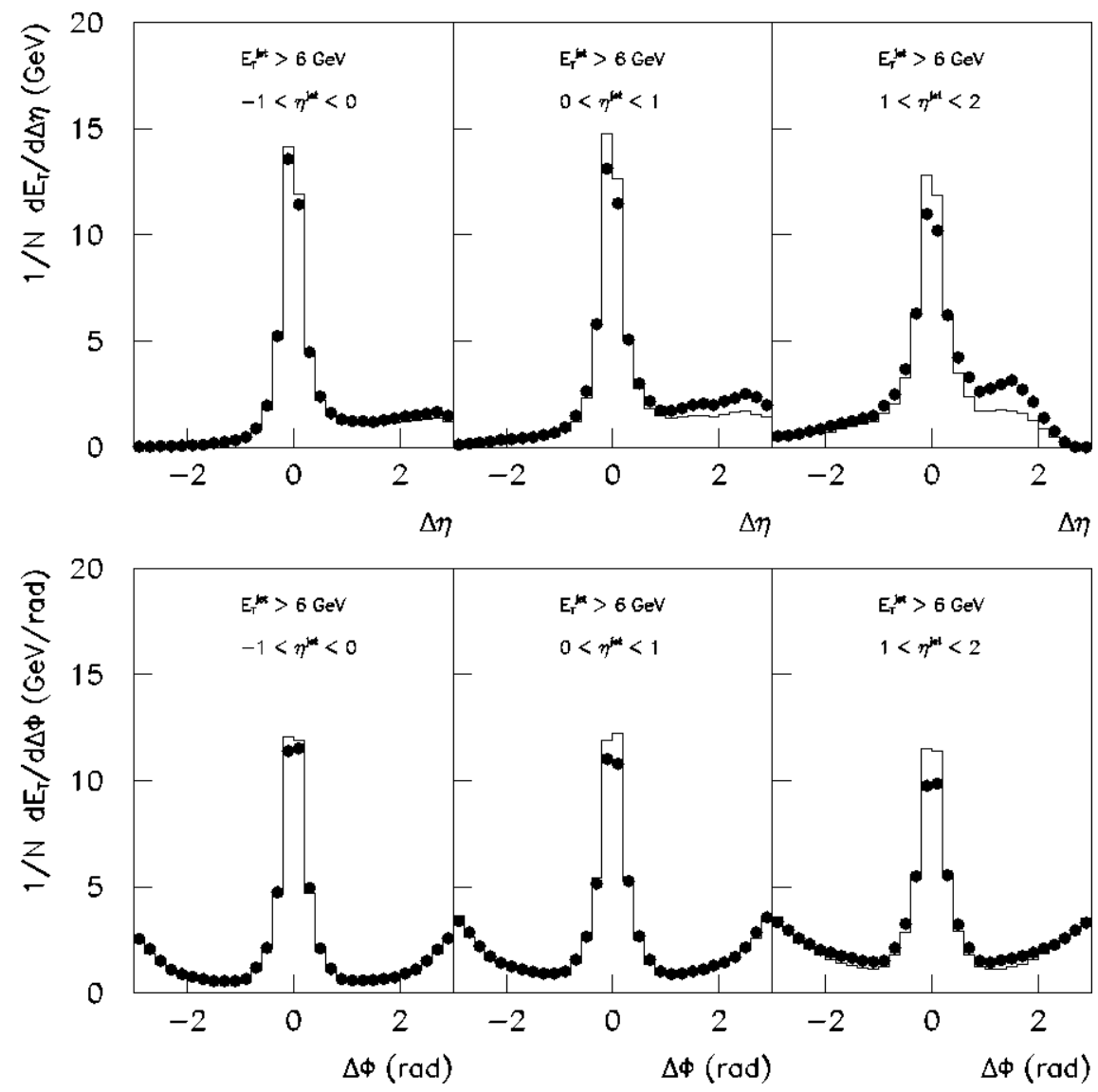

Figure 110: Transverse energy profiles as functions of $\delta \eta$ integrated over $|\delta \phi|<\pi / 2$ (top row) and $\delta \phi$ (bottom row) (see the text). Results from the PYTHIA simulation (with both resolved and direct processes) are shown (from [81)). 
ZEUS 1993

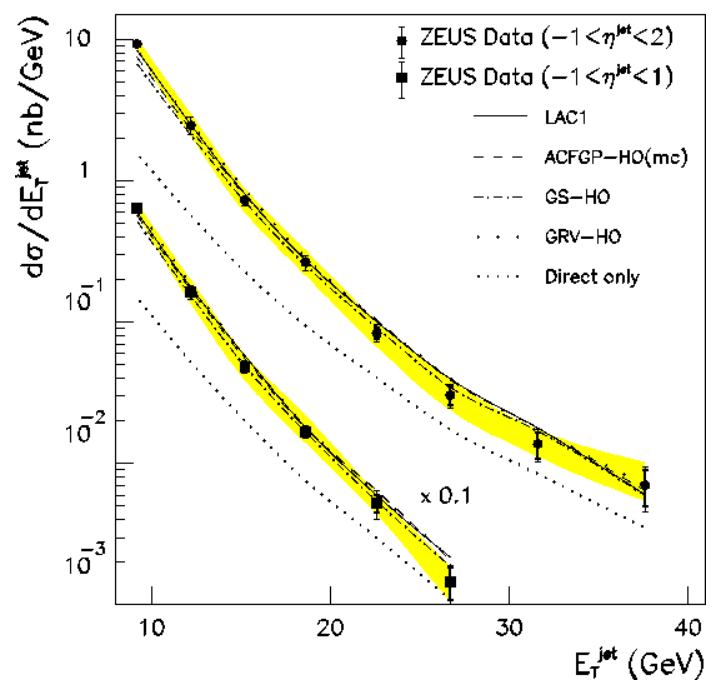

Figure 111: The $E_{T}$ distributions for jets (see text). Results of the PYTHIA simulations with the LAC1, ACFGP HO, GS HO and GRV HO parametrizations of the parton densities in the photon are shown (from [81]).
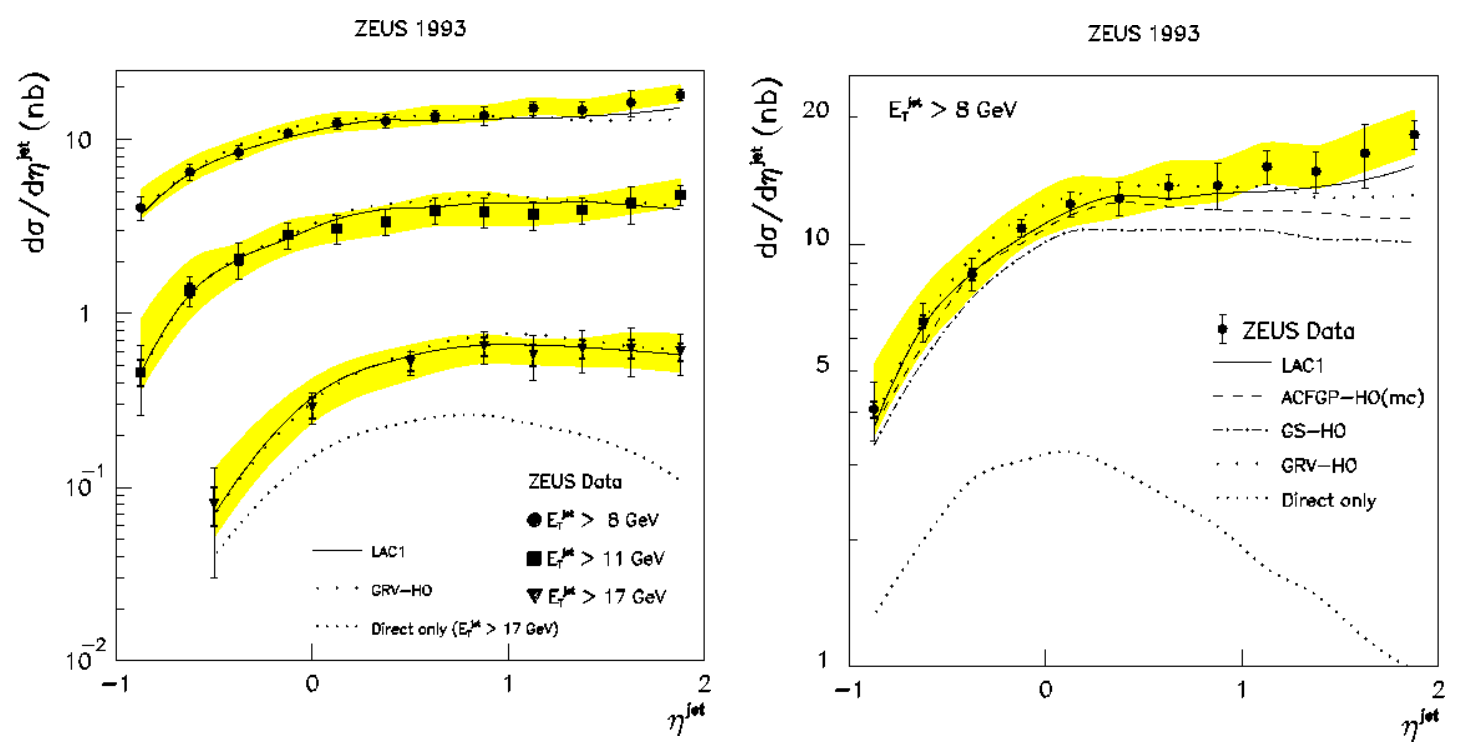

Figure 112: The jet pseudorapidity distributions: (left) integrated above three energy thresholds, $E_{T}>8,11$ and $17 \mathrm{GeV}$ (see text); the comparison with the PYTHIA simulations using the LAC1 and GRV HO parametrizations for the photon is shown; (right) the $E_{T}>8$ sample compared in addition with the ACFGP HO and GS HO parametrizations for the photon (from 81]).

Comment: "In the jet profiles, there is a significant excess of the transverse energy density in the data with respect to the Monte Carlo expectations for jets in the region $1<\eta<2$. This excess is located outside of the jet in the forward direction, i.e. $\Delta \eta>1$."

"Except for the region of very forward, low $E_{T}$ jets, these measurements are fully consistent with LO QCD in this new kinematic regime of the structure of the photon" - The result (for $d \sigma / d \eta$ with $E_{T}>8 G e V^{2}$ and $-1<\eta<1$ ) "does not support 
the discrepancy of $d \sigma / d \eta$ with respect to LO QCD calculations observed by the H1 Collaboration [H1 93]".

\section{-ZEUS 95b [82] (HERA)}

The photoproduction of dijets, with at least two jets of $E_{T}$ larger than $6 \mathrm{GeV}$, is considered in the 1993 data. Events corresponding to the scaled energy $y$ between 0.2 and 0.8 and $P^{2}$ lower than $4 \mathrm{GeV}^{2}$, with median $\sim 10^{-3} \mathrm{GeV}^{2}$ (for $|\Delta \eta|<0.5$ ) were grouped in the resolved and direct processes samples. The cone algorithm with $\mathrm{R}=1$ was used within the HERWIG 5.7 and PYTHIA 5.6 generators with the GRV LO parametrization for the photon and the MRSD_ for the proton (with $p_{T}^{\min }=2.5 \mathrm{GeV}$ ).

The $x_{\gamma}$ distribution was studied, for more recent results see ZEUS 96, 98a. The cut on the $x_{\gamma}$, equal to 0.75 , was introduced later to enhance the resolved or the direct photon contributions, and a few distributions were studied separately for these samples.

In fig. 113 the transverse energy flow around the jet axis versus $\delta \eta$ is shown, for the first time separately for the resolved photon and direct photon contributions (with the failure to describe low $x_{\gamma}$ data). $\quad$ ZEUS 1993
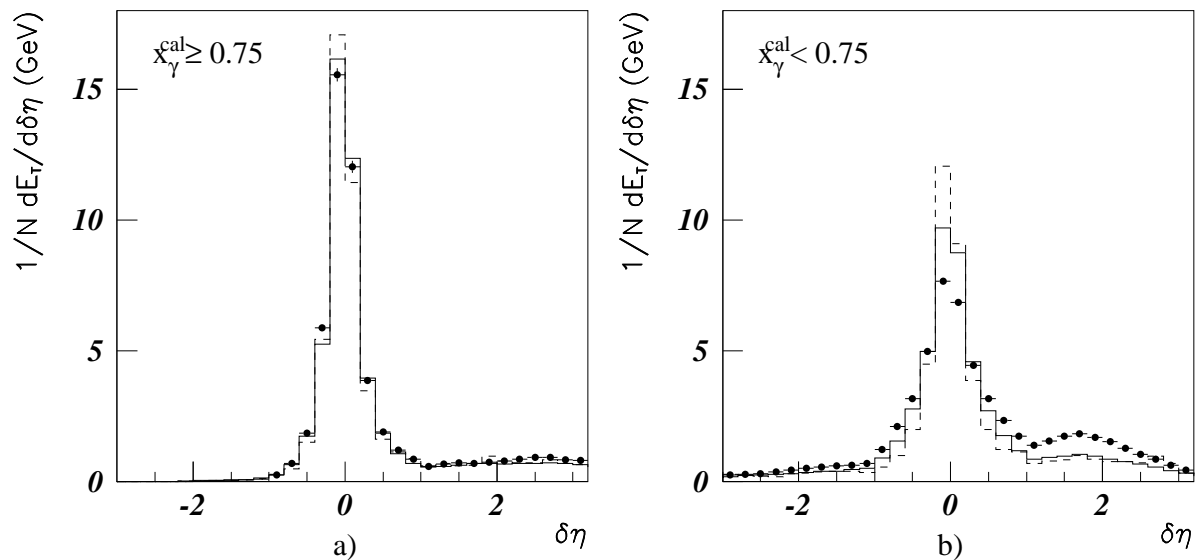

Figure 113: The transverse energy flow around the jet axis versus $\delta \eta$ : (left) $x_{\gamma}>0.75$ (direct events); (right) $x_{\gamma}<0.75$ (resolved events). The solid (dashed) line - the PYTHIA (HERWIG) simulation (from 82]).

The $d \sigma / d \bar{\eta}$ was also measured for the direct and resolved photon events (not shown, see below for comments and new data in ZEUS 96).

Comment: "Both simulations fail to describe the transverse energy flow in the forward region (see also ZEUS 95a and $\mathbf{H 1}$ 93)."

"The LO QCD predictions (with DG, GRV and GS2 parton parametrizations) lie below the dijet cross section $d \sigma / d \bar{\eta}$ data by factor 1.5-2".

The importance of the NLO calculation is stressed.

\section{•ZEUS 95c [83] (HERA)}

Photoproduction of three jets is studied for $\gamma p$ centre of mass energies in the range 130-270 GeV (1993 data). Events with two high- $p_{T}$ jets $\left(p_{T}>6 \mathrm{GeV}, \eta<1.6\right)$ were selected. A third cluster in the approximate direction of the electron beam is isolated using a $k_{T}$ clustering algorithm and identified as the photon remnant. Its properties 
(e.g. transverse and longitudinal energy flows around the axis of the cluster) were studied. The Monte Carlo PYTHIA both with default and with harder $p_{t}$ spectrum was applied (the power law with $\tilde{p}_{t 0}=0.66 \mathrm{GeV}$ ). For comparison also HERWIG was used. The GRV LO (also LAC1) and MRSD_ parton parametrizations were used for the photon and proton, respectively. The photon remnant jet was isolated for the first time.

Comment: Properties of the photon remnant jet isolated for the first time "are consistent with those commonly attributed to jets, and in particular with those found for the two jets in these events. The mean value of the photon remnant $p_{T}$ with respect to the beam axis is measured to be $2.1 \pm 0.2 \mathrm{GeV}$, which demonstrates substantial mean transverse momenta for the photon remnant."

"The leading order QCD Monte Carlo simulation, with default parameters, does not reproduce the pseudorapidity distribution or the transverse momentum distribution (with respect to the incident photon) of the photon remnant. The mean value of $p_{T}$ for the photon remnant, $2.1 \pm 0.2 \mathrm{GeV}$, is substantially larger than the Monte Carlo expectation. Better agreement can be obtained by increasing the average intrinsic transverse momenta of the partons in the photon to about 1.7 GeV."

\section{-ZEUS 96 [84] (HERA)}

Analysis of the 1994 data for dijets (in events with two or more jets) for $E_{T}$ above $6 \mathrm{GeV}$ and with the jet pair invariant mass above $23 \mathrm{GeV}$ was performed. Events correspond to the range of $y$ between 0.25 and 0.8 and $P^{2}$ below $4 \mathrm{GeV}^{2}$. The cone algorithm with $\mathrm{R}=1$ was used. The PYTHIA 5.7 and HERWIG 5.8 (with the multiple interaction) generators (with the MRSA parton parametrization for the proton and the GRV LO for the photon) were applied with the $p_{T}$ cutoff $2.5 \mathrm{GeV}$.

To obtain the scattering angle $\cos \theta^{*}$ distribution, sensitive to the parton dynamics and not parton densities as in analysis above, the cut was introduced not on $\Delta \eta$ (as in previous analysis) but on $\bar{\eta}$.

The results for the uncorrected $x_{\gamma}, x_{p}$ distributions and for the transverse energy flow as a function of $\delta \eta$ are shown in fig. 114. Both the HERWIG (with MI) and PYTHIA (without MI) describe the jet profiles data. Due to the cut on $\bar{\eta}$ the absolute value of $\eta$ is restricted to be below 1.8. Note that the applied cut on the invariant mass suppresses events with low $x_{\gamma}$. 


\section{ZEUS 1994}
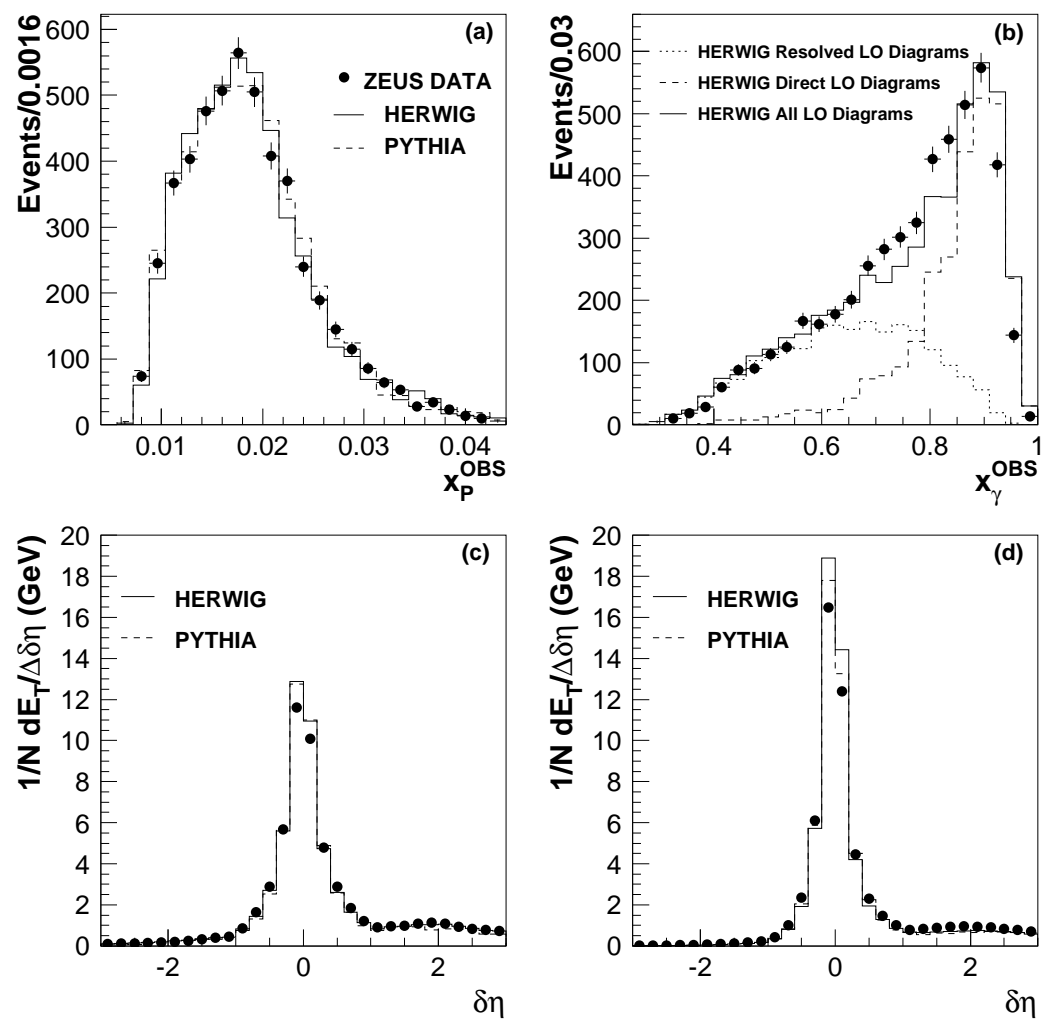

Figure 114: The uncorrected distributions in $x_{p}$ (a), $x_{\gamma}(b)$ and of the transverse energy flow $(c, d)$. The resolved $\left(x_{\gamma}<0.75\right)$ and the direct $\left(x_{\gamma}>0.75\right)$ events as a function of $\delta \eta$ are presented separately ( $c$ and $d$, respectively). The Monte Carlo (HERWIG) results are also shown (from [84]).

The important results concerning the angular distributions due to various partonic subprocesses were obtained for the first time in the large $p_{T}$ resolved photon processes. In fig. 115 the angular distributions $d \sigma / d \cos \theta^{*}$ for the resolved and direct processes together with the LO and NLO calculation based on the CTEQ3M parametrization for the proton and the GRV for the photon are presented. The comparison was also made with the HERWIG and PYTHIA simulations (not shown).

Comment: The transverse energy flow is described properly, the "requirements of high mass and small boost remove the disagreement in the forward flow between data and the simulations which has been reported elsewhere in hard photoproduction at HERA."

The dijet angular dependence is well described by the LO and NLO QCD calculations, and also by the HERWIG and PYTHIA models. 


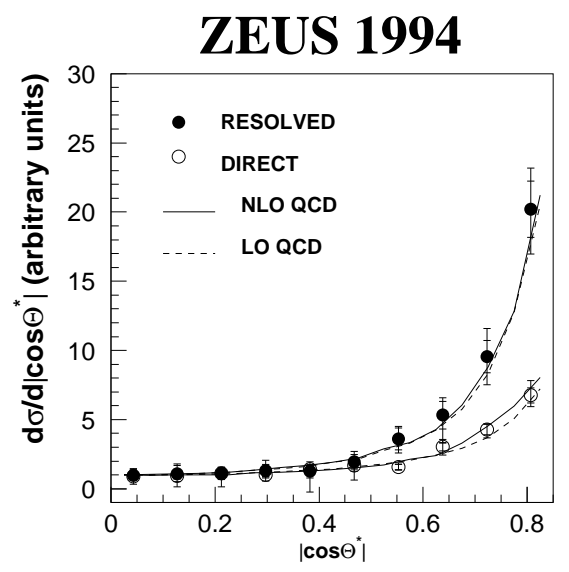

Figure 115: The dijet angular distributions for the resolved and the direct contributions (see text) (from 84]).

\section{-ZEUS 98a 85] (HERA)}

This is the extension of previous analyses (ZEUS 95b, 96) based also on the 1994 data on the dijet production with $P^{2}$ lower than $4 \mathrm{GeV}$ and $y$ between 0.2 and 0.8 . Results for the production of at least two jets with the pseudorapidity between -1.375 and 1.875 and for $E_{T}^{\min }=6,8,11$ and $15 \mathrm{GeV}$ are presented, assuming $|\Delta \eta|<0.5$. In the data analysis different jet-finding algorithms were applied: the cone algorithms EUCELL and PUCELL (both with $\mathrm{R}=1$ ) and the $k_{T}$ - cluster algorithm KTCLUS. The Monte Carlo simulation of HERWIG 5.8 and PYTHIA 5.7 with or without the multiple interaction was performed using MRSA and GRV LO parton parametrizations for the proton and the photon, respectively. The cutoff for no MI option was $p_{T}^{\text {min }}=2.5$ $\mathrm{GeV}$, while in the MI approach: $p_{T}^{m i}=2.5 \mathrm{GeV}$ (HERWIG) and $1.4 \mathrm{GeV}$ (PYTHIA). The data were compared to the predictions from a NLO QCD calculation, with the additional parameter describing the separation of jets $R_{\text {sep }}=R$ or $2 R$.

The resolved cross section was measured in the range $0.3<x_{\gamma}<0.75$ and the direct one - for $x_{\gamma}>0.75$. Analysis of the $x_{\gamma}$ distribution was performed; jet profiles in form of the transverse energy flow around the jet axis and the $d \sigma / d \bar{\eta}$ for the various jet definitions and transverse energy thresholds were studied.

The corrected $x_{\gamma}$ distribution obtained from the two-jet events indicates a need for the resolved photon contribution, see also ZEUS 95b and 96. The present analysis is based on the KTCLUS algorithm, for results see fig. 116. The small $x_{\gamma}$ region is not properly described by the Monte Carlo simulations both with and without the multiple interaction.

The transverse energy flow obtained using the KTCLUS algorithm is presented for different $E_{T}$ in fig. 117, with a similar discrepancy in comparison to MC (HERWIG, with and without MI) seen in the forward direction, as in previous measurements. 


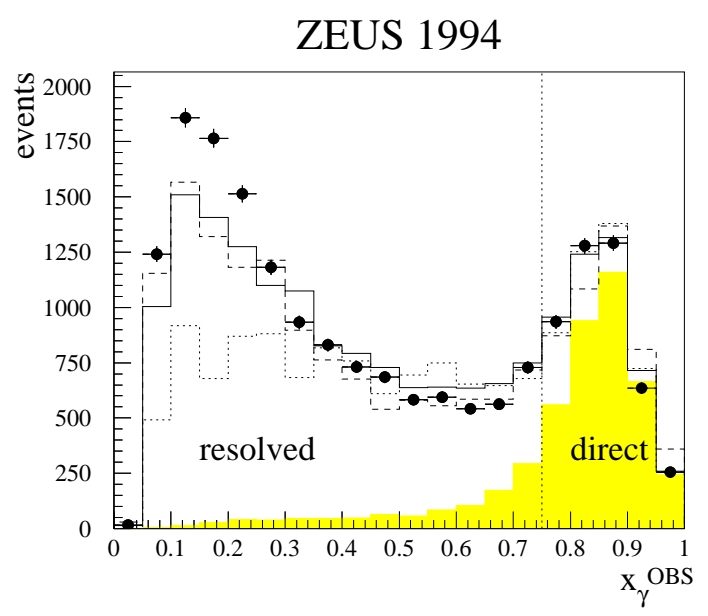

Figure 116: The corrected $x_{\gamma}^{\text {obs }}$ distribution. Solid line - HERWIG with the multiple interaction, dashed line - PYTHIA with the multiple interaction, dotted line - HERWIG without the multiple interaction (from 85]).

ZEUS 1994

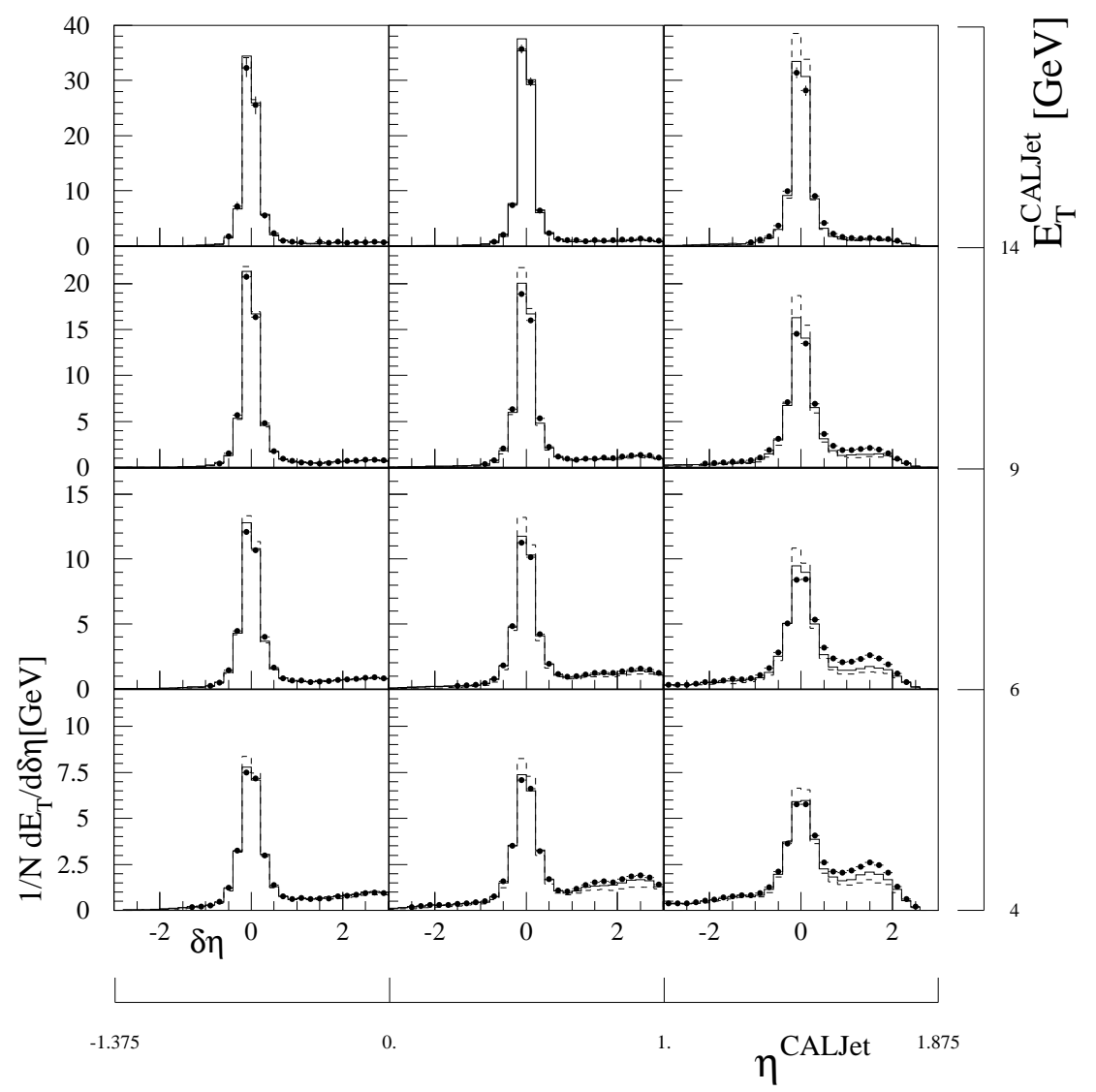

Figure 117: The uncorrected transverse energy flow around the jet. Solid line - HERWIG with the multiple interaction, dashed line - HERWIG without the multiple interaction (from [857).

The dijet cross section $d \sigma / d \bar{\eta}$ obtained under condition $|\Delta \eta|<0.5$, for $E_{T}>6 \mathrm{GeV}$ was measured and the effect of the MI was studied (not shown). This cross section was also studied for different $E_{T}$ thresholds and with different jet definitions, separately for $x_{\gamma}>$ above and below 0.75 . The results are plotted in fig. 118 (left) together with 
the predictions of the NLO QCD approach (the CTEQ3M parton parametrization for the proton and GRV HO for the photon; $\mathrm{R}_{s e p}=2$ or 1) [183].

The same cross section, now with the KTCLUS jet definition, is plotted in fig. 118 (right). The predictions of the NLO QCD approach [183 with the different parton parametrizations for the photon (GS and GRV HO) are compared with the data.

\section{ZEUS 1994}
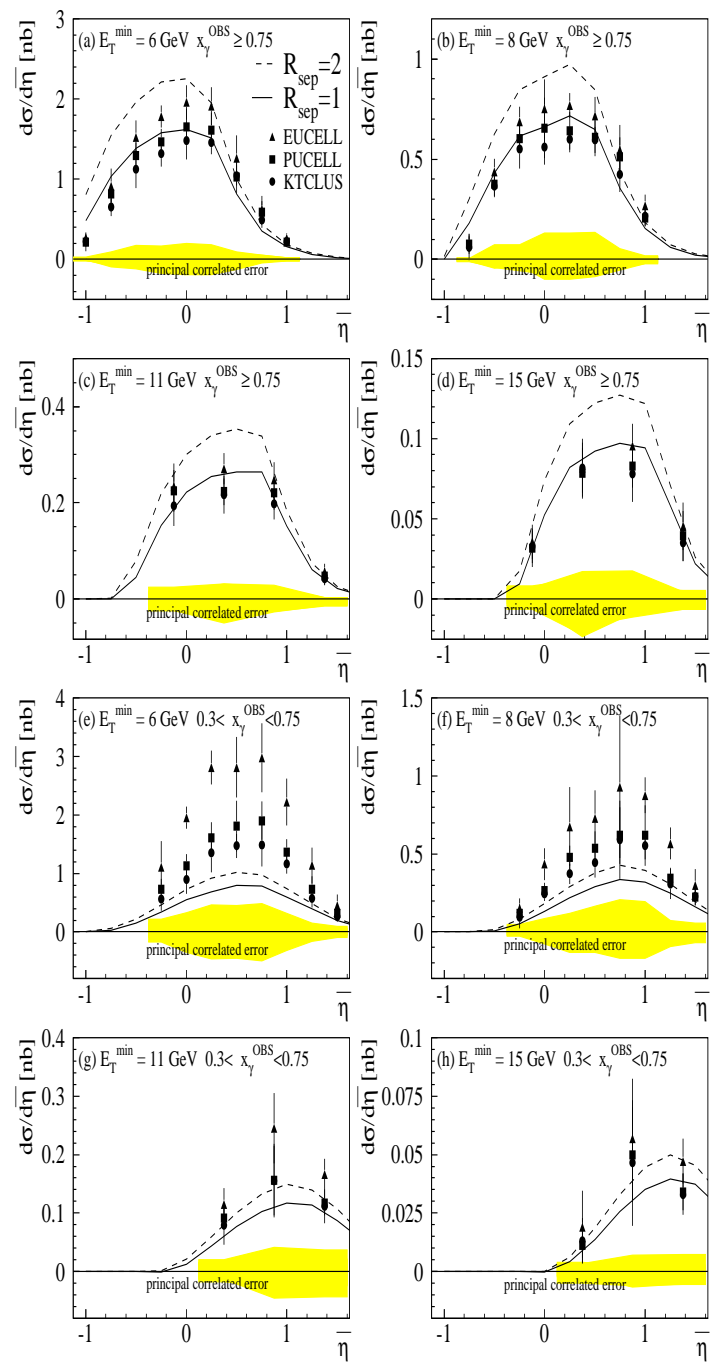

ZEUS 1994
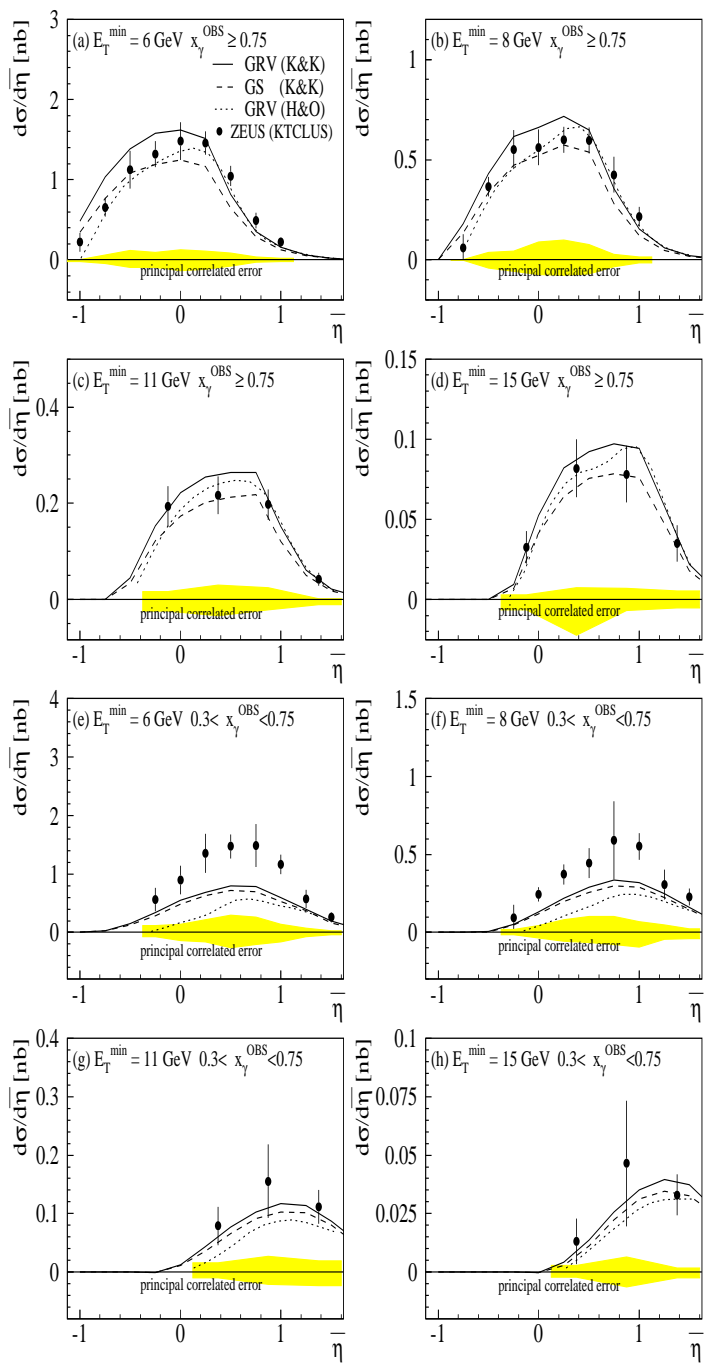

Figure 118: Left: Dijet cross sections obtained using different jet algorithms: EUCEL, PUCEL and KTCLUS. The curves show the results of the NLO calculation [183] using GS parton parametrization with $R_{\text {sep }}=1$ (solid line) and $R_{s e p}=2$ (dashed). Right: Dijet cross sections obtained using the KTCLUS jet algorithm. The curves show the results of the NLO calculations $K \& K$ [183] and $H \& O$ [186] with $R_{\text {sep }}=1$, using different NLO parton parametrizations: $G R V$ ( $K \& K$ - solid, $H \& O$ - dotted line) and $G S$ ( $K \& K$ - dotted line) (from [857).

"The difference between the parton distributions is largest in the direct photoproduction region. This is due to differences between the quark distributions in the photon for $x_{\gamma}>0.8 "$.

Comment: There is a lack of a good description of the $x_{\gamma}$ distribution for low $x_{\gamma}$. 
The discrepancy in the $\bar{\eta}$ distributions for resolved photon contributions was found for events with $E_{T}^{\text {min }}>6 \mathrm{GeV}$.

\section{-ZEUS 98b [86] (HERA)}

The inclusive single jet cross section for the $P^{2}$ below $4 \mathrm{GeV}^{2}$ with $y$ between 0.2 and 0.8 and for three energy regions for $W$ between 134 and $277 \mathrm{GeV}$ was studied. The measurement of the transverse energy of the jets bigger than $14 \mathrm{GeV}$ and the pseudorapidity range from -1 to 2 was performed. The Monte Carlo generators HERWIG 5.8 and PYTHIA 5.7 (default) $\left(p_{T}^{m i}=1 \mathrm{GeV}\right.$, and $p_{T}^{m i}$ for two partons equal to $\left.8 \mathrm{GeV}\right)$, including the multiparton interaction, were introduced (using jet cone algorithm with $\mathrm{R}=1,0.7$ and 0.5 ) in the analysis. Events were generated with the MRSA parton parametrization describing the proton structure and the GRV HO and LAC1 parton parametrizations for the photon.

Transverse energy profiles for jets with $E_{T}>14 \mathrm{GeV}$ are given for $\mathrm{R}=1.0$ and 0.7 using the PYTHIA (with and without MI), see fig. 119. The discrepancies are observed for the jets with the lowest $E_{T}$ and for $\eta>1$ for $\mathrm{R}=1$, they are reduced when $R=0.7$ is used. The multiple interaction improves the description of the data for forward jets with low $E_{T}$, but is worse in describing the smaller $\eta$ region for $\mathrm{R}=1$, and for whole $\eta$ region when $\mathrm{R}=0.7$ is used. For jets with $E_{T}>21 \mathrm{GeV}$ no significant discrepancies for jet profiles are observed between data and the Monte Carlo simulations (not shown).

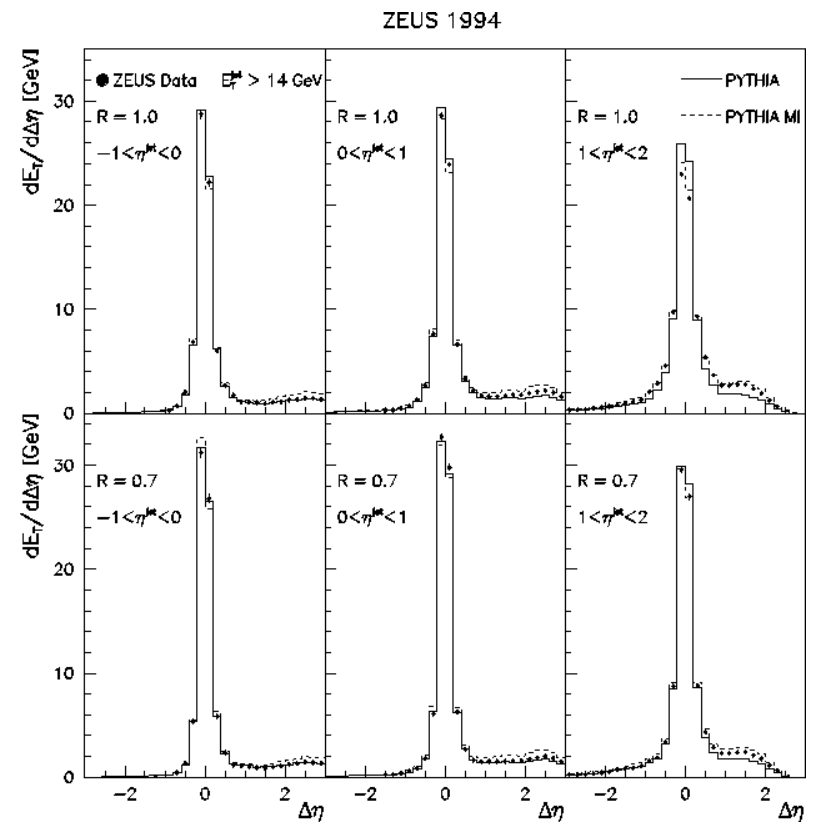

Figure 119: The uncorrected jet profiles as a function of $\delta \eta$ (integrated over $|\delta \phi|<\pi / 2$ ), for $E_{T}>14 \mathrm{GeV}$, in three $\eta$ regions, compared with PYTHIA (with and without MI) for $R=1$ and 0.7 (from [86]).

The distributions of jets with $E_{T}^{\min }=14,17,21$ and $25 \mathrm{GeV}$ as a function of $\eta$ $(-1<\eta<2)$ were measured, see for results fig. 120. They are not properly described in the forward low $E_{T}$ region by the NLO calculation [183 if $R=1$ is used (upper figures), for $R=0.7$ the agreement is obtained (lower figures). In the analysis $R_{\text {sep }}=R$ or $2 R$ was applied. 


\section{ZEUS 1994}
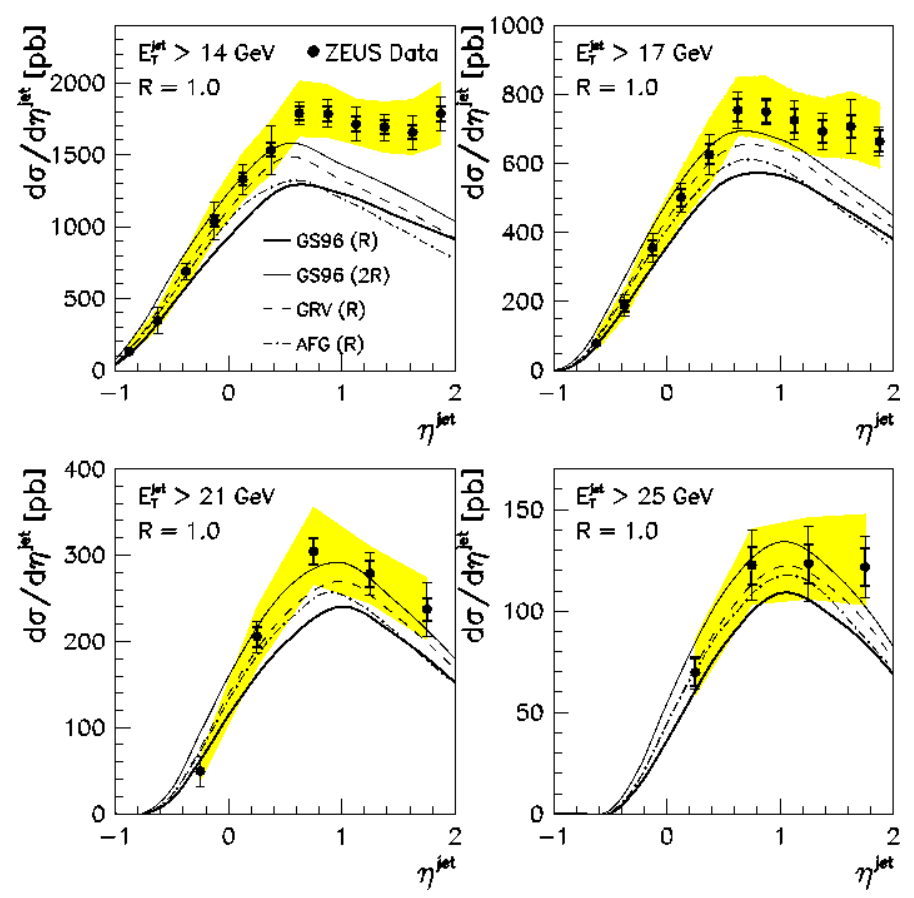

ZEUS 1994
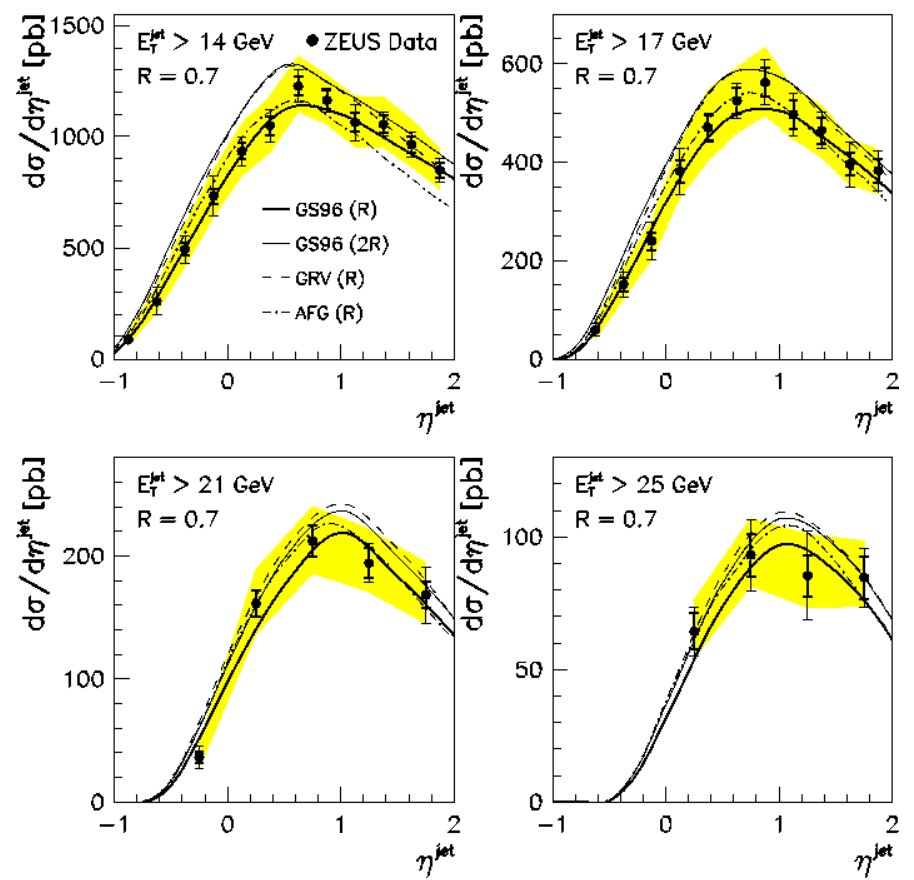

Figure 120: The differential cross section $d \sigma / d \eta$ integrated over $E_{T}$ for four thresholds: $E_{T}>14,17,21$ and $25 \mathrm{GeV}$. Curves based on the NLO calculations [183] using the GRV $H O\left(R_{\text {sep }}=R\right)$, GS HO $\left(R_{\text {sep }}=R\right.$ and $\left.2 R\right)$ and $A G F\left(R_{\text {sep }}=R\right)$ parton parametrizations for the photon and the CTEQ4M for the proton with $R=1$ (upper figures), $R=0.7$ (lower figures) are shown (from 86]).

The $\eta$ distributions in three regions of energy $W$ are not in agreement with the NLO calculation for the $R=1$, as can be seen in fig. 121. The data for $R=0.7$ (not shown) are in agreement with a QCD calculation, the same as in the previous analysis, ZEUS 98a. 


\section{ZEUS 1994}

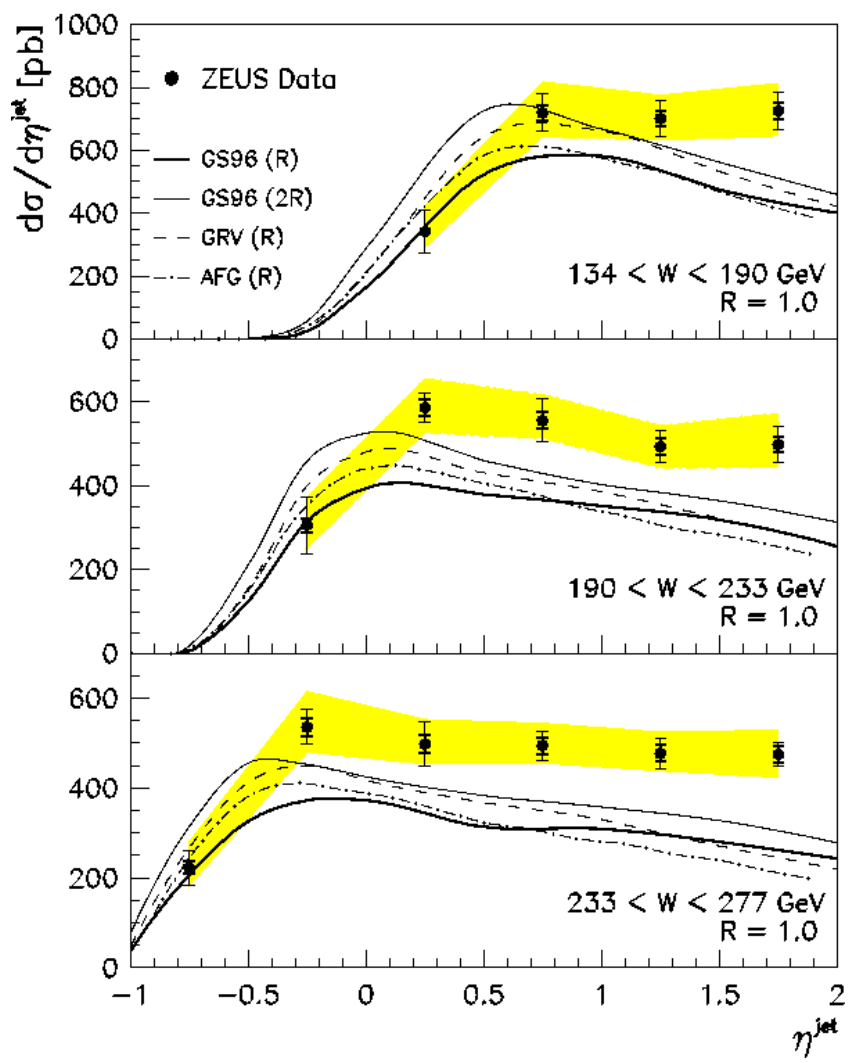

Figure 121: The differential cross section $d \sigma / d \eta$ for three regions of the energy $W$. The PYTHIA results with the MRSA for the proton and with different parton distributions in the photon (with $R_{\text {sep }}=R$ or $2 R$ ) are shown (from [86]).

The cone radius dependence of the cross section was studied as well to elucidate effects of a possible underlying event, see fig. 122 .

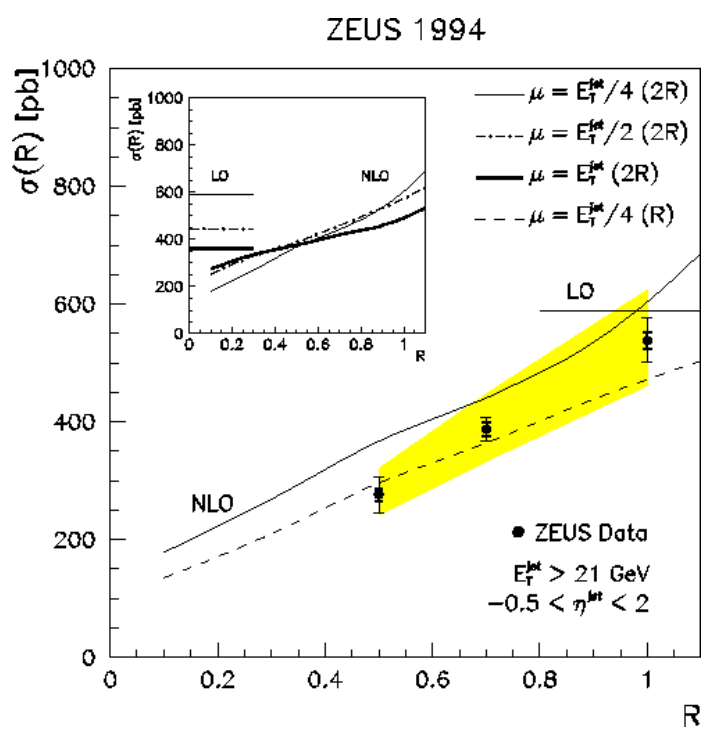

Figure 122: The cross section dependence on the $R$ parameter for $E_{T}>21 \mathrm{GeV}$ and -0.5 $<\eta<2$ obtained using various factorization scale $\tilde{Q}(=\mu)$ in the LO and NLO calculations (with the CTEQ4 and GS96 parton parametrization for the proton and photon, respectively) [183] (from [86]). 
Comment: "...the uncertainties on the jet measurements due to possible underlying event contributions become reduced at high $E_{T}\left(E_{T}>21 \mathrm{GeV}\right)$ or when using a reduced cone radius $(R=0.7)$."

"The measured cross sections for jets with $R=0.7$ are well described by the [NLO $Q C D]$ calculations in the entire measured range of $\eta$ and $E_{T}$."

\section{-ZEUS 98c,conf [87] (HERA)}

The $E_{T}$ measurement for jet photoproduction $\left(P^{2}<4 \mathrm{GeV}^{2}\right)$ at $134<W<277 \mathrm{GeV}$ is presented. Data collected in 1995-1997 lie in the large $E_{T}$ range: $17-74 \mathrm{GeV}$ and $-0.75<\eta<2.5$. Two methods of jet identification were used - with the iterative cone algorithm $(\mathrm{R}=0.7$ and 1$)$ and with the $k_{T}$ cluster algorithm. Comparison was made with the NLO calculation (with $\mathrm{R}_{s e p}=\mathrm{R}$ ), based on the CTEQ4M and the GRV HO, GS 96 and AGF parton parametrization for the proton and photon, respectively.

The $E_{T}$ distributions are presented in fig. 123 for various jet definitions: cone algorithm with $\mathrm{R}=1$ (left), cone algorithm with $\mathrm{R}=0.7$ (center) and $k_{T}$ cluster method (right). Fig. 124 shows the comparison of different ratios of $d \sigma / d E_{T}$. The cone algorithm with $\mathrm{R}=1$ and the $k_{T}$ cluster approach give similar results, larger than results obtained with $\mathrm{R}=0.7$.
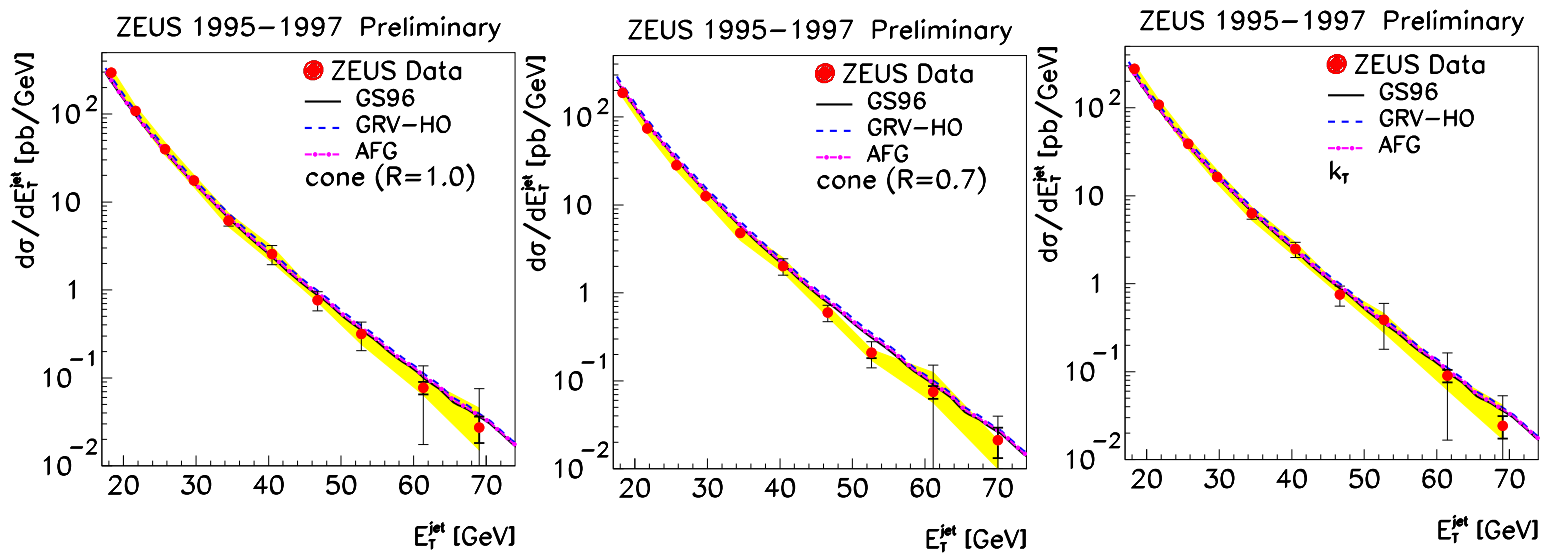

Figure 123: The $E_{T}$ distributions obtained using cone algorithm (left with $R=1$ and center with $R=0.7$ ) and the $k_{T}$ cluster method (right), compared to the NLO calculation [183] (from [87]).

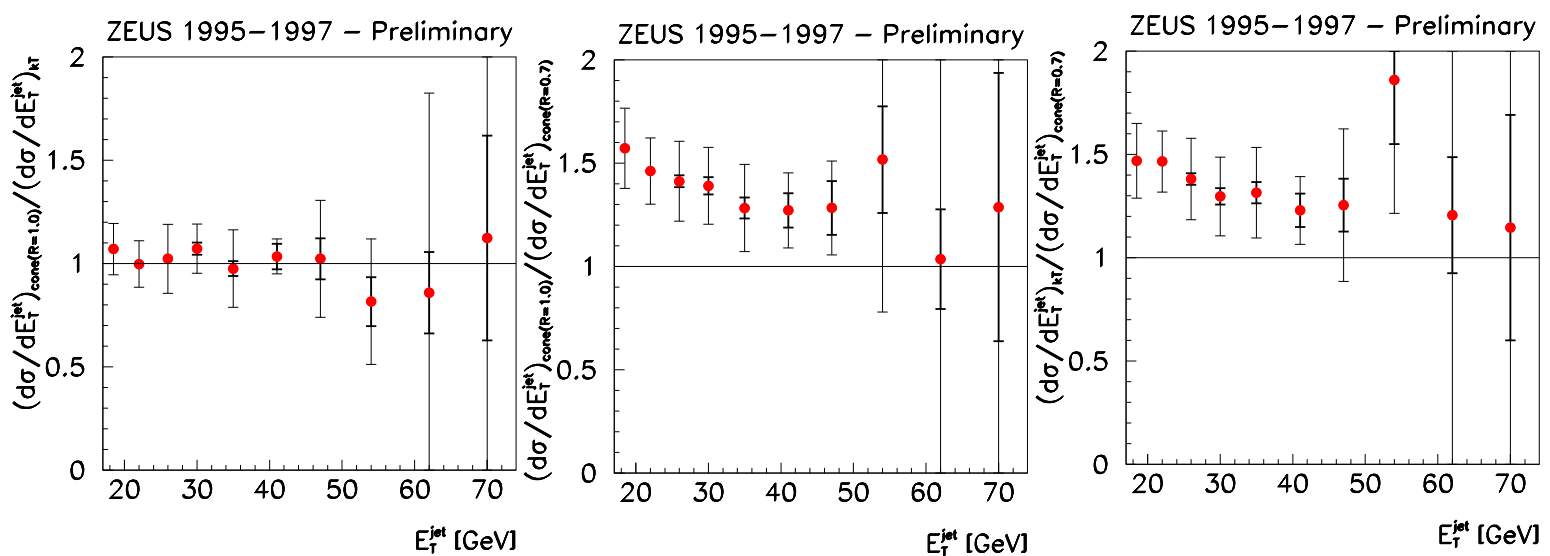

Figure 124: The ratios of the $E_{T}$ distributions obtained with the jet definitions, as used in fig. 123 (from [87]). 
The comparison with the theoretical predictions using the CTEQ4M parton parametrization for the proton and the GRV HO, GS96 and AGF densities for the photon, with $R_{\text {sep }}=R$, and different jet definitions is presented in fig. 125.
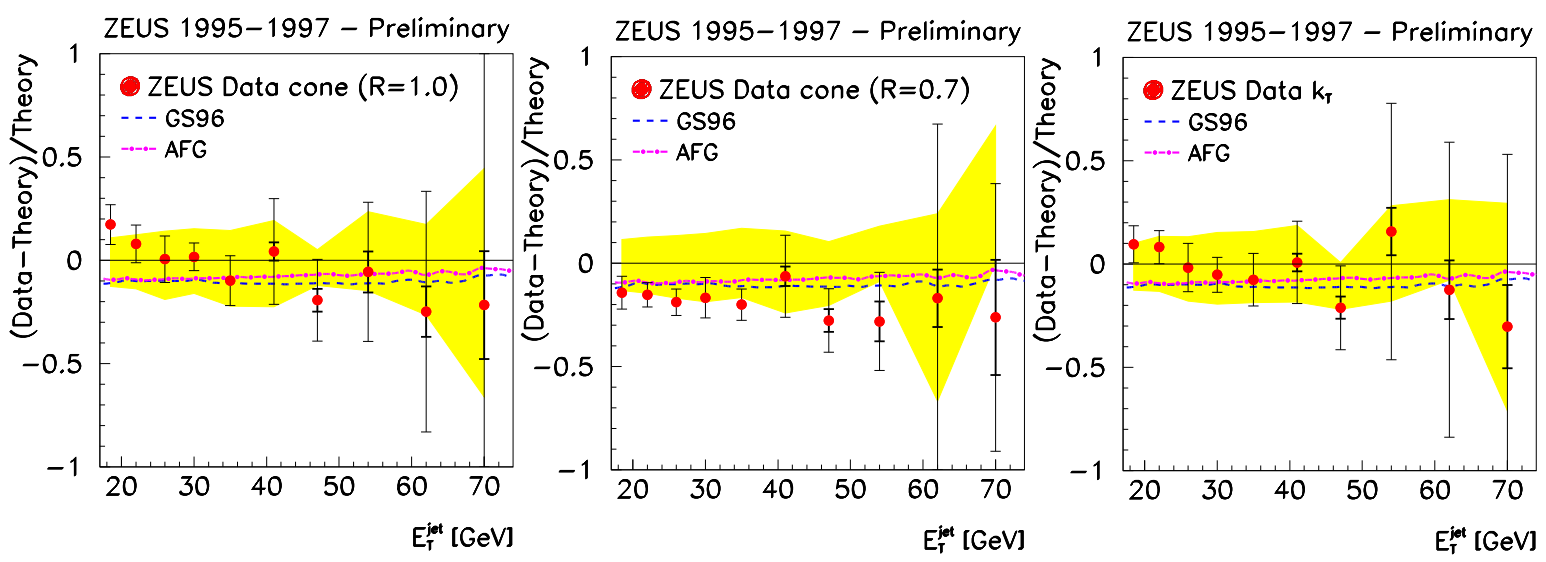

Figure 125: The fractional differences between the measured $d \sigma / d E_{T}$ for jets and the NLO calculations (using the GRV HO parametrizations - black points) with the cone algorithm (left with $R=1$, center with $R=0.7$ ) and the $k_{T}$ cluster method (right). The comparison with the NLO calculations with different parton parametrization for the photon: GS96 (dashed lines) and AGF (dash-dotted lines) is also shown (from [907).

Comment: "The NLO calculations give a reasonable description of the measured differential cross section in magnitude and shape."

\section{-ZEUS 99a [88] (HERA)}

The 1995 measurement of the photoproduction of dijets for $P^{2}<1 \mathrm{GeV}^{2}$, and $y$ between 0.2 and 0.85 , corresponding to $134<W<277 \mathrm{GeV}$ was performed, also a narrower $y$ range 0.50 to 0.85 was studied. The aim was to constrain the parton densities in photon at high $x_{\gamma}$. Events were separated in to the direct sample $\left(x_{\gamma}>\right.$ $0.75)$ and the resolved photon sample $\left(x_{\gamma}<0.75\right)$. The asymmetric cut is applied on the two highest transverse energy jets. The cross section is symmetrized in the pseudorapidities of these jets. Events correspond to the highest $E_{T}$ jets with the threshold $E_{\text {Tleading }}=14 \mathrm{GeV}$ and for the second jet the threshold is given by $E_{\text {Tsecond }}=11$ $\mathrm{GeV}$. The rapidity range $-1<\eta<2$ is considered. Two Monte Carlo generators were used: HERWIG 5.9 and PYTHIA 5.7 with the $k_{T}$ clustering method for the jet identification.

The uncorrected $x_{\gamma}$ distribution is shown in fig. 126, together with Monte Carlo predictions for a direct and the full (direct+resolved) contributions. 


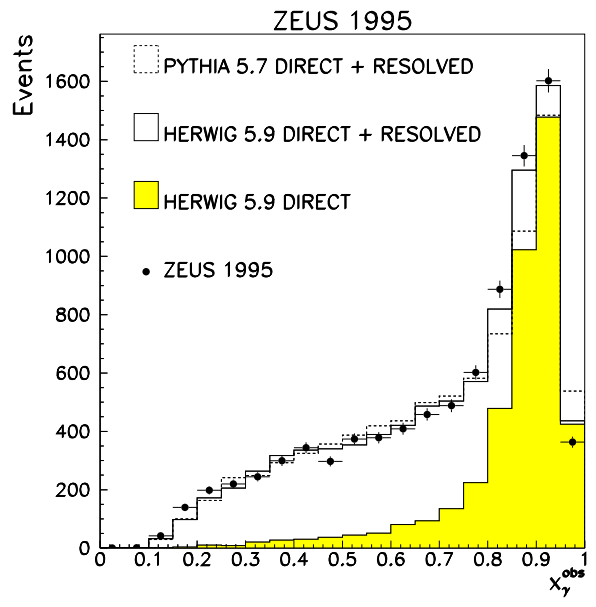

Figure 126: The uncorrected $x_{\gamma}$ distribution for the direct (defined as $x_{\gamma}>0.75$ ) and the full (direct+resolved) contributions obtained in the PYTHIA 5.7 and HERWIG 5.9 generators for $E_{T}>14 \mathrm{GeV}$ (from [88]).

The energy flow around the jet was studied for different regions of $x_{\gamma}$ and for different $E_{T, \text { leading }}$ thresholds, see fig. 127.

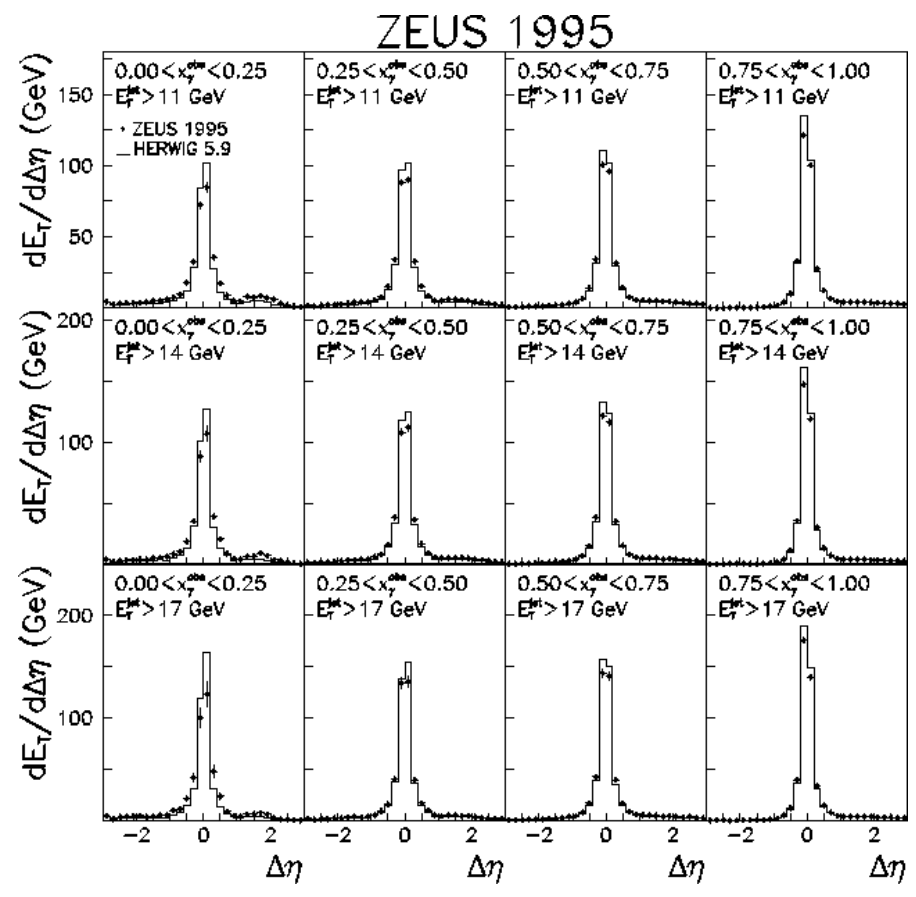

Figure 127: The transverse energy flow around the jet axis (integrated over $|\delta \phi|<1$ ), in different ranges of transverse energy and $x_{\gamma}$ (from [88]).

Below the results for the $E_{T}$ distribution in different regions of $\eta_{1,2}$ are presented (fig. 128), with the direct contributions displayed separately. Comparison with the NLO calculation (with CTEQ4M and the GRV HO, GS96 HO, AGF HO parton 
parametrizations for the proton and photon, respectively) is shown.
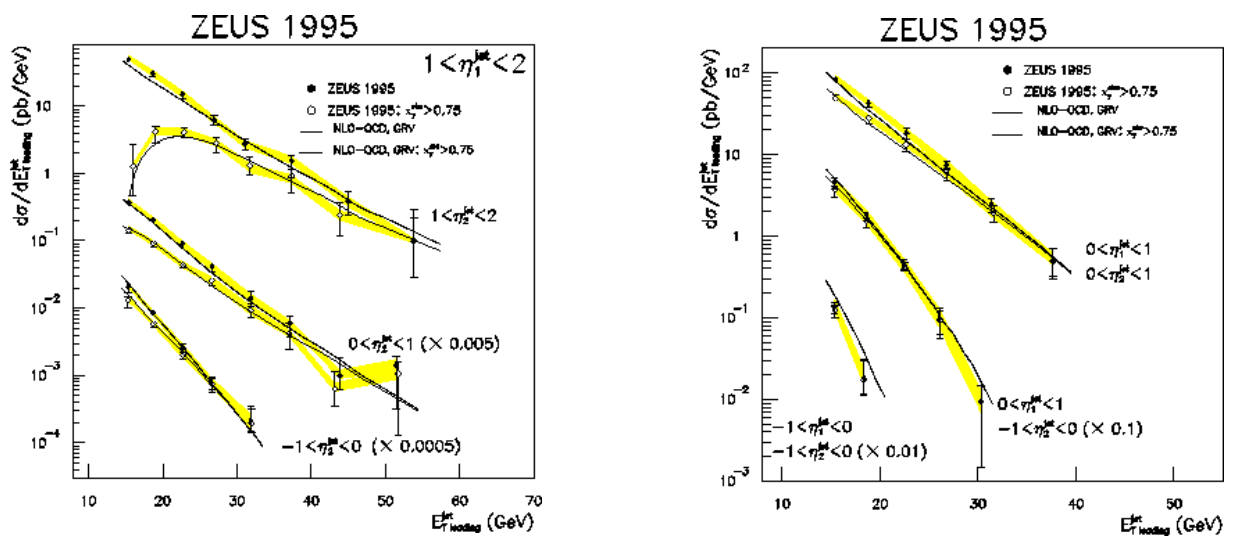

Figure 128: Left: Dijet cross section $d \sigma / d E_{\text {Tleading }}$ with one jet within the rapidity range $1<\eta_{1}<2$ and the other jet in three rapidity ranges, described in the figure. Right: Dijet cross section $d \sigma / d E_{T}$ with one jet within rapidity range $0<\eta_{1}<1$ and the other jet in three rapidity ranges, described in the figure. Comparison with the NLO calculation (with GRV HO) [183] - thick line (thin line shows the direct contribution) (from 88]).

For the leading jet, with $E_{\text {Tleading }}>14 \mathrm{GeV}$, the rapidity distribution $d \sigma / d \eta_{2}$ was measured as well. It was done for a full $y$ region measured in this experiment and, to enhance the sensitivity to the choice of parton density in the photon, for a large $y$ region (i.e. $0.5<y<0.85$ corresponding to $212<W<277 \mathrm{GeV}^{2}$ ). Three $\eta_{1}$ jet rapidity ranges were studied, see fig. 129 for results. Here the comparison was made with the NLO calculation [183] with the GRV HO, AGF HO and GS96 HO parton parametrization for the photon (the CTEQ4M parametrization for the proton). Also the comparison between the different NLO calculations ([176, 188, 185, 186, 187]) is made using the particular (GRV-HO) parton parametrization. 

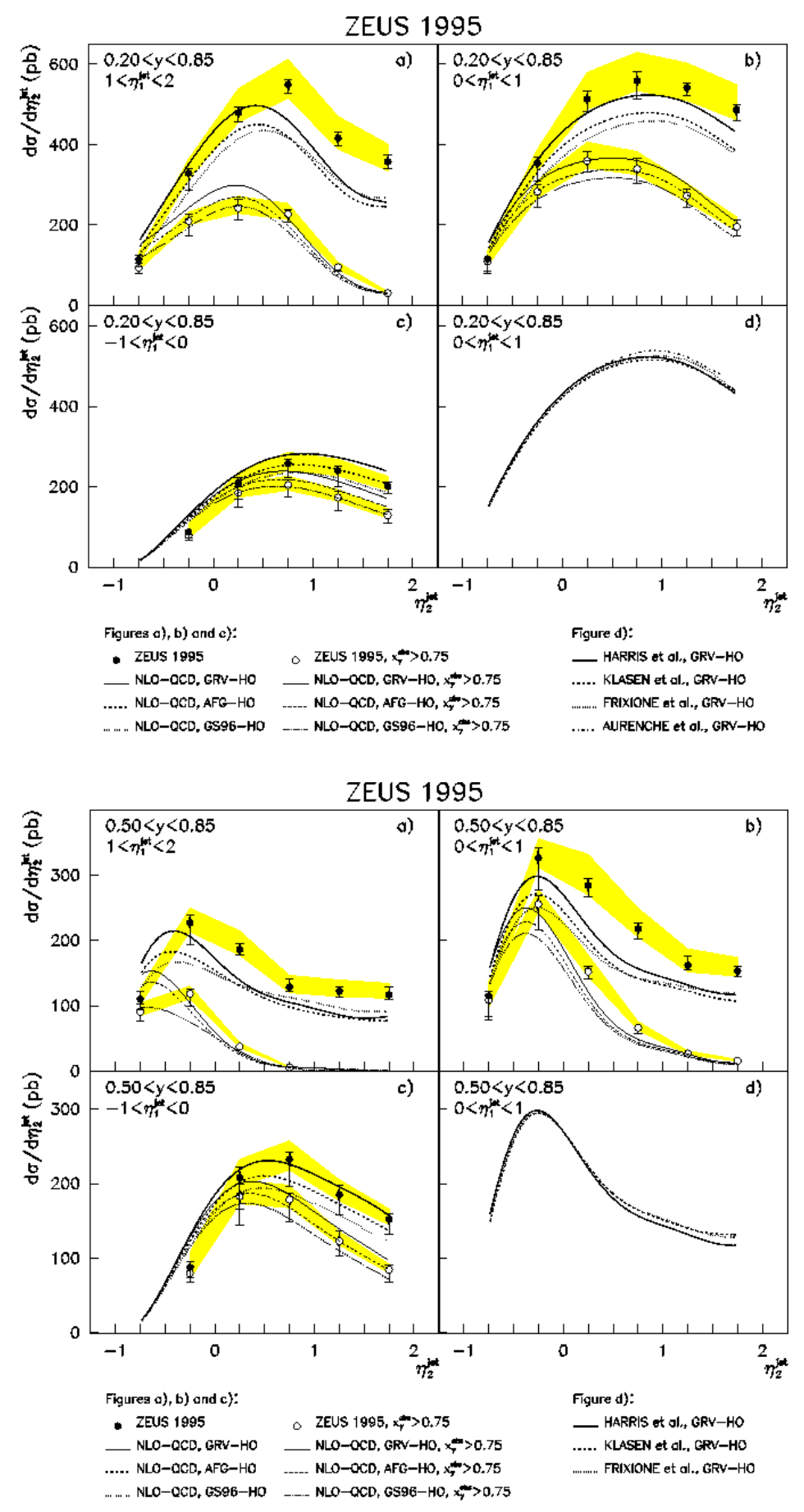

Figure 129: The rapidity distribution $d \sigma / d \eta_{2}$ for $0.2<y<0.85$ (upper set of figures) and $0.5<y<0.85$ (lower set of figures). The extra cut for the leading jet, $E_{\text {Tleading }}>14 \mathrm{GeV}$, was applied. Three $\eta_{1}$ jet rapidity ranges were considered. The comparison with the NLO calculation with different parton parametrization for the photon is made in a), b) and c). In d) comparison of different $N L O$ calculations with the particular parton parametrization for the photon (GRV-HO) is shown, see text (from [88]).

Comment: The underlying events "play no role in the present kinematic regime." For the full and for the higher energy range NLO QCD calculations using the GRV 
HO, AGF HO and GS96 HO parametrizations for the parton densities in the photon can describe properly the distribution in $E_{\text {Tlaeding. }}$ However "at central and forward pseudorapidities, both for the full and for the high $x_{\gamma}$ range, the data lie above the $N L O$ calculations". It "suggests that in this kinematic region the parton densities in the photon are too small in the available parametrizations". "This region has not been studied in $F_{2}^{\gamma}$ measurements."

\section{-ZEUS 99b,conf 89 (HERA)}

This is the continuation of the previous analysis of the dijet events for $E_{T}$ up to 75 $\mathrm{GeV}$. The data were collected in 1996-1997 with the kinematical cuts as in ZEUS 99a. The $k_{T}$ clustering algorithm was used for jet identification. The thresholds on $E_{\text {Tleading }}$ from 14 to $55 \mathrm{GeV}$ was introduced.

The $x_{\gamma}$ distributions for different transverse energy thresholds are presented in fig. 130; they are well described by Monte Carlo generators (also the transverse energy flows, not shown).

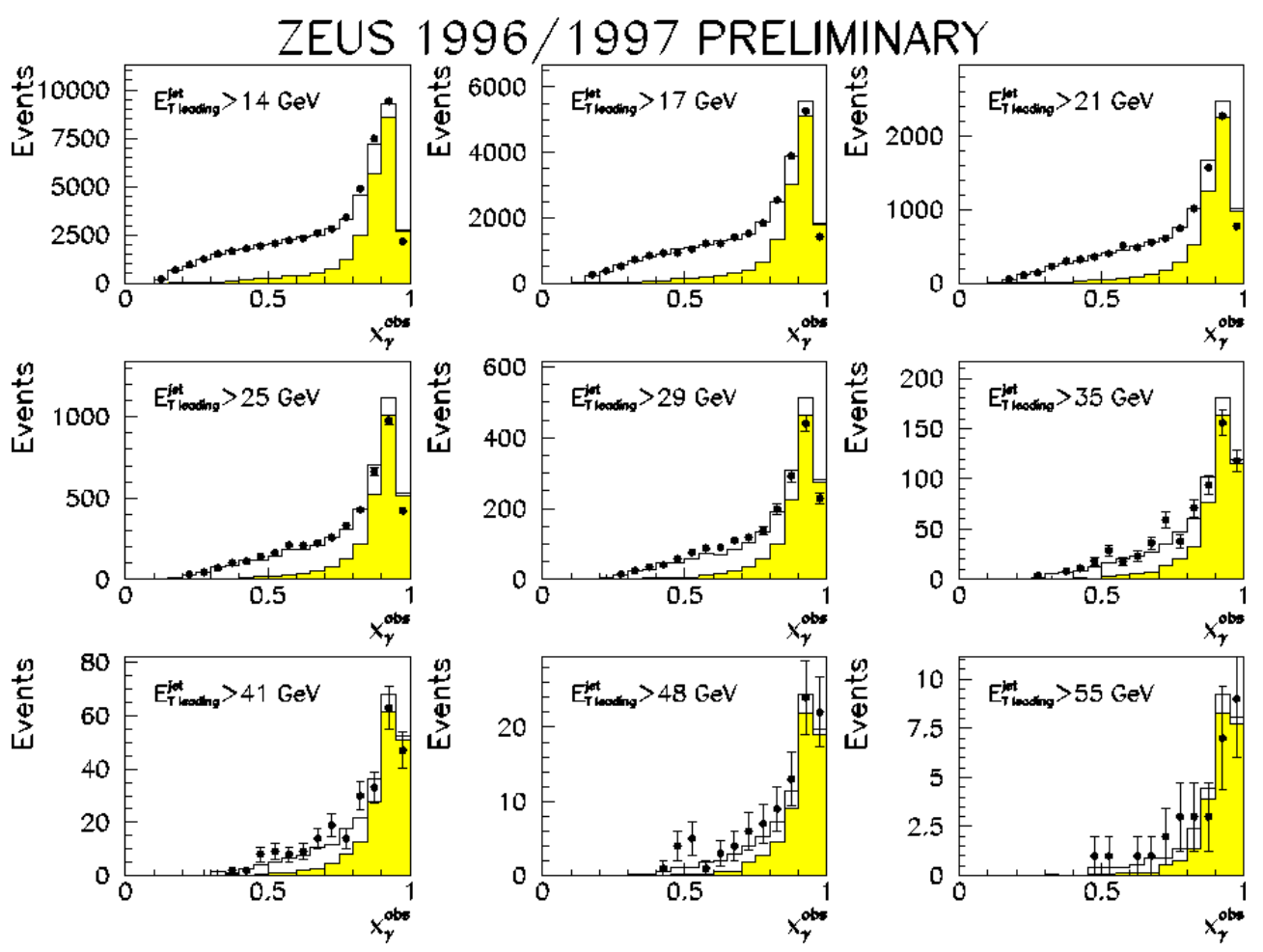

Figure 130: The $x_{\gamma}$ distribution (with the statistical errors only) for different thresholds on the highest transverse energy of the leading jet. The second one has $E_{T s e c o n d}>11 \mathrm{GeV}$. The solid histograms represent predictions of HERWIG 5.9, the shaded ones represent the direct contributions (from 897)).

Both the transverse energy distribution for $E_{\text {Tleading }}$ and the rapidity distribution were studied. Below, the results for the $E_{\text {Tleading }}$ distribution in different regions of $\eta_{1,2}$ are presented (fig. 131). The direct (defined as $x_{\gamma}>0.75$ ) contributions are displayed separately. The results for the rapidity distributions for various thresholds on $E_{\text {Tleading }}$ from 14 to $29 \mathrm{GeV}$ are shown in fig. 131, and in fig. 132 for a narrower high $y$ region. The data are compared with the NLO calculation using CTEQ4M (proton) and AGF $\mathrm{HO}$ (photon) parton parametrizations, and some discrepancies with data are found. 

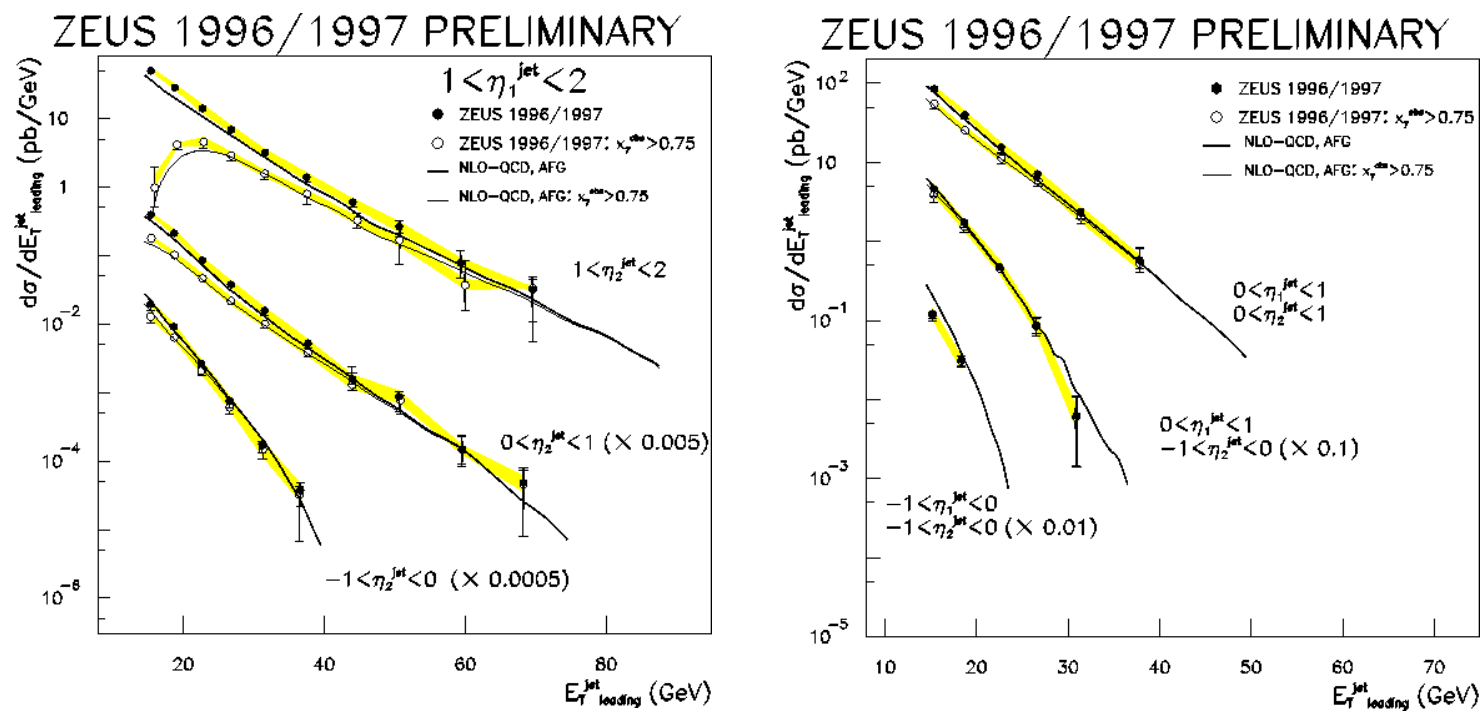

Figure 131: Left: Dijet cross section $d \sigma / d E_{\text {Tleading }}$ with one jet within the rapidity range $1<\eta_{1}<2$ and the other jet in three rapidity ranges, described in the figure. Right: Dijet cross section $d \sigma / d E_{\text {Tleading }}$ with one jet within rapidity range $0<\eta_{1}<1$ and the other jet in three rapidity ranges, described in the figure. Comparison with the NLO calculation [183] with the AGF HO parametrization for the photon and the CTEQ4M parametrization for the proton - solid line (dashed line shows the direct contribution) (from 889]).

Comment: These results confirm those in ZEUS 99a.

"...the measured cross sections remain higher than the NLO QCD predictions when the transverse energy threshold on the leading jet is raised up to 29 GeV" suggesting "that, in the kinematic region of the measurement presented here [high $x_{\gamma}$ and high $\left.E_{T}\right]$, the available parametrizations of the parton densities in the photon are too small." 


\section{ZEUS 1996/1997 PRELIMINARY}
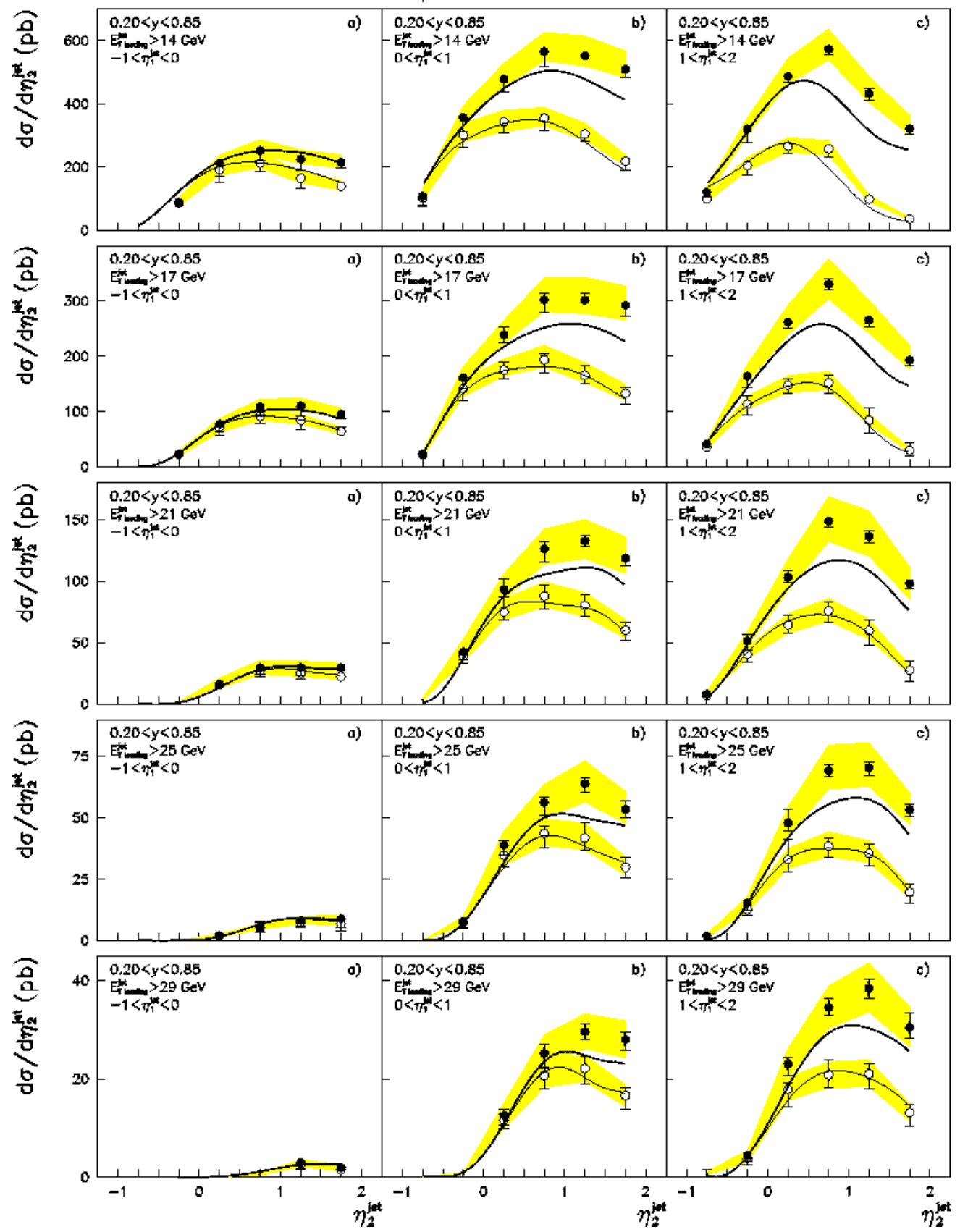

Figure 132: The rapidity distribution $d \sigma / d \eta_{2}$ for $0.2<y<0.85$. Five different cuts for the leading jet $E_{\text {Tleading }}$ were applied from 14 to $29 \mathrm{GeV}$. Three $\eta_{1}$ jet rapidity ranges were considered in a), b) and c), with the AGF HO parametrization for the photon and the CTEQ4M parametrization for the proton - solid line (dashed line shows the direct contribution). The particular parton parametrization for the photon (AGF HO) was used (from 89]). 
ZEUS 1996/1997 PRELIMINARY
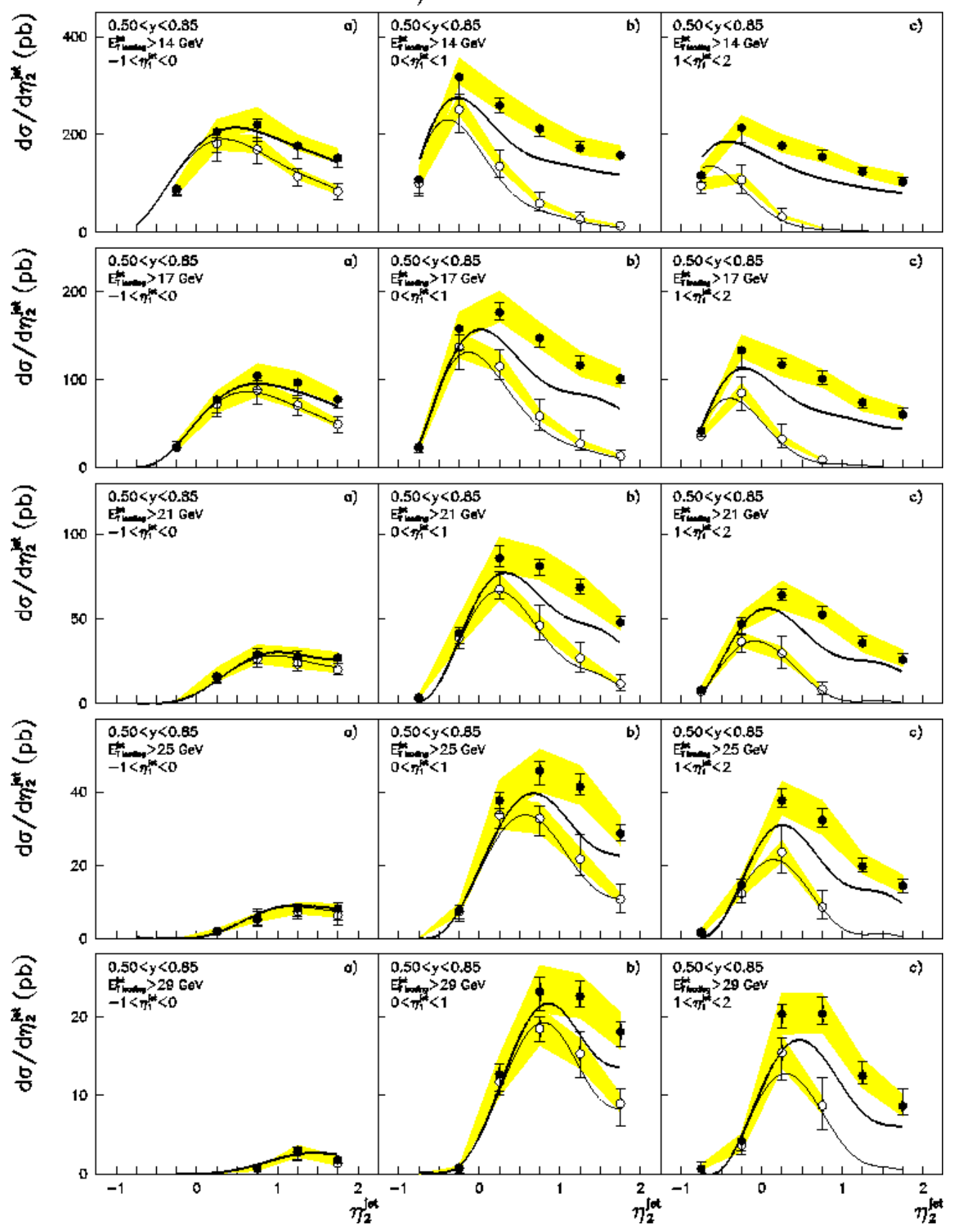

Figure 133: The rapidity distribution $d \sigma / d \eta_{2}$ for $0.5<y<0.85$, see fig. 132 (from [89]).

\section{•ZEUS 98d,conf 90] (HERA)}

The high mass $\left(M_{j j}>47 \mathrm{GeV}\right.$, up to $\left.140 \mathrm{GeV}\right)$ dijet cross section was measured for $134<W<277 \mathrm{GeV}$ (the 1995-1997 sample). Events with $P^{2}$ smaller than 4 $\mathrm{GeV}^{2}$ were collected and compared with the same NLO calculation 183 as in ZEUS 99b,conf. In the present analysis the cone algorithm with $R=1$ was used as well as $k_{T}$ algorithm.

Results for the $\cos \theta^{*}$ distribution obtained within these two-jet algorithms are presented in fig. 134. In the fig. 135 the invariant mass distribution (integrated over the $\left.\left|\cos \theta^{*}\right|<0.8\right)$ is shown. 

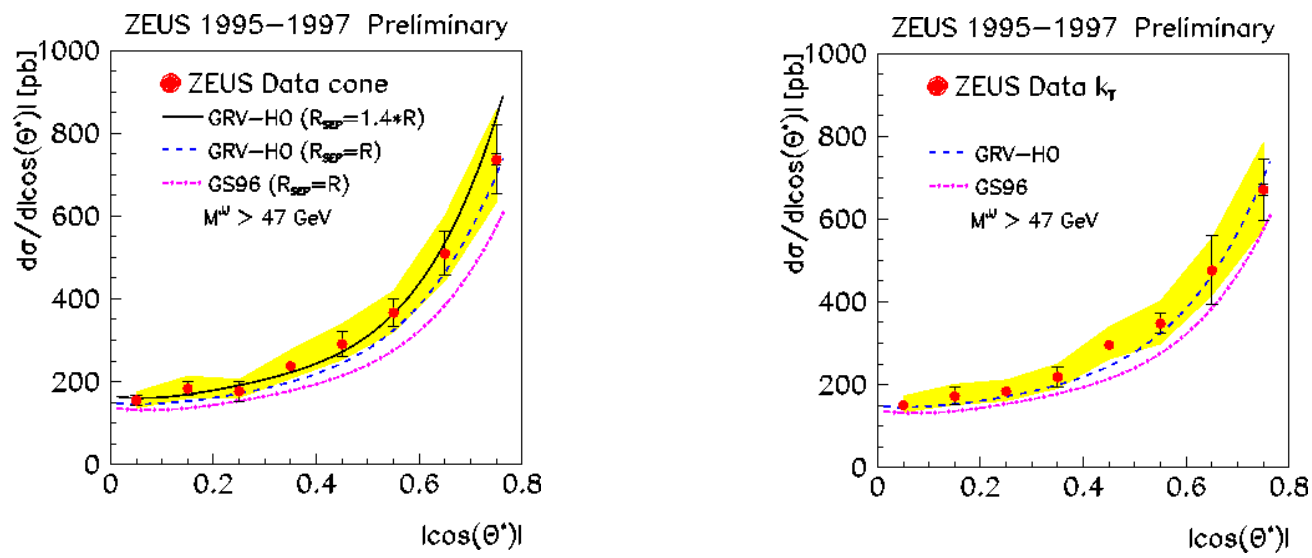

Figure 134: The $\cos \theta^{*}$ distribution for events with $M_{j j}$ above $47 \mathrm{GeV}$ obtained using twojet algorithms: the cone algorithm (left), the $k_{T}$ clustering (right). The comparison with the NLO calculation using the GRV HO parton densities (with $R_{\text {sep }}=R, 1.4 R$ for the cone algorithm) and GS96 ( $R_{\text {sep }}=R$ for the cone algorithm) is made (from 907).
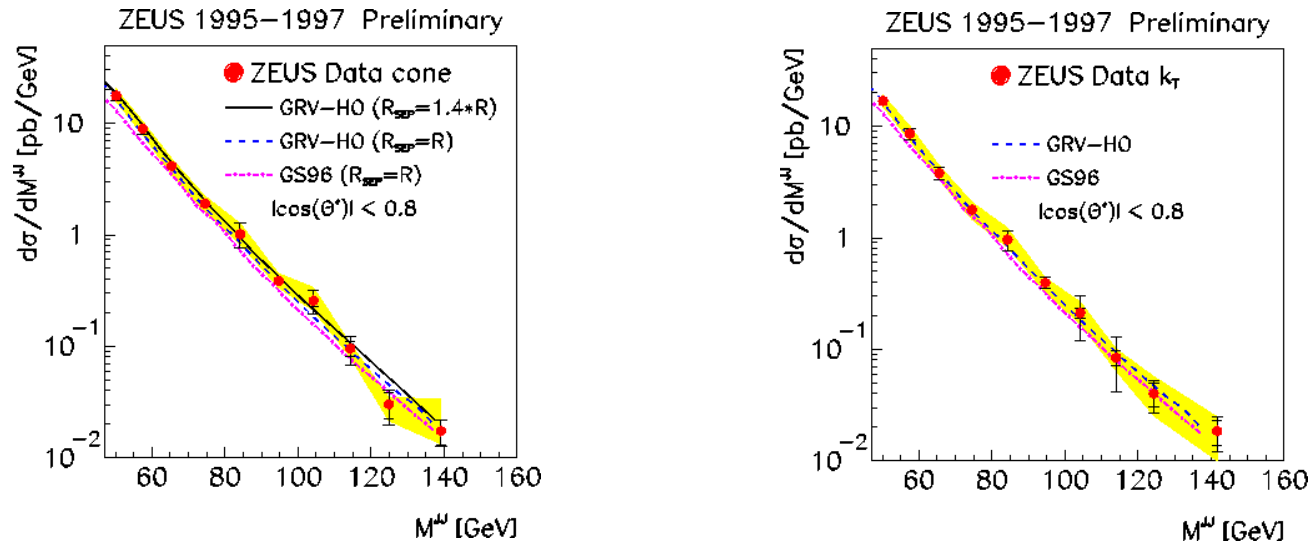

Figure 135: The $M_{j j}$ distribution for events with $\left|\cos \theta^{*}\right|<0.8$ obtained using two-jet algorithms: the cone algorithm (left), the $k_{T}$ clustering (right). The comparison with the NLO calculation as in fig. 134 (from 907).

Comment: "NLO QCD calculations account reasonably well for the shape and magnitude of the measured $d \sigma / d \cos \theta^{*}$ and $d \sigma / d M_{J J}$ ". 


\subsubsection{Prompt photon production in the resolved $\gamma p$ collision}

The prompt photons. i.e. large- $p_{T}$ photons (predominantly direct), produced in the $\gamma p$ collision 20 give an additional information as compared to jet(s) production discussed in the previous sections. Although the corresponding rate is much smaller $\left(\sim \alpha^{2}\right)$, the advantage is the possible direct access to the hard subprocess. However, there are also indirect contributions due to the quark and gluon fragmentation into photon [189. In practice the isolated photon production is considered. It corresponds to the restriction of the hadronic production around the photon direction. Usually the cone algorithm, with $\mathrm{R}$ defined as for the jets, is used with a limit on the allowed hadronic energy $\left(\right.$ e.g. $\left.\sum E^{h}<0.1 E^{\gamma}\right)$ within the cone [190].

The photoproduction of a hard direct photon may occur due to the direct interaction of the initial $\gamma$, or due to the resolved initial $\gamma$. The sensitivity to the particular subprocesses and to the form of parton density in the photon differs in such processes from that in the jet production, discussed in secs. 2.4.3 and 2.4.4. The domination of one contribution due to the gluonic content of the initial photon leading to the production of the forward, very energetic photons was pointed out in 189]g. This can be particularly useful in extraction of the gluonic content of the real (and also slightly virtual photon) 191. Also there is a chance to test a special photon-gluon fusion process, namely the $\gamma g \rightarrow \gamma g$ box diagram contribution.

All these make prompt photon processes a source of valuable independent information on the photon-hadron interaction at high energies. New data which appeared recently (ZEUS 2000a) can be compared to the two versions of the NLO calculations 192. The agreement of the MC models and the NLO calculation with data is reasonably good for rapidities of the final photon in the range $0.1<\eta<0.9$, while for $-0.7<\eta<0.1$ the excess of the data over the predictions is seen.

\section{DATA}

\section{•H1 97a,conf [91] (HERA)}

The measurement of the high $E_{T}$ photons $\left(E_{T}^{\gamma}>5 \mathrm{GeV}\right.$ and $\left.-1.2<\eta_{\gamma}<1.6\right)$ in quasi-real $\gamma p$ collisions was performed, based on 1996 data. The isolation of the photon within a cone with $\mathrm{R}=0.8$ around the photon direction was imposed. The total hadronic energy in this cone was restricted to be not higher than $10 \%$ of the photon energy. The balancing hadronic jet with transverse energy $E_{T}$ was also measured. The PYTHIA generator with the GRV LO parton densities in the proton and in the photon was used. The results for the transverse energy and for the pseudorapidity of the final photon distributions are presented in fig. 136.

\footnotetext{
${ }^{26}$ called also the deep inelastic Compton (DIC) process, as it corresponds to the $\gamma q \rightarrow \gamma q$ subprocess in the Born approximation
} 
H1 preliminary
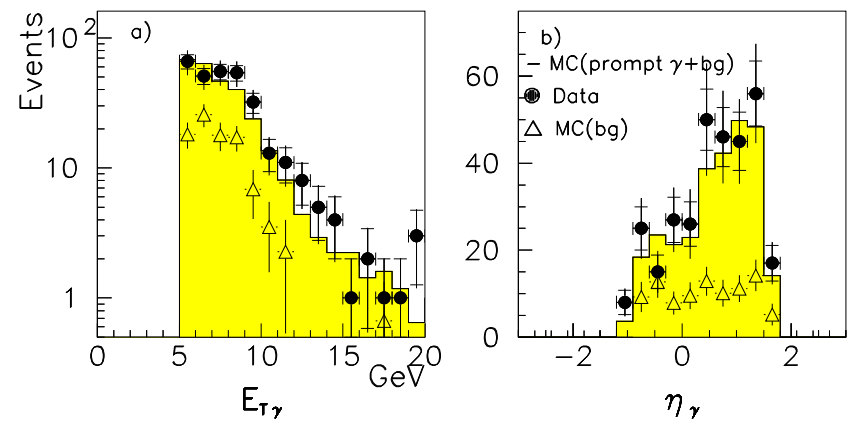

Figure 136: Transverse energy $E_{T}^{\gamma}$ (a) and the pseudorapidity $\eta^{\gamma}$ (b) distribution of the produced photon (from [91]).

The number of events as a function of the azimuthal angle between the final photon and the jet $\left(\Delta \phi_{(\gamma, j e t)}\right)$ and the transverse energy of the accompanying jet are presented in fig. 137.
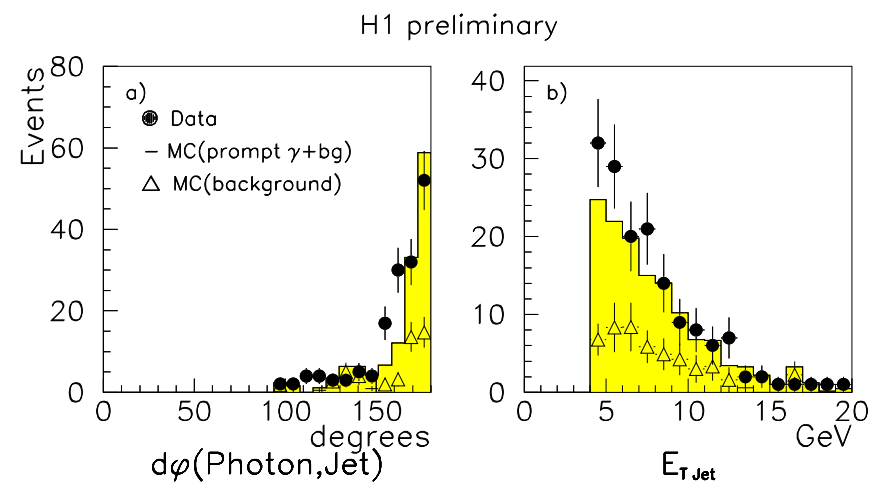

Figure 137: a) The angle between the final photon and the jet, $\Delta \phi_{(\gamma, j e t)}$, distribution, b) the transverse energy of the accompanying jet distribution (from [91]).

The measurement of the photon and the jet allows to reconstruct the $x_{\gamma}$ and $x_{p}$ variables. Their distributions are shown in fig. 138. 

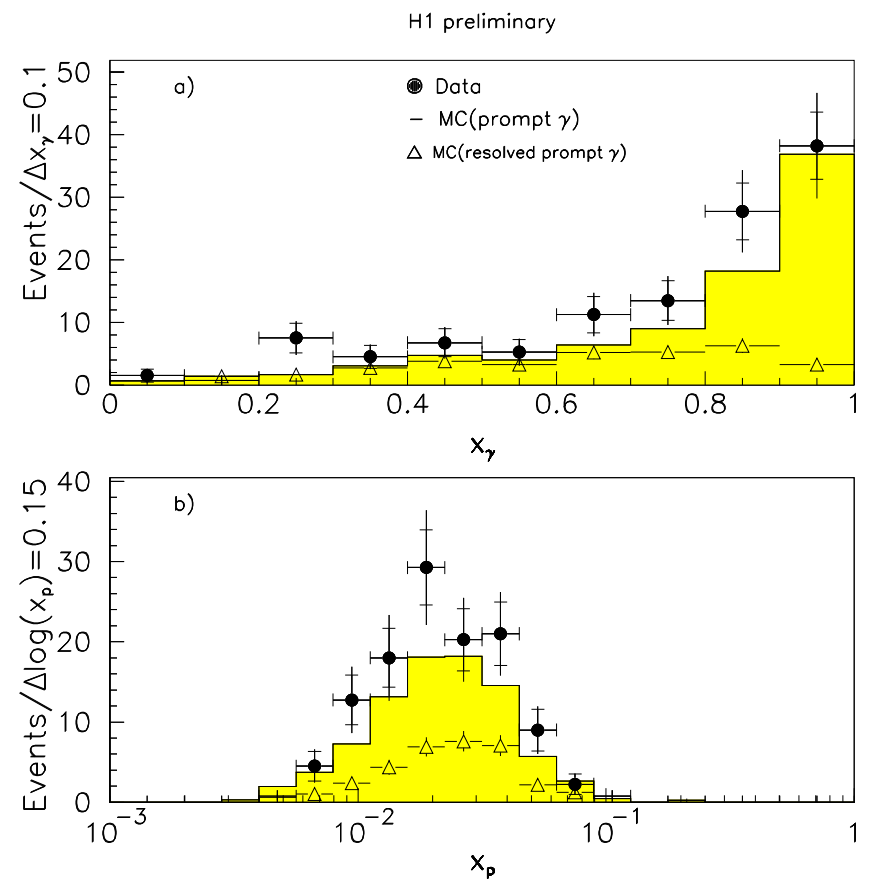

Figure 138: The $x_{\gamma}$ (a) and $x_{p}$ (b) distributions compared with the MC predictions (from [91]).

Comment: "It is shown that the prompt photon process at HERA is dominated by the direct $\gamma p$ interaction as expected from QCD."

\section{•ZEUS 97 [92] (HERA)}

The photoproduction of the isolated prompt photon with high $E_{T}^{\gamma}$, together with a balancing jet (described by $E_{T}$ and $\eta$ ) has been observed for the first time at HERA. The data (collected in 1995) correspond to $P^{2} \lesssim 1 \mathrm{GeV}^{2}, 5 \leq E_{T}^{\gamma}<10 \mathrm{GeV}$ and $E_{T} \geq 5 \mathrm{GeV}$, and in addition: $-0.7<\eta^{\gamma}<0.8$ and $-1.5<\eta<1.8$.

The isolation cone was imposed around the photon candidate; within the cone with $\mathrm{R}=1$ the hadronic energy was assumed to be smaller than $10 \%$ of the photon energy. The Monte Carlo PYTHIA program with the MRSA (proton) and GRV LO (photon) parton densities was applied. Results are presented in fig. 139 for $\Delta \phi(\gamma, j e t)$, and $\Delta E_{T}(\gamma$, jet $)$ distributions. In fig. 140 the $x_{\gamma}$ and $x_{p}$ distributions are shown. 

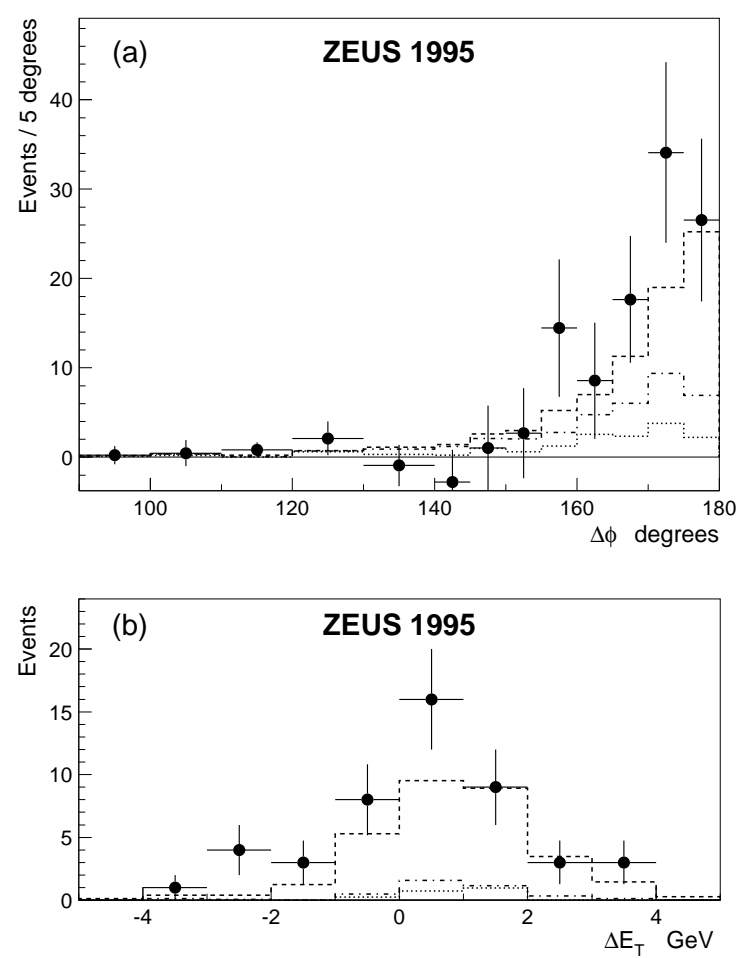

Figure 139: The $\Delta \phi(\gamma, j e t)$ (a) and $\Delta E_{T}(\gamma, j e t)$ (b) distributions (from 92]).
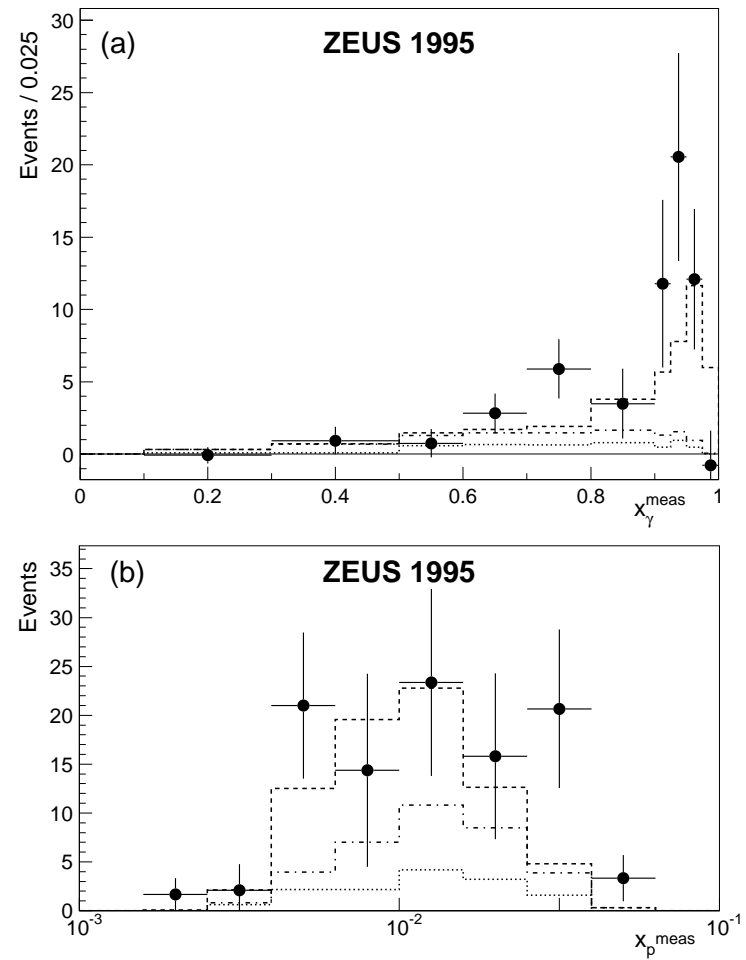

Figure 140: The $x_{\gamma}$ and $x_{p}$ distributions are shown for the isolated $\gamma$ plus jet production (from 92]).

Comment: For the first time the isolated high $-E_{T}$ photons accompanied by balancing 
jets were observed at HERA. The $x_{\gamma}$ distribution is in good agreement with the $L O$ QCD prediction of the PYTHIA generator.

\section{•ZEUS 2000a 93] (HERA)}

The first inclusive measurement of photoproduction of isolated prompt photon obtained from run 1996-1997 $\left(P^{2}<1 \mathrm{GeV}^{2}\right.$ and $\left.0.2<y<0.9\right)$ is reported. Events correspond to the following ranges of kinematical variables: $E_{T}^{\gamma}>5 \mathrm{GeV}$ and $-0.7<\eta^{\gamma}<$ 0.9 for the photon. The PYTHIA 5.7 and HERWIG 5.9 generators, with $p_{T}^{\min }=2.5$ $\mathrm{GeV}$ and no implementation of multi-parton interactions, were used. The proton and photon parton parametrizations, MRSA and GRV LO, respectively, were used in the analysis.

The isolation cut for the photon was imposed: in the cone $\mathrm{R}=1$ the total hadronic energy was restricted to be below $0.1 E_{T}^{\gamma}$. The $E_{T}^{\gamma}$ distribution is presented in Fig. 141 together with the MC predictions and the NLO calculations by Gordon and by Krawczyk \& Zembrzuski [192]. In Fig. 142 the data for the $\eta^{\gamma}$ distribution together with the predictions of the MC models and of the NLO calculations 192 are shown. In order to find an origin of the discrepancy between data and both MC and NLO calculations the rapidity distributions were studied in three $y$ bins (not shown). The disagreement is strongest for the smallest $y$ bin ( $y$ between 0.2 and 0.32 )

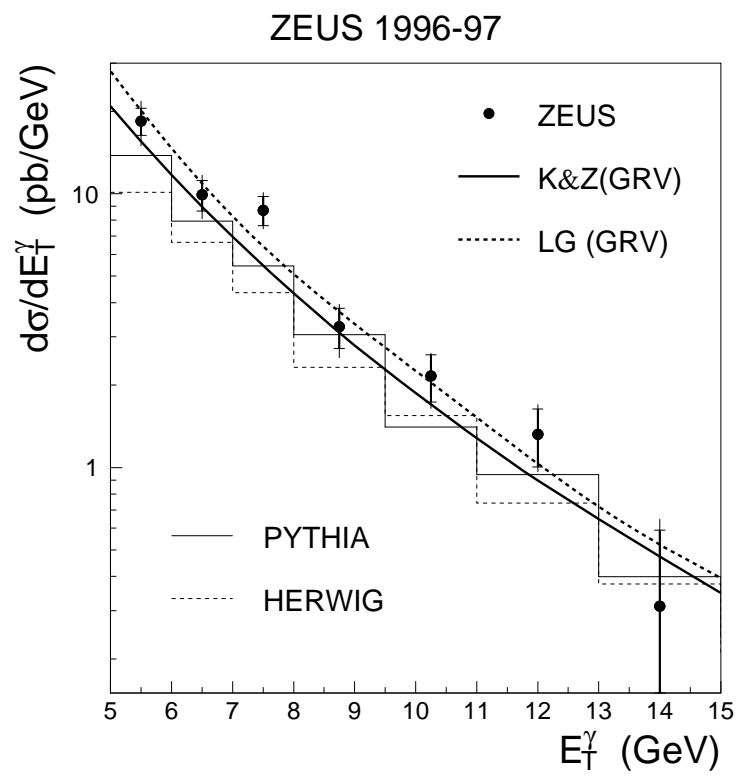

Figure 141: The $E_{T}^{\gamma}$ distributions for $-0.7<\eta^{\gamma}<0.9$ and $0.2<y<0.9$, is presented together with the MC predictions and the NLO calculations by Gordon and by Krawczyk\&Zembrzuski [192] with the GRV HO parton parametrizations (from 93]). 

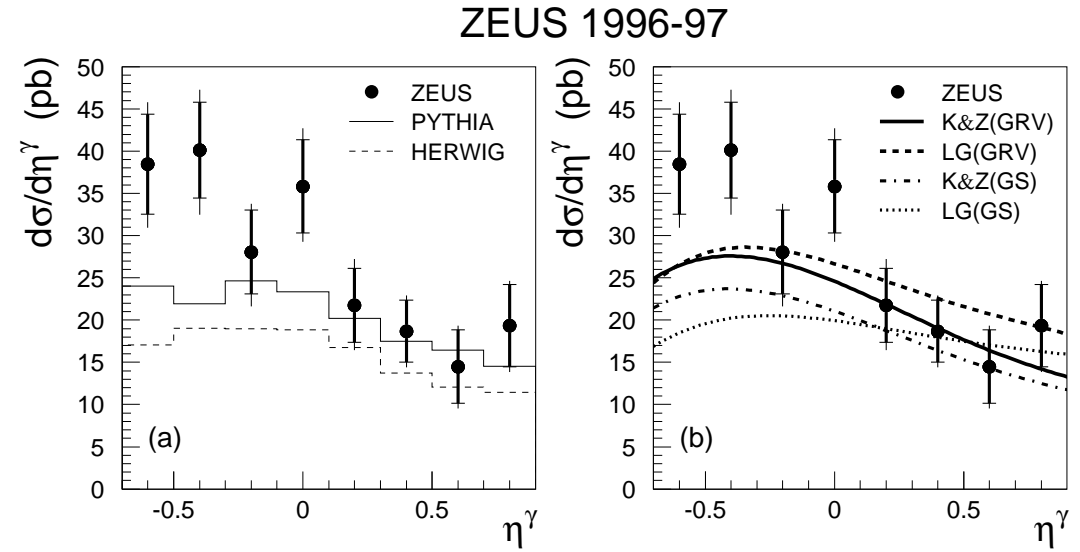

Figure 142: The $\eta^{\gamma}$ distribution for $5<E_{T}^{\gamma}<10 \mathrm{GeV}$ and $0.2<y<0.9$, together a) with the predictions of the PYTHIA and HERWIG models, b) with the NLO calculations by Gordon (LG) and by Krawczyk\&Zembrzuski (K\&Z) 192 with the GRV HO and GS parton parametrizations for the photon. (from [93]).

Comment: "The [MC] models are able to describe the data well for the forward (proton direction) photon pseudorapidities, but are low in the rear direction. The disagreement is strongest in the W interval 134-170 GeV. This result, together with the disagreements with the NLO predictions seen also in recent dijet results at HERA [ZEUS 98a], would appear to indicate a need to review the present theoretical modelling of the parton structure of the photon." 


\section{Partonic content of the virtual photon}

The notion of partonic content of the virtual photon has appeared in high energy interactions soon after the related concept for the real one ([2] a, [22]). From the very beginning it has a status of a unique test of QCD, as without additional experimental or model assumption the definite, singularity free predictions can be derived for both the $x$ and $Q^{2}$ dependence with the LO and NLO accuracy ${ }^{27}$. By virtue of the analytical continuation (dispersion relation) one expects that a limit of zero virtuality will lead to the partonic content of the real photon.

Not only the $\mathrm{DIS}_{e \gamma^{*}}$ can be performed for the virtual photon, where the corresponding structure functions of the $\gamma^{*}$ are measured, but also the resolved virtual photon processes sensitive to the individual parton densities in $\gamma^{*}$ can be studied.

The deep inelastic scattering on the virtual photon with hadronic final state was studied experimentally already in 1984 (PLUTO 84). Recently new data on DIS e $^{*}$ have appeared from the LEP accelerator (L3 2000). Also, the measurement of the leptonic final state in the deep inelastic scattering on the virtual $\gamma^{*}$ has been performed at LEP (OPAL 99b). It shows the importance of the interference terms which makes

the extraction of the corresponding structure functions $F_{i}^{\gamma^{*}(Q E D)}$ unfeasible in some kinematical regions (see sec. 4).

The bulk of the resolved virtual photon data, with $P^{2}$ up to $80 \mathrm{GeV}^{2}$ is just arriving from the HERA collider (single and double jet production), see sec. 3.3. The early analyses treated these events only as a special class of $\mathrm{DIS}_{e p}$ events - therefore the standard (for such analyses) types of Monte Carlo generators were used. In the recent analyses the generators incorporating resolved virtual photon interaction are used. The recent measurement of jet production in the virtual photon-proton scattering allowed to extract the effective parton density in $\gamma^{*}(\mathbf{H 1}$ 2000b). Still some discrepancies are observed, e.g. in ZEUS 2000b, where the dijet production in the $\gamma^{*} p$ collision for $P^{2}$ below $4.5 \mathrm{GeV}^{2}$ cannot be properly described by Monte Carlo models nor by the NLO QCD.

A dedicated study of the forward production of particles and jets, performed at HERA with the aim to establish a dynamics of the parton radiation at small $x_{B j}$ uses a large sample of the Monte Carlo generators - those typical for $\mathrm{DIS}_{e p}$ events and those for processes with a resolved virtual photon. Here the possible signal from the BFKL evolution for the partons from a proton is confronted with the importance of the contribution due to the resolved $\gamma^{*}$ interaction. We discuss this topic in the separate section (sec. 3.4).

\subsection{The virtual photon-induced processes}

The description of hadronic high energy processes with the initial virtual photon depends on its characteristic virtuality: on how much it differs from zero and how big it is as compared to other relevant scales, e.g. $\Lambda_{Q C D}$ or $p_{T}$. From these relations one can derive the role of the virtual photon in the considered process. However, the interpretation is highly nontrivial, as it may depend on the reference frame, and even on the chosen gauge. We will not discuss these theoretical aspects any further. Below

\footnotetext{
${ }^{27}$ However, the importance of nonperturbative components of the virtual photon was pointed out, see below (sec. 3.2.1).
} 
we try to underline only the roles in which the photon appears in the experimental analyses.

We start with the short discussion of the flux of virtual photons.

\subsubsection{The flux of virtual photons}

As for today, we always deal with virtual photons arising from the leptonic beam(s) ${ }^{28}$. Assuming the factorization holds between the leptonic source and the hadronic hard subprocess, one can introduce the flux of the transversely polarized virtual photons (e.g. [2] a, 94])

$$
f_{\gamma^{*} / e}^{T}\left(y, P^{2}\right)=\frac{\alpha}{2 \pi P^{2}}\left[\frac{1+(1-y)^{2}}{y}-\frac{2(1-y) P_{\min }^{2}}{y P^{2}}\right] .
$$

The flux of longitudinal virtual photons,

$$
f_{\gamma^{*} / e}^{L}\left(y, P^{2}\right)=\frac{\alpha}{2 \pi P^{2}}\left[\frac{2(1-y)}{y}\right],
$$

is expected to be small for the present experimental setup and it is usually neglected in the analyses [198, 27.

Both the flux of virtual photons emitted by the electron (eqs. 26, 27), and the cross sections involving initial virtual photons (e.g. $\sigma^{\gamma^{*} e}$ and $\sigma^{\gamma^{*} p}$ ) depend on convention used in the definitions 29 . This ambiguity is however absent in the ee or ep cross sections, where a convolution of the virtual photon flux and the virtual photon cross section appears $\left(e . g . \sigma^{e p} \sim f_{\gamma^{*} / e} \otimes \sigma^{\gamma^{*} p}\right)$. Note that the concept of the resolved electron may happen to be very useful here, see sec. 6 .

The virtual photons described by eqs. (26, 27) undergo further interaction. They may interact directly, or via their partonic agents, provided that in a process that follows a probe exists, hard enough to resolve the virtual photon.

\subsubsection{The virtual photon as a (non-elementary) probe}

The description of the inclusive processes with $\gamma^{*}$ as a probe of another object, e.g. of a real photon in DIS $_{e \gamma}$, is relatively simple if global quantities, like total $\gamma^{*} \gamma$ cross sections or $F_{2}^{\gamma}\left(x_{B j}, Q^{2}\right)$ for a real photon, are considered. Here the virtuality of the photon probe (we denote it consequently through the survey by $Q^{2}$ ) provides the hardest scale in the process, and a direct interaction of $\gamma^{*}$ with partonic content of the target dominates. However, when one tries to reconstruct the different classes of hadronic final states in the DIS-type of measurements, among them the rare events with $p_{T}^{2} \gg Q^{2}$, one should include the interaction due to the partonic content of the probe $-\gamma^{*}$ (see also discussion in sec. 2.3).

Similarly, in the case of ep collision the highly virtual photon, being a probe for the proton target $\left(\mathrm{DIS}_{e p}\right.$ events with standard variables $Q^{2}$ and $x_{B j}$ ), can be resolved itself by the large $p_{T}$ jet (particle) production邽 (see below).

\footnotetext{
${ }^{28}$ Protons, due to their larger mass, are much weaker source of the virtual photons.

${ }^{29}$ as they contain the convention - dependent flux of the initial virtual photons.

${ }^{30}$ This problem ("Who is probing who?") was underlined e.g. in 96].
} 


\subsubsection{The virtual photon as a target}

Sec. 3 covers the dedicated measurements of the "structure" of the virtual photon. Such a photon, with virtuality $P^{2}$, plays here a role of a target - with the structure to be resolved. The structure functions for such object are measured in the $\mathrm{DIS}_{e \gamma^{*}}$ experiments at $e^{+} e^{-}$colliders in the double-tag events, with $Q^{2} \gg P^{2}$. Jet production resolving the initial virtual photon, i.e. with $p_{T}^{2} \gg P^{2}$, corresponds in $e^{+} e^{-}$colliders to the single $\left(\gamma^{*} \gamma\right)$ and double-tag $\left(\gamma^{*} \gamma^{*}\right)$ events, and in the ep case - to the single tagged events $\gamma^{*} p$. In order to pin down the partonic content of the virtual photon also large $p_{T}$ particles (among them a real photon [191]) can be used.

In practice an admixture of the resolved virtual photon contribution is always present in measurements on the "real" photon discussed in the previous section. Data are usually corrected for this effect (see sec. 2).

As it was mentioned above, there is a possibility to introduce here the structure function of the electron (see sec. 6), given as a convolution of the flux for virtual photons (eqs. 26, 27) and the structure function of $\gamma^{*}$.

\subsection{DIS $_{e \gamma^{*}}$ experiments}

Measurements of the virtual photon structure functions in the deep inelastic scattering at $e^{+} e^{-}$colliders are performed using double-tag events. These events are selected in the kinematic region where one of the virtual photons (the probe) has, on the average, a large virtuality $Q^{2}$ and the other, the target, a small one, $P^{2} \ll Q^{2}$.

For general double-tag events the hadronic state $X$ is produced in the lepton beams collision, $e\left(p_{1}\right) e\left(p_{2}\right) \rightarrow e\left(p_{1}^{\prime}\right) e\left(p_{2}^{\prime}\right) X$, via the $\gamma(q) \gamma(p)$ collision with definite helicity states of the photons. The corresponding cross section for the unpolarized lepton beams is given by (see [2]a,b,c)

$$
\begin{aligned}
& E_{1}^{\prime} E_{2}^{\prime} \frac{d \sigma(e e \rightarrow e e X)}{d^{3} p_{1}^{\prime} d^{3} p_{2}^{\prime}}= \\
& =\frac{\alpha^{2}}{16 \pi^{4} q^{2} p^{2}}\left[\frac{(q \cdot p)^{2}-q^{2} p^{2}}{\left(p_{1} \cdot p_{2}\right)^{2}-m_{e}^{4}}\right]^{1 / 2}\left(4 \rho_{1}^{++} \rho_{2}^{++} \sigma_{T T}+2\left|\rho_{1}^{+-} \rho_{2}^{+-}\right| \tau_{T T} \cos 2 \bar{\phi}+\right. \\
& \left.\quad+2 \rho_{1}^{++} \rho_{2}^{00} \sigma_{T L}+2 \rho_{1}^{00} \rho_{2}^{++} \sigma_{L T}+\rho_{1}^{00} \rho_{2}^{00} \sigma_{L L}-8\left|\rho_{1}^{+0} \rho_{2}^{+0}\right| \tau_{T L} \cos \bar{\phi}\right),
\end{aligned}
$$

where $\mathrm{T}$ denotes transverse (+ or - ) and $\mathrm{L}-$ longitudinal helicity states $(0)$. The $q$ and $p$ stand for the photons four-momenta: $q \equiv p_{1}-p_{1}^{\prime}, p \equiv p_{2}-p_{2}^{\prime}$. The $\rho_{1}$ and $\rho_{2}$ are the photon (with $q$ and $p$ four-momentum, respectively) density matrices. The

$\sigma_{T T, T L, L T, L L}$ and $\tau_{T T, T L}$ denote the corresponding cross sections and interference terms (the first subscript corresponds to the photon with four-momentum $q$ ). The $\bar{\phi}$ is the angle between two scattering planes of the scattered electrons in the $\gamma \gamma \mathrm{CM}$ system.

For large virtualities of both photons, $Q^{2}=\left|q^{2}\right|, P^{2}=\left|p^{2}\right| \gg 4 m_{e}^{2}$, eq. 28 can be written in the following form:

$$
\begin{aligned}
& E_{1}^{\prime} E_{2}^{\prime} \frac{d \sigma(e e \rightarrow e e X)}{d^{3} p_{1}^{\prime} d^{3} p_{2}^{\prime}}=L_{T T}\left(\sigma_{T T}+\epsilon_{1} \sigma_{L T}+\epsilon_{2} \sigma_{T L}+\epsilon_{1} \epsilon_{2} \sigma_{L L}+\right. \\
& \left.\quad+\frac{1}{2} \epsilon_{1} \epsilon_{2} \tau_{L L} \cos 2 \bar{\phi}-2 \sqrt{\epsilon_{1}\left(1+\epsilon_{1}\right)} \sqrt{\epsilon_{2}\left(1+\epsilon_{2}\right)} \tau_{T L} \cos \bar{\phi}\right)
\end{aligned}
$$


where

$$
L_{T T}=\frac{\alpha^{2}}{16 \pi^{4} q^{2} p^{2}}\left[\frac{(q \cdot p)^{2}-q^{2} p^{2}}{\left(p_{1} \cdot p_{2}\right)^{2}-m_{e}^{4}}\right]^{1 / 2} 4 \rho_{1}^{++} \rho_{2}^{++}
$$

and

$$
\epsilon_{i}=\frac{1}{2} \rho_{i}^{00} / \rho_{i}^{++}
$$

For $\epsilon_{i}$ close to 1 , which is a typical value in present experiments, one can furthermore simplify the above formulae, and obtain:

$$
E_{1}^{\prime} E_{2}^{\prime} \frac{d \sigma(e e \rightarrow e e X)}{d^{3} p_{1}^{\prime} d^{3} p_{2}^{\prime}}=L_{T T}\left(\sigma_{e f f}+\frac{1}{2} \tau_{L L} \cos 2 \bar{\phi}-4 \tau_{T L} \cos \bar{\phi}\right)
$$

where an effective cross section $\sigma_{\text {eff }}=\sigma_{T T}+\sigma_{L T}+\sigma_{T L}+\sigma_{L T}$ was introduced.

For the deep inelastic scattering, where $Q^{2}>P^{2}$, the corresponding structure functions for a polarization-averaged photon target with virtuality $P^{2}$ can be introduced

$$
\begin{gathered}
2 x F_{1}^{\gamma^{*}}=\frac{-q^{2}}{4 \pi^{2} \alpha} \frac{\sqrt{(q \cdot p)^{2}-q^{2} p^{2}}}{q \cdot p}\left(\sigma_{T T}\left(x, q^{2}, p^{2}\right)-\frac{1}{2} \sigma_{T L}\left(x, q^{2}, p^{2}\right)\right), \\
F_{2}^{\gamma^{*}}=\frac{-q^{2}}{4 \pi^{2} \alpha} \frac{q \cdot p}{\sqrt{(q \cdot p)^{2}-q^{2} p^{2}}}\left(\sigma_{T T}\left(x, q^{2}, p^{2}\right)+\right. \\
\left.+\sigma_{L T}\left(x, q^{2}, p^{2}\right)-\frac{1}{2} \sigma_{L L}\left(x, q^{2}, p^{2}\right)-\frac{1}{2} \sigma_{T L}\left(x, q^{2}, p^{2}\right)\right), \\
\text { with } \quad F_{L}^{\gamma^{*}}=F_{2}^{\gamma^{*}}-2 x F_{1}^{\gamma^{*}},
\end{gathered}
$$

and $x=Q^{2} / 2 p q$.

\subsubsection{Theoretical description}

The structure function of the virtual photon can be obtained in the Parton Model assuming the production of the $q \bar{q}$ pairs in the $\gamma^{*}\left(Q^{2}\right) \gamma^{*}\left(P^{2}\right)$ collision. In the mass range

$$
Q^{2} \gg P^{2} \gg m_{q}^{2}
$$

it has the form [22]:

$$
F_{2}^{\gamma^{*}}\left(x, Q^{2}, P^{2}\right)=N_{c} N_{f}<Q^{4}>\frac{\alpha}{\pi} x\left\{\left[x^{2}+(1-x)^{2}\right] \ln \frac{Q^{2}}{P^{2} x^{2}}+6 x(1-x)-2\right\},
$$

where

$$
<Q^{4}>=\frac{1}{N_{f}} \sum_{i=1}^{N_{f}} Q_{i}^{4}
$$

to be compared with the eq. (10).

One can clearly see that the scale of the probe, $Q^{2}$, has to differ from the $P^{2}$ in order to 
test the structure of the virtual photon in the leading logarithmic accuracy (LL). The corresponding quark density in the virtual photon defined in the LL approximation has the form:

$$
\left.q_{i}^{\gamma}\left(x, Q^{2}, P^{2}\right)\right|_{P M} ^{L L}=\frac{\alpha}{2 \pi} N_{c} Q_{i}^{2}\left[x^{2}+(1-x)^{2}\right] \ln \frac{Q^{2}}{P^{2}}
$$

The QCD evolution equations for the virtual photon are analogous to those for the real photon with the inhomogeneous term given by the corresponding splitting function $P_{q \gamma^{*}}$. In the case of the virtual photon there is a hope that to solve evolution equations the initial conditions are not needed, since for $Q^{2} \gg P^{2} \gg \Lambda_{Q C D}^{2}$ the nonperturbative effects should be absent (see ref.[22]). Indeed, the virtual photon may play a unique role in testing the QCD.

However, recently there appeared papers [162, 170, 163] showing that the parton content of the virtual photon is not solely described by purely perturbative contributions in this region, in contrast to the expectation from [22].

Recently the LO 170, 193, 198] and the NLO QCD [194, 195, 196, 197 calculations have appeared for the DIS $_{e \gamma^{*}}$ and the jet production in the considered processes involving virtual photon(s) $\gamma^{*} \gamma, \gamma^{*} \gamma^{*}$ and $\gamma^{*} p$.

The existing parton parametrizations for the virtual photon are described in the Appendix.

\subsubsection{Measurements of $F_{2}^{\gamma^{*}}$}

For the DIS $\gamma^{*}$ measurements, the quantity

$$
x_{v i s}=Q^{2} /\left(Q^{2}+P^{2}+W_{v i s}^{2}\right)
$$

needs to be converted to the true $x_{B j}=Q^{2} / 2 p q$ variable $\$$.

We start with the old data from PLUTO, the first "DIS"-type measurements for the virtual photon.

\footnotetext{
${ }^{31}$ At finite $P^{2}$ a modified variable $x_{B j}$, which extends over the whole range between 0 and 1 may be introduced. For small ratio $P^{2} / Q^{2} \ll 1$ they coincide.
} 


\section{DATA}

-PLUTO 84 [6] (PETRA)

The double-tag events were measured in $e^{+} e^{-}$collision, with the energy of the beam equal to $17.3 \mathrm{GeV}$. One of the virtual photons (the probe) had, on the average, virtuality $<Q^{2}>=5 \mathrm{GeV}^{2}$ and the other (the target) $<P^{2}>=0.35 \mathrm{GeV}^{2}$. For the cross sections, the assumptions $\sigma_{T L} \approx \sigma_{L T}$ and $\sigma_{L L} \approx 0$ were made. The experiment was sensitive to the following combination of the virtual photon structure functions (the effective structure function):

$$
F_{\text {eff }} \equiv F_{2}+(3 / 2) F_{L} \text {. }
$$

Results for the extracted $F_{\text {eff }}$ as a function of $x_{B j}$ are presented in fig. 143 together with the theoretical predictions. In fig. 144 the quantity $Q^{2} \sigma_{\gamma \gamma} / 4 \pi^{2} \alpha^{2} \approx F_{\text {eff }} / \alpha$, averaged over both $x_{B j}$ and $Q^{2}$, is shown as a function of the measured $P^{2}(0.2-0.8$ $\mathrm{GeV}^{2}$ ), in comparison with QPM and VMD (for the target) contributions.

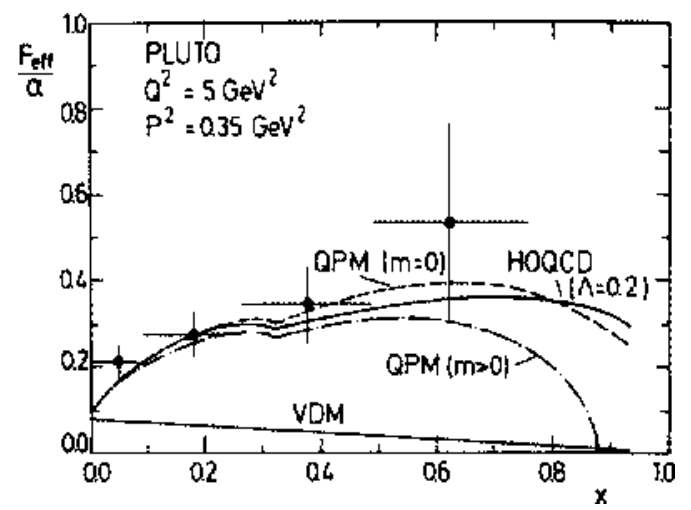

Figure 143: The data for the effective structure function $F_{\text {eff }} / \alpha$ for the virtual photon. For the averaged $Q^{2}=5 \mathrm{GeV}^{2}$, and $P^{2}=0.35 \mathrm{GeV}^{2}$, the dependence on $x_{B j}$ is shown in comparison with the QPM (with massless and massive quarks) and QCD calculations, both with the VMD for the target contributions added (from [6]).

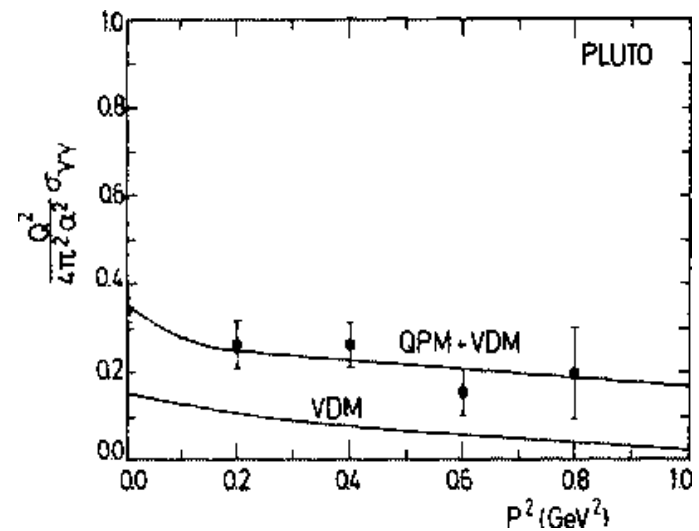

Figure 144: The results for the $\frac{Q^{2}}{4 \pi^{2} \alpha^{2}} \sigma_{\gamma \gamma}$ for virtual photon as a function of $P^{2}$ (averaged over $x_{B j}$ and $Q^{2}$ ), compared with QPM and VMD contributions (from [6]).

-L3 2000 42] (LEP 1)

The measurement of the structure function for the virtual photon $\left(<P^{2}\right\rangle=3.7$ 
$\mathrm{GeV}^{2}$ ) at $\sqrt{s}=89-92 \mathrm{GeV}$ was performed based on the 1991-1995 data. The large $Q^{2}$ region, between 40 and $500 \mathrm{GeV}^{2}\left(<Q^{2}>=120 \mathrm{GeV}^{2}\right)$, was analysed. The real photon structure function was studied as well, see sec. 2.2.2.

In the analysis the effective structure function:

was measured.

$$
F_{\text {eff }} \equiv \frac{Q^{2}}{4 \pi^{2} \alpha}\left(\sigma_{T T}+\sigma_{L T}+\sigma_{T L}+\sigma_{L L}\right)
$$

The data were analysed using the JAMVG generator modelling the QPM with $N_{f}=4$, the PHOJET $1.05 \mathrm{c}$ with a cutoff $p_{T}^{m i n}=2.5 \mathrm{GeV}$ and TWOGAM generating the three processes: QPM, VMD and QCD resolved photon contributions. The hadronic final state was investigated (see section 2.3 for details).

The $F_{\text {eff }}^{\gamma}$ data are presented in table 26 and fig. 145, where the results are compared with the predictions of the QPM and QCD calculations performed for the structure function of transverse photon only. The QCD contribution is needed to describe the $x_{B j}$ region below 0.5 .

Table 26:

\begin{tabular}{|c|c|c|}
\hline $\begin{array}{c}<Q^{2}> \\
{\left[\mathrm{GeV}^{2}\right]}\end{array}$ & $x_{B j}$ & $\begin{array}{c}F_{\text {eff }}^{\gamma} / \alpha \\
\text { (stat. }+ \text { syst. })\end{array}$ \\
\hline 120 & $0.05-0.20$ & $0.42 \pm 0.16 \pm 0.05$ \\
& $0.20-0.40$ & $0.71 \pm 0.24 \pm 0.09$ \\
& $0.40-0.60$ & $0.72 \pm 0.34 \pm 0.09$ \\
& $0.60-0.80$ & $1.27 \pm 0.51 \pm 0.16$ \\
& $0.80-0.98$ & $1.48 \pm 0.66 \pm 0.19$ \\
\hline
\end{tabular}

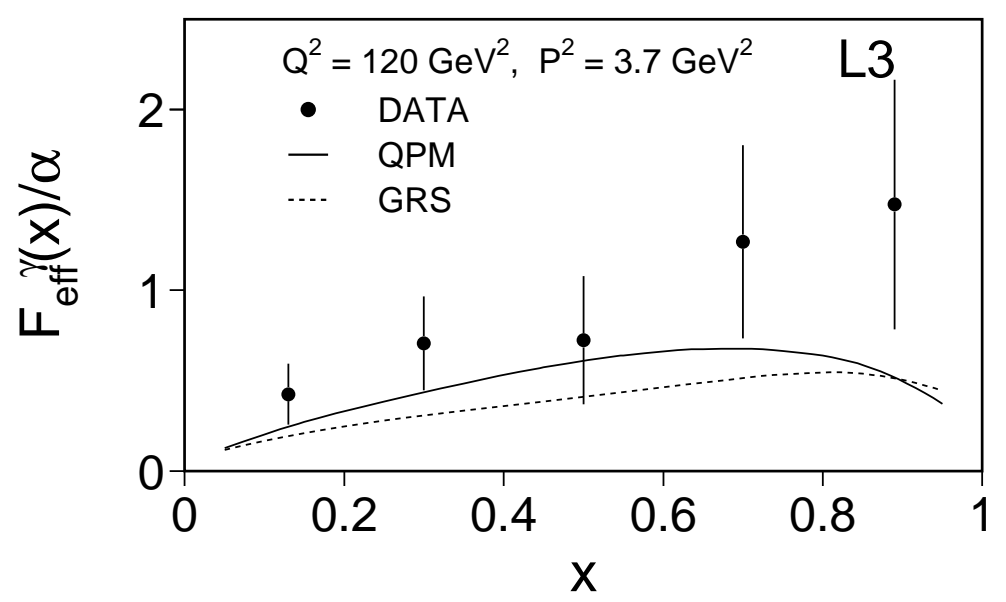

Figure 145: $F_{\text {eff }}^{\gamma} / \alpha$ as a function of $x_{B j}$ for $<Q^{2}>=120 \mathrm{GeV}^{2}$ and $<P^{2}>=3.7 \mathrm{GeV}^{2}$. The solid line corresponds to the QPM prediction. The QCD prediction (only for transverse target photon states) using GRS LO parton parametrization is given by the dashed line. (from [42]).

The averaged values of $F_{\text {eff }}^{\gamma} / \alpha$ as a function of $\left\langle P^{2}>\right.$ are presented in table 27 and in fig. 146. 
Table 27:

\begin{tabular}{|c|c|}
\hline $\begin{array}{c}<P^{2}> \\
{\left[G e V^{2}\right]}\end{array}$ & $\begin{array}{c}<F_{\text {eff }}^{\gamma} / \alpha> \\
\text { stat. }+ \text { syst. }) \\
x_{B j}=0.05-0.98\end{array}$ \\
\hline 0 & $0.83 \pm 0.06 \pm 0.08$ \\
2.0 & $0.87 \pm 0.25 \pm 0.11$ \\
3.9 & $1.00 \pm 0.32 \pm 0.13$ \\
6.4 & $1.02 \pm 0.70 \pm 0.13$ \\
\hline
\end{tabular}

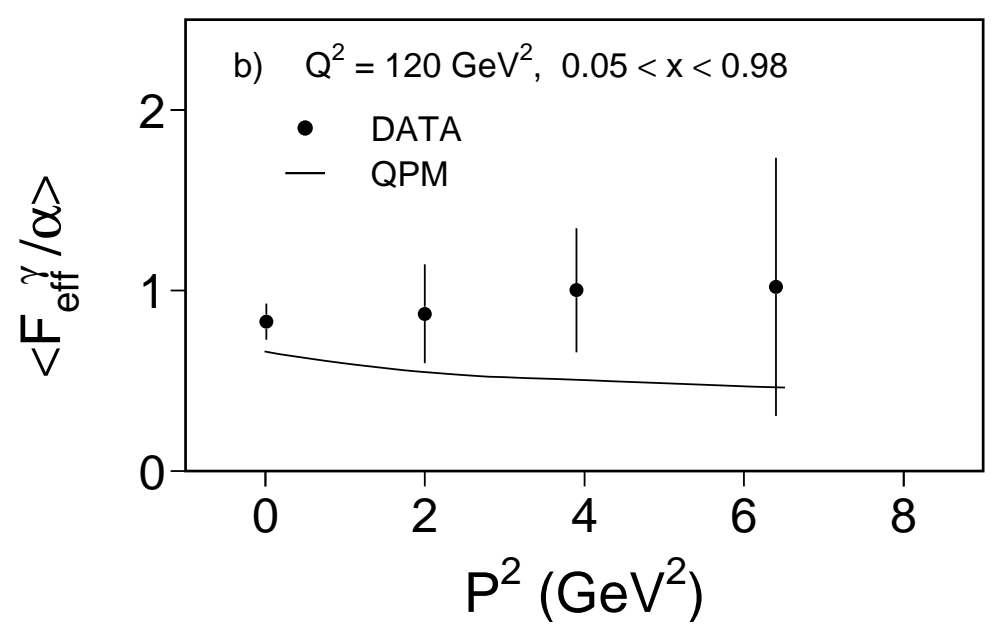

Figure 146: The $P^{2}$ dependence of $F_{\text {eff }}^{\gamma} / \alpha$ averaged over $x_{B j}=0.05-0.98$ for the double-tag data, compared with the QPM prediction (solid line) (from [42]).

\subsection{Measurements of resolved virtual photon(s) processes}

As in the case of the real photon, the large $p_{T}$ jets or particles may resolve the virtual photon(s). Provided the corresponding mass relation $\tilde{Q}^{2} \sim p_{T}^{2} \gg P_{1}^{2}\left(P_{2}^{2}\right) \gg \Lambda_{Q C D}^{2}$ holds, one introduces the contribution due to a partonic content of $\gamma^{*}$ and a direct $\gamma^{*}$ subprocesses, as for the real photon (eqs. (20, 22)). For the same reason as in sec. 2 we will limit ourselves to the jet(s) production. The exception is the hard forward production, where a single particle production is also considered, see sec. 3.4.

As we already discussed in sec. 2.3, in analyses of hadronic final state, accompanying the $F_{2}^{\gamma}$ measurements, jet production in $\gamma^{*} \gamma$ collision was studied, e.g. TOPAZ 94, DELPHI 96b,conf, OPAL 97d,conf. In some of these analyses, e.g. TOPAZ 94, there were events with the hard scale $\tilde{Q}^{2} \sim p_{T}^{2}$ much larger than the virtuality of $\gamma^{*}$, so $\gamma^{*}$ can be considered as being resolved.

However, so far there are no data from dedicated experiments on the resolved virtual photon processes in $e^{+} e^{-}$collisions at LEP and TRISTAN, whereas a large amount of data from the ep collider HERA have appeared recently. The single and double jets produced in the resolved virtual photon processes are being studied in detail at HERA. Three and four jets including remnant jets, and jet shapes were also 
studied, however we will omit these results in our presentation. For the first time the effective parton density, based on polarization-averaged parton densities in the virtual photon, was extracted from the data (H1 2000b).

The transition region between the interaction of an almost real photon and of a virtual photon with the proton is studied by the H1 and ZEUS collaborations in the ep collisions at HERA. In such analysis various Monte Carlo generators (LEPTO, ARIADNE, MEPS, DISJET, PROJET, RAPGAP and PHOJET, HERWIG), used to describe the photoproduction and the DIS $_{e p}$ events as well as the rapidity gap events at HERA, are used.

There are some discrepancies observed between the virtual photon cross sections for the dijets production at HERA and the NLO QCD [194, 195, 196, 197] calculations, see ZEUS 2000b.

\subsubsection{Jet production in $\gamma^{*} \gamma$ and $\gamma^{*} \gamma^{*}$ collisions}

The jet production in $\gamma^{*} \gamma$ collision were studied in DIS e $^{*}$ experiments, e.g. ALEPH 97a,conf, TOPAZ 94, see sec. 2.3. There are no data so far for the jet production in the double-tag events.

\subsubsection{Jet production in $\gamma^{*} p$ collisions}

In the experimental analyses at HERA the flux of virtual photons is introduced $(\mathbf{H 1}$ $\mathbf{9 7} \mathbf{b}$ ) in the form of the integral of the transversely polarized virtual photons over the relevant range of $y$, and over its positive squared mass (virtuality), $P^{2}$ :

$$
F_{\gamma^{*} / e}^{T}=\int_{y_{\min }}^{y_{\max }} d y \int_{P_{\min }^{2}}^{P_{\max }^{2}} d P^{2} f_{\gamma^{*} / e}^{T}\left(y, P^{2}\right) .
$$

The kinematical variables used in the analysis of the single or double jet production are defined as for the real photon case (secs. 2.4.2 and 2.4.4). In the resolved virtual photon processes some kinematical variables are defined also in the $\gamma^{*} p$ CM system, and are denoted below by a star, e.g. $E_{T}^{*}$. Note that here we use the notation $P^{2}$

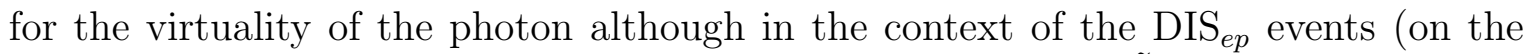

proton) at HERA it plays the role of the $Q^{2}$. The hard scale $\tilde{Q}^{2}$ is usually provided by the transverse energy or transverse momentum of jets. 


\section{DATA}

$\bullet$ H1 94 97 (HERA)

The jet rate measurement in DIS $_{e p}$ events with $Q^{2} \gg p_{T}^{2}$ was reported. The JADE jet clustering algorithm was used.

-H1 97b 98 (HERA)

The single jet cross section for the events with $0.3<y<0.6$ was studied in the transition between the photoproduction and the standard $\mathrm{DIS}_{e p}$ regime at HERA. The three ranges of the squared mass of the virtual photon were studied: $P^{2}<10^{-2}$ $\mathrm{GeV}^{2}$ (1994 data), $0.65<P^{2}<20 \mathrm{GeV}^{2}$ (the 1995 shifted vertex data) and $9<P^{2}<$ $49 \mathrm{GeV}^{2}$ (1994 data). The jets with $E_{T}^{*}>4 \mathrm{GeV}$ (for the photoproduction $E_{T}^{*}>5$ $\mathrm{GeV}$ only) for $-2.5<\eta^{*}<-0.5$ were measured. The $k_{T}$ - clustering algorithm was used. For the photoproduction PHOJET 1.03, for DIS $S_{e p}$ events - LEPTO 6.5 and ARIADNE 4.08 were used in simulation. RAPGAP and HERWIG 5.9 (with $p_{T}^{\text {min }}=$ $1.5 \mathrm{GeV}$ ) were applied to model direct and resolved real and virtual photon processes. The GRV94 HO parton parametrization was taken for the proton. GRV HO with the Drees - Godbole parametrization $\left(\omega^{2}=1 \mathrm{GeV}^{2}\right)$, denoted GRV HO/Drees-Godbole, and SaS2D parametrizations for the virtual photon were used.

The measured $d \sigma_{e p} / d E_{T}^{*}$ as a function of the transverse energy of the jet for various $P^{2}$ ranges integrated over $y$ and $\eta^{*}$ ranges is presented in fig. 147. It was found to be in agreement with the HERWIG model (using the GRV HO/Drees-Godbole parton parametrization). In fig. 148 the corresponding data for the rapidity distribution for jets with $E_{T}^{*}>5 \mathrm{GeV}$ are shown in the various virtuality ranges. 

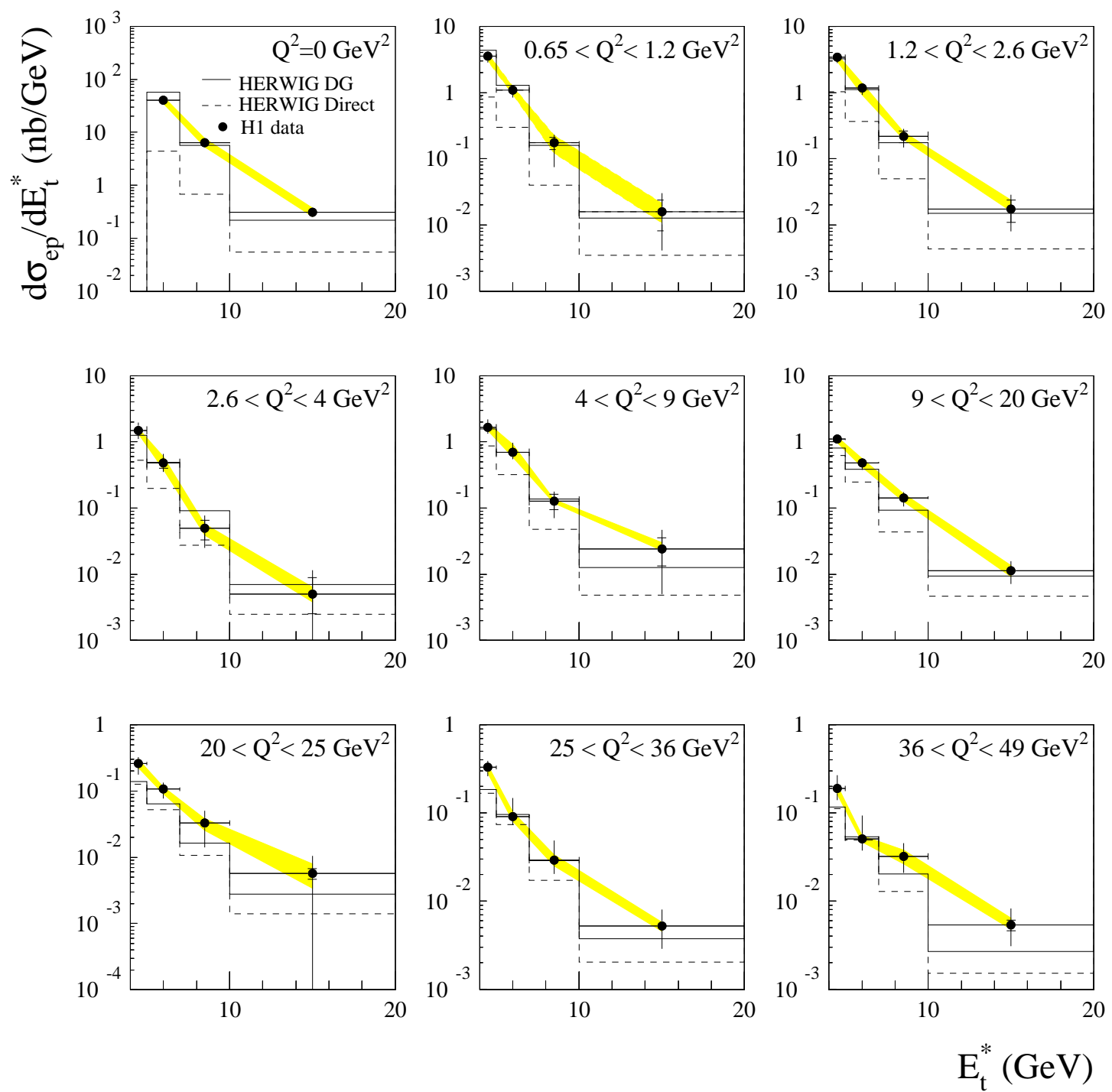

Figure 147: The inclusive $d \sigma_{e p} / d E_{T}^{*}$ jet cross section as a function of the transverse energy $E_{T}^{*}$ for various initial photon virtuality $P^{2}$ (denoted as $Q^{2}$ ) ranges and for $-2.5<\eta^{*}<-0.5$. The HERWIG GRV HO/Drees-Godbole prediction is denoted by the solid line, the dashed line corresponds to the direct contribution to this prediction (from [98]). 

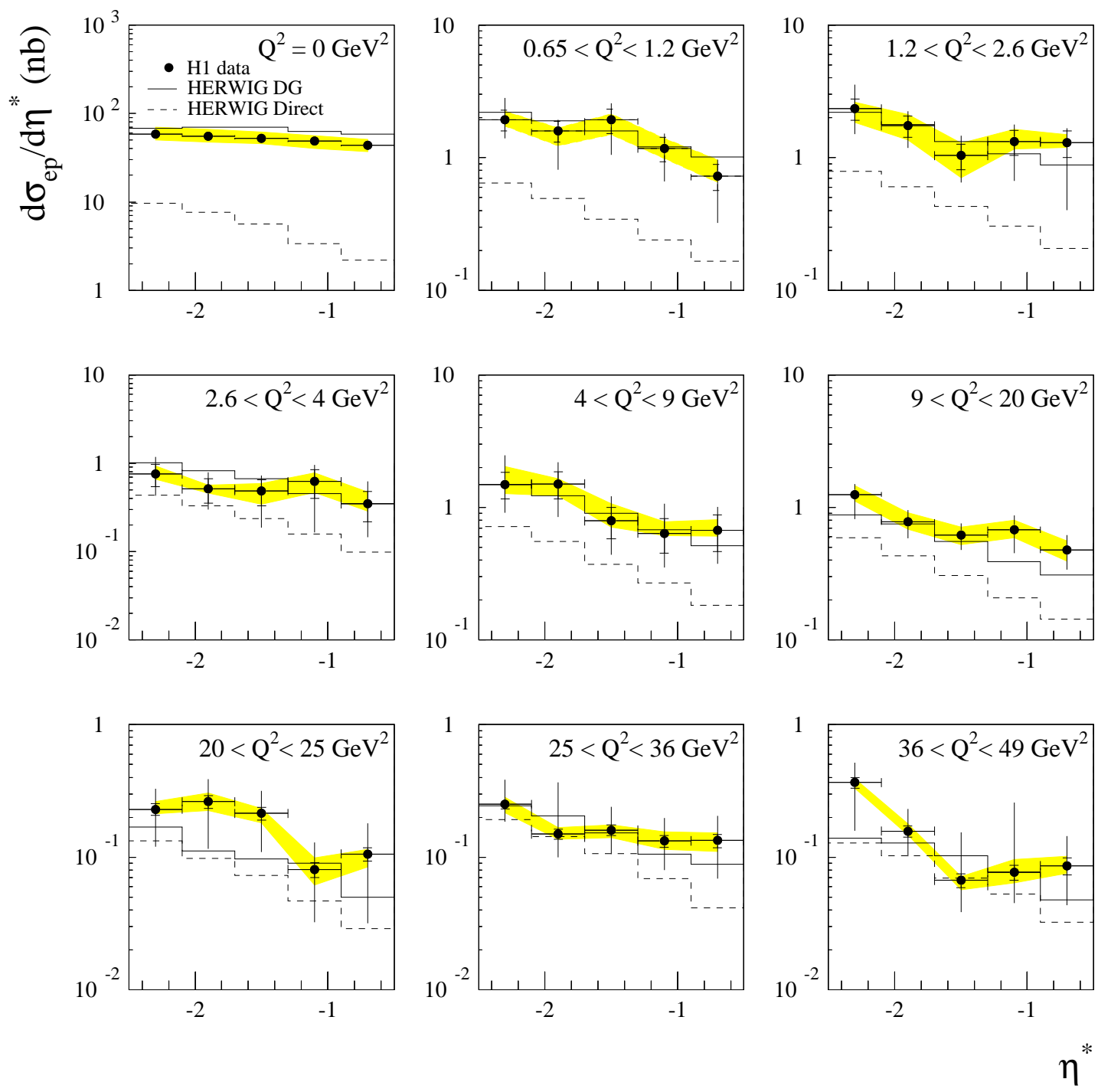

Figure 148: The inclusive $d \sigma_{e p} / d \eta^{*}$ jet cross section as a function of the rapidity $\eta^{*}$ for various initial photon virtuality $P^{2}$ (denoted as $Q^{2}$ ) ranges for the $E_{T}^{*}>5 \mathrm{GeV}$. The HERWIG (with the GRV HO/Drees-Godbole parton parametrization) prediction is denoted by the solid line, the dashed line denotes the direct contribution (from [98]).

To study the dependence of the virtuality of the photon, the flux (34) is introduced and the cross section $\sigma_{\gamma^{*} p}$ is calculated,

$$
\sigma_{\gamma^{*} p \rightarrow j e t+X}=\frac{\sigma_{e p \rightarrow j e t+X}}{F_{\gamma^{*} / e}^{T}} .
$$

Note that it is not certain that the implied factorization really holds for the whole range of kinematical variables.

The results for $\sigma_{\gamma^{*} p}$ agree with the HERWIG and RAPGAP predictions based on the GRV HO/Drees-Godbole and SaS2D parton parametrizations in the virtual photon, as shown in fig. 149. Similar comparison with LEPTO and ARIADNE was made (not shown) and "neither model can describe the data when $Q^{2}<E_{T}^{* 2}$ and the virtual photon can be resolved". 

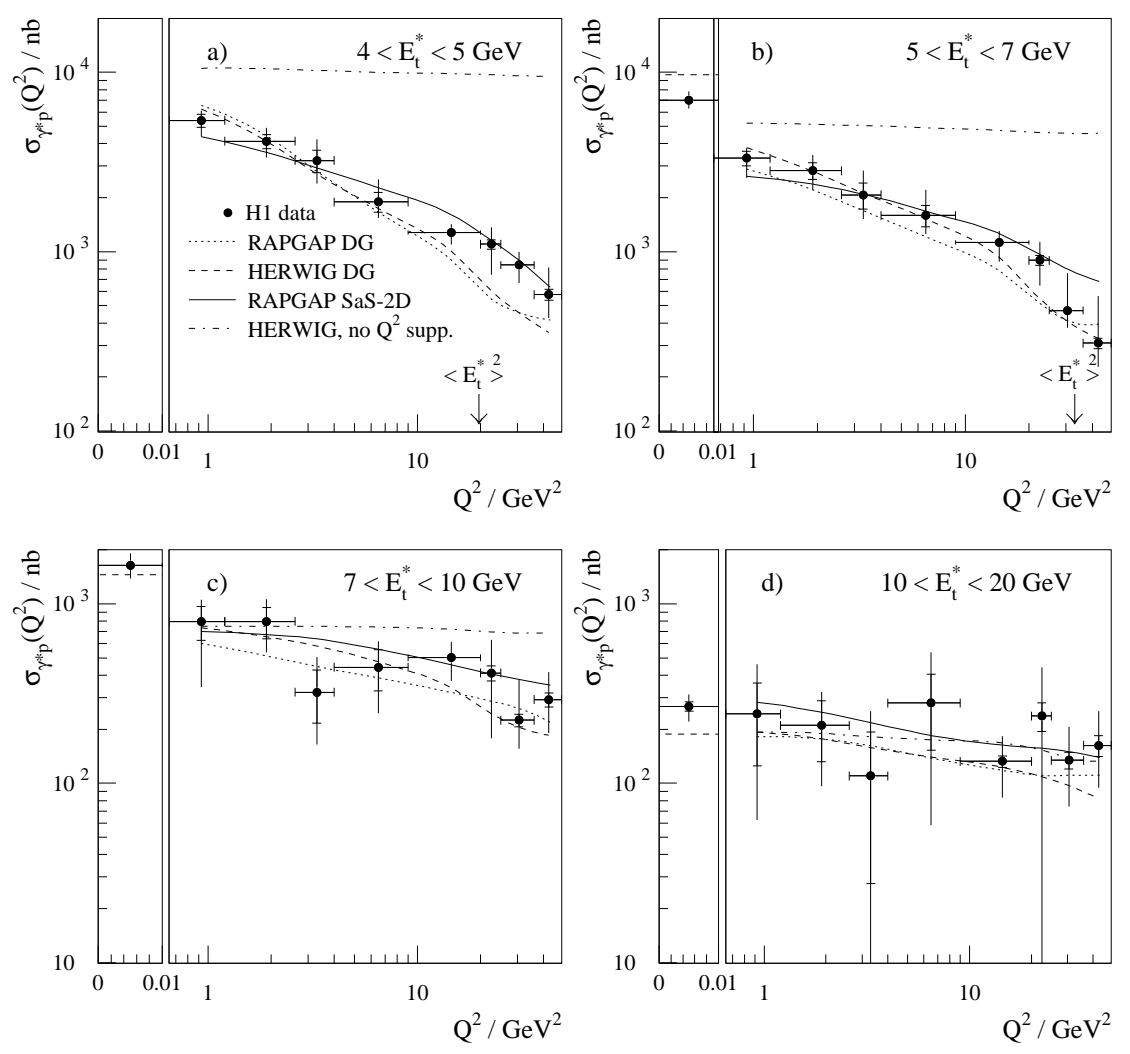

Figure 149: The inclusive $\gamma^{*} p$ jet cross section as a function of $P^{2}$ (denoted as $Q^{2}$ ) for various ranges of the transverse jet energy $E_{T}^{* 2}$, for $-2.5<\eta^{*}<-0.5$. The HERWIG (with the GRV HO/Drees-Godbole distribution) prediction is denoted by the dashed line, RAPGAP (with the GRV HO/Drees-Godbole distribution) - the dotted line, RAPGAP (with the SaS2D distribution) - the solid line, and the dot-dashed line corresponds to HERWIG with the GRV HO parametrization as for the real photon ("no $P^{2}$ suppression") (from [98]).

Comment: "The inclusive jet cross-section can therefore be understood if a partonic structure is ascribed to the virtual photon.

The data are best described by the RAPGAP model using the SaS2D parametrization of the virtual photon."

•H1 2000b 99 (HERA)

The dijet event rates have been measured for $5 \lesssim P^{2} \lesssim 100 \mathrm{GeV}^{2}$ and $10^{-4} \lesssim x_{B j} \lesssim$

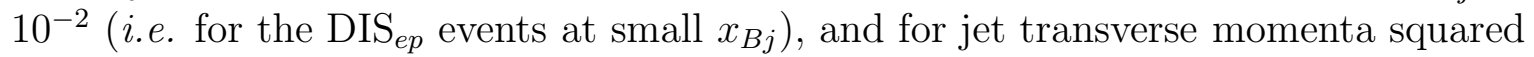
$p_{T}^{2} \gtrsim P^{2}$ (see also H1 94). The data collected in 1994 correspond to $y>0.05$. The condition $\left|\Delta \eta^{*}\right|<2$ was imposed. The jet $p_{T}$ was assumed to be at least $5 \mathrm{GeV}$, with the following requirements: for the symmetric case $p_{T 1,2}^{*} \geq 5 \mathrm{GeV}$, for the asymmetric case $p_{T 1}^{*} \geq 5 \mathrm{GeV}$ and $p_{T 2}^{*} \geq 7 \mathrm{GeV}$, and for the sum scenario $p_{T 1}^{*}+p_{T 2}^{*} \geq 13 \mathrm{GeV}$. The cone algorithm with $R=1$ was applied in the $\eta^{*}-\phi^{*}$ plane.

The standard DIS $e p$ Monte Carlo generators: LEPTO and ARIADNE were used to describe in LO the direct $\gamma^{*} q$ contribution with two sets of parton parametrization for the proton: MRS-H and GRV94 HO. The additional mechanism based on the resolved virtual photon interaction neglecting the longitudinal $\gamma^{*}$ was introduced in the analysis. Its contribution was obtained using the RAPGAP Monte Carlo model 
with the factorization and renormalization scale $\tilde{Q}^{2}=Q^{2}+p_{T}^{2}$. To describe this contribution also the NLO calculations (for partons) in JeTViP program with SaS1D parton parametrization for $\gamma^{*}$ were studied. For comparison the DISENT program was used also to calculate the NLO direct virtual photon contribution. The factorization and renormalization scale $\tilde{Q}^{2}=Q^{2}+50 \mathrm{GeV}^{2}$ was used in both NLO calculations.

The transverse energy flow with respect to the jet axis was studied. There is a good agreement between the data and the events simulated with LEPTO and ARIADNE, except for the $\eta^{*}$ distribution of the jets (not shown).

The comparison of the fraction of dijet events in all DIS $_{e p}$ events with the RAPGAP and ARIADNE models prediction can be found in fig. 150, where the rates as a function of $P^{2}$ (integrated over $x_{B j}$ ) and $x_{B j}$ (integrated over $P^{2}$ ) are shown. The same distributions are shown in fig. 151 for the NLO calculations obtained using DISENT (the direct photon contribution) and JeTViP, where also the resolved photon component is included. The $x_{\gamma}^{o b s}$ distribution is shown in fig. 152.

Comment: The agreement with the data at low $x_{B j}$ and moderate $P^{2}$ is improved when beside the direct also contributions from the resolved virtual photon are included in the Monte Carlo model (RAPGAP) and in the NLO calculations. "The CDM model, as implemented in ARIADNE, is also able to describe the dijet rate well." 

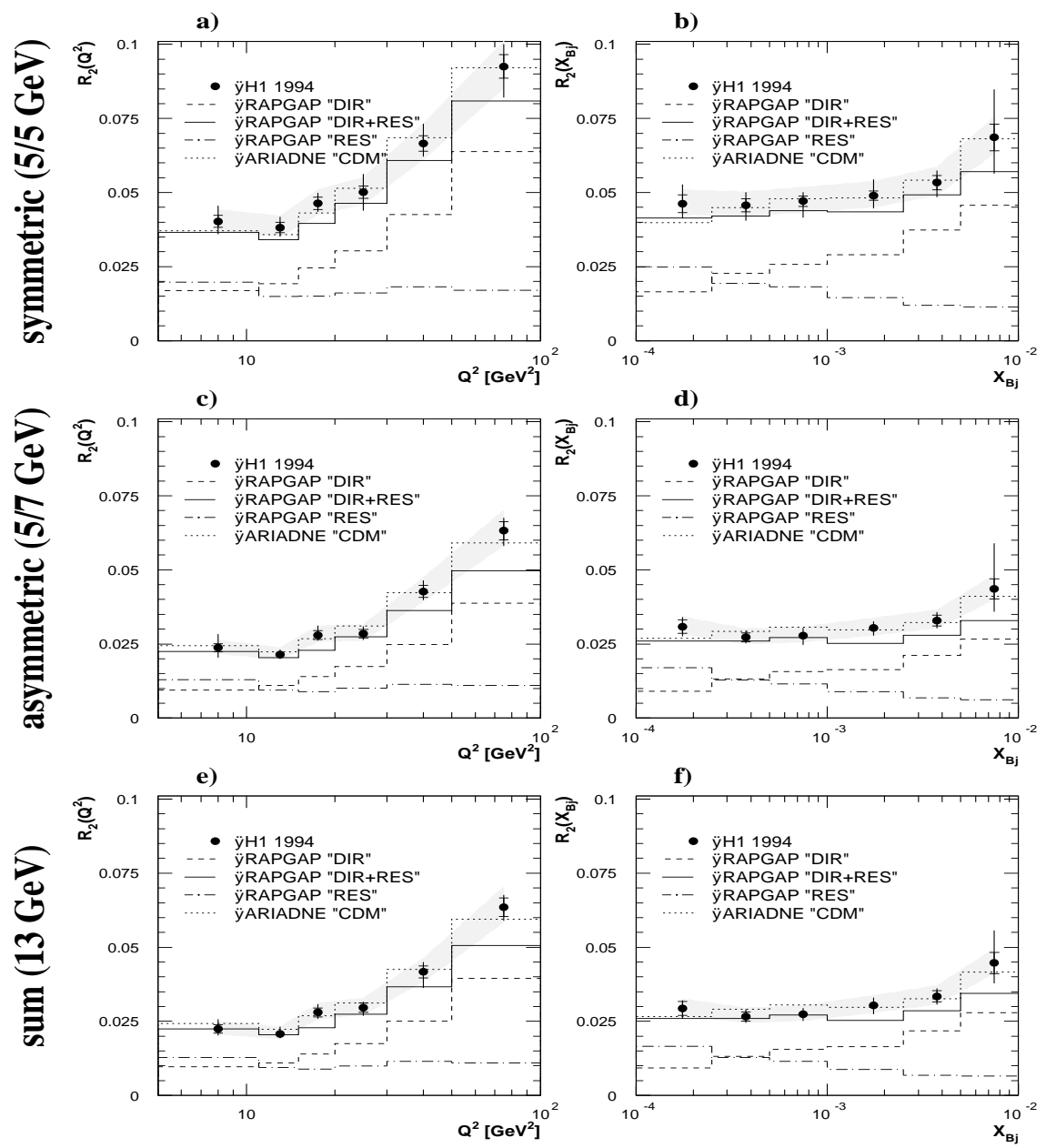

Figure 150: The dijet rate as a function of $P^{2}$ (denoted as $Q^{2}$ ) (a,c,e), integrated over $x_{B j}$, and as a function of $x_{B j}(b, d, f)$, integrated over $P^{2}$. The symmetric $(a, b)$, asymmetric $(c, d)$ and sum $(e, f)$ cut scenarios on the $p_{T}^{*}$ of the two jets are presented. The comparison is made with RAPGAP and ARIADNE predictions for the direct and resolved virtual photon contributions (from [99]). 

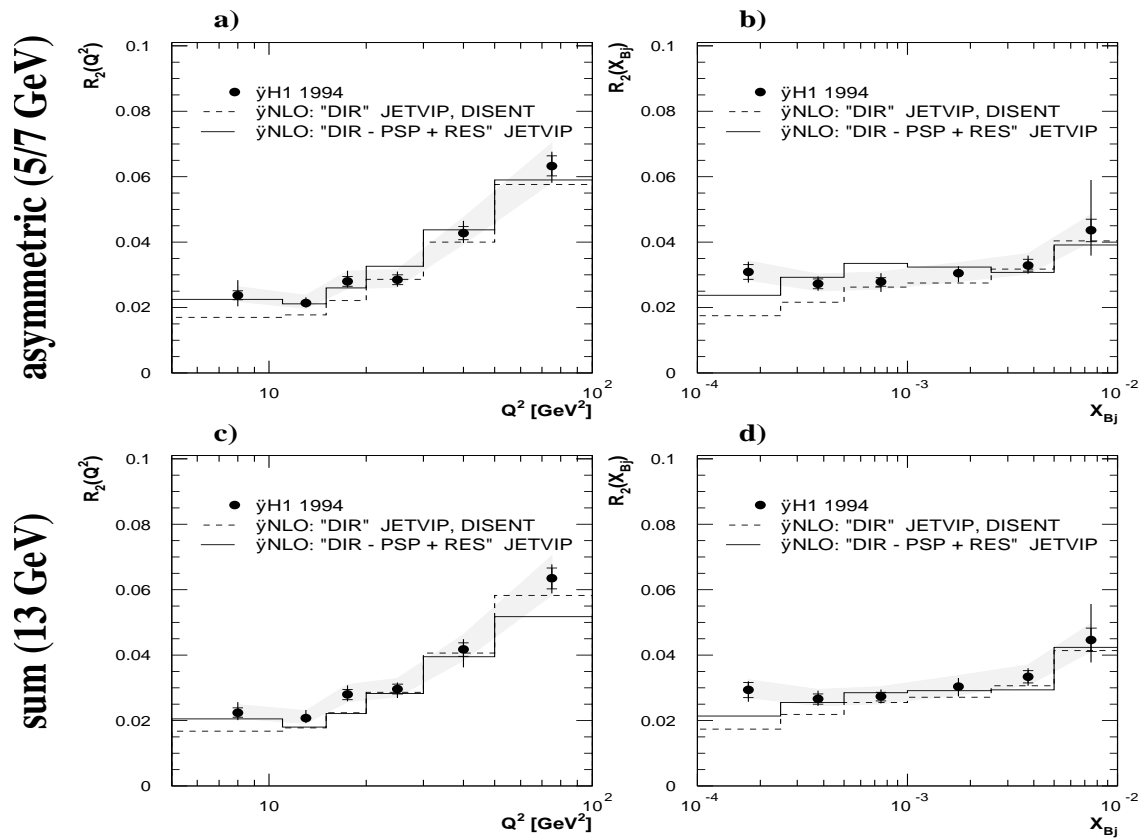

Figure 151: The dijet rate as a function of $P^{2}$ (denoted as $\left.Q^{2}\right)(a, c)$, integrated over $x_{B j}$, and as a function of $x_{B j}(b, d)$, integrated over $P^{2}$. The data (the same as in fig. 150c-f) for the asymmetric $(a, b)$ and the sum $(c, d)$ cut scenario are compared to different NLO calculations for the direct and resolved virtual photon contributions (from [99]).
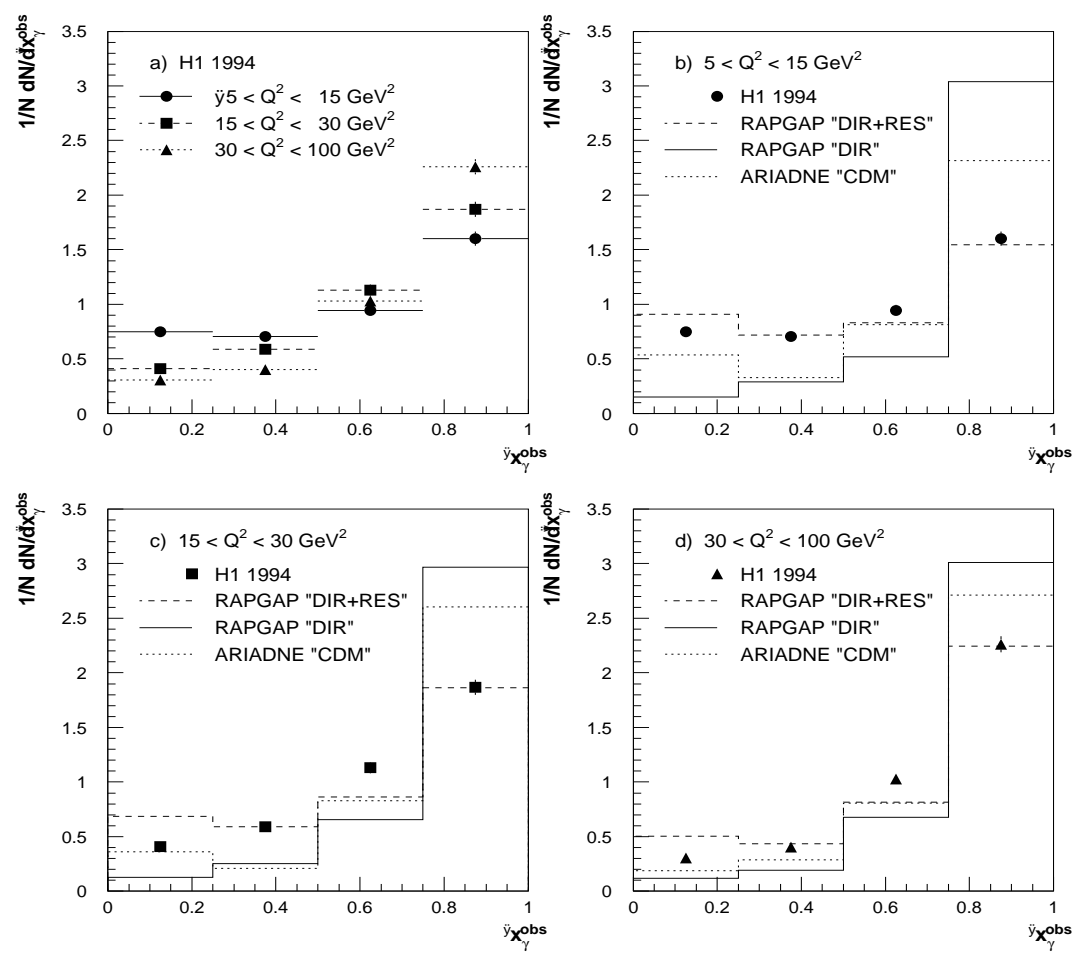

Figure 152: The uncorrected distribution of $x_{\gamma}^{\text {obs }}$ in three different $P^{2}$ (denoted as $Q^{2}$ ) bins (a). In b), c) and d) the data in different $P^{2}$ bins are compared to the RAPGAP and $A R I A D N E$ models for the direct and resolved virtual photon contributions (from 99]. 
•H1 2000c [100] (HERA)

The dijet cross sections were measured in 1996 for $1.6<P^{2}<80 \mathrm{GeV}^{2}, 0.1<y<0.7$, and $30<\bar{E}_{T}^{2}<300 \mathrm{GeV}^{2}$. For jets the ranges $|\Delta \eta|<1,-3.0<\bar{\eta}<-0.5$, and $2|\Delta E| / \bar{E}<0.25$ were assumed. Two Monte Carlo generators were used, HERWIG and RAPGAP, with different (LO and NLO) virtual photon parton densities: GRV HO/Drees-Godbole $\left(p_{T}^{\min }=3 \mathrm{GeV}\right)$, GRV LO/Drees-Godbole $\left(p_{T}^{\min }=2 \mathrm{GeV}\right)$, and $\mathrm{SaS1,2D}\left(p_{T}^{\min }=2 \mathrm{GeV}\right)$. For proton, the GRV HO and GRV LO parton parametrizations were used. The $k_{T}$ - clustering algorithm for jets was applied. Only the transversely polarized photon flux was assumed in the analysis.

The data were used to extract (for the first time) an effective LO parton density for the virtual photon.

The transverse energy flow was studied as a function of $\delta \phi$ and compared with the Monte Carlo predictions with and without soft underlying event (HERWIG and RAPGAP, respectively), see fig. 153.

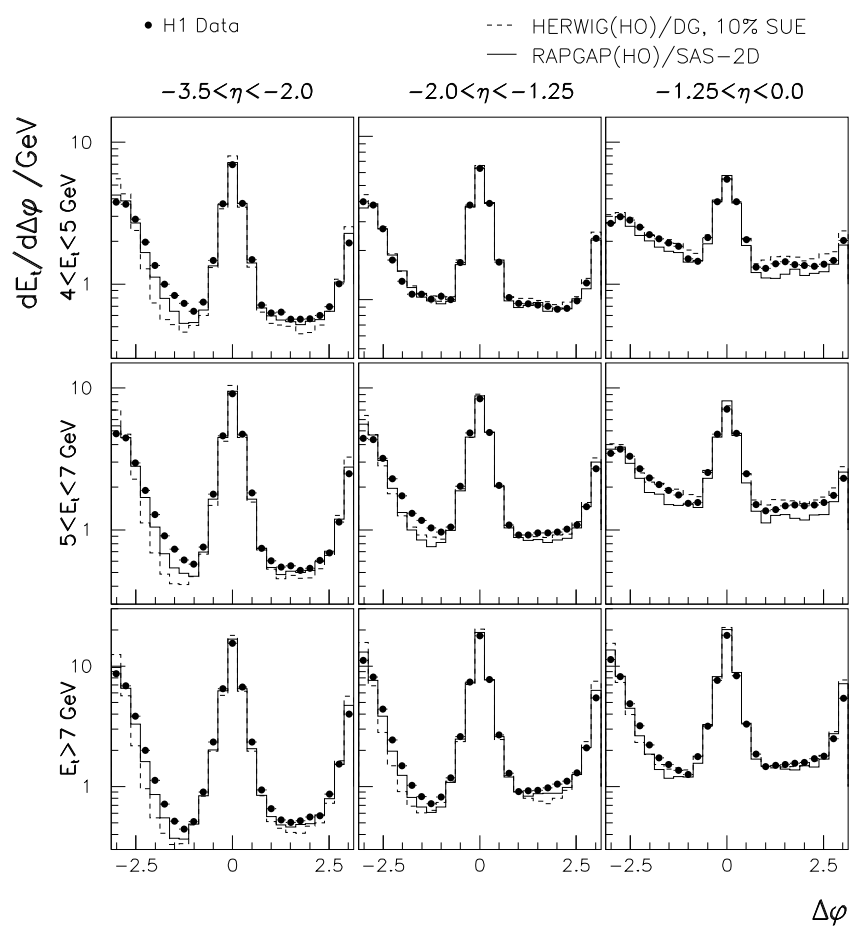

Figure 153: The transverse energy flow as a function of $\delta \phi$ for different $\eta$ bins. Comparison with predictions of HERWIG (HO) using the GRV HO/Drees-Godbole parton distribution (dashed line) and RAPGAP (HO) using the SaS2D parton distribution (solid line) is shown (from [100]).

The triple differential cross sections as a function of $x_{\gamma}, \bar{E}_{T}^{2}$ and $P^{2}\left(=Q^{2}\right)$ are presented in figs. 154, 155 and 156, respectively, together with predictions of the HERWIG and RAPGAP Monte Carlo programs. 


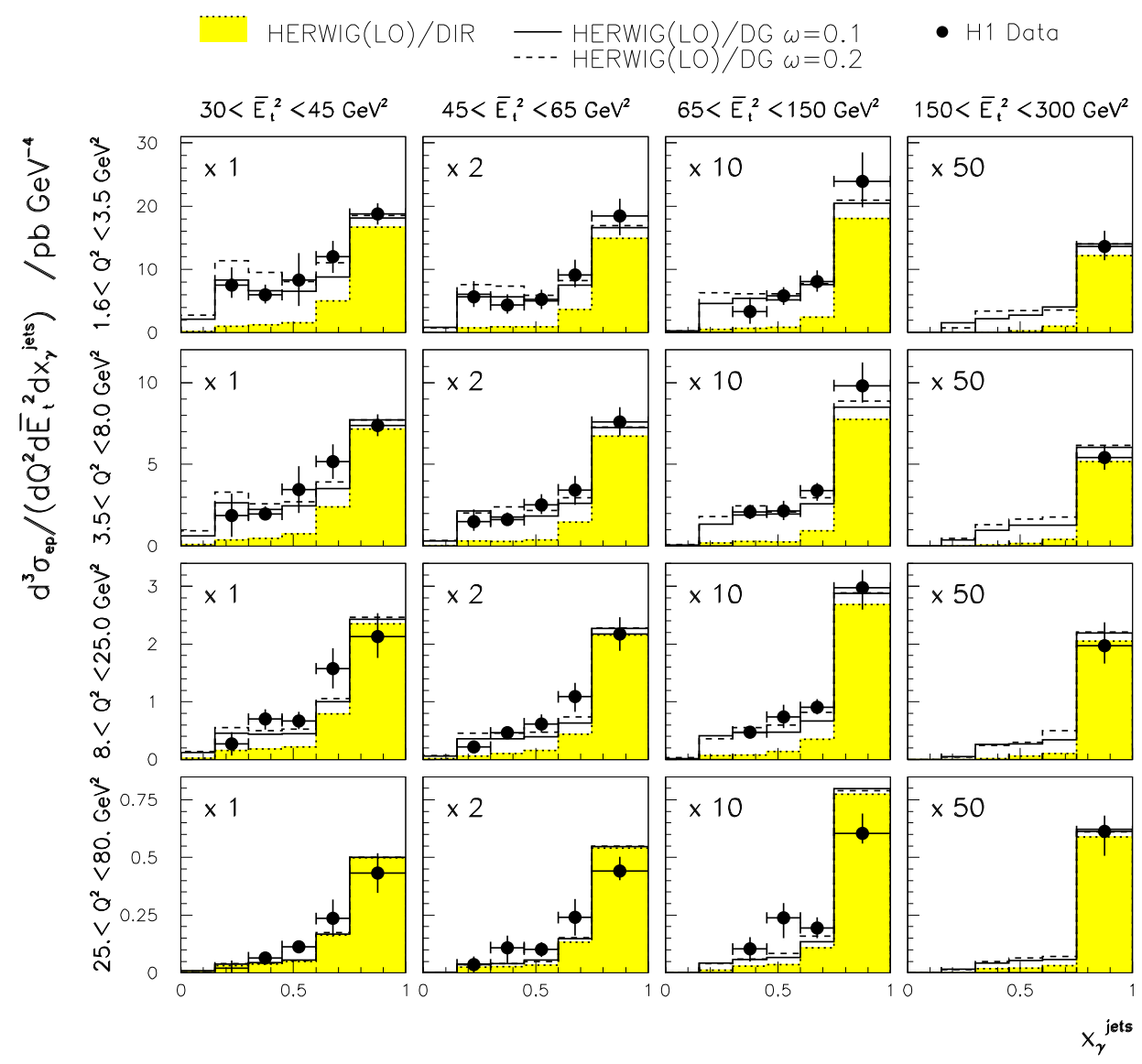

Figure 154: The differential cross section as a function of $x_{\gamma}$ in four bins of the virtuality $P^{2}$ (denoted as $Q^{2}$ ) and four bins of $\bar{E}_{T}^{2}$. The comparison with the LO prediction of the HERWIG Monte Carlo based on the GRV LO/Drees-Godbole parametrization with $\omega=0.1$ (solid line) and $\omega=0.2$ (dashed line) is shown. Also the direct contribution is displayed (dotted line) (from [100]). 
Table 28:

\begin{tabular}{|c|c|c|c|}
\hline $\begin{array}{c}P^{2} \\
{\left[G e V^{2}\right]}\end{array}$ & $\begin{array}{c}\tilde{Q}^{2}\left(=p_{t}^{2}\right) \\
{\left[G e V^{2}\right]}\end{array}$ & $x_{\gamma}$ & $\begin{array}{c}f\left(x_{\gamma}\right) / \alpha \\
(\text { stat. }+ \text { syst. })\end{array}$ \\
\hline \multirow[t]{8}{*}{2.4} & \multirow[t]{3}{*}{40.0} & 0.275 & $0.55 \pm 0.02_{-0.19}^{+0.23}$ \\
\hline & & 0.425 & $0.60 \pm 0.02_{-0.12}^{+0.15}$ \\
\hline & & 0.6 & $0.95 \pm 0.03_{-0.29}^{+0.17}$ \\
\hline & \multirow[t]{3}{*}{52.0} & 0.275 & $0.59 \pm 0.02_{-0.19}^{+0.32}$ \\
\hline & & 0.425 & $0.57 \pm 0.02_{-0.15}^{+0.20}$ \\
\hline & & 0.6 & $0.93 \pm 0.03_{-0.18}^{+0.19}$ \\
\hline & \multirow[t]{2}{*}{85.0} & 0.425 & $0.53 \pm 0.02_{-0.18}^{+0.29}$ \\
\hline & & 0.6 & $0.98 \pm 0.03_{-0.26}^{+0.21}$ \\
\hline \multirow[t]{8}{*}{5.3} & \multirow[t]{3}{*}{40.0} & 0.275 & $0.33 \pm 0.01_{-0.15}^{+0.15}$ \\
\hline & & 0.425 & $0.49 \pm 0.02_{-0.13}^{+0.14}$ \\
\hline & & 0.6 & $0.82 \pm 0.03_{-0.30}^{+0.13}$ \\
\hline & \multirow[t]{3}{*}{52.0} & 0.275 & $0.36 \pm 0.01_{-0.16}^{+0.20}$ \\
\hline & & 0.425 & $0.50 \pm 0.02_{-0.15}^{+0.14}$ \\
\hline & & 0.6 & $0.85 \pm 0.03_{-0.25}^{+0.14}$ \\
\hline & \multirow[t]{2}{*}{85.0} & 0.425 & $0.64 \pm 0.02_{-0.21}^{+0.19}$ \\
\hline & & 0.6 & $0.87 \pm 0.03_{-0.23}^{+0.15}$ \\
\hline \multirow[t]{8}{*}{12.7} & \multirow[t]{3}{*}{40.0} & 0.275 & $0.16 \pm 0.01_{-0.08}^{+0.07}$ \\
\hline & & 0.425 & $0.42 \pm 0.02_{-0.19}^{+0.09}$ \\
\hline & & 0.6 & $0.54 \pm 0.02_{-0.25}^{+0.10}$ \\
\hline & \multirow[t]{3}{*}{52.0} & 0.275 & $0.18 \pm 0.01_{-0.09}^{+0.09}$ \\
\hline & & 0.425 & $0.45 \pm 0.02_{-0.20}^{+0.09}$ \\
\hline & & 0.6 & $0.62 \pm 0.03_{-0.23}^{+0.10}$ \\
\hline & \multirow[t]{2}{*}{85.0} & 0.425 & $0.46 \pm 0.02_{-0.21}^{+0.12}$ \\
\hline & & 0.6 & $0.74 \pm 0.03_{-0.27}^{+0.14}$ \\
\hline \multirow[t]{2}{*}{40.0} & \multirow[t]{2}{*}{85.0} & 0.425 & $0.43 \pm 0.04_{-0.27}^{+0.09}$ \\
\hline & & & $0.65 \pm 0.04_{-0.41}^{+0.19}$ \\
\hline
\end{tabular}




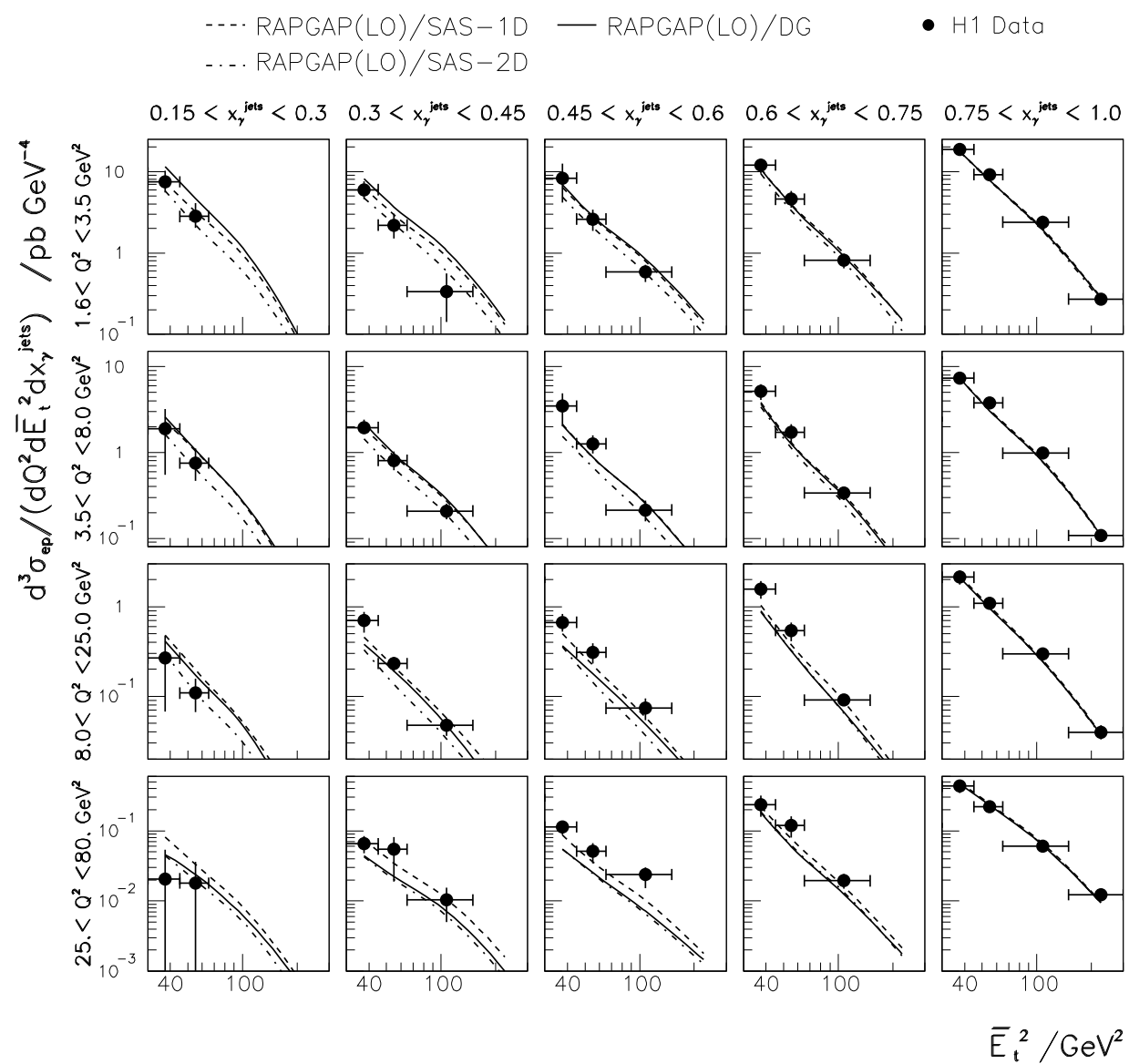

Figure 155: The differential cross section as a function of $\bar{E}_{T}^{2}$ in four bins of the virtuality $P^{2}$ (denoted as $Q^{2}$ ) and five bins of $x_{\gamma}$. The comparison with the LO prediction of the RAPGAP Monte Carlo using the SaS1D (dashed line), SaS2D (dash-dotted line) and GRV LO/Drees-Godbole (solid line) parton distributions in the photon is shown (from [100]). 


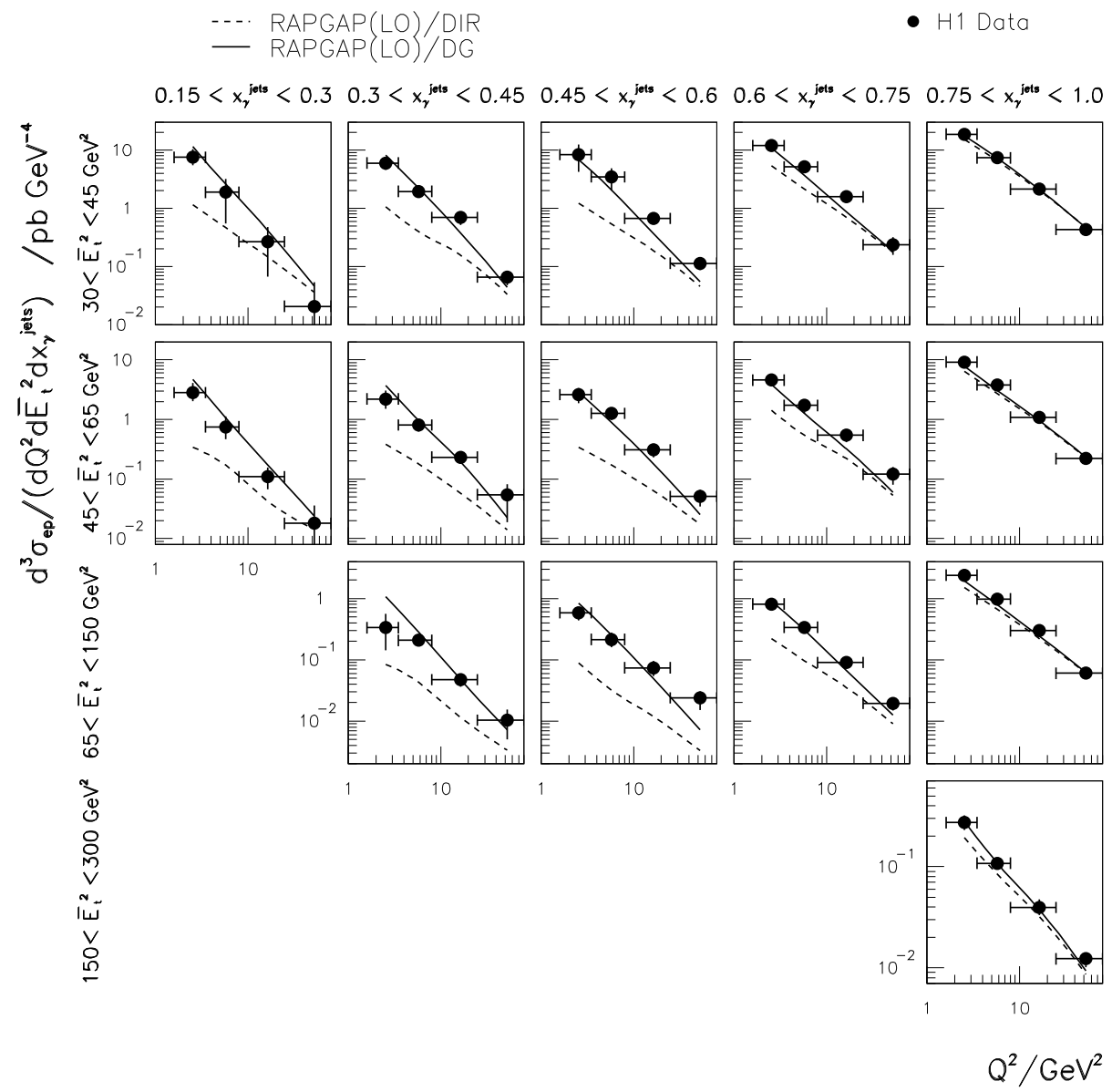

Figure 156: The differential cross section as a function of virtuality $P^{2}$ (denoted as $Q^{2}$ ) in four bins of $\bar{E}_{T}^{2}$ and five bins of the $x_{\gamma}$. The comparison with the $L O$ prediction of the RAPGAP Monte Carlo: using the GRV LO/Drees-Godbole parton distributions in the photon (solid line) and the the direct contribution only (dashed line) is shown (from 100]).

The Single Effective Subprocess Approximation [54 was assumed in the analysis and the effective (LO) parton density of the virtual photon

$$
\alpha^{-1} x_{\gamma}\left(\tilde{q}^{\gamma}+\frac{9}{4} G^{\gamma}\right)
$$

with $\tilde{q}^{\gamma}=\sum\left(q^{\gamma}+\bar{q}^{\gamma}\right)$, was extracted for the first time. Here $q^{\gamma}\left(\bar{q}^{\gamma}\right)$ and $G^{\gamma}$ are photon polarization-averaged parton densities. This effective density is presented in table 28 . In figs. 157, 158 and 159 it is displayed as a function of $x_{\gamma}, p_{t}^{2}$ (playing the role of the hard scale $\tilde{Q}^{2}$ ) and $P^{2}$, respectively. Only points where averaged $p_{t}^{2}$ is larger than $<P^{2}>$ are shown.

In fig. 160 the obtained LO effective parton density as a function of the virtuality $P^{2}$ is shown for $x_{\gamma}=0.425$ and 0.6 , at $\tilde{Q}^{2}=p_{t}^{2}$. 


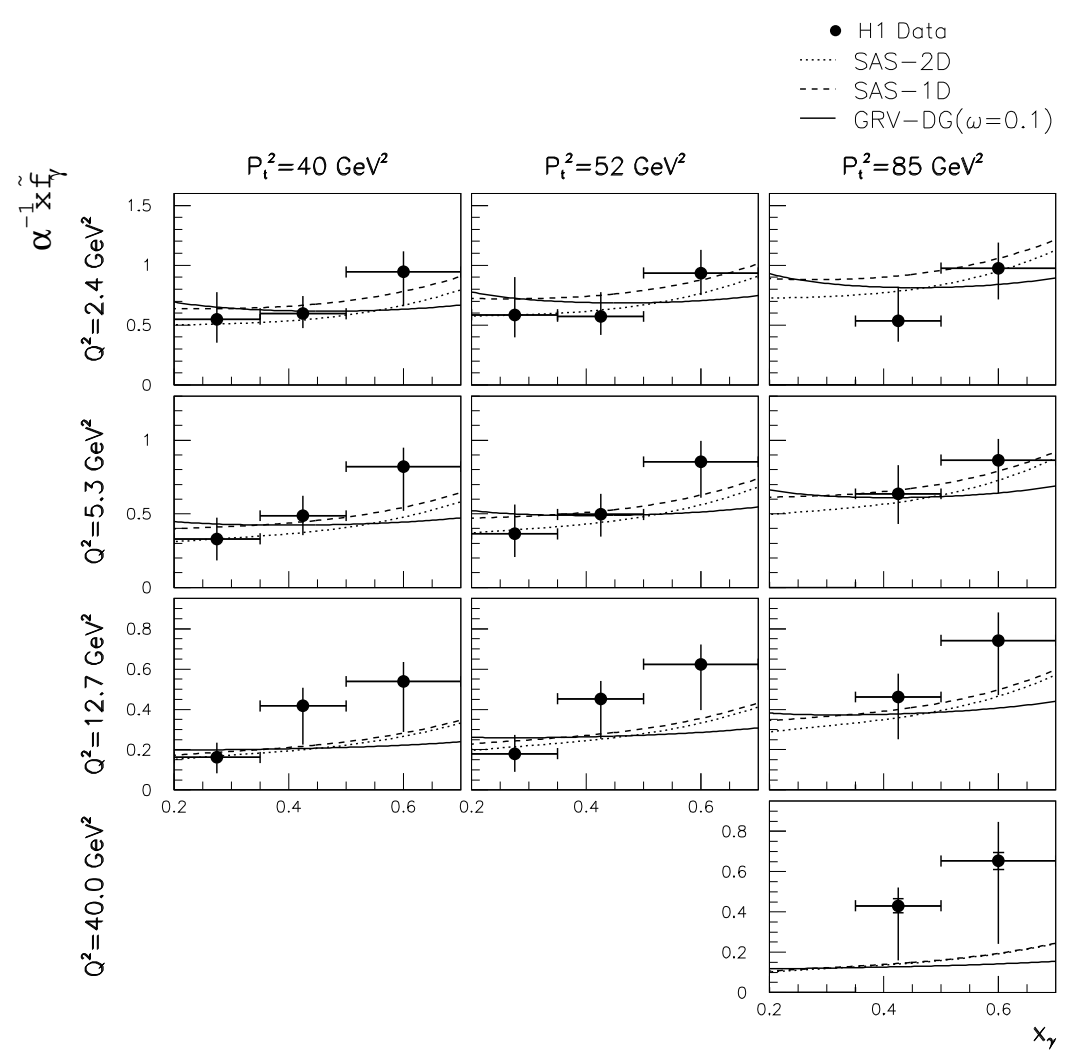

Figure 157: The effective parton density as a function of $x_{\gamma}$ for various values of the parton transverse momentum squared $\tilde{Q}^{2}\left(=p_{t}^{2}\right)$ and virtuality $P^{2}$ (denoted as $Q^{2}$ ). The comparison with predictions of the SaS2D (dotted line), SaS1D (dashed line) and DreesGodbole (with GRV LO parametrization for the real photon and using $\omega=0.1$, solid line) parton distributions in the photon is shown (from [100]).

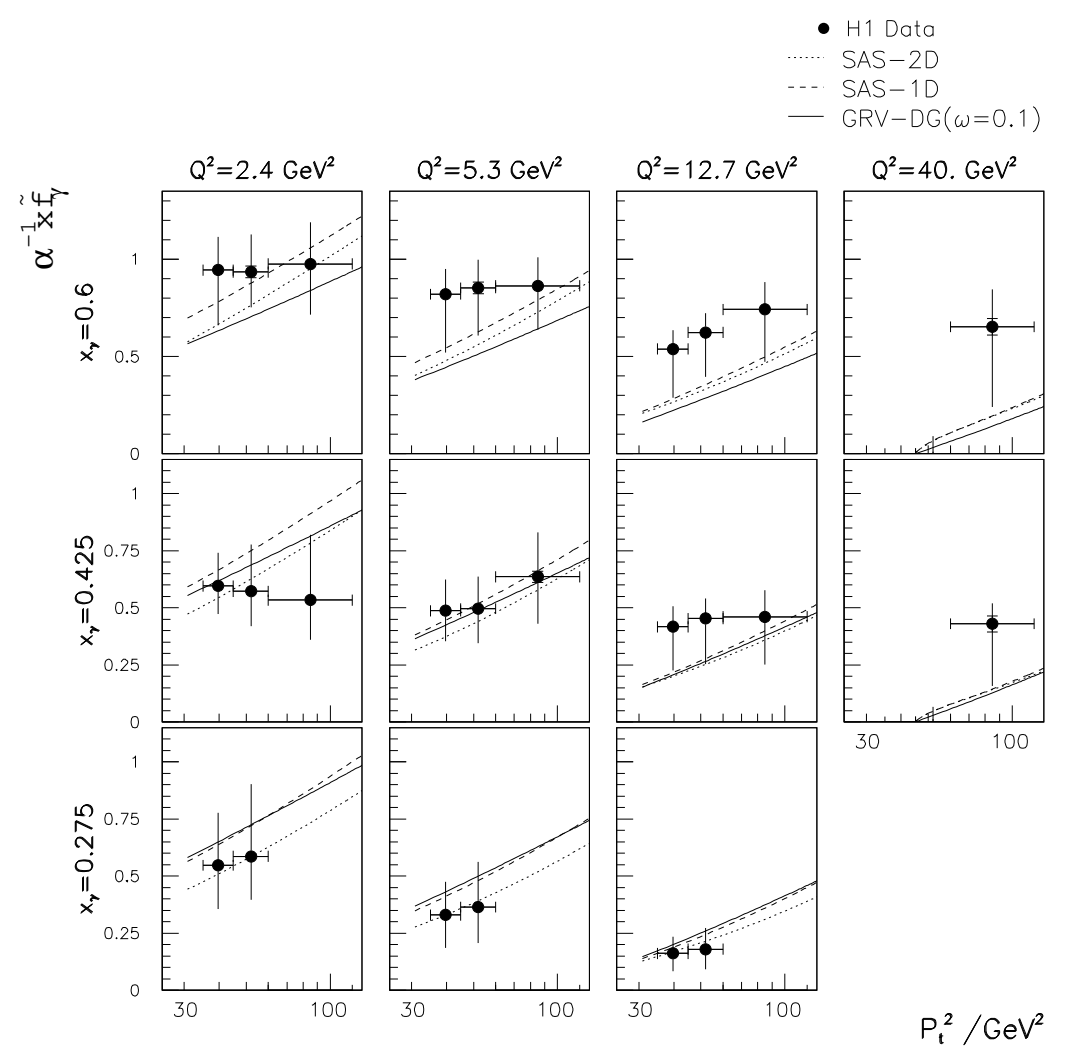

Figure 158: The effective parton density as a function of $\tilde{Q}^{2}\left(=p_{T}^{2}\right)$ for various values of $x_{\gamma}$ and virtuality $P^{2}$ (denoted as $Q^{2}$ ). Comparison with predictions as in fig. 157 (from [100). 


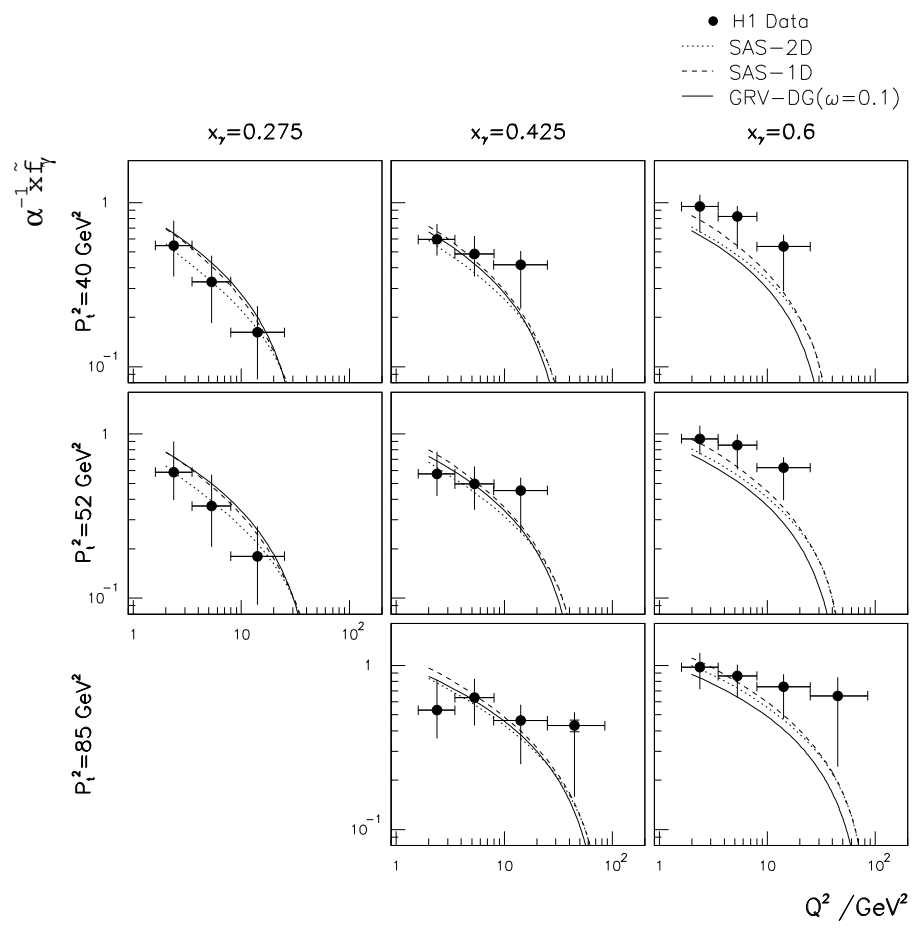

Figure 159: The effective parton density as a function of $P^{2}$ (denoted as $Q^{2}$ ) for various $\tilde{Q}^{2}$ $\left(=p_{t}^{2}\right)$ and $x_{\gamma}$. Comparison with predictions as in fig. 157 (from [100]).
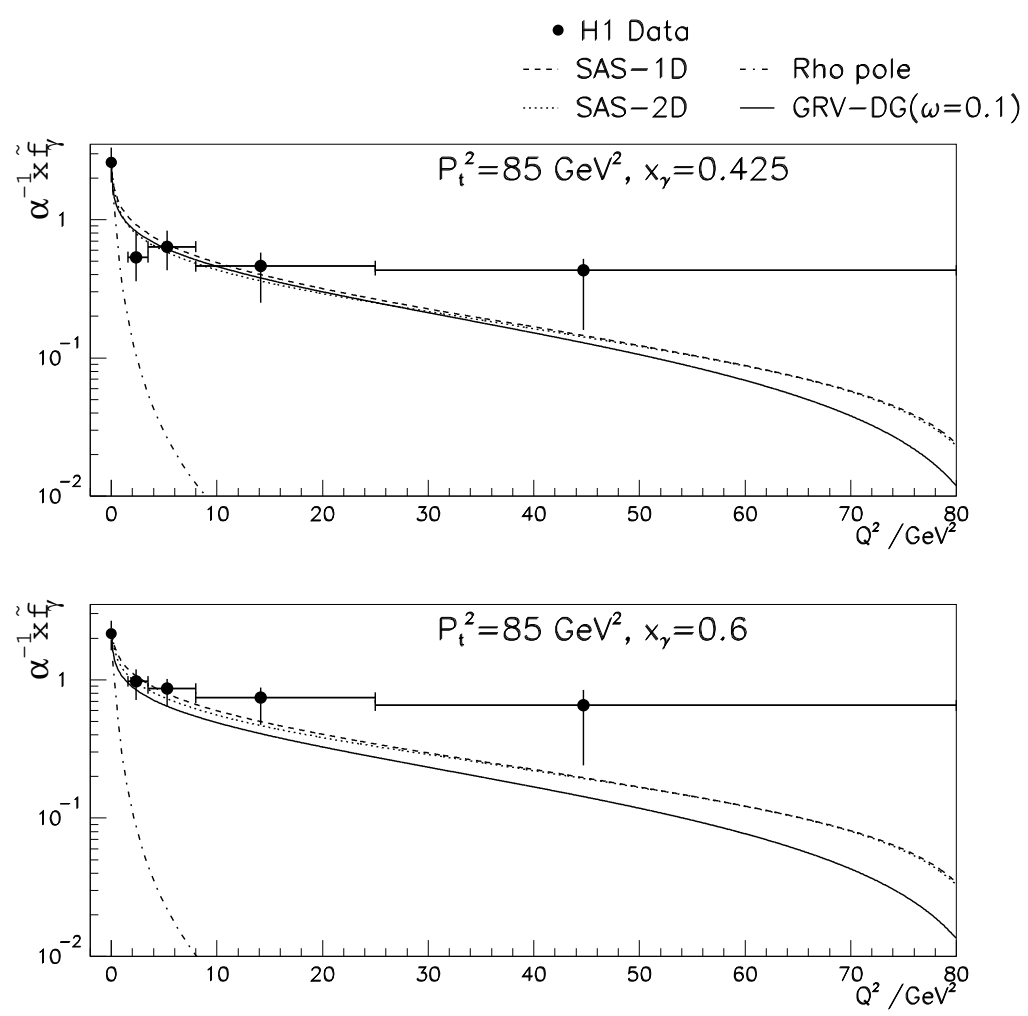

Figure 160: The LO effective parton density as a function of $P^{2}$ (denoted as $\left.Q^{2}\right)$ for $\tilde{Q}^{2}$ (= $\left.p_{t}^{2}\right)=85 \mathrm{GeV}^{2}, x_{\gamma}=0.425$ and 0.60. Comparison with predictions as in fig. 157 (from 100).

Comment: "Both simulations give a good description of the energy flow in the core of 
the jets", neither Monte Carlo model is able to describe the pedestal for all ranges of $E_{T}$ and $\eta$ (fig. 159).

-ZEUS 93 [101] (HERA)

The evidence for events with two or more jets in $\operatorname{DIS}_{e p}$ (neutral current interaction) is presented for $Q^{2}>4 \mathrm{GeV}^{2}$ (1992 data).

\section{-ZEUS 95d [102] (HERA)}

The two-jet production in $\mathrm{DIS}_{e p}$ for $160<Q^{2}<1280 \mathrm{GeV}^{2}, 0.01<x_{B j}<0.1$ and $0.04<y<0.95$ was studied. In reconstructing jets the JADE algorithm was used (in the HERA laboratory frame). The LEPTO 6.1 generator was used in the analysis. The comparison was made with the NLO calculations.

\section{- ZEUS 2000b [103] (HERA)}

Dijet production for photon virtualities $P^{2}$ from 0 up to $4.5 \mathrm{GeV}^{2}$ was measured, based on the data collected in 1995. Events corresponding to $0.2<y<0.55, E_{T 1,2}>5.5$ $\mathrm{GeV}$ and $-1.125<\eta<2.2$ were studied in three $P^{2}$ bins: $P^{2} \approx 0$ (a quasi-real photon sample obtained by antitagging condition, which corresponds to $P^{2}<1.0$ $\mathrm{GeV}^{2}$ with median $\left.10^{-3} \mathrm{GeV}^{2}\right), 0.1-0.55 \mathrm{GeV}^{2}$ and $1.5-4.5 \mathrm{GeV}^{2}$. The $k_{T}$ algorithm and the HERWIG 5.9 program (with $p_{T}^{\min }=2.5 \mathrm{GeV}$ ), with the GRV LO (photon) and MRSA (proton) parton parametrizations were used in the analysis of data. The possible multiple parton interaction ('underlying event') was simulated for the quasireal photon sample (denoted MI). To estimate uncertainties due to modelling of the jet fragmentation the PYTHIA model was used in addition. The LEPTO model for the direct $\gamma^{*} q$ events and the NLO prediction for the resolved $\gamma^{*}$ processes (JeTViP) are compared with data.

Uncorrected $x_{\gamma}$ distributions are presented in fig. 161 for real and for virtual photons (in two bins), and compared to the HERWIG predictions for the direct and resolved (LO) photon contributions.

In the low $x_{\gamma}$ region the data for quasi-real photons disagree with $\mathrm{MC}$ prediction. "Disagreement is also observed in the $\eta, y_{B j}$, and $E_{T}$ distributions (not shown) and can be attributed to the presence of underlying event effects or uncertainty in the PDFs of the photon." The inclusion of MI is not enough to reproduce the shape of the low $x_{\gamma}$ data (fig. 161a), so a reweighting has to be performed to include this fact in the correction of the data for migrations and acceptance. The obtained results (showing an agreement with data) are presented in fig. 161a (dashed histogram), also the $\eta$, $y_{B J}$, and $E_{T}$ distributions (not shown) "agree well with data". 

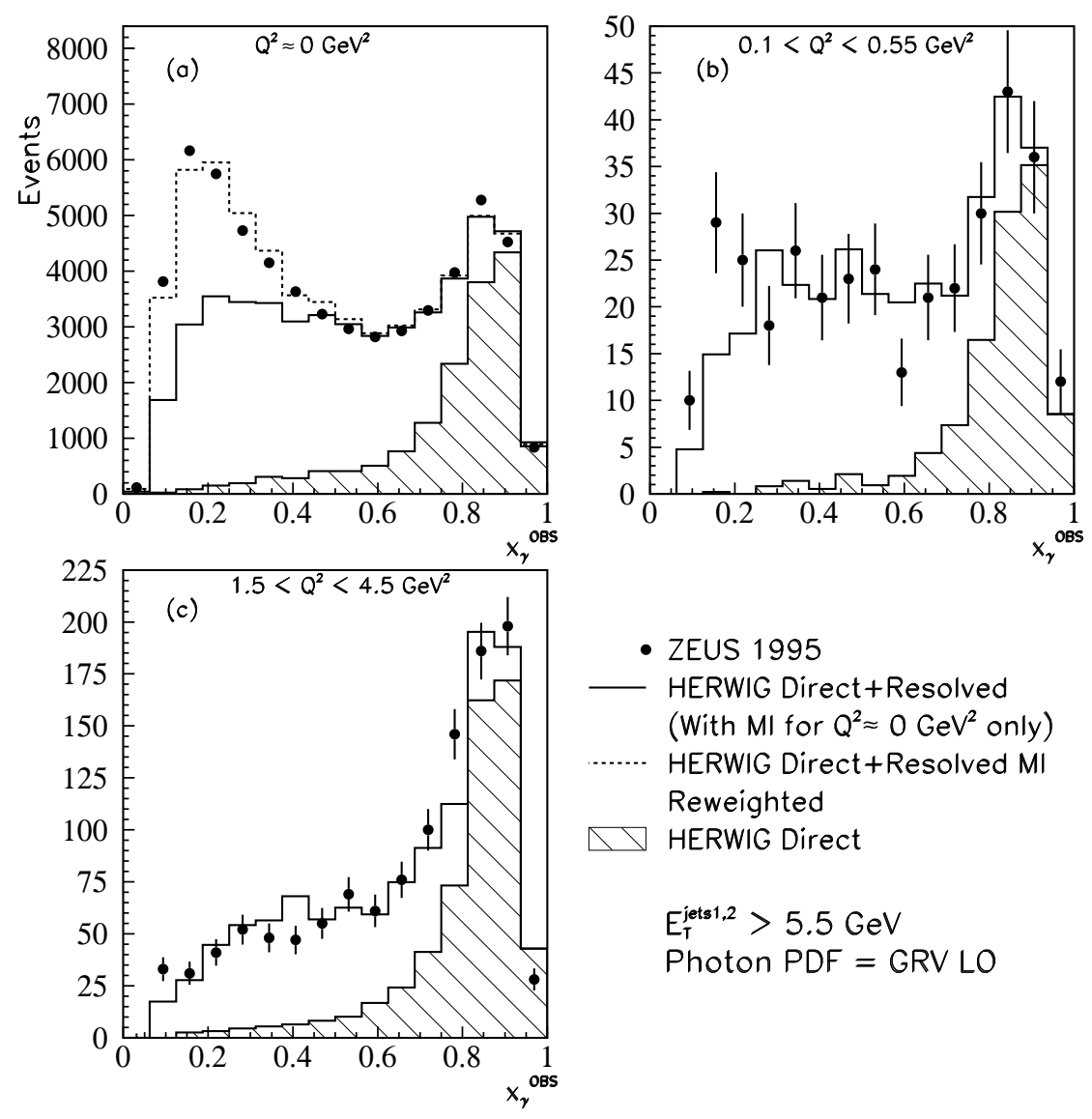

- ZEUS 1995

- HERWIG Direct+Resolved (With $\mathrm{Ml}$ for $\mathrm{Q}^{2} \approx 0 \mathrm{GeV}^{2}$ only) HERWIG Direct+Resolved MI Reweighted HERWIG Direct

$E_{\mathrm{T}}^{\mathrm{jets} 1,2}>5.5 \mathrm{GeV}$

Photon PDF $=$ GRV LO

Figure 161: Uncorrected $x_{\gamma}^{\text {obs }}$ distributions for various photon virtualities: a) $P^{2}$ (denoted here as $\left.Q^{2}\right)=0$, b) $0.1 \leq P^{2} \leq 0.55 G e V^{2}$, and c) $1.5 \leq P^{2} \leq 4.5 G e V^{2}$. Events with $E_{T 1,2}>$ $5.5 \mathrm{GeV},-1.125<\eta<2.2$ were included. The predictions of the HERWIG program (GRV LO) are shown for: a direct contribution (shaded histogram), a direct + resolved + MI (only for $P^{2}=0$ sample) contribution (solid histogram), in a) a reweighted prediction for a direct + resolved + MI contribution (dashed histogram) (from [103]).

The dijet cross section $d \sigma / d x_{\gamma}$, corrected to the hadron level, is presented in fig. 162 for a low $E_{T}$ sample, where, as above, $E_{T 1,2}>5.5 \mathrm{GeV}$ and $-1.125<\eta<2.2$. The data are compared with prediction of the HERWIG program, with different parton parametrizations for the photon: GRV LO and WHIT2 (without MI and with MI, then assuming $p_{T}^{m i}=2 \mathrm{GeV}$ ), which are valid for a real photon, and SaS1D. In addition a comparison with prediction of the LEPTO program is shown for events with $P^{2}>1.5$ $\mathrm{GeV}^{2}$. 

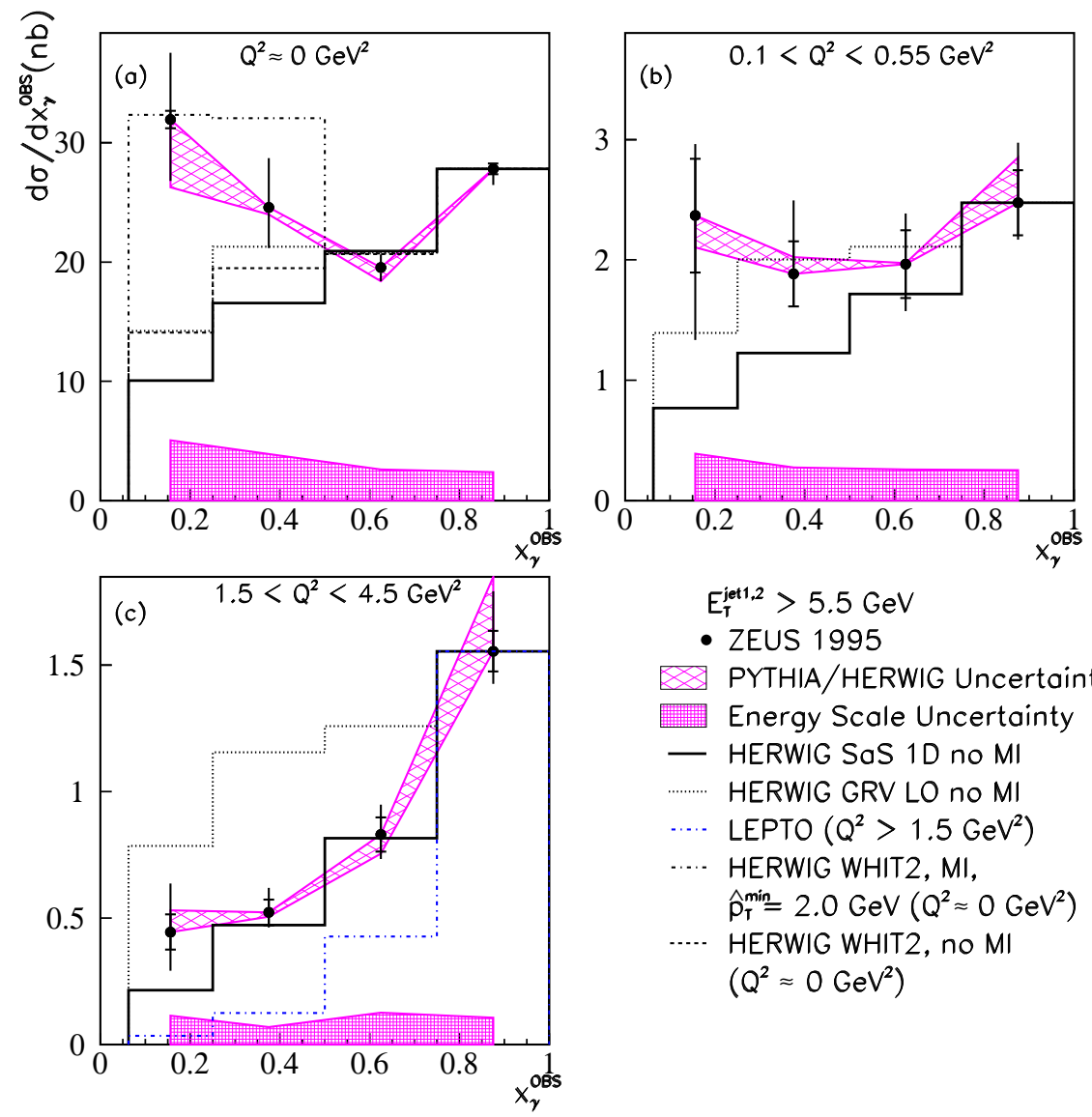

$$
\begin{aligned}
& E_{T}^{\text {jet1,2 }}>5.5 \mathrm{GeV} \\
& \text { - ZEUS } 1995 \\
& \text { PYTHIA/HERWIG Uncertainty } \\
& \text { Energy Scale Uncertainty } \\
& \text { - HERWIG SaS 1D no MI } \\
& \text { …... HERWIG GRV LO no MI } \\
& \cdots \operatorname{LEPTO}\left(\mathrm{Q}^{2}>1.5 \mathrm{GeV}^{2}\right) \\
& \text {.... HERWIG WHIT2, MI, } \\
& \hat{\mathrm{P}}_{\mathrm{T}}^{\text {min }}=2.0 \mathrm{GeV}\left(\mathrm{Q}^{2} \approx 0 \mathrm{GeV}^{2}\right) \\
& \text {.... HERWIG WHIT2, no MI } \\
& \left(Q^{2} \approx O \mathrm{GeV}^{2}\right)
\end{aligned}
$$

Figure 162: The $d \sigma / d x_{\gamma}^{\text {obs }}$ cross section for various photon virtualities: a) $P^{2}$ (denoted here as $\left.Q^{2}\right)=0$, b) $0.1 \leq P^{2} \leq 0.55 \mathrm{GeV}^{2}$, and c) $1.5 \leq P^{2} \leq 4.5 \mathrm{GeV}^{2}$, for low $E_{T}$ sample: $E_{T 1,2}>5.5 \mathrm{GeV}$, and $-1.125<\eta<2.2$. The predictions of the HERWIG program with GRV LO (SaS1D) are shown by dotted (solid) histograms. In a) the HERWIG predictions obtained using the WHIT2 parton paramerization with and without MI (dot-dashed and dashed histograms) are shown, in c) the LEPTO prediction (dot-dashed) is given. The shaded bound represents uncertainties in the simulation (from [103]).

Similar analysis has been performed for the high $E_{T}$ sample, where $E_{T 1}>7.5 \mathrm{GeV}$, $E_{T 2}>6.5 \mathrm{GeV}$ and $-1.125<\eta<1.875$, see fig. 163 for results. 

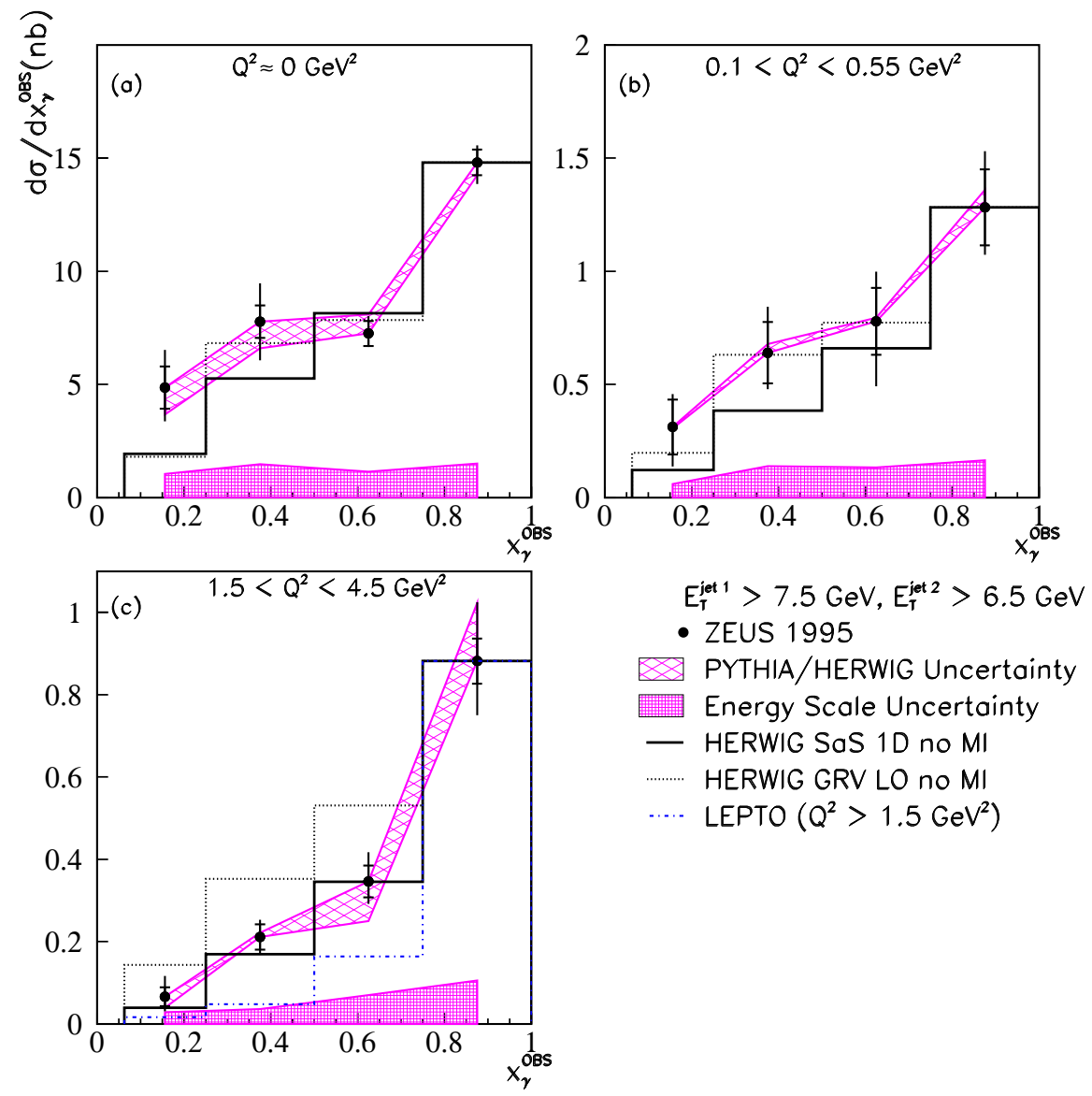

Figure 163: The $d \sigma / d x_{\gamma}^{\text {obs }}$ cross section for various photon virtualities: a) $P^{2}$ (denoted here as $\left.Q^{2}\right)=0$, b) $0.1 \leq P^{2} \leq 0.55 \mathrm{GeV}^{2}$, and c) $1.5 \leq P^{2} \leq 4.5 \mathrm{GeV}^{2}$, for high $E_{T}$ sample: $E_{T 1}>7.5 \mathrm{GeV}, E_{T 2}>6.5 \mathrm{GeV}$, and $-1.125<\eta<1.875$. The predictions of the HERWIG program with GRV LO (SaS1D) are shown by dotted (solid) histograms. In c) the LEPTO prediction (dot-dashed histogram) is given. The shaded bound represents uncertainties of the simulation (from [103]).

The ratio $\sigma\left(x_{\gamma}<0.75\right) / \sigma\left(x_{\gamma}>0.75\right)$ as a function of the photon virtuality $P^{2}$ is shown in fig. 164 for both sets of $E_{T}$ cuts. Here a comparison with the prediction of the HERWIG program with the GRV LO and SaS1D parton parametrizations is shown. The small $P^{2}$ region is not properly modelled. Also the LEPTO program predictions are shown for $P^{2}>1.5 \mathrm{GeV}$. The NLO QCD predictions obtained by using the JeTViP program with the SaS1D and GS96/Drees-Godbole parton parametrizations and the squared hard scale equal to $P^{2}+p_{T}^{2}$, are compared to high $E_{T}$ data. They "lie well below the data." 


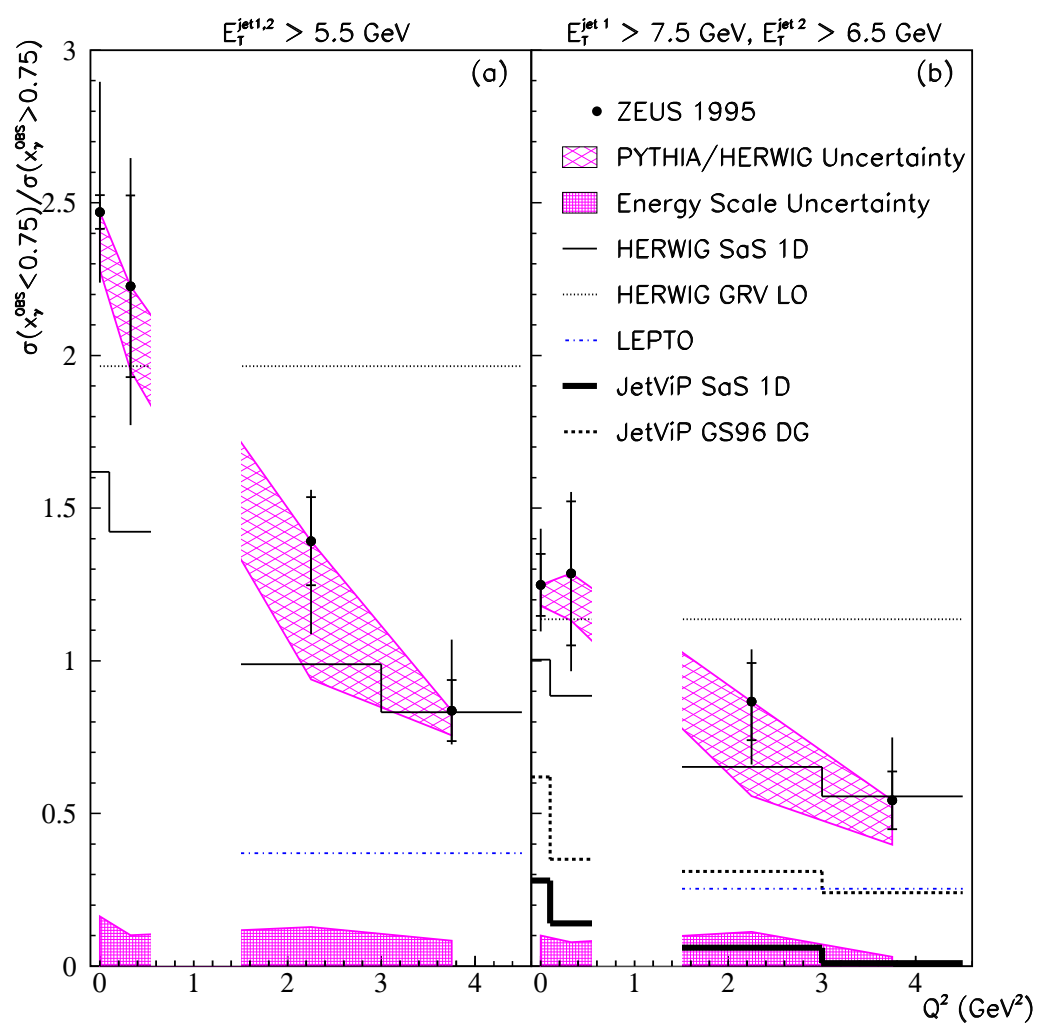

Figure 164: The ratio $\sigma\left(x_{\gamma}^{o b s}<0.75\right) / \sigma\left(x_{\gamma}^{o b s}>0.75\right)$ as a function of the photon virtuality $P^{2}$ (denoted as $Q^{2}$ ) for both $E_{T}$ cuts. The predictions of the HERWIG program with the GRV LO $($ SaS1D) parton parametrization are presented as dotted (solid) histograms. The LEPTO program predictions are shown for $P^{2}>1.5 \mathrm{GeV}$ (dot-dashed histograms). In b) the NLO QCD prediction of the JeTViP program with the SaS1D (GS96/Drees-Godbole) parton parametrization with the squared hard scale $P^{2}+p_{T}^{2}$ is shown as thick solid (dashed) histogram (from [103]).

Comment: "The shape of the dijet cross sections, $d \sigma / d x_{\gamma}$, is compared to the prediction of HERWIG MC for a variety of photon PDFs. None of these models is able to explain the data for both high- and low- $E_{T}$ cuts in all $P^{2}$."

For the dependence of the ratio $\sigma\left(x_{\gamma}<0.75\right) / \sigma\left(x_{\gamma}>0.75\right)$ on the photon virtuality $P^{2} \ldots$ " none of the LO model, or the NLO calculation examined here, gives a good description of the data across the full kinematic region."

Note, that data were corrected for acceptance, smearing and kinematical cut using HERWIG 5.9 with the GRV LO parton parametrization, valid for a real $\gamma$. 


\subsection{Forward jet (particle) production in $\gamma^{*} p$ collisions and the resolved virtual photon contribution}

The production of high $E_{T}$ jets (particles) in the direction of the proton in events with large virtualities, i.e. DIS $S_{e p}$ events, small $x_{B j}$ and $E_{T}^{2} \sim Q^{2}$ was studied by both the $\mathrm{H} 1$ and ZEUS groups at the HERA collider.

The contribution due the standard DGLAP evolution based on the strong $p_{t}$ ordering of parton emission along the whole chain between the proton and the photon is expected to be strongly suppressed for such events. In contrast, signals from the non-strongly ordered in transverse momenta partons from the proton (the BFKL 28 or CCFM [30] evolution) are expected to show up [200]. At first the BFKL calculation for the forward jet (particle) production was based on the LO approach, recently the modified LO BFKL approach has appeared including the kinematical constraint, called the consistency constraint, on the gluon emission. This constraint presumably embodies a major part of the NLO $1 / x$ corrections [203], see also discussion on this

point in [104]. Besides the BFKL, the mechanism with the two DGLAP evolution chains corresponding to the resolved virtual photon-proton interaction may also lead to forward jets (or particles), as was pointed out in 201. The NLO prediction of this contribution, based on the parton level generator JeTViP [196], was performed in 199. Several Monte Carlo programs were used to model the radiation characteristics for a particular evolution. The HERWIG and LEPTO models are based on DGLAP, while LDC (called also LDCMC) - on the CCFM equation. In the ARIADNE program, which implements the Color Dipole Model, there is no $p_{t}$ ordering, so it has the properties of the BFKL evolution. The RAPGAP generator (also JeTViP) contains the resolved virtual photon contribution with the DGLAP cascades.

Both forward jet and forward single particle production are measured. The advantage of studying single particles is the potential to reach a smaller angle than it is possible with jets.

The scale for the deep inelastic scattering on the proton $\left(\mathrm{DIS}_{e p}\right), Q^{2}$, plays the role of $P^{2}$, if the contribution due to the resolved virtual photon is considered. For the sake of transparency, in this section we keep $Q^{2}$ notation for the virtuality of the photon. 


\section{DATA}

•H1 95b 105 (HERA)

The first analysis of the forward jets in DIS $_{e p}$ was done.

•H1 97c [106] (HERA)

The $p_{T}$ distribution of charged particles in $\mathrm{DIS}_{e p}$ events was studied. The hadronic final state was analysed in order to test the different schemes for parton evolution in the proton (DGLAP and BFKL).

Comment: The large parton radiation between the current and the proton remnant system was found.

$\bullet H 1$ 99b 107] (HERA)

The production of forward jets and forward particles (the charged particles and the neutral pions) with large $p_{T}$ is analysed in the DIS D $_{e}$ events at small $x_{B j}$ (1994 data). The forward particles were measured for $5^{0}<\theta<25^{0}$ (LAB), and for the energy ratio $x_{c p(\pi)}=E_{c p(\pi)} / E_{p}$ between 0.01 and 0.15 (for charged particles below 0.015 only) . Two $p_{T}$ thresholds were used, 1 and $2 \mathrm{GeV}$ (only for $\pi^{0}$ ), with additional requirement $0.0002<x_{B j}<0.00235$. Forward jets with the energy ratio $x_{j e t}=E_{\text {jet }} / E_{p}>0.035$, and $0.5<p_{T}^{2} / Q^{2}<2,7^{\circ}<\theta_{\text {jet }}<20^{\circ}$ and $p_{T}>3.5$ and $5 \mathrm{GeV}$ were studied $\left(10^{-4}<x_{B j}<4 \cdot 10^{-3}\right)$. A simple cone algorithm with $\mathrm{R}=1$ was used for jets.

The predictions of the LO BFKL calculation [202 and of the Monte Carlo generators: LEPTO 6.5 (with renormalization scale $Q^{2}$ ), LDC 1.0 and ARIADNE 4.08 (scale $p_{T}^{2}$ ) were compared with data. The RAPGAP 2.06 generator with resolved photon processes (with SaS2D parton parametrization for the photon, assuming scale $Q^{2}+p_{T}^{2}$ ) was also used in comparison. To describe a proton the GRV HO parton densities were used in all the programs. The transverse energy flow as a function of $\delta \eta$ was measured for jets and results are presented in fig. 165 .
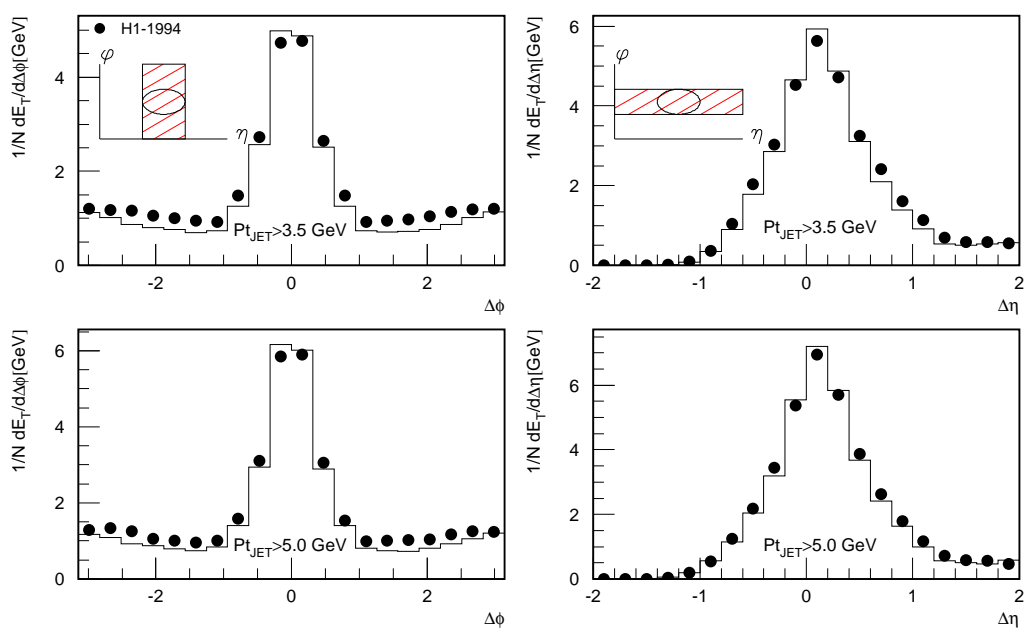

Figure 165: The transverse energy flow as a function of $\delta \phi$ and $\delta \eta$ for two $p_{T}$ cuts for jets (from [107]).

The comparison of the measured $x_{B j}$ distribution with prediction of different types 
of Monte Carlo models for single particle spectra is shown in fig. 166. Here also the comparison with the results of the analytical calculation based on the BFKL approach [202], with the factor $\frac{1}{2}$ to describe the data, is made.
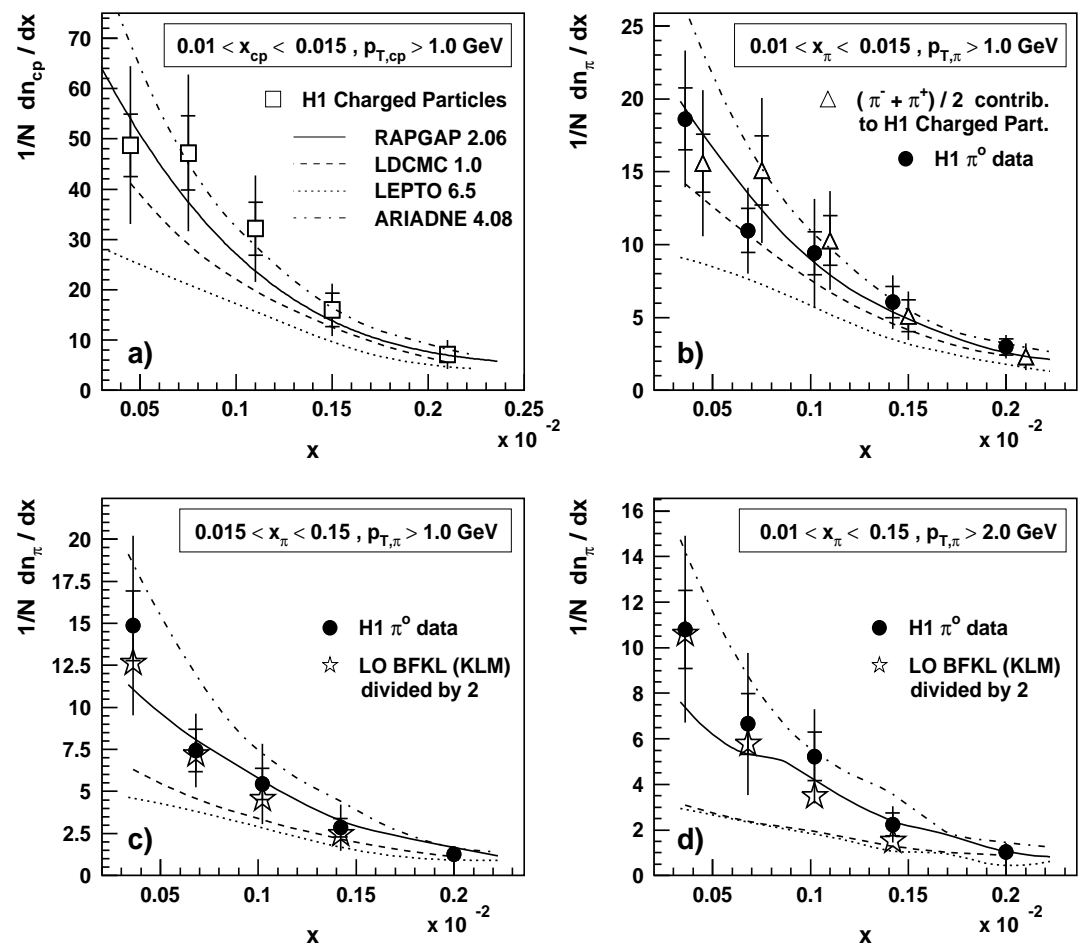

Figure 166: The single particle spectra as a function of $x_{B j}$ : a) for charged particles, b) for charged pions and $\pi^{0}$ 's; $c$ ), d) for $\pi^{0}$ 's with different cuts on $x_{\pi}$ and $p_{T}^{\pi}$. Comparison with the Monte Carlo predictions, and in panels $(c, d)$ in addition with the LO BFKL calculation [202], is presented (from [107]).

In fig. 167 the forward jet data for $p_{T}>3.5 \mathrm{GeV}$ and $p_{T}>5 \mathrm{GeV}$ are shown and compared to the Monte Carlo predictions and analytical calculations, LO BFKL and the NLO direct contribution (implemented in the DISENT program). 

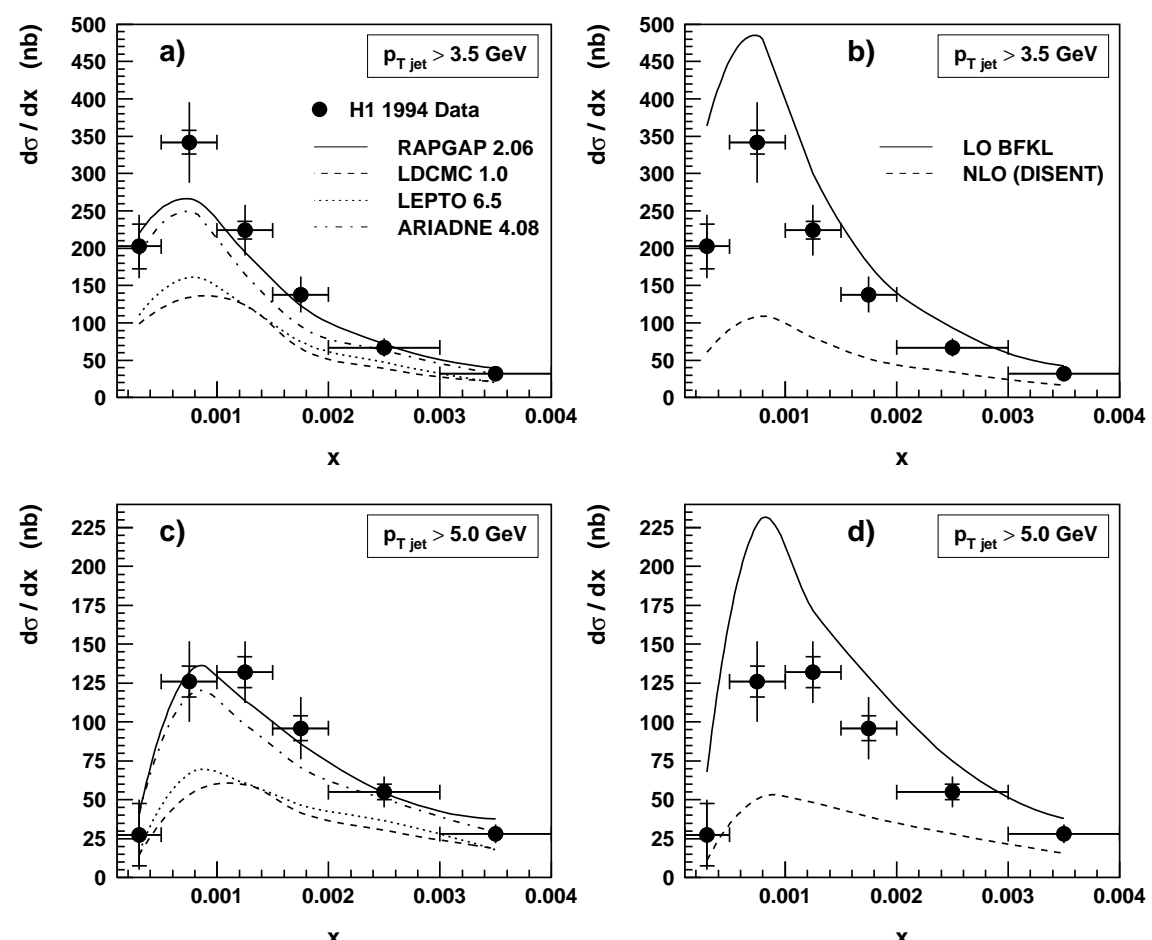

Figure 167: The forward jet cross section as a function of $x_{B j}$ for $p_{T}$ cuts: $3.5 \mathrm{GeV}$ (a, b) and $5 \mathrm{GeV}(c, d)$. a) and c) Comparison with the Monte Carlo predictions: RAPGAP, $L D C M C, L E P T O$ and ARIADNE. b) and d) Comparison with the LO BFKL calculation [204] and NLO direct prediction (DISENT) (from [107]).

Fig. 168 (taken from 199]) shows the data together with the standard DIS ${ }_{e p}$ NLO predictions, and the NLO calculation by Kramer and Pötter [199], where the interaction due to partonic content of the virtual photon is included. Note that in this calculation the hard scale $\tilde{Q}^{2}$ is assumed in the form $\tilde{Q}^{2}=k M^{2}+P^{2}$, with $M^{2}=<E_{T}>^{2}$ fixed to be $50 \mathrm{GeV}^{2}$ and parameter $k$ between $\frac{1}{3}$ and 3 . 

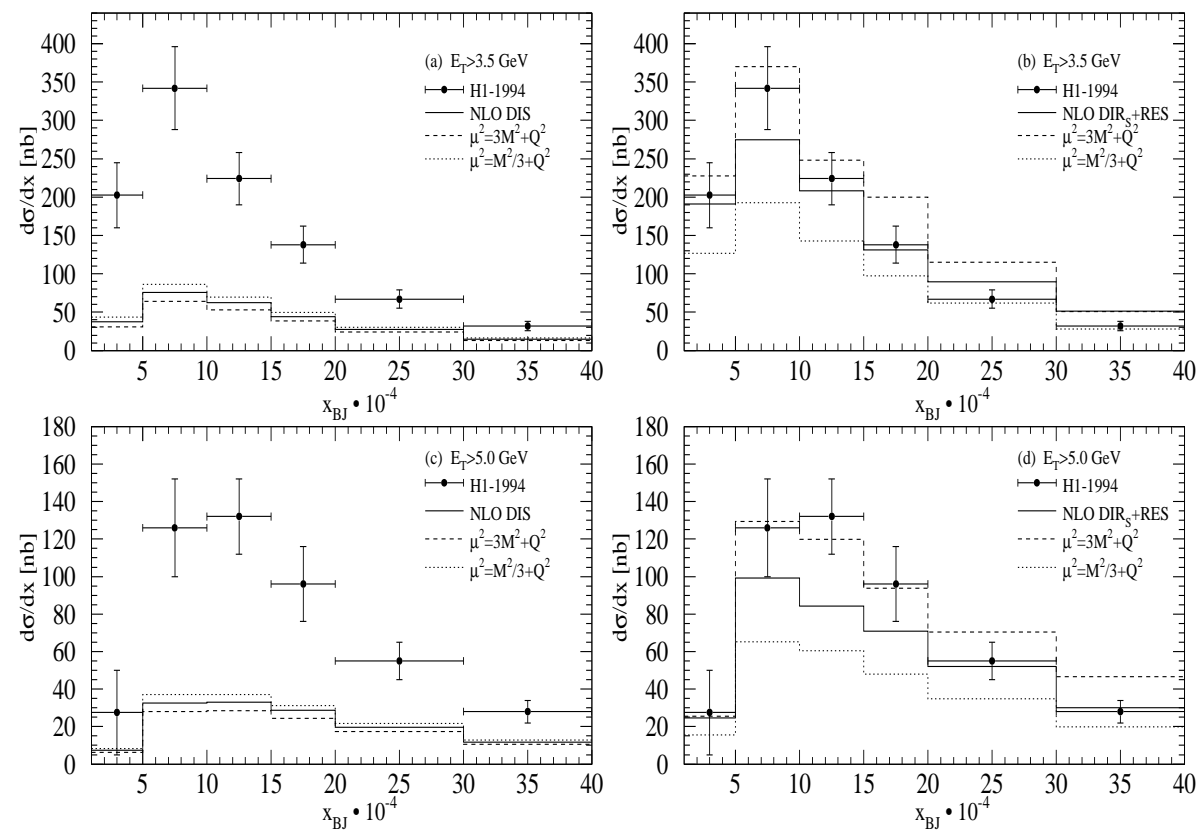

Figure 168: Results of the NLO calculations of the forward jet cross section as a function of $x_{B j}$, compared to the $H 1$ data for $E_{T}>3.5 \mathrm{GeV}(\mathrm{a}, \mathrm{b})$ and $E_{T}>5 \mathrm{GeV}(\mathrm{c}, \mathrm{d})$. The NLO description within the DIS $S_{e p}$ approach is presented in a) and c), while b) and d) show the NLO description based on the direct (DIR) and the resolved (RES) virtual photon contributions. Three different renormalization scales are used: $\tilde{Q}^{2}\left(=\mu^{2}\right)=M^{2}+Q^{2}$ (solid line), $\tilde{Q}^{2}\left(=\mu^{2}\right)=3 M^{2}+Q^{2}$ (dashed line) and $\tilde{Q}^{2}\left(=\mu^{2}\right)=M^{2} / 3+Q^{2}$ (dotted line), with $M^{2}=<E_{T}>^{2}=50 \mathrm{GeV}^{2}$ (from 199$]$ ).

The azimuthal correlation $\Delta \phi$ between the jet and the scattered electron was also studied (not shown).

Comment: "The conclusions obtained using the forward jet and the single particle measurements are in agreement".

Predictions based on the DGLAP approach fail to describe the data, except for those which allow for resolved virtual photon contributions. The RAPGAP model gives a good description of data. The BFKL calculation is above the measurements for the data. ARIADNE model reproduces the rise towards low $x$.

"Predictions of a CCFM evolution, which should smoothly interpolate between the DGLAP and BFKL regimes, give a poor description of all measured distributions."

\section{•H1 99c [108] (HERA)}

The forward production of large $p_{T} \pi^{0}$-mesons was measured in 1996 in DIS $_{e p}$ events at small $x_{B j}$. The events correspond to $2.0<Q^{2}<70 \mathrm{GeV}^{2}, 0.1<y<0.6$ and small polar angle between $5^{\circ}$ and $25^{\circ}$ (LAB). Events with forward $\pi^{0}$ production were collected for $p_{T}^{* \pi}>2.5 \mathrm{GeV}$ (in the hadron CMS) and $x_{\pi}=E_{\pi} / E_{p}>0.01$.

The comparison of the data is made with the prediction of the modified LO BFKL calculation 203] and with the Monte Carlo models: LEPTO 6.5 based on the DGLAP description of the direct $\gamma^{*} q$ scattering, and RAPGAP 2.06, which includes the resolved photon contributions and uses the DGLAP evolution for $\gamma^{*}\left(\operatorname{SaS} 1 \mathrm{D}\right.$ with $\left.\tilde{Q}^{2}=Q^{2}+p_{T}^{2}\right)$. For the proton all models use the CTEQ4M parton parametrization. 
In fig. 169/a the $x_{B j}$ dependence is presented for $p_{T}^{* \pi}>2.5 \mathrm{GeV}$ as a function of $x_{B j}$ in bins of $Q^{2}$. The observed strong rise of the cross section towards small $x_{B j}$ in the forward $\pi^{0}$ production is identical to the rise of the inclusive $e p$ cross section for all events, see fig. 169b. Comparison with the modified LO BFKL calculation [203], and with LEPTO and RAPGAP Monte Carlo models are also present in the figure.
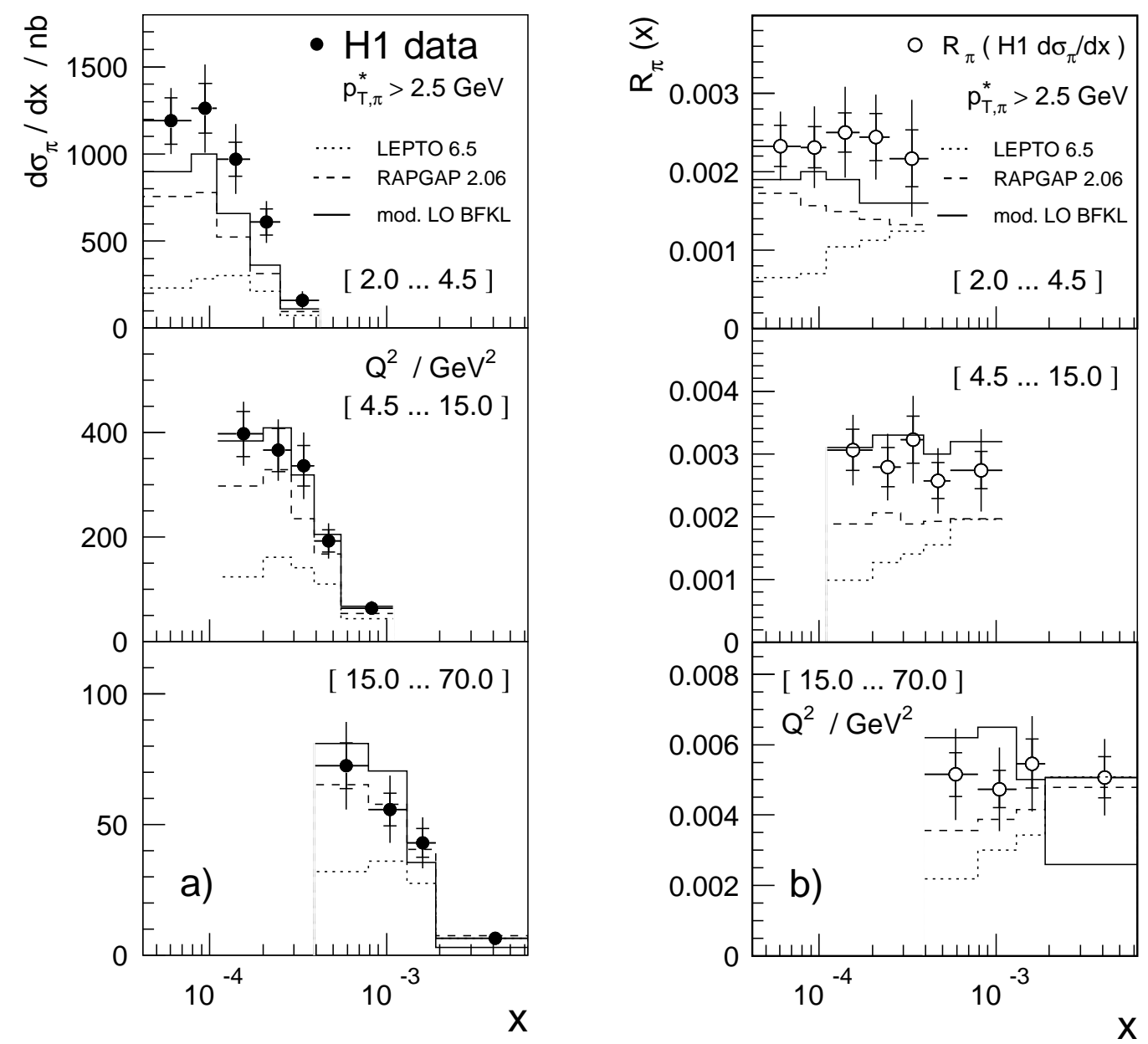

Figure 169: The inclusive forward $\pi^{0}$ cross section for $p_{T}^{* \pi}>2.5 \mathrm{GeV}$ as a function of $x_{B j}$ in bins of $Q^{2}$. Comparison with the modified LO BFKL calculation (solid line) [203], with the LEPTO (dotted line) and RAPGAP (dashed line) Monte Carlo models. a) The $x_{B j}$ distributions for $\pi^{0}$. b) The ratio of the $x_{B j}$ distributions presented in a) and the corresponding ones for the full sample of ep events (from [108]).

The analysis of the $p_{T}^{* \pi}$ and $\eta$ distributions in the $Q^{2}$ bins was performed and the results are presented in fig. 170 . 

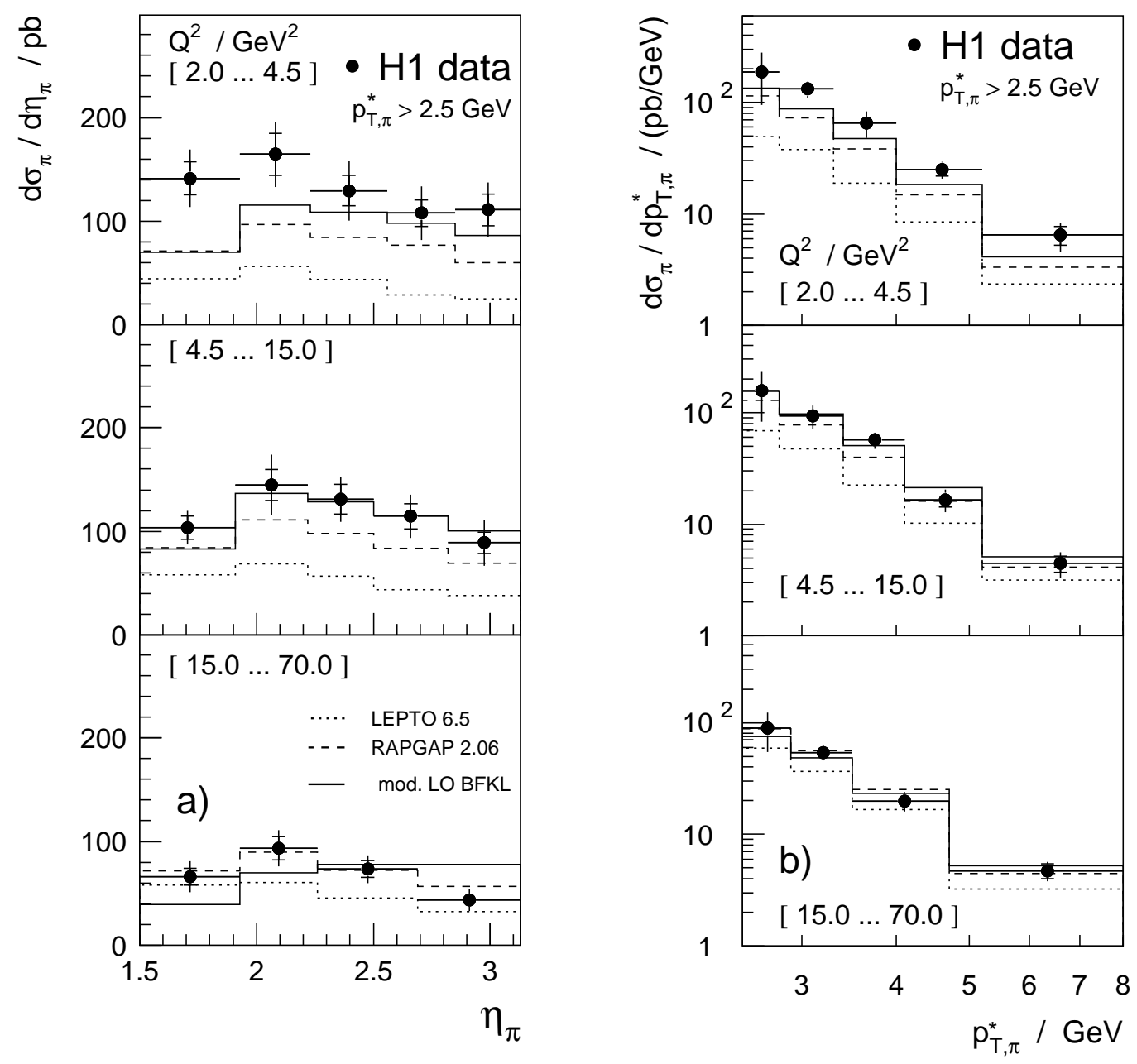

Figure 170: The inclusive forward $\pi^{0}$ cross section for $p_{T}^{* \pi}>2.5 \mathrm{GeV}$ as a function of $\eta_{\pi}$ (a) and of $p_{T}^{* \pi}(b)$, in bins of $Q^{2}$. Comparison with model predictions as in fig. 169 (from 108]). 
In fig. 171 the $Q^{2}$ dependence is presented for events with $p_{T}^{* \pi}>2.5 \mathrm{GeV}$, in comparison with the modified LO BFKL and Monte Carlo predictions.

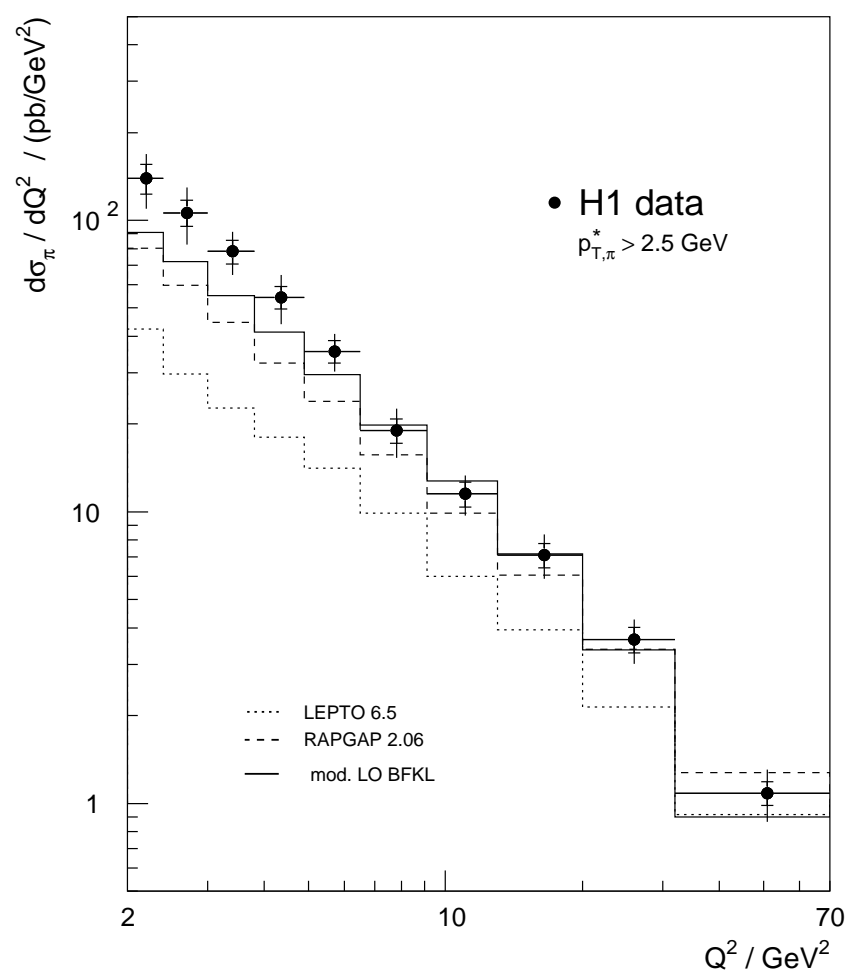

Figure 171: The inclusive forward $\pi^{0}$ cross section for $p_{T}^{* \pi}>2.5 \mathrm{GeV}$ as a function of $Q^{2}$. Comparison with model predictions as in fig. 169 (from [108]). 
For comparison also the $x_{B j}$ and $Q^{2}$ dependences are presented for the $p_{T}^{* \pi}$ threshold equal to $3.5 \mathrm{GeV}$, see fig. 172 .
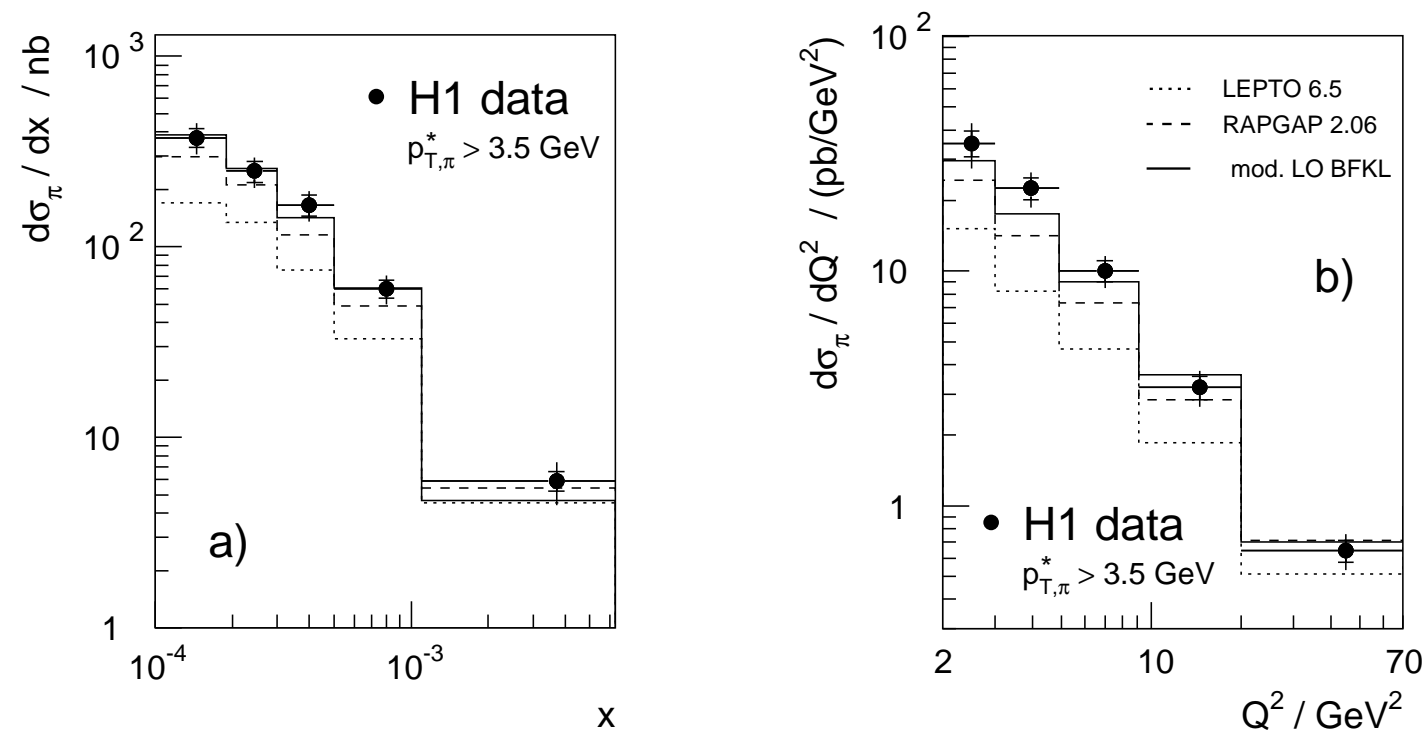

Figure 172: The inclusive forward $\pi^{0}$ cross section for $p_{T}^{* \pi}>3.5 \mathrm{GeV}$ as a function of (a) $x_{B j}$ and (b) $Q^{2}$. Comparison with model predictions as in fig. 169 (from [108]).

Comment: The measurement extends down to $x_{B j}=5 \cdot 10^{-5}$, lower than the previous values. It covers lower polar angles and larger $p_{T}^{* \pi}$ range, as compared with $\mathbf{H 1} \mathbf{9 9} \mathbf{b}$.

"Inclusion of processes in which the virtual photon is resolved improves the agreement with the data, but does not provide a satisfactory description in the full $x_{B j}$ and $Q^{2}$ range. A calculation based on the BFKL formalism is in good agreement with the data, particularly for the shape description, but the absolute normalization remains strongly affected by the scale uncertainty."

-ZEUS 99c 109] (HERA)

In the $e^{+} p$ scattering the forward jets produced with $y>0.1, E_{T}>5 \mathrm{GeV}$ and $x_{B j}$ between $4.5 \cdot 10^{-4}$ and $4.5 \cdot 10^{-2}$ are studied for the $0.5<E_{T}^{2} / Q^{2}<2$. Data were taken in 1995. The motivation was the search for effects of the BFKL type parton shower evolution. The jet was defined according to the cone algorithm $(\mathrm{R}=1)$. The LO BFKL and few Monte Carlo programs (ARIADNE 4.08, LEPTO 6.5, HERWIG 5.9, LDC 1.0 and MEPJET for the NLO direct contribution) predictions were compared with data.

The parton-level predictions for forward jet cross sections as a function of $x_{B j}$ are shown in fig. 173 (left) and for the hadron level in fig. 173 (right). The comparison with the LO BFKL and LO BFKL $1^{\text {st }}$ term (i.e. with one parton rung in the gluon ladder) and the Monte Carlo predictions is also given.

The $E_{T}^{2} / Q^{2}$ distribution was studied as well (see ZEUS 2000c for the new results). 

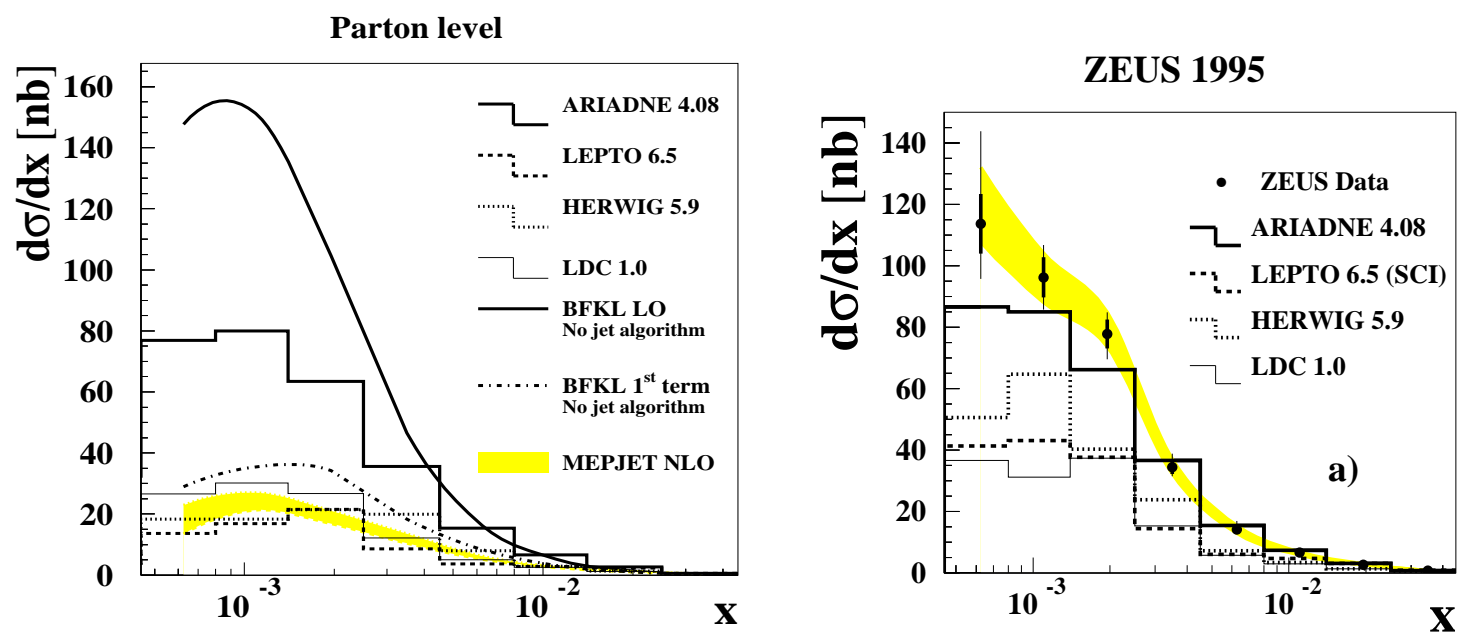

Figure 173: Left: The $x_{B j}$ distribution at the parton level obtained in various approaches (Monte Carlo and QCD analytical calculations). The shaded band corresponds to the range of results obtained with MEPJET using a renormalisation scale between $0.25 k_{T}^{2}$ and $2.0 k_{T}^{2}$. Right: The $x_{B j}$ distribution at the hadron level: data points and various Monte Carlo predictions (from [109]).

Comment: "Three regions are identified in the $E_{T}^{2} / Q^{2}$ distribution: i) the standard $D G L A P$ region with $E_{T}^{2} \ll Q^{2}$, where all Monte Carlo models are in agreement with the data; ii) the region of phase space where BFKL dynamics is expected to contribute significantly with $E_{T}^{2} \approx Q^{2}$, where only the Colour Dipole model describes the data well; iii) the region with $E_{T}^{2} \gg Q^{2}$, where none of the models reproduces the data." No contribution due to partonic content of the virtual photon was considered.

\section{-ZEUS 2000c 110 (HERA)}

The forward jet production (1995 data) was studied based on in DIS ev $_{\text {events with }}$ $Q^{2}>10 \mathrm{GeV}^{2}, y>0.1$. Events with $E_{T}>5 \mathrm{GeV}, 0.00025<x_{B j}<8.0 \cdot 10^{-2}$ and $E^{2} / Q^{2}$ between $10^{-2}$ and $10^{2}$ were considered, in addition the cut $x_{\text {jet }}=p_{z, \text { jet }} / p_{\text {proton }}>$ 0.036 was used to select forward jets. The cone algorithm was applied to jets in LAB frame with $\mathrm{R}=1$.

The LO MC models, standard for the description of the DIS $_{e p}$ events: LEPTO 6.5, HERWIG 5.9, LDC 1.0 (the linked-dipole chain as in CCFM), and ARIADNE 4.08 were used. In addition the RAPGAP 2.06 Monte Carlo program and the JeTViP (NLO calculation) program were included in the analysis to describe the resolved virtual photon interaction, with SaS2D and SaS1D parton parametrization for the photon, respectively. Both introduce the renormalization/factorization scale $\tilde{Q}^{2}=E_{T}^{2}+Q^{2}$. In fig. 174 the $E_{T}^{2} / Q^{2}$ distribution, and in fig. 175 the $x_{\gamma}$ distribution is shown and compared with the Monte Carlo models, with and without the resolved photon contribution. 

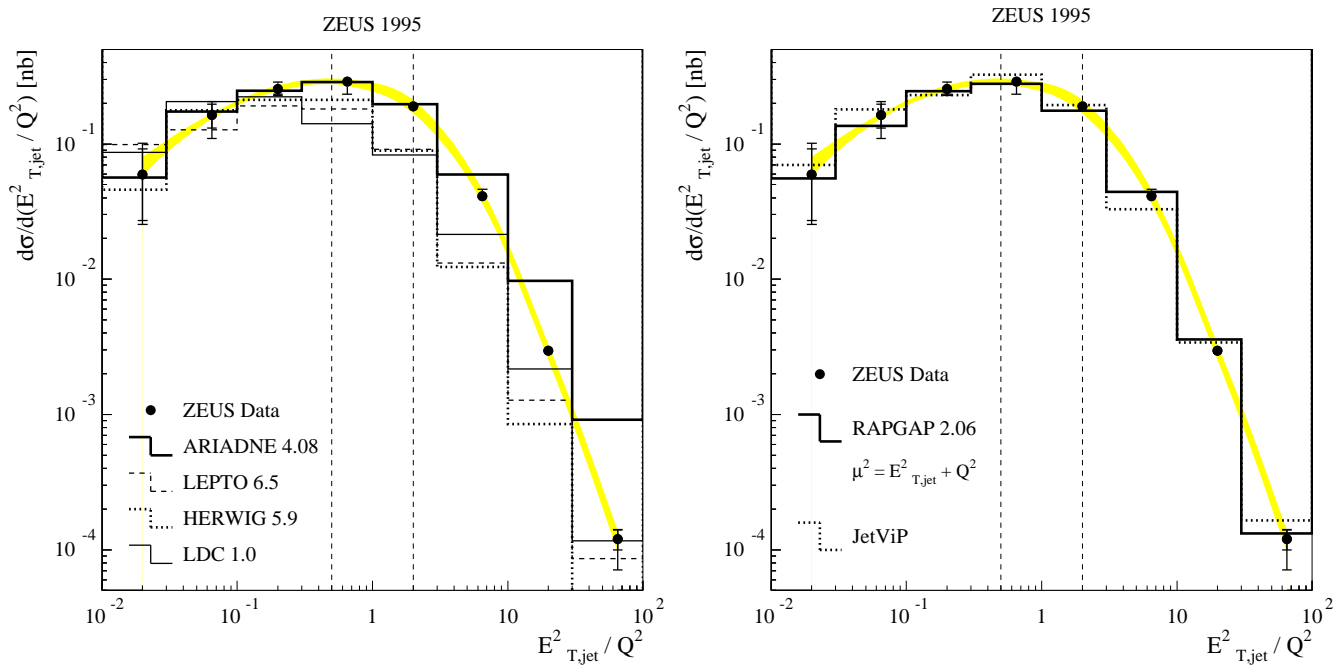

Figure 174: Forward jet production cross section as a function of $E_{T}^{2} / Q^{2}$. Comparison with predictions of the various MC models, left: ARIADNE 4.08, LEPTO 6.5, HERWIG 5.9, LDC 1.0, and right: RAPGAP 2.06, JeTViP (from [110]).

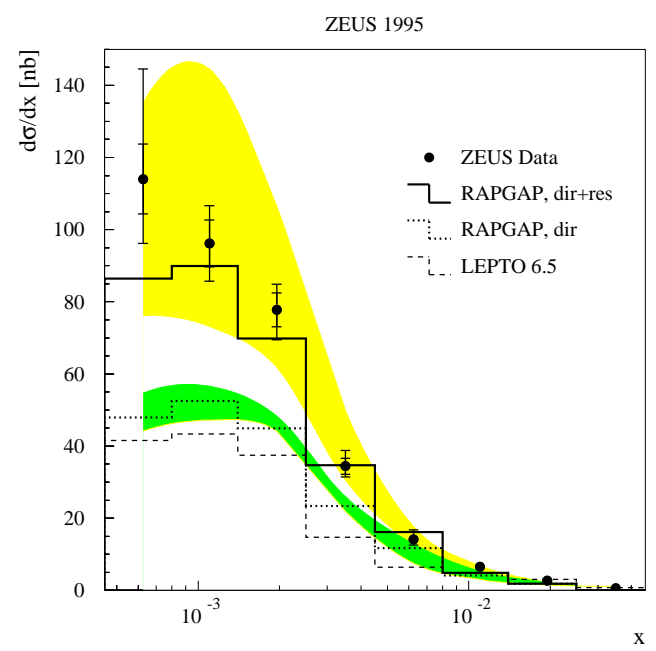

Figure 175: Forward jet production cross section as a function of $x_{B j}$ : data compared to the various Monte Carlo predictions: LEPTO based on the standard DGLAP evolution (dashed histogram), RAPGAP with the direct virtual photon contribution (dotted histogram) and RAPGAP with the direct and the resolved photon contribution (solid histogram) (from [110]). 
In fig. 176 (taken from [199]) the data are compared to the NLO calculation [199, with and without resolved virtual photon interaction (and with different renormalization scales).
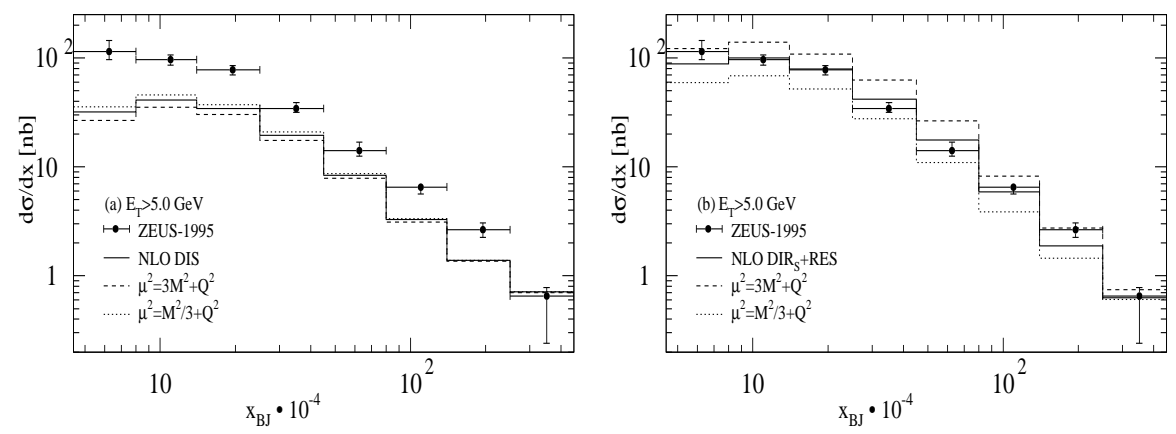

Figure 176: Results of the NLO calculations of the forward jet cross section for $E_{T}>5$ $G e V$, without (left) and with (right) resolved virtual photon interaction, compared to the ZEUS data. Three different renormalization scales are used: $\mu^{2}=M^{2}+Q^{2}$ (solid line), $\mu^{2}$ $=3 M^{2}+Q^{2}$ (dashed line) and $\mu^{2}=M^{2} / 3+Q^{2}$ (dotted line), with $M^{2}=<E_{T}>^{2}=50$ $\mathrm{GeV}^{2}$ (from [199]).

Comment: "All models describe DIS regime. In the BFKL regime $\left(Q^{2} \approx E_{T}^{2}\right) A R I$ $A D N E$ reproduces the measured distributions well. Only model with resolved photon component, RAPGAP, describes data everywhere." 


\section{Leptonic structure functions of the photon}

The lepton pair production by two photons in $e^{+} e^{-}$scattering, $e^{+} e^{-} \rightarrow e^{+} e^{-} l^{+} l^{-}$, is one of the basic test of QED 32 . It serves also as a test of experimental methods applied to more complicated two-photon reactions involving hadrons, especially to the process $e^{+} e^{-} \rightarrow$ hadrons, where the hadronic structure functions of the photon are measured. To study the reliability of unfolding and tagging applied in these measurements including the effect of the (small) virtuality $P^{2}$ of the quasi-real target photon, the lepton pair productions can be used as an ideal tool.

Single-tag events with lepton pair in the final state can be described in the unpolarized $e^{+} e^{-}$collision in terms of leptonic structure functions, $F_{1}^{\gamma(Q E D)}\left(=F_{T}^{\gamma(Q E D)}\right)$, $F_{2}^{\gamma(Q E D)}$ or $F_{L}^{\gamma(Q E D)}$, in analogy to the hadronic case. The leptonic structure functions were measured already in early eighties ${ }^{\text {34 }}$. Although the production of final states with electrons, muons and even taons have been measured, only the muonic case allows for the extraction of the structure function from the data. Also nowadays experiments at LEP measure only muonic structure functions. The invariant mass of the $\mu^{+} \mu^{-}$ pair, and hence $x_{B j}$, can be determined very accurately and the measurements of the muonic structure function $F_{2}^{\gamma}$, denoted below as $F_{2}^{\gamma(Q E D)}$, are only statistically limited in contrast to the hadronic $F_{2}^{\gamma}$. Recently also two other (muonic) structure functions, $F_{A}^{\gamma(Q E D)}$ and $F_{B}^{\gamma(Q E D)}$, have been measured at LEP.

Recently, for the first time, a measurement of the leptonic final state mediated by two highly virtual photons (double-tag events) was performed at LEP. For both photons with high virtualities the presence of large interference terms was established (OPAL 99b) even for events with very different virtualities $Q^{2} \gg P^{2}$. Thus in this case the (effective) leptonic structure function for a virtual photon cannot be introduced, nevertheless we report these results together with leptonic structure functions of the real photon.

\subsection{Theoretical description}

The QED structure functions for photon: $F_{2}^{\gamma(Q E D)}, F_{1}^{\gamma(Q E D)}, F_{L}^{\gamma(Q E D)}$, etc., unlike in the hadronic case, can be reliably calculated in QED. Moreover all the particles in the final state can, in principle, be directly observed.

The $F_{L}^{\gamma(Q E D)}$ structure function is much harder to measure than $F_{2}^{\gamma(Q E D)}$, because its contribution to the relevant cross section is weighted by the small factor $y^{2}$ (as for hadronic $F_{L}^{\gamma}$, see eq. (丑)). However, this longitudinal structure function is not the only structure function that contains additional information about the structure of the photon. It has been shown that there are azimuthal correlations in the final state particles from two-photon collisions which are sensitive to additional structure functions [113, 114]. Below we discuss some of them for the real photon.

Beside the cross section $\frac{d \sigma^{e \gamma \rightarrow e X}}{d x_{B j} d y}$ (eqs. (3), (4)), one can also measure in the twobody $\gamma^{*} \gamma$ process one final state particle $a$. Then additional structure functions $\tilde{F}_{A}^{\gamma}$ (resulting from interference between the amplitudes involving both longitudinal and

\footnotetext{
${ }^{32}$ See ref. [111] for the review of the early experiments.

${ }^{33}$ See discussion in secs. 2.2 and 2.3

${ }^{34}$ The earliest measurements are summed up in [112] (see also [2] $\mathrm{j}$ ).
} 
transverse photons) and $\tilde{F}_{B}^{\gamma}$ (describing interference between amplitudes for transverse photons only) appear [114:

$$
\begin{array}{r}
\frac{d \sigma(e \gamma \rightarrow e a X)}{d x_{B j} d y d \Omega_{a} / 4 \pi}=\frac{2 \pi \alpha^{2}}{Q^{2}} \frac{1+(1-y)^{2}}{x_{B j} y}\left[\left(2 x_{B j} \tilde{F}_{T}^{\gamma}+\epsilon(y) \tilde{F}_{L}^{\gamma}\right)\right. \\
\left.-\rho(y) \tilde{F}_{A}^{\gamma} \cos \phi_{a}+\frac{1}{2} \epsilon(y) \tilde{F}_{B}^{\gamma} \cos 2 \phi_{a}\right]
\end{array}
$$

Here $\Omega_{a}$ describes the solid angle for the particle $a$ in the $\gamma^{*} \gamma$ centre of mass frame, and $\phi_{a}$ is its azimuthal angle around the $\gamma^{*} \gamma$ axis, relative to the electron (tag) plane. The corresponding polar angle relative to the $\gamma^{*} \gamma$ collision axis is denoted by $\theta$. The functions $\epsilon(y)=2(1-y) /\left(1+(1-y)^{2}\right)$ and $\rho(y)=(2-y) \sqrt{1-y} /\left(1+(1-y)^{2}\right)$ are close to 1 , as they are $\sim\left(1-\mathcal{O}\left(y^{2}\right)\right)$. The standard functions $F_{T}^{\gamma}$ and $F_{L}^{\gamma}$ (and also $\left.F_{A, B}^{\gamma}\right)$ are obtained from the corresponding $\tilde{F}_{T, L}^{\gamma}\left(\tilde{F}_{A, B}^{\gamma}\right)$ by integration over the solid angle $\Omega_{a}$. Note that the formula (37) holds for two leptons (or two partons) produced in the final state.

The expression for $F_{T}^{\gamma(Q E D)}$ and $F_{L}^{\gamma(Q E D)}$ (or $F_{2}^{\gamma(Q E D)}=F_{L}^{\gamma(Q E D)}+2 x_{B j} F_{T}^{\gamma(Q E D)}$ ) in the lowest order QED is given by the Bethe-Heitler type formula (see eq. 6). The functions $F_{A}^{\gamma(Q E D)}$ and $F_{B}^{\gamma(Q E D)}$ calculated in the same approximation and with the full dependence on the muon mass up to terms of order $m_{\mu}^{2} / W^{2}$ 205], have the following form:

$$
\begin{array}{r}
F_{A}^{\gamma(Q E D)}\left(x_{B j}, \beta\right)=\frac{4 \alpha}{\pi} x_{B j} \sqrt{x_{B j}\left(1-x_{B j}\right)}\left(1-2 x_{B j}\right)\left\{\beta\left[1+(1-\beta)^{2} \frac{1-x_{B j}}{1-2 x_{B j}}\right]\right. \\
\left.+\frac{3 x_{B j}-2}{1-2 x_{B j}} \sqrt{1-\beta^{2}} \arccos \left(\sqrt{1-\beta^{2}}\right)\right\}, \\
F_{B}^{\gamma(Q E D)}\left(x_{B j}, \beta\right)=\frac{4 \alpha}{\pi} x_{B j}^{2}\left(1-x_{B j}\right)\left\{\beta\left[1-\left(1-\beta^{2}\right) \frac{1-x_{B j}}{2 x_{B j}}\right]\right. \\
\left.+\frac{1}{2}\left(1-\beta^{2}\right)\left[\frac{1-2 x_{B j}}{x_{B j}}-\frac{1-x_{B j}}{2 x_{B j}}\left(1-\beta^{2}\right)\right] \log \left(\frac{1+\beta}{1-\beta}\right)\right\},
\end{array}
$$

where

$$
\beta=\sqrt{1-\frac{4 m_{\mu}^{2}}{W^{2}}} .
$$

The collection of other useful formulae for the above structure functions in the lowest order QED approximation with the full dependence on the fermion mass can be found in [205], see also 206] for the first calculation in the lowest order QED.

The function $F_{B}^{\gamma(Q E D)}$ is, in a specific logarithmic approximation [115] and zero muon mass limit, accidentally equal to $F_{L}^{\gamma(Q E D)}$, although it involves quite different photon helicity structures. Thus extracting $F_{B}^{\gamma(Q E D)}$ can give us indirectly information on $F_{L}^{\gamma(Q E D)}$ (in this approximation).

The above description concerns only the leptonic production in $\gamma^{*} \gamma$ collisions, so with one (almost) real photon. Similar consideration can be performed for $\gamma^{*} \gamma^{*}$ collisions, when the notion of leptonic structure functions of the virtual photon can in principle be applied if $Q^{2} \gg P^{2}$, in full analogy to hadronic structure functions for the virtual photon (sec. 3). The extraction of such functions from the corresponding cross section, e.g. $F_{2}^{\gamma^{*}(Q E D)}$, is feasible if the interference terms can be neglected. 


\subsection{Measurements of single and double-tag events}

A measurement of single-tag events allows to extract $F_{2}^{\gamma(Q E D)}$, as well as $F_{A}^{\gamma(Q E D)}$ and $F_{B}^{\gamma(Q E D)}$. The function $\tilde{F}_{A}^{\gamma(Q E D)}$ is antisymmetric in $\cos \theta^{*}$. 3 . To extract it from the data, the $x_{B j}$-dependence of the term in the differential cross section proportional to $F_{A}^{\gamma(Q E D)}$ has to be studied separately for the ranges $\cos \theta^{*}<0$ and $\cos \theta^{*}>0$ in order to avoid cancellation.

In the last few years measurements of the leptonic structure function $F_{2}^{\gamma(Q E D)}$ have been performed at LEP 1 by the four collaborations; the ratios of structure functions $F_{A, B}^{\gamma(Q E D)}$ to $F_{2}^{\gamma(Q E D)}$ have also been measured by all the groups. Only the L3 and OPAL groups provided also the results for individual structure functions $F_{A}^{\gamma(Q E D)}$ and $F_{B}^{\gamma(Q E D)}$.

The Vermaseren, Galuga and BDK Monte Carlo generators, which have implemented full dependence on both photons' virtualities, were used in analyses (for background simulation also other generators were used).

Results of all $F_{2}^{\gamma(Q E D)}$ measurements discussed here will be shown together in figs. 189 and 190. A similar compilation is also presented for the ratios $F_{A, B}^{\gamma(Q E D)} / F_{2}^{\gamma(Q E D)}$ (fig. 191) and for individual functions $F_{A}^{\gamma(Q E D)}, F_{B}^{\gamma(Q E D)}$ (fig. 192).

The relevant cross section for the double-tag events, i.e. for the production of the leptonic $\left(\mu^{+} \mu^{-}\right)$final state in $\gamma^{*} \gamma^{*}$ collision, is as for the hadronic final state (eq. 28), with the same definition of kinematical variables. Note that for these events the additional angle $\bar{\phi}$ between two scattering planes for the two scattered electrons appears.

\section{DATA}

\section{-ALEPH 97b,conf [115 (LEP 1)}

$F_{2}^{\gamma(Q E D)}$ has been measured (data from 1994) for two samples: $0.6<Q^{2}<6.3 \mathrm{GeV}^{2}$ $\left(<Q^{2}>=2.8 \mathrm{GeV}^{2}\right)$ with $<P^{2}>$ equal to 0.15 and $3.0<Q^{2}<60.0 \mathrm{GeV}^{2}\left(<Q^{2}>=\right.$ $\left.14.6 \mathrm{GeV}^{2}\right)$ with $<P^{2}>=0.22 \mathrm{GeV}^{2}$. Results for these two samples are shown in fig. 177 .
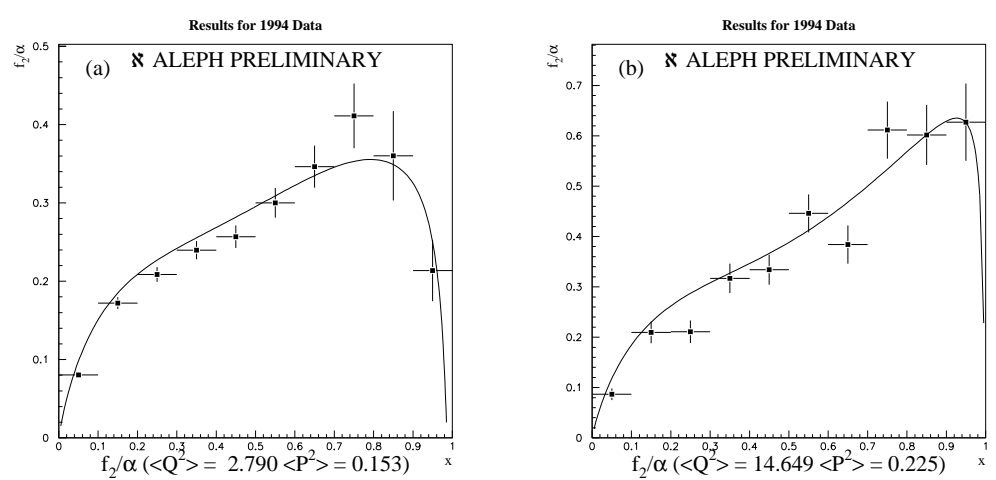

Figure 177: The structure functions $F_{2}^{\gamma(Q E D)} / \alpha$ as a function $x_{B j}$ for a) $<Q^{2}>=2.79$ $\mathrm{GeV}^{2},<P^{2}>=0.153 \mathrm{GeV}^{2}$ and $\left.\mathrm{b}\right)<Q^{2}>=14.65 \mathrm{GeV}^{2},<P^{2}>=0.225 \mathrm{GeV}^{2}$. The solid lines are the $Q E D$ expectations (from [115]).

\footnotetext{
${ }^{35}$ Often variables referring to the $\gamma^{*} \gamma$ centre of mass frame are denoted by adding a star
} 
In this experiment also the azimuthal angle distributions have been measured and ratios $F_{A}^{\gamma(Q E D)} / F_{2}^{\gamma(Q E D)}$ and $F_{B}^{\gamma(Q E D)} / F_{2}^{\gamma(Q E D)}$ extracted (fig. 178).
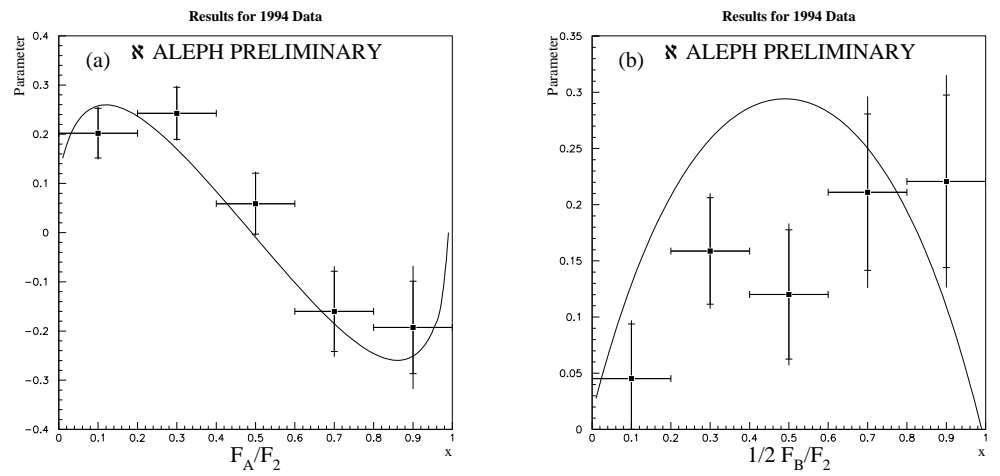

Figure 178: Results for a) $F_{A}^{\gamma(Q E D)} / F_{2}^{\gamma(Q E D)}$ and b) $\frac{1}{2} F_{B}^{\gamma(Q E D)} / F_{2}^{\gamma(Q E D)}$ as a function $x_{B j}$ at $\left\langle Q^{2}>=8.8 \mathrm{GeV}^{2}\right.$. The solid lines are the $Q E D$ expectations (from [115]).

\section{-DELPHI 96a [35] (LEP 1)}

$F_{2}^{\gamma(Q E D)}$ has been measured at $<Q^{2}>=12 \mathrm{GeV}^{2}$, as a test for the unfolding and tagging methods in extraction of the hadronic $F_{2}^{\gamma}$ (see sec. 2.2.2). The effect of nonzero target virtuality has been studied. A satisfactory fit to the measured $F_{2}^{\gamma(Q E D)}$ is obtained for the fixed value of $P^{2}=0.04 \mathrm{GeV}^{2}$.

\section{-DELPHI 99,conf 116 (LEP 1)}

The single-tag events with the muon pairs (data from 1992-1995) for $Q^{2}$ between 2.5 and $750 \mathrm{GeV}^{2}$ are used to extract the leptonic photon structure functions. The SAT (1991-1993) and the STIC (after 1994) samples were studied corresponding to $<Q^{2}>=12.5 \mathrm{GeV}^{2}$, and the FEMC sample for $<Q^{2}>=120 \mathrm{GeV}^{2}$.

The effect of nonzero target virtuality has been studied, a fit to $F_{2}^{\gamma}$ data gives for SAT (STIC) events $P^{2}=0.032 \pm 0.007(0.022 \pm 0.007) \mathrm{GeV}^{2}$. The results for various distributions for SAT and FEMC samples are presented in fig. 179 . 

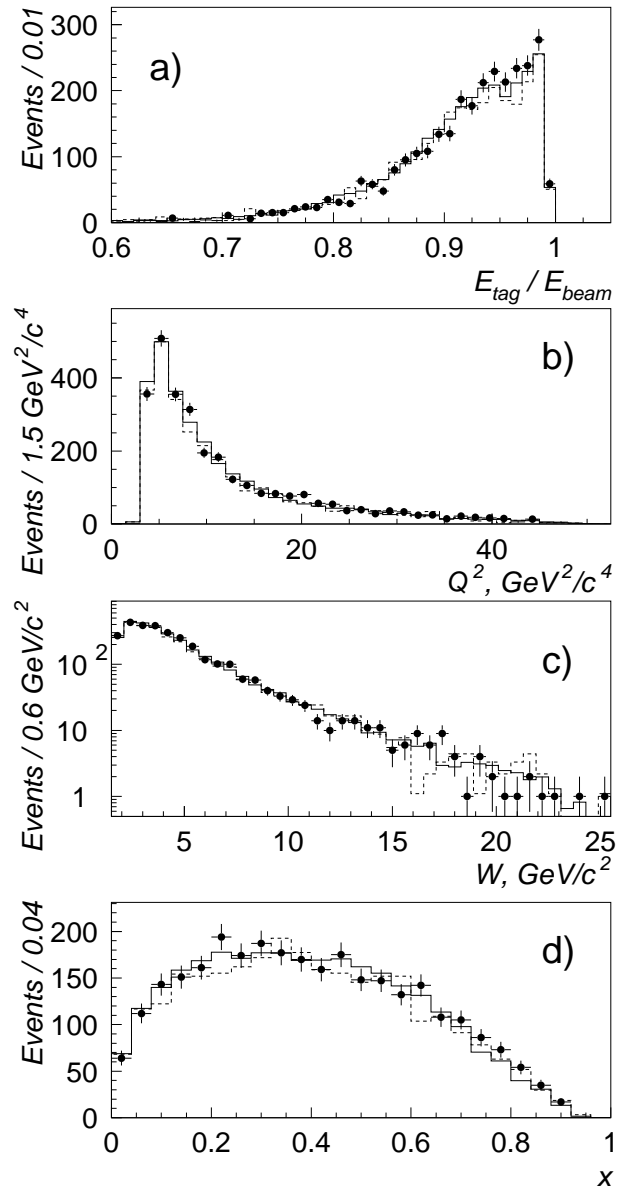
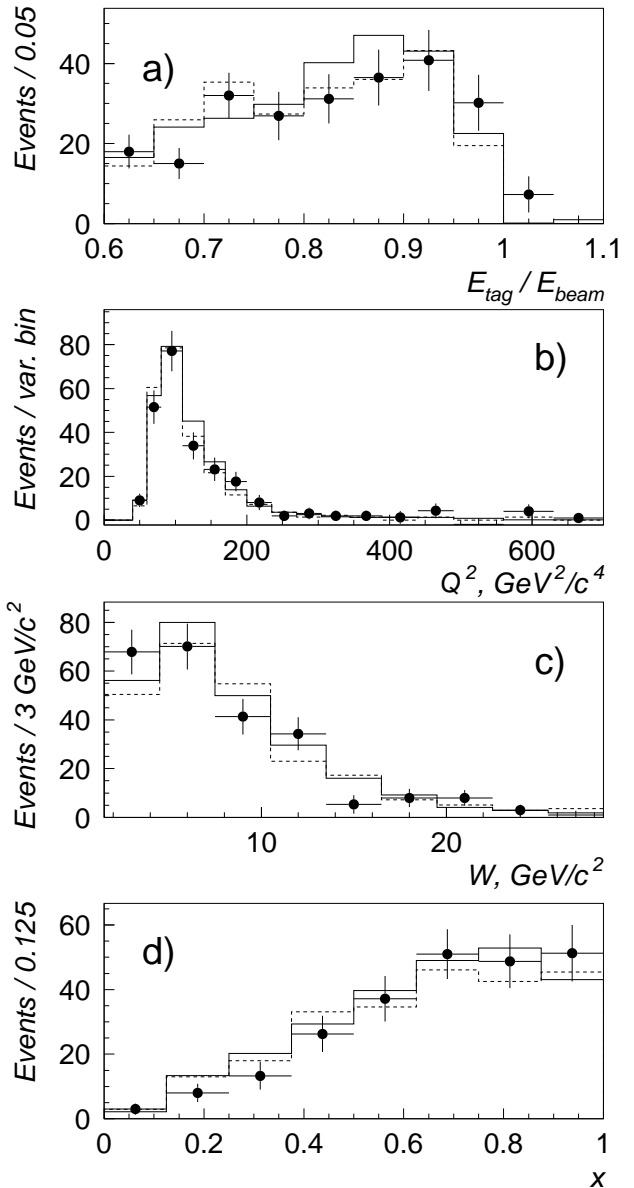

Figure 179: Left: Distributions for STIC sample: a) $\left.E_{\text {tag }} / E_{\text {beam }}, b\right) Q^{2}$, c) $W$, d) $x_{B j}$, and a comparison with BDKRC and DIAG36 generators. Right: The same for the FEMC sample (from [11]]).

The results for $F_{2}^{\gamma(Q E D)} / \alpha$ as a function of $x_{B j}$ for the combined SAT and STIC data $\left(<Q^{2}>=12.5 \mathrm{GeV}^{2}\right)$ and for FEMC sample $\left(<Q^{2}>=120 \mathrm{GeV}^{2}\right)$, together with QED predictions are shown in fig. 180.

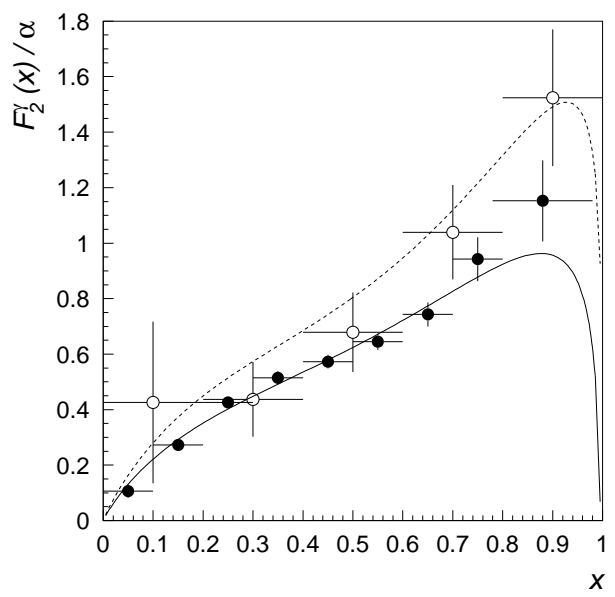

Figure 180: The results for $F_{2}^{\gamma(Q E D)} / \alpha$ as a function of $x_{B j}$ for the SAT and STIC combined data $\left(<Q^{2}>=12.5 \mathrm{GeV}^{2}\right)$ and for FEMC sample $\left(<Q^{2}>=120 \mathrm{GeV}^{2}\right)$, together with QED predictions (from [116]). 
Azimuthal correlations have been studied to derive $F_{A}^{\gamma(Q E D)}$ and $F_{B}^{\gamma(Q E D)}$. In order to increase the observed correlation only events with $20^{\circ}<\theta^{*}<160^{\circ}$ were taken into account. The azimuthal angle distributions (not shown) were fitted to obtain the factors multiplying the $\cos \phi_{a}$ and $\cos 2 \phi_{a}$ contributions. Next the extrapolation in these factors was made and the final results for ratios $F_{A}^{\gamma(Q E D)} / F_{2}^{\gamma(Q E D)}$ and $F_{B}^{\gamma(Q E D)} / F_{2}^{\gamma(Q E D)}$ obtained, see fig. 181.

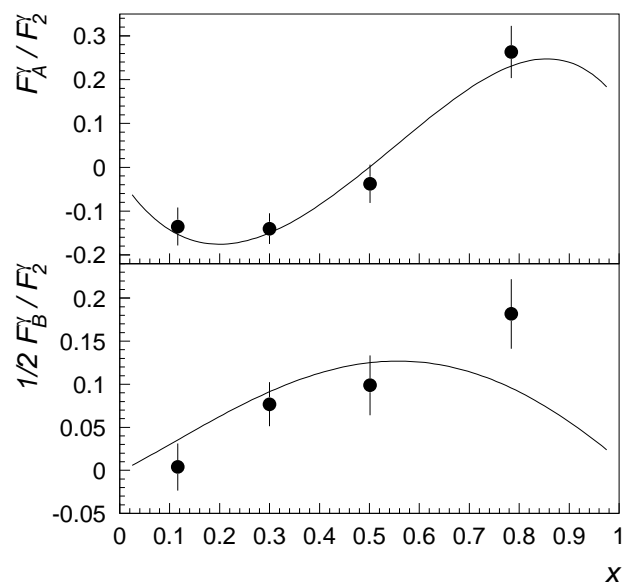

Figure 181: The results for $F_{A}^{\gamma(Q E D)} / F_{2}^{\gamma(Q E D)}$ (top) and $F_{B}^{\gamma(Q E D)} / 2 F_{2}^{\gamma(Q E D)}$ (bottom) as a function of $x_{B j}$. The $Q E D$ predictions are also shown (from [110]).

\section{-L3 98b [117 (LEP 1)}

The production of $e^{+} e^{-}$and $\mu^{+} \mu^{-}$pairs for $1.4 \mathrm{GeV}^{2} \leq Q^{2} \leq 7.6 \mathrm{GeV}^{2}$ was studied, based on the 1991-1994 run. For a muonic final state, data on $F_{2}^{\gamma(Q E D)}$, and on the ratios $F_{A}^{\gamma(Q E D)} / F_{2}^{\gamma(Q E D)}$ and $\frac{1}{2} F_{B}^{\gamma(Q E D)} / F_{2}^{\gamma(Q E D)}$, as well as on $F_{A}^{\gamma(Q E D)}$ and $F_{B}^{\gamma(Q E D)}$ were obtained.

The effect of the photon target virtuality has been studied and is clearly seen in all the three structure functions (see fig. 182 for $F_{2}^{\gamma(Q E D)}$ only). 


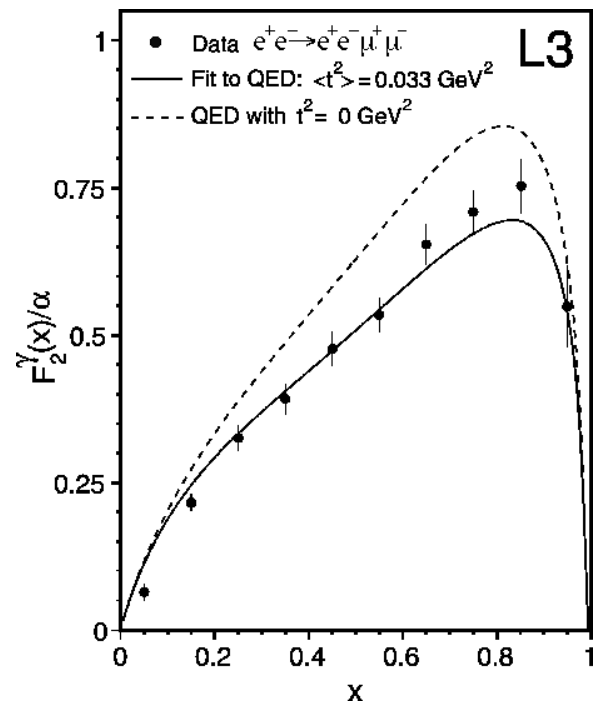

Figure 182: $F_{2}^{\gamma(Q E D)}\left(x_{B j}, Q^{2}, P^{2}\right) / \alpha$ for $1.4<Q^{2}<7.6 \mathrm{GeV}^{2}$ as a function of $x_{B j}$ compared to $Q E D$ predictions at $P^{2}=0$ (dashed line) and at $<P^{2}>_{\text {fit }}=0.033 \mathrm{GeV}^{2}$ (solid line) (from [117]).

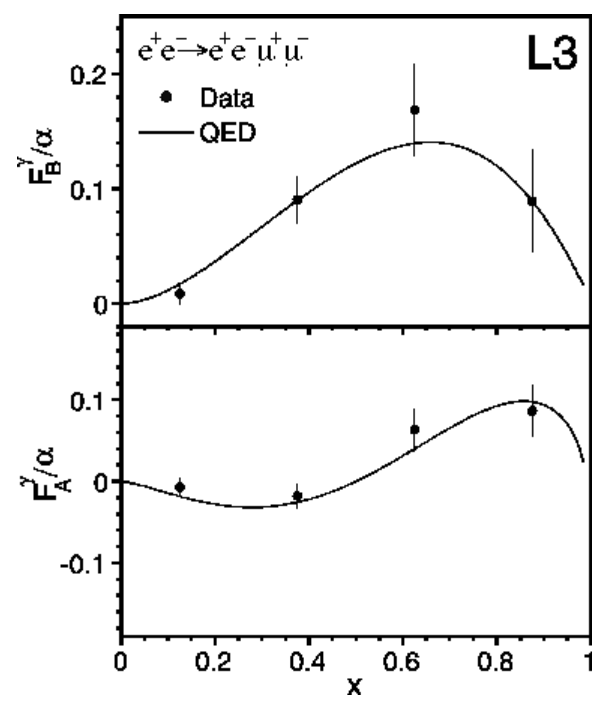

Figure 183: Results for $F_{B}^{\gamma(Q E D)} / \alpha$ and $F_{A}^{\gamma(Q E D)} / \alpha$ measured at $1.4<Q^{2}<7.6 \mathrm{GeV}^{2}$. The curves are the $Q E D$ predictions for $P^{2}=0$ (from [117]).

-OPAL 93 [118] (LEP 1)

The QED structure function $F_{2}^{\gamma(Q E D)}$ for $<Q^{2}>=8.0 \mathrm{GeV}^{2}$ was extracted from singletag events at $\mathrm{CM}$ energy $\sim M_{Z}$, for the first time at LEP.

-OPAL 97f [119] (LEP 1)

Here the extraction of $F_{B}^{\gamma(Q E D)}$ was performed for $0.85<Q^{2}<31 \mathrm{GeV}^{2}\left(<Q^{2}>=5.2\right.$ $\left.\mathrm{GeV}^{2}\right)$.

-OPAL 99b [120] (LEP 1)

The complete OPAL dataset taken in years 1990-95 at $e^{+} e^{-}$CM energies close to the 
mass of the $Z$ boson has been used to determine the QED structure functions $F_{2}^{\gamma(Q E D)}$, $F_{A}^{\gamma(Q E D)}$ and $F_{B}^{\gamma(Q E D)}$ of quasi-real photon (i.e. single tagged events, with $y<0.5$ and $P^{2}<1.4 \mathrm{GeV}^{2}$, with $<P^{2}>=0.05 \mathrm{GeV}^{2}$ ) and, for the first time, the differential cross section $\frac{d \sigma}{d x_{B j}}$ for highly virtual photons with $y<0.5$ and $1.5<P^{2}<20 \mathrm{GeV}^{2}$. For single-tag events the different samples (SW, FD, EE) were collected, depending on the $Q^{2}$ range (from 1.5 till $30 \mathrm{GeV}^{2}$ ) and the calorimeter used to detect the scattered electron. The double-tag sample is denoted by DB and corresponds to $Q^{2}$ between 1.5 and $300 \mathrm{GeV}^{2}$.

The various distributions for the single and double-tag events were measured and compared with MC (Vermaseren generator) predictions. In fig. 184 the results for DB sample are presented. Here the energy and the polar angle of the second electron, as well as the $Q^{2}$ and $P^{2}$ distributions, are shown.

\section{OPAL}
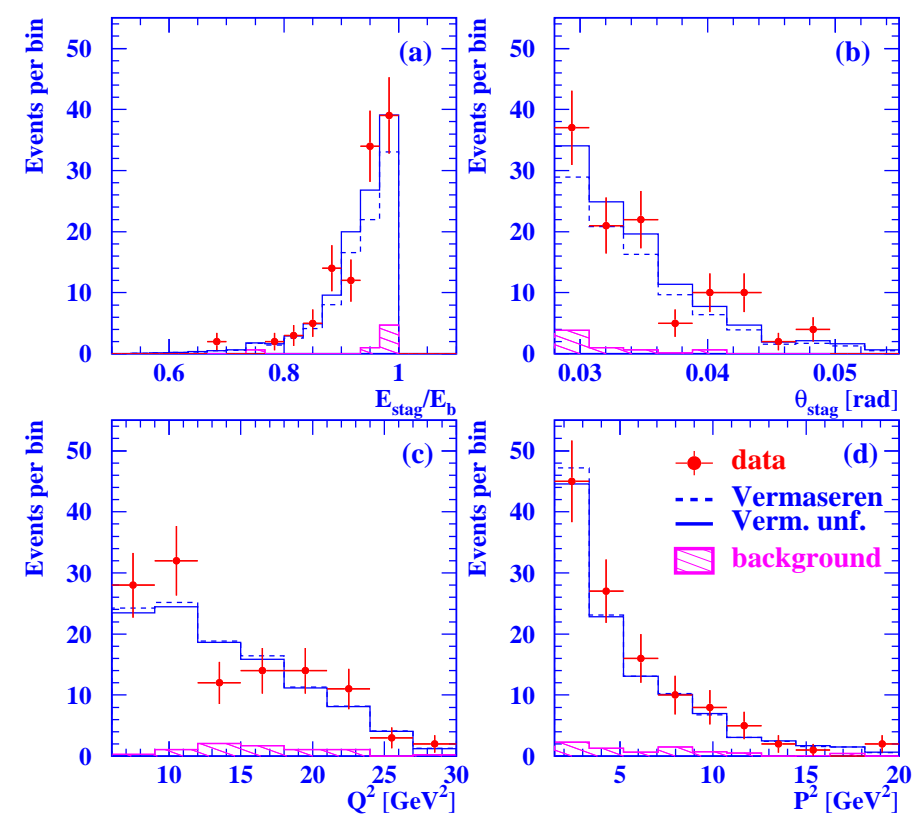

Figure 184: Various distributions for double-tag events (from [120]).

For single tagged events (with $<P^{2}>=0.05 \mathrm{GeV}^{2}$ ) the differential cross section $\frac{d \sigma}{d x_{B j}}$ have been measured and the $F_{2}^{\gamma(Q E D)}$ was extracted. Results for various samples are shown in fig. $185\left(\frac{d \sigma}{d x_{B j}}\right)$ and $186\left(F_{2}^{\gamma(Q E D)}\right)$, together with the corresponding QED predictions. 


\section{OPAL}
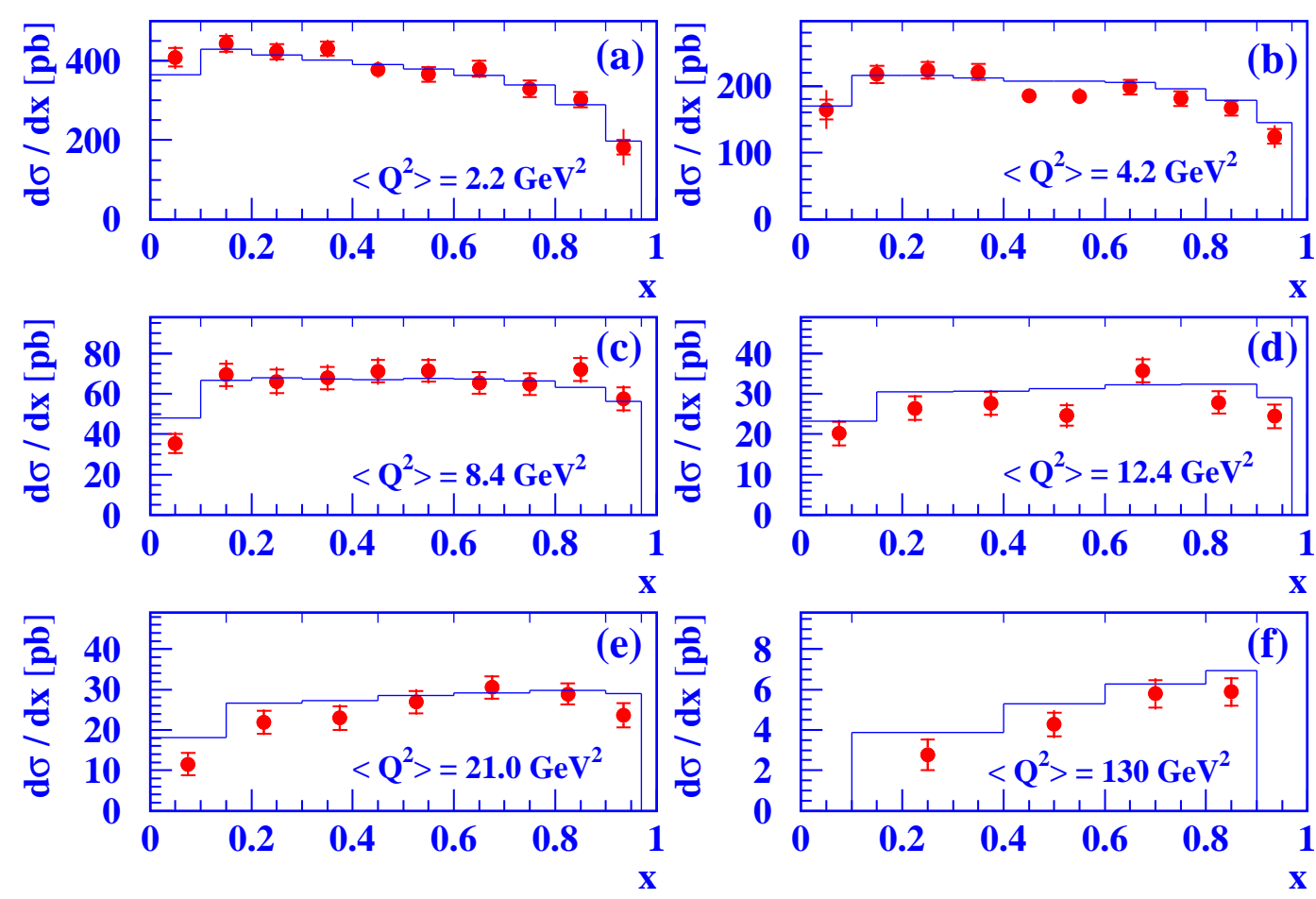

Figure 185: Differential cross section $d \sigma / d x_{B j}$ for various $\left\langle Q^{2}\right\rangle$ samples of singly tagged events. The full line is the differential cross section as predicted by $Q E D$ within Vermaseren Monte Carlo (from [120]). 


\section{OPAL}
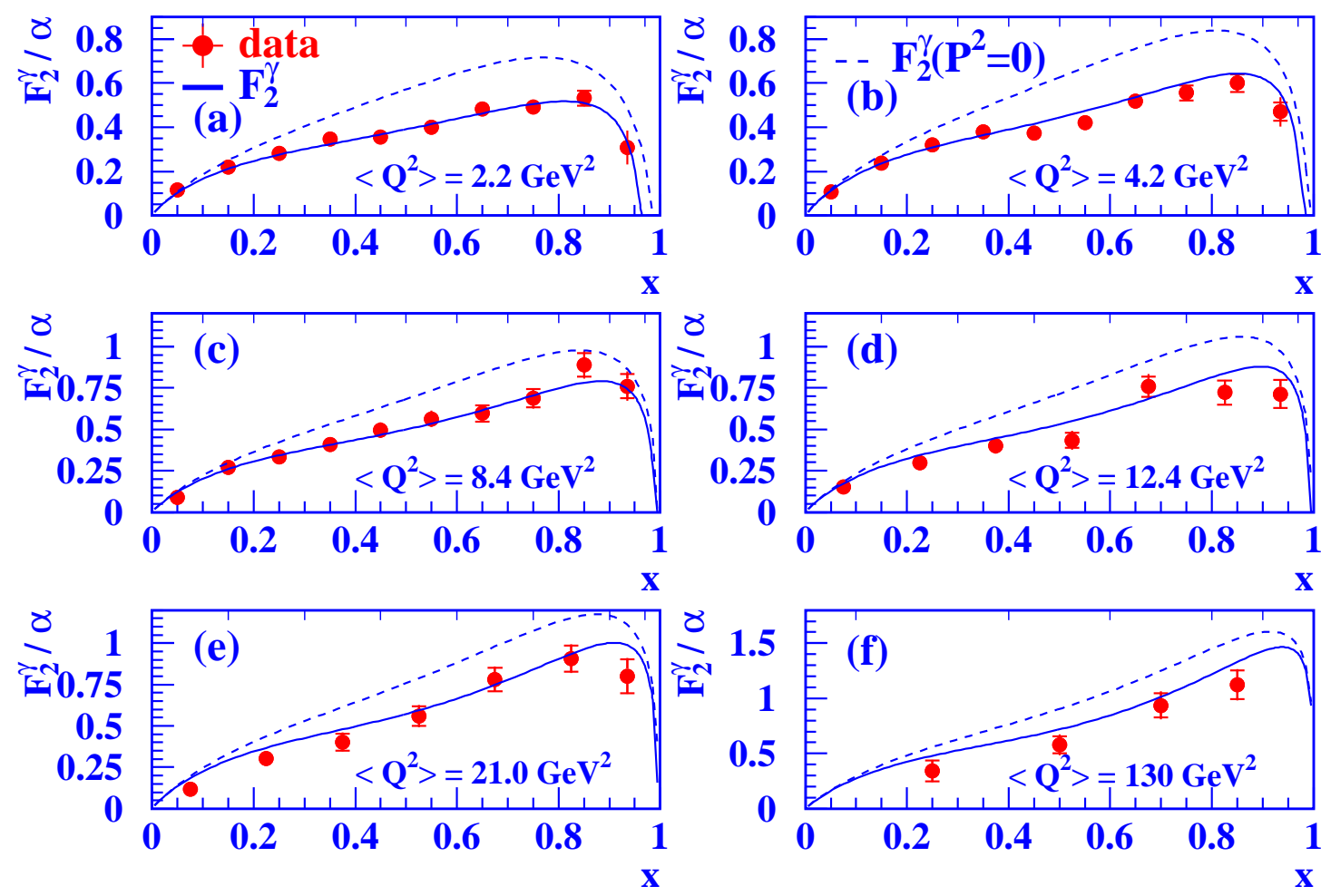

Figure 186: Structure functions $F_{2}^{\gamma(Q E D)} / \alpha$ for various $\left\langle Q^{2}>\right.$ samples of singly tagged events. The full line is the QED prediction for $\left\langle P^{2}\right\rangle=0.05 \mathrm{GeV}^{2}$, the dashed line - for $<P^{2}>=0$ (from 120 ).

For the first time the differential cross section has been measured for two highly virtual photons and the importance of the interference terms $\tau_{T T}$ and $\tau_{T L}$ (see eq. 28) has been shown.

If, after integration of the differential cross section (eq. 28) over $\bar{\phi}$, the contributions of the terms $\tau_{T T}$ and $\tau_{T L}$ vanish, one can introduce an effective structure function of virtual photon (see sec. 3.2.2, PLUTO 84 and L3 2000). This is not the case in the present measurement, as can be seen in fig. 187 where Monte Carlo predictions also for the options with $\tau_{T T}=0$ and $\tau_{T T}=\tau_{T L}=0$ are displayed and compared with the data.

Based on azimuthal correlations, the QED structure functions $F_{A}^{\gamma(Q E D)}$ and $F_{B}^{\gamma(Q E D)}$ for quasi-real photons have been determined for $<Q^{2}>=5.4 \mathrm{GeV}^{2}$. First, ratios $F_{A}^{(Q E D)} / F_{2}^{\gamma(Q E D)}$ and $F_{B}^{(Q E D)} / F_{2}^{\gamma(Q E D)}$ were obtained from a fit to the $\phi_{a}$ distribution. Then from the measurements of $F_{2}^{\gamma(Q E D)}$ also the individual structure functions $F_{A}^{\gamma(Q E D)}$ and $F_{B}^{\gamma(Q E D)}$ were calculated. The obtained results are shown in fig. 188 . 
OPAL
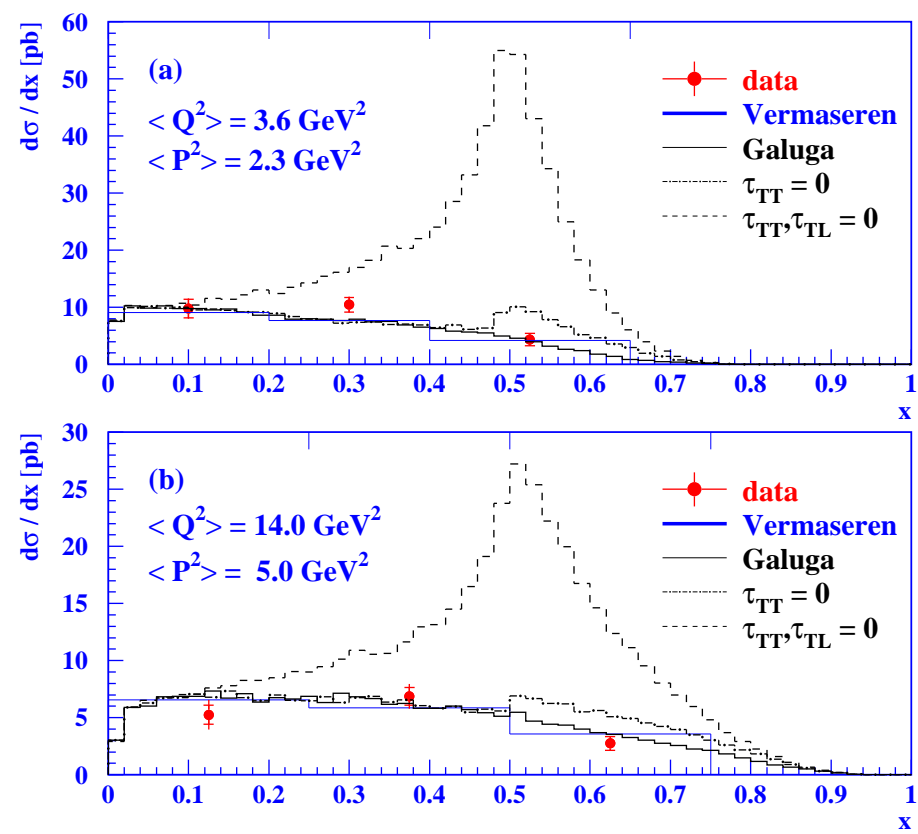

Figure 187: Differential cross section for $\mu^{+} \mu^{-}$production with two virtual photons, for two $<Q^{2}>,<P^{2}>$ samples, as a function of $x_{B j}$. The full line is the differential cross section predicted by the Vermaseren Monte Carlo. The additional three histograms represent the cross section calculations with the GALUGA Monte Carlo for the full cross section (solid line), cross section obtained for $\tau_{T T}=0$ (dot-dashed line) and that for $\tau_{T T}=\tau_{T L}=0$ (dashed line) (from 120]).

\section{OPAL}
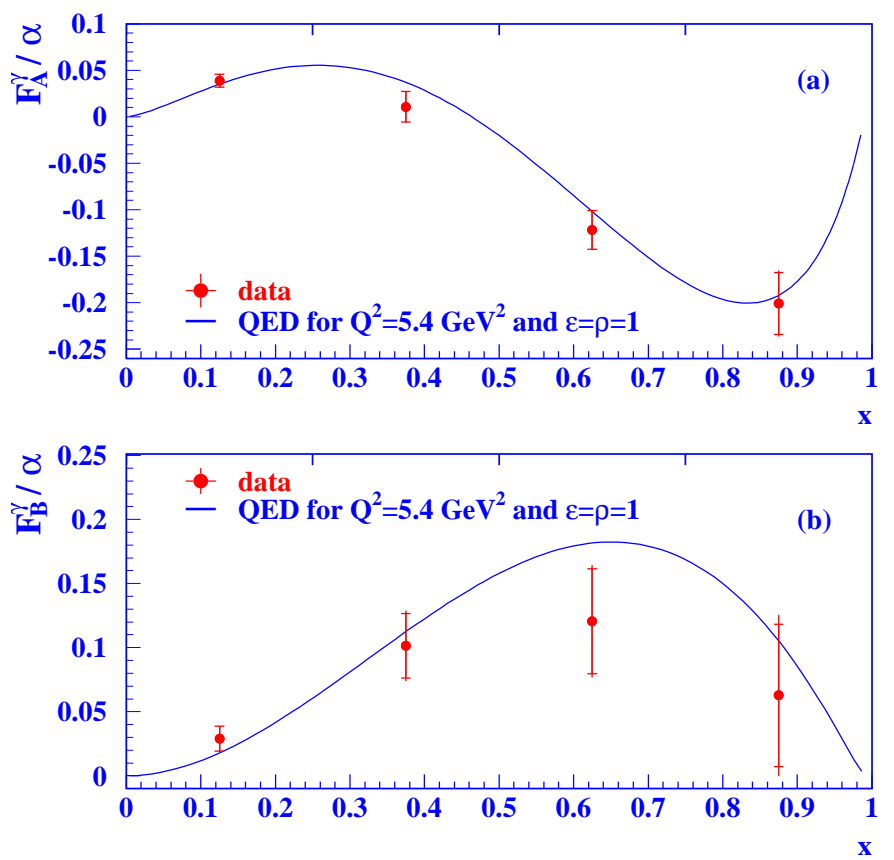

Figure 188: The measured structure functions $F_{A}^{\gamma(Q E D)} / \alpha$ and $F_{B}^{\gamma(Q E D)} / \alpha$ as a function of $x_{B j}$ for $<Q^{2}>=5.4 \mathrm{GeV}^{2}$. The solid lines are the $Q E D$ predictions for $Q^{2}=5.4 \mathrm{GeV}^{2}$ and $\epsilon=\rho=1$ (from [120]).

Comment: For the single-tag events leptonic structure functions were extracted in the 
wide range of $Q^{2}$. The effect of $P^{2}$ dependence was studied.

For the first time the leptonic final state was studied for the deep inelastic scattering on the highly virtual photon. Due to large interference terms there is no clear relation between structure functions and cross sections.

The definition of $F_{A}^{\gamma(Q E D)}$ adopted here differs by sign and a factor 2 from that used by $\mathbf{L 3} \mathbf{9 8 b}$. It is worth noting that the analytical formulae used here with the muon mass dependence, give noticeably different values from those with zero mass.

"The measurements presented here supersede the earlier structure function results" (OPAL 93, 97f).

$* * * * *$

For an overall comparison we present below collective figures (from ref. [2]j) of the QED structure functions for the real photon together with corresponding QED predictions for $P^{2}=0$. Data on $F_{2}^{\gamma(Q E D)}$ from old and new experiments are presented in fig. 189 as a function of $x_{B j}$, for $<Q^{2}>$ between 0.14 and $130 \mathrm{GeV}^{2}$, and in fig. 190 as a function of $Q^{2}$ for various $<x_{B j}>$ bins. Also the summary of structure functions ratios $F_{A}^{(Q E D)} / F_{2}^{\gamma(Q E D)}$ and $F_{B}^{(Q E D)} / F_{2}^{\gamma(Q E D)}$ and of the individual structure functions $F_{A}^{(Q E D)}$ and $F_{B}^{(Q E D)}$ is shown in fig. 191 and 192, respectively. 

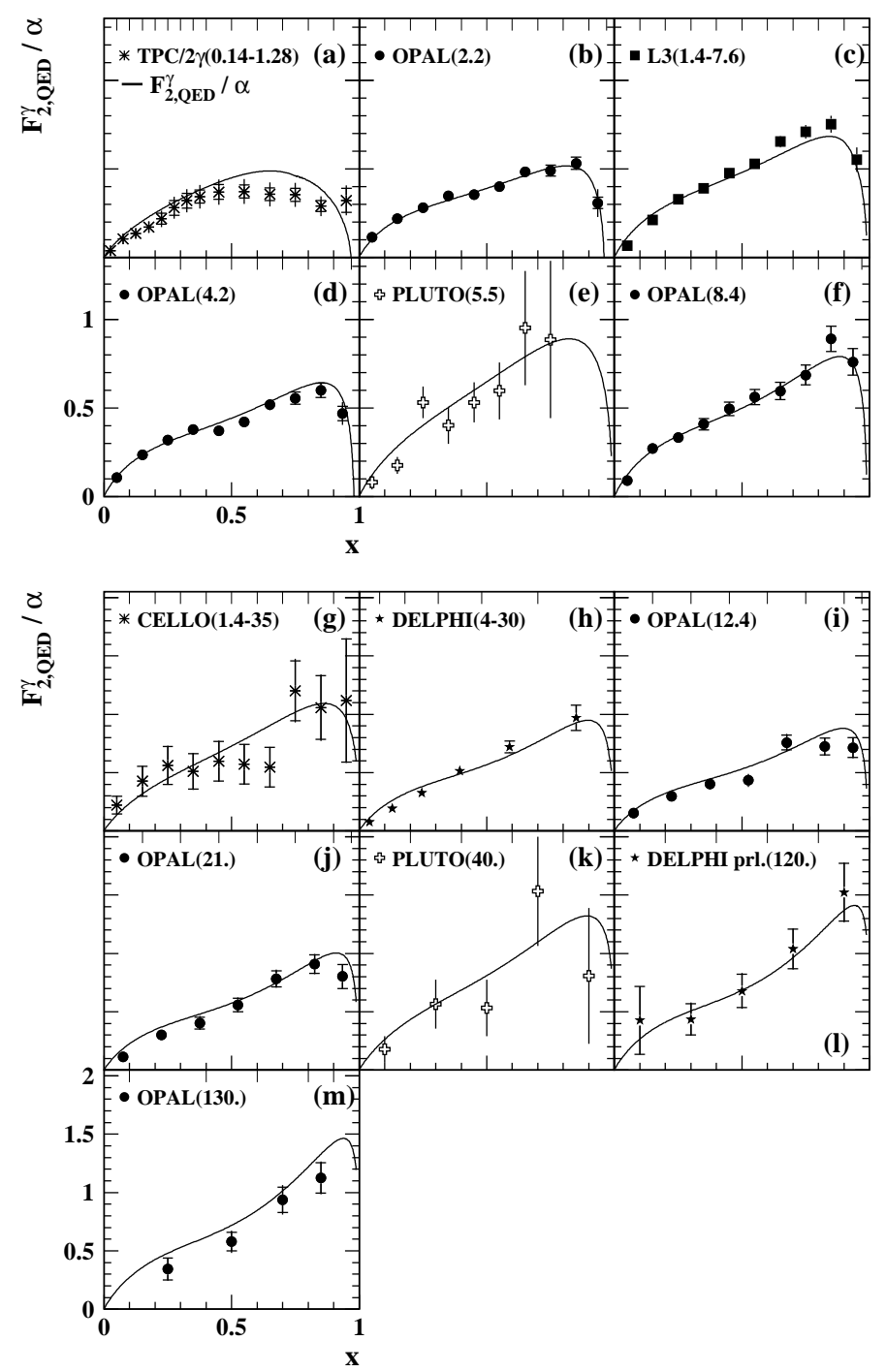

Figure 189: Summary of existing $F_{2}^{\gamma(Q E D)} / \alpha$ data as a function of $x_{B j}$ for broad $<Q^{2}>$ range shown with $Q E D$ predictions for $P^{2}=0$ (from [2] $]$ ). 


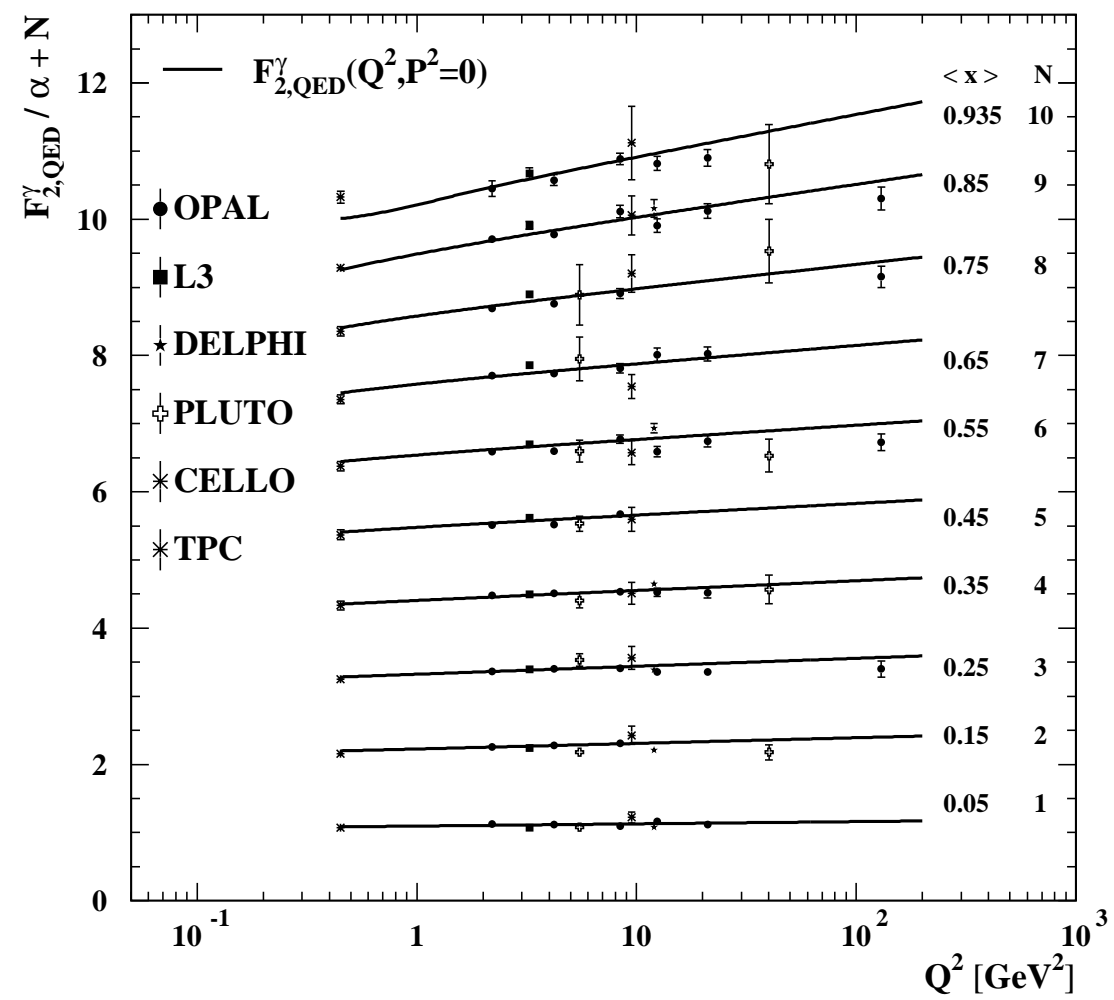

Figure 190: Summary of existing $F_{2}^{\gamma(Q E D)} / \alpha$ data as a function of $Q^{2}$ for fixed $\left\langle x_{B j}>\right.$ bins shown with $Q E D$ predictions for $P^{2}=0$ (from [2] $j$ ). 

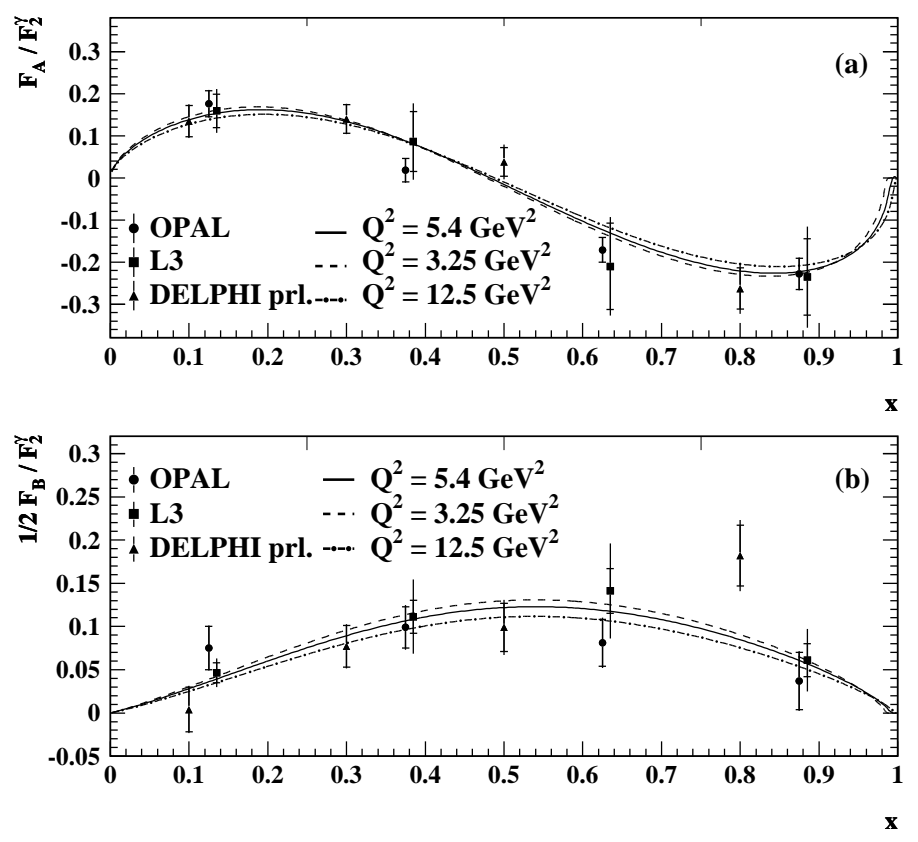

Figure 191: Summary of existing data: a) $F_{A}^{(Q E D)} / F_{2}^{\gamma(Q E D)}$ and b) $F_{B}^{(Q E D)} / F_{2}^{\gamma(Q E D)}$ as a function of $x_{B j}$ for $\left\langle Q^{2}>=3.25,5.4,12.5 \mathrm{GeV}^{2}\right.$, shown with $Q E D$ predictions for $P^{2}=0$ (from [a]j).
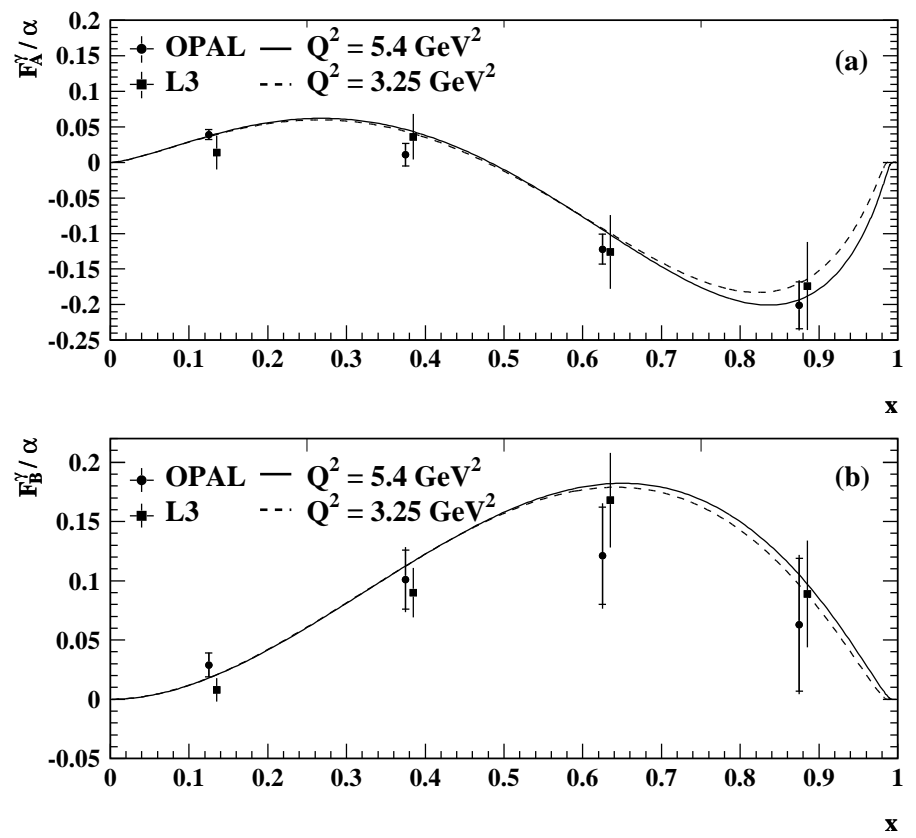

Figure 192: Summary of existing data: a) $F_{A}^{\gamma(Q E D)} / \alpha$ and b) $F_{B}^{\gamma(Q E D)} / \alpha$ as a function of $x_{B j}$. Data for $\left\langle Q^{2}>=3.25\right.$ and $5.4 \mathrm{GeV}^{2}$ are shown with corresponding $Q E D$ predictions for $P^{2}=0$ (from [2]j). 


\section{Selected results on the heavy quark content of the photon}

An additional information on the "structure" of the photon is coming from the production of heavy quarks in a photon-induced processes ( open heavy-flavor or heavy-flavor hadron production). In such processes heavy quark mass provides in a natural way the hard scale allowing to apply the perturbative QCD. However, the question how to describe within the perturbative QCD the heavy quark (hadron) production in hadronic processes is still open. Both the so called "massive" (no heavy parton) and "massless" (with heavy quark treated as a massless parton) schemes are being applied [207]-213]. For hard processes initiated by photons, where direct and resolved photon contributions are possible, this problem is particularly complex (see ref. [214]- [224]). In both approaches calculations for the heavy quark production in $\gamma \gamma$ and $\gamma p$ collisions were performed up to the NLO accuracy. The fragmentation of heavy quarks into heavy-flavor hadrons is usually described by a simple Peterson function [226] (sometimes with QCD evolution (DGLAP), see e.g. [223]). The NLO calculation of the charm quark production in the DIS D (contributions to $F_{2}^{\gamma}$ and $F_{L}^{\gamma}$ ) was done in 165. The progress in the theoretical description of heavy quark processes is necessary as large discrepancies in the description of the data involving heavy quarks are observed. This progress is needed not only for understanding of hadronic interaction. It is also crucial in searches of "new physics", e.g. for reliable estimation of the background.

On the experimental side - new results from LEP and HERA colliders have appeared recently on the production of $c$-quark (and $D^{* \pm}$ and $D_{s}^{ \pm}$mesons) as well as $b$ -quark, in the DIS e $_{\text {and }}$ an the resolved photon processes in $\gamma \gamma$ and $\gamma p$ collisions.

As it was already mentioned in sec. 2, in early $F_{2}^{\gamma}$ experiments the heavy quark distributions, mainly c-quark, were subtracted from $F_{2}^{\gamma}$ data, see e.g. [5], AMY 95 and TOPAZ 94 and references in [13. The recent DIS-type measurement at LEP 121 led to the extraction, for the first time, of the charm contribution to $F_{2}^{\gamma}$, denoted

$F_{2, c}^{\gamma}$. In fig. 193 the OPAL data in comparison with the NLO calculation [165] are shown. 

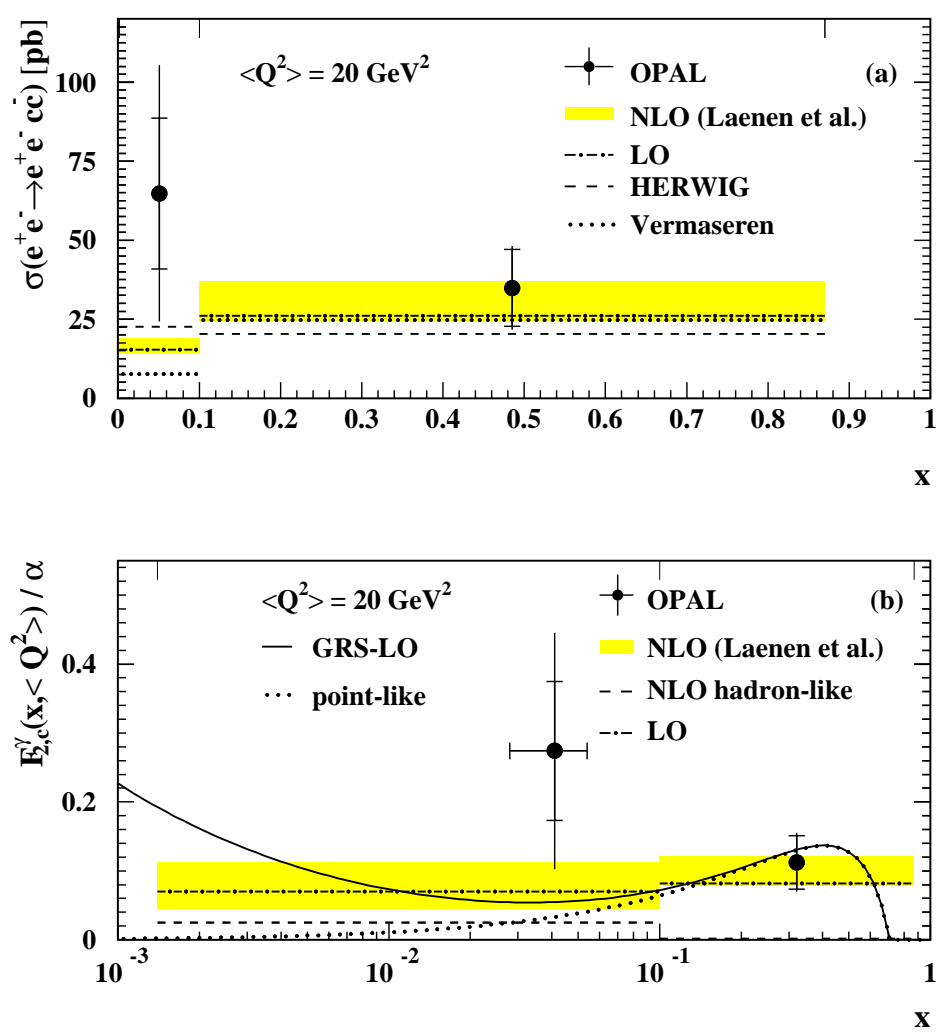

Figure 193: $O P A L$ data at $\left\langle Q^{2}\right\rangle=20 \mathrm{GeV}^{2}$ in comparison with predictions of $L O$ and NLO calculations and Monte Carlo models. a) The cross section for c $\bar{c}$ production as a function of $x_{B j}$. b) The extracted structure function $F_{2, c}^{\gamma}\left(x_{B j},<Q^{2}>\right) / \alpha$ (from [121]).

The first hint that the charm-quark production in $\gamma \gamma$ collisions requires the resolved photon processes can be found in AMY 1.5 94, [122], [123]; for other early results see ref. [124] - [129]. The new measurements of this type were performed recently at LEP by L3 group [130], for the first time with $b$-quarks production, see the collective figure, fig. 194. The data arrive also from the $c$-quark (also $D^{* \pm}$ and $D_{s}^{ \pm}$mesons) and $b$-quark photoproduction measurements at HERA [131, 132]. Some disagreement with the Monte Carlo predictions and the NLO QCD calculations, especially for the $b$-quark production, is observed, see fig. 195 for ZEUS preliminary results. 


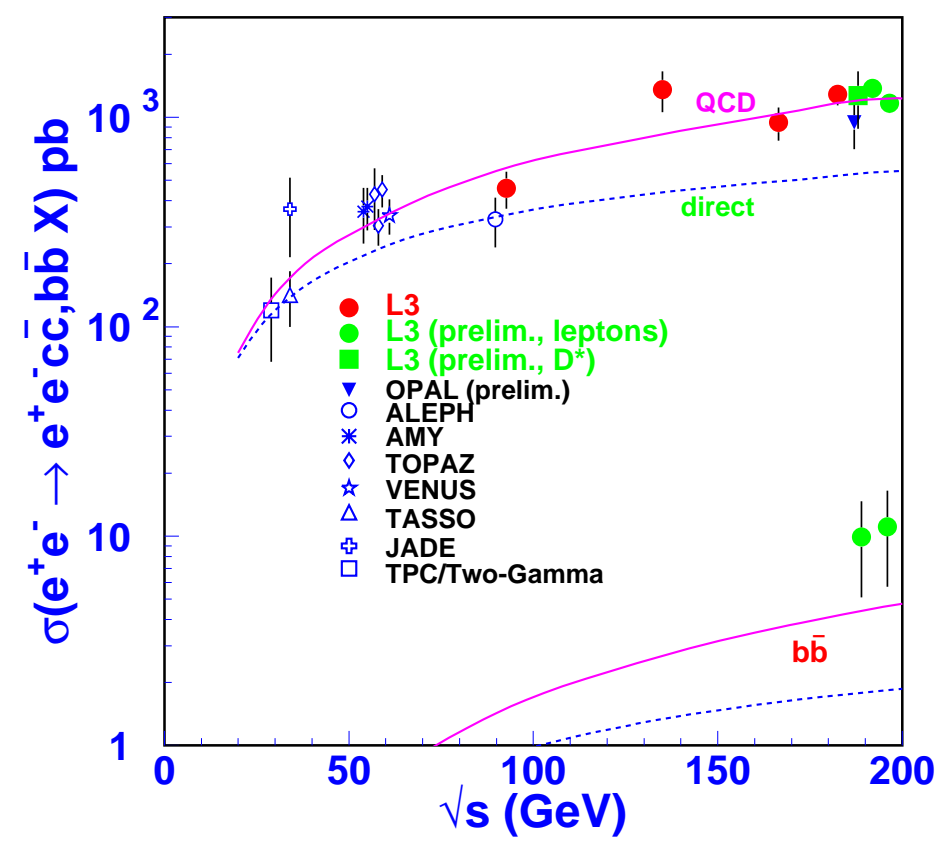

Figure 194: Summary of charm and beauty production in $\gamma \gamma$ collision at $e^{+} e^{-}$collisions (from [130]).
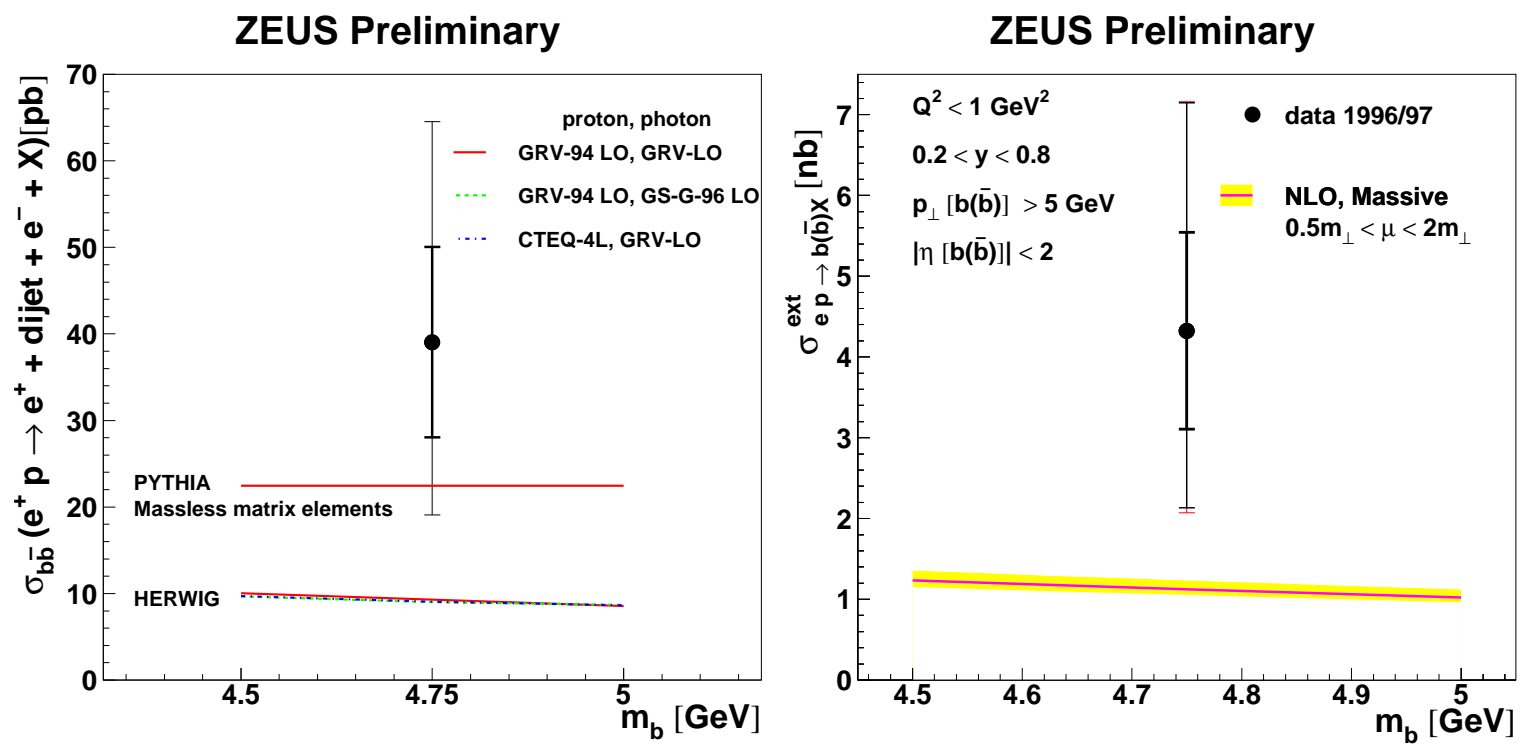

Figure 195: ZEUS preliminary data. Left: Measured $b \bar{b}$ cross section for ep collision with $P^{2}\left(=Q^{2}\right)<1 \mathrm{GeV}^{2}, 0.2<y<0.8$ and for $E_{T 1,2}>7,6 \mathrm{GeV},|\eta|<2.4, p_{T}^{e^{-}}>1.6 \mathrm{GeV},\left|\eta^{e^{-}}\right|<$ 1.1, compared to HERWIG and PYTHIA MC predictions. Right: Extrapolated $b(\bar{b})$ cross section in ep collisions for events with $P^{2}<1 \mathrm{GeV}^{2}, 0.2<y<0.8, p_{T b(\bar{b})}>5 \mathrm{GeV},\left|\eta_{b(\bar{b})}\right|<2$, compared to the "massive" NLO predictions (from [132]). 


\section{Related topics}

\subsection{On the polarized photon structure functions}

The polarized photon structure function $g_{1}^{\gamma}$ (another name is the spin dependent structure function, i.e. not spin-averaged like $F_{1}^{\gamma}$ and $F_{2}^{\gamma}$ ) both for a real and virtual photon can be studied in the DIS-type experiments with polarized electron beams and polarized photon target. The other spin dependent function, $F_{3}^{\gamma}$, can be studied in the conventional experimental setup with unpolarized electron beams, see sec. 2.2. The structure function $g_{1}^{\gamma}$ is of great importance, since its first moments (a sum rule for the "spin " of the photon) involve strong and electromagnetic anomalies, and it is deeply connected with the chiral properties of QCD [230].

The calculation of the structure function $g_{1}^{\gamma}$ for polarized real and virtual photon has been done in NLO in [227] and 228, 229], respectively. The NLO calculations of the jet production in the polarized real $\gamma$-hadron collision can be found in [231]- 234].

There is no data on the polarized photon structure functions $F_{3}^{\gamma}, g_{1}^{\gamma}$ nor on the polarized partonic content of the real and virtual photons from resolved photon processes. The future measurements in linear colliders, both in $e^{+} e^{-}$and $\gamma e$ or $\gamma \gamma$ options , may be crucial for testing the structure of polarized photons, see e.g. 2235, 133.

\subsection{On the structure function of the electron}

In the DIS experiments in $e^{+} e^{-}$collisions the inclusive hadron production can be ascribed not to the photon target, but rather to the parent electron or positron target. This in some cases may be more straightforward than pinning down the structure of the virtual photon, as was mentioned before.

This topic is discussed in e.g. Ref. [2] f, 228]. See also the results in 236], where the structure function of the electron (in general - lepton) is related not only to the structure function of photon but also to the electroweak gauge bosons $W$ and $Z$. The structure of weak bosons which appears in this approach was introduced and discussed in Ref. 237.

\subsection{On the photon content of the proton}

The photonic content of the proton relevant for the elastic and inelastic processes with an initial proton has been studied in 238] and 239, 240], respectively. 


\section{Summary and outlook}

The status of recent measurements ( 1990 and later $)$ of the "structure" of unpolarized photon in "DIS ${ }_{e \gamma}$ " experiments as well as in large $p_{T}$ jet production processes involving resolved photon(s) at $e^{+} e^{-}$and $e^{ \pm} p$ colliders is presented. In updating our previous work [2] i we have kept its form of the collection of selected data, presenting the main results of individual measurements. The final content of the survey has been dictated by the large amount of pioneering data that have appeared lately.

For the real photon the data from LEP 1 and higher energy data from LEP 1.5 and 2 , with the higher statistics and improved unfolding methods are now published in final or preliminary form. Final results from TRISTAN appeared. The basic features predicted by the QCD are observed with higher than previous accuracy, as qualitative change has appeared. The hadronic structure functions for real photon have been measured in the $\gamma^{*} \gamma$ processes in a wide range of $Q^{2}$ and down to very small $x_{B j}$. On the other hand impressive progress has been obtained in pinning down the parton densities, in particular the gluonic content, in large $p_{T}$ resolved photon processes in ep collisions. The data are not only from the larger than before kinematical range but are also more precise, which allowed to pose more quantitative questions, e.g. on the rise of the $F_{2}^{\gamma}$ or gluon density at the small $x_{B j}$. The prompt photon production has been measured in the resolved $\gamma p$ collisions at HERA (and also at TRISTAN in $\gamma \gamma$ collisions). This process can give an additional information on the partonic content of the photon as compared to the jet(s) production. The heavy $(c$ and $b)$ quark contribution to the photon structure functions has been measured in both $e^{+} e^{-}$ and $e^{ \pm} p$ collisions.

The survey contains a large amount of data related to the "structure" of the virtual photon from the hard photon-proton collisions at HERA, where the effective parton distribution has been extracted from the dijet production. More than 15 years after the first measurement at PLUTO the corresponding new precise DIS $_{e \gamma^{*}}$ measurement of the structure functions of virtual photon have been performed at LEP.

The new measurements of the partonic content of the photon are accompanied by the impressive progress made in the NLO QCD calculations for the resolved real and for virtual photon processes.

The discrepancies in describing the hadronic final states in the $\mathrm{DIS}_{\text {er }}$ experiments as well as in the resolved photon processes in $\gamma \gamma$ and $\gamma p$ collisions may have common origin. The need of additional $p_{T}$ in the standard QCD picture in the distribution of produced hadrons and jets may suggest an extra interaction involving the constituents of photon(s) - a multiple interaction. The newest data seem to give us a message that the partonic content of the photon is not properly described by existing parton parametrizations.

The future high-energy linear $e^{+} e^{-}$colliders (LC) 134 as well as related $e \gamma$ and $\gamma \gamma$ colliders, based on the backward Compton scattering on the laser light, will offer a unique opportunity to measure the structure of photon in a new kinematical regime [135. Moreover, at these colliders measurements of structure functions for photon with a definite polarization should become feasible with a good accuracy. To what

extent the new generation of $e p$ or $\gamma p$ accelerators under discussion (e.g. THERA [136]) may extend our present knowledge, remains to be seen. 


\section{Acknowledgments}

We express our special thanks to Aharon Levy for his encouragement, critical reading of the early version of the manuscript and important comments. We would like to thank R. Nisius for reading the earlier version of the updated paper, and his very useful, critical and constructive comments. We thank also I. Tyapkin, M. Kienzle, F. Kapusta, A. Finch and S.Söldner-Rembold for many important explanations and suggestions. We are very grateful to Stefan Söldner-Rembold for compilations of the newest results on $F_{2}^{\gamma}$. We are grateful to our colleagues from all collaborations carrying the $\gamma p$ and $\gamma \gamma$ analyses, especially to A. Finch, I. Tyapkin, F. Kapusta, M. Kienzle, M. Chamizo, J. A. Lauber, B. Surrow, S. Schlenstedt, P. Bussey and S.W. Lee for discussion and for providing us with their new data. Thanks are due to H. Hayashii and T. Nozaki for making available to us the postscript versions of older TRISTAN figures.

We thank T. Sjöstrand for illuminating discussion, and also D. Miller and L. Jonsson for important information.

We appreciate the collaboration with Jerzy Rowicki, Jan Żochowski, Paweł Jankowski and Urszula Jezuita-Dạbrowska. We would like to thank Mike Whalley for sending us copies of their "Compilation..." [13.

Supported in part by Polish Committee for Research, grant number 2P03B18209 (1996-1997), 2P03B18410 (1998-1999) and 2P03B11414 (2000), and by Interdisciplinary Centre for Mathematical and Computational Modelling, Warsaw University, Grant No G16-10 (1999). 


\section{Appendix}

\subsection{Parton parametrizations for the real photon}

Parton densities are given for fixed number of massless flavours (DO,LAC,GRSch,GIKO,IO) or for the number of flavours dependent on the scale $Q^{2}$ (DG,GRV,GS,AGF,WHIT,SaS).

Duke - Owens (DO) 154

A leading logarithmic parametrization of the parton distributions in an asymptotic form. Quarks with equal charges have the same distribution functions: $f_{u}=f_{c}$, $f_{d}=f_{s}\left(N_{f}=4\right)$.

Drees - Grassie (DG) 155]

A parametrization of the full solution of the leading order evolution equations. The input parton distributions with free parameters are assumed at $Q_{0}^{2}=1 \mathrm{GeV}^{2}$ and fitted to the only data on $F_{2}^{\gamma}$ existing at that time, at $Q^{2}=5.9 \mathrm{GeV}^{2}$, from PLUTO. Field - Kapusta - Poggioli (FKP) [32]

In this approach, there are two contributions to $F_{2}^{\gamma}$ : the nonperturbative ("hadronic") part, $F_{2}^{\gamma H A D}$, and the perturbative (pointlike) one, $F_{2}^{\gamma P L}$. The $F_{2}^{\gamma P L}$ arises from the basic direct $\gamma^{*} \rightarrow q \bar{q}$ coupling (and higher order QCD corrections), and it is included if the intrinsic quark $p_{T}$ is above a cutoff $p_{T}^{0}$. If $p_{T}$ is smaller than $p_{T}^{0}$ then the $q \bar{q}$ pair creates a bound state leading to $F_{2}^{\gamma H A D}$, usually taken from the VMD model. The perturbative $F_{2}^{\gamma P L}$ is calculated using the first order splitting functions and the one-loop $\alpha_{S}$.

Levy - Abramowicz - Charchuła (LAC) 156

A parametrization of the full solution of the leading order evolution equation fitted to all available in 1991 measurements of $F_{2}^{\gamma}$ for $Q^{2} \geq Q_{0}^{2}$. The number of flavours is fixed to $N_{f}=4$. Three sets are provided with the different choices of an input scale $Q_{0}^{2}$, and the $x \rightarrow 0$ behaviour of a gluon distribution $G(x)$, namely:

- LAC1: $Q_{0}^{2}=4 \mathrm{GeV}^{2}$

- LAC2: $Q_{0}^{2}=4 \mathrm{GeV}^{2}, x G(x) \rightarrow$ const.

- LAC3: $Q_{0}^{2}=1 \mathrm{GeV}^{2}$.

Gordon - Storrow (GS) 157

The LO and NLO parametrizations. The input structure function at the scale $Q_{0}^{2}=5.3$ $\mathrm{GeV}^{2}$ [157] a and $Q_{0}^{2}=3 \mathrm{GeV}^{2}$ [157]b is chosen in the LO analysis as a sum of a hadronic part from the VMD model and of a pointlike part based on the Parton Model. Free parameters (also light quarks masses) are fitted to the data with $Q^{2} \geq Q_{0}^{2}$. The input gluon distribution in LO [157] a is assumed in two different forms (set GS1 and GS2). The NLO distributions in the $\overline{\mathrm{MS}}$ scheme are obtained by matching of the $F_{2}^{\gamma}$ in the LO and the NLO approaches at the $Q_{0}^{2}$ scale. $N_{f}=3,4$, and 5 is used in [157]a. In 1157 b the number of flavours equal to $N_{f}=3$ for $Q_{0}^{2} \leq Q^{2} \leq 50 \mathrm{GeV}^{2}$ and $N_{f}=4$ for $50 \mathrm{GeV}^{2} \leq Q^{2}$ was used.

Glück - Reya - Vogt (GRV) [158]

The LO and NLO parametrizations of the parton distributions generated dynamically with the boundary conditions at $Q_{0}^{2}$ given by a VMD input. The physical photon is then assumed to be a coherent superposition of vector mesons, whose parton distributions are further assumed to be similar to those of a pion. The low initial scale $Q_{0}^{2}(\mathrm{LO})=0.25, Q_{0}^{2}(\mathrm{NLO})=0.3 \mathrm{GeV}^{2}$ is universal for structure functions of $p, \pi, \gamma$ 
etc. The DIS ${ }_{\gamma}$ scheme is introduced to avoid the large- $x_{B j}$ instability problems. The one free parameter, which is the VMD input normalization constant relative to $\pi$, is fixed by the data.

Aurenche - Chiapetta - Fontannaz - Guillet - Pilon (ACFGP) 159

A solution of the NLO evolution equation with the boundary condition taken at $Q_{0}^{2}$ $=0.25 \mathrm{GeV}^{2}$. The input parton distributions were obtained from the VMD model at $Q^{2}=2 \mathrm{GeV}^{2}$ and evaluated down to $Q_{0}^{2}$.

Aurenche - Guillet - Fontannaz (AGF) [160]

The NLO parton distributions obtained with the input distributions (shown to be scheme-dependent) at $Q_{0}^{2}=0.5 \mathrm{GeV}^{2}$. The input distributions are based on the VMD model modified to agree with the $\overline{\mathrm{MS}}$ scheme used in this analysis.

Hagiwara - Izubuchi - Tanaka - Watanabe (WHIT) [161]

A set of six LO parametrizations obtained by fitting the input distributions to all available data for $4 \mathrm{GeV}^{2} \leq Q^{2} \leq 100 \mathrm{GeV}^{2}$. The parametrizations WHIT1-WHIT6 are based on different input gluon distributions. A massive charm contribution is calculated from the Quark Parton Model below $Q^{2}=100 \mathrm{GeV}^{2}$, above this scale from the massive quark evolution equations.

Schuler - Sjöstrand (SaS) 162

Four sets of the LO parametrizations. The nonperturbative input distributions at $Q_{0}=0.6 \mathrm{GeV}$ in the SaS1D, SaS1M and $Q_{0}=2 \mathrm{GeV}$ in the SaS2D, SaS2M sets are based on the VMD model (their normalization is fixed by the momentum sum rule, and the $x$-dependence is obtained from the fits to the data). The fully calculable pointlike contribution to $F_{2}^{\gamma}$ is expressed as an integral over the $\gamma^{*} \rightarrow q \bar{q}$ virtuality $k^{2}$. The nonleading term $C_{\gamma}$ is included into the $F_{2}^{\gamma}$, leading to the $\overline{\mathrm{MS}}$ distributions (SaS1M, SaS2M). $C_{\gamma}=0$ gives distributions in the DIS scheme (SaS1D, SaS2D).

Glück - Reya - Schienbein (GRSch) [163

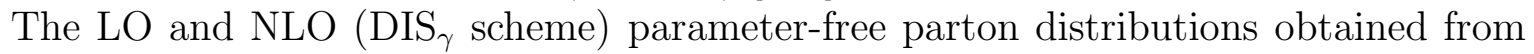
the VMD input based on a coherent superposition of vector mesons applying the new Glück - Reya - Schienbein pionic distributions [164]. The low initial scale (universal for the proton, pion and photon distributions) $Q_{0}^{2}(\mathrm{LO})=0.26, Q_{0}^{2}(\mathrm{NLO})=0.40 \mathrm{GeV}^{2}$ is assumed. The parton distributions are calculated for light quarks, $N_{f}=3$ (contribution of heavy quarks is taken into account in the structure function $F_{2}^{\gamma}$ ).

Laenen - Riemersma - Smith - Neerven (LRSN) 165

The NLO calculation of the structure function $F_{2}^{\gamma}$ and $F_{L}^{\gamma}$, including the heavy quark contribution.

Gorski - Ioffe - Khodjamirian - Oganesian (GIKO) 166

The structure function $F_{2}^{\gamma}$ of the real photon, obtained from the results for the virtual photon, where beside PM also a gluon condensate contribution is included (see below). Only the ligh quarks contributions are considered (i.e. $N_{f}=3$ ). This approach is valid for $Q^{2}$ below $10 \mathrm{GeV}^{2}$ due to the lack of $Q^{2}$ evolution, and for intermediate $x_{B j}$ values: $0.2-0.7$.

Ioffe - Oganesian (IO) 167

Gluon distributions in the real photon calculated using the LO and NLO evolution equations, with the light quark densities input from GIKO. Two sets of gluon density are studied: 1) without the intrinsic gluons at the input scale $Q_{0}^{2}=1 \mathrm{GeV}^{2}, 2$ ) with the gluon density at the input scale equal to gluonic content of vector mesons $\rho, \omega, \phi$. The range of applicability: $x_{B j}$ below 0.6 down to $0.03-0.05$, and $1<Q^{2}<100 \mathrm{GeV}^{2}$. 
Here also results for $F_{2}^{\gamma}$ of the real photon, with the LO and NLO evolutions in $Q^{2}$ are given (with $N_{f}=3$ ), valid for $0.2<x_{B j}<0.7$ and $1<Q^{2}<50 \mathrm{GeV}^{2}$.

H. Abramowicz, E. Gurvich, A. Levy (GAL) 169

The LO [169 a and NLO [169 b parton densities in the photon are extracted from the existing $F_{2}^{\gamma}$ measurements and the low- $x$ proton structure function (related to the $F_{2}^{\gamma}$ by Gribov factorization at low- $x$ ).

\subsection{Parton parametrizations for the virtual photon}

The notion of the partonic content of the virtual photon $\left(P^{2} \neq 0\right)$ can be applied if $Q^{2}$ scale is larger than $P^{2}$ (typically $P^{2} \lesssim 0.2 Q^{2}$ ). The parton distributions in the virtual photon can be calculated in the perturbative QCD for $\Lambda^{2} \ll P^{2} \ll Q^{2}$ without any input, for lower $P^{2}$ extra assumptions are needed. The parametrizations described below are valid for $0 \leq P^{2}$ and become the corresponding parametrizations for the real photon in the limit $P^{2} \rightarrow 0$. In the low- $P^{2}$ region some of them are based on interpolation between parton distributions in the real photon $\left(P^{2}=0\right)$ and the QCD predictions in high- $P^{2}$ region $\left(\Lambda^{2} \ll P^{2}\right)$. All the parametrizations presented below deal with the transverse polarized virtual photon, with one exception (Chýla parametrization).

\section{Drees - Godbole [2]f}

The parametrization of the parton distributions in the electron and in the virtual photon. The parton distributions in the virtual photon are equal to the corresponding distributions in the real photon (e.g. DG, GRV) multiplied by a factor dependent on virtuality $P^{2}$. In the limit $P^{2} \rightarrow Q^{2} \gg \Lambda^{2}$ the quark distributions in the virtual photon approach the leading logarithmic $\left(\ln \frac{Q^{2}}{P^{2}}\right)$ parton model predictions arising from $\gamma^{*}\left(Q^{2}\right) \gamma^{*}\left(P^{2}\right) \rightarrow q \bar{q}$ subprocess. The gluon distributions in this limit approach the $Q^{2}$ - behaviour $\left(\sim \ln ^{2} \frac{Q^{2}}{P^{2}}\right)$ following from the $\gamma^{*}\left(Q^{2}\right) \gamma^{*}\left(P^{2}\right) \rightarrow g q \bar{q}$ cross section. The parametrization contains one free parameter $P_{c}^{2}$, a typical hadronic scale (in applications denoted also as $\left.\omega^{2}\right)$.

Glück - Reya - Stratmann (GRS) [170]

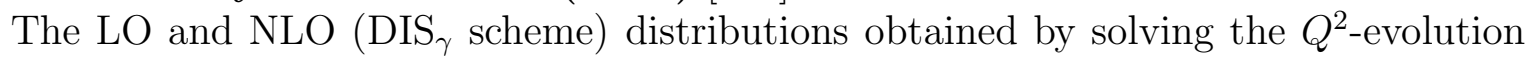
equation with boundary conditions being a smooth interpolation between boundary conditions valid at high and low $P^{2}$. For high $P^{2} \rightarrow Q^{2} \gg \Lambda^{2}$ the solutions correspond to the cross section for $\gamma^{*}\left(Q^{2}\right) \gamma^{*}\left(P^{2}\right) \rightarrow q \bar{q}$ subprocess. For $P^{2}=0$ they are given by VMD input parton distributions at the scale $Q_{0}^{2}(\mathrm{LO})=0.25$ or $Q_{0}^{2}(\mathrm{NLO})=0.3 \mathrm{GeV}^{2}$, as for the real photon in [158]. The applicability of the virtual photon parametrizations is assumed for $0 \leq P^{2} \leq 10 \mathrm{GeV}^{2}$ and $5 P^{2} \lesssim Q^{2}$. The number of active flavours is fixed to $N_{f}=3$.

Schuler - Sjöstrand (SaS) 162

An extension of the SaS parton distributions in the real photon to the virtual photon case, valid for $0 \leq P^{2} \leq Q^{2}$. In the pointlike contribution, the integral over the virtuality $k^{2}$ of the $\gamma^{*} \rightarrow q \bar{q}$ state, is modified by a factor $\left(\frac{1}{k^{2}}\right)^{2}$ with the lower limit of the integration equal to a cutoff scale $P_{0}^{2}$ (several choices for $P_{0}^{2}$ have been considered, e.g. $\left.P_{0}^{2}=\max \left\{P^{2}, Q_{0}^{2}\right\}\right)$. In the hadronic contribution a factor $\left(\frac{m_{V}^{2}}{m_{V}^{2}+P^{2}}\right)^{2}$ is introduced, where $m_{V}$ is a vector-meson mass. The notation SaS1D, SaS2D, SaS1M, and SaS2M is the same as in the real photon case. 
Glück - Reya - Schienbein (GRSch) [163

The LO and NLO parton distributions valid for $0 \leq P^{2} \ll Q^{2}$ and $N_{f}=3$. It is argued that whenever the virtual photon is probed at $Q^{2} \gg P^{2}$ it may be treated as the real one: the longitudinal polarization should be neglected and a partonic cross section involving $\gamma\left(P^{2}\right)$ should be calculated as if $P^{2}=0$ (in this approach the virtuality $P^{2} \neq 0$ is present in the flux $e \rightarrow e \gamma^{*}$ and in the virtual photon parton distributions). The boundary conditions in the limit $P^{2} \rightarrow Q^{2} \gg \Lambda^{2}$ should be calculated from $\gamma^{*}\left(Q^{2}\right) \gamma\left(P^{2}=0\right) \rightarrow q \bar{q}$ subprocess instead of $\gamma^{*}\left(Q^{2}\right) \gamma^{*}\left(P^{2}\right) \rightarrow q \bar{q}$; this implies changing the boundary conditions compared to the previous approach [170]. Now, at $P^{2}=Q^{2} \gg \Lambda^{2}$ the parton distributions vanish in both LO and NLO in DIS scheme. $^{2}$ The input distributions at the initial scale $Q^{2}=\max \left\{P^{2}, Q_{0}^{2}\right\}$ are assumed to be equal to the VMD parton distributions in the real photon (see sec. 8.1) multiplied by the factor $\left(\frac{m_{\rho}^{2}}{m_{\rho}^{2}+P^{2}}\right)^{2}$, where $m_{\rho}=0.59 \mathrm{GeV}^{2}, Q_{0}^{2}(\mathrm{LO})=0.26 \mathrm{GeV}^{2}$ and $Q_{0}^{2}(\mathrm{NLO})=0.4$ $\mathrm{GeV}^{2}$.

Gorski - Ioffe - Khodjamirian - Oganesian (GIKO) 166

The structure function $F_{2}^{\gamma}$ of the virtual photon, for $P^{2}>0.5 \mathrm{GeV}^{2}$, calculated using the Operator Product Expansion as a series in $1 / P^{2}$, where beside PM also a gluon condensate contribution is included. Using a dispersion relation the structure function is then presented in terms of the contributions from physical states $(\rho, \omega, \phi)$ and continuum. The proper continuation to $P^{2}=0$ can be performed, leading to prediction for the real photon (see sec. 8.1). Only the light quark contributions are considered. This approach is valid for intermediate $x_{B j}$ values: $0.2-0.7$, and for $Q^{2}$ larger than $P^{2}$ but below $10 \mathrm{GeV}^{2}$, due to the lack of $Q^{2}$ evolution.

Ioffe - Oganesian (IO) 167

Gluon distributions in the (transverse) virtual photon calculated using the LO and NLO evolution equations, with the light quark densities input from GIKO. Two types of gluon density are studied: 1) without the intrinsic gluons at the input scale, $Q_{0}^{2}=1$ $\left.\mathrm{GeV}^{2}, 2\right)$ with the gluon density at the input scale, $Q_{0}^{2}=1 \mathrm{GeV}^{2}$, equal to the gluonic content of the vector mesons $\rho, \omega, \phi$. The range of applicability: $x_{B j}$ below 0.6 down to $0.03-0.05$ and $1<Q^{2}<100 \mathrm{GeV}^{2}$.

Here also results for $F_{2}^{\gamma}$ of the (unpolarized and transverse) virtual photon, for 50 $\mathrm{GeV}^{2}>P^{2}>0.5 \mathrm{GeV}^{2}$ with the $\mathrm{LO}$ and NLO evolutions in $Q^{2}$ are given (with $\left.N_{f}=3\right)$. Results for $F_{2}^{\gamma}$ for a real photon are also given (see above).

Chýla [168]

The LO parametrization of the pointlike quark and gluon densities in the longitudinal virtual photon, $1 \mathrm{GeV}^{2}<P^{2}<0.2 Q^{2}$, obtained from the homogeneous evolution equation, without extra input. For $P^{2} \rightarrow Q^{2}$ the gluon distribution $G_{L}$ in the longitudinal virtual photon vanishes, while the quark densities $q_{L}^{S}=q_{L}^{N S}$ approach the PM formula for $\gamma^{*}\left(Q^{2}\right) \gamma^{*}\left(P^{2}\right) \rightarrow q \bar{q}$. Parton densities satisfy the momentum sum rule. 


\section{References}

[1] T.H. Bauer et al., Rev. of Modern Phys. 50 (1978) 262 (Err. ibid 51 (1979) 407)

[2] a) V.M. Budnev et al., Phys. Rep. C15 (1975) 181

b) H. Kolanoski, Springer Tracts in Modern Physics 105 (1984) 187

c) Ch. Berger and W. Wagner, Phys. Rep. C146 (1987) 1

d) H. Abramowicz et al., Int. J. of Modern Physics A8 (1993) 1005

e) M. Drees and R.M. Godbole, Pramana - J. of Physics 41 (1993) 83;

J. of Phys. G: Nucl. and Part. Phys. 21 (1995) 1559

f) M. Drees and R.M. Godbole, Phys. Rev. D50 (1994) 3124

g) S. Brodsky and P. Zerwas, Nucl. Instr. and Methods in Phys.Res. A355 (1995) 19

h) M. Erdmann, Springer Tracts in Modern Physics 138 (1997)

i) M. Krawczyk, M. Staszel and A. Zembrzuski, IFT 97/14, DESY 98-013 hepph/9806291)

j) R. Nisius, Phys. Rep. 332 (2000) 166

[3] G.A. Schuler and T. Sjöstrand, Nucl. Phys. B407 (1993) 539;

G.A. Schuler and T. Sjöstrand, Proc. of the Workshop on Two-Photon Physics from DA $\Phi$ NE to LEP200 and Beyond, Paris, February 1994, eds. F. Kapusta and J. Parisi, World Scientific 1994, p. 163

[4] PLUTO Coll., a) Ch. Berger et al., Phys. Lett. B107 (1981) 168; b) Ch. Berger et al., Phys. Lett. B142 (1984) 111; c) Ch. Berger et al., Z. Phys. C26 (1984) 353; d) Ch. Berger et al., Nucl. Phys. B281 (1987) 365

CELLO Coll., H.-J. Behrend et al., Phys. Lett. B126 (1983) 391

JADE Coll., W. Bartel et al., Z. Phys. C24 (1984) 231

TASSO Coll., M. Althoff et al., Z. Phys. C31 (1986) 527

TPC/2 $\gamma$ Coll., H. Aihara et al., Z. Phys. C34 (1987) 1; Phys. Rev. Lett. 58 (1987) 97

[5] AMY Coll., T. Sasaki et al., Phys. Lett. B252 (1990) 491

[6] PLUTO Coll., Ch. Berger et al., Phys. Lett. B142 (1984) 119

[7] M. N. Kienzle-Focacci, Proc. of the Int. Conf. on the Structure and Interactions of the Photon and the 12th Int. Workshop on Photon-Photon Collisions, Freiburg im Breisgau, Germany, May 1999, ed. S. Söldner-Rembold, Nucl. Phys. B (Proc. Suppl.) 82 (2000) 447

[8] a) D.J. Miller, Proc. of the Workshop on Two-Photon Physics at LEP and HERA, Lund, May 1994, eds. G. Jarlskog, L. Jönsson, p. 4

b) D.J. Miller, Proc. of the Int. Conf. on the Structure and Interactions of the Photon and the 11th Int. Workshop on Photon-Photon Collisions, Egmond aan Zee, The Netherlands, May 1997, eds. A. Buijs and F.C. Erné, World Scientific, 1997, p. 431

c) D.J. Miller, J. Phys. G24 (1998) 317 
[9] S. Cartwright et al., J. Phys. G24 (1998) 457;

G. Covan, Statistical Data Analysis, Clarendon Press, Oxford 1998

[10] M. N. Kienzle-Focacci, private communication

[11] A. J. Finch, Proc. of the Int. Conf. on the Structure and Interactions of the Photon and the 12th Int. Workshop on Photon-Photon Collisions, Freiburg im Breisgau, Germany, May 1999, ed. S. Söldner-Rembold, Nucl. Phys. B (Proc. Suppl.) 82 (2000) 156

[12] J. M. Butterworth and R. J. Taylor, Proc. of the Int. Conf. on the Structure and Interactions of the Photon and the 12th Int. Workshop on Photon-Photon Collisions, Freiburg im Breisgau, Germany, May 1999, ed. S. Söldner-Rembold, Nucl. Phys. B (Proc. Suppl.) 82 (2000) 112

[13] D. Morgan, M.R. Pennington, M.R. Whalley, J. Phys. G: Nucl. Part. Phys. 20 (1994) A1

[14] C.F. von Weizsäcker, Z.Phys. 88 (1934) 612; E.J. Williams, Kgl. Danske Vidensk. Selskab. Mat.-Fiz. Medd. 13 (1935) N4; E.J. Williams, Phys. Rev. 45 (1934) 729

[15] G. Schuler, Improving the equivalent-photon approximation in electron-positron collisions, CERN-TH/96-297 (hep-ph/9610406)

[16] V. Telnov, Nucl. Instr. and Methods in Phys. Res. A355 (1995) 3;

R. Brinkmann et al., Nucl. Instr. and Methods in Phys. Res. A406 (1998) 13

[17] V.E. Balakin, V.M. Budnev, I.F. Ginzburg, JETP Lett. 11 (1970) 388;

V.M. Budnev, I.F. Ginzburg, Phys. Lett. B37 (1971) 320;

S.J. Brodsky, T. Kinoshita, H. Terazawa, Phys. Rev. Lett. 27 (1971) 280;

T.F. Walsh, Phys. Lett. B36 (1971) 121;

C.E. Carlson, Wu-Ki Tung, Phys. Rev. D4 (1971) 2873;

T.F. Walsh, P. Zerwas, Phys. Lett. B44 (1973) 195; P. Zerwas, Phys. Rev. D10 (1974) 1485;

R.L. Kingsley, Nucl. Phys. B60 (1973) 45;

V.L. Chernyak, V.G. Serbo, Nucl. Phys. B71 (1974) 395;

M.A. Ahmed, G.G. Ross, Phys. Lett. B59 (1975) 369;

R.P. Worden, Phys. Lett. B(1974) 57;

W.R. Frazer, G. Rossi, Phys. Rev. D25 (1982) 843;

[18] E. Witten, Nucl. Phys. B120 (1977) 189

[19] R.J. DeWitt et al., Phys. Rev. D19 (1979) 2046;

[20] C.H. Llewellyn-Smith, Phys. Lett. B79 (1978) 83;

W.R. Frazer, J.F. Gunion, Phys. Rev. D20 (1979) 147;

C. Peterson, T.F. Walsh, P.M. Zerwas, Nucl. Phys. B174 (1980) 424; 229 (1983)

301;

K. Sasaki, Phys. Rev. D22 (1980) 2143; F. Delduc et al., Nucl. Phys. B174 (1980)

147, 157; D. W. Duke and J.F. Owens, Phys. Rev. D22 (1980) 2280 
[21] W.A. Bardeen, A.J. Buras, Phys. Rev. D20 (1979) 166; Phys. Rev. D20 (1980) 2041

[22] C.T. Hill, G.G. Ross, Nucl. Phys. B148 (1979) 373;

T. Uematsu, T.F. Walsh Phys. Lett. B101 (1981) 263; Nucl. Phys. B199 (1982) 93;

G. Rossi, Ph.D.Thesis, UCSD-10p10-227, Phys. Lett. B130 (1983) 105

[23] M. Glück, K. Grassie and E. Reya, Phys. Rev. D30, (1984) 1447

[24] J.J. Sakurai, Ann. Phys. 11 (1960) 1; J.J. Sakurai and D. Schildknecht, Phys. Lett. B40 (1972) 121

[25] Yu.L. Dokshitzer, Sov. Phys JETP 46 (1977) 641;

V.N. Gribov, L.N. Lipatov, Sov. J. Nucl. Phys. 15 (1972) 438 and 675;

G. Altarelli, G. Parisi Nucl. Phys. 126 (1977) 297

[26] M. Gluck and E. Reya, Phys. Lett. B443 (1998) 298;

L.L. Frankfurt and E.G. Gurvich, hep-ph/9505406; J.Phys. G22 (1996)903; Phys. Lett. B386 (1996)379

[27] J. Chýla, JHEP 04 (2000) 007

[28] E.A. Kuraev, L.N. Lipatov and V.S. Fadin, Sov. Phys JETP 45 (1977) 199; Y.Y. Balitsky and L.N. Lipatov Sov. J. Nucl. Phys. 28 (1978) 822

[29] V.S. Fadin, L.N. Lipatov Phys. Lett. B429 (1998) 127

[30] M. Ciafaloni, Nucl. Phys. B296 (1988) 49;

S. Catani, F. Fiorani, G. Marchesini, Phys. Lett. B234 (1990) 339, Nucl. Phys. B336 (1990) 18

[31] J.J. Ward, Proc of the Workshop on Two-Photon Physics from DA $\Phi$ NE to LEP200 and Beyond, Paris, 1994, eds. F. Kapusta and J. Parisi, World Scientific 1994, p. 199

[32] J.H. Field, F. Kapusta and L. Poggioli, Phys. Lett. B181 (1986) 362; Z. Phys. C36 (1987) 121;

F. Kapusta, Z. Phys. C42 (1989) 225

[33] ALEPH Coll., R. Barate et al. Phys. Lett. B458 (1999) 152

[34] ALEPH Coll., K. Affholderbach et al., contribution to the Int. Europhysics Conf. on High Energy Physics, Tampere, Finland, 1999, No 2-617 (ALEPH 99-038, CONF 99-022)

[35] DELPHI Coll., P. Abreu et al., Z. Phys. C69 (1996) 223

[36] DELPHI Coll., I. Tyapkin, Proc. of the 28th Conf. on High Energy Physics ICHEP'96, Warsaw 1996, eds. Z. Ajduk and A.K. Wróblewski, World Scientific 1997, p. 729; F. Kapusta et al., DELPHI 96-77 Conf 9, Contribution to ICHEP'96 (pa02-021) 
[37] DELPHI Coll., F. Kapusta et al., submitted to the Int. Europhysics Conf. on High Energy Physics, Jerusalem, 1997 (N416, DELPHI 97-122 CONF 104);

I. Tyapkin, Proc. of the Int. Conf. on the Structure and Interactions of the Photon and the 11th Int. Workshop on Photon-Photon Collisions, Egmond aan Zee, The Netherlands, May 1997, eds. A. Buijs and F.C. Erné, World Scientific, 1997, p. 26

[38] DELPHI Coll., I. Tyapkin, Proc. of Workshop on the Photon Interaction and the photon structure, Lund, Sweden, Sept. 1998, eds. G. Jarlskog and T. Sjöstrand, p. 59

[39] DELPHI Coll., Courtesy of I. Tyapkin

[40] L3 Coll., M. Acciarri et al., Phys. Lett. B436 (1998) 403

[41] L3 Coll., M. Acciarri et al., Phys. Lett. B447 (1999) 147

[42] L3 Coll., M. Acciarri et al., Phys. Lett. B483 (2000) 373

[43] C.H. Lin, L3 Note 2298 (1998)

[44] OPAL Coll., R. Akers et al., Z. Phys. C61 (1994) 199

[45] OPAL Coll., K. Ackerstaff et al., Z. Phys. C74 (1997) 33

[46] OPAL Coll., K. Ackerstaff et al., Phys. Lett. B412 (1997) 225

[47] OPAL Coll., K. Ackerstaff et al., Phys. Lett. B411 (1997) 387

[48] OPAL Coll., G. Abbiendi et al., CERN-EP-2000-082, submitted to Eur. Phys. J. C

[49] AMY Coll., S.K. Sahu et al., Phys. Lett. B346 (1995) 208

[50] AMY Coll., T. Kojima et al., Phys. Lett. B400 (1997) 395

[51] TOPAZ Coll., K. Muramatsu et al., Phys. Lett. B322 (1994) 447

[52] Courtesy of S. Söldner-Rembold

[53] K. Müller, Proc. of the Int. Europhysics Conf. on High Energy Physics, Jerusalem, August 1997, eds. D. Lelouch, G. Mikenberg and E. Rabinovici, Springer Verlag 1999, p. 464

[54] B.L. Combridge and C.J. Maxwell, Nucl. Phys. B239 (1984) 429

[55] F. Kapusta, LPNHE-92-08, Proc. of the 9th Int. Workshop on Photon-Photon Collisions, eds. D.O. Caldwell and H.P. Paar, World Scientific 1992, p. 142

[56] ALEPH Coll., submitted to the Int. Europhysics Conf. on High Energy Physics, Jerusalem, August 1997, No 592, and to the XVIII Int. Symposium on LeptonPhoton Interactions, Hamburg, July-August 1997 (LP253) 
[57] DELPHI Coll., P. Abreu et al., Phys. Lett. B342 (1995) 402

[58] OPAL Coll., submitted to the XVIII Int. Symposium on Lepton-Photon Interactions, Hamburg, July-August 1997 and to the Int. Europhysics Conf. on High Energy Physics, Jerusalem, August 1997 (OPAL Physics Note PN293)

[59] J.A. Lauber, L. Lönnblad, M.H. Seymour, Proc. of the Int. Conf. on the Structure and Interactions of the Photon and the 11th Int. Workshop on Photon-Photon Collisions, Egmond aan Zee, The Netherlands, May 1997, eds. A. Buijs and F.C. Erné, World Scientific, 1997, p. 52

[60] B. L. Combridge et al., Phys. Lett. B70 (1977) 234;

D. W. Duke and J. F. Owens [154];

M. Krawczyk and A. F. Żarnecki, IFT 08/91

[61] OPAL Coll., B. Surrow, Proc. of the Workshop on Photon Interaction and the Photon Structure, Lund, Sept. 1998, eds. G. Jarlskog and T. Sjöstrand, p. 83

[62] ALEPH Coll., D. Buskulic et al., Phys. Lett. B313 (1993) 509

[63] DELPHI Coll., P. Abreu et al., Z. Phys. C62 (1994) 357

[64] DELPHI Coll., N. Zimin, Proc. of the Int. Conf. on the Structure and Interactions of the Photon and the 11th Int. Workshop on Photon-Photon Collisions, Egmond aan Zee, The Netherlands, May 1997, eds. A. Buijs and F.C. Erné, World Scientific, 1997, p. 74

[65] OPAL Coll., K. Ackerstaff et al., Z. Phys. C73 (1997) 433

[66] OPAL Coll., Abbiendi et al., Eur. Phys. J. C10 (1999) 547

[67] AMY Coll., R. Tanaka et al., Phys. Lett. B277 (1992) 215

[68] AMY Coll., B.J. Kim et al., Phys. Lett. B325 (1994) 248

[69] TOPAZ Coll., H. Hayashii et al., Phys. Lett. B314 (1993) 149

[70] TOPAZ Coll., H. Hayashii, Proc. of the 10th Workshop on Gamma - Gamma Collisions and Related Processes, Sheffield, UK, April 1995, eds. D.J. Miller, S.L. Cartwright and V. Khoze, World Scientific 1995, p. 133

[71] TOPAZ Coll., H. Hayashii, Proc. of the Workshop on Photon Interactions and the Photon Structure, Lund, Sept. 1998, eds. G. Jarlskog and T. Sjöstrand, p. 297

[72] H1 Coll., T. Ahmed et al., Phys. Lett. B297 (1992) 205

[73] H1 Coll., I. Abt et al., Phys. Lett. B314 (1993) 436

[74] H1 Coll., T. Ahmed et al., Nucl. Phys. B445 (1995) 195

[75] H1 Coll., S. Aid et al., Z. Phys. C70 (1996) 17 
[76] H1 Coll., C. Adloff et al., Eur. Phys. J. C1 (1998) 97

[77] H1 Coll., C. Adloff et al., Eur. Phys. J. C10 (1999) 363

[78] H1 Coll., C. Adloff et al., Phys. Lett. B483 (2000) 36

[79] ZEUS Coll., M. Derrick et al., Phys. Lett. B297 (1992) 404

[80] ZEUS Coll., M. Derrick et al., Phys. Lett. B322 (1994) 287

[81] ZEUS Coll., M. Derrick et al., Phys. Lett. B342 (1995) 417

[82] ZEUS Coll., M. Derrick et al., Phys. Lett. B348 (1995) 665

[83] ZEUS Coll., M. Derrick et al. Phys. Lett. B354 (1995) 163

[84] ZEUS Coll., M. Derrick et al., Phys. Lett. B384 (1996) 401

[85] ZEUS Coll., J. Breitweg, et al., Eur. Phys. J. C1 (1998) 109

[86] ZEUS Coll., J. Breitweg et al., Eur. Phys. J. C4 (1998) 591

[87] ZEUS Coll., submitted to the 29th Int. Conf. on High Energy Physics, ICHEP'98, Vancouver, Canada, July 1998, abstract:812

[88] ZEUS Coll., J. Breitweg et al., Eur. Phys. J. C11 (1999) 35

[89] ZEUS Coll., submitted to the Int. Europhysics Conf. on High Energy Physics, Tampere, Finland, July 1999, abstract:540

[90] ZEUS Coll., submitted to the 29th Int. Conf. on High Energy Physics, ICHEP'98, Vancouver, Canada, July 1998, abstract:805

[91] H1 Coll., submitted to the Int. Europhysics Conf. on High Energy Physics, HEP97, Jerusalem, August 1997, abstract:265

[92] ZEUS Coll., J. Breitweg et al., Phys. Lett. B413 (1997) 201

[93] ZEUS Coll., J. Breitweg et al., Phys. Lett. B472 (2000) 175

[94] F. Halzen, A.D. Martin "Quarks and Leptons: an Introductory Course in Modern Particle Physics", John Wiley \& Sons, New York 1984;

A. Zembrzuski, M. Krawczyk, Proc. of the Workshop "Physics at HERA", Hamburg, 1991, eds. W. Buchmüller and G. Ingelman, Hamburg 1992, p. 617 Nucl. Phys. B (Proc. Suppl.) 82 (2000) 124

[95] L.N. Hand, Phys. Rev. 129 (1963) 1834

[96] A. Levy Phys. Lett. B404 (1997) 369

[97] H1. Coll., I. Abt et al., Z. Phys. C61 (1994) 59

[98] H1 Coll., C. Adloff et al. Phys. Lett. B415 (1997) 418 
[99] H1 Coll., C. Adloff et al., Eur. Phys. J. C13 (2000) 415

[100] H1 Coll., C. Adloff et al., Eur. Phys. J. C13 (2000) 397

[101] ZEUS Coll., M. Derrick et al., Phys. Lett. B306 (1993) 158

[102] ZEUS Coll., M. Derrick et al., Z. Phys. C67 (1995) 81

[103] ZEUS Coll., J. Breitweg at al., Phys. Lett. B479 (2000) 37

[104] K.Charchula and M. Krawczyk, A note on gluon distribution at small x, DESY 90-122

[105] H1 Coll., S. Aid et al., Phys. Lett. B356 (1995) 118

[106] H1 Coll., C. Adloff et al., Nucl. Phys. B485 (1997) 3

[107] H1 Coll., C. Adloff et al., Nucl. Phys. B538 (1999) 3

[108] H1 Coll., C. Adloff et al., Phys. Lett. B462 (1999) 440

[109] ZEUS Coll., J. Breitweg et al., Eur. Phys. J. C6 (1999) 239.

[110] ZEUS Coll., J. Breitweg et al., Phys. Lett. B474 (2000) 223

[111] A. Courau, Proc. of the 4th Int. Colloq. on Photon-Photon Interactions, Paris, 1981, ed. G.W. London, World Scientific 1981, p. 39

[112] M. Pohl, Proc. of the 5th Int. Workshop on Photon-Photon Collisions, Aachen 1983, ed. Ch. Berger, Lecture Notes in Physics, Vol. 191, Springer Verlag 1983, p. 234;

W.R. Frazer, ibid., p. 377

[113] N. Arteaga et al., Phys. Rev. D52 (1995) 4920 and Err. Phys. Rev. D53 (1996) 2854

[114] P. Aurenche et al., Proc. of the Workshop "Physics at LEP2", eds. G. Altarelli, T. Sjöstrand and F. Zwirner, CERN 96-01 (1996) 301

[115] ALEPH Coll., C.A. Brew, S. Cartwright, M. Lehto, Proc. of the Int. Conf. on the Structure and Interactions of the Photon and the 11th Int. Workshop on Photon-Photon Collisions, Egmond aan Zee, The Netherlands, May 1997, eds. A. Buijs and F.C. Erné, World Scientific, 1997, p. 21

[116] DELPHI Coll., W. Da Silva et al., Proc. of the Int. Conf. on the Structure and Interactions of the Photon and the 12th Int. Workshop on Photon-Photon Collisions, Freiburg im Breisgau, Germany, May 1999, ed. S. Söldner-Rembold, Nucl. Phys. B (Proc. Suppl.) 82 (2000) 43

[117] L3 Coll., M. Acciari et al., Phys. Lett. B438 (1998) 363.

[118] OPAL Coll., R. Akers et al., Z. Phys. C60 (1993) 593. 
[119] OPAL Coll., K. Ackerstaff et al., Z. Phys. C74 (1997) 49.

[120] OPAL Coll., G. Abbiendi et al., Eur. Phys. J. C11 (1999) 409

[121] OPAL Coll., G. Abbiendi et al., CERN-EP/99-157, (hep-ex/9911030), to be published in Eur. Phys. J. C

[122] TOPAZ Coll., M. Iwasaki et al., Phys. Lett. B341 (1994) 99

[123] TOPAZ Coll., R. Enomoto et al., Phys. Rev. D50 (1994) 1879

[124] JADE Coll., W . Bartelet al., Phys. Lett. B184 (1987) 288

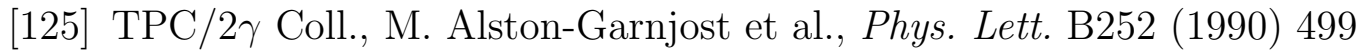

[126] TASSO Coll., W. Braunschweig et al., Z. Phys. C47 (1990) 499

[127] VENUS Coll., S. Uehara et al., Z. Phys. C63 (1994) 213

[128] AMY Coll., T. Aso et al., Phys. Lett. B363 (1995) 249, Phys. Lett. B381 (1996) 372

[129] ALEPH Coll., D. Buskulic et al., Phys. Lett. B355 (1995) 595

[130] L3 Coll., M. Acciarri et al., Phys. Lett. B453 (1999) 83; presentation at LEPC, 9 November 1999

[131] H1 Coll., submitted to the 29th Int. Conf. on High Energy Physics ICHEP'98, Vancouver, Canada, July 1998, abstract:540;

H1 Coll., C. Adloff et al., Nucl. Phys. B545 (1999) 21; Phys. Lett. B467 (1999) 156

[132] ZEUS Coll., J. Breitweg et al., Eur. Phys. J. C6 (1999) 67 and the contribution to EPS'99 (abstract 498); Eur. Phys. J. C12 (2000) 35

[133] M. Stratman, Proc. of the Int. Conf. on the Structure and Interactions of the Photon and the 12th Int. Workshop on Photon-Photon Collisions, Freiburg im Breisgau, Germany, May 1999, ed. S. Söldner-Rembold, Nucl. Phys. B (Proc. Suppl.) 82 (2000) 400

[134] E. Accomando et al., Phys. Rep. 299 (1998) 1

[135] A. Vogt, Proc. of the Int. Conf. on the Structure and Interactions of the Photon and the 12th Int. Workshop on Photon-Photon Collisions, Freiburg im Breisgau, Germany, May 1999, ed. S. Söldner-Rembold, Nucl. Phys. B (Proc. Suppl.) 82 (2000) 394

[136] M. Klein, talk at the 8th Int. Workshop on Deep-Inelastic Scattering, DIS 2000, April 2000, Liverpool, Great Britain; http://hep.ph.liv.ac.uk/ disproc/plenary2/klein/ 


\section{Monte Carlo generators}

[137] Vermaseren generator, JAMVG J. Smith et al., Phys. Rev. D15 (1977) 3280; R. Bhattacharya et al., Phys. Rev. D15 (1977) 3267;

J.A.M. Vermaseren et al., Phys. Rev. D19 (1979) 137;

J.A.M. Vermaseren, Nucl. Phys. B229 (1983) 347

[138] TPC $/ 2 \gamma$ H. Aihara et al. (the TPC $/ 2 \gamma$ Coll.), Z. Phys. C34 (1987) 1; Phys. Rev. Lett. 58 (1987) 97

[139] TWOGAM S. Nova, A. Olchevski and T. Todorov, "MONTE-CARLO Event Generator for two Photon Physics", DELPHI Note 90-35 PROG 152 (1990)

[140] F2GEN A. Buijs et al., Comp. Phys. Commun. 79 (1994) 523

[141] HERWIG G. Marchesini et al., Comp. Phys. Commun. 67 (1992) 465

HERWIG 5.9 G. Marchesini et al., hep-ph/9607393

HERWIG 6.1 G. Corcella et al., hep-ph/9912396

HERWIG + power law $p_{t}$, see S. Cartwright et al., 9

[142] PYTHIA T. Sjöstrand, Comp. Phys. Commun. 82 (1994) 74;

see also http://www.thep.lu.se/staff/torbjorn/Pythia.htm]

[143] PHOJET R. Engel, Z. Phys. C66 (1995) 203;

R. Engel and J. Ranft, Phys. Rev. D54 (1996) 4244

[144] GALUGA G.A. Schuler, Comp. Phys. Commun. 108 (1998) 279

[145] LEPTO 6.1 G. Ingelman, LEPTO 6.1 - The Lund Monte Carlo for deep inelastic lepton - nucleon scattering, Proc. of the DESY Workshop "Physics at HERA", Hamburg, 1991, eds. W. Buchmüller and G. Ingelman, Hamburg, 1992, p. 1366 LEPTO 6.5 G. Ingelman et al., Comp. Phys. Commun. 101 (1997) 108; http://www3.tsl.uu.se/thep/lepto/

[146] ARIADNE L. Lönnblad, Comp. Phys. Commun. 71 (1992) 15

[147] LDC H. Kharraziha and L. Lönnblad, JHEP 03 (1998) 006

[148] RAPGAP H. Jung, Comp. Phys. Commun. 86 (1995) 147;

"The RAPGAP Monte Carlo for DIS", version 2.06, Lund Univ., 1998, http://www-h1.desy.de/ jung/rapgap.htm]; version 2.08, Lund Univ., 1999, http://www.quark.lu.se/ hannes/rapgap.htm]

[149] JetViP see 196

[150] LUND B. Andersson et al., Phys. Rep. 97 (1983) 31

[151] JETSET T. Sjöstrand, Comp. Phys. Commun. 82 (1994) 74 
[152] cone jet algorithms J.E. Huth et al., Proc. of the 1990 DPF Summer Study on High Energy Physics, Snowmass, Colorado, ed. E.L. Berger, World Scientific, 1992, p. 134

[153] KTCLUS S. Catani et al., Nucl. Phys. B406 (1993) 187;

S.D. Ellis et al. Phys. Rev. D48 (1993) 3160

\section{Parton parametrizations}

[154] D.W. Duke and J.F. Owens, Phys. Rev. D26 (1982) 1600; Err: Phys. Rev. D28 (1983) 1227

[155] M. Drees and K. Grassie, Z. Phys. C28 (1985) 451

[156] H. Abramowicz, K. Charchuła and A. Levy, Phys. Lett. B269 (1991) 458

[157] a. L.E. Gordon and J.K. Storrow, Z. Phys. C56 (1992) 307

b. L.E. Gordon and J.K. Storrow, Nucl. Phys. B489 (1997) 405

[158] M. Glück, E. Reya and A. Vogt, Phys. Rev. D46 (1992) 1973, Phys. Rev. D45 (1992) 3986

[159] P. Aurenche, P. Chiapetta, M. Fontannaz, J.P. Guillet, E. Pilon, Z. Phys. C56 (1992) 589; M. Fontannaz and E. Pilon Phys. Rev. D45 (1992) 382

[160] P. Aurenche, J.P. Guillet and M. Fontannaz, Z. Phys. C64 (1994) 621

[161] K. Hagiwara, T. Izubuchi, M. Tanaka and I. Watanabe Phys. Rev. D51 (1995) 3197

[162] G.A. Schuler and T. Sjöstrand, Z. Phys. C68 (1995) 607; Phys. Lett. B376 (1996) 193

[163] M. Glück, E. Reya and M. Schienbein, Phys. Rev. D60 (1999) 054019

[164] M. Glück, E. Reya and M. Schienbein, Eur. Phys. J. C10 (1999) 313

[165] E. Laenen, S. Riemersma, J. Smith and W.L. van Neerven, Phys. Rev. D49 (1994) 5753 ;

E. Laenen and S. Riemersma, Proc. of the 10th Int. Workshop on Photon-Photon Collisions, Sheffield, U.K., April 1995, eds. B. Cartwright, D.J. Miller and V.A. Khoze, World Scientific 1995, p. 117

[166] A.S. Gorski, B.L. Ioffe, A. Yu. Khodjamirian and A. Oganesian, Z. Phys. C44 (1989) 529

[167] B.L. Ioffe and A. Oganesian, Z. Phys. C69 (1995) 119

[168] J. Chýla, "QCD improved parton distribution functions of $\gamma_{L}^{*}$, PRA-HEP 00-03, hep-ph/0006232 
[169] a) H. Abramowicz, E. Gurvich, A. Levy, Phys. Lett. B420 (1998) 104

b) H. Abramowicz, E. Gurvich, A. Levy, Proc. of the 29th Int. Conf. on High Energy Physics, ICHEP'98, Vancouver, Canada, July 1998, eds. A. Astbury, D. Axen and J. Robinson, World Scientific 1999, p. 885

[170] M. Glück, E. Reya and M. Stratmann Phys. Rev. D51 (1995) 3220, Phys. Rev. D54 (1996) 5515

\section{Theoretical papers}

\section{One and two jets production}

[171] a) T.-S. Tu, Nucl. Phys. B161 (1979) 417

b) J.F. Owens, Phys. Rev. D21 (1980) 54

c) I. Kang and C.H. Llewellyn Smith, Nucl. Phys. B166 (1980) 413

d) M. Fontannaz et al. Z. Phys. C6 (1980) 214

[172] M. Drees, R. Godbole, Phys. Rev. Lett. 61 (1988) 683; Phys. Rev. D39 (1989) 169

[173] H. Baer, J. Ohnemus, J.F. Owens, Phys. Rev. D40 (1989) 2844

[174] D. Bödeker, Phys. Lett. B292 (1992) 164, Z. Phys. C59 (1993) 501

[175] L.E. Gordon, J.K. Storrow, Phys. Lett. B291 (1992) 320

[176] P. Aurenche, M. Fontannaz, J.P. Guillet, Phys. Lett. B338 (1994) 98

[177] D. Bödeker, G. Kramer, S.G. Salesch, Z. Phys. C63 (1994) 471

[178] M. Greco, A. Vicini, Nucl. Phys. B415 (1994) 386

[179] G. Kramer, S.G. Salesch, Z. Phys. C61 (1994) 277

[180] M. Klasen, G. Kramer, S.G. Salesch, Z. Phys. C68 (1995) 113

[181] P. Aurenche, L. Bourhis, M. Fontannaz, J.P. Guillet, Proc. of the Workshop "Future Physics at HERA", Hamburg, 1995/6, eds. G. Ingelman , A. de Roeck, R. Klanner, DESY 1996, p. 570

[182] T. Kleinwort, G. Kramer, Nucl. Phys. B477 (1996) 3; Phys. Lett. B370 (1996) 141

[183] M. Klasen, G. Kramer, Phys. Lett. B366 (1996) 385; Z. Phys. C76 (1997) 67; M. Klasen, Ph. D. Thesis, 1996

[184] M. Klasen, G. Kramer, Z. Phys. C72 (1996) 107

[185] B.W. Harris, J.F. Owens, Proc. of the Meeting of the Division of Particles and Fields of the APS, Minneapolis, 1996, Vol. 1, p. 449 
[186] B.W. Harris, J.F. Owens, Phys. Rev. D56 (1997) 4007, D57 (1998) 5555

[187] M. Klasen, T. Kleinwort, G. Kramer, Eur. Phys. J. C1 (1998) 1

[188] S. Frixione, Nucl. Phys. B507 (1997) 295; S. Frixione, G. Ridolfi, Nucl. Phys. B507 (1997) 315

\section{Prompt photon production}

[189] a) T. Tu, C. Wu, Nucl. Phys. B 1, 5 (6) (1979) 493

b) M. Fontannaz and D. Schiff, Z. Phys. C14 (1982) 151

c) D.W. Duke and J.F. Owens 154

d) P. Aurenche, A. Douiri, R. Baier, M. Fontannaz, D. Schiff Z. Phys. C24 (1984) 309

e) M. Krawczyk, Acta Physica Polonica B21 (1990) 999

f) A.C. Bawa, M. Krawczyk and W.J. Stirling, Z. Phys. C50 (1991) 293

g) A.C. Bawa, M. Krawczyk, Phys. Lett. B262 (1991) 492

h) P. Aurenche, P. Chiappetta, M. Fontannaz, J.P. Guillet, and E. Pilon, Z. Phys. C56 (1992) 589

i) L.E. Gordon and J.K. Storrow, Z. Phys. C63 (1994) 581

[190] a) E.L. Berger and J. Qiu, Phys. Lett. B248 (1990) 371; Phys. Rev. D44 (1991) 2002

b) L.E. Gordon and W. Vogelsang, Phys. Rev. D50 (1994) 1901

c) M. Glück, L.E. Gordon, E. Reya and W. Vogelsang, Phys. Rev. Lett. 73 (1994) 388

d) L.E. Gordon Nucl. Phys. B501 (1997) 175

[191] a) A. Bawa, M. Krawczyk, IFT 17/91, Proc. of the Workshop "Physics at HERA", Hamburg 1991, eds. W. Buchmüller and G. Ingelman, Hamburg 1992 , p. 579

b) M. Krawczyk, A. Zembrzuski, Phys. Rev. D57 (1998) R10; Proc. of the Int. Conf. on the Structure and Interactions of the Photon and the 11th Int. Workshop on Photon-Photon Collisions, Egmond aan Zee, The Netherlands, May 1997, eds. A. Buijs and F.C. Erné, World Scientific, 1997, p. 162

c) M. Krawczyk, A. Zembrzuski, Proc. of the Int. Conf. on the Structure and Interactions of the Photon and the 12th Int. Workshop on Photon-Photon Collisions, Freiburg im Breisgau, Germany, May 1999, ed. S. Söldner-Rembold, Nucl. Phys. B (Proc. Suppl.) 82 (2000) 167

[192] a) L.E. Gordon and W. Vogelsang, Phys. Rev. D52 (1995) 58

b) L.E. Gordon, Phys. Rev. D57 (1998) 235

c) M. Krawczyk and A. Zembrzuski, Proc. of 29th Int. Conf. on High Energy Physics, ICHEP'98, Vancouver, Canada, July 1998, p.895; IFT-99-14

\section{Resolved virtual photon processes}


[193] a) D. de Florian, C. Garcia Canal, R. Sassot, Z. Phys. C75 (1997) 265

b) J. Chýla, J. Cvach, Proc. of the Workshop "Future Physics at HERA", Hamburg, 1995/6, eds. G. Ingelman , A. de Roeck, R. Klanner, DESY 1996, vol. 1, p. 545

[194] M. Klasen, G. Kramer, B. Pötter, Eur. Phys. J. C1 (1998) 261

[195] B. Pötter, Eur. Phys. J. C5 (1999) 1

[196] G. Kramer, B. Pötter, Eur. Phys. J. C5 (1998) 665; B. Pötter, Comp. Phys. Commun. 119 (1999) 45 (JetViP)

[197] B. Pötter, Nucl. Phys. B559 (1999) 323

[198] C. Friberg, Proc. of the Int. Conf. on the Structure and Interactions of the Photon and the 12th Int. Workshop on Photon-Photon Collisions, Freiburg im Breisgau, Germany, May 1999, ed. S. Söldner-Rembold, Nucl. Phys. B (Proc. Suppl.) 82 (2000) 124; C. Friberg and T. Sjöstrand, Eur. Phys. J. C13 (2000) 151

Forward jets, BFKL dynamics and the resolved virtual $\gamma$ processes

[199] G. Kramer and B. Pötter, Phys. Lett. B453 (1999) 295

[200] a) A.H. Mueller, Nucl. Phys. B (Proc. Suppl.) 18C (1990) 125, J. of Physics G17 (1991) 1443

b)J. Kwieciński, A.D. Martin, P.J. Sutton, Phys. Rev. D46 (1992) 921

c)J. Bartels, A. de Roeck, H. Loewe, Z. Phys. C54 (1992) 635

d)W.K. Tang Phys. Lett. B278 (1992) 363

[201] H. Jung, L. Jönsson, H. Küster, hep-ph/9805396v2; Proc. of the Workshop on Photon Interactions and the Photon Structure, Lund, Sept. 1998, eds. G. Jarlskog and T. Sjöstrand, p. 229; Eur. Phys. J. C9 (1999) 383

[202] J. Kwieciński, C.A.M. Lewis, A.D. Martin, Phys. Rev. D57 (1998) 496

[203] J. Kwieciński, A.D. Martin, J.J. Outhwaite, Eur. Phys. J. C9 (1999) 611

[204] J. Bartels et al., Phys. Lett. B384 (1996) 300

\section{Leptonic structure functions}

[205] R. Nisius and M.H. Seymour, Phys. Lett. B452 (1999) 409

[206] C. Peterson, P.M. Zerwas, T.F. Walsh, Nucl. Phys. B229 (1983) 301.

\section{Heavy quarks production}

[207] F.I. Olness and S.T. Riemersma, Phys. Rev. D51 (1995) 4746

[208] M.A.G. Aivazis, F.I. Olness and W. Tung, Phys. Rev. D50 (1994) 3085 
[209] M.A.G. Aivazis, J.C. Collins, F.I. Olness and W. Tung, Phys. Rev. D50 (1994) 3102

[210] S. Frixione, M.L. Mangano, P. Nason and G. Ridolfi, Phys. Lett. B348 (1995) 633

[211] B.A. Kniehl, M. Krämer, G. Kramer and M. Spira, Phys. Lett. B356 (1995) 539

[212] P. Agrawal, F.I. Olness, S.T. Riemersma and W. Tung, Proc. of 30th Rencontres de Moriond: QCD and High Energy Hadronic Interactions, France, March 1995, ed. J. Trân Thanh Vân, Editions Frontières 1995, p. 353

[213] M. Buza, Y. Matiounine, J. Smith and W.L. van Neerven, Eur. Phys. J. C1 (1998) 301

[214] M. Drees, M. Krämer, J. Zunft and P.M. Zerwas, Phys. Lett. B306 (1993) 371

[215] M. Cacciari, M. Greco, B.A. Kniehl, M. Krämer, G. Kramer and M. Spira, Nucl. Phys. B466 (1996) 173

[216] J. Binneweis et al., Phys. Rev. D58 (1998) 034016

[217] J. Binnewies, B.A. Kniehl, G. Kramer Z. Phys. C76 (1997) 677

[218] M. Cacciari et al. , Nucl. Phys. B466 (1996) 173

[219] M. Cacciari and M. Greco, Phys. Rev. D55 (1997)7134

[220] S. Frixione, M. Krämer and E. Laenen, Nucl. Phys. B571 (2000) 169

[221] B. Kamal and Z. Merebashvili, Phys. Rev. D58 (1998) 074005; B. Kamal, Z. Merebashvil and A. P. Contogouris, Phys. Rev. D51(1995) 4808

[222] S. Frixione et al. Nucl. Phys. B412 (1994) 225,Phys. Lett. B348 (1995) 633, Nucl. Phys. B454 (1995) 3

[223] B. A. Kniehl et al. Z. Phys. C76 (12997) 689;

J. Binnewies et al. Phys. Rev. D58 91998) 014014

[224] M. Cacciari and M. Greco, Z. Phys. C69 (1996) 459

[225] A. V. Berezhnoy at al., hep-ph/9908483; hep-ph/9905555

[226] C. Peterson et al. Phys. Rev. D27 (1983) 105

\section{Polarized photon structure functions and polarized parton densities for real and virtual photon}

[227] M. Stratmann and W. Vogelsang, Phys. Lett. B386 (1996) 370

[228] Prakash Mathews and V. Ravindran, Int. J. of Modern Physics A11 (1996) 2783 
[229] K. Sasaki, T. Uematsu, Phys. Rev. D59 (1999) 114011, Phys. Lett. B473 (2000) 309, hep-ph/007055

[230] A. V. Efremov and O.V. Teryaev, Phys. Lett. B240 (1990) 200;

S. D. Bass, Int. J. of Modern Physics A7 (1992) 6039;

S. Bass et al., Phys. Lett. B437 (1998) 417;

S. Narison, G. M. Shore and G,. Veneziano, Nucl. Phys. B381(1993) 69, G. Shore and G. Veneziano, Mod. Phys. Lett. A8 (1993) 373

[231] M. Stratmann and W. Vogelsang, Z. Phys. C74 (1997) 641; Proc. of the Workshop "Future Physics at HERA", Hamburg, 1995/6, eds. G. Ingelman , A. de Roeck, R. Klanner, DESY 1996, p. 815

[232] D. de Florian and S. Frixione, Phys. Lett. B457 (1999) 236

[233] A.P. Contogouris, Z. Merebashvili and G. Grispos, Phys. Lett. B482 (2000) 93

[234] G. Grispos, A.P. Contogouris, G. Veroponlos, Phys. Rev. D62 (2000) 014023

[235] J. Kwieciński and B. Ziaja, hep-ph/0006292

\section{Structure functions of the electron}

[236] W. Słomiński and J. Szwed, Acta Physica Polonica B27 (1996) 1887, B29 (1998) 1253; Phys. Lett. B387 (1996) 861; hep-ph/9802449; W. Słomiński, Acta Physica Polonica B30 (1999) 369

[237] W. Słomiński and J. Szwed, Phys. Lett. B323 (1994) 427

\section{Photon content of the proton}

[238] B. A. Kniehl, Phys. Lett. B254 (1991) 267

[239] M. Glück, M. Stratmann, W. Vogelsang, Phys. Lett. B343 (1995) 399

[240] A. de Rújula and W. Vogelsang, Phys. Lett. B451 (1999) 347 